\title{
Identifying the Unique Ground Motion Signatures of Supershear Earthquakes: Theory and Experiments
}

\author{
Thesis by \\ Michael Mello \\ In Partial Fulfillment of the Requirements \\ for the Degree of \\ Doctor of Philosophy
}

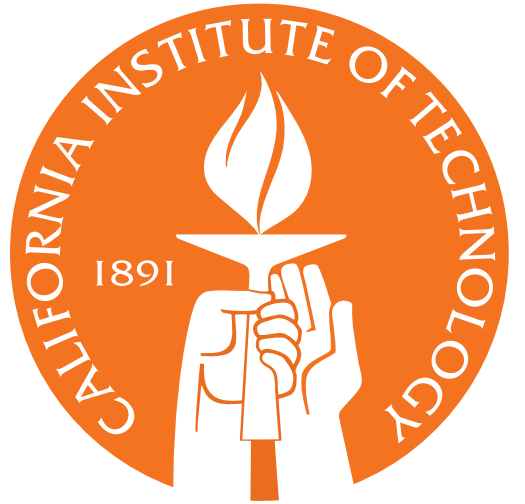

California Institute of Technology

Pasadena, California

2012

(Defended April 24, 2012) 
(c) 2012

Michael Mello

All Rights Reserved 
Dedicated in loving memory to my father Manuel C. Mello (7 September 1923 - 26 December 1998) 


\section{Acknowledgments}

The opportunity to return to graduate school to pursue my $\mathrm{PhD}$ after working for nearly 20 years was the opportunity of a lifetime. The one person I must thank above all for this opportunity is my PhD advisor Professor Ares J. Rosakis. Very few people in this world would have taken a chance on a 43 year old graduate student. Ares did exactly that without a single reservation by encouraging me to leave my job at Intel and apply to Caltech. I'll never know for sure, but I can well imagine that he had to convince a few (understandably) skeptical colleagues to accept me into the entering GALCIT class of 2006. It was a privilege to earn one of those seats, and I never took it lightly. I'm

eternally grateful for this opportunity, and it was an honor to be Ares' student and contribute a small part to his academic legacy. Beyond all of this, Ares has always been incredibly supportive, and we have developed a real friendship over time which means as much, if not more, to me than anything else.

The solid mechanics community is an extended family of sorts. If anyone embodies the heart and soul of this community, it is Professor Guruswami "Ravi" Ravichandran. I knew of Ravi even from afar, well before I came to Caltech, and was well aware of the respect others have for him both as a great human being and for his unassuming, yet profoundly forceful influence within the community. Ravi played an equally pivotal role in encouraging me to apply to Caltech. He was the reassuring voice when I expressed some initial anxieties about handling the coursework and passing qualifying exams. I have a deep respect for Ravi as a researcher and teacher. My notebooks from the solid mechanics courses that I took with him are among my greatest treasures. Coming to Caltech and becoming his friend has made this experience that much more memorable for me. Ravi, thanks for being there for me every single step of the way. I value our friendship and am grateful for it. 
An often overlooked aspect of the culture here is the extraordinary caliber of post doctoral scholars, who often play such a prominent role behind the scenes. It is safe to say that my experience here would not have been the same were it not for Dr. Harsha Bhat, who I had the fortune to work with during his tenure here. It was Harsha who framed my $\mathrm{PhD}$ thesis. He also taught me much of what I know today about Matlab programming and dynamic rupture theory. We worked on nearly everything together from the interpretation of particle velocity records to the development of ground motion scaling relationships. I cannot possibly imagine what this thesis would look like today had it not been for Harsha's influence. He was the anchor of my PhD research experience, and I'll never forget what he did for me. Beyond all of this, we were just plain old good buddies. I'll always value the talks we had at the Red Door cafe and the friendship we shared.

I would also like to extend a special thanks to my thesis committee members Professors Nadia Lapusta and Hiroo Kanamori. I am extremely grateful to both of them for all of their support over the years starting with my candidacy exam and finally ending with my $\mathrm{PhD}$ defense. Hiroo was part of our regular weekly meetings throughout the entire duration of my $\mathrm{PhD}$ experience. It was an honor and a privilege to have the most famous seismologist in the world be part our small team. His presence unquestionably raised the bar for all of us. Hiroo was always very quick to process the latest experimental findings. He would always ask just the right questions which often challenged my understanding of things. This only motivated me to learn more about seismology and fracture mechanics. I have an equally deep respect for Nadia. She was not only an esteemed committee member but was also my professor in the classroom. Her course on Dynamic fracture and frictional faulting (Ae266), served as my introduction to seismology. It was without a doubt one of the finest courses that I ever took at Caltech and I dove into it headlong. The notes from that course are a treasure that I will always cherish and refer to throughout the course of my career.

I was also lucky enough to cross paths with Shuman Xia during his post doctoral tenure here. Shuman and I connected immediately, and I quickly came to realize that he is a rare "five-tool player" when it comes to experimental solid mechanics. It is a privilege in the course of a life to meet such people who still manage to be humble and helpful to others. We became loyal friends, 
and I look forward to meeting up with him soon at Georgia Tech.

There were two senior, graduate students in particular that helped shape my PhD experience. I was darned lucky to meet and make friends with Christian Franck. Christian inspired me with his quiet confidence and the deep sense of pride he had for his work. It also did not take long to see that he is an extraordinary person, mature beyond his years, and someone that I could trust. Christian was the one I relied upon as I transitioned between the coursework and research phases of my graduate experience. It was Christian who nudged me out of my Microsoft comfort zone by convincing me to learn Latex and purchase my first Macintosh computer. Little did I realize at the time the profound impact that these tools would have on my $\mathrm{PhD}$ experience. We remain strong friends until this day.

Sharlotte Kramer was another person who I was extremely fortunate to know during my early years here. I admired her determination and ability to learn as much about optics as she did in such a short time. I enjoyed helping her with her project and the frequent talks we had about CGS, photoelasticity and phase-shifting interferometry. I thought I understood interferometry when I came to Caltech, but I learned a lot more as a result of my interactions with Sharlotte. I am also proud of the Ae104 photoelasticity lab that we developed together. It was hard work to construct that lab module in time for the GALCIT $80+$ celebration. We did it together, and in the end, it became was an immense source of pride between us. I only hope this contribution to the course will live on in the years to come.

I will always be grateful for the help that I received from my classmates Angie Capece, Daegyoum Kim, and Philipp Boettcher during the first couple of years when we all mainly took classes together. Among those who I grew closer to and will always remain in touch with are Paul Hebert and Chris Kovalchick. Paul and I worked together pretty closely on assignments during the first year, and I'll always be incredibly grateful for his help and friendship. He is one of the sharpest people I have ever met, and I know he will be extremely successful in his career beyond Caltech.

Chris and I have grown to become extremely close friends over the years. It is remarkable as I think back to the way we met by chance at the 2005 SEM conference, well before I had any notions 
of leaving Intel and returning to graduate school. Little did we know that one day soon we would be classmates at Caltech. Booyah! Chris' friendship meant and continues to mean so much to me. I always looked forward to meeting him in the morning for coffee or catching up with him later in the day. There always something new to talk about whether it was about research, the Red Sox, his job search, or latest adventures in ultramarathoning. It was a joy to watch him grow up during this time and become the man that he is today. I am so immensely proud of him and will always be amazed at the circumstances that became the basis of our friendship.

I also want to acknowledge my friends and group mates Jon Mihaly and Vahe Gabuchian in the Rosakis research group. It has been a pleasure knowing Vahe Gabuchian. He put his extraordinary mechanical design skills to use along with a fierce, focused determination, to design custom optical mounts for a spatial-phase-shifting interferometer that I designed and had hoped to construct here at Caltech. We have not quite gotten there yet for various reasons but are certainly a lot closer thanks to Vahe's efforts. I have also truly enjoyed getting to know Vahe over the years and admire his spirit, determination, and zest to grab life by the cojones. I'm happy to call him my friend.

It has also been an absolute pleasure to know and work with Jon Mihaly. Jon has single-handedly brought Ares' hypervelocity impact lab to the edge of international recognition. John has a quiet confidence about him and an unassuming demeanor which masks a fierce determination and a strong fire in his belly to succeed and become an astronaut one day. I will be the least surprised of all when I learn that he has succeeded in this regard. This country could use a lot more talented and dedicated people like Jon. I am proud to call him my friend.

Among all the people I have met at Caltech, my dearest friend Abha is the one truly special person who impacted my life the most. I was extremely lucky to meet her and be graced by her caring and sensitive personality. We developed a deep friendship and a strong bond of the rarest kind. During our time together I managed to find my smile again after many years. Our short time together in Pasadena was precious, and it changed my life for the better. Even from afar, she has managed to remain at my side in spirit and has been a constant and supportive companion throughout the period that I have spent writing this thesis. Regardless of the circumstances and 
whatever the future holds, I will always have the memories, and in my heart she will always be my Lovie.

George Raiser and Kris Frutschy are two of my closest friends, who trace back to my early years at Brown University, where they were both graduate students, and where I worked as a research engineer in Prof. Rodney J. Clifton's plate impact lab. Our friendships were forged back in that lab, and refined in later years by time spent working together at Intel. What an extraordinary thing it was to get to work with my two best friends for all of those years. They have two distinctly different personalities, and yet they have both been incredible role models to me. They are both extraordinary engineers who I learned so much from during the time we worked together.

I attribute a great deal of my success at Intel to George. He is without a doubt one of the brightest people and greatest problem solvers that I have ever known. George taught me so much when I joined Intel, and I was a sponge to any and all advice he offered me. He was my anchor in the early years and gave me the foundation I needed to eventually succeed on my own.

Kris has always been there for me through thick and thin, good times and bad. He has shown the meaning of true friendship. His encouragement, and the confidence he expressed went a long way with me as I contemplated leaving Intel to pursue my PhD. I have always cherished our talks and the way we can share and connect on nearly every facet of our lives. I am sure it will be that way forever. Last but not least, I have always admired Kris's spirit and work ethic, and in many ways, I have tried to emulate him here at Caltech by drawing from my memory of the younger Kris I knew at Brown. Thanks man!

This thesis is dedicated my father Manuel who was a tremendous source of inspiration to me each and every day for the first 35 years of my life. My dad was the hardest working man I have ever known, and any semblance of a work ethic that I possess is attributed to him. My father was a self taught man of exceedingly limited formal education who respected and valued the power of knowledge. I can recall him seated upright at his desk on most evenings reading the Kings James bible (either that or the local newspaper), while preparing a sermon for the upcoming Sunday night church service. Watching him deliver those small sermons was an education unto itself, even if I 
did struggle with the fact that they were in Portuguese. The devotion to his bible studies was a marvelous example of scholarship within the home that I emulated and applied when it came to my own school work. Dad also did his best to support my interest in science within the limits of a rather limited financial budget. He bought me a simple microscope in the third grade and a 2.5" refracting telescope when I was in the eighth grade. I treasured each of these gifts and used them to discover an entire universe in my backyard. Beyond all of this, my dad was also my best friend, and I still miss him dearly. Thanks to my dad for allowing me to pursue my studies and passion for science, even when it was far from clear where it would take me. That was the greatest gift of all.

I want to express my deepest gratitude to my sister Nancy, who has been a constant beacon in my life. Nancy has never stopped supporting and encouraging me and has always been my greatest cheerleader. I am grateful for her unconditional love and support. She gave me the greatest christmas gift in the winter of 1979 when she bought me a subscription to Sky and Telescope magazine. That gift meant so much to me at the time, and I will remember it all the days of my life. While growing up it was Nancy who I always looked up to, especially as a young man leading up through my college years. She was the heroine of my youth and among the first (if not the first) in our extended family of immigrants and first generation Americans to obtain a college education, having graduated from Providence College in 1976. She was a true pioneer and risk taker who broke away and assimilated more deeply into the American culture at large. There are no words that can adequately describe the great teacher and role model she was to me in this regard.

I also want to acknowledge my younger sister Betty who I love dearly and who has also been equally loving, encouraging, and supportive of me over the years. I see our dad's legacy in her and her three beautiful children Paul, Aaron and Jaymie.

There were so many more unique individuals that I was fortunate to know and learn from throughout the years who have profoundly shaped my life. It is simply not possible to list them all here, but the influence these folks have had upon me, and the gratitude that I have for all of them will never fade.

Finally, I would like acknowledge all of my former teachers in the Somerset, Massachusetts school 
system, of which I am most proud. Many of these teachers served as role models for me during some very confusing years of my childhood. That small town school system from yesteryear is my standard when I think of what public education should look like in this country.

I have relished every waking moment over the past five months that it has taken me to complete this thesis. Every day wasn't spent typing words on a page, as I was naive enough to believe when all of this started. Little did I know how much work lay ahead of me in the form of additional data analysis, generation of plots and figures, and even new discoveries, well before a single word was typed. This journey has spanned the spectrum of human emotion for me, characterized at various stages by doubt, fear, and anxiety which gradually transitioned to faith, exhilaration, and a sense of pride. Writing this thesis has been the single most challenging task that I have ever undertaken, bar none. The experience has been nothing short of a mental ultramarathon, and it is an understatement to say that it has taken a piece of me, both physically and otherwise, that I may never get back. Having said this, I would not have done it any other way, because I do not know any other way other than to leave it all on the field. No thesis is perfect, and mine is certainly no exception. I do not know when or if anyone will ever even read this thesis, but I have assumed all the while that someone might, and so have strived to be as clear and as complete as possible. The Devil is truly in the details, as the old saying goes. No words ring truer than these when it comes to achieving any measure of success with experiments. To that end I have obsessed to document the details of this experiment, regardless of how dry some of those sections of the thesis may be. Above and beyond the research findings presented here, of which I am proud, It will make me equally proud to learn one day that a future PhD student has benefited from this information and used it to improve the experiment and leave it behind better than he or she found it. 


\section{Abstract}

The near-field ground motion signatures associated with sub-Rayleigh and supershear ruptures are investigated using the laboratory earthquake experiment originally developed by Rosakis and coworkers (Xia et al., 2004, 2005a; Lu et al., 2007; Rosakis et al., 2007). Heterodyne laser interferometers enable continuous, high-bandwidth measurements of fault-normal (FN), fault-parallel (FP), and vertical (V) particle velocity "ground motion" records at discrete locations on the surface of a Homalite-100 test specimen as a sub-Rayleigh or a supershear rupture sweeps along the frictional fault. Photoelastic interference fringes, acquired using high-speed digital photography, provide a synchronized, spatially resolved, whole field view of the advancing rupture tip and surrounding maximum shear stress field.

The first phase of experimental investigations examine and verify the ground motion signatures of supershear ruptures. Experimental results demonstrate that a shear Mach front produced by a stable supershear rupture is characterized by a dominant FP velocity component. The situation is shown to reverse in the sub-Rayleigh rupture speed regime whereby the FN particle velocity component dominates the ground motion record. Additional distinguishing particle velocity signatures, consistent with theoretical and numerical predictions, and repeatedly observed in experimental records are, (1) a pronounced peak in the FP velocity record induced by the leading dilatational field, which sweeps the measurement station in advance of the shear Mach front, and (2) a pronounced velocity swing in the FN record associated with the arrival of a trailing Rayleigh sub-Rayleigh (secondary) rupture, which follows the arrival of the shear Mach front. Analysis of the particle velocity records also confirms 2D steady-state theoretical predictions pertaining to the separation, attenuation, and radiation partitioning of the shear and dilatational portions of the rupture velocity field components. 
The second phase of our experimental investigations re-examine the $2002, M_{w} 7.9$, Denali fault earthquake and the remarkable set of near-source ground motion records obtained at (PS10), located approximately $85 \mathrm{~km}$ east of the epicenter and just $3 \mathrm{~km}$ north of the fault along the Alaska pipeline. Motivated by the analysis and interpretation of these records by (Ellsworth et al., 2004; Dunham and Archuleta, 2004, 2005), we attempt to mimic the Denali strike-slip rupture scenario and replicate the PS10 ground motion signatures using a laboratory earthquake experiment. The experiments feature a left-to-right (west-to-east) propagating right lateral rupture within a Homalite-100 test specimen with particle velocity data collected at a near-field station situated just above (north of) the fault. Both sub-Rayleigh and supershear laboratory earthquake experiments are conducted using the Denali PS10 configuration in order to compare and contrast the resulting particle velocity signatures. Supershear laboratory records capture all of the prominent features displayed within the PS10 ground motion records. Noted velocity signatures are correlated to the location of the rupture fronts and their noted arrival times in the synchronized photoelastic image sequence. Scaling relationships are also presented which transform the laboratory records through six orders of magnitude in time, to match the scale of the PS10 ground motion records. The strong correlation between the scaled experimental records and the actual PS10 ground motion records supports the hypothesis that the Denali strike-slip fault exhibited a supershear burst.

Finally, we present a 2D steady state, stress-velocity formulation that relates the FP and FN particle velocity records measured close to the fault, to the evolution of the stress tensor at the same location. A locally steady-state condition is assumed within a restricted time interval in order to invoke these relationships and estimate the dynamic stresses, $\sigma_{x x}(t)$ and $\tau(t)$, at the nearfault station. Dynamic stress measurements enable a new class of friction investigations using the laboratory earthquake configuration. Experimental findings are presented, which capture the temporal and spatial distributions of $\sigma_{x x}$ and $\tau$, evolution of the dynamic friction coefficient, and velocity weakening behavior of a supershear slip-pulse. 


\section{Contents}

Abstract

2 Theoretical Foundations: Near-source ground motion signatures of sub-Rayleigh and supershear fault ruptures

2.1 Spectral representation of radiated wave fields arising from a steady-state shear dis-

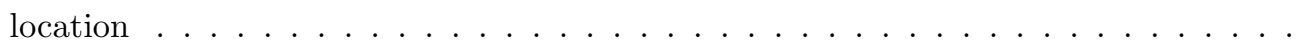

2.1.1 Characteristic decay of shear and dilatational radiation fields for sub-shear $\left(V_{r} \leq C_{s}\right)$ and intersonic $\left(C_{s} \leq V_{r} \leq C_{p}\right)$ dislocation speed regimes $\ldots$

2.1.2 Non-decaying nature of shear radiation fields emitted by a supershear dislocation $\left(C_{s} \leq V_{r} \leq C_{p}\right) \ldots \ldots \ldots \ldots \ldots$

2.1.3 Analysis and interpretation of dislocation shear Mach fronts in the plane wave

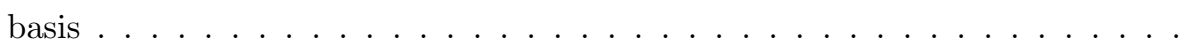

2.2 2D elastodynamic theory: Singular linear elastic solution for a propagating shear crack 21 
2.2.1 Particle velocity field arising from a shear crack in the sub-Rayleigh rupture speed regime $\left(V_{r}<C_{R}\right) \ldots \ldots \ldots \ldots \ldots$

2.2.1.1 Dominance of the fault-normal particle velocity component in the sub-Rayleigh rupture speed regime $\left(V_{r}<C_{R}\right) \ldots \ldots . . . . . .23$

2.2.2 Particle velocity field from a shear crack in the supershear regime $\left(C_{s} \leq V_{r} \leq C_{p}\right) 25$

2.2.2.1 Particle motion along a shear Mach front and dominance of the faultparallel component in the stable supershear rupture speed regime $\left(\sqrt{2} C_{s} \leq V_{r} \leq C_{p}\right) \ldots \ldots \ldots \ldots$

2.3 Steady-state cohesive zone models . . . . . . . . . . . . . . . . . . . . . . . 28

2.3.1 Overview of general 2D steady-state supershear slip-pulse solution . . . . . . 29

2.3.2 Recovery of the slip function at an off-fault location . . . . . . . . . . . 32

2.3.3 Partitioning between shear and dilatational radiation fields by a supershear

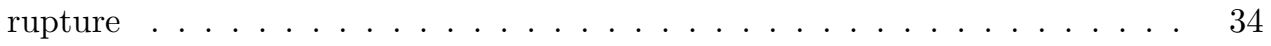

2.3.4 Overview of the $2 \mathrm{D}$ steady-state supershear model . . . . . . . . . . . 37

2.4 Unsteady cohesive zone models and the trailing Rayleigh rupture . . . . . . . . . . . 38

3 The Laboratory Earthquake Experiment

3.1 Early predictions of the sub-Rayleigh-to-supershear transition and the Burridge-Andrews

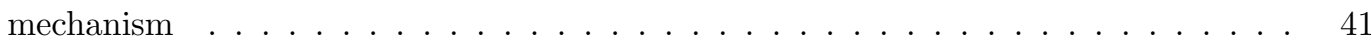

3.2 Brief historical background and introduction to the laboratory earthquake experiment 44

3.3 Step-by-step description of a laboratory earthquake experiment: From specimen preparation through dynamic rupture nucleation . . . . . . . . . . . . . . . . . 47

3.4 Physical dimensions of traditional laboratory earthquake specimens . . . . . . . . 60

3.4.1 Plate buckling analysis of $\mathrm{H}-100$ specimens . . . . . . . . . . . . . . . . . . 61

3.5 Fabrication and surface preparation of H-100 laboratory earthquake specimens . . . 62

3.5.1 Machining-induced surface defects . . . . . . . . . . . . . . . 67

3.5.2 Planarity and perpendicularity of machined fault surfaces . . . . . . . . . 68

3.6 H-100 specimen surface polishing procedure . . . . . . . . . . . . . . . . . 70 
3.7 Micro-bead blasting procedure for surface roughening of H-100 test specimens . . . 74

3.8 Surface roughness evaluation of H-100 specimen surfaces . . . . . . . . . . . . . 76

3.9 Introduction of new laboratory earthquake specimen featuring 3D fault geometry.$\quad 87$

3.9.1 Newfound control of laboratory earthquake experiments using 3D specimen fault geometry as revealed by experiments . . . . . . . . . . . . . . 89

3.9.2 Fabrication of 3D fault specimens . . . . . . . . . . . . . . . . 92

3.10 Observations and discussion of an anomalous secondary signal observed in supershear particle velocity records . . . . . . . . . . . . . . . . . . . . 96

3.10.1 Isochrone analysis of supershear ruptures in laboratory earthquake experiments101

3.11 Photoelasticity and high-speed digital photography for spatially resolved, whole-field

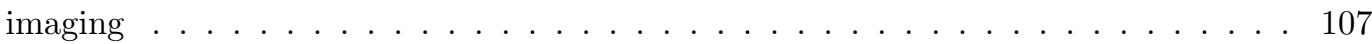

3.11.1 High-speed cameras . . . . . . . . . . . . . . . . . . . . . 111

3.11.2 Measurement of wave speeds and rupture speeds in laboratory earthquake

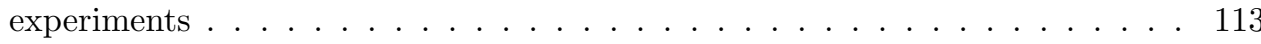

3.11.3 Analysis of a sub-Rayleigh and a supershear experiment . . . . . . . . . . 114

3.12 Estimation of sub-Rayleigh-to-supershear transition length in laboratory earthquake

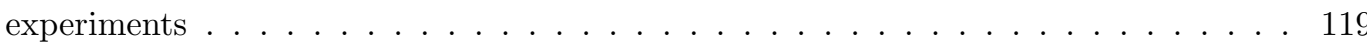

3.12.1 On the use of H-100 in laboratory earthquake experiments . . . . . . . . . . . 120

3.13 Control of rupture speed regime in laboratory earthquake experiments . . . . . . . . 121

3.13.1 High-speed photoelastic image sequence of sub-Rayleigh-to-supershear rupture

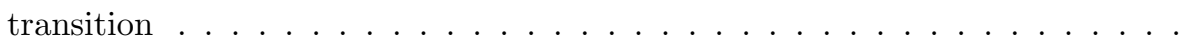

3.14 Fiber-optic heterodyne interferometers for continuous particle velocity measurements

3.14.1 Principle of heterodyne interferometry . . . . . . . . . . . . . .

3.14.2 Particle velocity measurement configurations adopted in laboratory earthquake experiments . . . . . . . . . . . . . . . . . 130

3.14.3 Non-dimensional analysis of particle velocity fields . . . . . . . . . . . . . . 131 
4.1 Introduction . . . . . . . . . . . . . . . . . . . . . . . 133

4.2 Identification of sub-Rayleigh and supershear ground motion signatures through a frame-by-frame comparison of two laboratory earthquake experiments . . . . . . . . 135

4.2.1 Design of experiment ....................... 135

4.2.2 Results, analysis, and interpretation of particle velocity records and photoe-

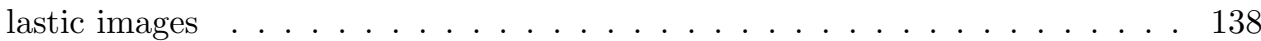

4.3 Examination of $\left|\delta \dot{u}_{y}(x, y)\right| /\left|\delta \dot{u}_{x}(x, y)\right|$ in sub-Rayleigh particle velocity records using the $(\mathrm{FN}, \mathrm{FP})$ laser interferometer probe configuration . . . . . . . . . . . . . 163

4.3.1 Design of experiment ...................... 163

4.3.2 Results, analysis, and interpretation of particle velocity records and photoelastic images . . . . . . . . . . . . . . . . . . . 163

4.4 Investigation of sub-Rayleigh particle velocity field decay using an (FN1,FN2) laser interferometer probe arrangement . . . . . . . . . . . . . . . . . . 169

4.4 Design of experiment . . . . . . . . . . . . . . . . . . 169

4.4.2 Results, analysis, and interpretation of particle velocity records and photoe-

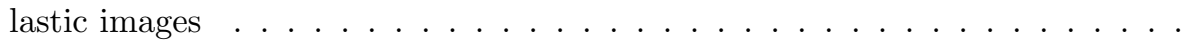

4.5 Examination of the ratio $\beta_{s}=\left|\delta \dot{u}_{x}^{s}\left(z_{s}\right)\right| /\left|\delta \dot{u}_{y}^{s}\left(z_{s}\right)\right|$ along a shear Mach front using the $(\mathrm{FN}, \mathrm{FP})$ laser interferometer probe configuration . . . . . . . . . . . . . . . 176

4.5.1 Design of experiment ..................... 176

4.6 Examination of the partitioning relationship between the motion recorded at the fault and the motion propagated by a shear Mach front into the surrounding medium. . .

4.6.1 Results, analysis, and interpretation of particle velocity records and photoe-

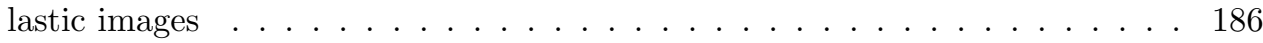

5 Scaling Relationships between Laboratory Earthquake Measurements and Real- 
xvii

5.1 The November 3, $2002\left(M_{w} 7.9\right)$ Denali, AK earthquake $\ldots \ldots \ldots \ldots$

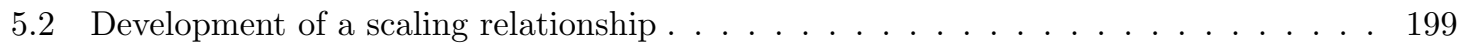

5.3 Scaled laboratory earthquake simulation of the $2002 M_{w} 7.9$ Denali fault, PS10 ground motion recordings . . . . . . . . . . . . . . . . . . . 203

5.3.1 Distance-time plots resulting from the scaled PS10 experiment . . . . . . . 205

5.3.2 High-speed photoelastic image sequence obtained during scaled supershear

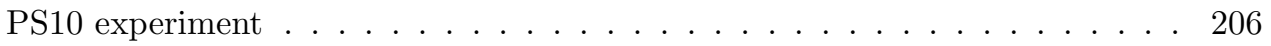

5.3.3 Distance-time plots resulting from the scaled sub-Rayleigh PS10 experiment . 210

5.3.4 Analysis and discussion of particle velocity records obtained from the scaled PS10 laboratory earthquake experiments . . . . . . . . . . . . . . . . . . 211

5.3.5 Analysis and discussion of sub-Rayleigh particle velocity records obtained in

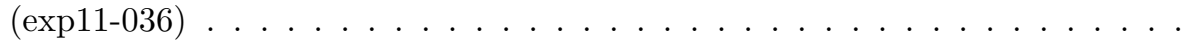

5.3.6 Analysis and discussion of supershear particle velocity records obtained in

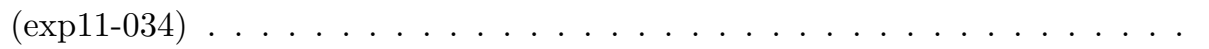

5.4 Scaling of laboratory earthquake supershear experiment and direct comparison with PS10 ground motion records . . . . . . . . . . . . . . . . . . . . 217

6 Dynamic Stress Measurements within the Supershear Slip Zone

6.0.1 Experimental verification of anti-symmetric fault-parallel particle velocity records in laboratory earthquake experiments . . . . . . . . . . . . . . . . 223

6.0.2 Summary of particle displacement for right- and left-lateral supershear ruptures229

6.0.3 Extension of 2D steady-state supershear slip-pulse solution to left-traveling

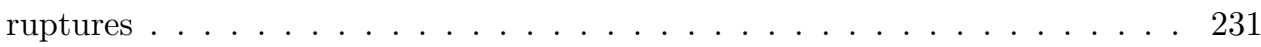

6.0.4 Relationship between the slip velocity $\Delta \dot{u}\left(z_{s}\right)$ and $\Im N\left(z_{s}\right) \ldots \ldots \ldots . \ldots 233$

6.0.5 Relationship between the slip velocity $\Delta \dot{u}\left(z_{s}\right)$ and $\Re N\left(z_{p}\right) \ldots \ldots \ldots . \ldots 235$

6.0.6 Experimental estimation of $\sigma_{x x}$ from fault-parallel particle velocity records . 235

6.0.7 Experimental estimation of $\tau(x)-\tau_{0}$ within the supershear slip zone $(-L<$

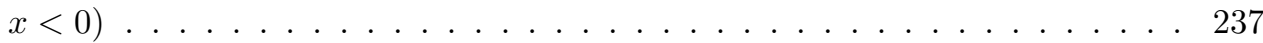


xviii

6.0.8 Alternative derivation for $\tau(x)-\tau_{0}$ within the supershear slip zone $(-L<x<$ 0) using the integral equation for $N(z) \ldots \ldots \ldots \ldots . \ldots \ldots$

6.0.9 Determination of $\tau(x)-\tau_{0}$ within a sub-Rayleigh slip zone $(-L<x<0) \quad \ldots \quad 242$

6.0.10 Dynamic stress measurements in laboratory earthquake experiments . . . . . 243

6.0.11 Dynamic stress and friction investigations of a supershear slip-pulse (exp010-014)245

6.0.12 Estimates of $\sigma_{x x}$ and $\tau$ obtained from the supershear slip-pulse particle velocity

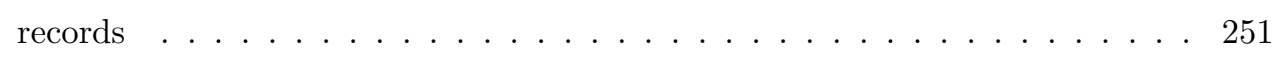

Bibliography 


\section{List of Figures}

1.1 Formation of sub-Rayleigh rupture and shear Mach fronts by the superposition of shear wavelets emitted by a steady-state shear dislocation . . . . . . . . . . . . .

1.2 Supershear rupture with shear Mach front, as revealed by photoelastic interference fringes, shown crossing a particle velocity measurement station during a laboratory earthquake experiment . . . . . . . . . . . . . . . . . . .

2.1 S-wave radiation from a supershear slip pulse propagated out to infinity along charac-

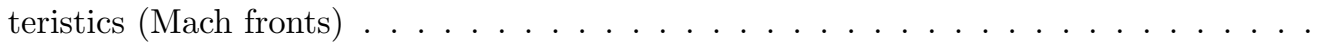

2.2 Curved Mach-front profiles resulting from decelerating or accelerating supershear ruptures

2.3 Mach fronts arising from a supershear source propagated out to infinity along charac-

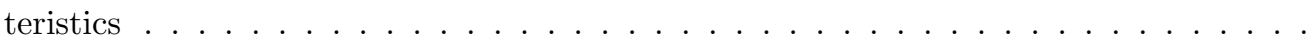

2.4 Range of Mach angles corresponding to each of the supershear rupture speed regimes

2.5 The near-tip particle velocity field for a shear crack propagating at $V_{r}=0.875 C_{s} \ldots$.

2.6 Singular elastic particle velocity field plots for a supershear crack propagating at (a) $V_{r}=1.005 C_{s}$, (b) $V_{r}=1.250 C_{s}$, (c) $V_{r}=\sqrt{2} C_{s}$, (d) $V_{r}=1.650 C_{s}$. The white arrows of constant magnitude form a streak plot, which indicates the sense of motion throughout

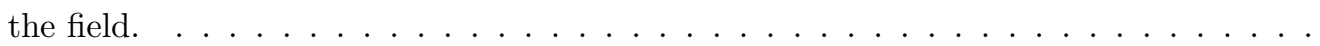

2.7 2 steady-state sliding rupture pulse subject to linear distance-weakening friction. Swave radiation is emitted exclusively from the slip zone. . . . . . . . . . . . . . . 30

2.8 Mixed boundary value problem corresponding to general 2D steady-state supershear slip-pulse problem (Dunham and Archuleta, 2005) . . . . . . . . . . . 
2.9 Radiation partitioning of the slip function as predicted by the general 2D steady-state supershear solution . . . . . . . . . . . . . . . . . . . . . . 36

2.10 Sense of particle motion for a spontaneous right-lateral sub-Rayleigh rupture. The contour plot shows the magnitude of the velocity field and the vectors show only the sense of motion.

2.11 Sense of particle motion for a spontaneous right-lateral supershear rupture. The contour plot shows the magnitude of the velocity field and the vectors show only the sense of motion. . . . . . . . . . . . . . . . . . . . . . . . .

3.1 Linear slip-weakening friction law . . . . . . . . . . . . . . . . . . .

3.2 Laboratory earthquake experimental setup: Dynamic photoelasticity for stress-field visualization and heterodyne fiber optic interferometers for continuous, point-wise measurements of particle velocity . . . . . . . . . . . . . . . . .

3.3 Schematic representations of traditional H-100 specimen geometry, static loading configuration, and dynamic rupture nucleation scheme ................

3.4 Inspection of fault surface planarity and perpendicularity using the USB microscope camera. See Figure 3.15 and Figure 3.16 for representative images of fault interfaces. .

3.5 Small rectangular strip of retro-reflective tape adhered to specimen surface to promote efficient scattering of focused laser light by fiber optic laser interferometer probes . .

3.6 $8 " \times 8 "$ H-100 laboratory earthquake specimen prepared and positioned within mount-

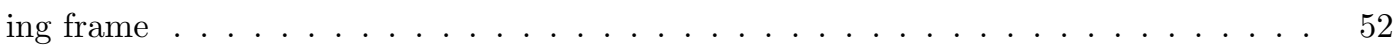

3.7 H-100 specimen assembly positioned in Carver@ model 3925 hydraulic press . . . . .

3.8 General dimensions of traditional H-100 laboratory earthquake specimens with uniform frictional fault extending throughout the entire specimen thickness . . . . . . . . . 56

3.9 Firing box voltage calibration curve. Rectifier DC voltage used to charge the capacitor bank plotted versus the AC voltage supplied by the variable transformer . . . . . . .

3.10 Physical dimensions of H-100 test specimens used in the laboratory earthquake experiments . . . . . . . . . . . . . . . . . . . . . . 
3.11 Critical buckling stress versus plate base dimension $w$ for a square H-100 plate of thickness $t_{1}=3 / 8^{\prime \prime}$ and $t_{2}=1 / 2^{\prime \prime} \ldots$. . . . . . . . . . . . . . . . . . 62

3.12 Overview of H-100 specimen fabrication and surface preparation . . . . . . . . . 63

3.13 Machined H-100 quadrilateral test sections: $\mathrm{s}=6$ " and $\mathrm{s}=8$ " mated pairs . . . . . 64

3.14 Surface texture of H-100 specimen surfaces resulting from conventional-cut and end-cut

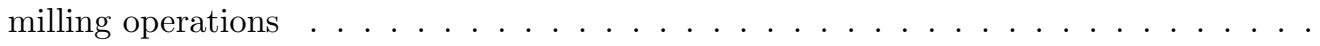

3.15 Optical inspection of H-100 test specimen revealing a well-machined laboratory earth-

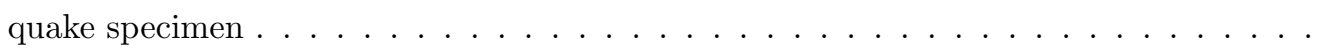

3.16 Optical machining inspection of H-100 test specimen revealing a wedge gap resulting from non-perpendicular fault surfaces . . . . . . . . . . . . . . . . . .

3.17 (a) Mated pair of H-100 quadrilateral test sections $(\mathrm{s}=8 "$, thickness $=0.5 ")$ mounted in steel polishing jig. (b) 12" diameter polishing wheel with Buehler UltraPo ${ }^{T M} 40-$ 7452 PSA-backed polishing cloth and $1 \mu m$ cerium oxide suspension (Buehler Ltd.

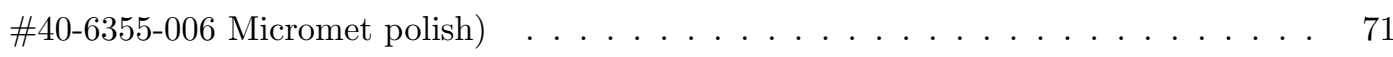

3.18 H-100 specimen fault surfaces polished to a near-optical-grade finish . . . . . . . . 73

3.19 H-100 specimens positioned within micro-bead blasting chamber . . . . . . . . . . 76

3.20 Parallelepiped H-100 specimens submitted for surface roughness evaluation . . . . . . 77

3.21 Dektak profilometer surface height line scan of an "as machined" H-100 surface. Plots in left column were obtained using a high pass cutoff wave length $\lambda_{c}=\mathrm{L} / 15$. Plots in right column were obtained using a high pass cutoff wave length $\lambda_{c}=\mathrm{L} / 100$. . . . . .

3.22 Dektak profilometer surface height line scan of a surface-roughened (micro-bead blasted) H-100 surface. Plots in the left column were obtained using a high-pass cut-off wave length $\lambda_{c}=\mathrm{L} / 15$. Plots in the right column were obtained using a high-pass cut-off wave length $\lambda_{c}=\mathrm{L} / 100 . \ldots \ldots \ldots \ldots \ldots \ldots$

3.23 Dektak profilometer surface height line scan of a fine-polished H-100 surface. Plots in the left column were obtained using a high-pass cut-off wave length $\lambda_{c}=\mathrm{L} / 15$. Plots in the right column were obtained using a high-pass cut-off wave length $\lambda_{c}=\mathrm{L} / 100$. . 
xxii

3.24 Dektak profilometer surface height line scan of a tested (ruptured) H-100 surface. Plots in the left column were obtained using a high-pass cut-off wave length $\lambda_{c}=\mathrm{L} / 15$. Plots in the right column were obtained using a high-pass cut-off wave length $\lambda_{c}=\mathrm{L} / 100$. .

3.25 One-sided amplitude spectra of the surface roughness profiles obtained through highpass filtering using $\lambda_{c}=\mathrm{L} / 15$ (a) as-machined, (b) bead0-blasted (70-140 mesh size), (c) polished, (d) tested (ruptured) surfaces $\ldots \ldots \ldots \ldots \ldots \ldots$

3.26 New H-100 laboratory earthquake specimen featuring 3D fault geometry . . . . . . 88

3.27 Close-up view of H-100 specimen prepared with 3D fault geometry $\ldots \ldots \ldots$. . . . 89

3.28 Masking of $\mathrm{H}-100$ specimen fault surface using $\mathrm{Cu}$ tape for protection of polished surface during bead-blasting step . . . . . . . . . . . . . . . . . .

3.29 (a) Specimen assembly masked and mounted just prior to bead-blasting with polished fault segments clearly visible. (b) Specimen assembly after bead-blasting procedure showing eroded $\mathrm{Cu}$ tape still intact with roughened fault segments clearly visible . . .

3.30 Mated pair of 3D laboratory earthquake specimens ready for testing . . . . . . . .

3.31 Left image: supershear rupture seen just crossing $\left(54,0^{+} \mathrm{mm}\right)$ at $t=33 \mu \mathrm{s}$. Right image: High-speed photoelasic image taken during exp 09-036 revealing faint secondary shear Mach fronts trailing the primary supershear rupture . . . . . . . . . . .

3.32 (a) Primary (on-fault) supershear particle velocity records acquired during exp 09-036 showing anomalous secondary signal disturbance. (b) S-wave isochrone plot corresponding to $\exp 09-036 \ldots \ldots \ldots \ldots \ldots \ldots \ldots$

3.33 (a) Primary (on-fault) supershear particle velocity records acquired during exp 09-050 showing anomalous secondary signal disturbance. (b) S-wave isochrone plot corresponding to $\exp 09-050 \ldots \ldots \ldots \ldots \ldots \ldots$

3.34 High-speed photoelastic image taken during exp 09-050 revealing secondary shear Mach fronts trailing primary supershear rupture . . . . . . . . . . . . . . . . . 101

3.35 S-wave isochrones computed for a sub-Rayleigh rupture propagating across the entire length of the specimen fault . . . . . . . . . . . . . . . . . . . 103 
xxiii

3.36 Upper right and left images: Laboratory earthquake experiment featuring fiber optic interferometers for continuous particle velocity measurements and photoelastic setup in conjunction with high-speed cameras for full-field imaging of a dynamic rupture with high spatial resolution. Below: Detailed schematic diagram of photoelastic interferom-

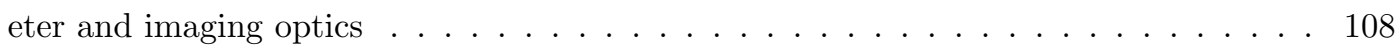

3.37 Right image: Coherent Verdi (V-10) solid state with variable attenuator optics. Left image: Keplerian beam expander with spatial filtering arrangement _ . . . . . . . . 109

3.38 Cordin 220 gated-intensified high-speed cameras . . . . . . . . . . . . . . . . . . 112

3.39 Sub-Rayleigh (left) and supershear (right) ruptures as revealed by photoelastic fringe patterns captured through high-speed photography . . . . . . . . . . . . . . . . 113

3.40 Plots of rupture position, $\mathrm{S}$ wave, and P-wave obtained during a sub-Rayleigh experi-

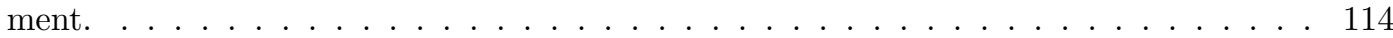

3.41 Measurement of shear Mach angles for determination of supershear rupture speed . . 118

3.42 Sequence of photoelastic images revealing sub-Rayleigh-to-supershear transition _ . . 122

3.43 Laboratory earthquake setup utilizing three fiber-optic probes focused at a common location for simultaneous measurement of fault-parallel, fault-normal, and vertical particle velocity components $\ldots \ldots \ldots \ldots \ldots \ldots$

3.44 Fiber-optic heterodyne laser interferometer configuration $\ldots \ldots \ldots \ldots \ldots \ldots$

3.45 Fiber-optic probe configurations adopted in laboratory earthquake experiments . . . 130

3.46 Particle velocity measurement configurations adopted in laboratory earthquake exper-

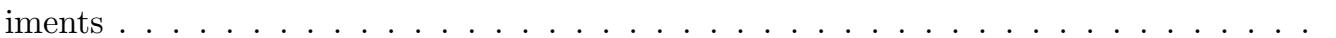

4.1 Laboratory earthquake experimental configuration used for the simultaneous measurement of fault-normal and fault-parallel particle velocity components at $(-55,10) \mathrm{mm}$

4.2 Displacement-time plots corresponding to the sub-Rayleigh and supershear experiments. Dots represent data points obtained from photoelastic image analysis and dashed lines represent a best-fit line passed through the points. . . . . . . . . . . . 
xxiv

4.3 Displacement-time plots corresponding to the sub-Rayleigh and supershear experiments shown with the best-fit lines extrapolated to their respective time axis intercepts. The supershear best-fit line was extrapolated to the point where it intersects the sub-Rayleigh best-fit line. . . . . . . . . . . . . . . . . . . . . 138

4.4 Contrast between sub-Rayleigh and supershear experiments: $(-55,10) \mathrm{mm}$ at $t=4 \mu \mathrm{s} 140$

4.5 Contrast between sub-Rayleigh and supershear experiments: $(-55,10) \mathrm{mm}$ at $t=8 \mu \mathrm{s} 141$

4.6 Contrast between sub-Rayleigh and supershear experiments: $(-55,10) \mathrm{mm}$ at $t=12 \mu \mathrm{s} 144$

4.7 Contrast between sub-Rayleigh and supershear experiments: $(-55,10) \mathrm{mm}$ at $t=16 \mu \mathrm{s} 145$

4.8 Contrast between sub-Rayleigh and supershear experiments: $(-55,10) \mathrm{mm}$ at $t=18 \mu \mathrm{s} 146$

4.9 Contrast between sub-Rayleigh and supershear experiments: $(-55,10) \mathrm{mm}$ at $t=21 \mu s 147$

4.10 Contrast between sub-Rayleigh and supershear experiments: $(-55,10) \mathrm{mm}$ at $t=25 \mu \mathrm{s} 148$

4.11 Contrast between sub-Rayleigh and supershear experiments: $(-55,10) \mathrm{mm}$ at $t=29 \mu \mathrm{s} 150$

4.12 Contrast between sub-Rayleigh and supershear experiments: $(-55,10) \mathrm{mm}$ at $t=33 \mu \mathrm{s} 152$

4.13 Contrast between sub-Rayleigh and supershear experiments: $(-55,10) \mathrm{mm}$ at $t=37 \mu \mathrm{s} 154$

4.14 Contrast between sub-Rayleigh and supershear experiments: $(-55,10) \mathrm{mm}$ at $t=41 \mu \mathrm{s} 156$

4.15 Contrast between sub-Rayleigh and supershear experiments: $(-55,10) \mathrm{mm}$ at $t=45 \mu \mathrm{s} 158$

4.16 Contrast between sub-Rayleigh and supershear experiments: $(-55,10) \mathrm{mm}$ at $t=50 \mu \mathrm{s} 159$

4.17 Contrast between sub-Rayleigh and supershear experiments: $(-55,10) \mathrm{mm}$ at $t=60 \mu \mathrm{s} 161$

4.18 Consecutive photoelastic image frames capture the sub-Rayleigh rupture crossing the measurement station located at $(-55,0) \mathrm{mm}$. The red lines in the image highlight the optical paths of the FP and FN interferometer beams, which simultaneously monitored the particle velocity at this location. . . . . . . . . . . . . . . . . 162

4.19 Pulse-like particle velocity profile of the sub-Rayleigh rupture portrayed in Figure 4.18.

The curves are characterized by a FN component, whose magnitude is only slightly greater than the magnitude of the FP velocity signal $\left(\left|\delta \dot{u}_{y}\right| /\left|\delta \dot{u}_{x}\right|^{+}=1.02\right) \ldots \ldots \ldots 164$ 
$\mathrm{XXV}$

4.20 Consecutive photoelastic image frames capture the sub-Rayleigh rupture crossing the particle velocity measurement station located at $(-55,0) \mathrm{mm}$. The $t=53 \mu \mathrm{s}$ image frame reveals the sudden emergence of a daughter crack and the onset of a supershear transition. ............................

4.21 Crack-like particle velocity profile of the sub-Rayleigh rupture portrayed in Figure 4.20. The curves are characterized by a dominant FN component, whose magnitude is $1.5 \times$ greater than the magnitude of the FP velocity signal $\left(\left|\delta \dot{u}_{y}\right| /\left|\delta \dot{u}_{x}^{+}\right|=1.50\right) \ldots \ldots . . .166$

4.22 Scatter plot of the $\left|\delta \dot{u}_{y}\right| /\left|\delta \dot{u}_{x}^{+}\right|$for sub-Rayleigh and trailing-sub-Rayleigh ruptures resulting from broad range of laboratory earthquake experiments . . . . . . . . . . . . 167

4.23 Laboratory earthquake experiment featuring the paired FN laser interferometer probe beam configuration for the characterization of sub-Rayleigh particle velocity field decay 169

4.24 Consecutive photoelastic image frames capture the sub-Rayleigh rupture and its surrounding stress field crossing the particle velocity measurement stations located at $(-55,0) \mathrm{mm}$ and $(-55,2.5) \mathrm{mm}$. The red lines in the image highlight the optical paths of the two FN interferometer probe beams, which simultaneously monitored the particle velocity at these locations. . . . . . . . . . . . . . . . . . . . 170

4.25 Paired FN particle velocity traces corresponding to the sub-Rayleigh rupture depicted in Figure 4.24. Virtually identical FN particle velocity profiles are observed at the on-fault and off-fault measurement stations. . . . . . . . . . . . . . . . . .

4.26 Consecutive photoelastic image frames capture the sub-Rayleigh rupture and its surrounding stress field crossing the particle velocity measurement stations located at $(-55,0) \mathrm{mm}$ and $(-55,10) \mathrm{mm}$. The red lines in the image highlight the optical paths of the two FN interferometer probe beams, which simultaneously monitored the particle velocity at these locations. . . . . . . . . . . . . . . . . . .

4.27 Paired FN particle velocity traces corresponding to the sub-Rayleigh rupture depicted in Figure 4.26. The FN record obtained at $(-55,10) \mathrm{mm}$ exhibits a signal amplitude which is $48 \%$ of its corresponding on-fault value. . . . . . . . . . . . . . . . . 173 
xxvi

4.28 Consecutive photoelastic image frames capture the sub-Rayleigh rupture and its surrounding stress field crossing the particle velocity measurement stations located at $(-55,0) \mathrm{mm}$ and $(-55,24) \mathrm{mm}$. The red lines in the image highlight the optical paths of the two FN interferometer probe beams, which simultaneously monitored the particle

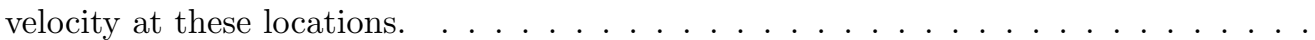

4.29 Paired FN particle velocity traces corresponding to the sub-Rayleigh rupture depicted in Figure 4.28. The FN record obtained at $(-55,24) \mathrm{mm}$ exhibits a signal amplitude which is $24 \%$ of its corresponding on-fault value . . . . . . . . . . . . . 175

4.30 Decay of the FN signal amplitude represented by the $\left|\dot{u}_{y}^{\text {off-fault }} / \dot{u}_{y}^{\text {on-fault }}\right|$ plotted versus the FN distance. Results were obtained from 10 independent laboratory earthquake experiments using the paired $\mathrm{FN}$ laser interferometer probe configuration, as depicted in the inset. . . . . . . . . . . . . . . .

4.31 Consecutive photoelastic image frames capture a supershear rupture crossing a particle velocity measurement station positioned at $(-55,5) \mathrm{mm}$. The red lines in the image highlight the optical paths of the FN and FP interferometer probe beams, which simultaneously monitored the FN and FP particle velocity components at this location. 177

4.32 Simultaneous FN and FP particle velocity records recorded at the at the off-fault measurement positioned at $(-55,5) \mathrm{mm}$ reveal the signature of the leading dilatational field lobe followed shortly thereafter by the sharp velocity jumps associated with the arrival of the shear Mach front.

4.33 Measurements of $\beta_{s}$ determined from supershear experiments plotted versus the normalized rupture speed. Excellent agreement between theory and experiment is demonstrated across a broad range of supershear rupture speeds, which nearly span the entire stable rupture speed regime. $\ldots \ldots \ldots \ldots \ldots \ldots$

4.34 Laboratory earthquake experiment featuring the (FP1,FP2) laser interferometer probe beam configuration for the simultaneous measurement of fault-parallel particle velocity components on the fault and away from the fault . . . . . . . . . . . . . 183 
xxvii

4.35 Series of FP particle velocity records obtained in five separate supershear experiments showing the progressive separation between the leading dilatational field lobe and the shear Mach front with increased FN distance . . . . . . . . . . . . . . . . . . . . . . . 184

4.36 Photoelastic image frames capture the supershear rupture at two different times in the experiment as the rupture tip and shear Mach front were about to cross the onand off-fault measurement stations at $\left(-55,0^{+}\right) \mathrm{mm}$ and $(-55,16) \mathrm{mm}$. Mach angle measurements yield an estimate of $V_{r} / C_{s}=1.67$ for the normalized rupture speed. . . 185

4.37 FP particle velocity records obtained the on- and off-fault measurement stations located at $\left(-55,0^{+}\right) \mathrm{mm}$ and $(-55,16) \mathrm{mm}$ during supershear experiment (exp 09-060) $\ldots 186$

4.38 Photoelastic image frames capture the supershear rupture at two different times in the experiment as the rupture tip and shear Mach front were about to cross the onand off-fault measurement stations at $\left(-55,0^{+}\right) \mathrm{mm}$ and $(-55,24) \mathrm{mm}$. Mach angle measurements yield an estimate of $V_{r} / C_{s}=1.85$ for the normalized rupture speed. . . 187

4.39 FP particle velocity records obtained the on- and off-fault measurement stations located at $\left(-55,0^{+}\right) \mathrm{mm}$ and $(-55,24) \mathrm{mm}$ during supershear experiment (exp 09-062). . . 189

4.40 FP particle velocity records obtained the on- and off-fault measurement stations located at $\left(-55,0^{+}\right) \mathrm{mm}$ and $(-62,10) \mathrm{mm}$ during supershear experiment (exp 09-079). Measurement stations were positioned along a line, which was very nearly aligned with the normal to the shear Mach front. . . . . . . . . . . . . . . . . . . 190

4.41 Photoelastic image frames capture the supershear rupture at two different times in the experiment as the rupture tip and shear Mach front were about to cross the onand off-fault measurement stations at $\left(-55,0^{+}\right) \mathrm{mm}$ and $(-62,10) \mathrm{mm}$. Mach angle measurements yield an estimate of $V_{r} / C_{s}=1.81$ for the normalized rupture speed. . .

4.42 Plot of the ratio $\dot{u}_{x}^{s} / \dot{u}_{x}\left(x, 0^{+}\right)$obtained from 11 supershear experimental records poltted against the normalized wave speed $V_{r} / C_{s}$. Results compare quite favorably with theoretical partitioning relationship corresponding to the blue curve. . . . . . . . . . . 192 
xxviii

5.1 Denali fault image montage: (a) Susitna Galcier, Denali, and Totschunda fault systems, (b) strike-slip fault segment extending from epicenter to Pump Station 10 located along the Alaska pipeline, (c) closeup view of central Denali fault and Pump Station 10, (d) Denali strike-slip segment and branch onto Totschunda fault (images obtained using Google earth Pro $\ldots \ldots \ldots \ldots \ldots \ldots \ldots$

5.2 From Eberhart-Phillips et al., 2003: Plate tectonic setting of southern Alaska and major tectonic elements. The Pacific and North American plates converge at $5.4 \mathrm{~cm} /$ year beneath Anchorage, and the Yakutat block collides with North America independently. Plate motion is indicated by green arrows. Blue open arrows schematically show lateral movement of broad region south of the Denali fault. . . . . . . . . . . . . . .

5.3 (a) From (Ellsworth et al., 2004): comparison of fault-parallel (top), fault-normal (middle) and vertical (bottom), PS10 data (gray) and synthetic (black) ground velocity predictions (b) From (Dunham and Archuleta, 2004)l: Comparison between recorded and synthetic ground motions at PS10 for dynamic model featuring slip weakening with healing feature, and slip to $10 \mathrm{~km}$ depth . . . . . . . . . . . . . . . . . 197

$5.4 \quad$ Laboratory earthquake scaling geometry . . . . . . . . . . . . . . . . . . . . . . 200

5.5 Locus of stations with the same $\Delta t^{S S-T R}$, assuming a fixed transition length and

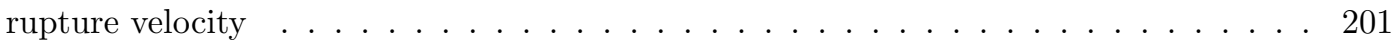

5.6 Close-up view of H-100 specimen prepared with 3D fault geometry used in scaled PS10 laboratory earthquake experiments . . . . . . . . . . . . . . . . . . . 204

5.7 Distance-time plots obtained from the scaled $2002 M_{w} 7.9$ Denali, PS10 supershear

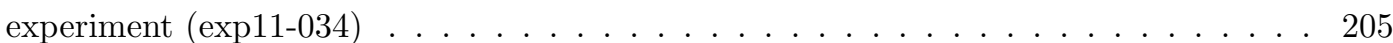

5.8 High-speed photoelastic image sequence of the sub-Rayleigh-to-supershear transition acquired during the scaled $2002 M_{w} 7.9$ Denali, PS10 laboratory earthquake experiment 207 
xxix

5.9 Image frame sequence following the superhsear transition captures the supershear rupture as it crosses the station followed by the emergence of the trailing Rayleigh rupture in the wake of the leading rupture. The two closely spaced shear Mach fronts define the supershear slip zone. . . . . . . . . . . . . . . . . . . . . . . . . . . 209

5.10 Distance-time plots obtained from the scaled $2002 M_{w} 7.9$ Denali, PS10 sub-Rayleigh

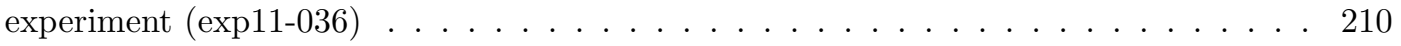

5.11 Comparison between the sub-Rayleigh and supershear laboratory earthquake experiments conducted using the scaled $2002 M_{w} 7.9$ Denali PS10 configuration . . . . . . . 212

5.12 Denali supershear event Recorded at PS10 (left) and Laboratory record obtained at the scaled measurement station located at $(57,3) m m$ (right) $\ldots \ldots \ldots$. . . . . . 217

5.13 Scaled experimental supershear record compared with Denali PS10 record . . . . . . . 218

6.1 Laboratory earthquake experimental configuration used for simultaneous measurement of fault-normal and fault-parallel particle velocity measurements at a near-fault measurement station positioned at $\left(-55,0^{-}\right) m m \ldots \ldots \ldots \ldots \ldots \ldots \ldots$

6.2 Supershear rupture experiment (exp10-036) featuring anti-symmetric fault-parallel particle velocity records $\mathrm{FP}^{+}\left(\dot{u}_{x}\left(60,0^{+}\right)\right.$and $\mathrm{FP}^{-}\left(\dot{u}_{x}\left(60,0^{-}\right) \ldots \ldots \ldots \ldots\right.$

6.3 Supershear rupture experiment (exp10-041) featuring anti-symmetric fault-parallel particle velocity records $\mathrm{FP}^{+}\left(\dot{u}_{x}\left(60,0^{+}\right)\right.$and $\mathrm{FP}^{-}\left(\dot{u}_{x}\left(60,0^{-}\right)\right.$along with $\mathrm{FN}\left(\dot{u}_{y}(60,0)\right.$ and vertical $\left(\dot{u}_{z}\left(60,0^{+}\right)\right.$records acquired at the same measurement station locations. . 227

6.4 (a) Sense of particle displacement for (stable) left-lateral supershear rupture rupture (b) Sense of particle displacement for (stable) left-lateral supershear rupture rupture . 229

6.5 Plot of speed dependent weighting factor $(B)$ versus the normalized rupture speed $\ldots 239$

6.6 Coefficient $C$ in Eq. 6.46 plotted as a function of the normalized rupture speed $V_{r} / C_{s} \quad 241$

6.7 Supershear rupture experiment (exp10-014) featuring an anti-symmetric slip-pulse followed by crack-like trailing sub-Rayleigh rupture . . . . . . . . . . . . . . . . . 245 
6.8 Position versus time of the supershear rupture tip determined from the photoelastic image sequence. Extrapolation of the best-fit line yields an estimated station crossing time of $t=33.0 \mu \mathrm{s}$. Extrapolation to $t=31.75 \mu \mathrm{s}$ (onset of sliding) reveals how the rupture tip (caustic) had not yet reached $(40,0) \mathrm{mm}$ at this time. . . . . . . . . . 247

6.9 (a) Expanded views of photoelastic images from supershear experiment (exp10-014) reveal the prominent dilatational field lobes extending out beyond the primary rupture tip which perturb the fault in advance of the primary rupture (b) Numerical simulation of photoelastic field obtained using ABAQUS validates and highlights the primary features of the supershear rupture field observed in experiments (c) Magnified view of the fault-parallel traces highlights the onset of slip and gradual ramping of the interfacial sliding speed prior to the arrival of the primary supershear rupture . . . . . 249

6.10 Temporal evolution and spatial distribution of dynamic stress components $\sigma_{x x}$ and $\tau$ accompanied by the FP and FN particle velocity records corresponding to the supershear slip-pulse recorded in $\operatorname{exp10-014.~.~.~.~.~.~.~.~.~.~.~.~.~.~.~.~.~.~.~} 251$

6.11 Temporal evolution and spatial distribution of the dynamic coefficient of friction $f_{d}$ obtained from supershear slip-pulse in exp10-014 . . . . . . . . . . . . . . . . . . . . 254

6.12 (a) Superimposed plots of the shear stress distribution and slip velocity corresponding to the supershear slip-pulse from exp10-014 (b) Plot of shear stress vs. slip rate (c) Superimposed plots of the shear stress distribution and accumulated slip corresponding to the supershear slip-pulse from exp10-014 (d) Plot of shear stress vs. accumulated slip 257 
xxxi

\section{List of Tables}

1.1 Natural earthquakes inferred to have been supershear events . . . . . . . . . . . .

3.1 Simple table for converting Mesh size to glass bead diameter . . . . . . . . . . . 75

3.2 Average roughness $\left(R_{a}\right)$ and root-mean-square roughness $\left(R_{q}\right)$ for (a) as-machined, (b) bead-blasted (70-140 mesh size), (c) polished, (d) tested (ruptured) surfaces . . . . . 84

3.3 Measured wave speed values and mean sub-Rayleigh rupture speed in Homalite ${ }^{\mathrm{TM}}$ H-100 obtained from laboratory earthquake experiments . . . . . . . . . . . . 116 


\section{Chapter 1}

\section{Introduction}

A natural earthquake is classified as supershear if it is surmised, generally with the aid of seismic inversions, that the primary rupture spent some portion of its history in the rupture speed regime $C_{s} \leq V_{r} \leq C_{p}$, where $V_{r}$ represents the rupture speed, and $C_{s}, C_{p}$ represent the crustal shear $(S)$ and dilatational, or pressure $(P)$ wave speeds, respectively. Rupture speed stability analyses have been performed within the context of either self-similar crack growth (Andrews, 1976; Burridge et al., 1979; Broberg, 1989) or by using steady-state, cohesive shear strength models (Samudrala et al., 2002a,b; Rosakis, 2002; Dunham and Archuleta, 2004, 2005) under uniform background stress and fault strength conditions. It is well established that once a rupture front has transitioned into the supershear domain it will tend to favor a stable rupture speed regime $V_{0}<V_{r} \leq C_{p}$ with $V_{r} \rightarrow C_{p}$, given a sufficient rupture propagation distance in the case of self-similar growth. The lower bound $V_{0}$ of the stable regime is a function of the fault strength and may also depend upon other system parameters, such as rate of velocity weakening and background stress. The minimum value that $V_{0}$ can assume, in the case of infinite fault strength, is $V_{0}=\sqrt{2} C_{s}$, in which case the maximum extent of the stable rupture speed regime becomes $\sqrt{2} C_{s}<V_{r} \leq C_{p}$. The rupture speed domain $C_{s} \leq V_{r}<V_{0}$ is consequently regarded as an unstable rupture speed regime. Earthquakes are otherwise generally classified as sub-Rayleigh, exhibiting a rupture speed bounded by $V_{r} \leq C_{R}$ with the rupture speed tending to approach the Rayleigh wave speed $C_{R}$ (Broberg, 1999; Rosakis, 2002). The rupture speed regime $C_{R}<V_{r}<C_{s}$ is forbidden since a rupture in this speed regime would otherwise become a source rather than a sink of fracture energy (Freund, 1979; Broberg, 1989; 


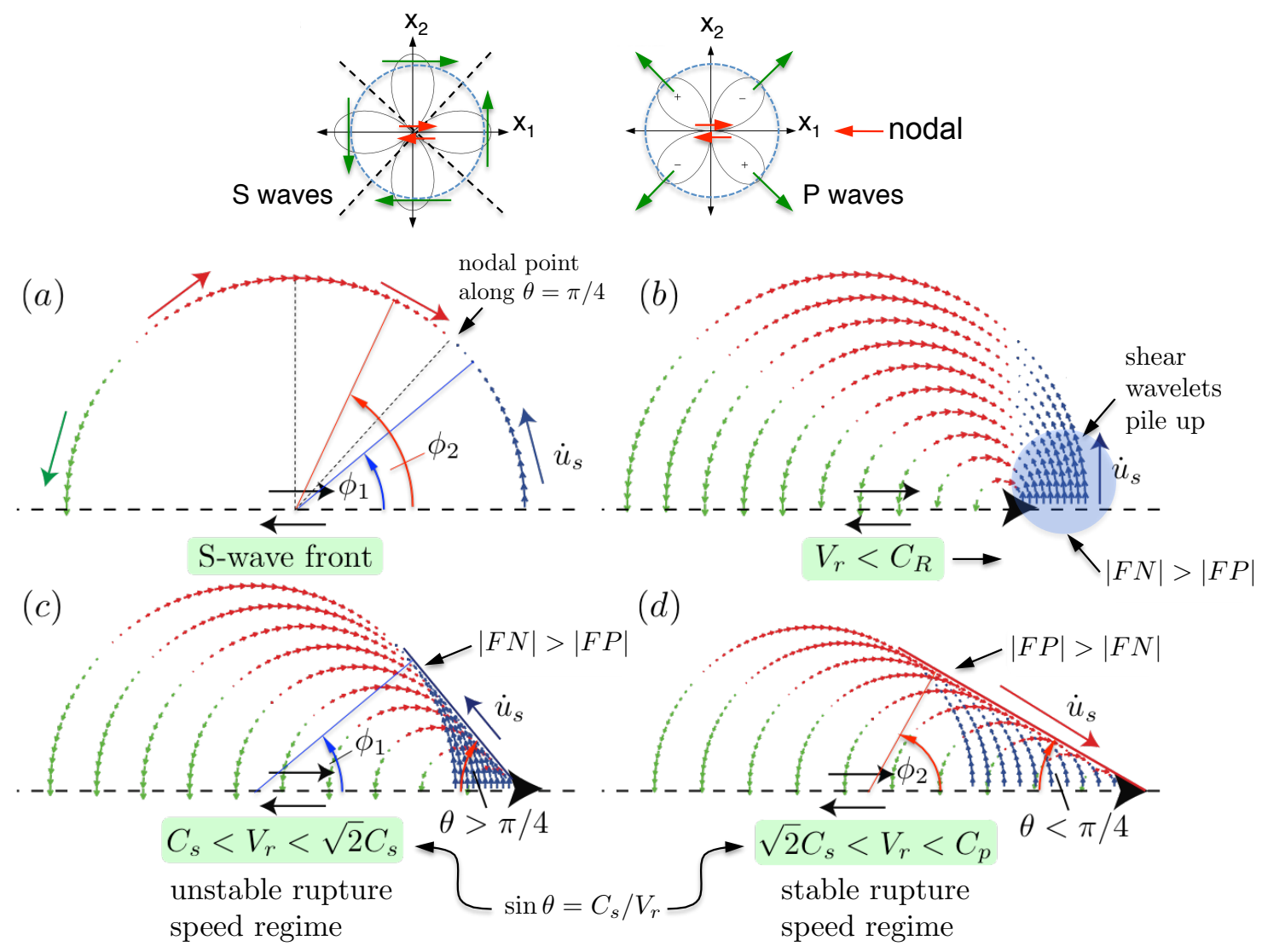

Figure 1.1: Formation of sub-Rayleigh rupture and shear Mach fronts by the superposition of shear wavelets emitted by a steady-state shear dislocation

Rosakis, 2002). The superposition of shear wavelets emitted by a steady-state, supershear rupture front leads to the formation of a shear Mach front, which stems from the advancing rupture tip and envelopes the surrounding medium along either side of the fault. Considerable insight into the formation of a shear Mach front and the nature of the resulting displacement field along the front is gained by recalling the solution for the far-field radiation pattern of the transverse component of displacement resulting from a right lateral shear dislocation, also commonly referred to as the double-couple solution in an infinite homogeneous medium (Aki and Richards, 2002)

The series of diagrams depicted in Figure 1.1 graphically demonstrate the formation of subRayleigh rupture and shear Mach fronts by the superposition of S-wave fronts emitted by a steadystate right-lateral rupture. Figure 1.1a shows how the displacement field vectors lying tangent to the arc of a circular S-wave front are oriented clockwise along the upper portion of the wave front 
corresponding to $\pi / 4<\phi \leq \pi / 2$. The displacement field is maximum at $\phi=\pi / 2$, and continuously decreases in magnitude along the S-wave front until vanishing at the nodal point corresponding to $\phi=\pi / 4$. The displacement field vectors along the upper quadrant are thus characterized by a dominant FP component. The nodal point at $\phi=\pi / 4$ also marks a reversal point in the displacement field. Displacement field vectors lying tangent to the lower portion of the circular S-wave front are now oriented counterclockwise as shown, featuring a continuous increase in magnitude starting from the nodal point at $\phi=\pi / 4$ until a maximum value is attained at $\phi=0$. Note that the displacement field vectors along the lower sector are now characterized by a dominant FN component.

Figure 1.1b depicts a steady-state, right-lateral shear dislocation emitting S-wave fronts as it propagates from left to right along the horizontal axis. When propagating at a speed $V_{r} \leq C_{R}$, (i.e., sub-Rayleigh rupture speed regime), the radiated S-wave fronts will tend to pile up to form a locally concentrated field just ahead of the advancing rupture, as shown. The resulting velocity field surrounding a sub-Rayleigh rupture, particularly in the region situated ahead of the rupture tip, is thus characterized by a dominant FN component, corresponding to the dominant component of motion along the contributing S-wave fronts.

The lower two diagrams in Figure 1.1 graphically depict how the circular S-wave fronts emitted by a steady-state supershear rupture combine to form an inclined Mach front, which is tangent to the continuum of shear wavelets, as shown. Any line drawn normal to the Mach front will necessarily pass through the center of a contributing circular S-wave front that was emitted by the rupture at an earlier time. The acute angle $\phi$ measured between the normal the Mach front and the fault plane is naturally the same for all of the S-waves that combine to form a given Mach front. The resulting displacement field $\vec{u}_{s}$ is thus oriented parallel to the shear Mach front, as shown.

Note that since the angle $\phi=\pi / 4$ corresponds to a nodal point along an S-wave front, it follows that the Mach angle $\theta=\pi / 4$ represents a natural boundary in the supershear regime whereby $\vec{u}_{s}$ is directed upward along the front for $\theta>\pi / 4$, as depicted in Figure 1.1c (i.e., $\phi<\pi / 4$ ), and is otherwise directed downward along the shear Mach front for $\theta<\pi / 4$, as depicted in Figure 1.1d (i.e., $\phi>\pi / 4)$. 
The propagation direction of the shear Mach front is defined by a wave vector $\vec{k}$, which is oriented at an angle $\phi$ with respect to the fault plane as shown, while the resulting Mach cone half-angle, measured with respect to the fault plane, is given by the familiar relation

$$
\theta=\sin ^{-1}\left(C_{s} / V_{r}\right)
$$

where $\theta=\pi / 2-\phi$.

Application of Eq. 1.1 reveals that $\theta=\pi / 4$ corresponds to the unique rupture speed $V_{r}=\sqrt{2} C_{s}$. Consequently, $\left|\vec{u}_{s}\right| \rightarrow 0$ as $V_{r} \rightarrow \sqrt{2} C_{s}$ since all of the contributing shear wavelets are nodal at the point of tangency to the Mach front when $\phi=\pi / 4$. The complete disappearance of the shear Mach front at this unique rupture speed and the resulting no-radiation condition is addressed in greater detail in a later section.

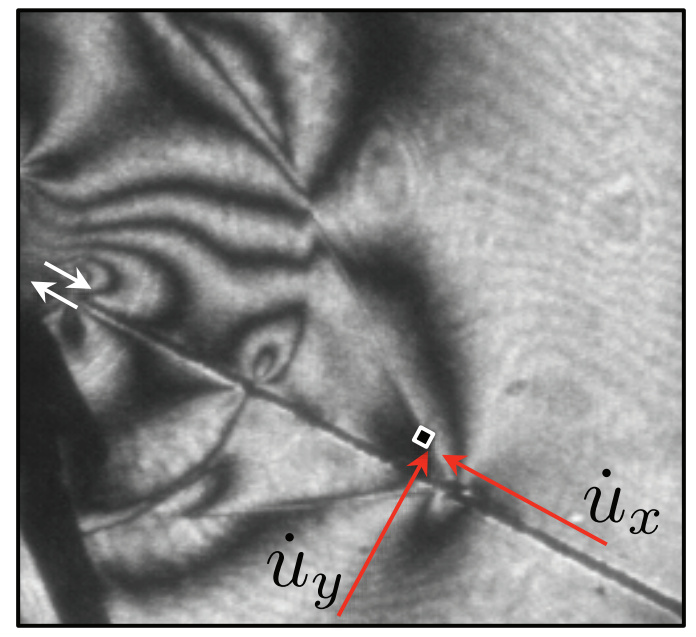

Figure 1.2: Supershear rupture with shear Mach front, as revealed by photoelastic interference fringes, shown crossing a particle velocity measurement station during a laboratory earthquake experiment

Continued application of Eq. 1.1 reveals that the Mach angle domain $\pi / 4<\theta \leq \pi / 2$ corresponds to the rupture speed domain $C_{s} \leq V_{r}<\sqrt{2} C_{s}$, while $\sin ^{-1}\left(C_{s} / C_{p}\right) \leq \theta<\pi / 4$ corresponds to the maximum stable rupture speed regime $\sqrt{2} C_{s}<V_{r} \leq C_{p}$. Special cases of interest that set the lower bound $\theta_{\min }=\sin ^{-1}\left(C_{s} / C_{p}\right)$ are $(1) C_{p}=\sqrt{3} C_{s}$, often a good approximation for crustal rock, where $\sin ^{-1}(1 / \sqrt{3}) \leq \theta<\pi / 4$, and $(2) C_{p} \approx 2 C_{s}$, applicable to H-100 test specimens used in laboratory 
earthquake experiments, where $\sin ^{-1}(1 / 2) \leq \theta<\pi / 4$.

Near-field locations, which are swept by a shear Mach front are subject to unique forms of ground motion, induced by various aspects of the radiated fields, which necessarily differ from classical subRayleigh ground motion. The nature of supershear ground motion and any features that distinguish it from sub-Rayleigh ground motion form the basis of the experimental investigation contained in this article. Ground motion signatures associated with sub-Rayleigh and supershear ruptures are investigated using the Laboratory Earthquake Experiment developed by Rosakis and co-workers (Xia et al., 2004; Rosakis et al., 2007; Lu et al., 2007, 2010). Heterodyne laser interferometers (Lykotrafitis et al., 2006a) enable continuous, high-bandwidth measurements of FN and FP particle velocity "ground motion" records at fixed locations on the surface of a Homalite TM $(\mathrm{H}-100)$ test specimen as a sub-Rayleigh or supershear rupture sweeps along the frictional fault. Photoelastic interference fringes, acquired using high-speed digital photography, provide a synchronized, spatially resolved, whole-field view of the advancing rupture tip and surrounding maximum shear stress field. Figure 1.2 captures the essence of the experimental strategy adopted in the current investigation. A photoelastic image reveals a supershear rupture and associated shear Mach fronts which sweep the medium to either side of the specimen fault. One of the Mach fronts is seen just crossing a particle velocity measurement station on the surface of the specimen. The red arrows highlight how a pair of laser beams are directed at nearly grazing incidence to the specimen surface and brought to focus at the lower-right-hand corner of a measurement station. Simultaneous FN and FP particle velocity records acquired at this location are analogous to ground motion measurements recorded by an accelerometer station situated in the immediate neighborhood of an active fault. Additional features such as a leading dilatational field lobe surrounding the rupture tip and the trailing secondary rupture in the wake of the shear Mach cone are also revealed in the image. A more-precise description of these features, along with a detailed discussion of the experimental arrangement and optical diagnostics, are addressed in later sections. Results obtained through experiments replicate and validate numerous supershear ground motion features predicted by both theory and numerical analysis. Knowledge gained through these investigations not only validates and 
6

\begin{tabular}{|c|c|c|}
\hline Year & Location & $M_{w}$ \\
\hline 18 April 1906 & San Francisco, CA & 7.9 \\
\hline 15 October 1979 & Imperial Valley, CA & 6.5 \\
\hline 17 August 1999 & Izmit, Turkey & 7.6 \\
\hline 12 November 1999 & Duzce, Turkey & 7.2 \\
\hline 14 November 2001 & Kunlunshan, Tibet & 7.8 \\
\hline 3 November 2002 & Denali, Alaska & 7.9 \\
\hline 6 April 2009 & L'Aquila, Abruzzo, Italy & 6.3 \\
\hline
\end{tabular}

Table 1.1: Natural earthquakes inferred to have been supershear events

ranks the fidelity of various theoretical and numerical predictions but can also provide an improved understanding of frictional faulting processes. Laboratory earthquake experiments also serve to emphasize the utility and potential payoff of acquiring ground motion measurements in the local vicinity of active faults on the surface of the Earth.

There have been at least seven natural earthquakes, chronologically listed in Table 1.1, that have been classified as, or at least conjectured to have been supershear events (Archuleta, 1984; Bouchon et al., 2001, 2002; Bouchon and Vallee, 2003; Bouin et al., 2004; Ellsworth et al., 2004; Dunham and Archuleta, 2004; Robinson et al., 2006; Song et al., 2008; Ellsworth and Chiaraluce, 2009). Most of these large-magnitude events occurred in long and relatively straight fault segments, and at least two ( $M_{w} 7.8$ Kunlunshan, Tibet, and $M_{w} 7.9$ Denali, Alaska) featured a well-documented transition from sub-Rayleigh-to- supershear speeds. Some of the necessary conditions, which increase the likelihood for a fault segment to host a supershear earthquake rupture have been studied by (Robinson et al., 2006) and (Das, 2007).

In all events listed in Table 1.1, finite fault inversion techniques, constrained by geodetic and/or seismic data, were applied to estimate the rupture velocity history, so as to infer where and when along the fault the rupture may have transitioned into the supershear domain. In some of these cases there is compelling field data to support this assertion. Generally speaking, however, there is an overall scarcity of reliable near-field ground motion records, thus placing a heavy reliance upon the use of seismic inversion techniques in order to conclude whether most of the events listed in Table 1.1 were supershear earthquakes. A notable exception in the list is the $2002 M_{w} 7.9$ Denali, 
Alaska, earthquake, in which case a unique set of near-field ground motion records signal the passage of a supershear rupture followed by a secondary slip pulse (Dunham and Archuleta, 2004, 2005). Motivated by the unique features contained in these ground motion records and by a dynamic rupture simulation of the event conducted by (Dunham and Archuleta, 2004, 2005), a series of laboratory earthquake experiments were conducted in an attempt to replicate the Denali near-source ground motion signatures using a model test specimen. Particle velocity records bearing a striking similarity to the Denali ground motion records are presented and discussed.

The final chapter presents a $2 \mathrm{D}$ steady-state, stress-velocity formulation derived from complex elasticity theory, which relates the in-plane particle velocity components measured close to the fault, to the evolution of the stress tensor at the same location. A locally steady-state condition is assumed within a restricted time interval in order to invoke these relationships and measure the dynamic stresses, $\sigma_{x x}(t)$ and $\tau(t)$, within the dynamic rupture slip zone. Dynamic stress measurements obtained in this manner represent a new dimension to the laboratory earthquake experiment originally developed by (Xia et al., 2004; Xia, 2005), and enable a new class of dynamic friction investigations. Experimental findings are presented, which capture the evolution of the dynamic coefficient of friction, and demonstrate velocity weakening behavior on the sub-microsecond time scale. 


\section{Chapter 2}

\section{Theoretical Foundations: Near-source ground motion signatures of sub-Rayleigh and supershear fault ruptures}

\subsection{Spectral representation of radiated wave fields arising from a steady-state shear dislocation}

Recent theoretical and numerical investigations of supershear ruptures in 2D (Dunham and Archuleta, 2005; Bhat et al., 2007) and in 3D (Dunham and Bhat, 2008) show that ground motion due to the passage of the Mach front is virtually unattenuated at large distances from the fault. This conclusion is qualitatively consistent with high-speed photoelastic images of supershear ruptures, which extend out to large distances from the rupture tip (Rosakis et al., 1999; Rosakis, 2002; Lu et al., 2007). In their numerical 2D steady-state supershear slip-pulse model, (Dunham and Archuleta, 2005), showed that the supershear rupture S-wave spectrum is transported out to infinity along Mach line characteristics emanating exclusively from within the slip zone. Supershear ground motion records gathered far from the fault should thus contain detailed information about the frictional faulting process, in stark contrast to the sub-Rayleigh ground motion records, which suffer rapid attenuation with increased off-fault distance and reveal little information about the rupture source spectrum (Dunham and Archuleta, 2005). 
The Fourier-transform-based spectral representation of the radiated wave fields arising from a steady-state sub-Rayleigh rupture pulse which is presented here augments these earlier studies. The analysis clearly reveals how an on-fault rupture pulse source spectrum is rapidly stripped of its high-frequency content with increased off-fault distance, thus leaving little chance of extracting detailed information about the breakdown zone from off-fault ground motion records. A similar analysis applied to a 2D supershear rupture pulse is also presented below, which reveals how the S-wave source spectrum remains fully preserved out to infinity, implying that off-fault ground motion records contain detailed information about the frictional faulting process.

\subsubsection{Characteristic decay of shear and dilatational radiation fields for sub-shear $\left(V_{r} \leq C_{s}\right)$ and intersonic $\left(C_{s} \leq V_{r} \leq C_{p}\right)$ dislocation speed regimes}

Elastic wave fields may be separated into dilatational (irrotational) and shear (rotational) parts, each governed by the wave equation and an appropriate wave speed. For the sake of clarification, we note that dilatational waves, primary waves, and pressure $(\mathrm{P})$ waves are all synonymous in the context of this discussion. Adopting the Poisson representation, the displacement field may be expressed as

$$
\vec{u}=\vec{u}^{p}+\vec{u}^{s}
$$

where $\vec{u}^{p}$ and $\vec{u}^{s}$ represent the dilatational (P) and shear (S) displacement fields, respectively. Each displacement field component is in turn governed by the wave equation (Freund, 1990) provided that

$$
\begin{aligned}
& \nabla \times \vec{u}^{p}=0 \\
& \nabla \cdot \vec{u}^{s}=0 .
\end{aligned}
$$

Consider a 2D rupture propagating along a frictional fault coinciding with the $\mathrm{x}$-axis of a Cartesian coordinate system. By imposing the steady-state assumption and taking the origin of a new coor- 
dinate system $\left(\xi_{1}, \xi_{2}\right)$ to coincide with the rupture front, the governing wave equation expressions become two-dimensional in $\xi_{1}=x-V_{r} t$ and $\xi_{2}=y$ under a Galilean coordinate transformation. A general field quantity $f\left(\xi_{1}, \xi_{2}\right)$ (which may represent any stress or particle velocity field component), associated with a rupture speed $V_{r}$, may therefore be expressed as

$$
f\left(\xi_{1}, \xi_{2}\right)=f^{p}\left(\xi_{1}, \xi_{2}\right)+f^{s}\left(\xi_{1}, \xi_{2}\right)
$$

where $f^{p}$ represents the dilatational contribution to the field quantity, and $f^{s}$ corresponds to the shear field contribution. The general 2D spectral representation of $f^{j}\left(\xi_{1}, \xi_{2}\right)$ is given by the inverse Fourier transform

$$
\begin{array}{r}
f^{j}\left(\xi_{1}, \xi_{2}\right)=\frac{1}{2 \pi} \int_{-\infty}^{\infty} \int_{-\infty}^{\infty} \hat{f}^{j}\left(k_{x}, k_{y}\right) \times \\
e^{i\left[k_{x} \xi_{1}+k_{y} \xi_{2}\right]} d k_{x} d k_{y}
\end{array}
$$

where $j=p$ or $j=s$, and the frequency variables $k_{x}$ and $k_{y}$ are the Cartesian components of the plane wave propagation vector

$$
\vec{k}=k_{x} \vec{e}_{1}+k_{y} \vec{e}_{2}
$$

corresponding to each of the field quantities $f^{s}$ or $f^{p}$, respectively. The angular frequency $\omega_{j}$ and the wave number $k=|\vec{k}|$ are related through the dispersion relation

$$
\omega=C_{j} k
$$

where we omit the use of subscripts for $\omega$ and $k$, and $C_{j}$ represents the wave speed $C_{p}$ or $C_{s}$, corresponding to the field component of interest. Next, consider that the propagating rupture is a source of waves moving along the $x$-axis at speed $V_{r}$, in which case the fault-parallel phase velocity equals the steady-state rupture speed, hence

$$
\omega=k_{x} V_{r}
$$


where $k_{x}=2 \pi / \lambda$. Combining Eq. 2.8 with the general dispersion relation Eq. 2.7 establishes a unique relationship between $k_{x}$ and $k_{y}$ given by

$$
k_{y}=i \alpha_{j} k_{x}
$$

where

$$
\alpha_{j}=\sqrt{1-\frac{V_{r}^{2}}{C_{j}^{2}}} .
$$

Note that $\alpha_{j}$ remains a real quantity if $V_{r} \leq C_{j}$. In particular, $\alpha_{s}$ remains real when rupture speeds are sub-shear, while $\alpha_{p}$ remains real when the rupture speed is sub-sonic. Moreover, since $k_{y}$ is now expressed in terms of $k_{x}$, the general 2D spectral representation of $f^{j}\left(\xi_{1}, \xi_{2}\right)$ given by Eq. 2.5 simplifies to

$$
f^{j}(x, y)=\frac{1}{\sqrt{2 \pi}} \int_{-\infty}^{\infty} e^{-\alpha_{j}\left|k_{x} y\right|} \hat{f}^{j}\left(k_{x}\right) e^{i k_{x}(x)} d k_{x}
$$

where $t=0$ is now taken for the sake of convenience such that $\xi_{1}=x-V_{r} t=x$, and $\xi_{2}=|y|$ assures an exponential decay of the spectral field components with increased FN distance. The form of Eq. 2.11 corresponds to the representation of an evanescent wave in the Fourier domain. Rigorous derivations of evanescent wave fields arising from sub-Rayleigh ruptures have been previously established for a dislocation source model (Aki and Richards, 2002), as well as for dynamic rupture models (Kostrov, 1966; Kostrov et al., 1969; Kostrov and Nikitin, 1970; Freund, 1990; Broberg, 1999). Next, note that the source spectrum representation of the field component $f^{j}(x, y)$ is obtained by setting $y=0$ in Eq. 2.11, in which case

$$
f^{j}(x, 0)=\frac{1}{\sqrt{2 \pi}} \int_{-\infty}^{\infty} \hat{f}^{j}\left(k_{x}\right) e^{i k_{x}(x)} d k_{x}
$$

It then follows from the convolution theorem that

$$
f^{j}(x, y)=\frac{1}{\sqrt{2 \pi}} \int_{-\infty}^{\infty} f^{j}(x-\xi, 0) h(\xi, y) d \xi
$$


where $\xi$ is the integration variable and

$$
h(\xi, y)=\frac{1}{\sqrt{2 \pi}} \int_{-\infty}^{\infty} e^{-\alpha_{j}\left|k_{x} y\right|} e^{i k_{x} \xi} d k_{x}
$$

represents the effective "impulse response" of the surrounding 2D linear elastic medium. An explicit form of the impulse response function, obtained by evaluating the Fourier transform expression (Eq. $2.14)$, is given by

$$
h(\xi, y)=\sqrt{\frac{2}{\pi}}\left[\frac{\alpha_{j} y}{\left(\alpha_{j} y\right)^{2}+\xi^{2}}\right] .
$$

The magnitude and subsequent decay of the radiated P- and S-wave field contributions of a given stress or particle velocity field component with increased FN distance $y$ from a steady-state subRayleigh rupture is thus governed by the convolution of the corresponding on-fault (source) field strength with the $2 \mathrm{D}$ impulse response function $h(\xi, y)$.

The radiated $\mathrm{P}$-wave and S-wave fields arising from a rupture source are generally superimposed and not easily decomposed. The relative contribution of each radiation term at a given off-fault location is therefore difficult to assess unless a unique condition arises whereby one of the contributing field terms is absent. Such is the case, for example, at a field point taken sufficiently far from the fault where the dilatational field contribution has essentially vanished in comparison to the shear field radiation term. Another unique case applies to the dilatational field radiated by a supershear rupture $\left(C_{s} \leq V_{r} \leq C_{p}\right)$, in which case the field strength outside of the shear Mach cone is described by the sub-sonic $(j=p)$ form of the convolution integral Eq. 2.13. This point is re-addressed later in relation to experimental measurement of supershear ground motion signatures. Note that the evanescent wave field description, as expressed by Eq. 2.13, is strictly applicable to ruptures propagating under steady-state conditions. In fact, (Madariaga, 1977) showed that a decelerating or an accelerating rupture can emit high-frequency radiation into the medium and may no longer be subject to the above attenuation relationship. Bearing this point and other possible steady-state rupture scenarios in mind, Eq. 2.13 nonetheless effectively demonstrates how a stress or particle velocity field component arising from a sub-Rayleigh rupture is destined to attenuate with increased 
FN distance. The result further demonstrates how high-frequency content from a sub-Rayleigh rupture pulse source spectrum is rapidly stripped away with increased FN distance, thus making it very difficult to obtain detailed information about the breakdown zone from off-fault ground motion records (Dunham and Archuleta, 2005).

\subsubsection{Non-decaying nature of shear radiation fields emitted by a supers- hear dislocation $\left(C_{s} \leq V_{r} \leq C_{p}\right)$}

In the case of a supershear rupture $C_{s} \leq V_{r} \leq C_{p}$, a unique condition arises whereby $\alpha_{s}$ becomes imaginary while $\alpha_{p}$ remains real. It is then convenient to define the quantity

$$
\beta_{s}=\sqrt{\frac{V_{r}^{2}}{C_{s}^{2}}-1}
$$

in place of $\alpha_{s}$, which remains real for $C_{s} \leq V_{r} \leq C_{p}$, in which case

$$
k_{y}^{s}=\beta_{s} k_{x}^{s}
$$

where the superscript $s$ is now included in order to reinforce the fact that Eq. 2.17 applies strictly to the S-field contribution $f^{s}(x, t)$ while the dilatational field contribution $f^{p}$ is still subject to decay as previously described. Substituting Eq. 2.17 for $k_{y}$ in Eq. 2.5 leads to a spectral representation of the shear field contribution given by

$$
f^{s}\left(\xi_{1}, \xi_{2}\right)=\frac{1}{\sqrt{2 \pi}} \int_{-\infty}^{\infty} \hat{f}^{s}\left(k_{x}\right) e^{i k_{x} \xi_{1}} e^{i k_{x} \beta_{s} \xi_{2}} d k_{x}
$$

Note the absence of an attenuating factor in the Fourier transform relation, which clearly distinguishes this result from the sub-Rayleigh result Eq. 2.11. Application of the Fourier transform shift theorem now yields

$$
f^{s}\left(\xi_{1}, \xi_{2}\right)=f^{s}\left(\xi_{1}+\beta_{s} \xi_{2}\right)
$$




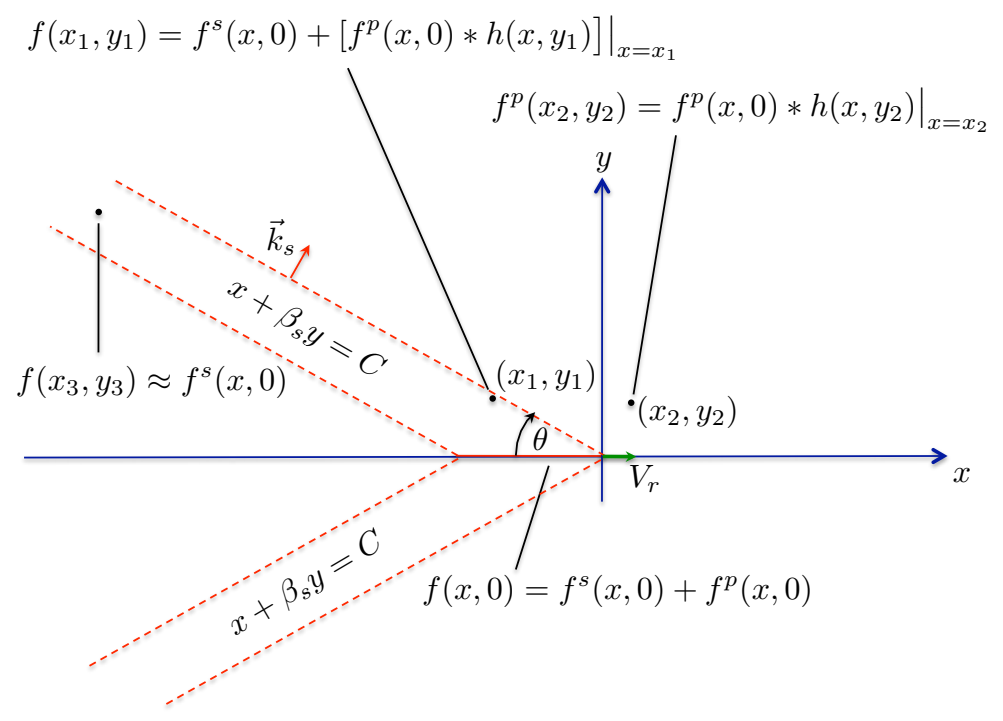

Figure 2.1: S-wave radiation from a supershear slip pulse propagated out to infinity along characteristics (Mach fronts)

implying that the S-wave field remains unattenuated and radiates out to infinity along characteristic lines stemming from the rupture front defined by

$$
\xi_{1}+\beta_{s} \xi_{2}=C
$$

Note that $C=0$ since the origin of the coordinate system $\left(\xi_{1}, \xi_{2}\right)$ is situated at the rupture tip. The equation of a characteristic line, which emanates from the rupture tip is thus given by

$$
\xi_{2}=-\frac{1}{\beta_{s}} \xi_{1}
$$

from which it follows that

$$
\tan \theta=\frac{1}{\beta_{s}}
$$

where $\theta$ is the acute angle between the $\mathrm{x}$-axis and the $\mathrm{S}$-wave characteristic as depicted in Figure 2.1. Finally, from the definition of $\beta_{s}$, as expressed through Eq. 2.16, it is straightforward to show that

$$
\tan \theta=\frac{C_{s} / V_{r}}{\sqrt{1-C_{s}^{2} / V_{r}^{2}}}
$$



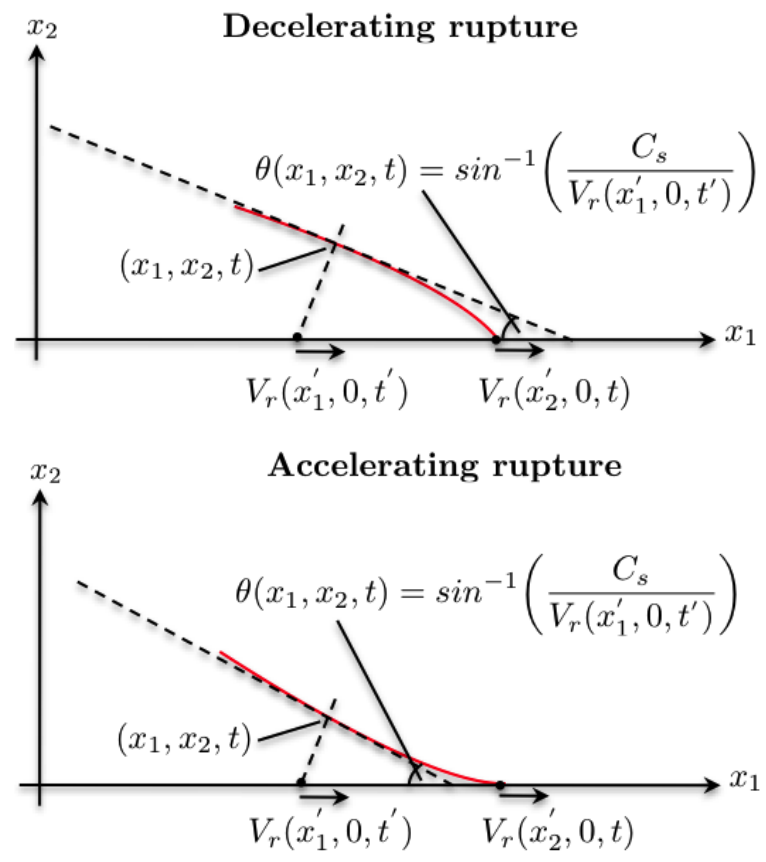

Figure 2.2: Curved Mach-front profiles resulting from decelerating or accelerating supershear ruptures

which corresponds with the Mach cone half-angle relation Eq. 1.1. The S-wave field characteristics extending from the front and rear of the slip zone out to infinity are evidently Mach fronts, which bound the radiated S-wave field, as depicted in Figure 2.1. The figure assumes $t=0$ for the sake of convenience such that $\xi_{1}=x-V_{r} t=x$ and $\xi_{2}=y$. Note that the particle velocity and stress field components lying outside of the shear Mach cone must be purely dilatational in character and are therefore subject to attenuate in accordance with Eqs. 2.13 and 2.15, since $\alpha_{p}$ remains a real quantity. This point is emphasized by the point labeled $\left(x_{2}, y_{2}\right)$ in Figure 2.1. The results further imply that the near-field region bounded by the leading and trailing slip zone Mach fronts comprises the unattenuated S-wave field and a superimposed, attenuating dilatational field as depicted at $\left(x_{1}, y_{1}\right)$ in Figure 2.1. Finally, at field points located far from the source where the dilatational field has decayed away, such as the point labeled $\left(x_{3}, y_{3}\right)$ in Figure 2.1, the field quantity $f\left(x_{3}, y_{3}\right)$ is shear-field dominated and is essentially a scaled replica of the on-fault slip distribution (Dunham and Archuleta, 2005).

The notion of perfectly planar Mach fronts arising from a steady-state 2D rupture is a useful idealization. In actuality, a propagating supershear rupture may very well exhibit an unsteady 
rupture velocity history, characterized by an accelerating or decelerating rupture front, thereby leading to a curved Mach-front profile as depicted in Figure 2.2. In such cases the angle $\theta$ formed by a line drawn tangent to the Mach front at $\left(x_{1}, x_{2}, t\right)$ is directly related to the rupture velocity at an earlier point in time $\left(x_{1}^{\prime}, 0, t^{\prime}\right)$ in accordance with Eq. 2.24. In geometric terms, $\left(x_{1}^{\prime}, 0, t^{\prime}\right)$ is the point of intersection along the fault with a normal line drawn from the point of tangency $\left(x_{1}, x_{2}, t\right)$ along the Mach front as depicted in the figures.

$$
\theta\left(x_{1}, x_{2}, t\right)=\sin ^{-1}\left[\frac{C_{s}}{V_{r}\left(x_{1}^{\prime}, 0, t^{\prime}\right)}\right]
$$

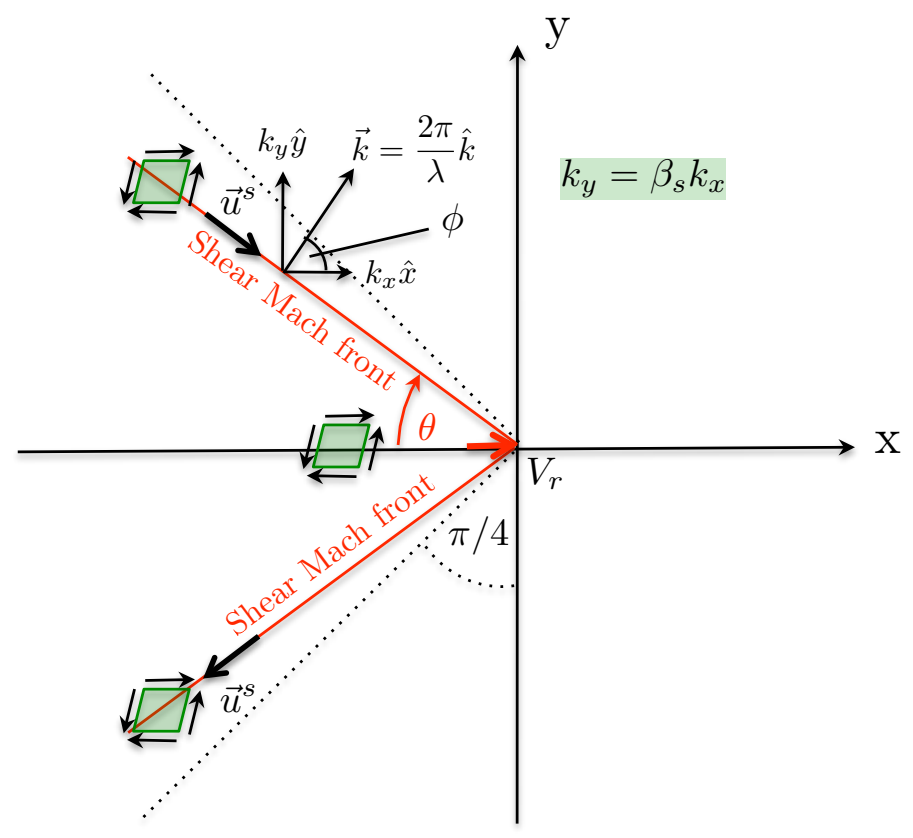

Figure 2.3: Mach fronts arising from a supershear source propagated out to infinity along characteristics 


\subsubsection{Analysis and interpretation of dislocation shear Mach fronts in the plane wave basis}

Recall once again the inverse Fourier transform representation of an S-wave field quantity $f^{s}\left(\xi_{1}, \xi_{2}\right)$ given by

$$
f^{s}\left(\xi_{1}, \xi_{2}\right)=\int_{-\infty}^{\infty} \hat{f}^{s}\left(k_{x}\right) e^{i\left[k_{x} \xi_{1}+\beta_{s} k_{x} \xi_{2}\right]} d k_{x}
$$

arising from a 2D steady-state supershear rupture where $k_{y}=\beta_{s} k_{x}$. The supershear source spectrum representation, expressed by Eq. 2.25 represents the integration of all plane wave components having wave number $k=2 \pi / \lambda$, which collectively comprise $f^{s}\left(\xi_{1}, \xi_{2}\right)$ along the resulting shear Mach front. The contribution from an individual plane wave component, having wave number $k=2 \pi / \lambda$, is therefore given by

$$
d f^{s}\left(\xi_{1}, \xi_{2}\right)=\hat{f}^{s}\left(k_{x}\right) e^{i\left[k_{x} \xi_{1}+\beta_{s} k_{x} \xi_{2}\right]} d k_{x}
$$

This representation effectively defines the plane wave basis, which provides a convenient means of analyzing the displacement or stress field components along a shear Mach front. Figure 2.3 depicts a pair of planar shear Mach fronts stemming from a supershear slip pulse propagating in the positive x-direction. A single plane wave contribution (of wavelength $\lambda$ ), to the shear displacement field along the Mach front may then be expressed as

$$
u^{s}\left(\xi_{1}, \xi_{2} ; \lambda\right)=\hat{u}^{s}(\lambda) e^{i\left[k_{x} \xi_{1}+\beta_{s} k_{x} \xi_{2}\right]}
$$

where the differential notation has been dropped for the sake of convenience and the symbol $\hat{u}^{s}(\lambda)=\hat{u}^{s}\left(k_{x}\right) d k_{x}$ has been adopted to denote the fact that we are actually referring to a single wavelength contribution of the entire plane wave spectrum. All of the wavelength contributions to the displacement field $u^{s}\left(\xi_{1}, \xi_{2}\right)$ along the shear Mach front are represented by similar plane wave expressions, which may differ in amplitude depending upon the functional nature of the rupture source spectrum. The individual plane wave components, each defined by a wave vector $\vec{k}=(2 \pi / \lambda) \hat{k}$, are

all superimposed in space with their unit vectors $\hat{k}$ oriented normal to the shear Mach front and 
inclined at an angle $\phi$ with respect to the $\mathrm{x}$-axis, as depicted in Figure 2.3. Note that $t=0$ is assumed for the sake of convenience, such that $\xi_{1}=x-V_{r} t=x$ and $\xi_{2}=y$. Next, consider the plane wave basis representation of the FP and FN displacement components, $u_{x}^{s}$ and $u_{y}^{s}$, which may be expressed as

$$
\begin{aligned}
& u_{x}^{s}\left(\xi_{1}, \xi_{2} ; \lambda\right)=\hat{u}_{x}^{s}(\lambda) e^{i\left[k_{x} \xi_{1}+\beta_{s} k_{x} \xi_{2}\right]} d k_{x} \\
& u_{y}^{s}\left(\xi_{1}, \xi_{2} ; \lambda\right)=\hat{u}_{y}^{s}(\lambda) e^{i\left[k_{x} \xi_{1}+\beta_{s} k_{x} \xi_{2}\right]} d k_{x} .
\end{aligned}
$$

Invoking Eq. 2.3 along with Eqs. 2.28 and 2.29 in the plane wave basis leads to

$$
\nabla \cdot \vec{u}_{\lambda}^{s}=i k_{x} u_{x}^{s}(\lambda)+i \beta_{s} k_{x} u_{y}^{s}(\lambda)=0
$$

Substituting Eq. 2.17 then leads to

$$
u_{x}^{s}(\lambda)=-\beta_{s} u_{y}^{s}(\lambda)
$$

The expression immediately reveals that $\left|u_{y}^{s}\right|>\left|u_{x}^{s}\right|$ (FN component dominates over FP component) when $0 \leq \beta_{s}<1$, corresponding to the rupture speed regime $\left(C_{s} \leq V_{r}<\sqrt{2} C_{s}\right)$, whereas $\left|u_{x}^{s}\right|>\left|u_{y}^{s}\right|$ (FP component dominates over FN component) when $1<\beta_{s} \leq \sqrt{\left(C_{p} / C_{s}\right)^{2}-1}$, corresponding to the speed regime $\left(\sqrt{2} C_{s}<V_{r} \leq C_{p}\right)$. Special cases of interest that limit the upper bound of $\beta_{s}$ in the stable rupture speed regime are (1) if $C_{p}=\sqrt{3} C_{s}$, often assumed for crustal rock, where $1<\beta_{s} \leq \sqrt{2}$, and (2) $C_{p} \approx 2 C_{s}$, applicable to H-100 test specimens used in laboratory earthquake experiments, where $1<\beta_{s} \leq \sqrt{3}$. The two Mach angle domains bounded to either side of $\theta=\pi / 4$ are graphically depicted in Figure 2.4. Once again, $t=0$ is assumed for the sake of convenience such that $\xi_{1}=x-V_{r} t=x$ and $\xi_{2}=y$. The right-hand diagram demonstrates how the faultparallel velocity component dominates over the fault-normal velocity component along the shear Mach front in the stable supershear regime $(\theta<\pi / 4)$, whereas the opposite case applies in the left-hand diagram, corresponding to the unstable rupture speed regime $(\theta>\pi / 4)$. Note as well how the direction of the displacement vector $\vec{u}_{s}$ is depicted pointing upward along the shear Mach 

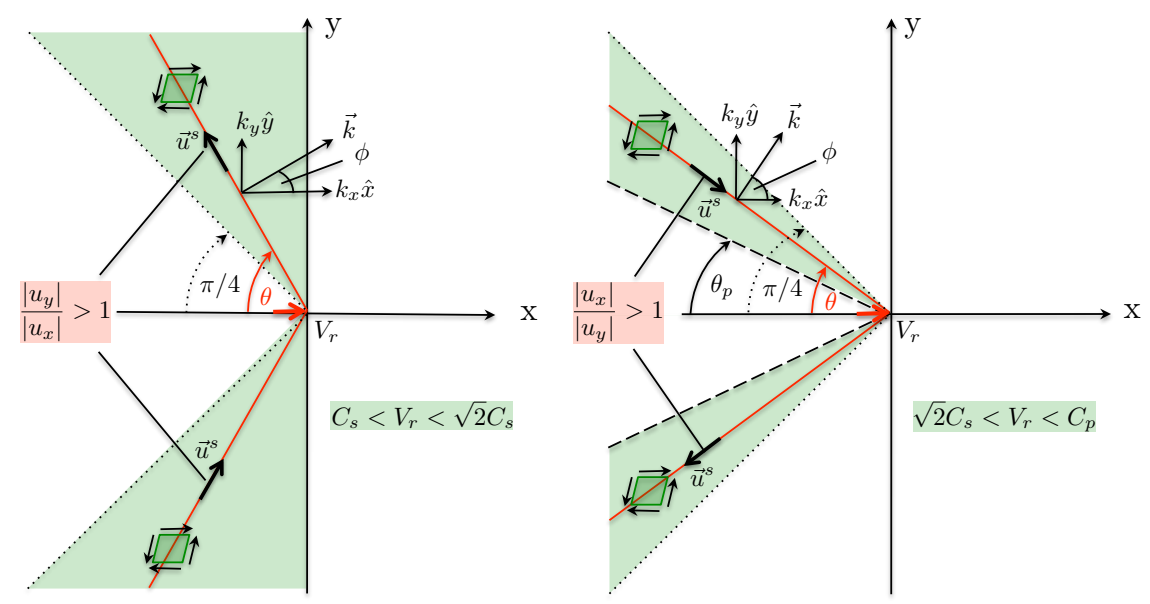

Figure 2.4: Range of Mach angles corresponding to each of the supershear rupture speed regimes

front in the left-hand diagram where $\theta>\pi / 4$, as opposed to pointing downward along the Mach front, as depicted in the right-hand diagram where $\theta<\pi / 4$, in accordance with the sense of motion of the contributing shear wavelets at points of tangency to the Mach front. Following the Poisson displacement field decomposition Eq. 2.1, the radiated strain field tensor arising from a supershear rupture may then also be expressed as the sum of shear and dilatational field contributions

$$
\epsilon_{i j}=\epsilon_{i j}^{s}+\epsilon_{i j}^{p}
$$

Application of the strain-displacement relations applied to the plane wave basis expressions (Eqs. 2.28 and 2.29) and combining with Eq. 2.31 leads to the strain field components of the S-wave field term given by

$$
\begin{gathered}
\epsilon_{x x}^{s}(\lambda)=i k_{x} u_{x}^{s}(\lambda) \\
\epsilon_{y y}^{s}(\lambda)=-i k_{x} u_{x}^{s}(\lambda)=-\epsilon_{x x}^{s}(\lambda) \\
\epsilon_{x y}^{s}(\lambda)=\frac{i \mu k_{x}}{2} u_{x}^{s}(\lambda)\left[\beta_{s}-\frac{1}{\beta_{s}}\right] .
\end{gathered}
$$

Application of Hooke's law and invoking the fact that $\epsilon_{k k}^{s}=0$ leads to S-wave field stress tensor

$$
\sigma_{i j}^{s}(\lambda)=2 \mu \epsilon_{i j}^{s}(\lambda)
$$


When $V_{r}=\sqrt{2} C_{s}$, corresponding to $\theta=\pi / 4$, it follows from Eq. 2.31 that $u_{x}^{s}=-u_{y}^{s}$, since $\beta_{s}=1$. Combining this with the fact that $\vec{u}^{s}$ reverses direction at $\theta=\pi / 4$, in accordance with the doublecouple solution, as graphically reinforced in Figure 1.1, implies that $u_{x}^{s}=u_{y}^{s}=0$ and thus $\vec{u}^{s}=0$ at this unique rupture speed. Hence, by Eqs. 2.33, 2.34, and 2.35 it follows that $\sigma_{i j}^{s}$, will also vanish when $V_{r}=\sqrt{2} C_{s}$, thus leaving only a dilatational stress field contribution, which curls around the slip zone and eventually attenuates with off-fault distance. Simply stated, a supershear rupture will not radiate any $\mathrm{S}$-waves into the surrounding medium when $V_{r}=\sqrt{2} C_{s}$. The result is consistent with the fact that the locus of points that collectively comprise the Mach front at this rupture speed are the nodal points corresponding to $\phi=\pi / 4$ on the shear wavelets, as depicted in Figure 1.1. The vanishing of all S-wave radiation at $V=\sqrt{2} C_{s}$, as motivated here using a simple spectral analysis, also emerges from more rigorous elastodynamic solutions for a 2D steady-state rupture as addressed in the following section.

A continuum-mechanics-based treatment of the significance of the rupture speed $V_{r}=\sqrt{2} C_{s}$ in isotropic, steady-state elastodynamics problems is found in (Liu et al., 1995) and (Gao et al., 1999). In particular, the paper by (Liu et al., 1995) uses continuum mechanics to show that a shear Mach front features jumps in shear stress and particle velocity. These jumps are shown to disappear when the rupture speed reaches $V_{r}=\sqrt{2} C_{s}$. Consistent with the above discussion, the work of (Gao et al., 1999) uses a continuum-mechanics approach to identify radiation-free stress states for various steady-state, linear elastodynamics problems, including dislocations and cracks. Here again the unique rupture speed $V_{r}=\sqrt{2} C_{s}$ emerges as one which corresponds to "radiation free" growth of dynamically propagating singularities. 


\section{$2.22 \mathrm{D}$ elastodynamic theory: Singular linear elastic solution for a propagating shear crack}

\subsubsection{Particle velocity field arising from a shear crack in the sub-Rayleigh rupture speed regime $\left(V_{r}<C_{R}\right)$}

(Freund, 1979, 1990) rigorously solved the 2D plane strain elastodynamic problem of a dynamically propagating shear (mode II) crack in a linear elastic half-space. The problem was posed in a Cartesian coordinate frame with the crack front oriented parallel to the $x_{3}$ axis such that the displacement field $\vec{u}=\vec{u}\left(x_{1}, x_{2}\right)$. The crack was assumed to propagate strictly along the $x_{1}$ direction with crack-tip coordinates given by $\left(x_{1}=l(t), 0\right)$, where $l(t)$ is a continuous function of time. The instantaneous crack-tip speed is then given by $V_{r}(t)=\dot{l}(t)$. A local coordinate system $\left(\xi_{1}, \xi_{2}\right)$ was then introduced with the origin selected to coincide with the crack tip, such that $\xi_{1}=x_{1}-l(t)$ and $\xi_{2}=x_{2}$. A local polar coordinate system $(r, \theta)$ was also introduced, whereby $r=\sqrt{\xi_{1}^{2}+\xi_{2}^{2}}$ and $\theta=\tan ^{-1}\left(\xi_{2} / \xi_{1}\right)$. By imposing the steady-state assumption and taking the origin of a new coordinate system to coincide with the crack tip, the governing wave equation expressions for the dilatational displacement potential $\phi$ and shear displacement potential $\psi$ become two-dimensional in $\xi=x-V_{r} t$ and $\xi_{2}=y$ under a Galilean coordinate transformation. The derivation ultimately leads to a standard Hilbert problem in analytic function theory, which is solved subject to the prescribed traction-free boundary conditions on the crack surfaces, in order to obtain the asymptotic stress and particle velocity field solutions. The singular elastic (asymptotic) solution for the particle velocity field components $\dot{u}_{x}(r, \theta)$ and $\dot{u}_{y}(r, \theta)$ is given by

$$
\begin{aligned}
\dot{u}_{x}= & -\operatorname{sgn}\left(\xi_{2}\right) \frac{V_{r} \alpha_{s} K_{I I}(t)}{\mu D \sqrt{2 \pi r}}\left[2 \frac{\sin \frac{1}{2} \theta_{p}}{\sqrt{\gamma_{p}}}-\right. \\
& \left.\left(1+\alpha_{s}^{2}\right) \frac{\sin \frac{1}{2} \theta_{s}}{\sqrt{\gamma_{s}}}\right]
\end{aligned}
$$


and

$$
\begin{aligned}
\dot{u}_{y}= & -\frac{V_{r} K_{I I}(t)}{\mu D \sqrt{2 \pi r}}\left[2 \alpha_{p} \alpha_{s} \frac{\cos \frac{1}{2} \theta_{p}}{\sqrt{\gamma_{p}}}-\right. \\
& \left.\left(1+\alpha_{s}^{2}\right) \frac{\cos \frac{1}{2} \theta_{s}}{\sqrt{\gamma_{s}}}\right]
\end{aligned}
$$

where $K_{I I}(t)$ is the instantaneous mode II stress intensity factor, $\mu$ is the shear modulus, and $\alpha_{s}$, $\alpha_{p}$ are given by Eq. 2.10. $\operatorname{sgn}(x)$ is the signum function, which is equal to +1 for $x>0$ and -1 for $x<0$. The remaining factors $D$ (known as Rayleigh function), $\theta_{p}, \theta_{s}, \gamma_{d}$, and $\gamma_{s}$ are given by

$$
\begin{gathered}
D=4 \alpha_{p} \alpha_{s}-\left(1+\alpha_{s}^{2}\right)^{2} \\
\theta_{p}=\tan ^{-1}\left(\alpha_{p} \tan \theta\right) ; \theta_{s}=\tan ^{-1}\left(\alpha_{s} \tan \theta\right)
\end{gathered}
$$

and finally,

$$
\begin{aligned}
& \gamma_{p}=\sqrt{1-\left(V_{r} \sin \theta / C_{p}\right)^{2}} \\
& \gamma_{s}=\sqrt{1-\left(V_{r} \sin \theta / C_{s}\right)^{2}} .
\end{aligned}
$$

The algebraic sign of the coefficient of the singularity in the mode II particle velocity (and stress) field expressions is controlled by the sign of the Rayleigh function $(D)$. Note how $D \rightarrow 0$ as $V_{r} \rightarrow C_{R}$, and furthermore that $D>0$ when $V_{r}<C_{R}$, and $D<0$ when $C_{R}<V_{r}<C_{s}$. Careful inspection of the (sub-Rayleigh) mode II field solutions (taking into account the sign of $D$ ) reveals that there will be a net flux of energy into the crack tip whenever $V_{R}<C_{R}$, whereas a net flux of energy will be directed out from the crack tip if $C_{R}<V_{r}<C_{s}$. The latter scenario is rejected on physical grounds leading to the conclusion that a sub-Rayleigh mode II crack is necessarily confined to the sub-Rayleigh speed rupture speed regime $V_{R}<C_{R}$. 


\subsubsection{Dominance of the fault-normal particle velocity component in the sub-Rayleigh rupture speed regime $\left(V_{r}<C_{R}\right)$}

Figure 2.5 displays the particle velocity field $\vec{u}\left(\xi_{1}, \xi_{2}\right)$ in the immediate neighborhood of a dynamic shear crack propagating at $V_{r}=0.875 C_{s}$. The colors correspond to $|\dot{\vec{u}}|$, while the white arrows of constant magnitude form a streak plot, which indicates the sense of motion throughout the field. The plot corresponds to a right lateral shear rupture, as indicated by the sense of particle motion above and below the crack plane (dashed black line in the figure). The most obvious and striking feature of the result is dominance of the FN particle velocity component over the FP velocity component for $\xi_{1}>0$, as revealed by the inclination of the velocity vectors, which are predominantly pointing in the vertical direction. Particle velocity records acquired at the two fictitious measurement stations displayed in the figure are thus expected to exhibit a FN velocity swing, which dominates in magnitude over the $\mathrm{FP}$ velocity swing, i.e.,

$$
\left|\dot{u}_{y_{\max }}-\dot{u}_{y_{\min }}\right|>\left|\dot{u}_{x_{\max }}-\dot{u}_{x_{\min }}\right|, \quad\left(V_{r}<C_{R}\right)
$$

as the sub-Rayleigh crack-tip field sweeps through each respective station. Apart from the fact

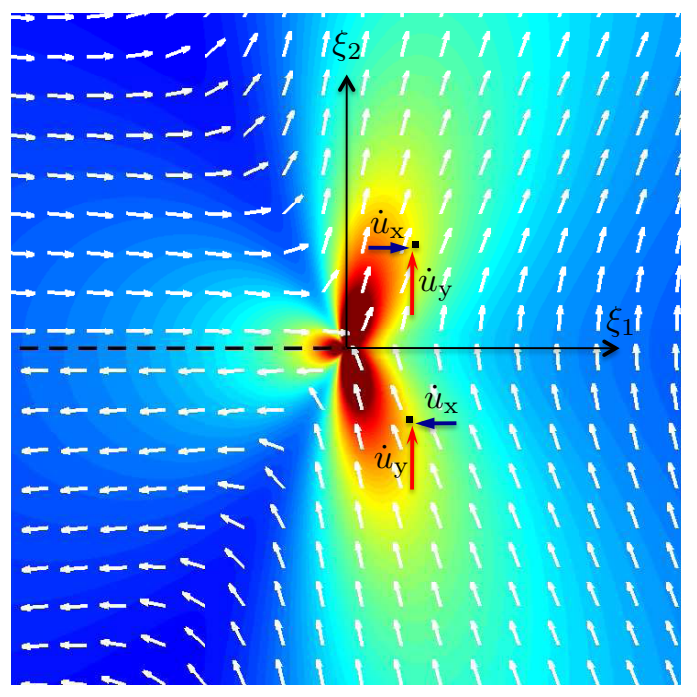

Figure 2.5: The near-tip particle velocity field for a shear crack propagating at $V_{r}=0.875 C_{s}$

that there is a sharp singularity at $r=0$ and that the equations lack a meaningful length scale, 

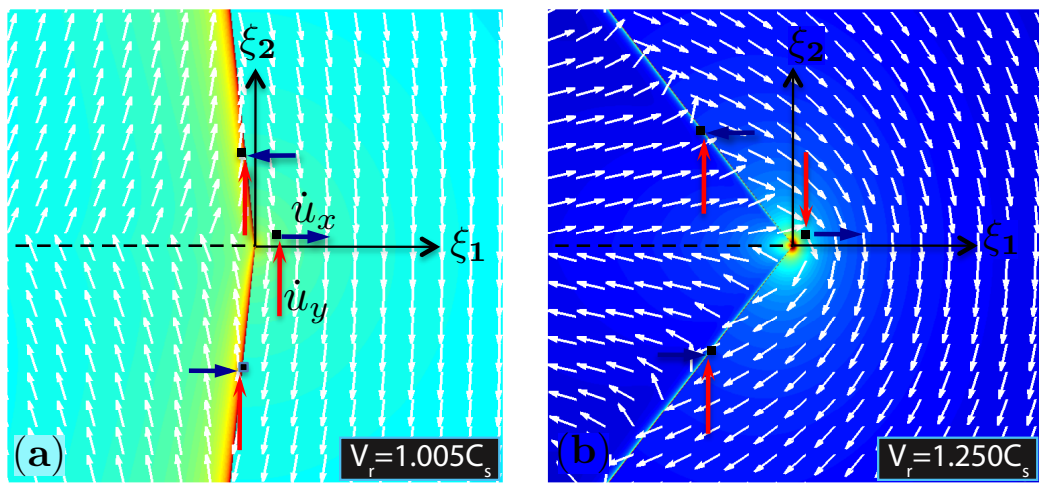

0

$2 \mathrm{~m} / \mathrm{s}$
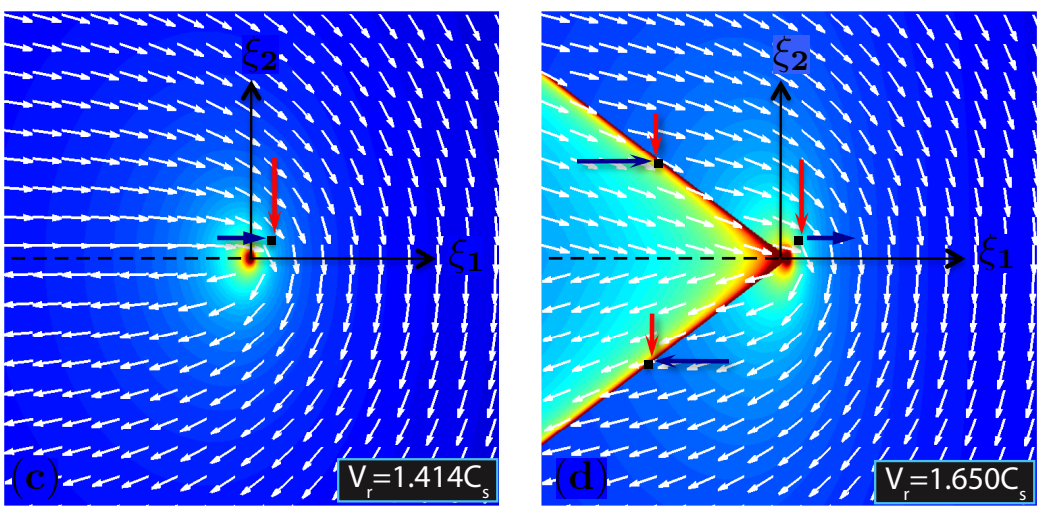

Figure 2.6: Singular elastic particle velocity field plots for a supershear crack propagating at (a) $V_{r}=1.005 C_{s}$, (b) $V_{r}=1.250 C_{s}$, (c) $V_{r}=\sqrt{2} C_{s}$, (d) $V_{r}=1.650 C_{s}$. The white arrows of constant magnitude form a streak plot, which indicates the sense of motion throughout the field.

the solution in the neighborhood of the advancing crack tip is nonetheless representative of the particle velocity field resulting from a sub-Rayleigh rupture. Similar ground motion signatures associated with sub-Rayleigh rupture propagation in natural earthquakes, which feature a dominant FN motion component, were numerically demonstrated by (Aagaard and Heaton, 2004) using a kinematic rupture model. Rigorous elastodynamic solutions, which consider the frictional fault resistance between the shear crack faces and a cohesive (breakdown) zone ahead at the rupture front, also lead to particle velocity field solutions featuring a dominant FN component, particularly in the half-space $\xi_{1}>0$ situated ahead of the advancing rupture front (Dunham and Archuleta, 2005). A major distinction of these solutions over the singular elastic solution for a propagating shear crack, is the emergence of a meaningful length scale, resulting from an assumed length of the break-down zone. 


\subsubsection{Particle velocity field from a shear crack in the supershear regime}

$$
\left(C_{s} \leq V_{r} \leq C_{p}\right)
$$

(Freund, 1979, 1990) also considered the elastodynamic solution for a dynamic shear crack propagating into the supershear rupture speed regime subject to the same coordinate frame description and traction-free boundary conditions as were assumed in the sub-Rayleigh problem. The closed-form solution that emerges for the particle velocity components $\dot{u}_{x}$ and $\dot{u}_{y}$ is conveniently expressed as the sum of a dilatational and shear field contribution

$$
\dot{u}_{j}=A V_{r}\left(\dot{u}_{j}^{p}+\dot{u}_{j}^{s}\right)
$$

where $j=1,2$, and $A$ represents a stress intensity factor. The dilatational field contributions to the FP and FN particle velocity components are given by

$$
\dot{u}_{x}^{p}=\frac{\sin \left(q \theta_{p}\right)}{r_{p}^{q}} ; \quad \dot{u}_{y}^{p}=-\alpha_{p} \frac{\cos \left(q \theta_{p}\right)}{r_{p}^{q}}
$$

with variables $r_{p}$ and $q$ given by

$$
\begin{gathered}
r_{p}=\sqrt{\xi_{1}^{2}+\left(\alpha_{p} \xi_{2}\right)^{2}} \\
q=\frac{1}{\pi} \tan ^{-1}\left[\frac{4 \alpha_{p} \beta_{s}}{\left(2-V_{r}^{2} / C_{s}^{2}\right)^{2}}\right] .
\end{gathered}
$$

Note that $0 \leq q \leq 1 / 2$ and that the value $q=1 / 2$ is only attained when $V_{r}=\sqrt{2} C_{s}$, where the S-wave field is known to vanish. Hence, the singularity introduced by the exponent $q$ is notably weaker than the $r^{-1 / 2}$ singularity, which arises in the sub-Rayleigh solution.

The shear-field contributions to the FP and FN particle velocity components are given by

$$
\begin{aligned}
\dot{u}_{x}^{s}= & -\operatorname{sgn}\left(\xi_{2}\right) \frac{\beta_{s}^{2}\left(2-\frac{V_{r}^{2}}{C_{s}^{2}}\right) \sin (\pi q)}{2 \beta_{s}\left(\left|\xi_{1}+\beta_{s} \xi_{2}\right|\right)^{q}} \times \\
& H\left(-\xi_{1}-\beta_{s}\left|\xi_{2}\right|\right)
\end{aligned}
$$


and

$$
\dot{u}_{y}^{s}=\frac{\left(2-\frac{V_{r}^{2}}{C_{s}^{2}}\right) \sin (\pi q)}{2 \beta_{s}\left(\left|\xi_{1}+\beta_{s} \xi_{2}\right|\right)^{q}} H\left(-\xi_{1}-\beta_{s}\left|\xi_{2}\right|\right)
$$

where $\operatorname{sgn}(x)$ is the signum function, which is equal to +1 for $x>0$ and -1 for $x<0$.

\subsubsection{Particle motion along a shear Mach front and dominance of the fault-parallel component in the stable supershear rupture speed regime $\left(\sqrt{2} C_{s} \leq V_{r} \leq C_{p}\right)$}

The particle velocity field plots depicted in Figure 2.6 correspond to four different crack-tip speeds, which collectively span the entire supershear rupture speed regime. Each plot corresponds to a rightlateral rupture of a shear crack, which is represented by the dashed black line. The colors correspond

to $|\dot{\vec{u}}|$, while the white arrows of constant magnitude form a streak plot, which only reveals the sense of motion throughout the field. The plot in Figure 2.6a, corresponding to $V_{r}=1.005 C_{s}$, highlights how the shear Mach angle approaches $\theta=\pi / 2$ in the limit that $V_{r} \rightarrow C_{s}$. Note the dominance of the FN particle velocity component over the FP component along the shear Mach front, as expected in the unstable portion of the supershear rupture speed regime $\left(C_{s} \leq V_{r}<\sqrt{2} C_{s}\right)$. Care should be taken not to confuse the sense of motion suggested by the vectors on the purely dilatational side of the velocity field as indicative of motion along the shear Mach front. The direction and sense of rotation of the vector field in the wake of the rupture, where the field is shear dominated, reveals how particle motion is directed upwards along the shear Mach front. The particle velocity field is evidently characterized by a sharp reversal in the particle velocity field across the Mach front between the shear-dominated side and the purely dilatational region ahead of the Mach front.

Figure $2.6 \mathrm{~b}$ corresponds to a supershear rupture speed $V_{r}=1.25 C_{s}$. Note how the Mach angle has decreased with increased rupture speed but is still inclined such that $\theta>\pi / 4$, and that the particle velocity field along the shear Mach front is still dominated by the FN component. Particle motion along the shear Mach front is still directed upwards along the shear Mach front, as revealed by the direction and sense of rotation of the velocity vector field in the wake of the rupture tip.

The shear Mach fronts in frames (a) and (b) are both shown crossing hypothetical particle velocity measurement stations situated above and below the crack plane, with red and blue arrows 
representing the FN and FP velocity components, respectively. Particle velocity records acquired at these locations are thus expected to exhibit a sharp positive FN velocity swing which dominates in magnitude over the FP velocity swing, i.e.,

$$
\begin{array}{r}
\left|\dot{u}_{y_{\max }}-\dot{u}_{y_{\min }}\right|>\left|\dot{u}_{x_{\max }}-\dot{u}_{x_{\min }}\right| \\
\left(C_{s} \leq V_{r}<\sqrt{2} C_{s}\right) .
\end{array}
$$

Finally, note the presence of the purely dilatational field, which circulates about the crack tip and extends out a short distance out beyond the Mach front before rapidly attenuating. A hypothetical near-field measurement station, lying close to the crack plane and situated ahead of the advancing Mach front, should thus record an initial velocity swing induced by the passage of the locally concentrated dilatational field, followed by a sharp, positive velocity jump in the FN component as the shear Mach front crosses the station.

Figure 2.6c corresponds to the unique case corresponding to $V_{r}=\sqrt{2} C_{s}$, where the shear Mach front has now completely vanished as expected and previously predicted using a simple 2D steadystate spectral analysis. At this unique rupture speed, energy that would be otherwise radiated into the medium in the form of shear waves is instead converted into fracture energy. Note that the only radiation term arising from the crack tip is in the form of dilatational waves, as revealed by the concentrated field surrounding the crack tip, which rapidly attenuates with off-fault distance.

Finally, consider Figure 2.6d, corresponding to a supershear rupture speed $V_{r}=1.65 C_{s}$, where the rupture speed has now crossed over the $V_{r}=\sqrt{2} C_{s}$ threshold. Note the dominance of the FP particle velocity component over the FN component along the shear Mach front for $\theta<\pi / 4$, as expected in the rupture speed regime $\left(\sqrt{2} C_{s}<V_{r} \leq C_{p}\right)$. Particle motion along the shear Mach front is now directed downward along the shear Mach front, as revealed by the direction and sense of rotation of the velocity vector field in the wake of the rupture, where the field is shear dominated. It is interesting to note how the particle velocity field vectors on either side of the Mach front are now aligned in the same direction, indicating that there is no longer a sharp reversal in the 
particle velocity field across the Mach front, which occurs in the unstable rupture speed regime $C_{s} \leq V_{r}<\sqrt{2} C_{s}$. As with frames (a) and (b), the shear Mach fronts are shown crossing fictitious particle velocity measurement stations situated above and below the crack plane, with red and blue arrows representing the FN and FP velocity components, respectively. Particle velocity records acquired at these locations are thus expected to exhibit a sharp positive FP velocity swing which dominates in magnitude over the FN velocity swing, i.e.,

$$
\begin{aligned}
&\left|\dot{u}_{x_{\max }}-\dot{u}_{x_{\min }}\right|>\left|\dot{u}_{y_{\max }}-\dot{u}_{y_{\min }}\right| \\
&\left(\sqrt{2} C_{s}<V_{r} \leq C_{p}\right) .
\end{aligned}
$$

Note the concentrated dilatational field, which is spread out over a larger spatial extent and visibly contracted along the propagation direction at this increased rupture speed. A hypothetical near-field measurement station lying close to the crack plane and situated ahead of the advancing Mach front should again record a dilatational precursor signal, followed by a sharp, positive velocity jump in the FP component as the shear Mach front crosses the station.

\subsection{Steady-state cohesive zone models}

The main underlying assumption in the singular elastic crack models discussed earlier is that the inelastic breakdown processes associated with the crack tip are confined to its vicinity. Such models do not allow, nor do they take into account the change in the tractions which occur in such breakdown zones. By adopting an approach that was analogous to the cohesive zone models for tensile cracks developed by (Dugdale, 1960) and (Barenblatt, 1962), (Ida, 1972) and (Palmer and Rice, 1973) introduced the non-singular slip-weakening model for shear, or Mode II, cracks that provides a description of the tractions in the cohesive zone. In these models the shear traction along the crack undergoes a weakening or degradation process which begins when the shear stress reaches a finite material peak strength ahead of the crack tip and continues to degrade with increasing amount of slip, approaching a residual level of shear strength. This results in a spatial degradation of the shear 
strength behind the crack tip. This also introduces a characteristic length scale into the analysis, which is missing in the singular elastic models. The resulting length scale is proportional to the size of the breakdown/process zone behind the crack tip and varies with the speed of the crack tip. (Samudrala et al., 2002a,b) applied a slip-velocity-dependent shear strength breakdown law, commonly referred to as the velocity-weakening friction law, and obtained steady-state solutions to the propagating sub-Rayleigh and supershear Mode II crack problems. All of the above problems feature steady-state solutions that depend on the real and imaginary parts of the following integral (Muskhelishvili, 1953; Broberg, 1978, 1989, 1999)

$$
N\left(z_{p / s}\right)=c_{1}\left[\Omega\left(z_{p / s}\right)\right]^{q} \int_{\Gamma} \frac{\tau(w)}{\left[\Omega_{+}(w)\right]^{q}\left(w-z_{p / s}\right)} d w
$$

where $\Omega$ is an analytic function with branch cuts along the crack (or pulse), $\Gamma, c_{1}$ depends on the rupture speed, and $q$ is the rupture speed dependent exponent (Eq. 2.46. $\tau(x)$ is either prescribed a priori in slip-weakening-like models, or is obtained for slip-velocity-weakening models by solving a singular integral equation (Samudrala et al., 2002a,b). This integral is evaluated in the Cauchy Principal Value sense and has analytical closed-form solutions for certain forms of $\tau(x)$ and subRayleigh rupture velocities. For supershear cases the closed-form solution exists only for constant $\tau(x)$, i.e., $\tau(x)=\tau_{p}$.

\subsubsection{Overview of general 2D steady-state supershear slip-pulse solution}

(Dunham and Archuleta, 2005), extended the steady-state sub-Rayleigh slip-pulse model of (Rice et al., 2005) to the intersonic rupture velocity domain $\left(C_{s}<V_{r}<C_{p}\right)$. A general solution was obtained for the stress and particle velocity fields generated by a right-lateral, steady-state slip pulse of finite length L, propagating to the right $(x>0)$, and subject to a prescribed shear traction $\tau(x)$ along the slip zone. Figure 2.7 depicts the case of a propagating slip-pulse subject to a linear distance-weakening friction relation characterized by a breakdown zone of length $R$ over which $\tau(x)$ degrades linearly before settling to a residual strength level. 


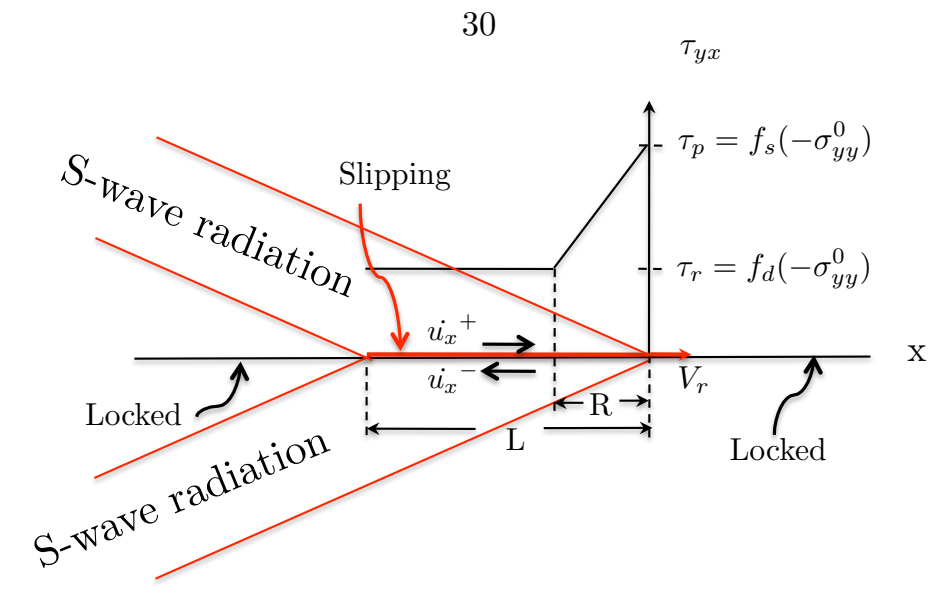

Figure 2.7: 2D steady-state sliding rupture pulse subject to linear distance-weakening friction. S-wave radiation is emitted exclusively from the slip zone.

The x-axis corresponds to the fault joining two identical, isotropic linear elastic half-spaces characterized by a shear modulus $\mu, \mathrm{P}$-wave speed $C_{p}$, and $\mathrm{S}$-wave speed $C_{s}$. The slip pulse is depicted propagating to the right in the positive $\mathrm{x}$ direction and corresponds to the case of right lateral slip. The mixed boundary value problem is summarized in Figure 2.8. $\tau(x)$ is a prescribed shear traction along the slip zone, while $\sigma_{y y}^{0}$ and $\sigma_{y x}^{0}$ represent the initial far field stresses exerted on the fault. The elastic fields are separated into dilatational and shear parts, each governed by the wave equation and the appropriate respective elastic wave speed. A complex elasticity solution to the steady-state slip-pulse problem leads to solutions for the stress and particle velocity fields in terms of a single integral expression for $\tau(x)$ over the slip zone, weighted by a singular kernel. The fault-parallel $\left(\dot{u}_{x}\right)$ and fault-normal $\left(\dot{u}_{y}\right)$ ground motion components are given by

$$
\begin{gathered}
\dot{u}_{x}=-\frac{V_{r}}{\mu}\left[\frac{1}{2 \alpha_{p}} \Im N\left(z_{p}\right)+\frac{\beta_{s}^{2}-1}{4 \alpha_{p}} \Im N\left(z_{s}\right)\right] \\
\dot{u}_{y}=-\frac{V_{r}}{\mu}\left[\frac{1}{2} \Re N\left(z_{p}\right)-\operatorname{sgn}(y) \frac{\beta_{s}^{2}-1}{4 \alpha_{p} \beta_{s}} \Im N\left(z_{s}\right)\right] \operatorname{sgn}(x)
\end{gathered}
$$

where the variables $z_{p}, z_{s}$ are given by

$$
z_{p}=x+i \alpha_{p} y \quad ; \quad z_{x}=x+\beta_{s} y
$$




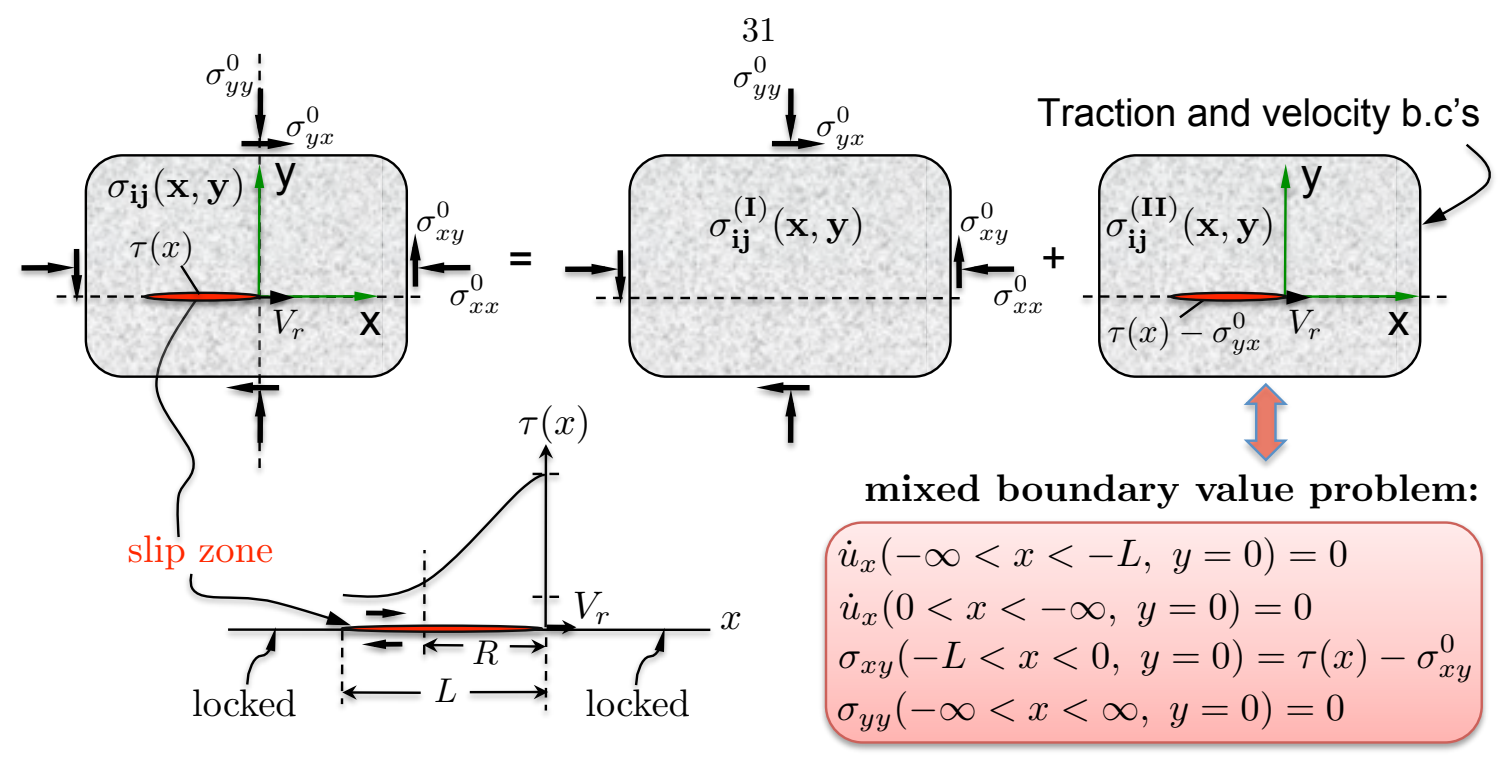

Figure 2.8: Mixed boundary value problem corresponding to general $2 \mathrm{D}$ steady-state supershear slip-pulse problem (Dunham and Archuleta, 2005)

and

$$
N(z)=-\frac{\sin (\pi q)}{\pi} z^{1-q}(z+L)^{q} \int_{-L}^{0} \frac{\tau(w)-\sigma_{x y}^{0}}{(-w)^{1-q}(w+L)^{q}(w-z)} d w
$$

represents a complex integral solution of the Hilbert problem, which is analytic everywhere except along a branch cut $(-L<x<0)$ corresponding to the slip zone. The variable $q$ corresponds to the same rupture-speed-dependent function in Eq. 2.46 as originally identified by (Freund, 1979, 1990). Furthermore, note that $z_{s}=x+\beta_{s} y$ represents a family of characteristics (shear Mach fronts), which propagate the on-fault shear field contribution in Eq. 2.52 and Eq. 2.53 out to infinity.

Finally, as noted by (Dunham and Archuleta, 2005), the stress and particle velocity field solutions are also expected to vanish at points far from the slip zone (outside of the radiation S-wave region). Enforcement of this boundary condition leads to a constraint on $\sigma_{x y}^{0}$ given by

$$
\sigma_{x y}^{0}=\frac{\sin (\pi q)}{\pi} \int_{-L}^{0} \frac{\sigma_{x y}^{0}(x)}{-x^{1-q}(x+L)^{q}} d x
$$

A careful examination of Eq. 2.55 at points very close to the fault (letting $z_{I} \rightarrow 0$ ) reveals that $N(z)$ is a complex function only within the interval $(-L<x<0)$, corresponding to the slip zone (Dunham and Archuleta, 2005). This implies that S-wave radiation must be confined within 
a region of space bounded by the leading and trailing Mach cones stemming from the points $x=0$ and $x=-L$. Moreover, due to the finite crack-tip stresses and a finite width process zone, the passage of the Mach front results in particle velocity jumps that are of finite width and magnitude, unlike the singular elastic crack models. Also, in stark contrast to the singular models, the crack-tip energy flux is non-zero and finite in the supershear regime (Broberg, 1989; Samudrala et al., 2002a,b; Rosakis et al., 2007).

\subsubsection{Recovery of the slip function at an off-fault location}

The standard definition for the slip function is given by

$$
\Delta \dot{u}_{x}(x, 0)=\dot{u}_{x}\left(x, 0^{+}\right)-\dot{u}_{x}\left(x, 0^{-}\right)
$$

where $\left(x, 0^{+}\right),\left(x, 0^{-}\right)$represent two closely spaced points located "just above and below" the fault. Furthermore $z_{p}=z_{s}=x^{+}$in the limit that $z_{I} \rightarrow 0^{+}$, and so Eqn. 2.52 assumes the form

$$
\dot{u}_{x}\left(x, 0^{+}\right)=-\frac{V_{r}}{\mu}\left[\frac{1}{2 \alpha_{p}} \Im N_{+}(x)+\frac{\beta_{s}^{2}-1}{4 \alpha_{p}} \Im N_{+}(x)\right]
$$

where

$$
N_{+}(x)=\frac{\sin (\pi q)}{\pi} e^{-i \pi q}\left[(-x)^{1-q}(x+L)^{q} \int_{-L}^{0} \frac{\tau(w)-\tau_{0}}{(-w)^{1-q}(w+L)^{q}(w-x)} d w+i \pi\left[\tau(x)-\tau_{0}\right]\right]
$$

is a complex expression containing a real integral, which is evaluated within the slip zone $(-L<$ $x<0)$.

Invoking anti-symmetry of the fault-parallel component, i.e., $\Delta \dot{u}_{x}(x, 0)=2 \dot{u}_{x}\left(x, 0^{+}\right)$in Eq. 2.58 leads to an expression for the slip function given by

$$
\Delta \dot{u}_{x}(x, 0)=-2 \frac{V_{r}}{\mu}\left(\frac{\beta_{s}^{2}+1}{4 \alpha_{p}}\right) \Im N_{+}(x) .
$$


Similarly, since $\Delta \dot{u}_{x}(x, 0)=-2 \dot{u}_{x}\left(x, 0^{-}\right)$(also by definition) and since $\Im N_{+}(x)=-\Im N_{-}(x)$ (mode II antisymmetry), it follows that

$$
\Delta \dot{u}_{x}(x, 0)=2 \frac{V_{r}}{\mu}\left(\frac{\beta_{s}^{2}+1}{4 \alpha_{p}}\right) \Im N_{-}(x) .
$$

and therefore,

$$
\Delta \dot{u}_{x}(x, 0)=\mp 2 \frac{V_{r}}{\mu}\left(\frac{\beta_{s}^{2}+1}{4 \alpha_{p}}\right) \Im N_{ \pm}(x)
$$

Next, invoke $\Delta \dot{u}_{x}(x, 0)=\Delta \dot{u}\left(z_{s}\right)$ and $\Im N_{+}(x)=\Im N\left(z_{s}\right)$, since $z_{s}=x+\beta_{s} y$ defines a characteristic extending from the fault out to infinity. Hence,

$$
\Delta \dot{u}_{x}\left(z_{s}\right)=-\operatorname{sgn}(y) 2 \frac{V_{r}}{\mu}\left(\frac{\beta_{s}^{2}+1}{4 \alpha_{p}}\right) \Im N\left(z_{s}\right)
$$

Substitution of Eq. 2.63 into Eq. 2.52 and Eqn. 2.53 yields the remarkable result that the shear contribution to both of the particle velocity field components scales directly with the slip function as expressed by Eq. 2.64 and Eq. 2.65 (Dunham and Archuleta, 2005).

$$
\begin{gathered}
\dot{u}_{x}(z)=-\frac{V_{r}}{\mu}\left[\frac{1}{2 \alpha_{p}} \Im N\left(z_{p}\right)\right]+\operatorname{sgn}(y) \frac{\beta_{s}^{2}-1}{2\left(\beta_{s}^{2}+1\right)} \Delta \dot{u}_{x}\left(z_{s}\right) \\
\dot{u}_{y}(z)=\left[-\frac{V_{r}}{\mu} \frac{1}{2} \Re N\left(z_{p}\right)-\frac{1}{2 \beta_{s}} \frac{\beta_{s}^{2}-1}{\beta_{s}^{2}+1} \Delta \dot{u}_{x}\left(z_{s}\right)\right] \operatorname{sgn}(x)
\end{gathered}
$$

Finally, far from the fault, where the dilatational field contribution has decayed away, the faultparallel and fault- normal particle velocity components along the shear Mach front reduce to

$$
\begin{gathered}
\dot{u}_{x}\left(z_{s}\right) \approx \operatorname{sgn}(y) \frac{1}{2} \frac{\beta_{s}^{2}-1}{\beta_{s}^{2}+1} \Delta \dot{u}_{x}\left(z_{s}\right) \\
\dot{u}_{y}\left(z_{s}\right) \approx-\operatorname{sgn}(x) \frac{1}{2 \beta_{s}} \frac{\beta_{s}^{2}-1}{\beta_{s}^{2}+1} \Delta \dot{u}_{x}\left(z_{s}\right) .
\end{gathered}
$$

The familiar relationship $\left|\dot{u}_{x}\right|=\beta_{s}\left|\dot{u}_{y}\right|$ is recovered far from the fault, as was previously obtained 
using a simple 2D steady-state spectral analysis. The sign and magnitude of the slip velocity function $\Delta \dot{u}_{x}$, along with $\beta_{s}$, in Eqs. 2.66 and 2.67, completely determine the sign and magnitude of the faultparallel and fault-normal ground motion components along the shear Mach front. Most remarkably is the fact that both far-field ground motion components carried by the shear Mach front are scaled replicas of the slip function back on the fault. This is a general feature of all steady-state Mode II cohesive zone solutions (Broberg, 1989; Samudrala et al., 2002a; Dunham and Archuleta, 2005) irrespective of the details of the cohesive zone law used. This characteristic of supershear S-wave radiation is in stark contrast to the nature of S-wave radiation arising from sub-Rayleigh ruptures, which is stripped of high-frequency content and attenuates rapidly with off-fault distance.

\subsubsection{Partitioning between shear and dilatational radiation fields by a supershear rupture}

The supershear slip-pulse equations from the previous section may be re-arranged into alternative forms, which provide insight into how radiation is partitioned between the shear and dilatational fields. Consider a fault-parallel particle velocity measurement $\left[\dot{u}_{x}\left(x, 0^{+}\right)\right]$, which is conducted at a point located arbitrarily close to the fault. As before, $z_{p}=z_{s}=x$ within the limit that $z_{I} \rightarrow 0^{+}$. Equation 2.52 then assumes the form given by

$$
\dot{u}_{x}\left(x, 0^{+}\right)=-\frac{V_{r}}{\mu}\left[\frac{1}{2 \alpha_{p}} \Im N_{+}(x)+\frac{\beta_{s}^{2}-1}{4 \alpha_{p}} \Im N_{+}(x)\right] .
$$

Substituting Eq. 2.60 into Eq. 2.68, and invoking antisymmetry through $\dot{u}\left(x, 0^{+}\right)=\frac{1}{2} \Delta \dot{u}_{x}(x, 0)$, yields

$$
\Delta \dot{u}_{x}(x, 0)=\left[\left(\frac{2}{\beta_{s}^{2}+1}\right) \Delta \dot{u}_{x}\right]_{\text {dilatational }}+\left[\left(\frac{\beta_{s}^{2}-1}{\beta_{s}^{2}+1}\right) \Delta \dot{u}_{x}\right]_{\text {shear }} .
$$

The first term still represents the dilatational field contribution, while the second term represents the shear field contribution. Nothing has changed in that regard other than the appearance of the equation through various substitutions. The speed-dependent functions that multiply the slip function in each respective term, herein referred to as $R_{p}\left(\beta_{s}\right)$ and $R_{s}\left(\beta_{s}\right)$, dictate how radiation is 
partitioned between the shear and dilatational fields. An alternative and simplified form for $R_{s}$ and $R_{p}$, given by

$$
R_{p}=\frac{2}{\beta_{s}^{2}+1}=2 \frac{C_{s}^{2}}{V_{r}^{2}}
$$

and,

$$
R_{s}=\frac{\beta_{s}^{2}-1}{\beta_{s}^{2}+1}=1-2 \frac{C_{s}^{2}}{V_{r}^{2}}
$$

is obtained by substituting for $\beta_{s}$ through Eqn. 2.16 , where $R_{s}+R_{p}=1$ is naturally observed. The slip function expression given by 2.69 can then finally be recast into the compact form given by

$$
\Delta \dot{u}_{x}(x, 0)=\left[\left(2 \frac{C_{s}^{2}}{V_{r}^{2}}\right) \Delta \dot{u}_{x}\right]_{\text {dilatational }}+\left[\left(1-2 \frac{C_{s}^{2}}{V_{r}^{2}}\right) \Delta \dot{u}_{x}\right]_{\text {shear }} .
$$

Plots of $R_{p}$ and $R_{s}$ as a function of normalized rupture speed are depicted in Figure 2.9. The normalized rupture speed $V_{r}=\sqrt{2} C_{s}$ marks the boundary between the unstable and stable rupture speed regimes. The maximum normalized rupture speed value of $V_{r} / C_{s}=2$ was set to correspond with $V_{r} \rightarrow C_{p}$ in the case of H-100 laboratory earthquake specimens (Table 3.3).

As expected, the radiation field surrounding a supershear rupture is purely dilatational when the rupture is propagating at $V_{r}=\sqrt{2} C_{s}$. The dilatational field contribution then decreases as the rupture speed increases, reaching a minimum value of $R_{p}=0.5$ at $V_{r}=2 C_{s}$. The shear field contribution $\left(R_{s}=1-R_{p}\right)$ ranges between $0<R_{s}<0.5$ across the same rupture speed interval. Typical normalized rupture speeds of $V_{r} / C_{s}=1.70$ in supershear experiments correspond to $R_{s}=0.308(30.8 \%)$.

The portion of the curves corresponding to the unstable rupture speed regime may appear somewhat confusing at first glance, especially since $-1<R_{s}<0$ within this domain. The situation is best understood by recalling Figure 1.1, which depicts the sense of motion along the shear wavelets radiated by a right-lateral supershear rupture propagating in the $+x$ direction. In the limit that $V_{r} \rightarrow C_{s}^{+}$the displacement vectors of the radiated shear waves are steeply inclined at the point of tangency where the shear Mach front is formed $(\theta \rightarrow \pi / 2)$. There is only a weak FP component 


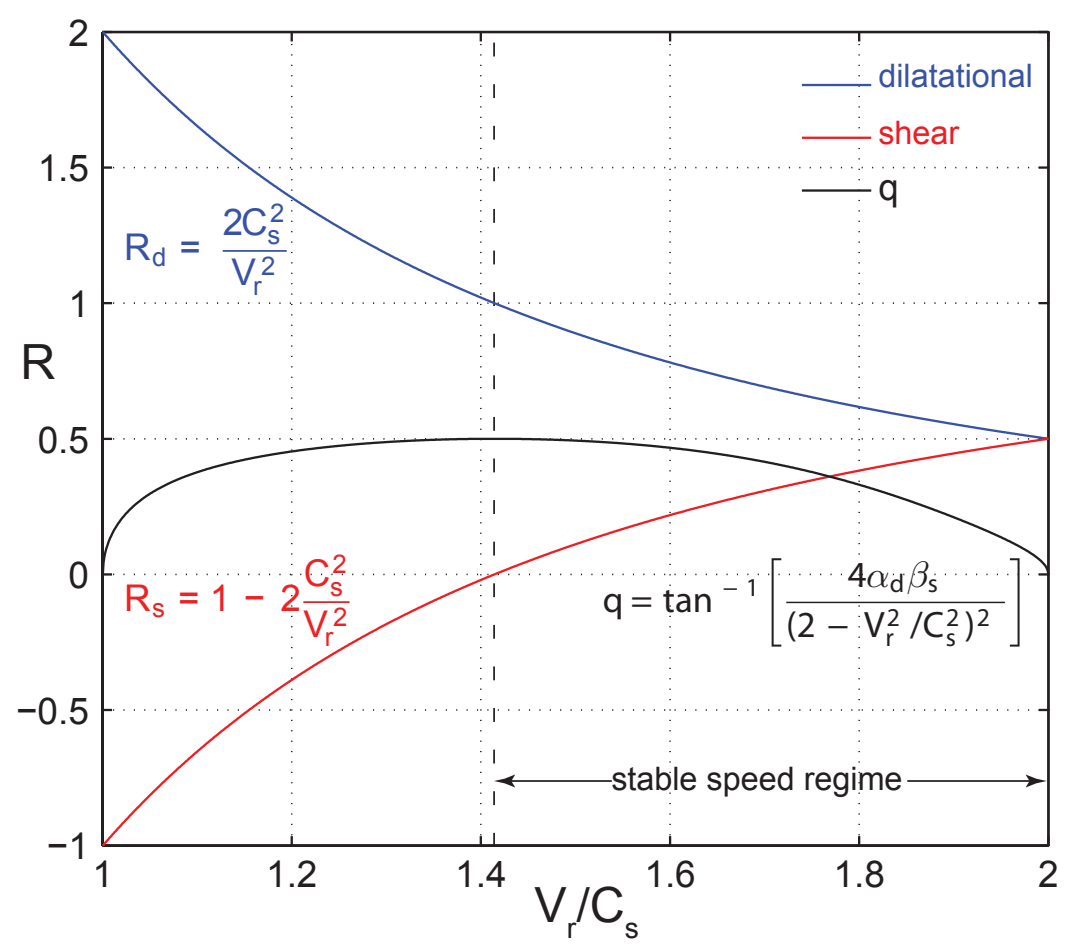

Figure 2.9: Radiation partitioning of the slip function as predicted by the general 2D steady-state supershear solution

directed along the $-x$ direction, which is actually opposite to the slip direction. Hence, the negative range of values exhibited by $R_{s}$ simply reflects the negative sign of the FP component in the shear wave displacement field when $C_{s}<V_{r}<\sqrt{2} C_{s}$. The corresponding positive range of values $1<R_{p}<2$ for the dilatational field contribution naturally offset the negative contribution of the shear field so as to satisfy $R_{s}+R_{p}=1$ throughout the entire rupture speed domain.

Finally, note that there are no analogous partitioning relationships, which arise in the case of the fault-normal component $\left(\dot{u}_{y}\right)$. The presence of $\Re N\left(z_{p}\right)$ in the dilatational field term and $\Im N\left(z_{s}\right)$ in the shear field term of Eq. 2.53 (and no anti-symmetry relation for $\dot{u}_{y}$ ) prohibits the types of substitutions and algebraic manipulations that were adopted in the case of the fault-parallel component $\left(\dot{u}_{x}\right)$. 


\subsubsection{Overview of the $2 \mathrm{D}$ steady-state supershear model}

The sense of particle motion along a shear Mach front was reasoned from rudimentary principles in Chapter 1 by considering the source spectrum of a simple shear dislocation. Sub-Rayleigh and supershear particle velocity signatures were also demonstrated on more-rigorous grounds by plotting the particle velocity fields for the 2D plane strain elastodynamic solution for a propagating shear (mode II) crack in a linear elastic half-space (Freund, 1979, 1990). Steady-state cohesive zone models were also examined, culminating with the general 2D steady-state solution obtained by (Dunham and Archuleta, 2004) for a supershear rupture pulse of finite length L, propagating with a rupture speed $V_{r}$, along a frictional fault with a prescribed shear traction $\tau(x)$.

The various rupture models discussed above show that in the rupture speed regime $\sqrt{2} C_{s}<$ $V_{r} \leq C_{p}$, the ground motion carried by the shear Mach front is characterized by a fault-parallel (FP) particle velocity swing which dominates in magnitude over the fault-normal (FN) velocity swing. The opposite case is shown to hold true in the rupture speed regime $C_{s} \leq V_{r}<\sqrt{2} C_{s}$, where the $\mathrm{FN}$ velocity swing dominates in magnitude over the $\mathrm{FP}$ velocity swing. A similar analysis applied to sub-Rayleigh ruptures predicts ground motion records, in regions ahead of the rupture

front, that are characterized by a FN velocity swing which dominates in magnitude over the FP velocity swing (Aki and Richards, 2002).

As a supershear rupture sweeps past an observation point within the near-field, the resulting ground motion records will be strongly influenced by the superimposed dilatational field, which circulates around the slip zone. Since S-wave radiation is confined to the region between the leading and trailing Mach fronts, the regions lying ahead will experience an initial disturbance, which is attributed to the leading dilatational field. This is a unique feature of the supershear regime where the dilatational and shear fields are separated. The effect is predicted by every analytical model tracing back to original solutions by (Freund, 1979). Synthetic ground motion records (Dunham and Archuleta, 2005), also predict a pronounced peak, particularly in the FP record, which signals the passage of the leading concentrated dilatational field prior to the arrival of the shear Mach front. A similar effect, although less pronounced, is observed in the corresponding FN ground motion 


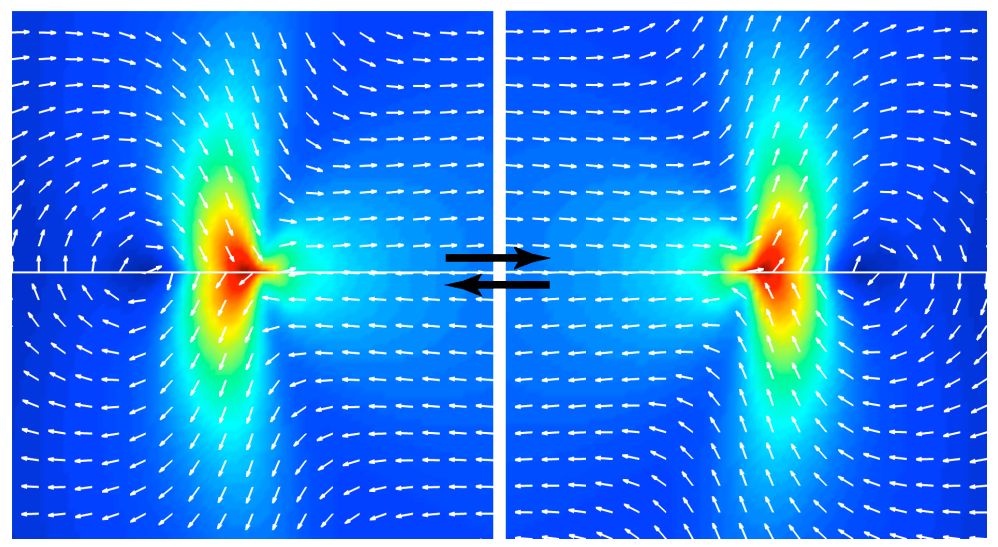

Figure 2.10: Sense of particle motion for a spontaneous right-lateral sub-Rayleigh rupture. The contour plot shows the magnitude of the velocity field and the vectors show only the sense of motion.

records. At greater and greater off-fault distances, there will also be an increased separation in time between the arrival of the leading dilatational field and the arrival of the fault-parallel velocity jump associated with the arrival of the shear Mach front. The dilatational field will tend to decay with increased off-fault distance in accordance with Eq. 2.15, leaving only an S-wave displacement field contribution to the Mach front in the far field, which resembles the slip function back at the fault.

\subsection{Unsteady cohesive zone models and the trailing Rayleigh}

\section{rupture}

By their very nature, 2D steady-state models generally assume that the rupture has existed for all time. Such models do not tend to address the dynamic events that lead to the emergence of the supershear rupture in the first place. Various supershear rupture transition mechanisms have been proposed in recent years. Each model typically assumes the existence of an initial sub-Rayleigh rupture, which proceeds to induce a supershear rupture transition. In the traditional Burridge-Andrews mechanism (Andrews, 1976; Burridge, 1973; Burridge et al., 1979), the sub-Rayleigh rupture stress field overcomes the strength of the fault to nucleate a daughter crack, which quickly transitions to a supershear rupture. Alternative, equally plausible models consider the dynamic interaction of the sub-Rayleigh rupture with an inclusion or high-strength barrier to produce stress concentrations and 
dynamic instabilities which can potentially spawn a supershear rupture (Dunham et al., 2003; Liu and Lapusta, 2008). Both types of supershear rupture transition mechanisms have now been observed in laboratory earthquake experiments. In all instances, the arrival of the leading supershear rupture is followed by a trailing sub-Rayleigh rupture.

Close scrutiny of the entire class of self-similar analytical solutions for supershear ruptures (for example Burridge, 1973; Broberg, 1994) also reveals that a secondary rupture travels behind the main crack tip at speeds approaching the Rayleigh wave speed. This feature, herein referred to as a trailing Rayleigh rupture, also accompanies spontaneously propagating ruptures and cannot be captured by steady-state models. The trailing Rayleigh rupture represents the region behind the crack tip where Rayleigh waves emitted from the process zone of the crack tip coalesce, anddepending on the friction conditions - result in a secondary rupture. Its strength is also expected to depend on the speed of the main crack tip, the nature of the main rupture (pulse-like or crack-like), the size of the process zone, and the strength/stress drop associated with it.

Figures 2.10 and 2.11 are useful for graphically illustrating the general sense of particle motion for spontaneous right lateral sub-Rayleigh and supershear ruptures subject to a slip-weakening friction law. These results were numerically simulated using a commercial Finite Element code, ABAQUS. Bilateral ruptures are depicted in the figure as generally encountered in laboratory earthquake experiments. The sense of particle motion is reasoned as follows.

In the case of sub-Rayleigh ruptures (Figure 2.10), the FN component is dominant near the rupture tip, as expected from earlier, more-elementary discussions. Since no opening is allowed, the FN component does not change sign as one crosses the fault. The direction of the velocity vector near the rupture tip depends, obviously, on whether the domain is under compression or extension in the FN direction. Also, since this is a crack-like rupture, there is continuous sliding behind the rupture tip.

For supershear ruptures (Figure 2.11) the dominant sense of motion near the crack tip is FP, as expected in the stable rupture velocity regime. The sense of motion is consistent with a rightlateral nature. The vector field plot also shows how the leading dilatational field (just beyond the 


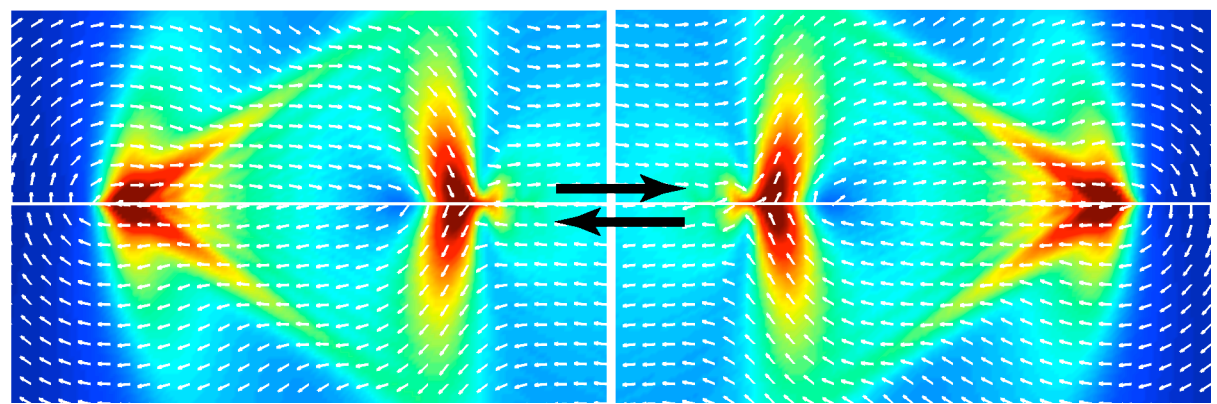

Figure 2.11: Sense of particle motion for a spontaneous right-lateral supershear rupture. The contour plot shows the magnitude of the velocity field and the vectors show only the sense of motion.

Mach front) exhibits a dominant FP component in the near-field. The supershear rupture tip is then followed by the secondary or "trailing Rayleigh" rupture, which trails in the wake of the shear Mach cone. The sense of motion exhibited by the secondary rupture is once again consistent with the ground motion signatures of a sub-Rayleigh rupture. Depending on the governing friction law, both the main rupture and the trailing Rayleigh rupture can be pulses in the strictest sense of the word (i.e., the fault is locked from slipping in their wake) or they could both be crack-like ruptures or be a combination of crack-like and pulse-like rupture. Regardless of whether it is a pulse or a crack, both theory and experiment show that supershear ruptures are often followed by a trailing (sub-Rayleigh) rupture propagating with a speed approaching the Rayleigh wave speed.

The analysis considered thus far is a 2D approximation of a 3D problem. In reality, geometrical attenuation and other dispersive mechanisms will ultimately conspire to attenuate the shear Mach front in the far field. (Bhat et al., 2007) estimated that a reasonable attenuation distance should be of the order of the depth of the seismogenic zone. A numerical 3D steady-state analysis of supershear ruptures by (Dunham and Bhat, 2008) resulted in a similar prediction. Nonetheless, it stands to reason that supershear ruptures should have a more far-reaching influence into the surrounding medium, compared to subshear ruptures of equivalent magnitude, and an increased propensity to perturb the stress state of neighboring faults. This point has been partly observed by (Bouchon and Karabulut, 2008) who showed that the aftershocks cluster in a region away from the fault at distances comparable to the depth of the seismogenic zone following the passage of a supershear rupture. 


\section{Chapter 3}

\section{The Laboratory Earthquake Experiment}

\subsection{Early predictions of the sub-Rayleigh-to-supershear tran- sition and the Burridge-Andrews mechanism}

The sub-Rayleigh-to-super-shear transition, originally theorized by (Burridge, 1973) and later numerically demonstrated and further interpreted by (Andrews, 1976), is commonly referred to as the Burridge-Andrews mechanism. (Burridge, 1973) was the first to theoretically describe the conditions under which a plain-strain, self-similar mode II crack can propagate in the supershear regime. The derivation assumed a simple Coulomb friction relation for the slip zone just ahead of the advancing rupture tip, which was enough to predict the existence of a peak in the shear stress field located on the S-wave front that could overcome the frictional strength of the interface and induce slip ahead of the primary crack tip. Burridge summarized his key findings as follows: "When a zone of slip governed by a Coulomb law of friction spreads steadily in an interface between two elastic half-spaces, only certain rupture speeds are admissible, the rupture speed, or crack speed, being the speed of the boundary of the region of slip. We cannot rule out any speed $V_{c}$ between $V_{R}$ and $V_{s}$ when static friction is high. When static friction is not so high only a sub-interval $V_{R}<V_{c}<V_{0}<V_{s}$ is available. If static friction is even lower not even $V_{R}$ is admissible and the crack may propagate at the P-wave speed" (Burridge, 1973). In Burridge's notation $V_{c}$ corresponds to the crack-tip speed, $V_{R}$ 
is the Rayleigh wave speed, and $V_{s}$ stands for the shear wave speed. The shortcoming of this early analytical model was its inability to account for the effects of inelastic (cohesive) energy losses at the crack tip, which lead to some ambiguous predictions regarding admissible crack speeds and the predicted range of the admissible supershear rupture speeds. In particular, the analysis predicted that once a rupture had transitioned into the supershear regime, it could only propagate at the P-wave speed. High-versus low-static-friction scenarios even admitted the possibility of crack speeds in the domain $C_{R}<V_{r}<C_{s}$, which was later shown to be a forbidden speed regime (Freund, 1979; Broberg, 1989, 1999).

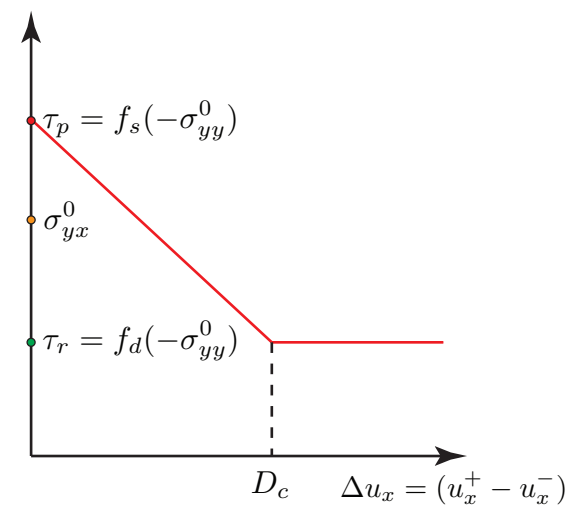

Figure 3.1: Linear slip-weakening friction law

(Andrews, 1976) followed with the very first numerical finite difference calculations that successfully simulated the sub-Rayleigh-to-supershear transition. Results of these numerical trials demonstrated the emergence of a secondary rupture at the leading S-wave front and its subsequent merger with the primary rupture, in a manner consistent with the original predictions by (Burridge, 1973).

Andrew's model featured a linear slip weakening friction law, graphically depicted in Figure 3.1, which provided both a finite strength criterion $\tau \leq \tau_{P}$ and accounted for fracture energy $G_{c}$ at the crack tip, given by

$$
G_{c}=\frac{1}{2}\left(\tau_{p}-\tau_{r}\right) D_{c}
$$

These features distinguished Andrew's work from the earlier analytical treatment by (Burridge, 1973), which failed to incorporate the effects of inelastic (cohesive) energy losses at the crack tip. 
(Andrews, 1976) also carefully examined the sub-Rayleigh-to-supershear transition distance $L$ and reasoned that it must be related to the critical (plane strain) crack half-length $L_{c}$ given by

$$
L_{c}=\frac{\mu\left(\tau_{p}-\tau_{r}\right) D_{c}}{\pi(1-\nu)\left(\sigma_{y x}^{0}-\tau_{r}\right)^{2}}
$$

where $\nu$ is Poisson's ratio, $\mu$ is the shear modulus value, and $D_{c}$ corresponds to the critical slip weakening distance. Moreover, it is easily shown that the normalized quantity $L_{c} / L$ is equivalent to the energy absorbed by the rupture front to the amount of available strain energy which has been released to propagate the crack to a new length $L$ (Andrews, 1976). Andrews incorporated these points and summarized his findings by plotting the dependence of $L_{C} / L$ on the seismic S-ratio

$$
S=\frac{\tau_{p}-\sigma_{y x}^{0}}{\sigma_{y x}^{0}-\tau_{r}}
$$

Points derived from numerical trials define the boundaries of a transitional region, which divide the sub-Rayleigh rupture speed regime on the right from the super-shear rupture speed regime on the left (Andrews, 1976). The final result can be restated as

$$
L=F(s) L_{c}=9.8(1.77-S)^{-3} L_{c}
$$

(Xia et al., 2004) where the function $\mathrm{F}(\mathrm{S})$ is numerically obtained from Andrews plot. The unique point $S \approx 1.77$, represents an upper value for the seismic $\mathrm{S}$ ratio, where $L \rightarrow \infty$, beyond which a sub-Rayleigh rupture cannot possibly transition into the super-shear regime. The lower limit $S \rightarrow 0$, equivalent to $\sigma_{y x}^{0} \rightarrow \tau_{p}$, corresponds to a lower bound for the transition length given by $L \approx 1.77 L_{c}$. Based upon the results of his numerical trials, Andrews concluded that subshear ruptures were confined to the sub-Rayleigh domain $0<V_{R}<C_{R}$ while intersonic ruptures always tended to favor the domain $\sqrt{2} C_{s}<V_{R}<C_{p}$, which he termed the supershear domain.

(Burridge et al., 1979) later revisited the problem of admissible rupture speeds by conducing a stability analysis of a steadily moving shear crack. The model assumed a shear crack was driven 
by a point load which remains at a constant distance $d$ from the crack tip. Energy dissipation was introduced through a Dugdale-type cohesive traction relation confined to a small region around the crack tip, and taken to be small in comparison to the distance $d$. The primary conclusions drawn from the shear crack stability analysis were found to be in agreement with Andrews' numerically based findings and are summarized as follows: (1) the velocity domain $C_{R}<V_{R}<C_{S}$ is strictly forbidden, since the crack tip would otherwise act as a source of energy if allowed to propagate at these speeds, (2) the intersonic velocity regime $C_{s}<V_{R}<\sqrt{2} C_{s}$ is generally unstable, (3) the intersonic velocity regime $\sqrt{2} C_{s}<V_{R} \leq C_{p}$ is stable with a unique local minima within this velocity domain, (4) the sub-Rayleigh velocity regime $V_{R}<C_{R}$ is classified as an unstable regime. This is strictly true mainly due to the fact that the stability curve lacks any local minima within this domain. The resulting curves actually exhibit a strong negative slope which quickly blows up in the limit $V_{R} \sim C_{R}$, thus implying that a rupture confined within this velocity domain will always tend to the Rayleigh wave speed. Similar conclusions regarding the nature of admissible rupture speeds and the stability of various rupture speed regimes, were rigorously demonstrated by (Freund, 1979) and later by (Broberg, 1989) by considering the energy flux into a dynamic shear crack.

\subsection{Brief historical background and introduction to the lab- oratory earthquake experiment}

The first high-speed camera images of an intersonic mode II rupture were obtained by (Lambros and Rosakis, 1995) in a series of dynamic shear impact studies conducted on PMMA/4340 steeledge cracked bimaterial plates. Interfacial crack propagation was observed using the optical technique of coherent gradient sensing and high-speed photography. Very high crack-tip speeds (up to $\left.1.5 C_{R}^{P M M A}\right)$ were reported. Numerous impact investigations followed, both on homogeneous and bimaterial systems, which provided additional confirmation of intersonic ruptures through the continued refinement of high-speed imaging techniques (Singh et al., 1997; Kavaturu et al., 1998; Rosakis et al., 1998, 1999; Coker and Rosakis, 2001; Samudrala and Rosakis, 2003; Coker et al., 2003). 


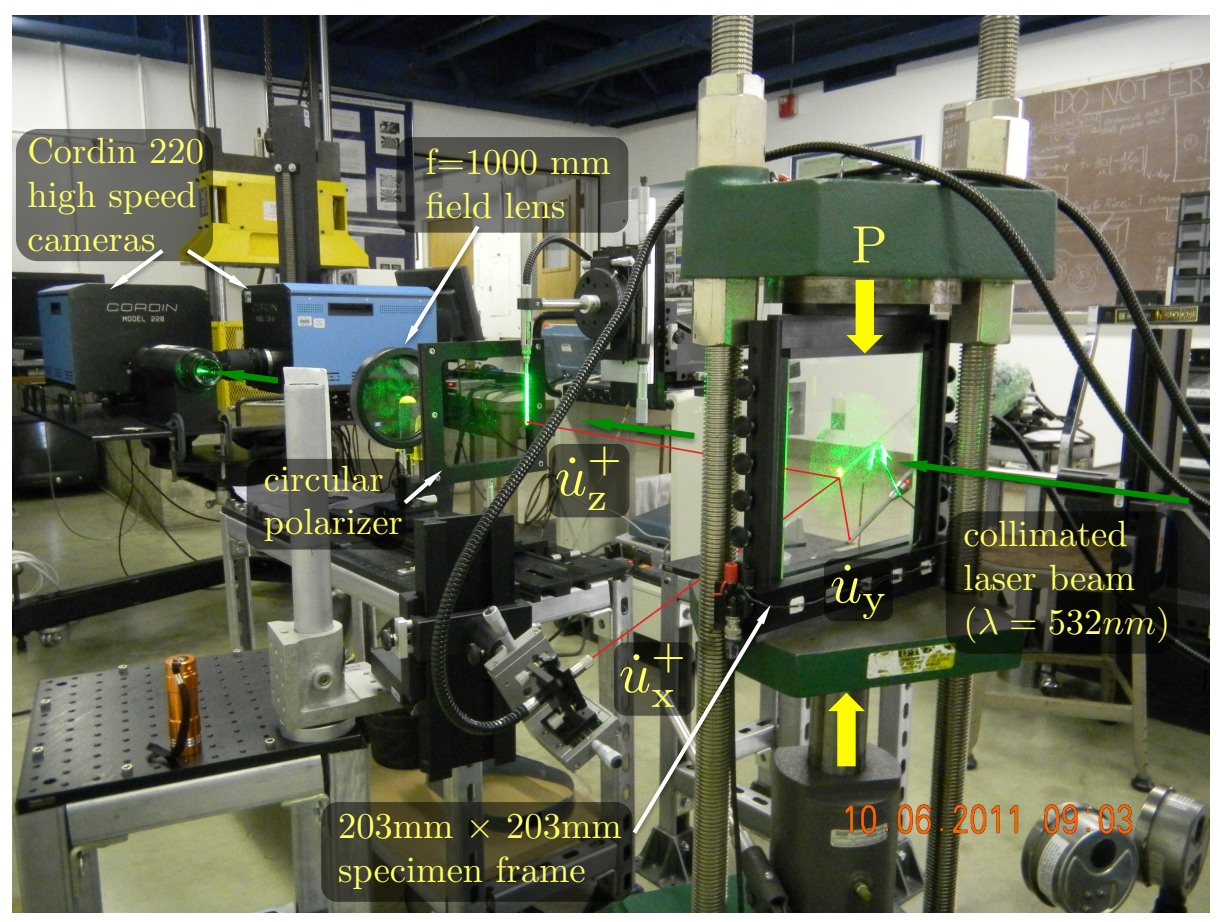

Figure 3.2: Laboratory earthquake experimental setup: Dynamic photoelasticity for stress-field visualization and heterodyne fiber optic interferometers for continuous, point-wise measurements of particle velocity

The early body of experimental work on intersonic ruptures was soon recognized within the geophysical community, where attention was focused on whether certain anomalous seismological records could be attributed to the propagation of an intersonic (supershear) rupture along a frictional faults within the Earth's crust (Bouchon et al., 2001). Experimental investigations and research directions gradually shifted away from dynamic shear impact studies to the investigation of spontaneous dynamic ruptures along frictional faults. This lead to the eventual creation of a unique "laboratory earthquake experiment" developed by Xia, Rosakis, and Kanamori (Xia et al., 2004, 2005a).

The original experiment featured an inclined, frictional interface formed by two compressed quadrilateral sections of Homalite ${ }^{\mathrm{TM}}$ (H-100), an optically transparent, stress-birefringent, and mildly rate-sensitive brittle polyester resin. A dynamic rupture was triggered by a surge of current, which was passed through a thin $\mathrm{NiCr}$ wire filament oriented across the specimen fault. Photoelastic interferometry and high-speed photography were used to image the propagating rupture front and yielded first-of-its-kind high-speed images of spontaneously generated supershear ruptures (Xia 
et al., 2004; Xia, 2005; Xia et al., 2005b,a; Rosakis et al., 2006, 2007).

Measurement diagnostics were later expanded to include fiber-optic-based laser interferometers, which were first used by Lykotrafitis and Rosakis to measure sliding speeds in dynamic shear impact investigations of frictionally held bimaterial interfaces (Lykotrafitis et al., 2006b; Lykotrafitis and Rosakis, 2006a,b; Lykotrafitis et al., 2006a). This capability was later successfully applied by Lu and Rosakis (Lu et al., 2007; Lu, 2009; Lu et al., 2010; ?) to verify the existence of sub-Rayleigh and supershear slip pulses in laboratory earthquake experiments. The laboratory earthquake experiment has been continuously refined and applied in subsequent years to investigate various problems of seismological relevance ranging from studies on the effects of off-fault damage (Grifith et al., 2009; Biegel et al., 2010; Bhat et al., 2010) to recent investigations on the ground motion signatures of supershear earthquakes (Mello et al., 2010) and thrust faults.

A landscape view of the laboratory earthquake experiment in its current form is depicted in Figure 3.2. An $8 " \times 8 " \times 0.5 " \mathrm{H}-100$ earthquake specimen is seen mounted within the black anodized Aluminum frame and compressed by a manually operated hydraulic press. The $26^{\circ}$ specimen fault is visible, as are the wire leads along the right side, which are clipped to either end of a short NiCr wire filament that passes through the specimen. Scattered laser light reveals the circular profile of the 5.5" diameter laser beam centered within the $8^{\prime \prime} \times 8^{\prime \prime}$ test specimen. The circularly polarized beam is transmitted through the specimen and processed by the circular polarizer to produce the familiar photolelastic fringe pattern. The field lens focuses the beam and directs it into the high-speed camera imaging optics.

Three fiber-optic probes are labeled in the image as $\dot{u}_{x}^{+}, \dot{u}_{y}$, and $\dot{u}_{z}^{+}$in order to highlight how the three motion components are simultaneously monitored. The thin red lines highlight the optical path of the individual laser beams from each fiber optic probe to the point on the specimen surface (at the corner of a retro-reflective tape strip) where the beams are focused and particle velocity measurements are obtained. Collected laser light is directed by optical fiber to heterodyne interferometers, which are positioned outside of the image. Interferometer signals are then processed by a controller module and sent out as analog voltage signals, which are acquired by a digital oscilloscope. The 
acquired signals are linearly proportional to the particle velocity and are scaled accordingly when the curves are analyzed.

The various sections of this chapter systematically address every major facet of the laboratory earthquake experiment. These include the best-known methods for (1) machining test specimens, (2) preparation of specimen fault surfaces (including a new 3D fault geometry), (3) inspection and quality control of test specimens, (4) final assembly and mounting of laboratory earthquake specimens, and (5) alignment of all optical diagnostics. A detailed discussion of the instruments and measurement diagnostics is also presented, followed by a description of the various methods of analysis which are routinely employed to process the data gathered in the experiments.

\subsection{Step-by-step description of a laboratory earthquake ex- periment: From specimen preparation through dynamic rupture nucleation}

A schematic depiction of the traditional H-100 specimen geometry, static loading configuration, and dynamic rupture nucleation scheme is shown in Figure 3.3. The mating edges of two H-100 quadrilateral test sections are initially polished to a near-optical-grade finish in order to remove surface defects from the original CNC milling operation. The surfaces are then roughened using a micro-bead blasting treatment using an abrasive media comprised of tiny glass beads with diameters typically ranging from $104 \mu m-211 \mu m$ (70-140 mesh size). Precise repetition of the bead-blasting procedure from specimen to specimen assures a consistent surface roughness from experiment to experiment. Specific details of the specimen fabrication and surface preparation procedures are provided in Section 3.5.

Fault surfaces are thoroughly cleaned using laboratory-grade methanol and acetone, and frequently inspected to protect the integrity of the fault surfaces. The frictional interface is visually inspected either with the aid of a magnifying loupe or a low-power microscope similar to a $130 \mathrm{x}$ USB microscope camera (Sharper Image CN311), which can be mounted directly upon the flat face 


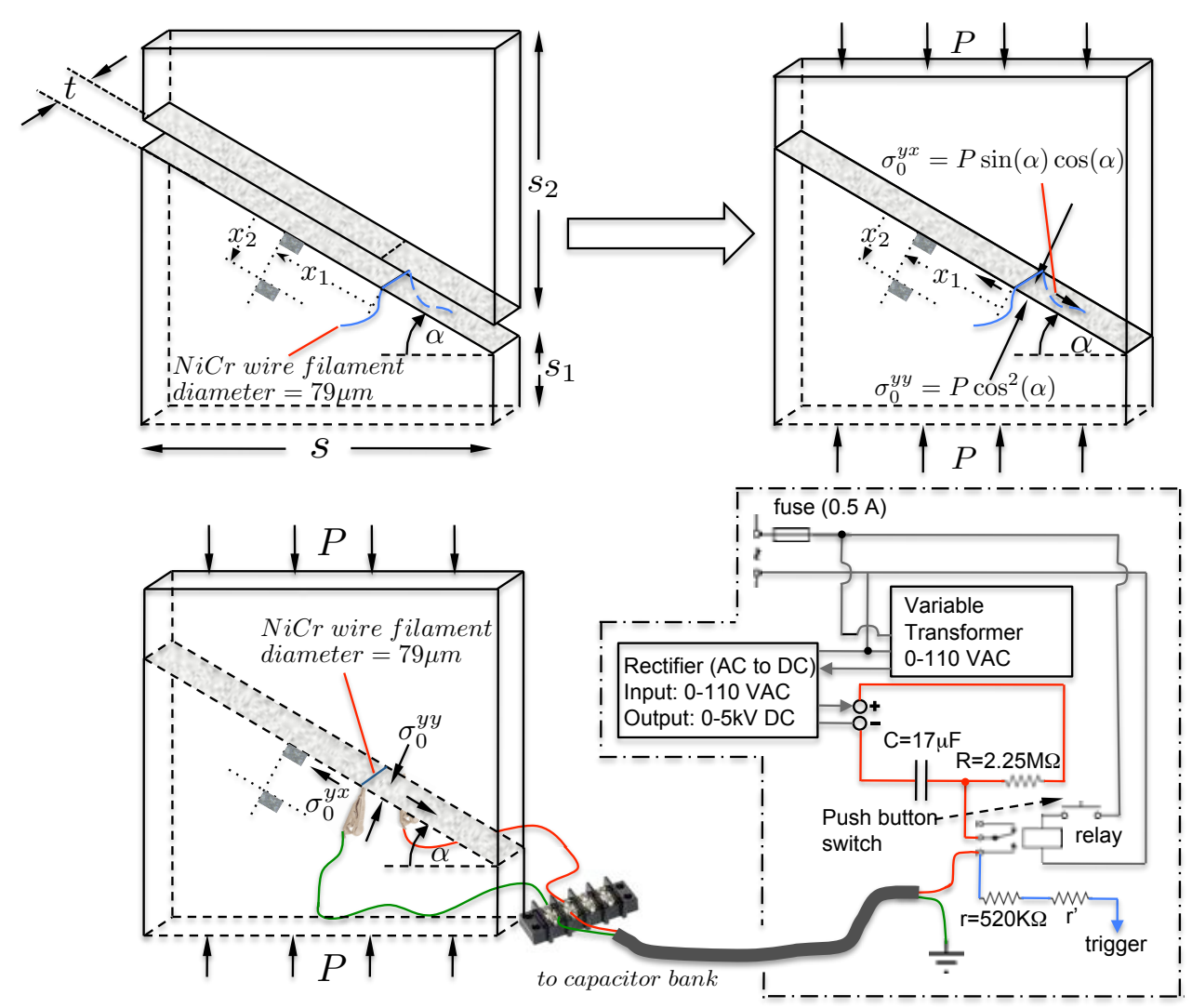

Figure 3.3: Schematic representations of traditional H-100 specimen geometry, static loading configuration, and dynamic rupture nucleation scheme

of a mated H-100 specimen assembly. The planarity and perpendicularity of specimen fault surfaces are examined by laying a mated pair of quadrilateral test sections on a flat glass plate and butting the two halves together. Two small pieces of clear cellophane tape placed across the fault interface near the edges of the assembly hold the two plates together and keep the parts from slipping or separating during this inspection process. The interface is then examined using the USB microscope camera as depicted in Figure 3.4

Parts are typically inspected this way following the machining, polishing, and surface roughening (micro-bead blasting) phases of specimen fabrication. A mated specimen assembly should exhibit a sharp interface without any visible gap between the plates. Additional details of this inspection procedure along with examples of representative "known good" H-100 specimen interfaces are addressed 


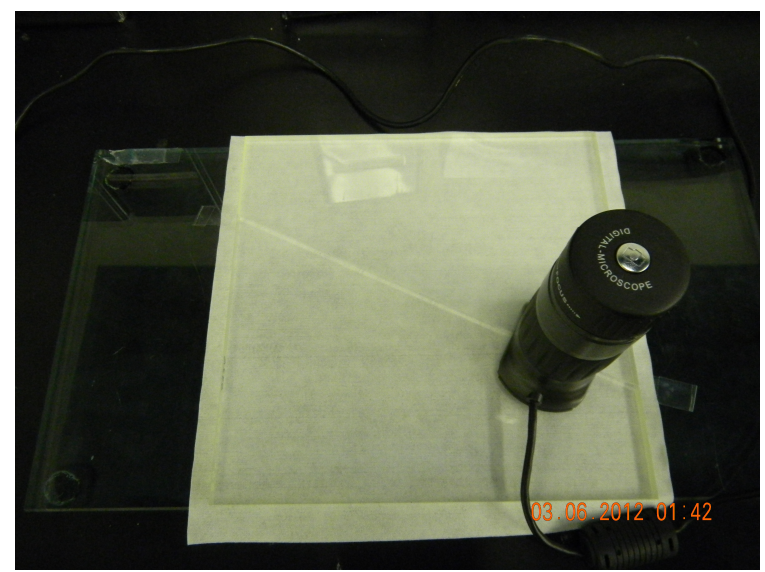

Figure 3.4: Inspection of fault surface planarity and perpendicularity using the USB microscope camera. See Figure 3.15 and Figure 3.16 for representative images of fault interfaces.

\section{in Section 3.5.2.}

Once the fault surfaces are visually inspected, an initial evaluation of the frictional interface is conducted by carefully mating the quadrilateral test sections together on the lab work bench in order to gage whether the assembly can hold together without slipping. The top and bottom test sections should be well aligned to form a flush surface across the fault interface, and there should be no static offset at the left or right extreme ends of the fault. Once carefully positioned in this manner, the static H-100 assembly should be able to hold together without slipping. A second test of the frictional interface involves slowly pivoting the mated assembly about its lower-right-hand corner and estimating the tilt angle at which slip ensues. The test is based upon the simple static coefficient of friction relationship given by $\tan \left(\alpha+\theta_{\text {rot }}\right)=f_{s}$, where $\theta$ is the tilt angle at the moment of slip, $\alpha$ is the specimen fault angle, and $f_{s}$ is the static coefficient of friction. In this case the slip angle is measured and the relationship is invoked in order to estimate the static coefficient of friction. The measurement is conducted with the aid of a clear plastic protractor mounted along the edge of the lab work table. The specimen assembly is aligned parallel to the protractor and positioned as closely as possible to the edge of the work bench so that the fault angle can be estimated by looking through the transparent protractor. Care must also be taken to coincide the lower-right-corner of the square specimen assembly with the rotational reference point along the base of the protractor 
(assuming a right lateral slip configuration). The frictional interface is then carefully monitored as the assembly is slowly rotated until the plates are observed to slip. As a good rule of thumb, the plates should hold together without slipping at least until $\alpha+\theta=31^{\circ}\left(f_{s}=0.6\right)$. As an example, H100 test sections with a specimen fault angle $\alpha=26^{\circ}$ and roughened by the standard bead-blasting treatment (glass bead mesh size 70-140), typically exhibited slip angles of $7^{\circ}-9^{\circ}$, corresponding to $\left(0.65<f_{s}<0.7\right)$. Note that if the plates slip too easily during this test then it might be an early indicator that they will not hold together under the applied static compressive load.

The next step in the specimen assembly process flow involves seating a thin $\mathrm{NiCr}$ wire filament within a 0.005 " diameter semi-circular channel that has been machined into the lower quadrilateral test section. The NiCr filament used in the experiments is 40 AWG high-temperature nickel wire with a diameter of $0.003 "(78.7 \mu \mathrm{m})$. The wire is purchased in spool form (catalog number: $8880 \mathrm{~K} 87$ ) from the McMaster-Carr company. The lower test section is clamped and held in place using a small table-top vise with the fault surface facing upwards and nominally level to the surface of the lab work-bench. A 30-cm-long strand of NiCr filament is then set within the channel, stretched tight, and bent around the edges of the H-100 test section. Two small pieces of cellophane tape are then used to affix the wire to each face of the test section and temporarily hold the wire tightly in place within the channel until the plates are actually mated together. Each of the cellophane tape sections should have one end folded back on itself in order to create a "handle", which prevents the entire section of tape from adhering to the surface of the specimen and facilitates removing the tape at a later stage. A magnifying lens should be used to carefully check whether the $\mathrm{NiCr}$ wire is properly positioned within the channel and whether it remains below the fault surface. A very practical technique is to clasp the two loose ends of the $\mathrm{NiCr}$ wire filament with a set of needle-nose pliers and pull the wire down into the channel. An image of how the wire is temporarily set in place is shown in Figure 3.27 of Section 3.9.

A final sample preparation step, prior to the mating and assembly of the H-100 test sections, involves adhering small rectangular strips of retroreflective tape at preselected positions on the face of the specimen where particle velocity measurements will be conducted during the experiment. 


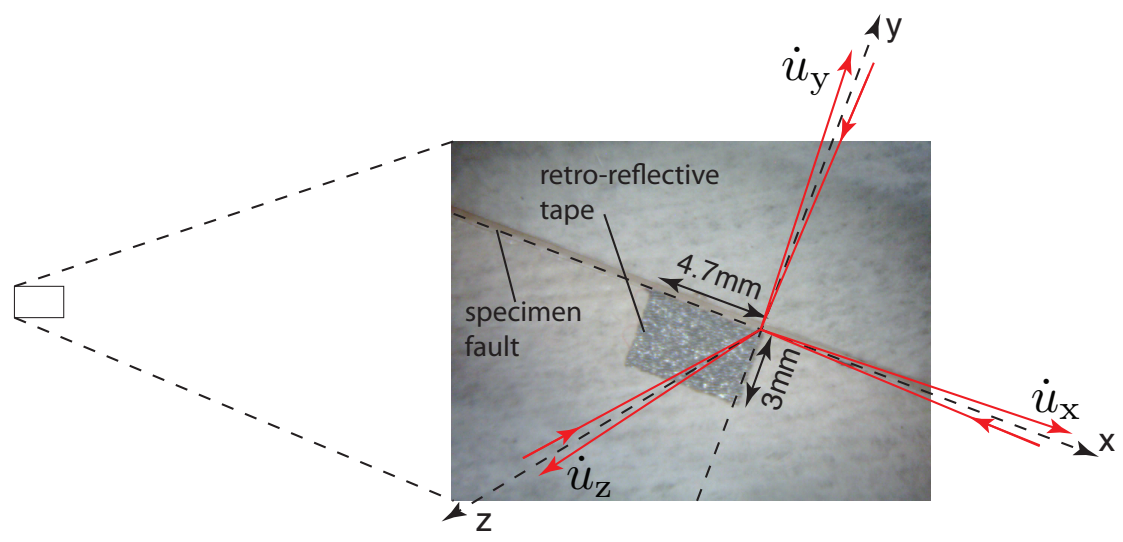

Figure 3.5: Small rectangular strip of retro-reflective tape adhered to specimen surface to promote efficient scattering of focused laser light by fiber optic laser interferometer probes

The two test sections are once again mated and held together by two small strips of clear cellophane tape while lying on a flat work surface. A clear plastic protractor with a metric rule scale is then aligned with the specimen interface and also temporarily affixed to the surface using clear cellophane tape. A thin rectangular strip of adhesive-backed retroreflective tape $\left(3 \mathrm{M}^{\circledR}\right.$ Scotchlite $\left.{ }^{\circledR}\right)$, measuring approximately $3 m m \times 4 m m$, is cut from sheet stock using an X-Acto knife equipped with a "Number 2" blade. Blades should be kept clean and sharp and be frequently replaced. The edge of the retroreflective tape strip must be cut clean and straight, as shown in the example in Figure 3.5. The small retroreflective strip is then accurately positioned and affixed to the $\mathrm{H}-100$ specimen with the aid of a mounted workbench magnifying lamp. The tape is positioned such that one of its corners coincides with the desired measurement coordinate, as depicted in the close-up view in Figure 3.5 where the upper-right-hand corner of the tape section has been precisely positioned at the coordinate of interest. The diagram also schematically depicts how the focused fiber-optic probe beams are focused to a minimum spot size and scattered back toward the probe from this corner location.

Toothless stainless-steel alligator clips used to clasp the ends of the $\mathrm{NiCr}$ wire filament are wired to an electrical terminal block at the base of the specimen-mounting frame, as depicted in Figures 3.3 and 3.6. Stainless-steel alligator clips with an overall length of $13 / 32$ ", maximum jaw opening of $3 / 16$ ", and current rating of $5 \mathrm{~A}$ were purchased in bulk quantity from McMaster-Carr ${ }^{\circledR}$ (catalog 


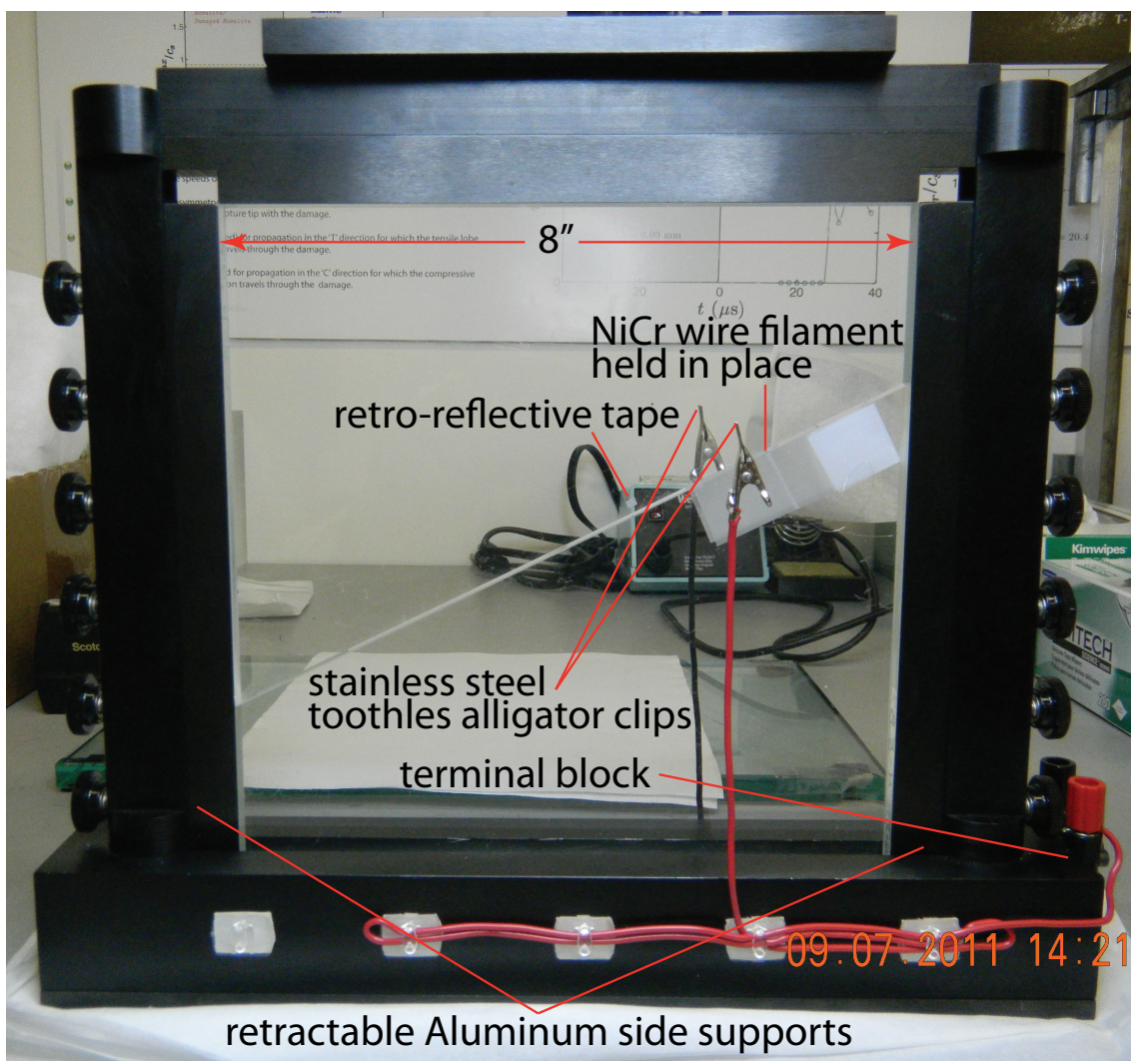

Figure 3.6: $8 " \times 8$ " H-100 laboratory earthquake specimen prepared and positioned within mounting frame

number: 7236K27). The clips should be cleaned before each experiment using a fine 600-grit $\mathrm{SiC}$ abrasive paper in order to remove any carbon deposits that may have accumulated during the previous test. The quality and cleanliness of the clips helps to maintain good electrical contact and a consistent value for the resistance, which is important to achieving a repeatable discharge from experiment to experiment. The clips are gradually eroded by the electrical discharge process and can also lose their elastic clamping force over time and should therefore be inspected at the start of each experiment and periodically replaced.

Specimen fault surfaces are gently cleaned one final time before the two sections are brought into contact and mounted within the specimen-mounting frame as shown in Figure 3.6. Special care is always taken to prevent acetone or any cleaning solvent from contacting the retro-reflective tape strip. The two H-100 test sections are brought into contact and carefully adjusted so as to establish a 
smooth, flush surface along the fault interface. There should be no detectable step height anywhere across the interface on either face of the specimen. The side bars of the specimen frame are gently adjusted to make light contact with the sides of the specimen assembly. The thumb screws within the left and right pillars of the specimen frame are then adjusted in order to gently snug the aluminum side bars against the sides of the specimen. Note that the side bars also should be positioned in such a way that they can be retracted from each edge once the assembly is placed under a pre-load. The entire specimen frame assembly is then carried over and seated on the base platen of the manually operated hydraulic press (Carver® model 3925), as depicted in Figure 3.7. The base compression platen is then slowly raised using the manual hydraulics until the test specimen is properly centered within the laser beam along the vertical direction. A partial reflection of the incident expanded laser beam is then used to establish perpendicularity by aligning the specimen frame assembly until the reflect beam is directed back into the laser beam expander. At the same time, care must also be taken to make sure that the specimen frame is also properly centered from left to right with respect to the incident laser beam in order to properly illuminate the region of interest. Last, but not least, the entire assembly must also be properly positioned "depth wise" within the press such that the top member of the specimen mounting frame is properly aligned with respect to the diameter of the top compression platen. The three adjustments are iterated until each of the critical alignment conditions are all met. The top compression platen is then slowly lowered and made as parallel as possible to the top member of the specimen frame assembly. The large stainless steel tie rod nuts, which support the entire top assembly of the hydraulic press, are slowly turned so as to nearly close the gap and adjust the relative tilt between the top compression platen and the top member of the specimen frame assembly. Parallelism between the two surfaces may be achieved by adjusting the tie rod nuts while peering through the gap and taking note of the slit of light between the two surfaces. From this point on any additional tilt compensation will be taken up by the lower base compression platen which is designed to naturally pivot.

A static compressive pre-stress of $P_{i}=3 M P a$ is applied to the assembly once it has been properly positioned and oriented with respect to the incident photoelastic imaging beam. Thumb 


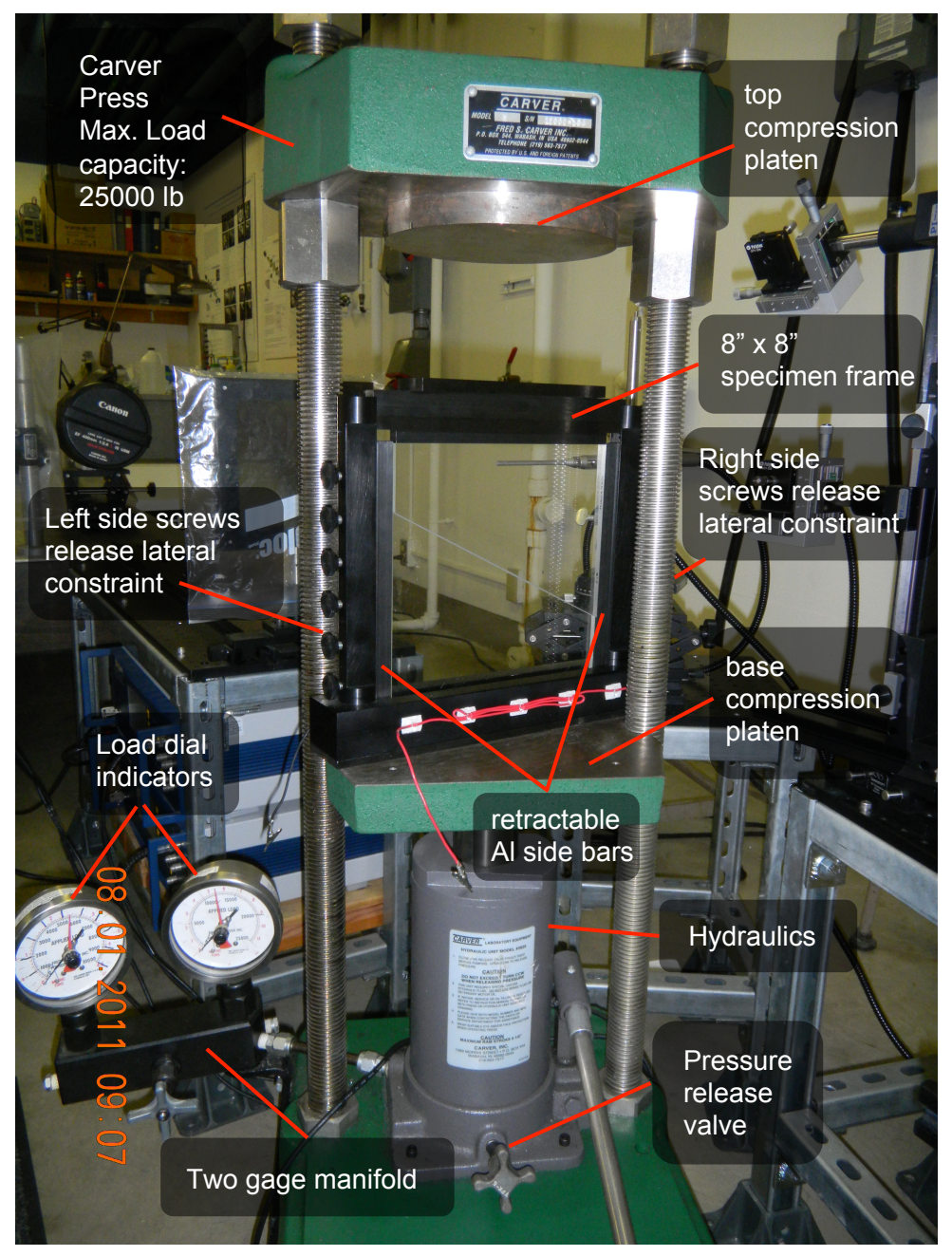

Figure 3.7: H-100 specimen assembly positioned in Carver@ model 3925 hydraulic press

screws in the left-and right-hand pillars of the specimen mounting frame are then loosened and the retractable aluminum side bars are pulled away from the left and right boundaries of the H-100 test specimen. Close attention is paid in order to detect whether the plates slip during this procedure. If any slip is detected, the specimen is generally removed from the mounting frame and the fault surfaces are either cleaned or completely reconditioned before attempting to reload the specimen assembly. Assuming that the plates hold together after releasing the Aluminum side supports (under the $3 \mathrm{MPa}$ pre-stress), the load is then gently ramped up to the desired static compressive stress. Photoelastic fringes may be observed during loading procedure by propping up a sheet of paper 
after the second circular polarizer. If the specimen is properly aligned, the resulting fringe pattern should exhibit symmetry with respect to the center of the specimen and should appear to expand out from this center of symmetry as the static compressive load is slowly ramped. The applied static compressive load translates into a normal traction

$$
\sigma_{y y}^{0}=P \cos ^{2} \alpha
$$

and resolved shear traction

$$
\sigma_{y x}^{0}=P \sin \alpha \cos \alpha
$$

along the inclined frictional interface, which mimics tectonic stresses imposed on a strike-slip frictional fault within the Earth's crust. Interfacial sliding is prevented by virtue of the fact that the resolved shear stress does not exceed the peak (static) frictional strength $\tau_{p}=f_{s} \sigma_{y y}^{0}$ of the interface, where $f_{s}$ represents the static coefficient of friction. The inclined specimen fault assures that once nucleated, a right-lateral or left-lateral rupture will propagate along the frictional fault in response to the applied far-field stress.

Once the static compressive load is applied, the final step is to clip the two ends of the $\mathrm{NiCr}$ wire filament with the toothless alligator clips in order to establish an electrical connection to the discharge circuit. This last step is accomplished by gently peeling one of the cellophane tape sections away (easily grasped using the tape handle created earlier) from the H-100 specimen face and then clasping the filament with the toothless alligator clip as closely as possible to the specimen face. The wire should be pulled taut with one hand, while the other hand is used to clasp the wire with the toothless alligator clip. The process is then repeated on the opposite side of the specimen. Care must be taken to insure that the clips are both as flush as possible to the front and rear surfaces of the specimen and that they are not left "dangling". A fine set of sharp wire cutters should then be used to gently clip away the lose strand of $\mathrm{NiCr}$ wire dangling to either side of the specimen. The wire should be clipped as closely as possible to the alligator clips. The electrical resistance of the wire filament path is then measured between the two connecting points of the electrical terminal 


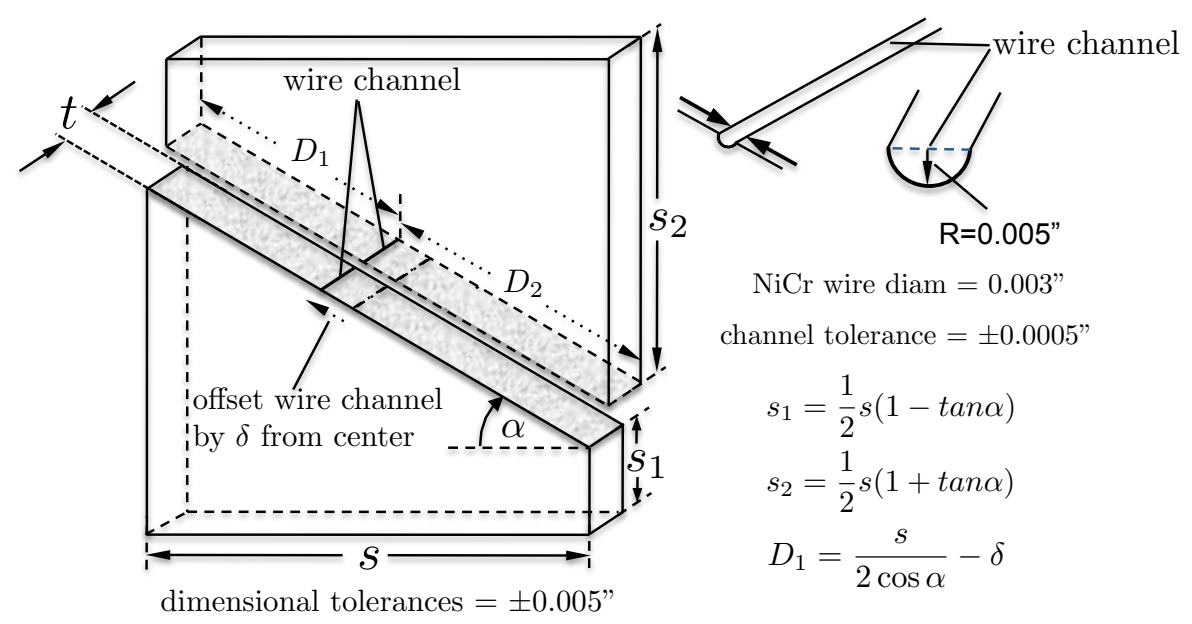

Figure 3.8: General dimensions of traditional H-100 laboratory earthquake specimens with uniform frictional fault extending throughout the entire specimen thickness

block. The BNC cable, which leads to the discharge circuit should be temporarily disengaged from the terminal block while the multimeter makes contact with the two points on the terminal block where each of the wires are attached. The measured resistance value should be approximately $\mathrm{R}=$ $3 \Omega$ and should remain very consistent from experiment to experiment as long as the final trimmed length of the $\mathrm{NiCr}$ wire filament is kept relatively constant. The path resistance value is dominated by the resistance of the $79-\mu \mathrm{m}$-diameter $\mathrm{NiCr}$ wire filament, and the measured resistance simply scales with length of the wire filament in accordance with the well-known relationship $R=\rho L / A$, where $\mathrm{R}$ is the measured resistance, $\rho$ is the resistivity of the $\mathrm{NiCr}$ filament, $\mathrm{L}$ is the length of the wire strand, and A corresponds to its cross-sectional area. The BNC cable leading to the discharge circuit is then reconnected to the terminal block once the resistance measurement is completed.

The next critical steps involve alignment of the optical diagnostics used in the experiment. Fiber-optic laser probes are mounted using appropriate optical rail carrier assemblies and multi-axis translation stages with fine-pitch screw adjustment capability. All probe beams other than the one currently being aligned are turned off in order to clearly see where the beam is being focused onto the specimen. A small magnifying lens is then propped up on the base platen of the hydraulic press and used to view the corner of the retro-reflective tape section while the beam's position is carefully 
adjusted. In-plane particle velocity beams $\left(\dot{u}_{x}\right.$ and $\left.\dot{u}_{y}\right)$ are directed toward the corner of the tape at an angle within $5^{\circ}-7^{\circ}$ of grazing incidence. The orientation of an in-plane beam can also be controlled by taking advantage of a series of internal reflections which are formed within the H-100 plate. The effect is easier to see by turning down the room lights. The fiber optic probe is translated and tilted in order to align the internally reflected beam, with the specimen fault. When the incident beam is properly adjusted, a "zig zag" light beam pattern is seen superimposed along the entire fault length. The incident beam is then translated parallel to fault by a distance $x_{2}$ and directed to focus at the appropriate corner of the retro-reflective tape strip. The fiber-optic probe variable focus knob is adjusted in order to achieve the smallest spot size at the location of interest. The focused beam should not be spread across the top surface of the tape but should instead be focused along the thin cross-sectional edge of the tape section. Only the laser beam used for the vertical component $\left(\dot{u}_{z}\right)$ should be focused onto the actual face of the tape section at the appropriate corner. The instrument features a live LED display containing a series of green indicators that light up once the scattered light is collected and interfered. An increase in the number of illuminated LED "bars" translates to an improved interference signal. With practice, and experience it is possible to align the beams well enough so that all of the the LED bars are illuminated for each of the three interferometer signals. Often it is the lack of fine mechanical adjustment capability that limits the optimization of the interference signals. How well the retro-reflective tape strip was cut also matters a great deal. A "ragged edge" will simply not exhibit good scattering efficiency. On some occasions it may not be possible to peak the signals and one may have to settle for 5-6 illuminated bars, which is still good enough to produce a good-quality output signal to the oscilloscope.

The high-speed cameras are also aligned by taking a series of static images using the actual dynamic framing rate and exposure time. Typical laser power levels of $1 \mathrm{~W}-1.25 \mathrm{~W}$ have been used to obtain bright, high-contrast images. The cameras are mounted on a custom table that has been designed with tilt, rotation, and height adjustments. Each camera is in turn mounted on its own vertical translation stage atop the table surface. The first major step in camera alignment is to cover the leading lens element using the customized reflective lens cap, which contains a series of 
concentric circles etched into its face. The $f=1000 \mathrm{~mm}$ plano-convex field lens should be positioned so as to focus the beam to the smallest admissible spot size onto the center of the reflective lens cap. The table surface is then adjusted (tilted, elevated, rotated) as required until the focused beam is reflected back through the field lens. Proper alignment requires that the beam must be focused precisely at the center of the reflective lens cap while also reflecting the laser beam back through the field lens. An iterative procedure must be patiently conducted until both of these conditions are satisfied. An excellent indication of alignment is the emergence of a speckle pattern that is observed on the black anodized lens mount. The focused beam position on the reflective lens cap is verified by viewing the cap while intermittently blocking the beam with a sheet of paper. This is the single most critical step of camera alignment and if it is achieved as specified here then there is a greater than $95 \%$ chance that all 16 image frames will appear properly centered within the computer display when the camera is triggered. Test images are acquired by properly arming the camera and forcing a trigger from the oscilloscope. Fine adjustments of each camera are then conducted through small perturbations of the individual translation stages upon which they are mounted.

A dynamic rupture is nucleated with the aid of a high-voltage "RC" discharge circuit, which is schematically depicted in Figure 3.3. An adjustable knob on the "firing box" is connected to a variable transformer, which controls the $\mathrm{AC}$ voltage that is supplied to the rectifier. The rectifier then produces a DC voltage, which is used to charge the capacitor bank. The range of knob settings on the firing box correspond to the (0-110 VAC) output range of the variable transformer, which translates to a $(0-5 \mathrm{kV}) \mathrm{DC}$ voltage output produced by the rectifier. High-voltage probes were used to characterize the voltage output of the rectifier as a function of the input AC voltage supplied by the variable transformer. The measurements were used to construct a calibration curve for the firing box, which is depicted in Figure 3.9. A standard voltage value of $\mathrm{V}=1.6 \mathrm{kV}$ is used in all laboratory earthquake experiments. The knob setting of " 45 " on the outside of the box corresponds very nearly to a $1.6 \mathrm{kV}$ DC voltage supplied to the capacitor bank.

The value of $C=17 \mu F$ corresponds to the series capacitance of 3 individual $50.8 \mu F$ capacitors contained in the actual circuit. Similarly, the value $\mathrm{R}=2.25 \mathrm{M} \Omega$ represents the total series resistance 
Firing Box Calibration Curve

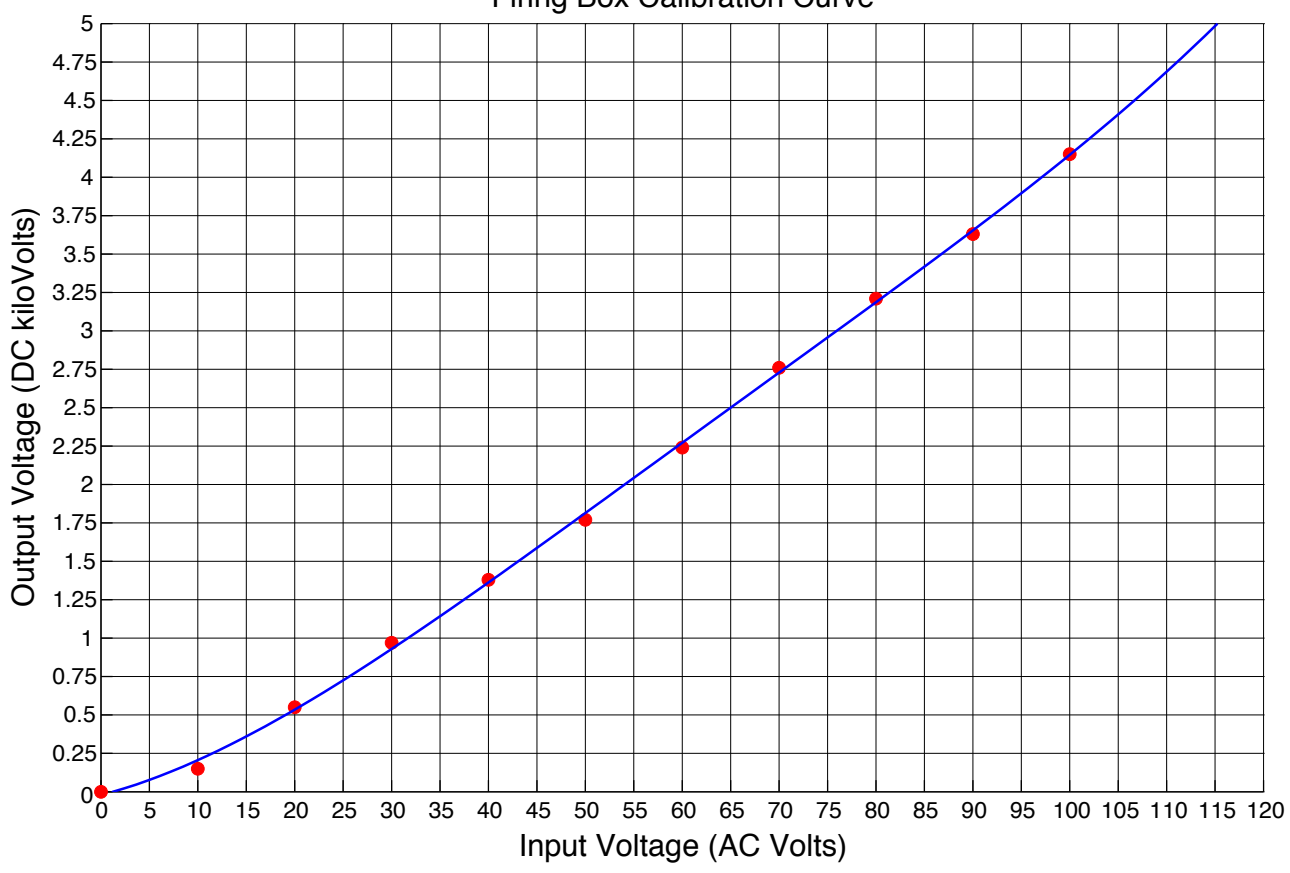

Figure 3.9: Firing box voltage calibration curve. Rectifier DC voltage used to charge the capacitor bank plotted versus the $\mathrm{AC}$ voltage supplied by the variable transformer

of 30 individual $75 \mathrm{k} \Omega$ resistors within the circuit. The effective $\mathrm{RC}$ time constant of the circuit is therefore given by $\tau=38.25 \mathrm{~s}$. An estimate for the time taken to fully charge the capacitor bank is obtained from the capacitor charging relation given by $Q=C V\left(1-e^{-t / \tau}\right)$, where $\mathrm{Q}$ is the total charge and $\mathrm{V}$ is the applied potential. Solving for the time taken to charge the capacitor bank to $90 \%$ of its full capacity and invoking the relationship $\lim _{t \rightarrow+\infty} Q(t)=C V$ then yields $\mathrm{t}=88 \mathrm{~s}$. The capacitor bank is thus fully charged in approximately 1.5 minutes.

The NiCr wire filament extends through the thickness of the H-100 test specimen and is seated within a small cylindrical cavity, as depicted in Figure 3.8 in order to prevent the wire from being pinched as the two plates are compressed. A DC voltage of $1.6 \mathrm{kV}$ is typically used to charge the capacitor bank. When the circuit is activated, the electrical charge stored within a capacitor bank is directed to ground and forced to pass through the thin $(79-\mu m$-diameter $)$ wire filament. The wire filament is unable to sustain the high current and is rapidly disintegrated. The resulting discharge produces a locally concentrated expansion of gas and material ejected by the reaction, 
which induce a small momentary reduction of the local normal traction $\sigma_{y y}^{0}$ while spreading out across a characteristic distance on the order of $1 \mathrm{~cm}-1.5 \mathrm{~cm}$. Somewhere within the course of $4.5 \mu s$, the applied shear traction $\sigma_{y x}^{0}$ becomes momentarily larger than the local static frictional strength and a mode II dynamic bilateral rupture is nucleated. A patch of discharge residue is always observed on both fault surfaces once the plates are unloaded and separated upon completion of the experiment. The precise role played by the discharge ejecta during the rupture nucleation process remains unclear at this time but it is reasonable to assume that it may also dynamically alter the local texture of the fault surface, in addition to partially unloading the normal traction.

A much scaled "replicate" of the high-current signal sent to along the NiCr wire path is simultaneously generated from the same circuit using a simple voltage divider. The sharp leading edge of the signal, which exhibits a sub-microsecond rise time, is fed into one channel of a digital oscilloscope (Tektronix model DPO3034) and used to trigger the oscilloscope. A standard TTL signal is then produced by the oscilloscope and used to trigger the high-speed gated-intensified CCD cameras. The remaining 3 channels of the oscilloscope are typically dedicated to acquiring the particle velocity signals.

\subsection{Physical dimensions of traditional laboratory earthquake specimens}

The general physical dimensions of the traditional laboratory earthquake specimen are depicted in Figure 3.8. The location of the NiCr wire channel is chosen based upon the desired rupture propagation distance and the time window of interest in a particular experiment. The position may be offset by a distance $\delta$ to the left of center as shown in Figure 3.8. Care is taken to calculate the arrival time of boundary waves reflected from the left free surface, which will necessarily arrive at the measurement station sooner than they would for the case when the wire filament is passed through the center of the specimen. There is also some risk of shifting the wire too far to one side into a region where the stress field becomes uniform, a condition which naturally arises as one shifts 


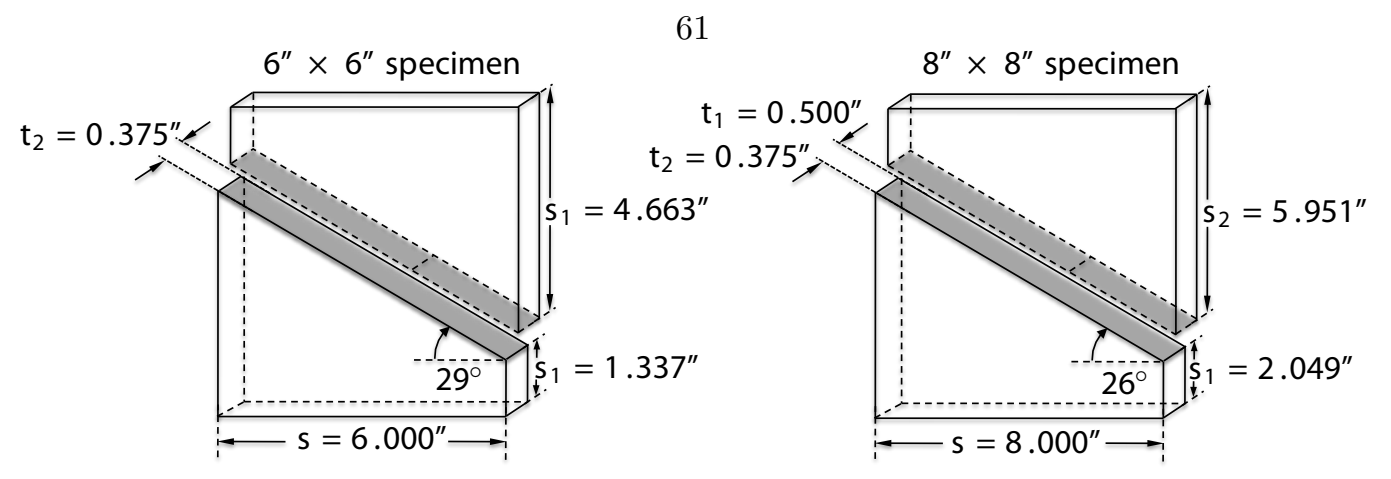

Figure 3.10: Physical dimensions of H-100 test specimens used in the laboratory earthquake experiments

closer to a free boundary. Schematic representations of the laboratory earthquake specimens that were machined for the experiments discussed and presented in this thesis are shown in Figure 3.10. The traditional $0.375^{\prime \prime} \times 6^{\prime \prime} \times 6^{\prime \prime}$ specimen dimensions were utilized by (Xia, 2005) and (Lu, 2009) and later by (Mello et al., 2010). Early experiments on ground motion investigations presented in this thesis were conducted using $0.375^{\prime \prime} \times 6^{\prime \prime} \times 6^{\prime \prime}$ specimens with a fault angle of $29^{\circ}$. The specimen dimensions were later extended to $8^{\prime \prime} \times 8^{\prime \prime}$ specimens of either $3 / 8^{\prime \prime}$ and $1 / 2^{\prime \prime}$ thickness with a fault angle of $26^{\circ}$. Increased specimen dimensions provided longer supershear transition lengths and expanded observation times of specific events that would otherwise be corrupted by reflected boundary waves in the case of $6^{\prime \prime} \times 6^{\prime \prime}$ specimens.

\subsubsection{Plate buckling analysis of $\mathrm{H}-100$ specimens}

Plate buckling ultimately limits the maximum static-compressive stress which can be applied to a square H-100 specimen assembly. The critical plate buckling stress $P_{c}$ for a square plate of width $w$ and thickness $t$ can be estimated using the relationship given by

$$
P_{c}=\frac{\pi^{2} E}{3\left(1-\nu^{2}\right)}(t / w)^{2}
$$

(Timoshenko and Woinowsky-Krieger, 1959) where $E$ and $\nu$ represent Young's modulus and Poisson's ratio, respectively. Figure 3.11 is a plot of the critical buckling load as a function of the plate width dimension $w$. Calculations were conducted by assuming a value of $E=5.2 \times 10^{9} G P a$ and $\nu=0.34$ 


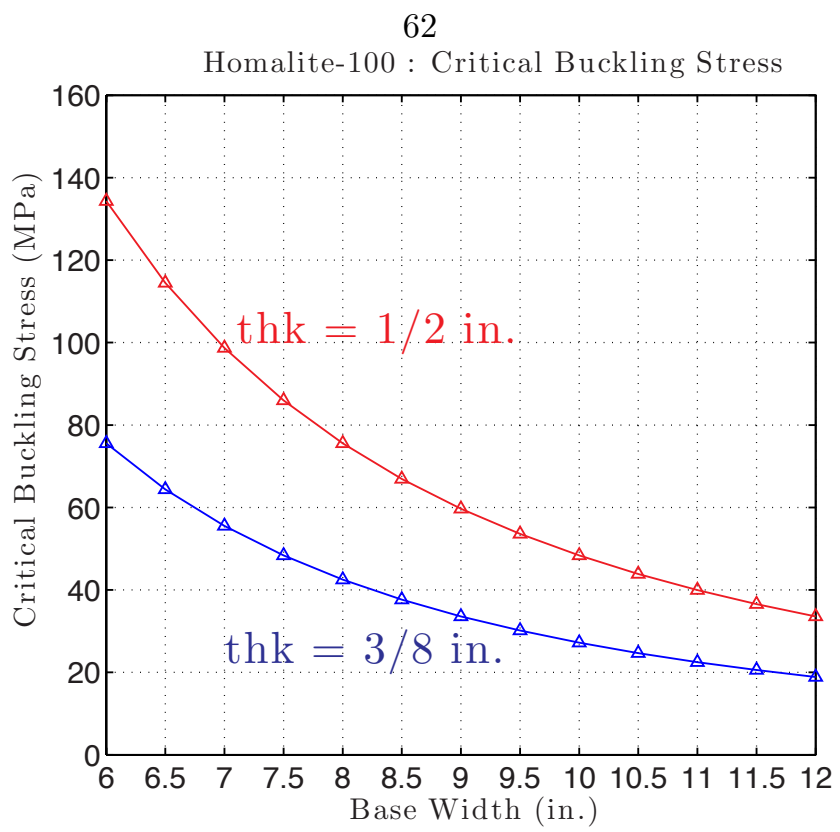

Figure 3.11: Critical buckling stress versus plate base dimension $w$ for a square H-100 plate of thickness $t_{1}=3 / 8^{\prime \prime}$ and $t_{2}=1 / 2^{\prime \prime}$

(Rousseau and Rosakis, 2009). The graph clearly shows how the critical buckling load is reduced by more than $50 \%$ when the base width of the H-100 assembly is extended from $6^{\prime \prime}$ to $8^{\prime \prime}$ for a plate thickness of $3 / 8^{\prime \prime}$. The plot also shows how the critical buckling load safety margin is easily recovered when the $8^{\prime \prime} \times 8^{\prime \prime}$ specimen plate thickness is increased from $3 / 8^{\prime \prime}$ to $1 / 2^{\prime \prime}$. Note however, that a $1.8 \times$ increase in the applied hydraulic force is now required to generate an equivalent static compressive stress in a $0.5^{\prime \prime} \times 8^{\prime \prime} \times 8^{\prime \prime}$ test specimen.

\subsection{Fabrication and surface preparation of H-100 laboratory earthquake specimens}

The general process flow for the fabrication of laboratory earthquake test specimens is depicted in Figure 3.12 and discussed in the following sections. The upper-left quadrant (I) depicts a mated pair of quadrilateral H-100 test sections cut from H-100 sheet stock. The parts are machined to specified tolerances by a CNC milling operation. Specimen dimensions and fault surface texture are carefully examined as detailed later in this section. The lower-left quadrant (II) depicts the 
63

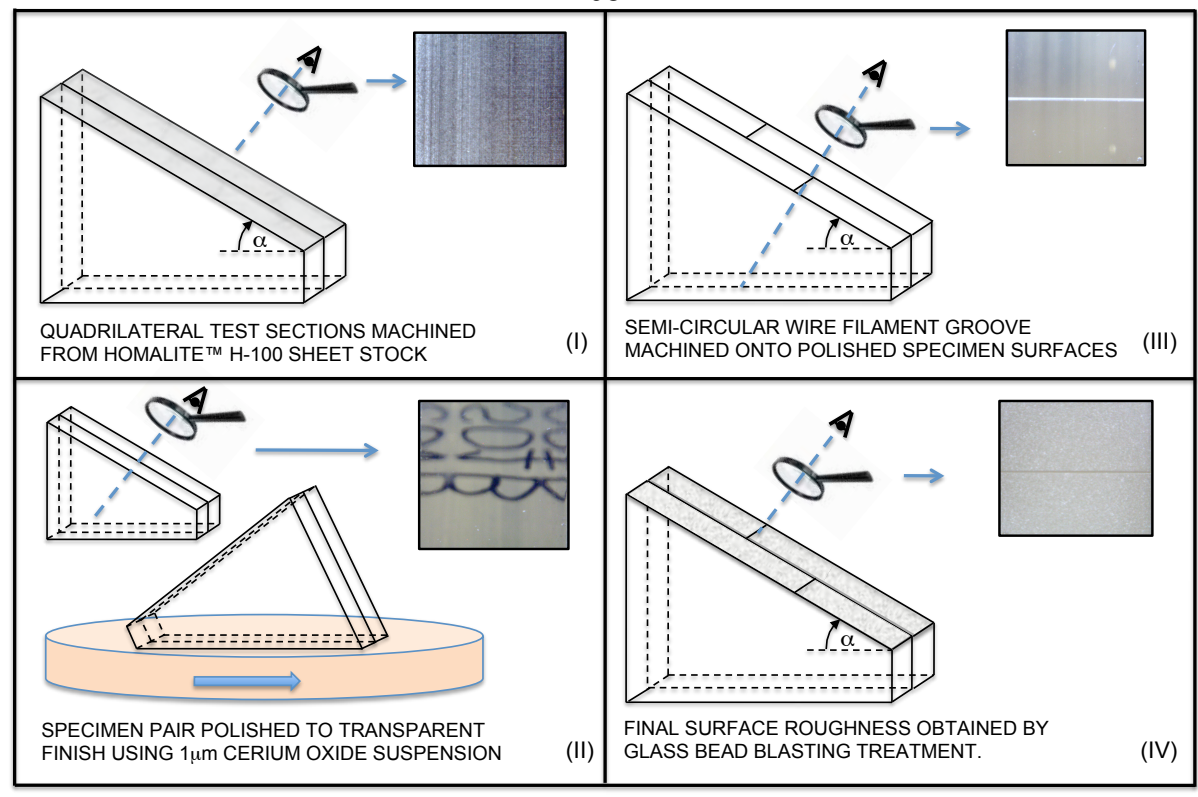

Figure 3.12: Overview of $\mathrm{H}-100$ specimen fabrication and surface preparation

polishing step used to remove all surface defects. The mating test surfaces are mounted together in a steel jig and polished together as a pair using a $1 \mu m$ cerium oxide suspension. The accompanying image in (II) reveals how the resulting test surfaces are polished to a near-optical-grade finish, free of the machining grooves and other surface defects. The inset depicts a specimen identification number written on the opposite edge of the specimen, which was imaged through the polished, optically transparent test surfaces. The upper-right quadrant (III) depicts two $127-\mu m$-diameter semi-circular channels that are machined into the polished surfaces at the desired rupture nucleation site. The two channels should meet to form a tiny hole passing through the frictional interface in the mated specimen assembly. The $79-\mu m$-diameter $\mathrm{NiCr}$ wire filament is seated within this cavity in the assembled specimen configuration and used to trigger a dynamic rupture. The lower-right-hand quadrant (IV) depicts the final roughened surface texture, which is achieved through a micro-bead blasting treatment. The abrasive media is typically comprised of tiny glass beads with diameters ranging from $105 \mu m-210 \mu m$ (70-140 mesh size). Precise repetition of the bead-blasting procedure from specimen to specimen assures a consistent surface roughness from experiment to experiment. A detailed description of these procedures is presented in the following sections. 


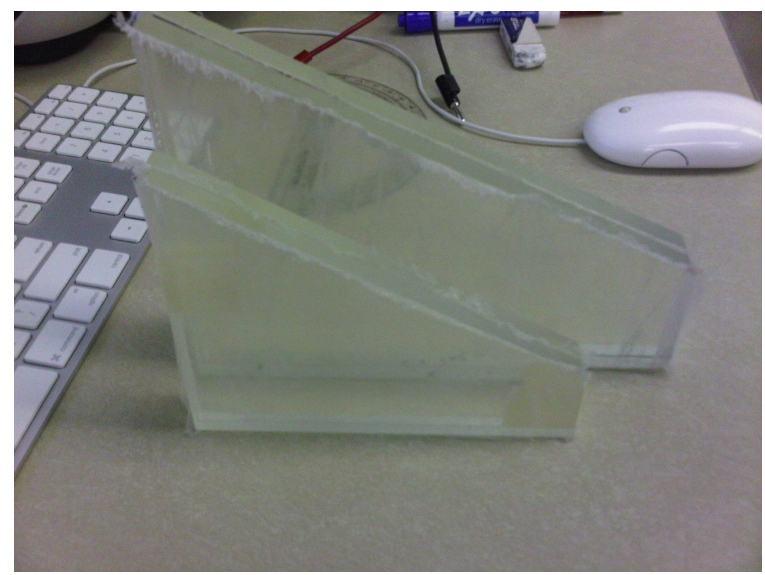

Figure 3.13: Machined H-100 quadrilateral test sections: $\mathrm{s}=6$ " and $\mathrm{s}=8$ " mated pairs

H-100 is obtained in sheet stock form from the Homalite Division of the Brandywine Investment Group Corporation. Typical sheet dimensions are $21^{\prime \prime} \times 31^{\prime \prime}$ and the material is made available in standard thicknesses of 0.031 in, 0.045 in, $0.062 \mathrm{in}, 0.093 \mathrm{in}, 0.125 \mathrm{in}, 0.25 \mathrm{in}, 0.375 \mathrm{in}$, and 0.500 in. H-100 sheet stock can exhibit inherent curvature resulting from fabrication processes or possibly even the manner in which it has been stored. It is for this reason that H-100 sheet stock should be kept stacked flat in a cool dry environment whenever possible.

A standard sheet of $\mathrm{H}-100$ is first cut into either $6.375^{\prime \prime} \times 7^{\prime \prime}$ sections or $8.375^{\prime \prime} \times 9^{\prime \prime}$ rectangular sections using a band saw operation, depending upon whether final specimen dimensions of $6^{\prime \prime} \times 6^{\prime \prime}$ or $8^{\prime \prime} \times 8^{\prime \prime}$ test specimens are desired. Each individual "rough cut" rectangular section is then firmly clamped onto a tooling fixture within the CNC milling machine. Detailed specifications obtained from machine drawings are encoded into a computer-aided machining (CAM) program that drives and controls the CNC milling machine. The feed rate and spindle speed of the CNC milling machine are set according to documented guidelines in order to prevent chipping and fracturing during this machining operation. The part is also constantly sprayed with machining oil in order to prevent overheating of the part and tool during the cutting operation. A diagonal cut of the clamped rectangular section is executed using a $3 / 16^{\prime \prime}$ flute-standard Carbide square end mill in order to create two quadrilateral sections. The two "rough cut" quadrilateral test sections are appropriately marked and maintained together as a mated set. After completing the diagonal cut, 
the outer perimeter of each clamped quadrilateral section is trimmed down using the same $3 / 16^{\prime \prime}$ flute-standard Carbide square end mill. After this first milling operation is completed, the Carbide square end mill is replaced by a newer and sharper $3 / 16^{\prime \prime}$ Carbide tool and the process is repeated in order to complete the operation using a conventional cutting operation. Each quadrilateral section is then re-clamped using additional clamps and trimmed down to specified dimensions using a $3 / 8^{\prime \prime}$ flute-standard Carbide square end mill. A final finishing operation is then executed using a $5 / 16^{\prime \prime}$ flute standard Carbide square end mill to produce a finely machined surface. Finish parts should be accurately machined to within $\pm 0.005^{\prime \prime}$ of the specified dimensions. The final angle of the diagonal edge (test specimen fault surface), measured with respect to the base edge of dimension $s$, should be machined to within $\pm 0.25^{\circ}$ of the desired fault angle. Figure 3.13 shows a typical pair of machined, H-100 quadrilateral test sections with base dimension $s=6$ inch, which are contrasted beside a set of larger quadrilateral test sections with base dimension $s=8$ inch. Note as well the protective plastic film, which is adhered to the parts in order to protect the integrity of the specimen faces during the machining phase. Both specimen sizes were utilized in the experimental investigations conducted in support of the results presented in this thesis. The justification for choosing one dimension over the other is addressed in the various sections where these experiments are presented and analyzed.

The final machining operation involves milling the cylindrical wire channels into the quadrilateral test sections. A $0.005^{\prime \prime}$ diameter cylindrical cavity is machined at the desired offset location as depicted in Figure 3.8. Precise location of the wire channel is specified by the distances $D_{1}$ and $D_{2}$, as shown. H-100 specimens have traditionally been machined with a pair of mated wire channels. In this case the wire is seated within one of the channels (typically the channel milled into the bottom test section), which is aligned directly opposite to a second identical cylindrical cavity. Inspection of the H-100 interface in the region of the milled wire channels with the aid of an optical microscope has revealed that the wire channels are typically misaligned by at least a full channel diameter, when the two test sections are mated together. This is mainly a consequence of dimensional tolerances. It is certainly possible to machine wire channels that will be properly registered when the plates are mated together but the procedure is manually intensive, which results in longer 
machining throughput time and increased machining costs. Having noted this issue, many successful experiments have been conducted using a single wire channel machined into only one of the H-100 plate test sections (typically the bottom plate). Dynamic ruptures have been successfully nucleated and the experimental results appear similar in all respects to previous data collected using specimens with two opposing wire channels. It remains inconclusive as to what role, if any, the second wire channel plays or whether it is actually critical in any way to the success of laboratory earthquake experiments

In anticipation of surface polishing and micro-bead blasting procedures, a decision must be made early on as to whether the wire channel should be milled right away or later on once the final desired surface texture has been achieved. Polishing is conducted in order to remove any grooves or other surface defects induced by machining as a prerequisite step to the surface-roughening (micro-bead blasting) procedure. However, excessive polishing of the fault surfaces can easily lead to the erosion of the wire channel, or at the very least a significant reduction of the wire channel depth. Furthermore, the experiments naturally depend upon the integrity of the final roughened fault surfaces and so it would seem unwise to resubmit bead-blasted specimens to the machine shop for the final wire channel milling step. Note as well that the depth of the wire channel relative to the fault surface is largely preserved during bead blasting even though the channel itself does become pitted and even slightly widened in the process. As it turns out, the decision whether to to mill the wire channel right away or until after the polishing step is completed depends upon the the surface quality of the "as machined" test specimens. The approach that was adopted in the vast majority of the experiments presented in this thesis was to mill the wire channels after completing the polishing step and prior to the bead-blasting procedure. However, alternative process flows, such as milling the channel right away and even milling the wire channel after bead blasting, were also experimented with on occasion. There appear to be no obvious anomalies associated with the data collected in these experiments that can be attributed to the surface preparation process flow. The common ingredient in all cases was a wire channel of sufficient depth such that the NiCr filament was properly seated and maintained just below the fault surface. The main point here is to be aware of these issues and 

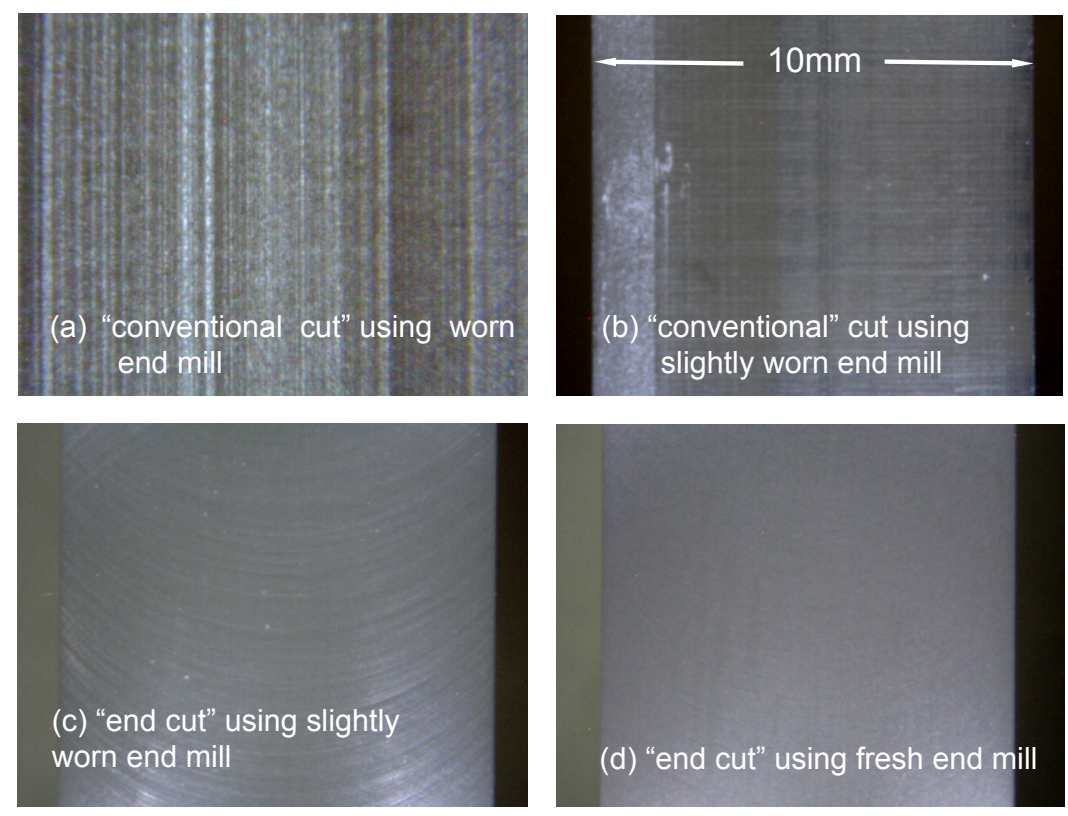

Figure 3.14: Surface texture of H-100 specimen surfaces resulting from conventional-cut and end-cut milling operations

to have solution paths in place depending upon the initial state of the "as machined" test specimens.

There are three key indicators that are used to judge the quality of "as machined" H-100 test specimens: (1) surface texture, (2) overall planarity of the fault surface, and (3) the perpendicularity of the fault surface with respect to the face of the test specimen. Each of these factors can have a direct bearing on the success or failure of an experiment.

\subsubsection{Machining-induced surface defects}

The use of worn end mills can induce deep machining grooves that run parallel to the fault surfaces. Surface defects of this type are depicted in Figure 3.14a. It can take considerable time and effort to polish out these surface defects. Besides the added time and effort, excessive polishing of the fault surfaces can potentially erode the wire channel and degrade the planarity of the fault surface. Figure 3.14b shows how a significantly smoother surface texture was achieved by the same conventional milling operation, but with a newer and sharper end mill. Even smoother surface textures can be achieved by the same conventional cutting operation if a brand new set of end mills are used for each H-100 mated pair. A second type of milling operation, referred to as an "end cut" milling procedure 
was also explored. Figure 3.14c reveals the characteristic circular swirl pattern that results from this type of milling operation. The circular grooves in this instance were found to be quite shallow and could be polished away with a reasonably minimal effort. The image in Figure 3.14d represents the smoothest surface that was attained using the "end cut" milling procedure. The surface quality in this instance was superb and polishing of the test piece was technically not even required.

\subsubsection{Planarity and perpendicularity of machined fault surfaces}

Strict attention was paid to how well the material was clamped during the machining and cutting operations. Care was also taken to position tooling clamps on either side of the planned diagonal cut, while keeping the clamps as close as possible to the path of the cutting tool, so as to prevent the edge of the part from curling and lifting up as residual stresses were relieved during the course of the cutting operation. Proper clamping of an H-100 work piece is critical in achieving planar fault surfaces that are also perpendicular to the face of the part. The planarity and perpendicularity of "as machined" H-100 test specimens were routinely checked by laying a mated pair of quadrilateral test sections on a flat glass plate and butting the two halves together, as schematically depicted in Figure 3.15. Two small pieces of clear cellophane tape were placed across the interface near the lower-left and upper-right-hand corners of the mated parts in order to keep the parts from slipping or separating during the inspection process. The interface was then examined using a $130 \mathrm{x}$ USB microscope camera (Sharper Image CN311), which features a variable magnification. Lowmagnification images of the interface obtained at 3 unique locations along the fault are depicted in Figure 3.15. The right and left lateral configuration description refers to the fact that both sides of the test specimen assembly were inspected. The images reveal a very tight interfacial seam in all 6 images, which is characteristic of a very well machined test specimen. The small semi-circular channel for seating the $\mathrm{NiCr}$ wire filament is seen at the center of the upper-right image in the left lateral configuration. The upper-left image of the right lateral imaging configuration was obtained from a region just beyond the wire channel, which explains its absence from that image.

Figure 3.16 contains images obtained from a different specimen interface, which featured a "wedge 

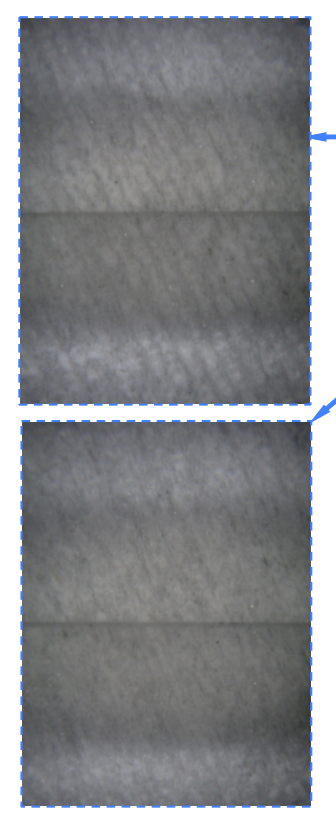
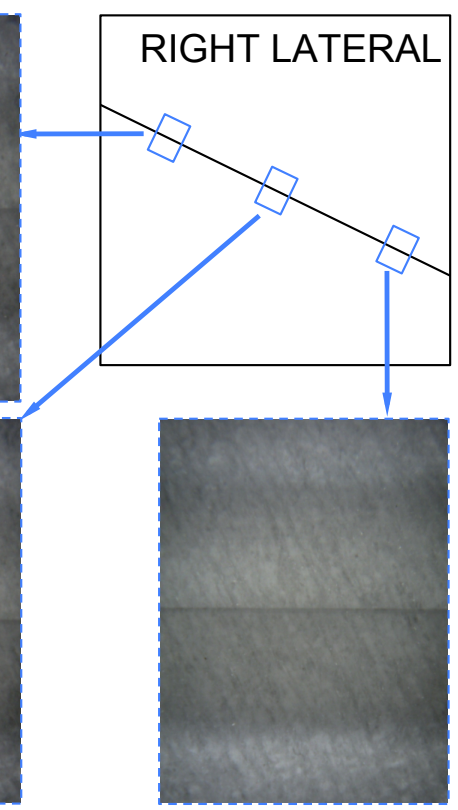

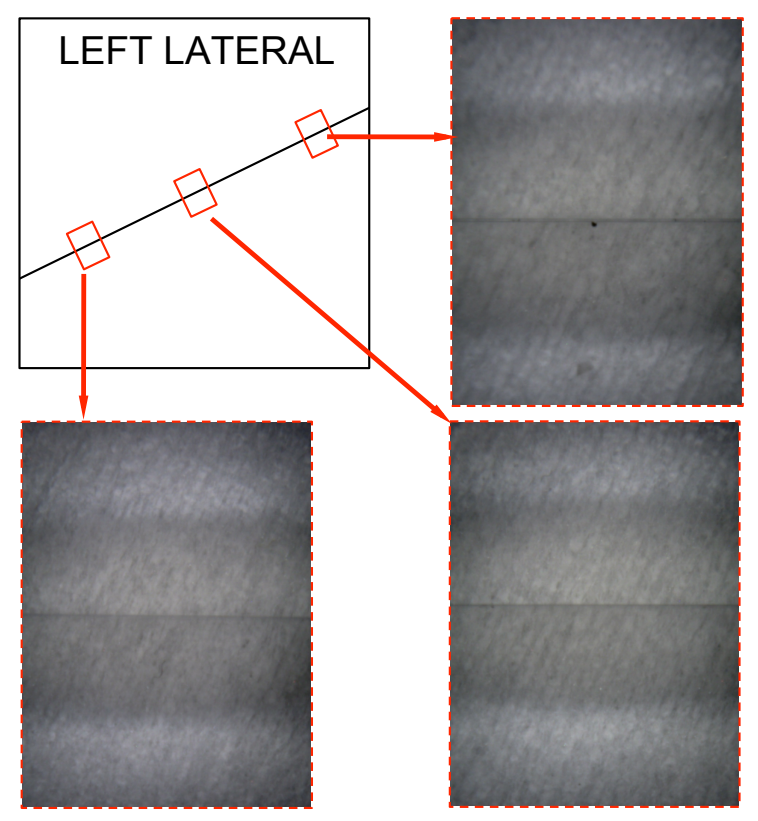

Figure 3.15: Optical inspection of H-100 test specimen revealing a well-machined laboratory earthquake specimen

gap". In this case, the microscope was deliberately tilted to produce the right lateral images.

The images provide a clear view of the frictional fault seen looking through the transparent $\mathrm{H}$ 100 specimen face. The image in the upper left corner reveals the milled semi-circular wire channel, which has been machined into the lower quadrilateral test section. A close-up view of this image and the accompanying two images reveals a very tight seam defining the frictional interface. The same test specimen was then turned over and imaged at the three corresponding locations, as depicted in the left-lateral image configuration. In this case only the upper right-image was obtained using a tilted imaging perspective. Note in particular how the interface is no longer as sharply defined compared to how it appeared on the opposite side when viewed in the right-lateral coinfiguration. The thick black region aligned with the interface corresponds to a physical gap between the edges of the two mated fault surfaces. The remaining two images in the left-lateral imaging configuration also reveal the same type of gap. The corresponding right- and left-lateral imaging perspectives collectively reveal a wedge-shaped gap, since the edges of the fault surface on the opposite side were seen to be in contact. Hence, in this particular case the highly planar fault surfaces are not quite 
perpendicular to the face of the test specimen. Although this situation appears less than ideal at first glance, it turns out that the wedge angle is actually quite small. Using the wire channel diameter as a length scale, the gap is estimated to be $100 \mu \mathrm{m}$, which translates to a wedge angle on the order of $10 \mathrm{mrad}$ (since the part is $10 \mathrm{~mm}$ thick). Specimens such as the one depicted in Figure 3.16 can therefore still be successfully tested, as the gap will close under the applied static compressive load. Nonetheless, this particular example begins to reveal some of the consequences that may arise when machining tolerances are not met. In the case of larger wedge angles the assembly may be prone to buckling. Another worst-case scenario (not depicted here) corresponds to the situation where the width of the gap starts out small at one end and continuously increases all the way to the opposite end of the fault. This scenario is generally associated with curved fault surfaces, which typically arise from excessive polishing whereby the initial machined planarity is lost and the parts have conformed more to the (concave) profile of the polishing surface. These worst-case specimens with curved fault surfaces require a considerable static compressive load before the curvature is fully overcome and a proper interface is formed. This is typically accompanied by severe stress gradients, which in the worst case can conspire to suppress rupture nucleation altogether. The importance of obtaining the flattest and smoothest possible "as machined" surfaces right from the outset and preserving or even improving the flatness of the part cannot be overstated.

\subsection{H-100 specimen surface polishing procedure}

A specimen-polishing procedure is typically conducted in order to remove any surface defects that may have resulted from the CNC milling process. As previously discussed, the polishing step should generally be conducted prior to milling of the wire channel unless the parts are consistently machined to a high-quality surface finish. If this is indeed the case it may be possible to have the wire channel machined before the polishing procedure without any risk of eroding the wire channel.

Two mated H-100 quadrilateral test sections are clamped together by a heavy steel jig as shown in Figure 3.17a. Two rectangular steel blocks measuring $12^{\prime \prime} \times 2.5^{\prime \prime} \times 1^{\prime \prime}$ are fastened together by a pair of 3/8"-16 socket head screws. A $9.625^{\prime \prime}$ center-to-center spacing between the screws provides 

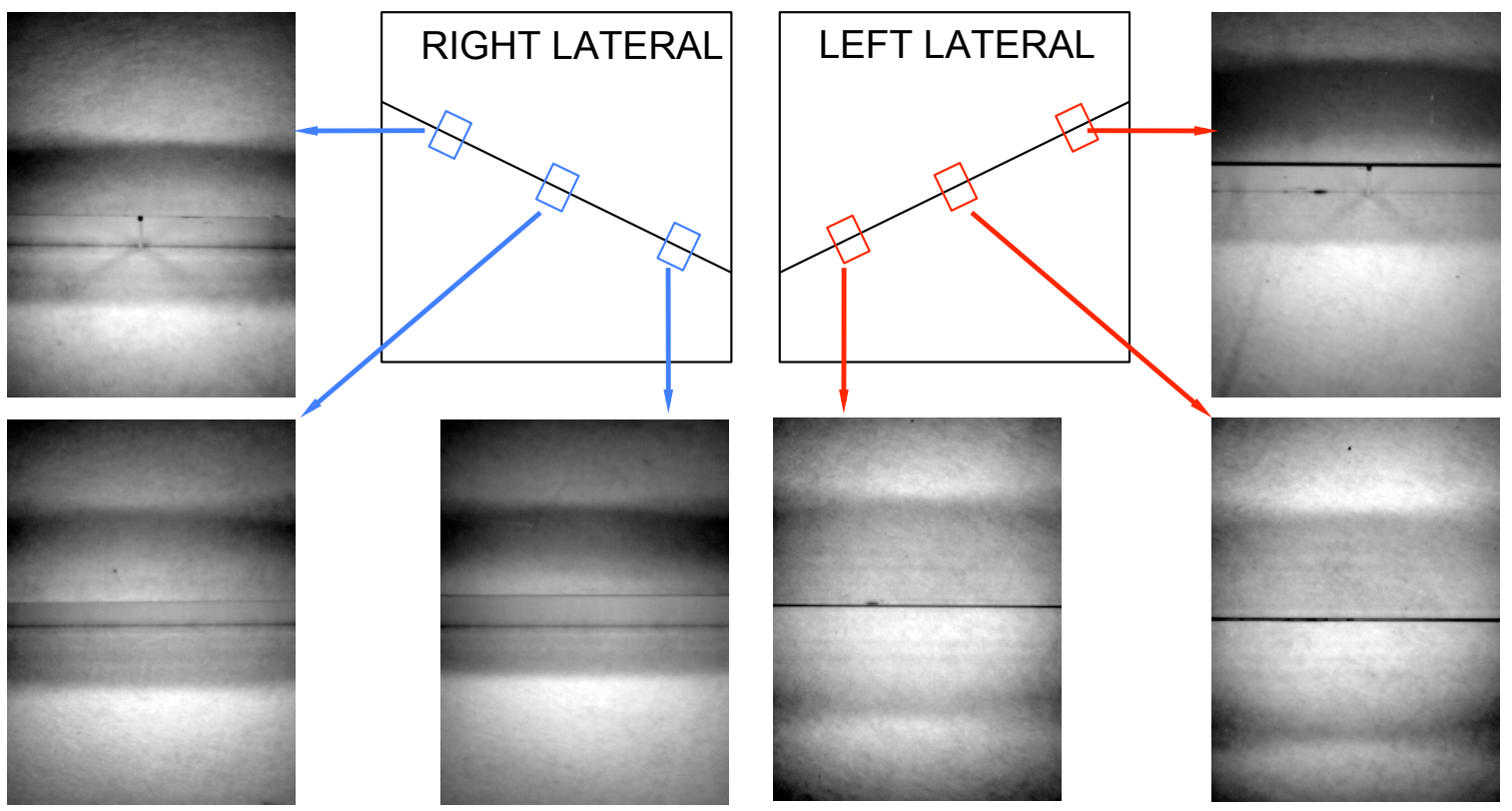

Figure 3.16: Optical machining inspection of $\mathrm{H}-100$ test specimen revealing a wedge gap resulting from non-perpendicular fault surfaces
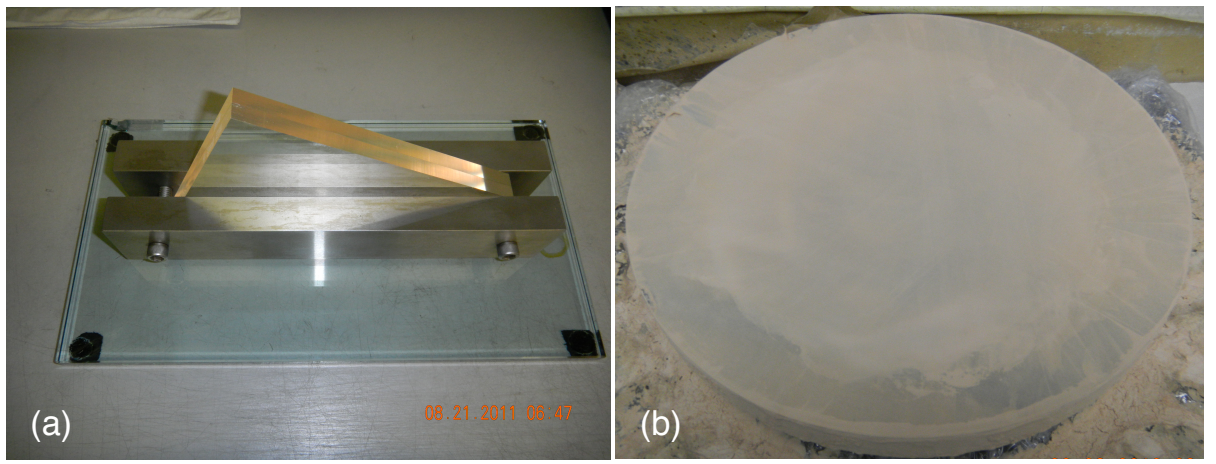

Figure 3.17: (a) Mated pair of H-100 quadrilateral test sections ( $=8$ ", thickness $=0.5 "$ ) mounted in steel polishing jig. (b) 12" diameter polishing wheel with Buehler UltraPo ${ }^{T M} 40-7452$ PSA-backed polishing cloth and $1 \mu \mathrm{m}$ cerium oxide suspension (Buehler Ltd. \#40-6355-006 Micromet polish)

enough clearance for mounting a mated pair of $8^{\prime \prime}$ quadrilateral test sections, as depicted in the image. The entire assembly is initially placed on a flat glass plate prior to tightening the bolts. The glass plate and H-100 specimen surfaces are carefully cleaned with acetone prior to this step in order to avoid the possibility of entrapping hard particulates that might otherwise scratch the H-100 fault surfaces. Other solvents such as such as methanol may also be used. Small thin strips of paper (not 
visible in the image) are placed between the steel blocks and the glass plate in order to create a very short stand-off distance. A slight downward pressure is then applied to the mated H-100 test sections in order to force the fault surfaces to become flush with the flat glass reference surface below. The entire assembly is gently squeezed and the bolts are hand tightened and then firmly snugged using a 5/16 Allen wrench. The jig assembly is then flipped over and carefully inspected. The first thing to note is whether the interface between the mated, clamped H-100 sections is smooth and continuous, or whether a step-height discontinuity can be detected. There is no better way to check this than to run your index finger along the seam. The second critical point to check is whether a proper stand-off distance has been established by the thin paper shims between the plane set by the H-100 fault surfaces and the bottom edges of the steel blocks. The short but finite stand-off height is critical to the polishing process and insures that the H-100 fault surfaces will rest upon the polishing surface during the manual polishing operation, while experiencing the weight imposed by the steel jig. Meanwhile the jig itself is kept from making any contact with the polishing surface. The mated H-100 test surfaces were polished together as a pair using a $1 \mu \mathrm{m}$ cerium oxide suspension (Buehler LTD \#40-6355-006 Micromet polish) with a Buehler UltraPo ${ }^{T M}$ 40-7452 PSA-backed polishing cloth on the 12" diameter polishing wheel depicted in Figure 3.17b.

Early attempts at polishing using a manually guided polishing procedure on a motorized polishing wheel resulted in highly transparent surfaces that unfortunately also exhibited significant curvature along the long axis of the fault surfaces. Use of an electrical-motor-driven polishing wheel induced greater instability, making it more difficult to maintain the test pieces normal to the plane of the polishing surface. The surface curvature is not immediately visible to the eye and the parts may appear extremely flat at first glance. Inspection of the interface as discussed in Section 3.5.2 is the ultimate indicator. Convex fault surfaces are revealed if one extreme edge of the fault displays a tight interfacial seam while the opposite end exhibits a pronounced gap or vice versa. This was the most common form of curvature that was detected and is likely an indicator that the polishing wheel itself exhibits a concave profile, which the parts generally conform to if excessively polished.

The jig assembly containing the mated test sections should be held firmly using two hands and 

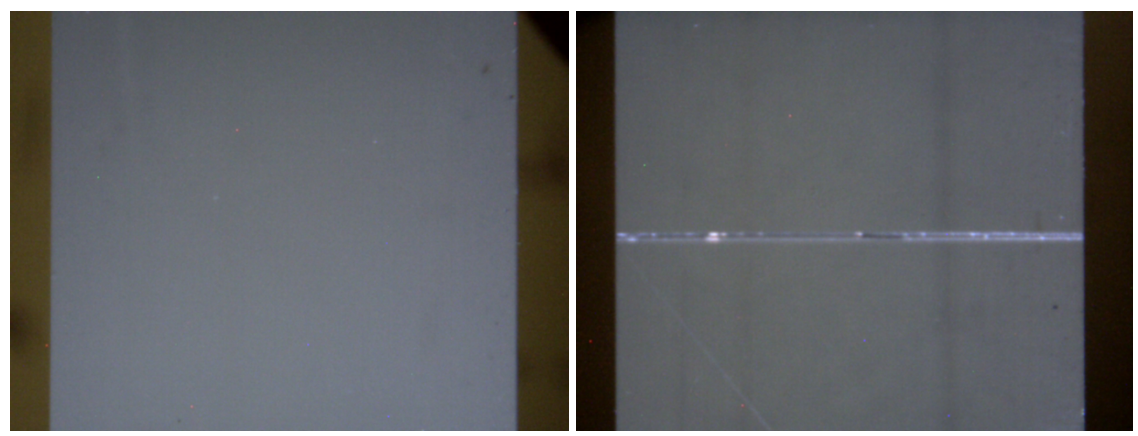

Figure 3.18: H-100 specimen fault surfaces polished to a near-optical-grade finish

slid around the circumference of the polishing surface in one continuous motion at approximately 1 $\mathrm{rev} / \mathrm{second}$. Only a gentle pressure is required and the weight of the steel blocks and relatively low center of gravity should be enough to maintain proper contact between the specimen surfaces and the polishing media. Polishing should be sustained for approximately 60 revolutions before stopping to evaluate progress. The assembly is then rinsed with water and dried, ideally using a centralized high-pressure air hose in order to thoroughly dry the specimen surfaces and prevent the formation of streaks that may result from wiping the surface dry. When examined under normal room-lit conditions, the specimen fault surfaces should already be transparent in certain regions. The most critical factor at this point is to note whether one plate is becoming more polished and transparent than the other. If so, then this is a clear indication of a step-height discontinuity between the test sections. The jig must be then be disassembled and cleaned and the parts once again remounted using the flat glass plate. With experience this process becomes routine and the two mated test surfaces become one continuous test surface with respect to the polishing wheel surface. Polishing is continued until the specimen fault surfaces become completely transparent. If the parts are properly machined and well aligned within the jig, then it should not take more than 5 minutes of polishing to achieve completely transparent test surfaces that are free of any major surface defects. Figure 3.18 shows two images taken by the 130x USB optical microscope under low magnification. Note the wire channel in the image on the right and the lack of any significant surface defects in either image. A nearly optical grade surface finish can be achieved using this simple polishing procedure. 


\subsection{Micro-bead blasting procedure for surface roughening of H-100 test specimens}

The polished fault surfaces of laboratory earthquake specimens are uniformly roughened in a microbead blasting chamber. The surfaces are pitted by a jet of tiny glass beads of a selected size range, which are accelerated toward the specimen fault surfaces by a blast pressure of 20-60 PSI. Glass bead blasting media is obtained from McMaster-Carr and is supplied according to "mesh size",

a designation that is commonly used in determining the particle size distribution of a granular material. The table in Figure 3.1 provides a conversion between the mesh-size range of available glass beads supplied by McMaster-Carr and the corresponding range of particle diameter. The use of one particular size within a given study is dependent upon the nature of the surface roughness desired in that particular investigation. All of the experimental data that is discussed and analyzed in this thesis was obtained using H-100 test specimens whose surfaces were prepared using a mesh size range (70-140) or particle diameter range $(105 \mu m-210 \mu m)$.

A mated set of polished $\mathrm{H}-100$ test specimens is mounted within the same steel jig used in the polishing procedure and positioned within the bead-blasting chamber, as depicted in Figure 3.19. A glass plate is once again used as a reference surface for assembling the polished test pieces within the steel jig. All surfaces are thoroughly cleaned prior to assembly in order to avoid scratching the polished surfaces. Note that the use of a thin paper shim is not required as it was in preparation for the polishing procedure. The objective in this case is to make all surfaces flush with the surface of the glass plate in order to avoid eroding the edges of the H-100 test sections during the bead-blasting procedure. The assembly should be squeezed together with light pressure applied from above while the $3 / 8$ " -16 socket head screws are finger tightened. The assembly is then flipped over and stood upright on the work bench. The seam formed between the two mated test sections should be smooth with no detectable step height discontinuity along the entire length of the mated assembly. The test pieces may be gently shifted if necessary in order to make the surfaces flush. Attention must always be paid to make sure that the fault surfaces do not extend out beyond the exposed surfaces of the 


\begin{tabular}{|c|c|}
\hline Mesh size range (US) & Particle diameter $(\mu m)$ \\
\hline $40-60$ & $250-400$ \\
$60-120$ & $125-400$ \\
$70-140$ & $105-210$ \\
$100-170$ & $149-210$ \\
$170-325$ & $44-88$ \\
\hline
\end{tabular}

Table 3.1: Simple table for converting Mesh size to glass bead diameter

steel jig. The $3 / 8$ " -16 socket head screws may then be tightened using a $5 / 16$ Allen wrench once the H-100 specimens have been properly adjusted within the jig.

A mask should be worn during all phases of the bead-blasting operation in order to avoid inhaling any fine dust particles. Glass beads are stored in a sealed plastic bin and transported from the laboratory to the machine shop where the bead-blasting unit resides. The bead-blast chamber should be thoroughly flushed of previously used bead-blast media at the start of each session. This is a very quick and easy process, which mainly involves dumping out whatever material is sitting in the chamber bin. The machine is then run for 30 seconds and residual material collected within the bin is once again dumped. Fresh glass beads are then poured into the chamber.

The clamped specimen assembly is then positioned against the rear wall of the bead blast chamber, as depicted in Figure 3.19. Once the jig assembly is properly positioned, the chamber door is closed and sealed tight by two latching clasps. The unit is turned on and a partial vacuum is created in the test chamber. A set of sealed rubber gloves allow the operator to hold and operate the gun assembly inside the chamber. A foot pedal is then used to apply air pressure to the gun nozzle. The nozzle is maintained at a fixed distance of 5-10 inches from the test surface and swept vertically up and down in order to uniformly roughen the H-100 surfaces. A systematic and repeatable procedure was established and applied for every specimen that was prepared in this manner. H-100 specimens were bead blasted by counting out 20 total strokes at approximately $4 \mathrm{sec} /$ stroke, where a stroke corresponds to a single directional sweep, either up or down.

Once completed, the specimen assembly is removed from the test chamber and cleaned off using a high-pressure air line. The glass bead blast media was then immediately re-ollected into the 


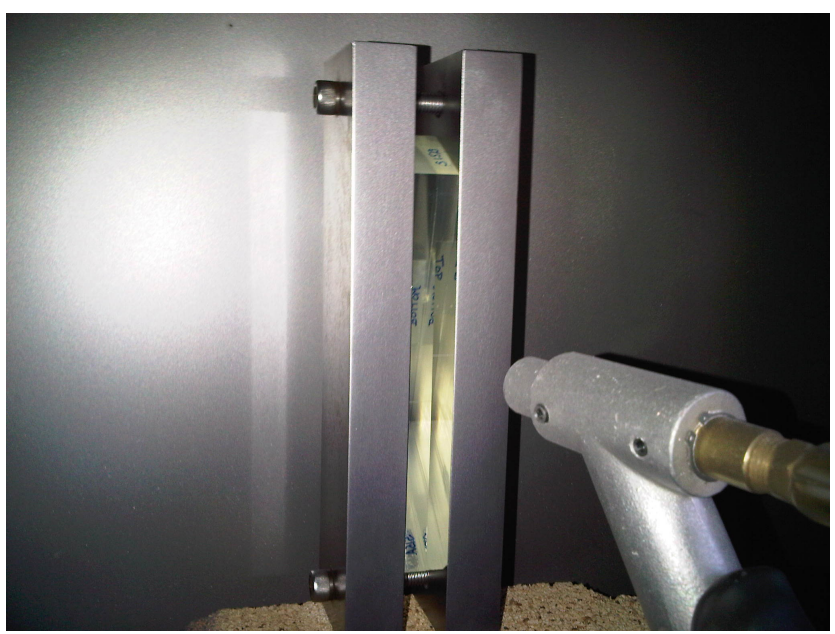

Figure 3.19: H-100 specimens positioned within micro-bead blasting chamber

plastic bin and transported back to the lab. Glass beads were never left unattended in the chamber for any prolonged period of time in order to avoid the possibility of the beads being inadvertently used to blast some other material, which might otherwise contaminate the media. The H-100 test sections were then removed from the jig and thoroughly cleaned with acetone. The steel jig was also thoroughly wiped and cleaned and stored away for later use.

\subsection{Surface roughness evaluation of $\mathrm{H}-100$ specimen surfaces}

Surface texture is typically characterized using either an optical (white-light interferometer) or stylusbased measurement technique which reports the surface height variation as a function of scan position. The generic term surface texture encompasses a number of topographical parameters such as "waviness" and "roughness". Waviness corresponds to low-frequency (long-wavelength) surface irregularities whose spacing is significantly greater than the data sampling period, while roughness pertains to higher-frequency (short-wavelength) surface irregularities. The surface waviness profile is obtained by subjecting the surface height data to a low-pass filtering operation. Surface roughness, on the other hand, is determined through a high-pass filtering operation. The choice of an appropriate low- or high-pass cut-off frequency must be carefully evaluated on a case-by-case basis. Measurement standards such as (ANSI/ASMEB46.1-2009, 2009) provide guidance for establishing 


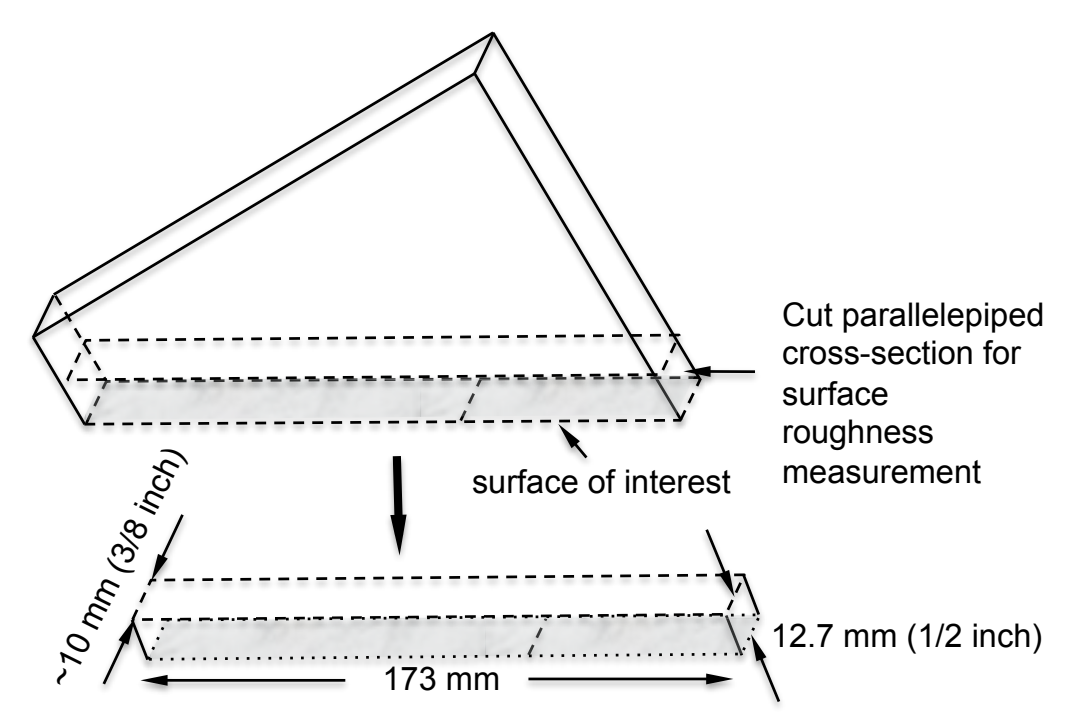

Figure 3.20: Parallelepiped H-100 specimens submitted for surface roughness evaluation

appropriate cut-off frequencies when attempting to extract waviness and roughness from surface height-data. A recommended "rule of thumb" for determining surface roughness is to select a highpass-cutoff wavelength equal to $1 / 100$ the entire length of the scan (Chi et al., 2004). Standard parameters that are commonly used to quantify surface roughness are the average roughness $(R a)$, given by

$$
R_{a}=\frac{1}{L} \int_{0}^{L}|z(x)| \mathrm{d} x
$$

and the root-mean-square $(R M S)$ roughness given by

$$
R_{q}=\sqrt{\frac{1}{L} \int_{0}^{L} z(x)^{2} \mathrm{~d} x}
$$

where $x$ represents the scan position, $z(x)$ is the surface height function, and $\mathrm{L}$ corresponds to the total scan length. The quantity $R_{a}$ is commonly quoted and corresponds to the arithmetic average surface deviation from the mean line within the scan length $(\mathrm{L})$. The quantity $R_{q}$, is a more statistically meaningful quantity, which corresponds to the root-mean-square average of the departures of the roughness from the mean line. Analogous definitions may also be applied to the characterization of waviness (Chi et al., 2004). 
A surface roughness evaluation was conducted on four individual H-100 samples corresponding to (a) a well-machined surface (b) a fine-polished surface (c) a bead-blasted surface prepared using $104 \mu m-211 \mu m$ (70-140 mesh size) glass beads, and (d) a tested (ruptured) H-100 test surface, which was initially bead blasted. Test samples were carefully sectioned from original quadrilateral test sections into parallelepiped shaped strips measuring $173 \mathrm{~mm} \times 12.7 \mathrm{~mm} \times 10 \mathrm{~mm}$, as depicted in Figure 3.20. The four H-100 surface roughness specimens were sent to the University of Colorado-Advanced Photonics Technology Center (CAPT) Lab for high-resolution surface roughness evaluations using a Dektak ${ }^{\mathrm{TM}}$ surface profilometer manufactured by Veeco Instruments, Inc. The instrument features a diamond tipped stylus with a $200 \mathrm{~nm}$ radius that maintains physical (low- force) contact with the test surface in order to detect height variations in the surface topography (Chi et al., 2004). The stylus remains fixed and in contact with the test surface at all times as the sample is physically displaced across a flat reference surface with the aid of a high-resolution translation stage. A linear variable differential transformer (LVDT), which is coupled to the stylus, produces an analog signal (proportional to displacement) that is properly amplified, filtered, and digitally recorded. A properly calibrated and vibration isolated Dektak ${ }^{\mathrm{TM}}$ profilometer is theoretically capable of resolving surface height variations as small as $10 \AA$ (Chi et al., 2004).

A $173 \mathrm{~mm}$-length line scan was conducted across the full length of each test sample with the instrument stylus positioned at the midpoint of the $10 \mathrm{~mm}$ cross-sectional width. Data was collected with a data sampling frequency of $F_{s}=378.0718$ points $/ \mathrm{mm}$, which translates into a sampling interval of $\delta X_{s}=2.645 \mu \mathrm{m}$. Raw data files representing the sample surface height as a function of the linear scan position were obtained from CAPT and analyzed at Caltech using an internally developed Matlab ${ }^{\circledR}$ data reduction algorithm. The raw "as received" data set contained scan line coordinates expressed in microns and the surface height $z(x)$ in Angstroms. Each scan began from an initial position $x_{0}=24 \mathrm{~mm}$ and terminated at a final position $x_{f}=196.9974 \mathrm{~mm}$, corresponding to a scan length $L=172.9974 \mathrm{~mm}$ between the initial and final positions of the Dektak ${ }^{\mathrm{TM}}$ translation stage.

The Matlab program reads in each data file and promptly converts the scan dimension to mil- 

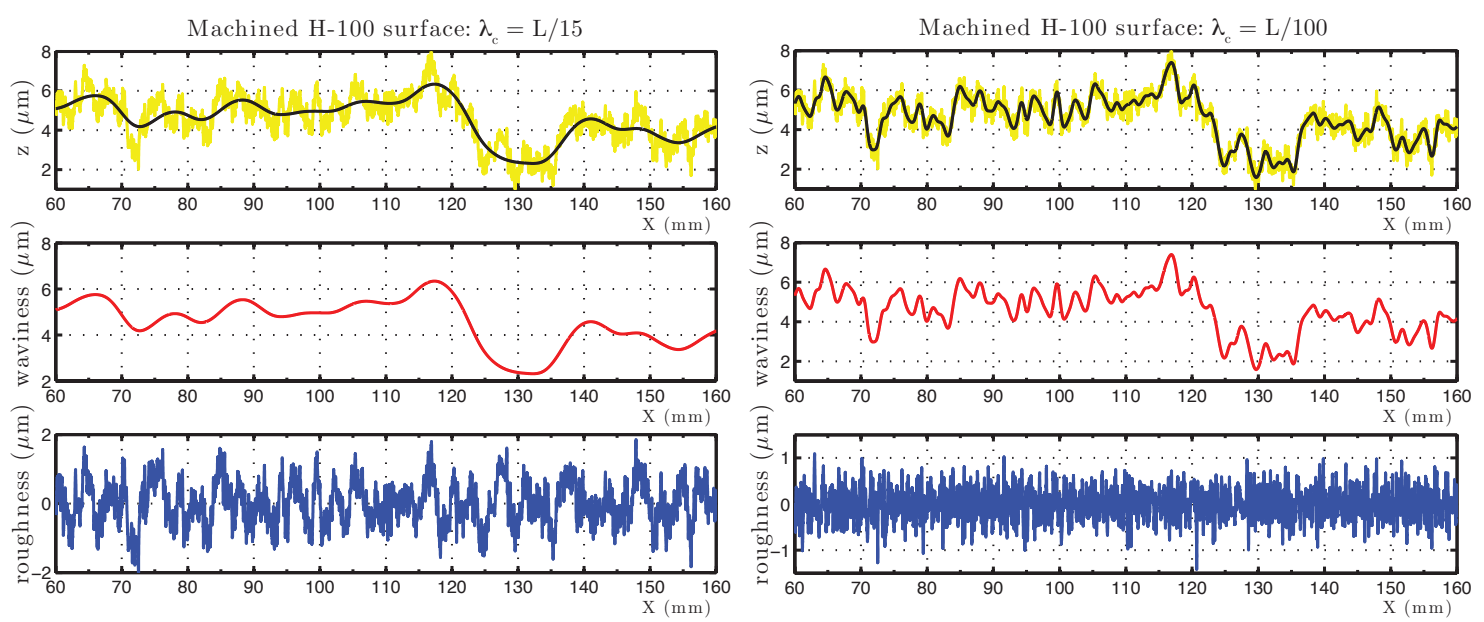

Figure 3.21: Dektak profilometer surface height line scan of an "as machined" H-100 surface. Plots in left column were obtained using a high pass cutoff wave length $\lambda_{c}=\mathrm{L} / 15$. Plots in right column were obtained using a high pass cutoff wave length $\lambda_{c}=\mathrm{L} / 100$.

limeters, and surface height data from angstroms to microns $\left(1 \stackrel{\circ}{A}=10^{-4} \mu \mathrm{m}\right)$. The raw files are then plotted and a specific region of the data is selected for roughness analysis. Filtering is achieved using an $\mathrm{n}^{\text {th }}$-order low pass digital Butterworth filter given by $[\mathrm{B}, \mathrm{A}]=\operatorname{BUTTER}(\mathrm{N}, \mathrm{Wn})$ in Matlab. The variable $\mathrm{N}$ represents the integer order of the Butterworth filtering function. Wn corresponds to a normalized cut-off frequency $\left(0<W_{n}<1\right)$, whereby the value $W_{n}=1$ corresponds to a cut-off frequency equal to one-half the data sampling rate in accordance with the Nyquist theorem. The normalized cut-off frequency was defined as

$$
W_{n}=f_{c} /\left(F_{s} / 2\right)
$$

within the code where $f_{c}$ represents the user-selected low-pass cut-off frequency and $F_{s}$ corresponds to the data-sampling frequency (in this case $F_{s}=378.0718$ point $/ \mathrm{mm}$ ). The presence of the factor 2 in the denominator assures that the user-selected cut-off frequency expressed within the numerator cannot exceed $F_{s} / 2$, since the function requires $(0<W n<1)$. The raw surface height data is filtered by imposing the selected cut-off wavelength in a fourth-order low-pass Butterworth filtering operation. 
The Butterworth filtering operation always results in a filtered curve that is shifted to the right with respect to the original surface profile. The induced phase shift appears constant across the data set and is a known residual effect of the Butterworth filtering operation. Although there are sophisticated techniques available for correcting this problem, a simple solution was adopted, which simply involved shifting the data array to the left using the "circshift" function in Matlab. The shift was checked by plotting the filtered data superimposed upon the original surface height profile. Corresponding peaks in the original and filtered curves were realigned after only a few iterations following an initial guess. An optimized and fully automated shift procedure can easily be implemented in the future if required.

Figure 3.21 depicts the surface height $\mathrm{z}(\mathrm{x})$, waviness, and surface roughness profiles obtained from the "as machined" specimen surface. Data corresponding to the central $100 \mathrm{~mm}$ portion of the scan length was analyzed and the distance units displayed within the plots correspond to the absolute scan position of the stylus in the instrument frame of reference. Plots along the left column were obtained using a cut-off wave length $\lambda_{c}=L / 15(11.53 \mathrm{~mm})$, while plots within the right column resulted from a cut-off wave length $\lambda_{c}=L / 100(1.73 \mathrm{~mm})$. The cut-off wavelength $\lambda_{c}=$ $\mathrm{L} / 15$ corresponds to a normalized frequency $W_{n}=0.00046$ in the Butterworth filter, while the cutoff wavelength $\lambda_{c}=\mathrm{L} / 100$ corresponds to a normalized frequency value $W_{n}=0.0031$, in accordance with Eq. 3.10.

The raw surface height data is represented by the yellow curves within the top-left and -right panels of Figure 3.21. The black curves shown superimposed upon these plots are the filtered and phase-shift-corrected waviness profiles, which are replotted and contrasted side by side within the central panels of Figure 3.21. As expected, the curve on the right incorporates more detail from the original surface height profile. The large-amplitude oscillations in the resulting waviness profile correspond to tooling marks introduced by the milling procedure.

Surface roughness profiles were obtained through a "high-pass filtering procedure", which simply involves subtracting the waviness profile from its corresponding surface height profile. The resulting surface roughness profiles are each plotted in the lower-most panel of Figure 3.21. The $\lambda_{c}=\mathrm{L} / 15$ 

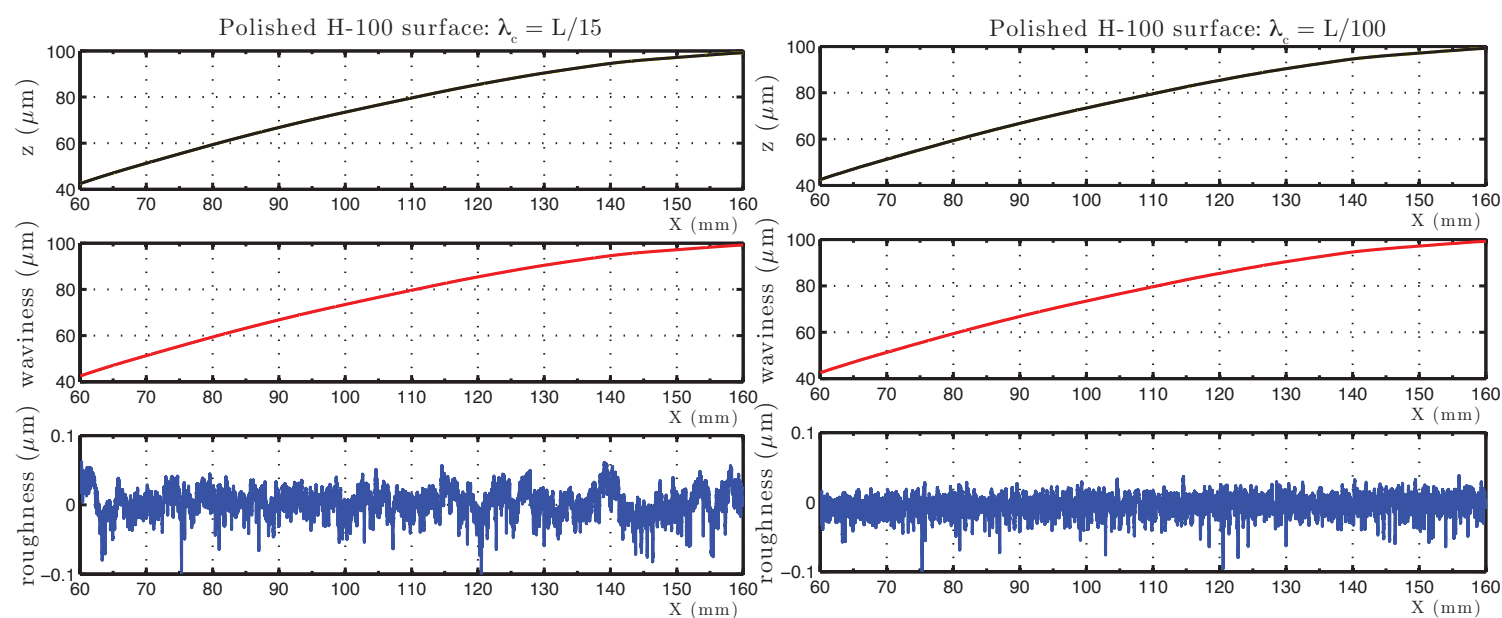

Figure 3.22: Dektak profilometer surface height line scan of a surface-roughened (micro-bead blasted) H-100 surface. Plots in the left column were obtained using a high-pass cut-off wave length $\lambda_{c}=\mathrm{L} / 15$. Plots in the right column were obtained using a high-pass cut-off wave length $\lambda_{c}=\mathrm{L} / 100$.

filtering criteria resulted in a surface roughness profile with an average surface roughness of $R_{a}=$ $0.502 \mu \mathrm{m}$ and RMS roughness value of $R_{q}=0.617 \mu \mathrm{m}$ while the $\lambda_{c}=L / 100$ criteria yielded average and RMS surface roughness values of $R_{a}=0.259 \mu \mathrm{m}$ and $R_{q}=0.324 \mu \mathrm{m}$, respectively. The measured values for $R_{a}$ and $R_{q}$ differ by almost a factor of two.

This particular case highlights how the choice of the cut-off wavelength can be somewhat ambiguous in the absence of any additional physical constraints. Imposing the criteria $\lambda_{c}=L / 100$ as per the ANSI/ASME B46.1 specification may be perfectly justified for a standard surface inspection procedure but it may be not make any sense at all to apply this criterion to a laboratory earthquake specimen. A more physically meaningful length scale should be considered, such as the frictional break down zone or the pulse length spanned by a propagating slip pulse (sub-Rayleigh or supershear). The idea being that at any given moment a rupture is spread across a characteristic distance and thus never directly encounters surface wavelengths that exceed this characteristic distance. It therefore seems reasonable to bound the low-pass cut-off wavelength such that $\lambda_{c}>V_{r} \Delta \tau$, where $V_{r}$ is the rupture speed and $\Delta \tau$ is the temporal pulse width. In laboratory earthquake experiments this effectively translates into characteristic lengths ranging from $1 \mathrm{~cm}-2 \mathrm{~cm}$ based upon typical pulse widths and rupture speeds that are routinely measured. A reasonable choice for the cut-off 

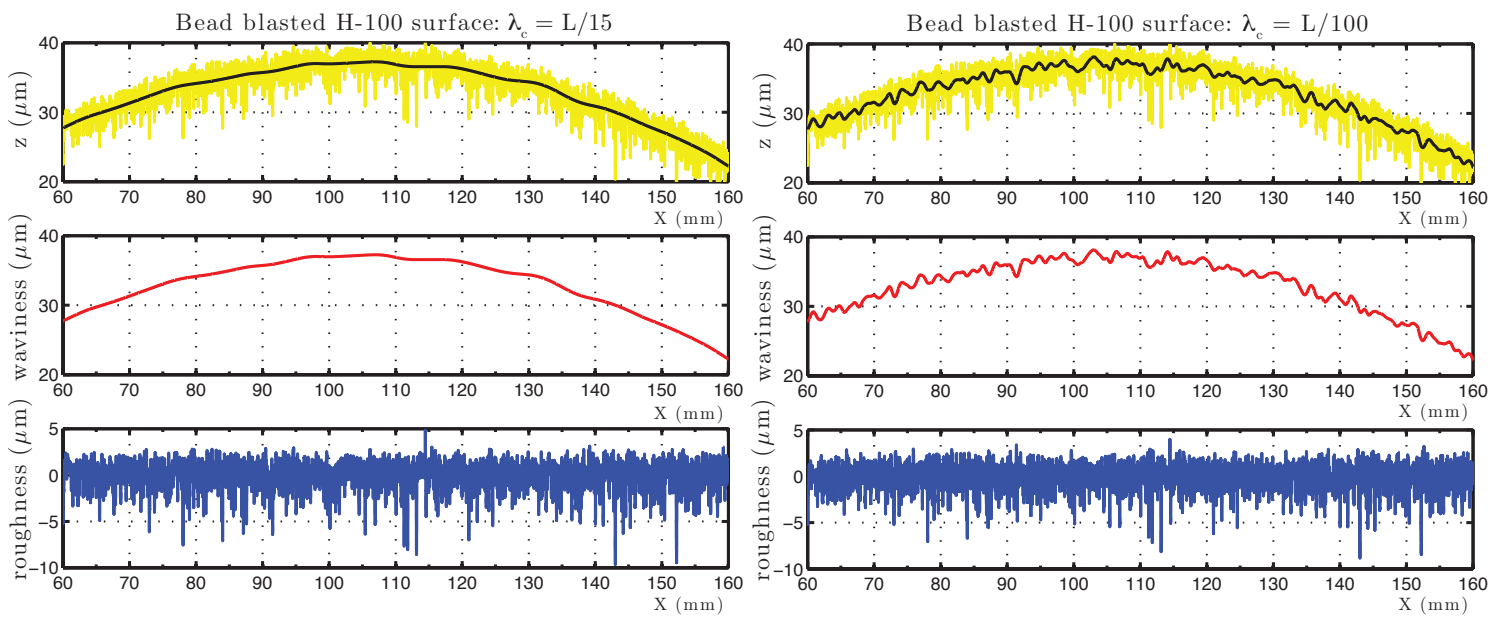

Figure 3.23: Dektak profilometer surface height line scan of a fine-polished H-100 surface. Plots in the left column were obtained using a high-pass cut-off wave length $\lambda_{c}=\mathrm{L} / 15$. Plots in the right column were obtained using a high-pass cut-off wave length $\lambda_{c}=\mathrm{L} / 100$.

wavelength might then be $\lambda_{c}=11.5 \mathrm{~mm}$, which in relation to the scan length $L=173 \mathrm{~mm}$ of the recently tested samples conveniently translates to a low-pass cut-off criteria $\lambda_{c}=L / 15$. The decision to contrast this particular cutoff wavelength with the "recommended" value of $\lambda_{c}=L / 100$ was motivated by this logic.

Figure 3.22 contrasts the surface height $\mathrm{z}(\mathrm{x})$, waviness, and surface roughness profiles obtained corresponding to the polished specimen. The waviness curve reveals a convex surface profile, which "climbs" $60 \mu \mathrm{m}$ across a specimen distance of $100 \mathrm{~mm}$. When viewed at this scale, the waviness curves appear nearly identical to the original surface height profiles. However, there are subtle differences between each respective set of curves, which appear across a much finer amplitude scale. The surface roughness plots displayed in the lower-left and -right panels reveal the very fine surface roughness measurements that resulted from the high-pass filtering procedures. The $\lambda_{c}=\mathrm{L} / 15$ filtering criteria resulted in a surface roughness profile with an average surface roughness of $R_{a}=$ $0.016 \mu m(160 \AA)$ and an RMS roughness value of $R_{q}=0.021 \mu m(210 \AA)$, while the $\lambda_{c}=L / 100$ criteria yielded average and RMS surface roughness values of $R_{a}=0.011 \mu m(110 \AA)$ and $R_{q}=$ $0.015 \mu m(150 \AA)$, respectively. Measured values are trustworthy, since they are still a full order of magnitude above the instrument's noise floor. Optical-grade surface finishes are clearly achieved by 

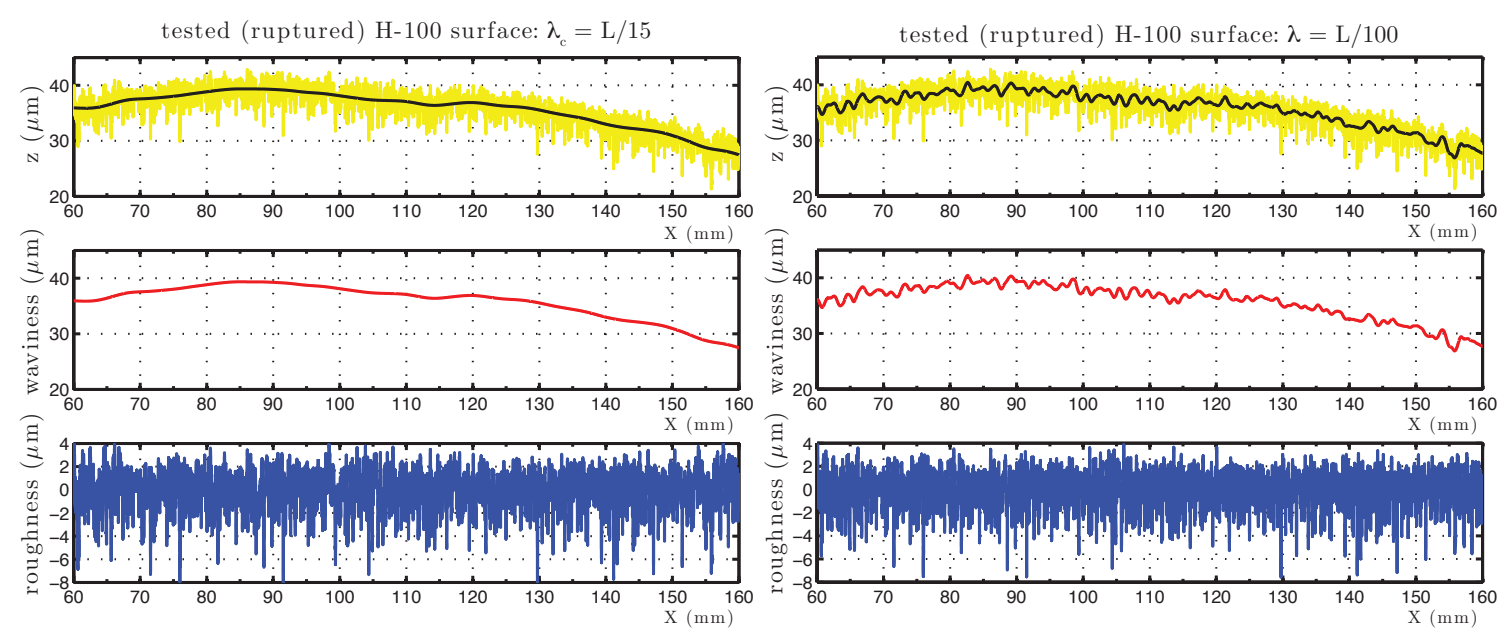

Figure 3.24: Dektak profilometer surface height line scan of a tested (ruptured) H-100 surface. Plots in the left column were obtained using a high-pass cut-off wave length $\lambda_{c}=\mathrm{L} / 15$. Plots in the right column were obtained using a high-pass cut-off wave length $\lambda_{c}=\mathrm{L} / 100$.

the polishing techniques discussed in Section 3.6. In this case note how the two filtering operations yielded similar surface roughness values and that there was not much gained by imposing the lower cut-off wavelength, since the magnitude of the surface roughness and waviness profiles are orders of magnitude apart.

The surface of the bead-blasted test piece was roughened using glass beads corresponding to a mesh size (70-140) using the standard micro-bead blasting procedure discussed in Section 3.7. Curves depicted in Figure 3.23 once again contrast the waviness and surface roughness profiles obtained at the two cut-off wavelength extremes. The surface height profile reveals a rich surface texture that contains a spread of high spatial frequencies. The waviness curves reveal a nominally symmetric and slightly convex global surface profile, which increases by approximately $15 \mu \mathrm{m}$ between the right edge of the specimen and the peak of the crown. The $\lambda_{c}=\mathrm{L} / 15$ filtering criteria resulted in a surface roughness profile with an average surface roughness of $R_{a}=1.157 \mu \mathrm{m}$ and an RMS roughness value of $R_{q}=1.504 \mu \mathrm{m}$, while the $\lambda_{c}=L / 100$ criteria yielded average and RMS surface roughness values of $R_{a}=1.080 \mu \mathrm{m}$ and $R_{q}=1.404 \mu \mathrm{m}$, respectively. These values represent the roughness of typical fault surfaces used in laboratory earthquake experiments. Note that the average (or RMS) depth of the surface irregularities is two orders of magnitude lower than the mean diameter of the glass beads 


\begin{tabular}{|l||l|l||l|l|}
\hline \multicolumn{1}{|c||}{} & \multicolumn{2}{c|}{$\lambda_{c}=L / 15$} & \multicolumn{2}{c|}{$\lambda_{c}=L / 100$} \\
\cline { 2 - 5 } & $R_{a}(\mu m)$ & $R_{q}(\mu m)$ & $R_{a}(\mu m)$ & $R_{q}(\mu m)$ \\
\hline \hline Machined & 0.502 & 0.617 & 0.259 & 0.324 \\
Polished & 0.016 & 0.021 & 0.011 & 0.015 \\
Bead Blasted & 1.157 & 1.504 & 1.080 & 1.404 \\
Tested (Ruptured) & 1.347 & 1.721 & 1.252 & 1.597 \\
\hline
\end{tabular}

Table 3.2: Average roughness $\left(R_{a}\right)$ and root-mean-square roughness $\left(R_{q}\right)$ for (a) as-machined, (b) bead-blasted (70-140 mesh size), (c) polished, (d) tested (ruptured) surfaces

used in the bead-blasting operation. There was also not much difference between the two filtering extremes in this case due to a reasonably smooth waviness profile in comparison to the amplitude of the surface height fluctuations.

The measured surface height, waviness, and surface roughness profile of the tested (ruptured) specimen are displayed in Figure 3.24. The $\lambda_{c}=\mathrm{L} / 15$ filtering criteria resulted in a surface roughness profile with an average surface roughness of $R_{a}=1.347 \mu \mathrm{m}$ and an RMS roughness value of $R_{q}=$ $1.721 \mu \mathrm{m}$, while the $\lambda_{c}=L / 100$ criteria yielded average and RMS surface roughness values of $R_{a}=1.252 \mu \mathrm{m}$ and $R_{q}=1.597 \mu \mathrm{m}$, respectively. The results are quite similar in all respects to the surface height data obtained from the bead-blasted test piece. Virtually identical observations and similar conclusions can be drawn from the two cases. As with the previous case there is no technically significant difference that resulted from using a much shorter cut-off wavelength due to a reasonably smooth waviness profile in comparison to the amplitude of the surface height fluctuations.

The measured average and RMS surface roughness values obtained for the four samples are summarized in Table 3.2 .

A discrete Fourier transform (DFT) of the surface roughness data was taken using a fast Fourier transform (FFT) algorithm made available within Matlab. Figure 3.25 is a compilation of one-sided amplitude spectra of the surface roughness profiles obtained through high-pass filtering using $\lambda_{c}$ $=\mathrm{L} / 15$. The spatial frequency axes extend out to $f_{\max }=12 \mathrm{~mm}^{-1}$ or equivalently a minimum wavelength $\lambda_{\min }=83 \mu \mathrm{m}$. Note that although the spatial frequency axis appears to start $f=$ 0 , the first prominent peak corresponds to the original cutoff frequency corresponding to $f_{c}=$ $(1 / 11.5) \mathrm{mm}^{-1}$. The amplitude spectrum of the "as machined" specimen's surface roughness profile 

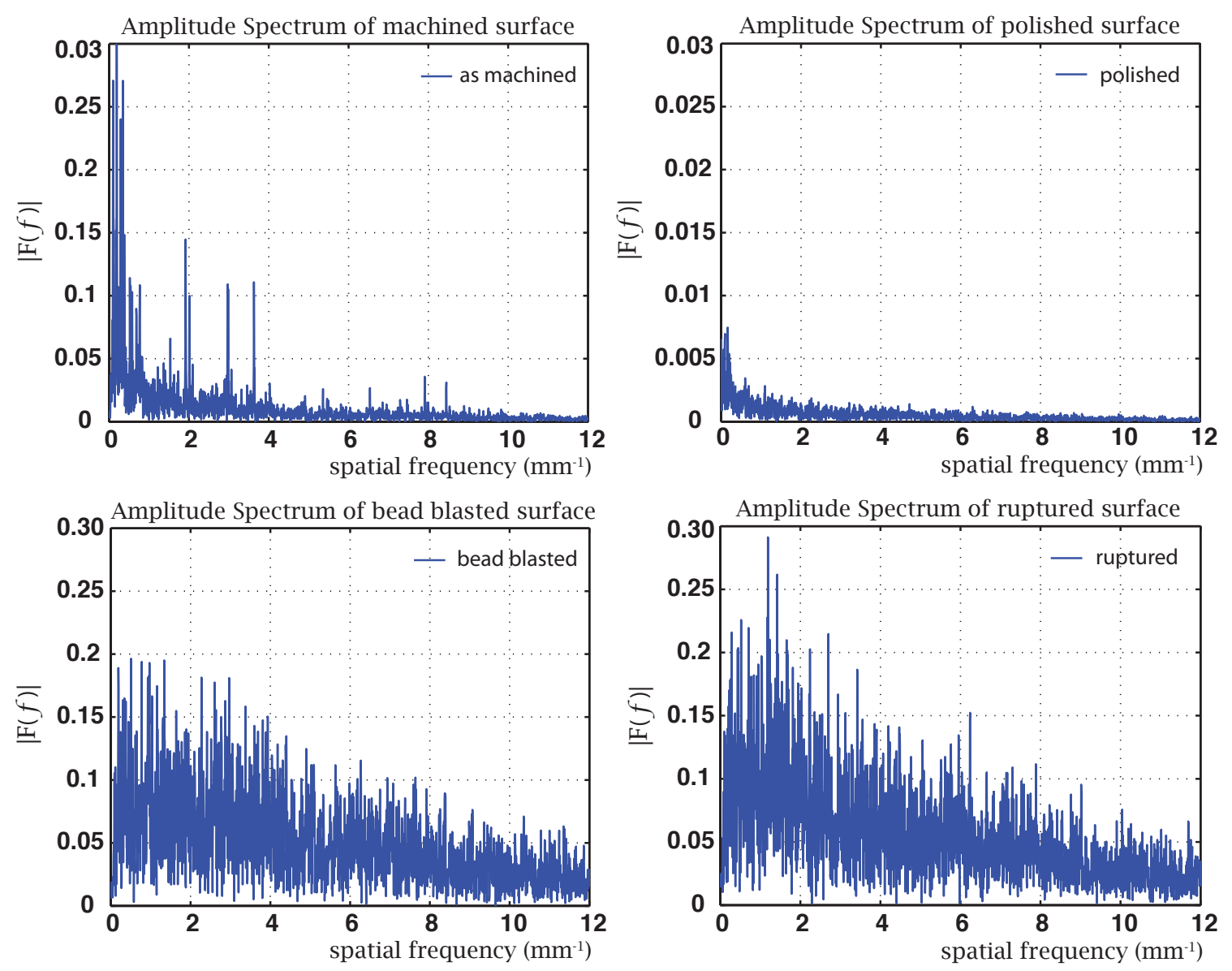

Figure 3.25: One-sided amplitude spectra of the surface roughness profiles obtained through highpass filtering using $\lambda_{c}=\mathrm{L} / 15$ (a) as-machined, (b) bead0-blasted (70-140 mesh size), (c) polished, (d) tested (ruptured) surfaces

is characterized by several distinct and prominent peaks that rise up above the rest of the spectrum. These peaks are a direct manifestation of the "chattered" surface profile depicted in Figure 3.21. Laboratory earthquake specimens exhibiting this type of surface profile are not ordinarily tested. Nonetheless the spectrum reveals the initial state of an "as machined" surface. The "as machined" spectrum in Figure 3.25 along with the corresponding surface profile, waviness, and roughness profile in Figure 3.21 collectively reinforce the importance of machining the smoothest possible surfaces and the additional requirement to polish machined surfaces prior to the micro-bead blasting surface treatment.

The amplitude spectrum of the polished specimen's surface roughness profile is two orders of mag- 
nitude lower than the spectra exhibited by the other three specimens. The polished surface spectrum is characterized by a thin spectral band lacking any prominent peaks. The diminished magnitude in the case of the polished surface spectrum simply reflects the same two-order-of-magnitude difference in surface roughness between the polished specimen surface and the other three surfaces as summarized in Table 3.2.

The amplitude spectrum of the bead-blasted specimen's surface roughness profile exhibits a dense array of peaks which gradually diminish in magnitude with increased spatial frequency. There appears to be no hint of a dominant, characteristic spatial frequency that correlates to the mean dimensions of the glass beads, as might be ideally assumed. The resulting spectrum reflects a broad spread of wavelengths spanning several orders of magnitude across the specimen fault surface. The longer wavelengths in the surface roughness profile may be a relic of the unsteady motion of the bead blast nozzle as it was swept back and forth across the specimen surface. Density variations associated with the divergent jet of beads emerging from the nozzle may have also contributed to an uneven erosion of the fault surface, which is reflected by longer wavelength surface height variations. The tail end of the spectra corresponding to the higher spatial frequencies may be the result of smaller, broken-glass particulates that accumulate over time within the glass bead reservoir. As previously indicated in Section 3.19, the glass beads are typically recollected and reused again in subsequent surface treatments. It is therefore reasonable to conclude that range of particle diameters can extended well below the stated glass bead diameter range over the course of time.

The amplitude spectrum of the tested (ruptured) specimen's surface roughness profile appears similar in many respects to the spectrum of the bead-blasted surface roughness profile. The slightly taller peaks in the tested surface spectrum simply result from a higher average (and RMS) surface roughness as summarized in Table 3.2. There appear to be no obvious spectral signatures linked to the rupture process itself. Finally, apart from a slight difference in overall magnitude, the strong similarities between the two spectra bode well for the repeatability of the surface roughening procedure. 


\subsection{Introduction of new laboratory earthquake specimen fea- turing 3D fault geometry}

The "contact bonding" technique is well known in the field of optics and has been leveraged for many years in optical shops for fusing flat prisms together or even temporarily bonding optical components during fabrication in order to eliminate the added dimensional uncertainty introduced by bonding

agents. The strong adhesion forces generated between flat, polished surfaces are attributed to attractive van der Waals forces. These intermolecular forces can dominate if a small separation distance on the order of a wavelength (of visible light) or even less is sustained across a macroscopic distance (Deryagin, 1967). There appears to be very little published scientific literature (if any) on the dynamic shearing resistance of intermolecularly bonded surfaces.

A variant of this technique has been recently implemented in order to lock certain portions of the fault within a laboratory earthquake specimen. The new technique takes full advantage of the static-compressive load that is ordinarily applied and naturally required in these experiments. Preliminary findings using the contact bonding method in laboratory earthquake specimens appears to demonstrate that the adhesion between two polished segments of the specimen fault surface act as a high-strength barrier to a propagating rupture front.

Figure 3.26 is a schematic representation of a new laboratory earthquake specimen that has been recently developed and successfully implemented in dynamic rupture investigations. The specimen fault surfaces contain finely polished segments that adhere under pressure so as to effectively "lock" the fault and suppress rupture propagation within these regions. The remaining roughened portions of the fault support rupture propagation and can undergo frictional sliding in the usual manner.

The new 3D fault geometry was originally devised in order to try to suppress a sharp, anomalous signal disturbance that is consistently observed in supershear particle velocity records. The sharp secondary signal typically arrives $5-7 \mu s$ after the arrival of the primary supershear rupture signal. There is no observed sub-Rayleigh signal counterpart to this phenomena. An example of a secondary supershear signal disturbance can be seen within fault-normal (FN) and fault-parallel (FP) particle 


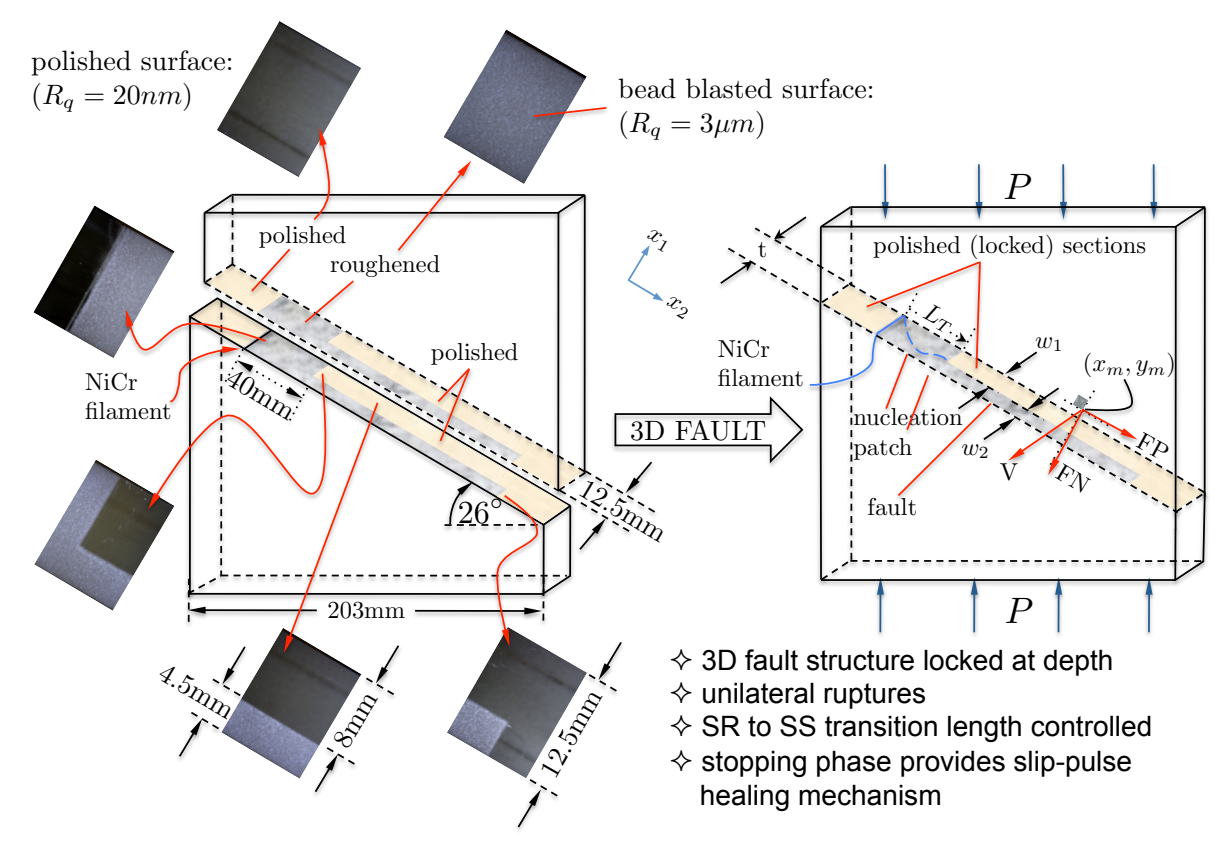

Figure 3.26: New H-100 laboratory earthquake specimen featuring 3D fault geometry

velocity records in Figure 3.32 of Section 3.10, where a more in-depth description and analysis of this feature is presented. The problem is only briefly mentioned here in order to properly highlight the role it played in motivating the development of the new 3D specimen fault geometry. The precise cause of this anomalous signal disturbance is not entirely clear at this time. One hypothesis is that it may be a direct consequence of the finite plate thickness manifest in the form of strong radiated waves emitted from the point where the supershear rupture encounters the free surface on the opposite side of the specimen. The basic premise behind the 3D fault geometry was to therefore try and lock the fault at depth in an effort to partially arrest the supershear rupture and prevent it from interacting with the specimen boundary on the opposite side. Experimental results presented in a later chapter describe how the new fault geometry was used to effectively dampen the secondary signal disturbance and produce a nearly pulse-like supershear rupture. The resulting particle velocity records bear some striking similarities to the celebrated $2002 M_{w} 7.9$ Denali, AK, ground motion records obtained at Pump Station 10 near the Alaska Pipeline.

Figure 3.27 depicts a close-up view of 3D fault specimen assembly, comprised of 0.5 " thick quadrilateral sections, shown mounted within the $8^{\prime \prime} \times 8^{\prime \prime}$ specimen frame. The roughened frictional 


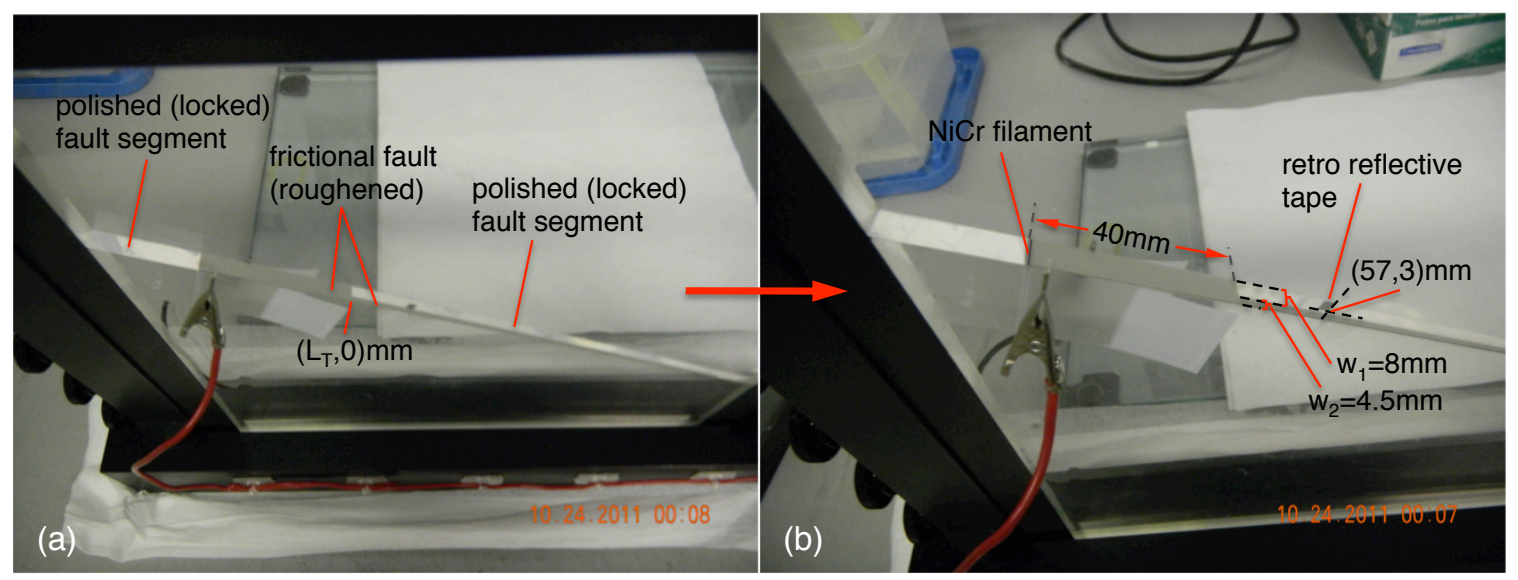

Figure 3.27: Close-up view of $\mathrm{H}-100$ specimen prepared with $3 \mathrm{D}$ fault geometry

fault segments are clearly seen in sharp contrast to the polished segments, which appear highly reflective as a consequence of total internal reflection (TIR). The TIR condition quickly disappears as soon as the fault surfaces are compressed, whereupon the polished fault segments become completely transparent. The $79-\mu m$-diameter NiCr filament, which is barely visible in the image, is seated within the channel with external strands temporarily affixed to the specimen faces by cellophane tape, as shown. Toothless alligator clips are not clasping the wire filament at this stage and are only engaged once the specimen is placed under load, at which point the tape is removed.

\subsubsection{Newfound control of laboratory earthquake experiments using 3D specimen fault geometry as revealed by experiments}

Additional benefits of the new 3D fault specimen have been realized during the course of development and experimentation. Among the most noteworthy of these newfound features are the following: (1) the ability to consistently nucleate unilateral ruptures, (2) the ability to partially arrest an advancing sub-Rayleigh rupture and leverage a stopping phase as a healing mechanism to produce highly repeatable slip pulses, (3) a new sub-Rayleigh- to-supershear transition mechanism, which also exhibits a tendency to produce more pulse-like supershear ruptures, and (4) the ability to precisely control the supershear transition length, which is set by the distance to $x=L_{T}$ depicted in Figures 3.26 and 3.27. These unique features, along with the increased repeatability and control of 
the experiment afforded by the new design, have now superseded the original reasons for exploring this type of specimen in the first place. Additional details of these new features are presented here and then highlighted again in later chapters in relation to specific experiments that were conducted using the this specimen design.

The 3D fault geometry configuration is naturally prone to nucleating unilateral ruptures by virtue of the locked fault segment located to the left of the nucleation site. Limitations traditionally imposed on the effective observation time window are relaxed somewhat since there is no rupture that terminates at the left boundary to produce strong waves that are normally directed back toward the particle velocity measurement station. High-speed photoelastic images have also shown that the circular p-wave and s-wave fronts that propagate into the locked fault segment rapidly attenuate and produce weak reflections from the left boundary of the specimen.

A unilateral sub-Rayleigh rupture initially propagates along a region of the fault designated as the "nucleation patch". The rupture propagates over a distance $x=L_{T}$ until it encounters the boundary of the polished rectangular fault segment of width $w_{1}$ as depicted in Figures 3.26 and 3.27. The width of the frictional fault is then abruptly restricted to a depth $w_{2}$ at this location, as shown.

Experiments have shown that if the static-compressive load is below a critical threshold (experimentally determined), then a sub-Rayleigh pulse-like rupture will generally emerge. Sub-Rayleigh pulses are now easier to produce and control compared to ruptures generated using the traditional specimen geometry, which more often than not result in crack-like ruptures. Although a detailed analytical or numerical analysis of this rupture scenario has not yet been conducted, it appears that a repeatable healing mechanism is introduced, perhaps through a stopping phase that is produced by the partial arrest of the rupture front at the fault boundary boundary. The stopping phase then propagates forward and assists in healing the trailing edge of the advancing sub-Rayleigh rupture to produce a pulse-like rupture. Additional details and observations from experiments that explored this rupture scenario are presented in a later chapter.

A second rupture scenario, which has been experimentally observed arises when the static com- 


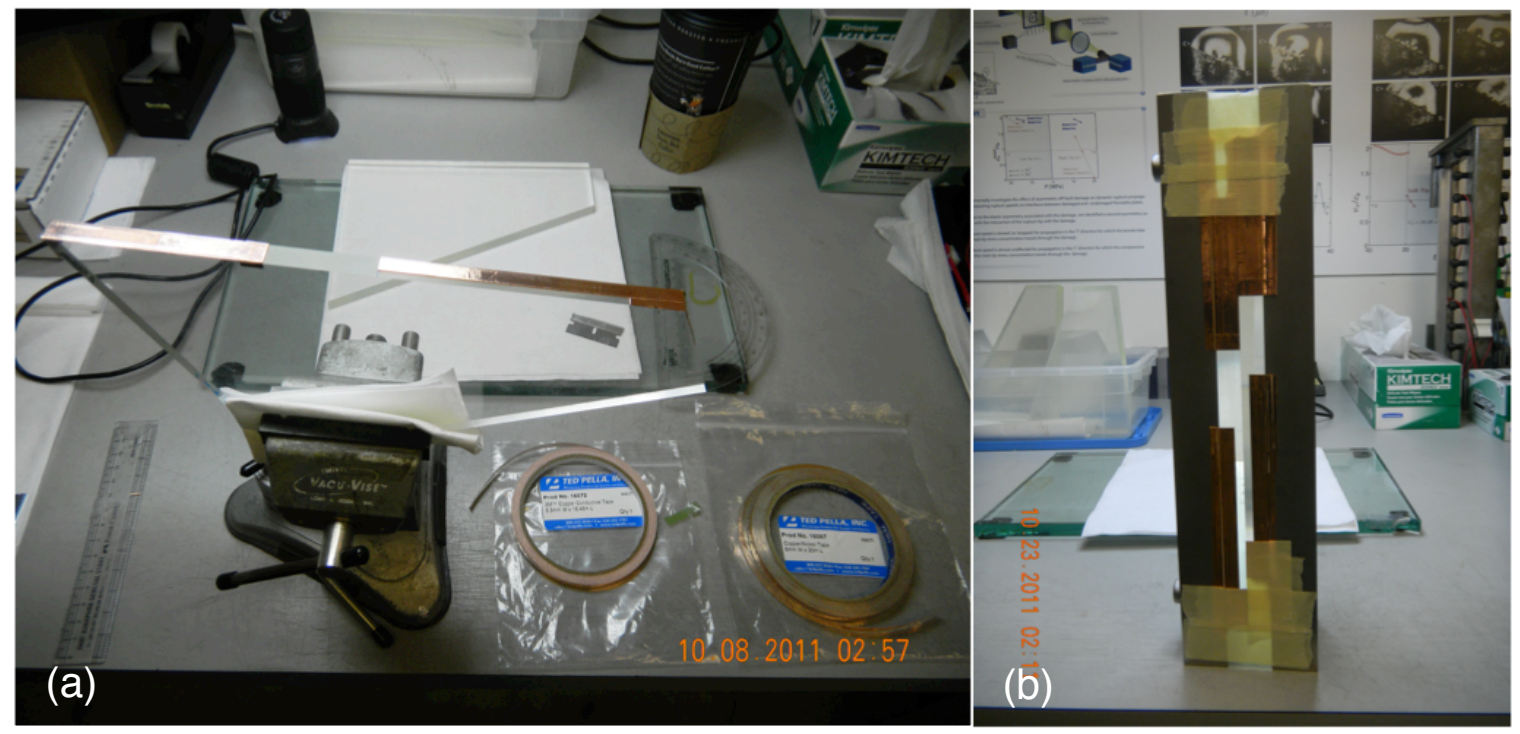

Figure 3.28: Masking of $\mathrm{H}-100$ specimen fault surface using $\mathrm{Cu}$ tape for protection of polished surface during bead-blasting step

pressive load exceeds the critical stress threshold, in which case the interaction of the sub-Rayleigh rupture with the sharp fault boundary at $x=L_{T}$ results in a supershear rupture transition at this location. The results appear to represent a new type of sub-Rayleigh-to-supershear -ransition that is unlike the classic Burridge-Andrews mechanism (Xia et al., 2004; Xia, 2005; Lu et al., 2009; Lu, 2009; Mello et al., 2010). The locked section of the fault appears to mimic the manner through which a high-strength barrier or patch of higher pre-stress can induce a supershear transition, as has been numerically and theoretically predicted (Dunham et al., 2003; Liu and Lapusta, 2008). The ability to set the supershear transition length a priori offers unique possibilities for the design of future laboratory earthquake investigations that would otherwise be difficult to execute based upon the uncertainty and inherent variation of the supershear transition distance. Additional details and results of supershear experiments conducted using the new 3D fault geometry are presented in a later chapter. 


\subsubsection{Fabrication of 3D fault specimens}

H-100 quadrilateral fault surfaces should machined to a fine surface finish similar to the surface depicted in Figure 3.14d. The benefit of an initially smooth machined surface finish is the minimal polishing time that is subsequently required in order to achieve a flat, transparent surface finish using a manual polishing procedure discussed in Section 3.6.

The H-100 quadrilateral test sections that were used to fabricate the first 3D laboratory earthquake specimens were sent to Valley Design Corporation located in Shirley, MA, which specializes in edge polishing. $8^{\prime \prime} \times 8^{\prime \prime}$ mated pairs of H-100 quadrilateral test sections, machined out of $3 / 8^{\prime \prime}$ and $1 / 2^{\prime \prime}$ sheet stock were sent out for polishing. According to the vendor, the parts were stacked and held together within a custom jig in order to lap all of the parts together at once on a flat lapping surface. A fine-grade diamond polishing compound was used to produce an optically and flat transparent surface finish. The fault surfaces polished by the vendor were quoted as meeting a flatness specification of two fringes or less when viewed using an optical flat under a monochromatic light source. The polished fault surfaces were highly transparent and exhibited excellent planarity and perpendicularity when mated together and evaluated with a low-power microscope, as previously detailed in section 3.5.2. The "in-house" manual polishing procedure described in Section 3.6 was developed at a later stage after having tested all of the parts polished by Valley Design, Inc. Based upon recent discoveries there is very little advantage gained by outsourcing the polishing work as long as attention to detail is paid in preparing the test specimens according to the guidelines discussed in this section and previous sections on polishing and surface inspection. It is highly recommended that an optically flat 12" diameter cast iron hand lapping plate be purchased (Lapmaster International) and used as a reference surface for mounting the PSA-backed polishing cloth. A large optical flat and monochromatic light source should also be obtained for quick and easy inspection of surface flatness.

A robust masking procedure was developed in order to protect polished segments of the fault surfaces while exposed sections are bead blasted. The H-100 fault surfaces are masked using $3 \mathrm{M}^{\circledR} \mathrm{Cu}$ tape that is ordinarily used for SEM microscopy. Individual rolls of $\mathrm{Cu}$ tape in $6.3 \mathrm{~mm}$ and $8 \mathrm{~mm}$ 

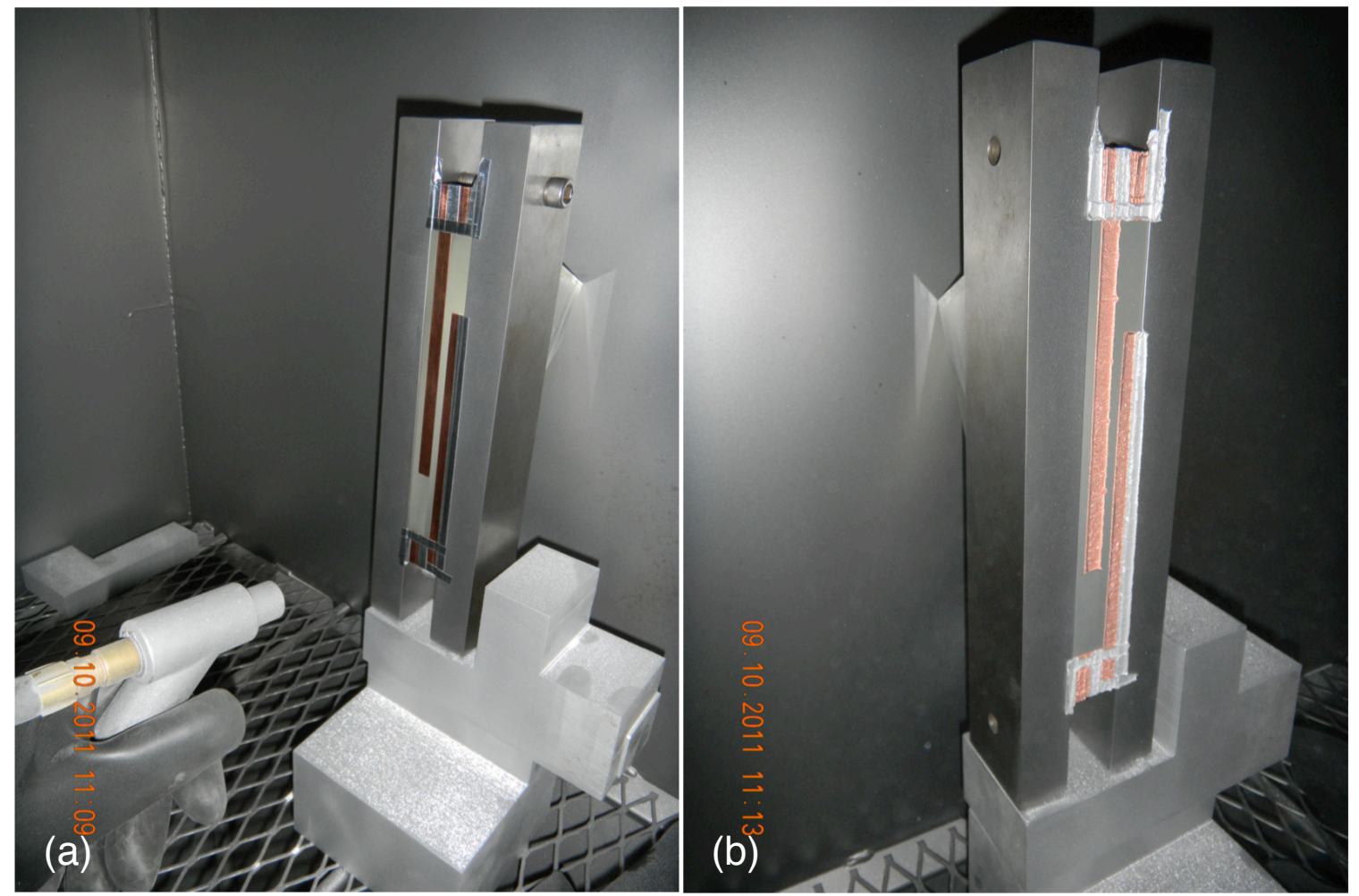

Figure 3.29: (a) Specimen assembly masked and mounted just prior to bead-blasting with polished fault segments clearly visible. (b) Specimen assembly after bead-blasting procedure showing eroded $\mathrm{Cu}$ tape still intact with roughened fault segments clearly visible

widths were purchased from Ted Pella, Inc. Figure 3.28a shows an H-100 quadrilateral section securely mounted in a clamping vise. In addition to its resilience against to the bead-blasting procedure, $\mathrm{Cu}$ tape is for all practical purposes inextensible and provides a very straight edge, which aligns well to the marked boundaries. The various tools used in the masking procedure along with rolls of $\mathrm{Cu}$ tape are also seen on the surface of the lab workbench in Figure 3.28a. Critical dimensions of the various fault segments are carefully marked off and the $\mathrm{Cu}$ tape is then carefully applied to the surface. Up to 4 layers of tape are typically applied in order to build up a secure layer that will protect the underlying polished surface. The procedure appears very simple at first glance, but can take considerable time to complete since the $\mathrm{Cu}$ tape must be very carefully aligned parallel to the marked boundaries. Care must also be taken not to inadvertently scratch the H-100 surfaces with the blade that is used to trim the tape.

The position of the $\mathrm{NiCr}$ wire channel was varied over the course of several experiments in order 
to establish its optimal position. A particular detail that is not easily conveyed by the figures and diagrams in this section is how the upper fault surface was sometimes masked such that its nucleation patch segment extended slightly over the edge of the lower polished section by $0.5 \mathrm{~mm}-1 \mathrm{~mm}$. This was only done in situations where the wire channel was milled into the polished segment of the lower plate as depicted in the inset of Figure 3.26. This small detail prevented the wire filament from being confined between two flat polished surfaces during nucleation and provided a little bit more "breathing room" for the nucleation process. Experiments were also conducted with the wire located within the nucleation patch of the lower test section and positioned $0.5 \mathrm{~mm}-1 \mathrm{~mm}$ to the right of the polished segment. In all cases, the wire channel was milled as a final step once the specimen had been masked and bead blasted. Considerable care was taken protect the integrity of all surfaces during the wire channel milling operation.

Figure $3.28 \mathrm{~b}$ shows how two masked, mated test sections are mounted in the steel jig in preparation for the bead-blasting surface-roughening procedure. Care must be taken to insure that the two H-100 fault surfaces are evenly aligned within the jig so as to form a flush surface across the interface where the parts meet together. An index finger is run up and down this seam and the parts are gently adjusted (before firmly tightening the screws) until there is no detectable step height discontinuity anywhere along the interface. Note that failure to establish a smooth seam between the H-100 test sections can result in severe erosion of the corner of the raised test section during the bead- blasting operation. The jig bolts are snugged tight once the parts are properly aligned. Masking tape can be applied at the end of the procedure, as shown, in order to prevent some of the bead blast media from passing thorough the upper and lower gaps between the steel blocks where it might work its way to the exposed surfaces of H-100 test sections.

Figure 3.29 shows the mounted jig assembly positioned within the bead-blasting chamber both before and after the bead-blasting operation. Note that in this particular case the upper and lower gaps between the steel blocks remained unblocked, as opposed to the sample assembly displayed in the previous image (taken at a later date), which shows the openings covered by masking tape. The silver-colored tape in the image was $3 \mathrm{M} \mathrm{Al}$ conductive tape also purchased from Ted Pella, 

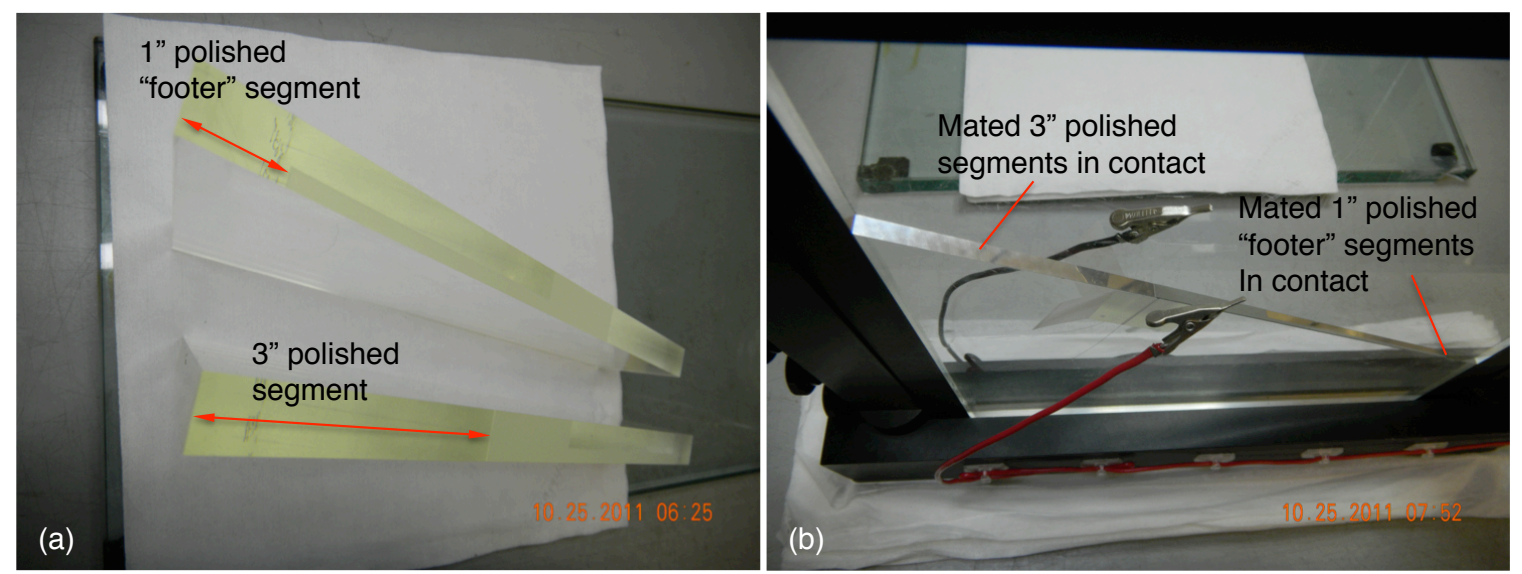

Figure 3.30: Mated pair of 3D laboratory earthquake specimens ready for testing

Inc. The same bead-blasting technique is applied as was previously detailed in Section 3.7. As previously discussed, care is taken to keep track of the number of strokes and the approximate speed of each stroke in order to establish a consistent process. Overly aggressive bead blasting should be avoided as it can lead to a significant step height between the polished and bead-blasted surfaces. Experimentation with this process is required in order to optimize the process. Typical specimens used in the experiments discussed in this thesis were bead blasted by applying 20 strokes at approximately $2-3 \mathrm{sec} /$ stroke, with a stroke defined as a single pass either up or down the fault surfaces.

Once the bead-blasting procedure is completed, the tape is promptly removed and the surfaces thoroughly cleaned using acetone. Figure 3.30 shows a mated pair of 3D laboratory earthquake specimens fabricated out of $1 / 2$ " thick H-100. Note the 3 " long polished segment in this particular instance, as well as the presence of a 1" length polished "footer" segment labeled on the other test section, which has no direct bearing on the rupture dynamics other than to help establish a more-uniform static-compressive load distribution across the length of the fault. 


\subsection{Observations and discussion of an anomalous secondary signal observed in supershear particle velocity records}

Figure 3.31 corresponds to a pair of high-speed photoelastic images obtained during a supershear experiment using a $6 " \times 6$ " laboratory earthquake specimen of $3 / 8$ " nominal thickness. The image frame on the left, acquired at $t=33 \mu \mathrm{s}$, depicts a right lateral supershear rupture just as it crossed the particle velocity measurement station positioned at $\left(-54,0^{+}\right) \mathrm{mm}$. The $(+)$ symbol simply indicates that the laser velocimeter beams were focused at a point $(y<0.2 \mathrm{~mm})$ positioned slightly above the fault. The image reveals the expanding circular s-wave front, propagating at $C_{s}=1.28 \mathrm{~mm} / \mu \mathrm{s}$, and the trailing sub-Rayleigh rupture within the wake of the shear Mach front, which propagates at a rupture speed $V_{r}=1.13 \mathrm{~mm} / \mu \mathrm{s}\left(V_{r} / C_{s}=0.88\right)$. An average supershear rupture speed of $V_{s s}=1.7 C_{s}$ was determined through a frame-by-frame analysis of the high-speed image sequence.

Particle velocity records obtained during the experiment are displayed in Figure 3.32a.The red vertical line labeled $t_{p}$, green line labeled $t_{s}$, and blue line labeled $t_{s}$ correspond to the theoretical arrival times of the p-wave, s-wave, and trailing sub-Rayleigh rupture, respectively. The vertical dashed line at $t=33 \mu \mathrm{s}$ corresponds to the arrival time of the supershear rupture at the particle velocity measurement station. Positive velocity jumps registered by each velocity component along with the observed dominance of the FP $\left(\dot{u}_{x}\right)$ component over the FN $\left(\dot{u}_{y}\right)$ mark the arrival of a stable, right lateral, supershear rupture. The rupture also constitutes a supershear slip pulse, as revealed by the healing phase of the $\mathrm{FP}$ signal, which is momentarily reduced to zero at $t=39.2 \mu \mathrm{s}$.

The sub-Rayleigh portions of the particle velocity records in Figure 3.32, which follow the arrival of the $\mathrm{S}$ wave front, are consistent with the signature of a right lateral sub-Rayleigh rupture at $\left(-54,0^{+}\right) \mathrm{mm}$. The sign of each respective velocity swing corresponds the anticipated sense of motion registered by a right lateral sub-Rayleigh rupture at this location, while the magnitude of the FN component dominates over the magnitude of the FP component (as it should). Small-velocity perturbations in the records following the passage of the sub-Rayleigh rupture are attributed to the 

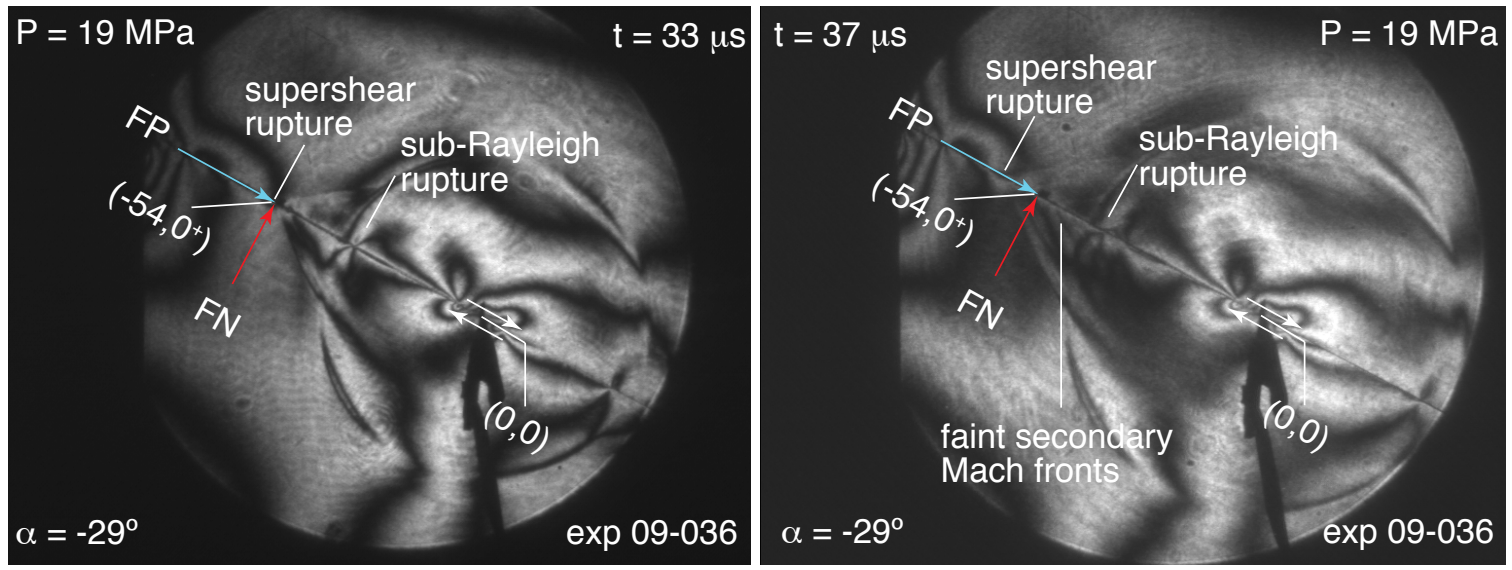

Figure 3.31: Left image: supershear rupture seen just crossing $\left(54,0^{+} \mathrm{mm}\right)$ at $t=33 \mu \mathrm{s}$. Right image: High-speed photoelasic image taken during exp 09-036 revealing faint secondary shear Mach fronts trailing the primary supershear rupture

arrival of reflected boundary waves, which effectively terminate the experiment.

The supershear particle velocity records are both abruptly interrupted by an anomalous disturbance, which appears at the tail end of the primary supershear slip pulse $(t=39.3 \mu s)$. The effect is commonly observed in supershear particle velocity records following the arrival of the primary rupture and there is no observed sub-Rayleigh counterpart to this phenomena. The secondary signal in the FP record appears to mimic the primary rupture signal that it follows and gives the impression of a second slip pulse. Meanwhile, the secondary signal in the FN record appears as an inverted and somewhat distorted replicate of the primary FN supershear signal that it follows. Note how the secondary FP and FN signals in Figure 3.32 appear $180^{\circ}$ out of phase with each other, while the magnitude of the secondary FP component dominates over the magnitude of the secondary FN component, just as it does for the leading supershear rupture signals. Experiments also appear to suggest that the secondary FN signal is anti-symmetric with respect to the leading signal that it follows, but this is difficult to establish with certainty due to temporal broadening and amplitude distortion effects, which are sometimes observed. Although not shown here, vertical $\left(\dot{u}_{z}\right)$ particle velocity records obtained in other supershear experiments also feature a secondary signal that appears inverted (and possibly anti-symmetric) with respect to the primary signal that it follows.

In the case of the first experiment (exp09-036), the leading supershear rupture was able to 


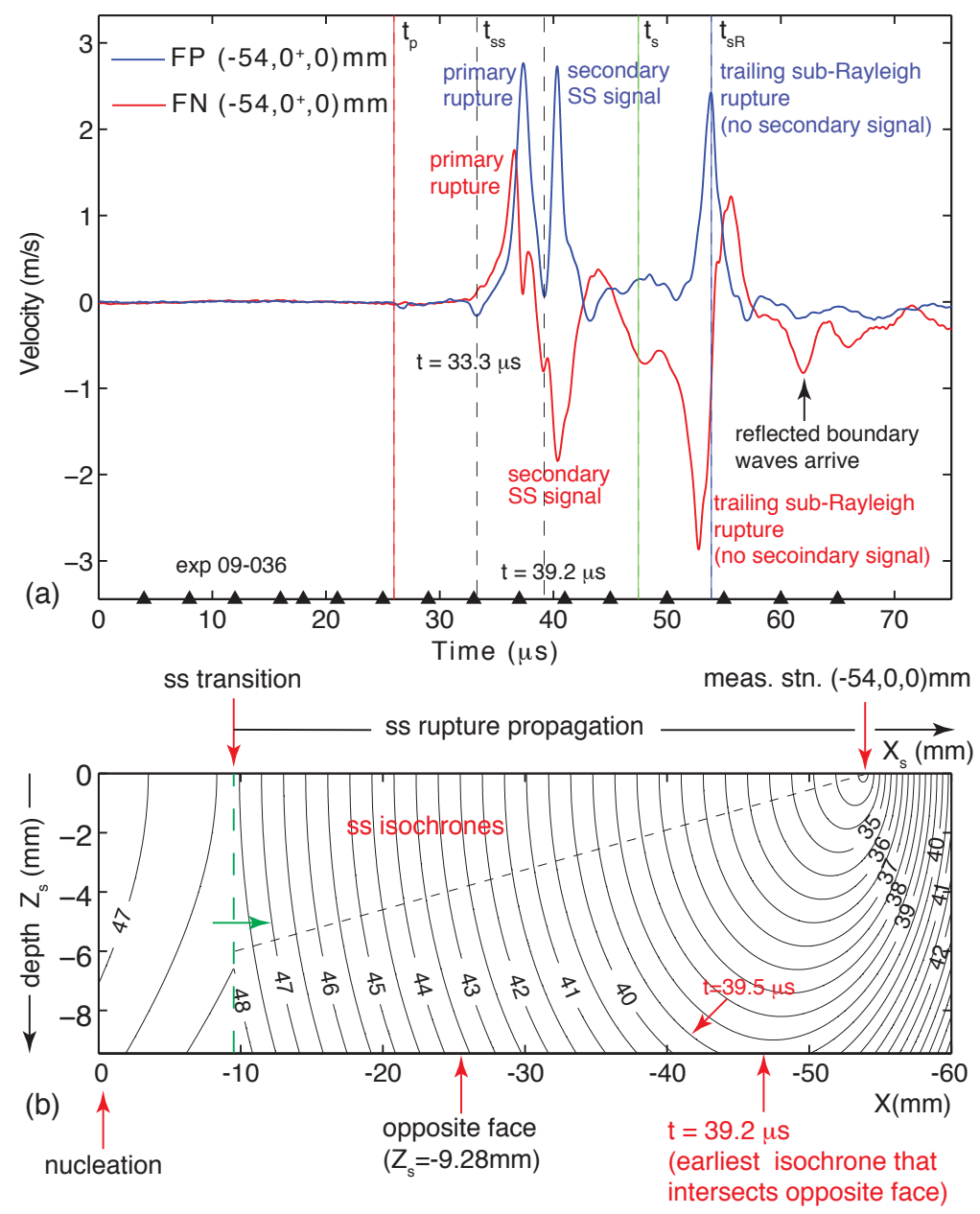

Figure 3.32: (a) Primary (on-fault) supershear particle velocity records acquired during exp 09-036 showing anomalous secondary signal disturbance. (b) S-wave isochrone plot corresponding to exp 09-036

momentarily heal before the secondary signal disturbance arrived and produce a second "replicate" slip-pulse profile. This is not always the case however, and there are instances where the secondary signal disturbance prematurely disrupts the trailing edge of the leading supershear rupture signal and makes it difficult to know whether the original signal was destined to heal or level off into a crack-like rupture profile. An example of one such case (exp 09-050) is depicted in Figure 3.33. Note how in this instance the trailing edge of the leading FP signal drops off sharply before being suddenly interrupted by the secondary signal starting at around $t=36.9 \mu \mathrm{s}$. The FP signal exhibits a second sharp velocity jump and reaches a peak velocity slightly beyond the first maxima before 


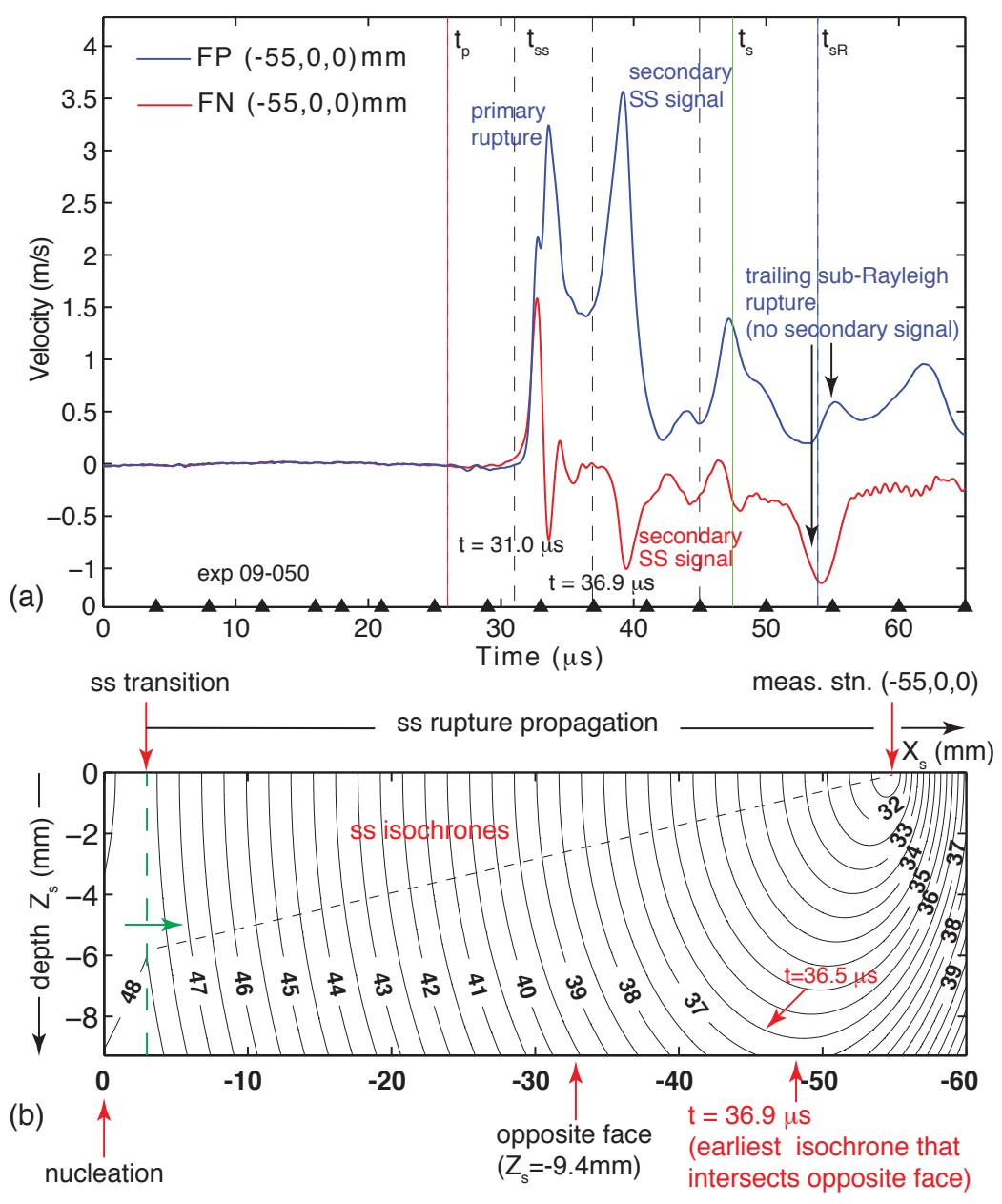

Figure 3.33: (a) Primary (on-fault) supershear particle velocity records acquired during exp 09-050 showing anomalous secondary signal disturbance. (b) S-wave isochrone plot corresponding to exp 09-050

finally dropping off to produce an odd "doubled peaked" slip-pulse velocity profile. Meanwhile, at $t=36.9 \mu \mathrm{s}$, the primary supershear FN signal had clearly settled down to zero before the secondary FN signal abruptly arrived to induce the negative velocity swing seen in the record. Note as well how secondary signal profile exhibits a diminished magnitude and a broadened temporal profile compared to the sharp profile of the leading FN signal that it follows. A careful examination of the secondary signals also reveals a slight offset in time between the peak in the FP record and the minima in the secondary FN record. This is attributed to a small horizontal separation between the focused laser velocimeter beams that were used to generate the records. There are no other peculiar secondary signal anomalies in the records other than the small velocity increase registered by each component 
starting at around $t=45 \mu \mathrm{s}$. The observed velocity increase at this time could possibly result from a supershear daughter crack, but this is unlikely due to the relatively long rise time of these features. The signal disturbance seen at this time is more than likely attributed to weak residual velocity field perturbations left over from the rupture nucleation process that propagate just ahead of the circular S-wave front.

Finally, note once again, as seen in the previous example, how the sub-Rayleigh portion of the record exhibits the correct sense of motion of a right lateral rupture with the magnitude of the FN component dominating over the magnitude of the FP component as it should. No secondary signal disturbances are observed within this portion of the particle velocity records.

A final set of independent and complementary observations related to the secondary signal observed in the supershear records, pertains to the observation of faint "secondary shear Mach fronts", which are frequently observed in high-speed photoelastic images at the same time that the secondary signals are registered. In most cases the secondary Mach fronts are quite faint and go almost unnoticed while in other instances they can be as rich in contrast as the leading rupture itself. An expanded view of the high-speed photoelastic image in Figure 3.31b, acquired at $t=37 \mu s$, reveals a faint pair of shear Mach fronts, which trail within the wake of the leading supershear rupture. Careful frame-by-frame tracking of these features yields a projected station crossing time that correlates with the time at which the secondary signals emerge in the supershear particle velocity records. The secondary shear Mach fronts in Figure 3.34 are much more obvious. Note that the image frame corresponds to $t=41 \mu s$ and that the corresponding tip of the Mach cone has already cleared the measurement station. Careful frame-by-frame tracking of this feature also shows that it is well correlated with the emergence of the secondary particle velocity signals depicted in Figure 3.33. The fact that the observed supershear secondary signal disturbances emerge in the time period following the passage of the primary rupture and yet prior to arrival of the sub-Rayleigh rupture signal is serendipitous. This circumstance has enabled numerous laboratory earthquake investigations where the presence of these secondary disturbances was largely inconsequential to the main objectives of the experiment. This was indeed the case for experiments on supershear ground motion that are 


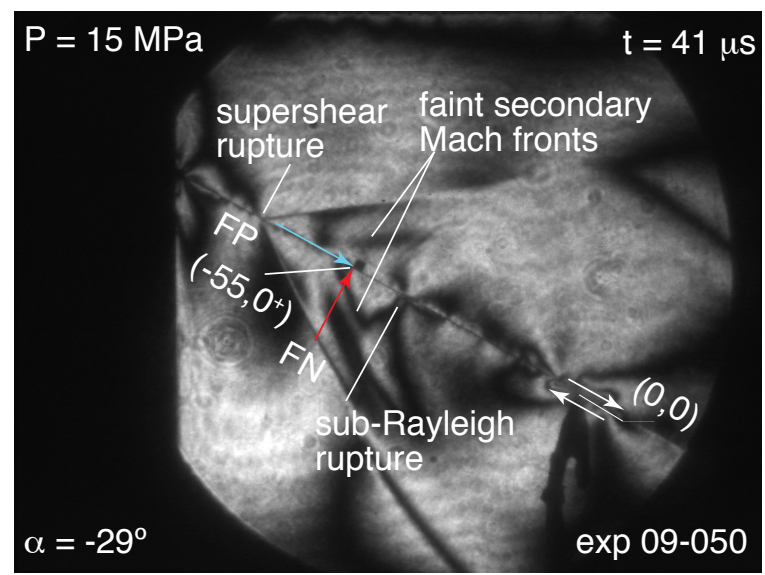

Figure 3.34: High-speed photoelastic image taken during exp 09-050 revealing secondary shear Mach fronts trailing primary supershear rupture

presented in this thesis, whereby only the leading portions of the particle velocity records leading up to the peak of the profile, were required. However, as has also been shown here, there can be instances where the secondary disturbance can interfere with the signal of interest so as to compromise the objective of the experiment. Such is the case in scaled laboratory earthquake investigations where the entire duration of the record is utilized and the total accumulated slip is scaled to the slip of a natural earthquake. It is therefore imperative to gain a better handle on the physical nature and root cause of the anomalous signals observed in supershear particle velocity records. The goal would be to either eliminate or at the very least partially suppress these effects. The central question is whether these collective observations correspond to additional supershear ruptures, which are spawned during the experiment, or whether they are an unusual artifact produced by the experiment itself, perhaps related to the finite thickness of the specimen.

\subsubsection{Isochrone analysis of supershear ruptures in laboratory earth- quake experiments}

It might appear reasonable to simply interpret the collective observations presented in the previous section as evidence of "secondary supershear ruptures" spawned during the experiment. Two of the observations that lend credence to this hypothesis are (1) the observed dominance of the magnitude 
of the secondary FP component over the magnitude of the secondary FN component (a defining signature of a stable supershear rupture), and (2) the photographic evidence of secondary shear Mach fronts within the wake of the primary supershear rupture, which are seen propagating in the same direction as the primary rupture.

The problem with the "secondary supershear rupture" hypothesis is in the observed sense of motion of the secondary FN (and vertical) signal, and the fact that it is always opposed to the sense of motion of the leading supershear rupture signal that it follows. The experiments in the previous section only demonstrated this feature for the case of a right lateral rupture propagating to the left, which always resulted in a secondary FN signal that exhibited a negative velocity swing. The result is actually quite general. The tendency of the secondary FN (and vertical) signal to exhibit an opposite sense of motion with respect to the leading FN (and vertical) signal is consistently observed in every supershear experiment, irrespective of whether the rupture is right lateral or left lateral or what direction the rupture travels along (left or right). Simply stated, the recorded net sense of motion exhibited by the secondary supershear disturbance is opposite to that which is theoretically predicted for a supershear rupture. Hence, the disturbance cannot correspond to an actual supershear rupture in the traditional sense.

Based upon this line of reasoning, an alternative hypothesis is considered for the root cause of the secondary signals and the observed secondary shear Mach fronts. The fact that the experiment is being conducted within a rectangular waveguide must be kept in mind. The observed signals may be a direct consequence of a finite plate thickness, as manifested by the delayed arrival of strong particle motion arising from the supershear rupture tip at the fault boundary located on the opposite side of the specimen. The information is propagated through the plate by strong $\mathrm{P}-, \mathrm{SV}-$, and $\mathrm{SH}-$ waves, which are then subject to symmetric and anti-symmetric modes of propagation in accordance with the Rayleigh-Lamb problem (Graff, 1991). The observed distortions to the amplitude and temporal profile of the secondary signals may simply be a consequence of the dispersion problem and the resulting cut-off frequencies of the various plate modes.

A pragmatic approach based upon isochrone theory was adopted to explore the plausibility of the 


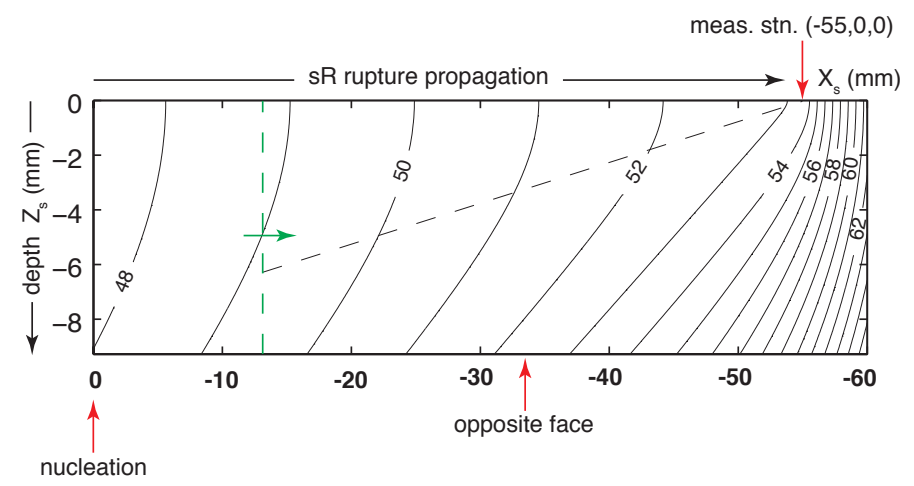

Figure 3.35: S-wave isochrones computed for a sub-Rayleigh rupture propagating across the entire length of the specimen fault

finite plate thickness hypothesis. A Matlab script was developed, which assumes a rupture velocity history and calculates all S-wave arrival times at a measurement station located at $\left(x_{1}, y_{1}, 0\right)$ on the surface of the specimen.

As the rupture front propagates it emits $\mathrm{P}$-waves and S-waves that arrive at the measurement station. The contribution from $\mathrm{P}$-waves are ignored in the analysis and it is only the $\mathrm{S}$-wave arrival times that are considered. The individual isochrone contours represent loci of constant S-wave arrival time at the measurement station location. A given isochrone $t=t\left(x_{s}, z_{s}\right)$ therefore represents the only points on the fault that contribute to the S-wave information that reaches the measurement station at time $t$.

The arrival time of an S-wave disturbance at $\left(x_{1}, y_{1}, 0\right)$, corresponding to the passage of a supershear rupture front at some point $\left(x_{s}, 0, z_{s}\right)$ on the fault, is given by

$$
t\left(x_{s}, 0, z_{s}\right)=\int_{0}^{L_{c}} \frac{1}{V_{r}\left(x_{s}^{\prime}\right)} \mathrm{d} x_{s}^{\prime}+\int_{L_{c}}^{x_{s}} \frac{1}{V_{s s}\left(x_{s}^{\prime}\right)} \mathrm{d} x_{s}^{\prime}+\frac{\sqrt{\left(x_{1}-x_{s}\right)^{2}+y_{1}^{2}+z_{s}^{2}}}{C_{s}}+t_{\text {delay }}
$$

where $L_{c}$ represents the supershear transition distance, and $V_{r}\left(x_{s}\right), V_{s s}\left(x_{s}\right)$ correspond to the rupture speed history of the sub-Rayleigh and supershear ruptures, respectively. The first term simply calculates the time that it takes for the sub-Rayleigh rupture to propagate a distance $x_{s}=L_{c}$. A straight rupture front is assumed and so all points along the advancing rupture front take this much time to reach the transition distance. Similarly, the second term accounts for the time taken by a 
straight supershear rupture front to propagate a distance $x_{s}-L_{c}$ where $L_{c} \leq x_{s} \leq x_{\max }$, with $x_{\max }$ corresponding to the length of the fault. The third term represents the time taken by an $\mathrm{S}$ wave to propagate along a straight line path from a point $\left(x_{s}, z_{s}\right)$ on the fault to the measurement station positioned at $\left(x_{1}, y_{1}, 0\right)$. The last term $t_{\text {delay }}$ corresponds to a well- characterized rupture delay time of $4.45 \mu \mathrm{s}$ that is observed in the experiments and addressed in a later section. Including $t_{\text {delay }}$ in the calculation of the isochrones was necessary in order to properly synchronize the isochrone plots with the particle velocity records. Finally, the fault is discretized into a $100 \times 1000$ point grid and the value of $\mathrm{t}\left(x_{s}, z_{s}\right)$, is calculated at each point within the grid. Isochrone plots corresponding to $t\left(x_{s}, z_{s}\right)=c$ such as those shown in Figures $3.32 \mathrm{~b}, 3.33 \mathrm{~b}$ are then immediately generated.

Figure 3.31b and Figure 3.34 represent isochrone contour maps corresponding to each of the supershear experiments that were previously discussed. The variable $z_{s}$ corresponds the fault depth where $z_{s}=0$ corresponds to the side of the specimen where the measurement station is located. A straight rupture front was assumed, which extends throughout the entire depth of fault. The rupture is nucleated at the left end of the figure and propagates to the right along the direction corresponding to the $x_{s}$ axis. Note that the $x_{s}$ axis in the diagram assumes negative values in an effort to remain consistent with the coordinates in the experiments. Positive values for $x_{s}$ were assumed in the isochrone calculations.

Before examining the supershear case, it is instructive to first consider what an isochrone plot looks like for the case of a sub-Rayleigh experiment. The isochrones in Figure 3.35 were computed by considering a sub-Rayleigh rupture, which traverses the entire length of the fault with a rupture speed $V_{r}=0.88 C_{s}$. A specimen thickness of $9.45 \mathrm{~mm}$ was assumed with the particle velocity measurement station positioned at $(-55,0,0) \mathrm{mm}$.

An S-wave emitted from $(0,0,0) \mathrm{mm}$ will be first S-wave to reach the measurement station at $(-55,0,0) \mathrm{mm}$ and is predicted to arrive at $t=47.4 \mu \mathrm{s}$. Likewise, an S-wave emitted from the rupture front at opposite edge of the fault at $(0,0,-9.45) \mathrm{mm}$ is predicted to reach the station at $t=48.048 \mu \mathrm{s}$. The latter time naturally corresponds to the unique isochrone which is the first to extend across the entire depth of the fault. $\mathrm{S}$ wave information reaching $(-55,0,0) \mathrm{mm}$ from 
this time onward can always be traced back to isochrones, such as those depicted in Figure 3.35, that extend throughout the entire depth of the specimen fault. The result shows that beyond what occurs during the first $0.6 \mu s$ of the experiment, there is never any distinction made between S-waves emitted by the rupture tips on either edge of the fault. The information received at $(-55,0,0) \mathrm{mm}$ is always a superposition of $\mathrm{S}$ waves from point sources spanning the entire depth of the fault that collectively define the observed signal.

The simple isochrone analysis, although extremely insightful, neglects to account for the inherent decay of S-wave amplitude with propagation distance, which must also be considered. The amplitude of the S-waves arriving from points on an isochrone lying closer to the measurement station is expected to dominate over the amplitude of S-waves arriving from points from the opposite edge of the fault. Hence, although S-waves emitted across the entire specimen thickness do indeed contribute at all times, there is an unequal weighting of their contributions at the measurement station, which naturally favors the contributions from the points in the local vicinity of the rupture. This would also help to explain why there are never any distinguishing signatures in sub-Rayleigh rupture signals that can be attributed to the finite thickness of the specimen.

The results of an isochrone analysis applied to the supershear rupture experiments exp 09-036 and exp 09-050 are now examined. Isochrone plots for each case are represented in Figure 3.32b and Figure 3.33b. A constant sub-Rayleigh rupture speed $V_{r}=0.88 C_{s}$ and a supershear rupture speed $V_{s s}=1.7 C_{s}$ were used in both calculations. The supershear speed estimate corresponds to an average rupture speed determined from the high-speed photoelastic image sequence. The supershear transition distance $L_{c}$ in each case was also estimated through an analysis of the photoelastic image sequence and then slightly adjusted within the code in order to more accurately match the observed supershear arrival times. Specimen thickness values were obtained from digital caliper measurements rather than simply assuming the nominal thickness. Specific values for specimen thickness and the supershear transition distance are given as each specific experiment is presented and discussed.

Figure 3.32b depicts the isochrone plot corresponding to exp 09-036. A specimen thickness of $9.28 \mathrm{~mm}\left(z_{\min }=-9.28 \mathrm{~mm}\right)$ and a supershear transition distance $L_{c}=9.5 \mathrm{~mm}$ were assumed in 
the analysis. Note the familiar isochrones confined within the region to the left of $\mathrm{x}=L_{c}$ (vertical dashed line), which correspond to the sub-Rayleigh isochrones discussed in the previous example. Contours to the right of this line naturally correspond to the supershear isochrones.

The supershear rupture is predicted to reach $(-54,0,0) \mathrm{mm}$ at $t=33.3 \mu \mathrm{s}$. This establishes the first supershear isochrone, which is represented by a single point positioned at $(-54,0,0) \mathrm{mm}$. The remaining supershear isochrones appear as elliptical contours, which surround the measurement station and become progressively larger with increased time. Note in particular the unique supershear isochrone corresponding to $t=39.2 \mu \mathrm{s}$, which first intersects the fault boundary on the opposite side of the specimen. The result implies that a $5.9 \mu s$ time span from $33.3 \mu s-39.2 \mu s$ in the particle velocity records must be free of any S-wave radiation emitted from the opposite fault boundary. Stated differently, there is a $5.9 \mu s$ time window following the arrival of the primary supershear rupture during which an infinite specimen depth may be assumed (P-waves ignored). As previously noted, and as revealed by the particle velocity plots, $t=39.2 \mu \mathrm{s}$ also corresponds to the arrival tine of the secondary signals.

Figure $3.33 \mathrm{~b}$ depicts the isochrone plot corresponding to the particle velocity data acquired in exp 09-050. A specimen thickness of $9.4 m m\left(z_{\min }=-9.4 \mathrm{~mm}\right)$ and a supershear transition distance $L_{c}=3 \mathrm{~mm}$ were assumed in the analysis. The dashed vertical line corresponding to the supershear transition distance once again delineates between the sub-Rayleigh isochrones to the left and the supershear isochrones to the right. The unique supershear isochrone corresponding to $t=36.9 \mu \mathrm{s}$, establishes the soonest time that S-wave information from the fault boundary on the opposite side can reach the measurement station at $(-55,0,0) \mathrm{mm}$. In this case the time $t=36.9 \mu \mathrm{s}$ is within $0.5 \mu s$ of the minima set between the two peaks in the FP record $(t=36.4 \mu s)$, which correlates very well with observed arrival time of the secondary signals in Figure 3.33a. Once again there appears to be an excellent correlation between the arrival of the secondary signals in supershear velocity records and the first isochrone to intercept with the fault boundary on the opposite side of the specimen.

In summary, the following four critical input parameters are required to conduct the $\mathrm{S}$ wave 
isochrone analysis: (1) measurement station coordinates, (2) rupture speed history, (3) supershear transition length, and (4) specimen thickness. All of these quantities must be accurately known in order to achieve the correlation demonstrated here. The simplest rupture history was assumed corresponding to a constant sub-Rayleigh rupture speed $V_{r}=0.88 \mathrm{Cs}$ for $0 \leq x_{s}<L_{c}$, followed by a constant supershear speed $V_{s s}=1.7 \mathrm{Cs}$ for $L_{c} \leq x_{s} \leq x_{\max }$, where $x_{\max }$ is the total length of the fault. The analysis correctly predicts the arrival time of the supershear rupture front and also identifies a unique S-wave isochrone, which corresponds to the soonest possible time that S-waves emitted by the rupture tip on the opposite side of the specimen, will be registered at the measurement station. The actual measured time difference between the arrival of the primary supershear rupture and the anomalous secondary signals was $\delta t=5.92 \mu s$ for exp09-036 and $\delta t=5.45 \mu s$ for exp09050. Time differences on the order of $(5.5-6 \mu s)$ are frequently observed in supershear experiments, particularly those involving on-fault measurements. The isochrone calculations accurately predicted the secondary signal arrival times for both experiments. The observed correlation between the time of the first supershear isochrone that extends to the fault boundary and the arrival time of the secondary signals lends credence to the hypothesis that the disturbance may be correlated to the supershear rupture tip on the opposite side of the specimen.

\subsection{Photoelasticity and high-speed digital photography for spatially resolved, whole-field imaging}

The stress-birefringent property of H-100 enables the application of photoelastic interferometry in conjunction with high-speed digital photography for full-field imaging in a dynamic rupture experiment. Images of the experimental setup, including a detailed schematic depiction of the photoelastic optical arrangement, are shown in Figure 3.36.

A vertically polarized output beam from a CW solid state laser is expanded to a nominal beam diameter $\approx 145 \mathrm{~mm}$, which establishes the field of view in the experiment. The primary laser light source is a Coherent Verdi (V-10) solid-state, air-cooled laser which emits a $532 \mathrm{~nm}$ (green) laser 


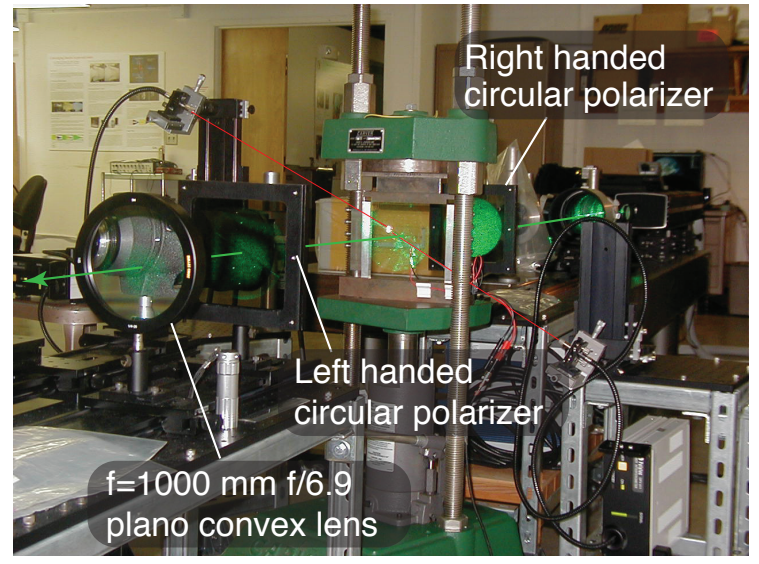

Laboratory earthquake setup

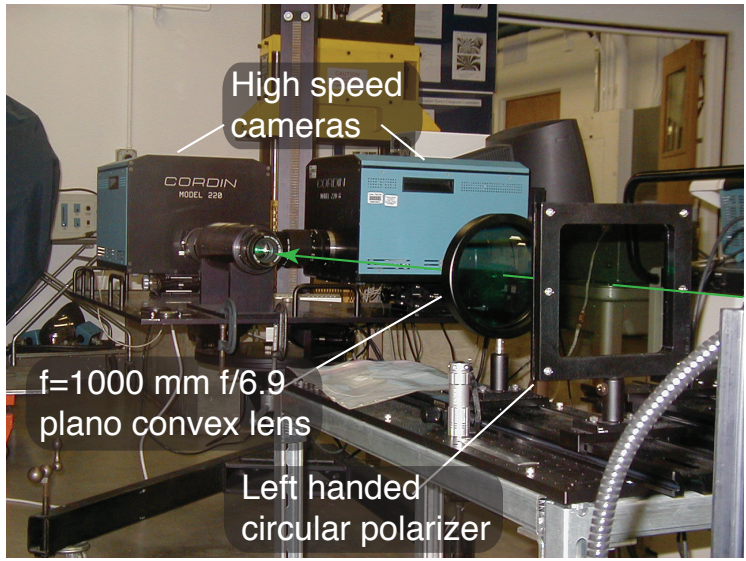

High-speed imaging configuration

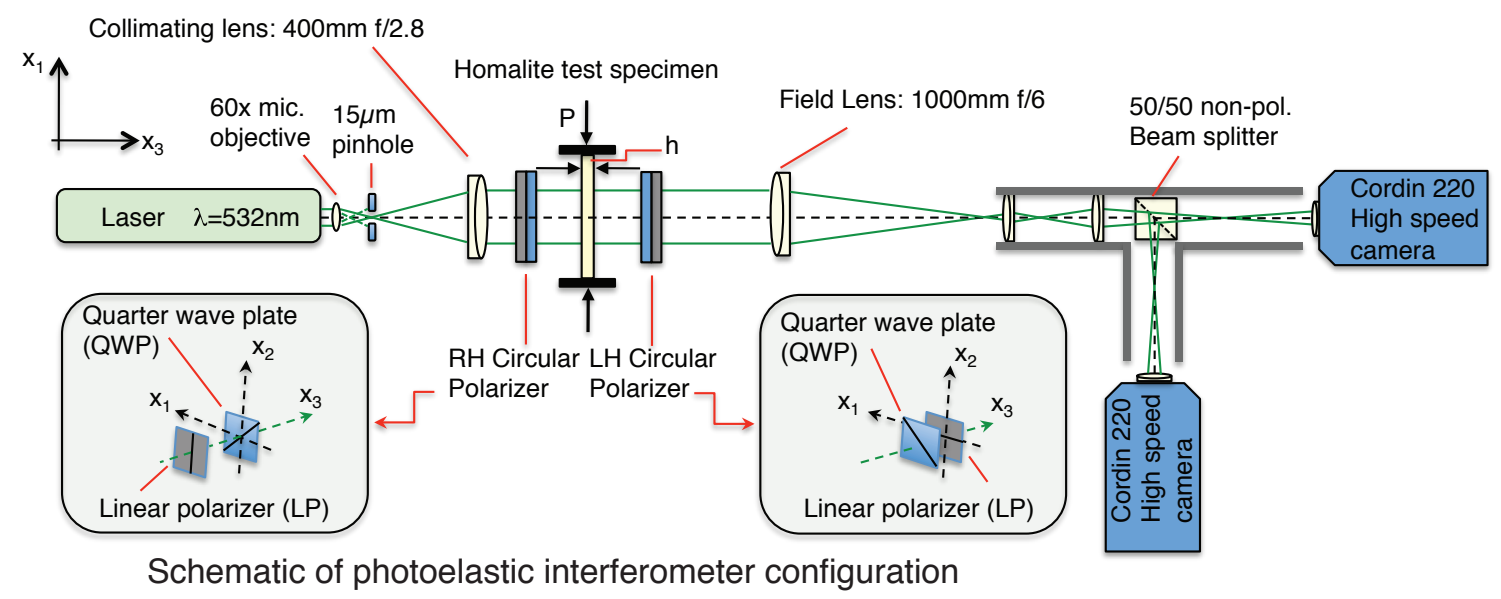

Figure 3.36: Upper right and left images: Laboratory earthquake experiment featuring fiber optic interferometers for continuous particle velocity measurements and photoelastic setup in conjunction with high-speed cameras for full-field imaging of a dynamic rupture with high spatial resolution. Below: Detailed schematic diagram of photoelastic interferometer and imaging optics

line. The laser and beam expander arrangement are depicted in Figure 3.37. Additional optical components seen in the right-hand image constitute a variable attenuator for external control of the laser power (Newport, 2006). The primary cooling mechanism is the aluminum heat sink upon which the laser is mounted. Chilled water, maintained at $20^{\circ} \mathrm{C}$ and circulated through the cooling block acts to further stabilize the laser wavelength. The power of the output beam is continuously adjusted from the main laser power supply. The laser is capable of generating up to $10 \mathrm{~W}$ in continuous wave mode, although typical power levels used in current laboratory earthquake experiments are generally less than $1.5 \mathrm{~W}$.

Photoelastic fringes are produced through the use of custom fabricated right- and left-handed 

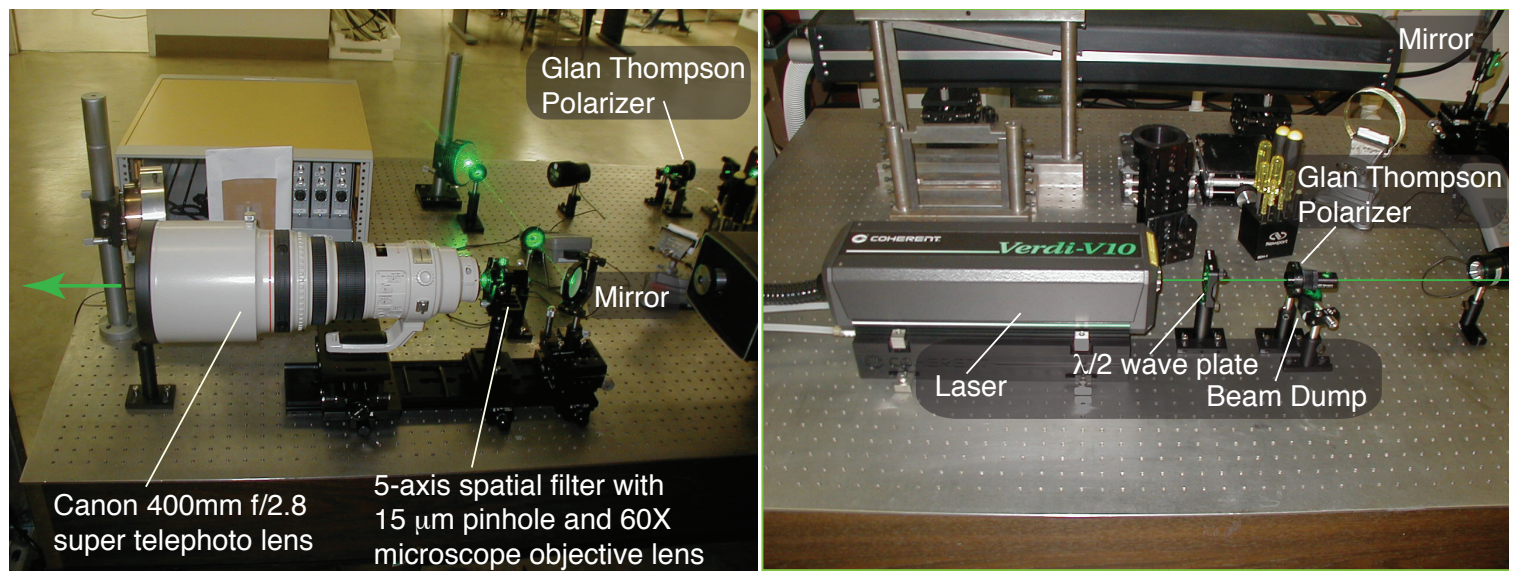

Figure 3.37: Right image: Coherent Verdi (V-10) solid state with variable attenuator optics. Left image: Keplerian beam expander with spatial filtering arrangement

circular polarizers obtained from American Polarizer, Inc. Square polarizers measuring $152 \mathrm{~mm} \times$ $152 \mathrm{~mm}$ are mounted in Al black-anodized mounting frames, as seen in Figure 3.36. The optical components are laminated assemblies of $\lambda / 4$ (quarter wave) and linear sheet polarizers, which are sandwiched and bonded between optical windows using an index-matching epoxy. The expanded laser beam is transmitted through a right- hand circular polarizer (RHCP) which converts the incident linearly polarized light into a circularly polarized state. The circularly polarized beam is then transmitted through the H-100 test specimen.

If the test specimen is unstressed, the $\mathrm{H}-100$ test sections remain optically isotropic and the transmitted beam remains circularly polarized. The transmitted beam is then passed through a left-hand circular polarizer (LHCP), whereupon the leading quarter wave plate nullifies the circular polarization state and restores the light field to its original, vertically oriented, linearly polarized state. The linearly polarized light is then extinguished by the horizontally oriented linear polarizer (often termed the analyzer) in the LHCP (see Figure 3.36). The resulting image in the case of an unstressed specimen is a dark field void of any interference fringes and the optical configuration is commonly referred to as a dark-field arrangement.

When mechanically stressed however, the H-100 test specimen becomes optically anisotropic and the transmitted light field is no longer circularly polarized. The dark-field image is then transformed 
into a photoelastic interference pattern featuring bright and dark fringes corresponding to isocontours of maximum shear stress within the plane of the test specimen. The resulting interference problem is best understood by appealing to the general time-averaged intensity representation of the photoelastic fringe pattern given by

$$
I\left(x_{2}, x_{2}\right)=I_{b}\left(x_{1}, x_{2}\right)+\frac{I_{a}\left(x_{1}, x_{2}\right)}{2}\left(1-\cos \delta\left(x_{1}, x_{2}\right)\right)
$$

where $I_{b}$ represents a background intensity and $I_{a}$ represents the amplitude (Ramesh, 2008). The stress-induced relative retardation, $\delta$, also commonly referred as the isochromatic phase, is given by

$$
\delta\left(x_{1}, x_{2}\right)=\frac{4 \pi h C}{\lambda} \Delta \tau_{\max }\left(x_{1}, x_{2}\right)
$$

where $C$ is the stress optic coefficient $\left(C=0.231 \times 10^{-10} \mathrm{~m}^{2} / N\right.$ for $\left.\mathrm{H}-100\right), \lambda$ represents the laser wavelength $(\lambda=532 \mathrm{~nm}), h$ is the specimen thickness $(h=9.5 \mathrm{~mm}$ is the nominal H-100 sheet thickness), and $\Delta \tau_{\max }$ corresponds to the relative change in maximum shear stress. The intensity variation $I=I(x, y)$ is modulated according to the spatial variation of the isochromatic phase term $\delta=\delta(x, y)$ and the resulting photoelastic fringes are often referred to as isochromats. Next, since the relative retardation $\delta$ is expressed in radians, the relative fringe order is therefore defined by $\Delta N=\delta / 2 \pi$, whereby a fringe corresponds to a fundamental period in the photoelastic fringe pattern characterized by a bright-to-bright or dark-to-dark intensity transition (or any other intermediate grey-scale level). The quantity $f_{\sigma}=\lambda / C$ appearing in (3.13) is often adopted in the photoelastic literature. Care must be taken to consider the assumed wavelength when using a tabulated value of $f_{\sigma}$. In the case of Homalite- 100 , a value $f_{\sigma}=23.6 \mathrm{kN} / \mathrm{m}$ is often reported, based upon a source wavelength $\lambda=546 \mathrm{~nm}$ (Kobayashi, 1993). Factoring the assumed wavelength into account and then taking $\lambda=532 \mathrm{~nm}$, leads to a corrected value $f_{\sigma}=23.03 \mathrm{kN} / \mathrm{m}$. The so called "stress optic law" relating the relative change in the maximum shear stress $(\Delta \tau)$ to a corresponding fringe shift 
in the photoelastic pattern is then given by

$$
\Delta \tau_{\max }=\frac{f_{\sigma}}{2 h} \Delta N
$$

The "fringe counting relationship" given by Eq. 3.14 thus demonstrates how photoelastic fringes directly correspond to isocontours of maximum shear stress. Note that the dark-field intensity expression Eq. 3.12 does not include any reference to the principle angle and that fringe counting using Eq. 3.14 must be done so with care since the photoelastic fringes correspond to isocontours of maximum shear stress in magnitude only.

\subsubsection{High-speed cameras}

The Cordin 220 gated intensified cameras are shown in Figure 3.38. The $f=1000 \mathrm{~mm} \mathrm{f} / 6.9$ planoconvex field lens focuses the expanded light beam down into an array of imaging lenses mounted within the baffled tube assembly, as depicted in Figure 3.36. The beam is focused to the smallest possible spot size onto the center of the reflective lens cap, which can also be seen at the front of the tube assembly in Figure 3.38. The camera assembly is then carefully aligned with respect to the incident beam by adjusting the tilt, rotation, and height of the camera table as outlined in Section 3.3. The reflective lens cap is removed once the alignment is complete. The beam is then passed through the first camera lens, at the main entrance of the tube assembly, followed by an additional lens (not seen) located within the tube, before reaching a 50/50 non-polarizing - 2" cube beam splitter. The beam splitter divides the imaging path and directs each laser beam to a final camera lens at the entrance to each camera. The beams are split yet again by a series of pellicle beam splitters to create eight imaging paths within each camera. Each beam is finally brought to a sharp focus onto an $18 \mathrm{~mm}$ diameter photocathode corresponding to the front element of the image intensifier. An image intensifier is comprised of a photocathode, microchannel plate (MCP), and a phosphor screen. The image of the test specimen is initially cast on the photocathode. As photons strike the cathode, electrons are emitted and attracted to the MCP by an electric field. 


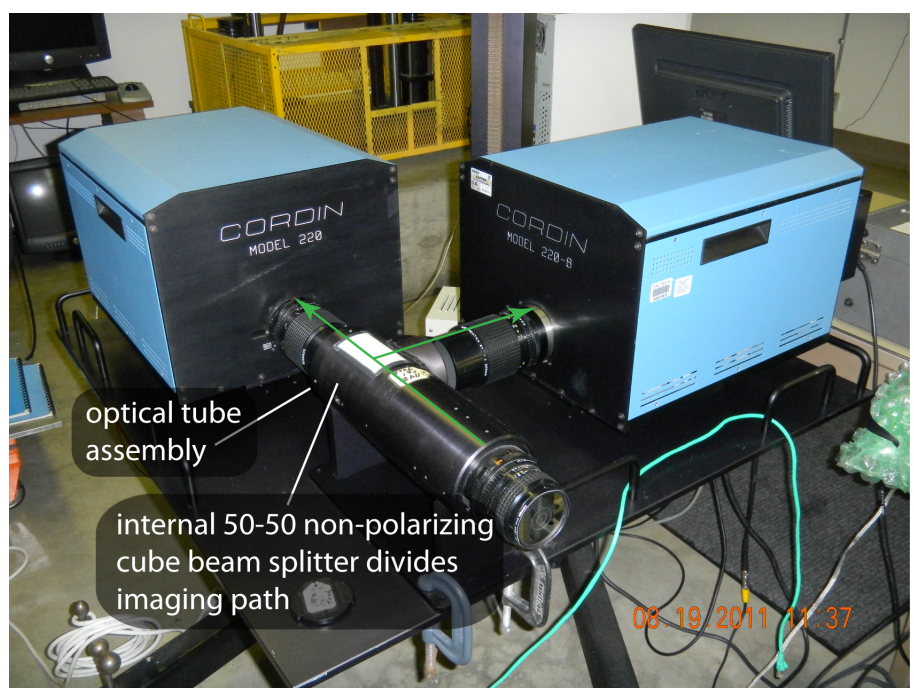

Figure 3.38: Cordin 220 gated-intensified high-speed cameras

The MCP is a thin, disk-shaped, honeycomb structure containing glass channels, which are typically $6-10 \mu m$ in diameter. A high potential is applied across the MCP, which induces the electrons to accelerate down the MCP channels. High-energy photoelectrons collide with the MCP walls and dislodge secondary electrons, which are in turn accelerated by the applied electric field. The process results in a tremendous multiplication of secondary electrons. The degree of electron multiplication is set by the "gain" applied across the MCP, which is controlled through the camera software. The multiplied electrons strike a phosphor screen which produces an amplified image of the original image that was initially projected onto the photocathode. The light emitted by the phosphor screen is then coupled to a CCD through a bundle of optical fibers. Image-intensified CCDs exploit this gain principle to overcome the inherent read noise limit of traditional CCD sensors. Another significant advantage of image intensifiers is that they can be operated as a very fast optical switch, capturing an optical signal in billionths of a second. The Cordin 220 features inter-frame times ranging from $10 \mathrm{~ns}-10 \mathrm{~ms}$ and exposure (integration) times ranging from $10 \mathrm{~ns}-1 \mathrm{~ms}$. Inter-frame times used in the laboratory earthquake experiments are typically in the range of $1-4 \mu s$, with exposure times of $50 \mathrm{~ns}$. 


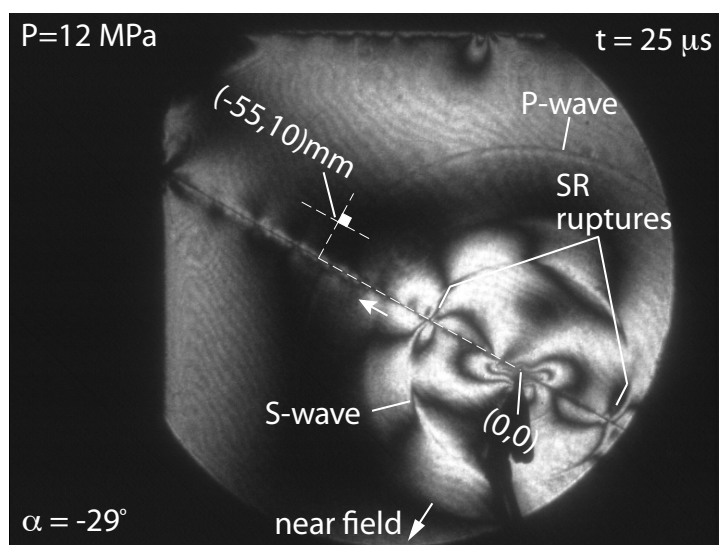

sub-Rayleigh experiment: $\exp 09-052$

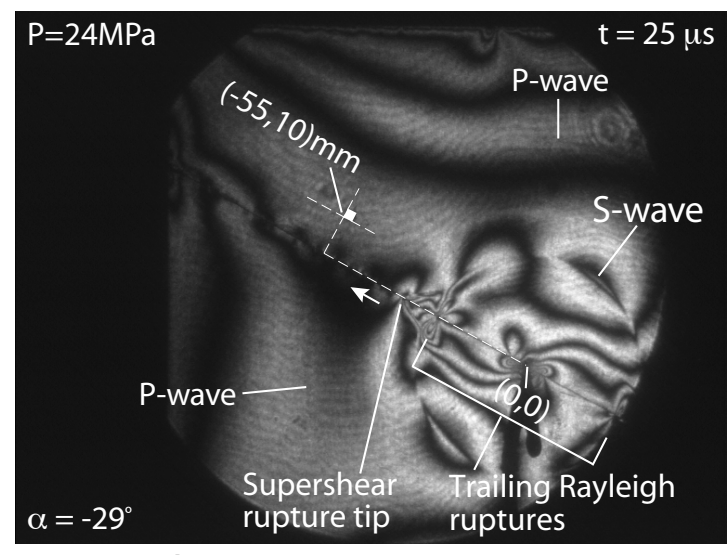

Supershear experiment: $\exp$ 09-047

Figure 3.39: Sub-Rayleigh (left) and supershear (right) ruptures as revealed by photoelastic fringe patterns captured through high-speed photography

\subsubsection{Measurement of wave speeds and rupture speeds in laboratory earthquake experiments}

Figure 3.39 contrasts high-speed photoelastic image frames of a sub-Rayleigh rupture and a supershear rupture, which resulted from two completely different experiments. The image on the left was obtained from a pure sub-Rayleigh experiment (exp 09-052) while the image on the right resulted from an experiment (exp 09-047) that was designed to undergo a sub-Rayleigh-to-supershear transition. Both image frames were acquired at $t=25 \mu \mathrm{s}$ in each respective experiment. Each experiment featured a specimen fault angle $\alpha=-29^{\circ}$, which naturally promoted a right-lateral rupture. A bi-lateral rupture resulted in both cases as is typically observed with the traditional laboratory earthquake specimen design. Particle velocity measurement stations were positioned at $(-55,10) \mathrm{mm}$ in both experiments and are each highlighted by a small white rectangle in the photoelastic images.

The sub-Rayleigh experiment (exp 09-052) was conducted under a static compressive load of $P=12 \mathrm{MPa}$, which was low enough to prevent the sub-Rayleigh rupture from transitioning to a supershear rupture. The resulting sub-Rayleigh rupture, along with the expanding P- and S-wave fronts are identified in the left-hand image. The precise location of the sub-Rayleigh rupture tip is identified by a small dark caustic zone, which can be best seen when the image is expanded. The 

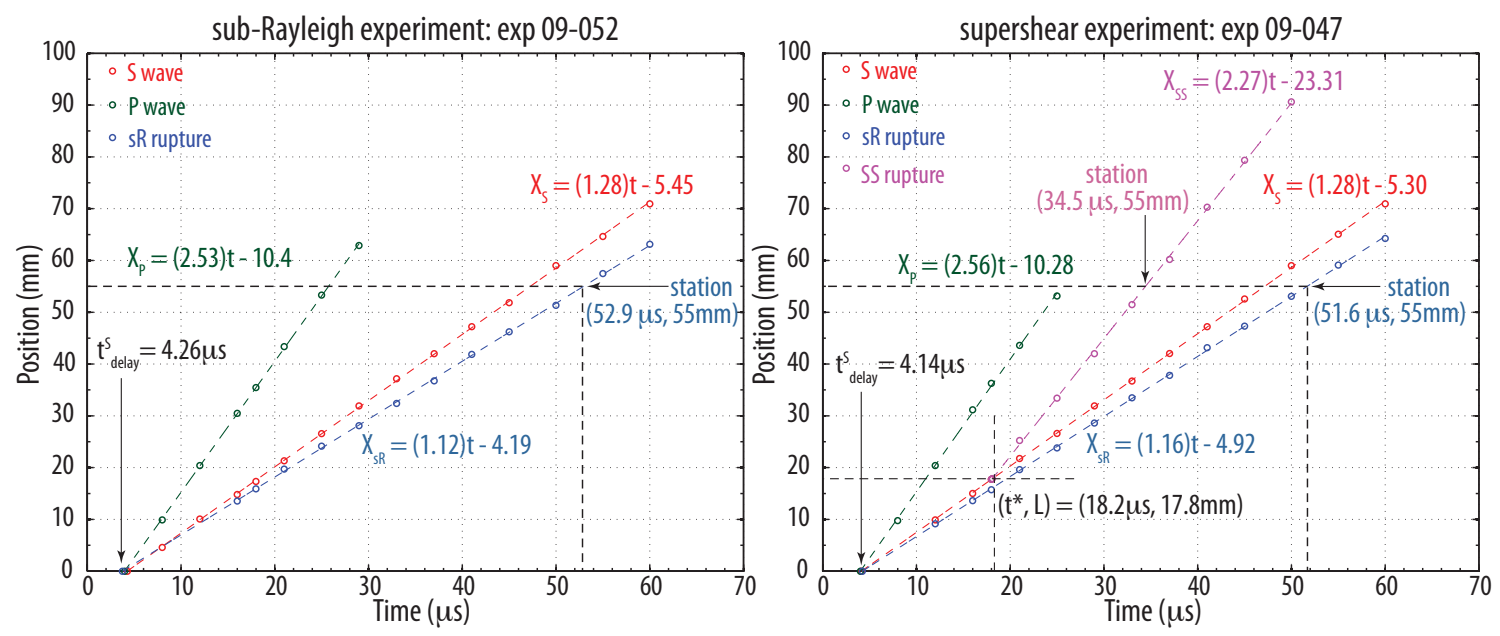

Figure 3.40: Plots of rupture position, $\mathrm{S}$ wave, and $\mathrm{P}$-wave obtained during a sub-Rayleigh experiment.

arc of the $\mathrm{P}$-wave front is far less visible than the $\mathrm{S}$-wave front but is still discernible at this time. P-wave fronts tend to decay faster than the S-wave fronts and are typically rarely observed beyond $t=30 \mu s$. Note how the near-field region, characterized by a band of eight bright radial lobes, is also clearly visible in the image.

The supershear experiment (exp 09-047) was conducted under a static compressive load of $P=$ $24 M P a$ in order to promote a supershear transition. A fairly early transition resulted, as revealed by the fully developed shear Mach fronts, which are clearly visible in the image on the right. The trailing sub-Rayleigh rupture can also be seen along with the $\mathrm{S}$ - and $\mathrm{P}$-wavefronts. The P-wave is barely visible in this image frame. Nonetheless a faint hint of the circular arc is all that is required to track its position (radius) at this particular time.

\subsubsection{Analysis of a sub-Rayleigh and a supershear experiment}

An image-length scale is established for each experiment (either immediately before or after) using a simple calibration procedure. A rectangular blade of known dimensions is temporarily affixed to the surface of the H-100 test specimen and aligned with its long edge parallel to the specimen fault. The specimen is illuminated by the expanded laser beam and sixteen sharply defined, silhouette images of the blade are obtained by the two high-speed cameras. The pixel dimensions of the blade image 
are determined by imaging software and a precise pixel-to-length calibration factor is established for each individual camera frame.

High-speed photoelastic images are analyzed using Adobe Illustrator. Circles drawn over the S-wave and P-wave fronts are carefully sized and positioned until they overlap the wave fronts and share a common center. The center of the two concentric circles establishes $(0,0) \mathrm{mm}$, which should coincide with the position of the NiCr filament. Distances to the rupture tips, radii of the Sand $\mathrm{P}$-wave fronts, and the distance to the particle velocity measurement station are all accurately measured with respect to this point. The procedure is carried out for all the image frames within a given photoelastic image sequence where the rupture and elastic wave fronts can be identified.

Figure 3.40 summarizes all of the data obtained through a frame-by-frame analysis of the subRayleigh experiment (exp 09-052) and the supershear experiment (exp 09-047). The plot on the left-hand side corresponds to the sub-Rayleigh experiment (exp 09-052). The position versus time of the S-wave, P-wave, and sub-Rayleigh rupture tip correspond to the red, green, and blue curves, respectively. The dots in each curve correspond to actual data obtained from the photoelastic image analysis. The curve corresponding to the $\mathrm{P}$-wave front is comprised of seven data points, with the last point obtained from the $t=29 \mu s$ image frame. The S-wave front data contains 14 discrete data points, which nearly span the entire duration of the experiment. The sub-Rayleigh rupture history is represented by 12 data points. In all three cases the dashed lines represent a best linear fit to the data. Accurate estimates for the average rupture speed and the elastic waves are determined from the slope of the best-fit lines.

Application of this measurement procedure to the analysis of 41 independent laboratory earthquake experiments has yielded new estimates for the shear (S) and dilatational (P) wave speeds in H-100. Values for the elastic wave speeds are listed in Table 3.3. The stated value for the measurement uncertainty corresponds to the standard error computed by the well-known formula: $S_{x}=\sigma / \sqrt{N}$, where $\sigma$ represents the standard deviation and N corresponds to the number of measurements. The measured values appear slightly higher (by $6.5 \%$ in the case of $\left(C_{s}\right)$ and $3.5 \%$ in the case of $\left(C_{p}\right)$ than values that are frequently cited in the literature (Bhat et al., 2010). Note as 
Table 3.3: Measured wave speed values and mean sub-Rayleigh rupture speed in Homalite ${ }^{\mathrm{TM}} \mathrm{H}-100$ obtained from laboratory earthquake experiments

\begin{tabular}{ccccc}
$C_{s}(\mathrm{~mm} / \mu s)$ & $C_{p}(\mathrm{~mm} / \mu s)$ & $C_{p} / C_{s}$ & $V_{r} / C_{s}\left(V_{r}<C_{R}\right)$ & $V_{r}(\mathrm{~mm} / \mu s)$ \\
\hline \hline $1.284 \pm 0.002$ & $2.590 \pm 0.010$ & $2.017 \pm 0.008$ & $0.883 \pm 0.003$ & $1.133 \pm 0.004$ \\
\hline
\end{tabular}

well that measurement of the shear wave speed enables a direct estimate of the shear modulus for H-100 using $\mu=\rho C_{s}^{2}$ assuming the density of H-100 is known. An estimate for the shear modulus of $\mu=1.96 \mathrm{GPa}$ is obtained assuming a value of $\rho=1190 \mathrm{~kg} / \mathrm{m}^{3}$ as listed by (Tippur, 2006). Table 3.3 also lists an average value for the sub-Rayleigh rupture speed of $V_{r}=1.133 \pm 0.004 \mathrm{~mm} / \mu \mathrm{s}$, which was obtained from 31 independent laboratory earthquake experiments. This value translates to $\bar{V}_{r} / C_{s}=0.883 \pm 0.003$ (assuming $\left.C_{s}=1.284 \mathrm{~mm} / \mu \mathrm{s}\right)$.

Dilatational (P) and shear $(\mathrm{S})$ elastic wave speed values corresponding to $C_{p}=2.53 \mathrm{~mm} / \mu \mathrm{s}$ and an $\mathrm{S}$ wave speed $C_{s}=1.28 \mathrm{~mm} / \mu \mathrm{s}$ were obtained from the analysis of the sub-Rayleigh experiment (exp 09-052). The S-wave speed estimate in this case is in agreement with the tabulated value. The P-wave speed estimate is seen to lie outside of the standard error range but lies well within the one standard deviation of the expected value $\left(\sigma_{P}=0.064\right)$.

An average rupture speed of $\bar{V}_{r}=1.12 \mathrm{~mm} / \mu \mathrm{s}$ was obtained from the sub-Rayleigh curve, which corresponds to $\bar{V}_{r} / C_{s}=0.875$ (assuming $C_{s}=1.284 \mathrm{~mm} / \mu \mathrm{s}$ ). The measured value lies outside of the standard error estimate but is within one standard deviation $\left(\sigma_{s R}=0.017\right)$ of the tabulated average value.

All of the plots in Figure 3.40 contain an additional data point $\left(t_{\text {delay }}, 0 \mathrm{~mm}\right)$ corresponding to their respective time-axis intercept. The curves were extrapolated back to this point in order to highlight an observed time delay between instrumentation trigger and the very first moment that elastic waves are emitted from the rupture nucleation site. The noted delay time $t_{\text {delay }}^{s}$ in Figure 3.40 corresponds to the S-wave curve intercept. The P-wave curve traces back to a slightly different intercept at $(3.97 \mu s, 0 \mathrm{~mm})$ while the sub-Rayleigh rupture curve projects back to $(3.75 \mu \mathrm{s}, 0 \mathrm{~mm})$. The difference between the S- and $\mathrm{P}$-wave curve intercepts is easily attributed to the increased measurement uncertainty of the $\mathrm{P}$-wave measurements. The time axis intercept for the sub-Rayleigh 
rupture curve is less meaningful since the rupture is never physically located at the origin, but is instead initiated at critical-crack-length distance located to the left and right of the origin.

The noted time delay between the instrumentation trigger time and the emergence of the very first elastic waves is consistently observed in every experiment and has been very well characterized. The precise cause of this delay is not fully understood, but it is more than likely related to the rupture nucleation process, as opposed to being an artifact of the electronic diagnostics. Measurements obtained from 49 independent laboratory earthquake experiments have yielded an average S-wave delay time of $4.449 \mu s \pm 0.047 \mu s$. This factor must always be taken into account when predicting P- and S-wave arrival times in relation to particle velocity records. The correct estimate for the first $\mathrm{P}$ - and S-waves to reach the measurement station at $(\mathrm{x}, \mathrm{y})$ is calculated by

$$
t_{j}=\sqrt{x^{2}+y^{2}} / C_{j}+t_{\text {delay }} \quad j=1,2 .
$$

A similar analysis was applied to the photoelastic image sequence corresponding to the supershear experiment (exp 09-047). The data pertaining to the elastic wave fronts and rupture histories is summarized by the curves on the right-hand side in Figure 3.40. The sub-Rayleigh rupture history is represented by 13 data points, which span the entire duration of the experiment. An average sub-Rayleigh rupture speed $\bar{V}_{r}=1.16 \mathrm{~mm} / \mu s$, corresponding to $\bar{V}_{r} / C_{s}=0.903$, is obtained from the slope of the best linear fit (blue dashed line). The position versus time of the supershear rupture tip was established by 7 data points spanning a $29 \mu \mathrm{s}$ interval from $t=21 \mu \mathrm{s}$ to $t=50 \mu \mathrm{s}$. A best linear fit to the data is represented by the violet dashed line passing through these points. An average supershear rupture speed of $\bar{V}_{s s}=2.27 \mathrm{~mm} / \mu \mathrm{s}$, corresponding to $\bar{V}_{s s} / C_{s}=1.77$, is directly inferred from the slope of the best-fit line. The intersection of the sub-Rayleigh and supershear curves (best-fit lines) uniquely determines the point $\left(\mathrm{L}, t^{*}\right)$, which represents an estimate for the location and time of the supershear transition. The supershear transition point $(17.8 \mathrm{~mm}, 18.2 \mu \mathrm{s})$ was added to the supershear curve in order to extend the curve to the point of intersection with the sub-Rayleigh curve. 
118

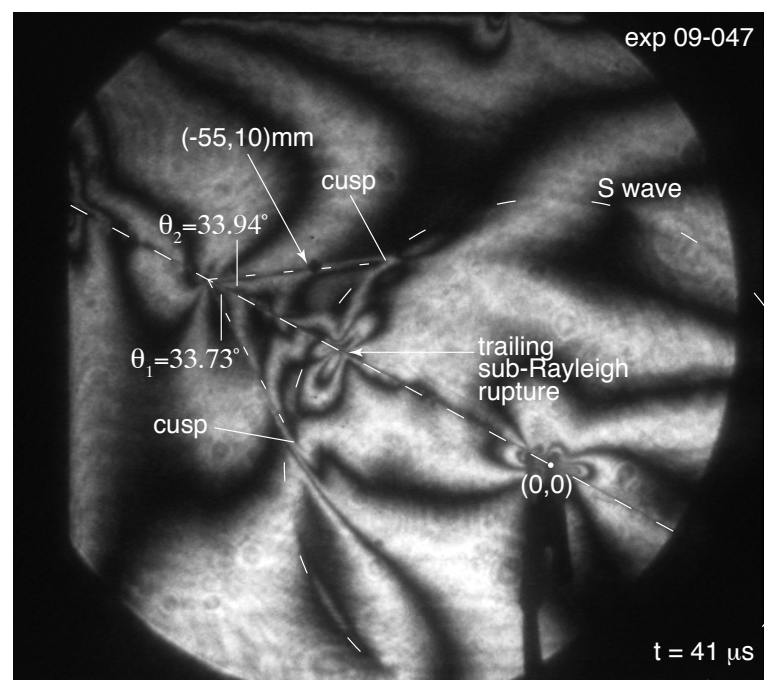

Figure 3.41: Measurement of shear Mach angles for determination of supershear rupture speed

An estimate of the instantaneous supershear rupture speed is obtained by measuring the shear Mach angle and applying the familiar Mach cone half-angle relation Eq. 1.1, restated as

$$
\sin \theta=C_{s} / V_{r}
$$

Figure 3.41 corresponds to a photoelastic image acquired at time $t=41 \mu s$ during the supershear experiment (exp 09-047). The shear Mach front is seen just crossing the measurement station at $(-55,10) \mathrm{mm}$ on the extensional side of the fault. The trailing sub-Rayleigh rupture is also clearly visible in the image positioned just behind the S-wave front. Sharp cusps in the fringe pattern, such as those labeled in the figure, are frequently observed. The cusps mark the location of the steepest stress gradient and thus provide a natural fiducial for estimating the Mach angle. Lines are drawn from the supershear tip to each of the sharp cusps as shown in Figure 3.41 to form the angles $\theta_{1}=33.73^{\circ}$ and $\theta_{2}=33.94^{\circ}$. Applying Eq. 3.16 to each of these angles and taking the average of the two values yields $V_{s s} / C_{s}=1.80$, which is within $2 \%$ of the average supershear rupture speed determined across a $29 \mu \mathrm{s}$ interval. The measured wave speed values collectively describe a nearly steady-state rupture. 


\subsection{Estimation of sub-Rayleigh-to-supershear transition length in laboratory earthquake experiments}

Original laboratory earthquake experiments conducted by (Xia et al., 2004) provided the first direct evidence of the sub-Rayleigh-rupture-to-supershear-rupture transition through high-speed photoelastic images. An analysis of the rupture history as revealed by photoelastic images concluded that the transition length $L$ exhibited a faster decay with increasing uniaxial pressure $P$, than the ex-

pected $L \propto P^{-1}$ dependence predicted by Eq. 3.4 and Eq. 3.2. The observed discrepancy was first attributed to a pressure dependency of the critical slip weakening distance $D_{c}$ in the slip-weakening friction law that was unaccounted for in Andrews' model. (Xia et al., 2004) combined results from rock friction experiments obtained by (Ohnaka, 2003) with a simple asperity-contact-based frictional model to show that $D_{c} \propto P^{-1 / 2}$. Specifically,

$$
D_{c}=C\left(\frac{\tau_{s}-\tau_{r}}{\tau_{r}}\right)^{M} \sqrt{H} a_{0} \cos \alpha P^{-1 / 2}
$$

where $C, M$ are constants, $H$ is the hardness of the material (defined as the ratio of the total normal force to the actual contact area), and $a_{0}$ represents the mean radius of contact asperities (Xia et al., 2004). Assuming plane stress loading conditions for the laboratory earthquake specimen then leads to a modified expression for the critical crack half-length given by

$$
L_{c}=\frac{\mu(1+\nu)\left(\tau_{p}-\tau_{r}\right) D_{c}}{\pi\left(\sigma_{y x}^{0}-\tau_{r}\right)^{2}}
$$

We can substitute the pressure dependent relation for Eq. 3.17 into Eq. 3.18, while observing that the supershear transition length $L$ should scale with $L_{c}$ and invoking Eqs. 3.5 and 3.6, to obtain a 
modified transition length relation given by

$$
\begin{aligned}
L= & F(s)\left[\frac{\mu(1+\nu) C \sqrt{H} a_{0} \cos \alpha}{\pi\left(\sin \alpha-f_{d} \cos \alpha\right)^{2}}\right] \times \\
& \left(\frac{f_{s}-f_{d}}{f_{d}^{M-1}}\right)^{M+1} P^{-3 / 2} .
\end{aligned}
$$

A detailed derivation of the revised transition-length expression Eq. 3.19 is given in (Rosakis et al., 2007), where an analytical approximation of the function $F(s)$ of the seismic S-ratio is provided. The modified expression given by Eq. 3.19 was found to be in good agreement with measured supershear transition lengths by using $a_{0}$ as a fitting parameter (Xia et al., 2004).

(?) later followed with numerical calculations, which examined the effect of the dynamic rupture initiation procedure and fault friction on supershear transition using a plane-stress model with an interface governed by linear slip weakening friction. A rupture initiation procedure that mimicked the dynamic nature of the discharging $\mathrm{NiCr}$ wire filament was achieved by reducing the normal stress over a part of the interface for a given time. Results demonstrated that dynamic rupture initiation procedure can, at times, significantly shorten the supershear transition length observed in experiments, compared to those predicted through the quasi-static rupture initiation process. Moreover, it was further determined that in some cases, the dynamic initiation procedure changes the very mode of transition, causing a direct supershear transition at the tip of the main rupture instead of the Burridge-Andrews, mother-daughter mechanism. Last, but not least, it is also possible, and even likely, based on the study conducted by (Lu et al., 2007), that the interface friction is better described by a rate and state-dependent law with significant rate-dependence at high slip rates rather than the linear slip-weakening law used in this work. The effect of such a law on transition length should be explored in future studies.

\subsubsection{On the use of H-100 in laboratory earthquake experiments}

It is instructive to note how the critical crack half-length relation Eq. 3.18 reinforces the use of Homalite-100 (or other brittle, mildly rate-sensitive polymeric materials) as a model material 
of choice in the laboratory earthquake experiment, as opposed to using test specimens fashioned from crustal rock material. The critical crack half-length relation given by Eq. 3.18, and thus the supershear transition length, are seen to scale in direct proportion to the shear modulus of the surrounding medium. H-100 has a shear modulus of $\mu=1.96 \mathrm{GPa}$ (Section 3.11.3), which is at least $30 \times$ less than the shear modulus value of crustal rock. Experiments have demonstrated that typical H-100 supershear transition lengths generally range from $10 \mathrm{~mm}-100 \mathrm{~mm}$ depending upon test conditions and the nature of the investigation. Thus, assuming a similar critical slip weakening distance $\left(D_{c}\right)$ between H-100 and crustal rock, a sub-Rayleigh rupture nucleated in a rock specimen would require a supershear transition length at least 30x greater $(0.3 m<L<3 m)$ than required

with H-100 test specimens. Hence, the use of rock specimens in the current loading arrangement requires considerably larger (and far more massive) test specimens compared to the current H-100 test specimen dimensions. Investigating dynamic ruptures in real rock samples will likely require a modified specimen design and loading arrangement.

\subsection{Control of rupture speed regime in laboratory earth-}

\section{quake experiments}

An alternative form for the seismic S-ratio, strictly applicable to the laboratory earthquake loading configuration, is given by

$$
S=\frac{f_{s}-\tan \alpha}{\tan \alpha-f_{d}}
$$

As previously noted (Andrews, 1976), a seismic S-ratio $S<1.77$ assures that a sub-Rayleigh rupture will transition into a supershear rupture. Assuming that this condition is satisfied in a laboratory earthquake experiment, the supershear transition length may then be controlled by varying the specimen fault angle $\alpha$ and the applied static compressive stress $P$, in accordance with Eq. 3.19. This assumes fixed values for the static and dynamic frictional coefficients $f_{s}, f_{d}$, elastic constants $\mu, \nu$ and contact asperity parameters $C, M, H, a_{0}$. The value of the specimen fault angle in a typical laboratory earthquake experiment ranges between $20^{\circ}<\alpha<30^{\circ}$. Specimen fault angles less 

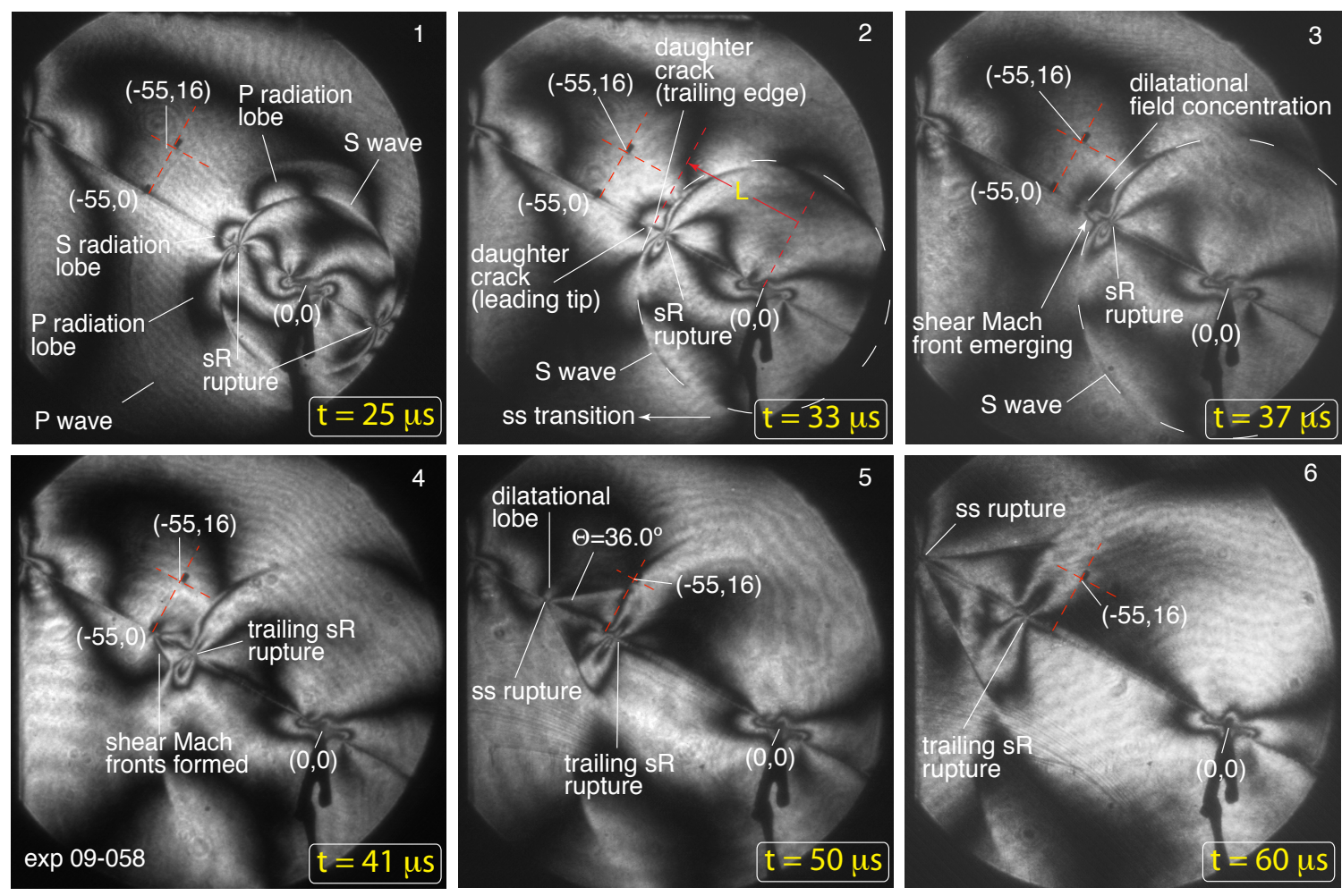

Figure 3.42: Sequence of photoelastic images revealing sub-Rayleigh-to-supershear transition

than $20^{\circ}$ require too much static pressure, which may induce buckling of the H-100 plate assembly, whereas specimen faults oriented at angles greater than $30^{\circ}$ are prone to slip under the applied static load. Taking $f_{s}=0.6$ and $f_{d}=0.2$ as representative values for the static and dynamic frictional coefficients leads to a practical bound on the seismic S-ratio given by $0.06<S<1.44$. The subRayleigh-to-supershear transition criterion $S<1.77$ is evidently satisfied over the entire range of specimen fault angles, thus implying that a sub-Rayleigh rupture will invariably transition into a supershear rupture. Whether or not a supershear transition will occur as $S \rightarrow 1.44$ is dictated by the physical dimensions of the test specimen and the available propagation distance $L^{*}$, measured from the nucleation site to the edge of the specimen fault.

Laboratory earthquake rupture propagation scenarios are therefore controlled by either fixing the fault angle $\alpha$ and varying the static pressure $P$ or vice versa so as to modulate $L$ in relation to $L^{*}$. The condition $L>L^{*}$ and Eq. 3.19 thus assure that a nucleated rupture will remain within the sub-Rayleigh rupture speed regime for the duration of the experiment, whereas $L<L^{*}$ assures 
that a sub-Rayleigh to supershear transition will occur between the rupture nucleation site and the edge of the frictional fault. The ability to adjust the supershear transition length in the laboratory earthquake experiment thus provides a versatile test platform for investigating various earthquake scenarios and frictional faulting processes involving both types of dynamic ruptures.

\subsubsection{High-speed photoelastic image sequence of sub-Rayleigh-to-supershear rupture transition}

The image sequence in Figure 3.42 demonstrates a sub-Rayleigh-to-supershear-transition, as captured through a sequence of high-speed photoelastic images. The experiment was conducted under a static compressive load of $P=12 M P a$ with a specimen fault angle of $\alpha=-29^{\circ}$, which naturally promoted a right-lateral rupture. Rich detail contained within the image frames is best seen at increased viewing magnification.

The P-wave front is clearly visible in frame $1(t=25 \mu s)$ and is seen approaching the measurement stations positioned at $(-55,0) \mathrm{mm}$ and $(-55,16) \mathrm{mm}$. The advancing sub-Rayleigh rupture corresponds to the fringe concentration situated just behind the S-wave front, as indicated in the figure. The annulus within the photoelastic fringe pattern, which is bounded on the interior by the S-wave front, corresponds to the near-field region, which is frequently observed during the earliest image frames. The dark fringe loops confined within this region correspond to the radial P- and S-lobes arising from the mode II (double couple) rupture nucleation process. The noted "fringe loop" corresponds the leading S-lobe while the adjacent dark looping fringes oriented at $\approx \pm 45^{\circ}$ with respect to the fault are the P-lobes. The near-field S- and P-lobes, and the annulus of the near-field, fade very rapidly and completely disappear by $t=37 \mu \mathrm{s}$.

The onset of a sub-Rayleigh-to-supershear transition is captured in frame $2(t=33 \mu \mathrm{s})$. A magnified view of the image reveals that the dark fringe loop coinciding with the S-lobe has been split with the emerging daughter crack corresponding to the white gap, between the two dark fringes. The supershear transition distance (L) appears to coincide with a point on the S-wave front, located just a few millimeters ahead of the sub-Rayleigh rupture tip. Note how the observed shift of the 
lower fringe segment to the left is consistent with the right-lateral rupture.

The first hint of the emerging shear Mach fronts appears in frame $3(t=37 \mu s)$ in the form of the shallow wedge- shaped fringe with its tip extending out beyond the S-wave front. The dark arching fringe reveals a dilatational field concentration surrounding the tip of the emerging supershear rupture.

A fully transitioned supershear rupture is visible in frame $4(t=41 \mu s)$. Well-defined shear Mach fronts are seen stemming from a supershear rupture tip, which has just reached the on-fault measurement station at $(-55,0) \mathrm{mm}$. The original sub-Rayleigh rupture is still clearly defined and seen trailing within the wake of the leading supershear rupture.

Straight and sharply defined shear Mach fronts are seen in frame $5(t=50 \mu s)$. The supershear rupture is clearly outrunning the trailing sub-Rayleigh rupture, as revealed by the increased distance between the two rupture tips. The sub-Rayleigh rupture is also still several millimeters away from the on-fault measurement station at this time. The measured Mach angle value of $\theta=36^{\circ}$ corresponds

to $V_{r} / C_{s}=1.70\left(V_{s s}=2.18 \mathrm{~mm} / \mu \mathrm{s}\right)$, which is only $3 \%$ greater than the average supershear rupture speed obtained through a frame-by-frame analysis from $t=37 \mu s \rightarrow t=55 \mu \mathrm{s}$. Finally, note how the shear Mach front has still not reached the off-fault station located at $(-55,16) \mathrm{mm}$. Particle velocity records confirm that the Mach front swept the station at $52.9 \mu \mathrm{s}$

The final image frame $(t=60 \mu s)$ captures the last moment before the supershear rupture tip reached the extreme edge of the fault. The subtle concave profile of the shear Mach fronts reveals that the supershear rupture has continued to accelerate. The sub-Rayleigh rupture and its surrounding stress field is seen to have crossed both measurement stations by this time.

\subsection{Fiber-optic heterodyne interferometers for continuous particle velocity measurements}

Polytec fiber-optic laser interferometers were first applied by (Lykotrafitis et al., 2006a) in dynamic shear impact studies, and later by (Lu et al., 2007) to the investigation of pulse-like and crack-like 
ruptures in laboratory earthquake investigations. Heterodyne interferometers enable continuous, high-bandwidth particle velocity measurement at fixed locations on the surface of a test specimen as a rupture propagates along the specimen fault. Particle velocity records obtained in this manner are entirely analogous to ground motion measurements recorded by seismograph stations on the surface of the Earth in the immediate neighborhood of an active fault.

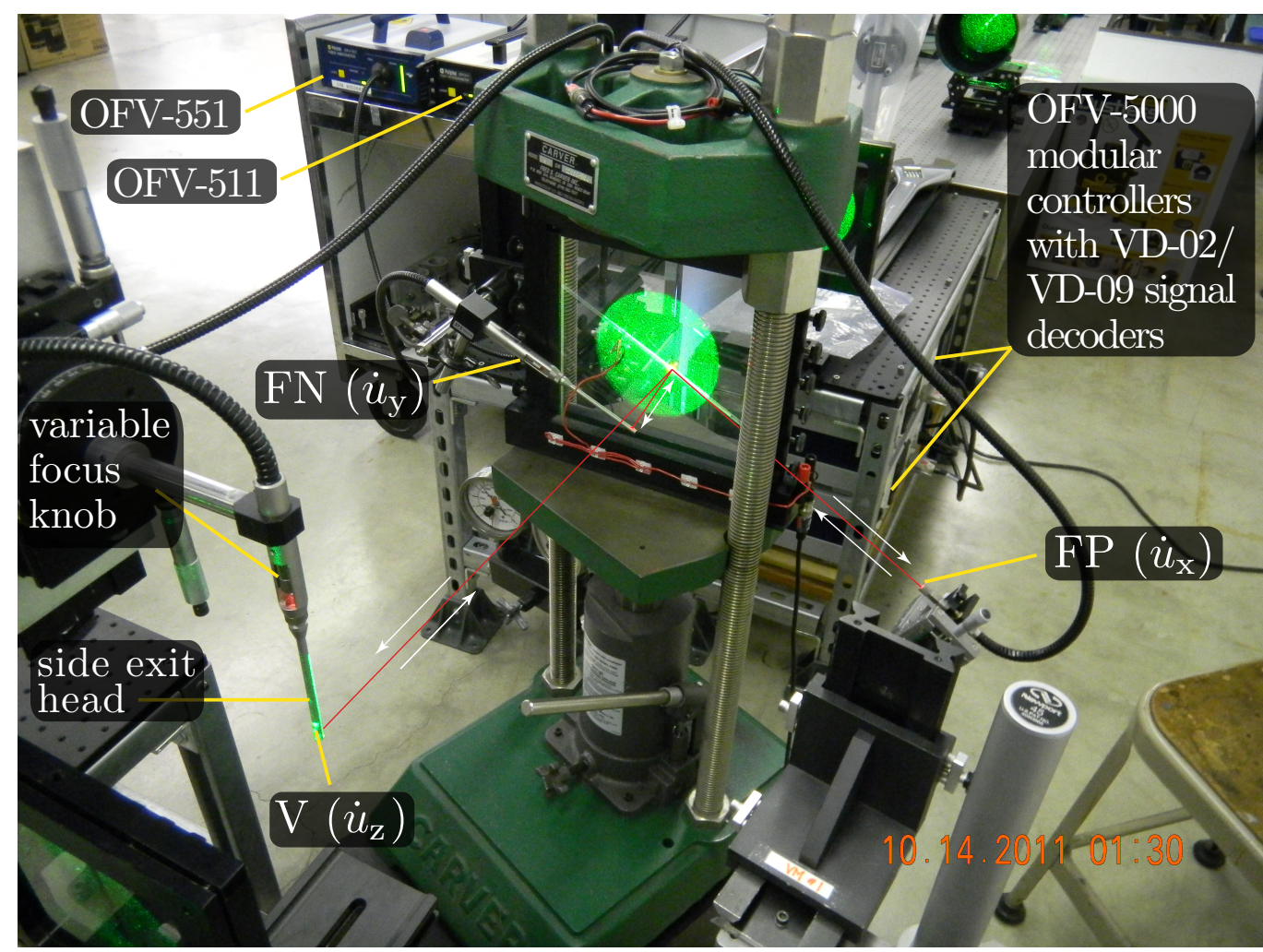

Figure 3.43: Laboratory earthquake setup utilizing three fiber-optic probes focused at a common location for simultaneous measurement of fault-parallel, fault-normal, and vertical particle velocity components

Figure 3.43 shows a laboratory earthquake setup, which utilized three Polytec fiber-optic heterodyne laser interferometers for simultaneous measurement of fault-parallel (FP), fault-normal (FN), and vertical (V) particle velocity components at an on-fault measurement station. The 3-component configuration shown here was used in experiments designed to simulate the 2002 Denali $M_{w} 7.9$ earthquake, which is discussed in Chapter 5. The red lines highlight the helium neon laser beam path from each fiber-optic probe (Polytec OFV-130-5 "spot sensor head") to the point on the specimen 
surface where the light is focused. The probes feature a variable working distance, which is precisely adjusted by a knurled focusing knob such as the one labeled in the image. Detailed specifications for the spot size of the focused beam as a function of working distance are provided by Polytec. In the case of the experiment depicted in Figure 3.43, the largest spot size corresponding to the most distant probe (vertical component) is estimated to be in the range of $150 \mu \mathrm{m}-200 \mu \mathrm{m}$.

The fault-parallel (FP) and fault-normal (FN) beams are focused at nearly grazing incidence onto the edges at the lower-right-hand-corner edge of the retro-reflective tape strip. The FP probe on the right is clamped within an X-Y "tip/tilt" positioning stage (Polytec OFV-033-TP), as shown. The FN probe is attached to a stainless steel "side exit probe" (OFV-C-102), which contains a tiny mirror inclined at $45^{\circ}$ to at the end of the stainless steel shaft. The mirror redirects the focusing beam to approach the corner of the tape strip from below. The vertical (V) probe beam is also mounted within a side exit probe, which directs the beam at nearly normal incidence onto the specimen surface. Scattered light, directed back along the incident light path, is collected by each fiber optic probe and directed back to the heterodyne interferometers (OFV-511 and OFV-551) where the laser light is optically interfered.

Figure 3.44 provides a schematic representation of the Polytec interferometer configuration. A fiber-optic probe focuses a HeNe laser beam to a point on the test specimen surface and collects scattered light which is directed back to the probe. The collected light is transmitted by optical fiber to the OFV-511 (or OFV-551) interferometers where it is interfered with a reference beam derived from the same HeNe laser. The two light beams are optically interfered in a Mach-Zehnder arrangement as shown to produce a modulating light signal, which a photodetector converts into a time-varying electrical signal.

\subsubsection{Principle of heterodyne interferometry}

Optical heterodyning is achieved through a frequency shift of the reference beam. In the case of the Polytec OFV-511, a Bragg cell (acousto-optic modulator) is used to modulate the reference beam at a constant reference frequency $f_{b}=40 \mathrm{MHz}$. The optical heterodyning technique and the manner 


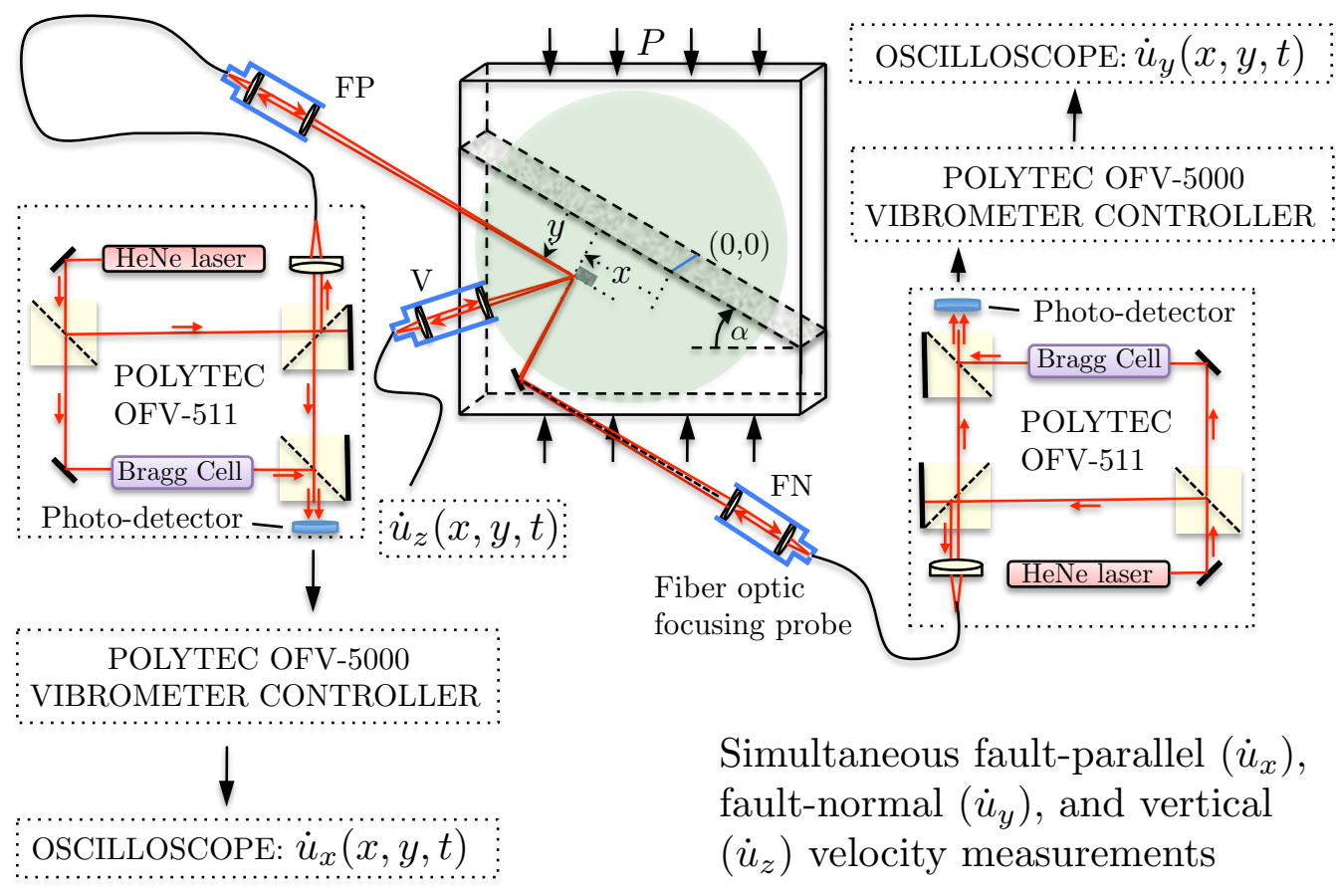

Figure 3.44: Fiber-optic heterodyne laser interferometer configuration

in which particle velocity is extracted from the heterodyned signal is best understood by considering the two-beam time-averaged interference expression given by

$$
I(t)=I_{b}+I_{m} \cos \left[2 \pi\left(f_{b} t-\frac{2 u_{i}(t)}{\lambda} \cos \alpha\right)+\left(\phi_{r}-\phi_{s}\right)\right]
$$

where $I_{b}$ is a constant background intensity, $I_{m}$ represents the amplitude of the modulating signal, $\lambda$ represents the HeNe laser wavelength $(\lambda=632.8 \mathrm{~nm}), u_{i}(t)$ corresponds to the surface displacement component of interest where $i=x, y, z, \phi_{r}-\phi_{s}$ is the difference between two (constant) arbitrary phase terms carried by the reference and specimen beams, and $\alpha$ represents the angle between the optic axis as defined by the focused laser beam and a unit vector parallel to the particle motion component of interest. The $\cos \alpha$ factor can theoretically be used to correct for the effect of nonnormal incidence. However, in most instances it is possible to accurately align the fiber optic probes within $5-10^{\circ}$ of normal incidence, in which case $\cos \alpha \rightarrow 1$. 
The intensity expression can then be recast as follows

$$
I(t)=I_{b}+I_{m} \cos \left[2 \pi\left(f_{b} t-\frac{2 u_{i}(t)}{\lambda \Delta t} \cos \alpha \Delta t\right)+\left(\phi_{r}-\phi_{s}\right)\right]
$$

where $\Delta t$ represents a discrete time interval over which surface displacement data is acquired. The accumulated phase change $\Delta \Phi$ in the interference signal occurring between time $\mathrm{t}$ and time $\mathrm{t}+\Delta t$ is then given by

$$
\Phi(t+\Delta t)-\Phi(t)=2 \pi\left[f_{b}-\frac{2}{\lambda}\left(\frac{u_{i}(t+\Delta t)-u_{i}(t)}{\Delta t}\right) \cos \alpha\right] \Delta t
$$

Taking the limit as $\Delta t \rightarrow 0$ yields

$$
\omega_{s}=\lim _{\Delta t \rightarrow 0} \frac{\Delta \Phi(t+\Delta t)-\Phi(t)}{\Delta t}=2 \pi\left(f_{b}-\frac{2 \dot{u}_{i}(t)}{\lambda} \cos \alpha\right)
$$

where $\omega_{s}$ represents the instantaneous angular frequency of the interference signal and $\dot{u}_{i}$ corresponds to the particle velocity. The resulting signal frequency $f_{s}$ is therefore given by

$$
f_{s}=f_{b}+f_{d}
$$

where

$$
f_{d}=-\frac{2 \dot{u}_{i}(t)}{\lambda} \cos \alpha
$$

represents the Doppler frequency shift induced by the particle velocity component. As the specimen surface displaces, the resulting signal frequency $f_{s}$ is either perturbed above or below the reference 
frequency $f_{b}$, depending upon the sign of $\dot{u}_{i}$. Frequency demodulation of the heterodyne interferometer signal frequency leads to a direct and unambiguous measure of particle velocity when processing the resulting waveforms. Particle motion directed away from the interferometer $\left(\dot{u}_{i}>0\right)$ results in a decreased signal frequency below the reference frequency $\left(f_{s}<f_{b}\right)$. Conversely, particle motion directed toward the interferometer $\left(\dot{u}_{i}<0\right)$ results in an increased signal frequency, i.e., $f_{s}>f_{b}$.

Each output signal from the photodetector in the OFV-511 and OFV-551 interferometer assemblies is sent to an OFV-5000 modular controller where the frequency content of the signals is analyzed in real time and converted into particle velocity signals. The two original OFV-500 modular controllers feature $1.5 \mathrm{MHz}$ bandwidth VD-02 velocity output decoders capable of tracking particle velocities up to $10 \mathrm{~m} / \mathrm{s}$ with a resolution limit of $0.15 \mu \mathrm{m} / \mathrm{s}$. The newest Polytec system obtained in 2010 features a newer VD-09 decoder with a $2.5 \mathrm{MHz}$ bandwidth and theoretical resolution of $0.02 \mu \mathrm{m} / \mathrm{s}$. Continuous particle velocity records in the form of an analog voltage signal, are output by the controllers and typically sampled at $2.5 \mathrm{Gs} / \mathrm{s}(0.4 \mathrm{~ns} /$ data point $)$ by a Tektronix DPO3034, $300 \mathrm{MHz}$ digital oscilloscope. The acquired signals are then converted to particle velocity records through a calibrated scaling factor $(1 \mathrm{~V}=1 \mathrm{~m} / \mathrm{s})$. Note that the $1.5 \mathrm{MHz}$ and $2.5 \mathrm{MHz}$ bandwidth specifications for the VD-02 and VD-09 deciders apply to the analog bandwidth of the final processed particle velocity record as captured by the oscilloscope. A bandwidth limit of $1.5 \mathrm{MHz}$ translates into a minimum resolvable velocity signal rise time of $\tau=0.233 \mu s$, while a bandwidth limit of $2.5 \mathrm{MHz}$ translates into a minimum resolvable velocity signal rise time of $\tau=0.140 \mu \mathrm{s}$ using $\left.t_{\text {rise }}=0.35 / B W\right)$. The VD-02 and VD-09 signal decoders are clearly very well suited for capturing particle velocity records generated in laboratory earthquake experiments.

Finally, a repeatable time delay has been observed between signals produced by the newer generation controller and VD-09 output decoder and the older VD-02-based controllers. All signals produced by the VD-09 output decoder are observed to arrive $5.23 \mu \mathrm{s}$ later. Polytec has also confirmed this value. The noted time delay is certainly significant in laboratory earthquake experiments and must be accounted for in the analysis and plots of particle velocity signals derived from the newer Polytec interferometer system. 


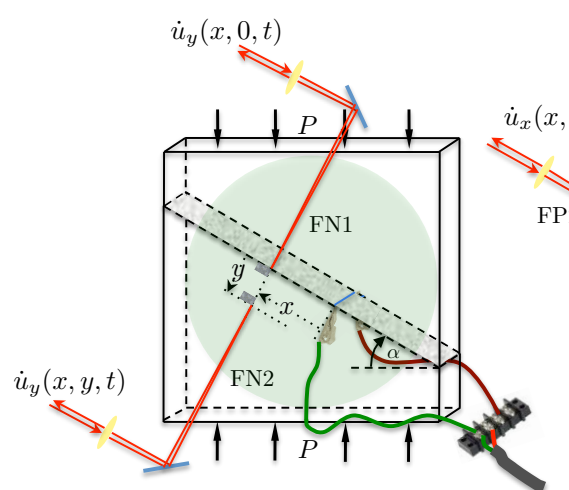

(a) Paired fault normal particle velocity measurements.

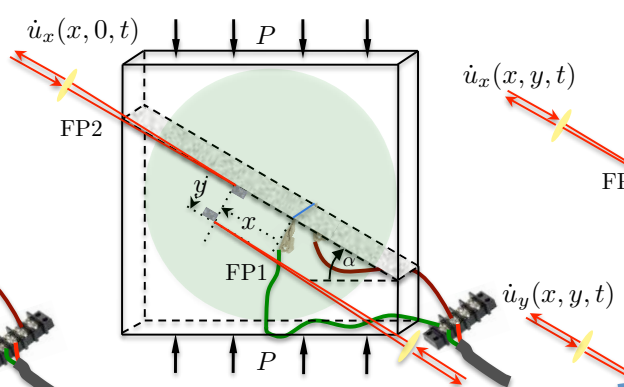

(b) Paired fault parallel $\quad \dot{u}_{x}(x, y, t)$ particle velocity measurements.

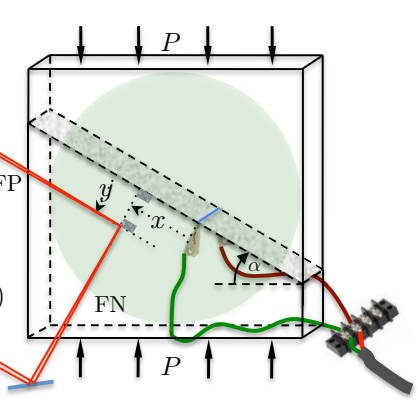

(c) Combined fault parallel and fault normal particle velocity measurements.

Figure 3.45: Fiber-optic probe configurations adopted in laboratory earthquake experiments

\subsubsection{Particle velocity measurement configurations adopted in labora- tory earthquake experiments}

Three other particle velocity measurement schemes commonly adopted in laboratory earthquake investigations are depicted in Figure 3.45. The light green circle in each figure represents the expanded laser beam, used for photoelastic imaging, which is simultaneously transmitted through the H-100 test specimen in order to obtain synchronized whole-field images of the event. Figure 3.45a depicts a paired fault-normal particle velocity measurement scheme. The fiber-optic probes are oriented such that one probe beam is focused at an on-fault station while the other probe beam is focused at an off-fault station as shown. This arrangement was used for investigating the decay of $\dot{u}_{y}(t)$ as a function of FN distance in sub-Rayleigh experiments. Figure 3.45b depicts a paired fault-parallel particle velocity measurement scheme. The fiber-optic probes are oriented such that one probe beam is focused at an on-fault station while the other probe beam is focused at an offfault station, as shown. The (FP1, FP2) scheme has been applied in supershear ground motion studies to investigate attenuation of supershear ground motion and the partitioning of $\dot{u}_{x}(t)$ as a function of rupture speed. The third configuration depicted in Figure 3.45c, which is a two-beam variant of the configuration in Figure 3.43 (also seen earlier in Figure 3.2), enables the simultaneous measurement of the fault- normal and fault-parallel particle velocity components at a single location.

This arrangement was used to investigate the ratio of the FP to the FN component, and vice versa, 

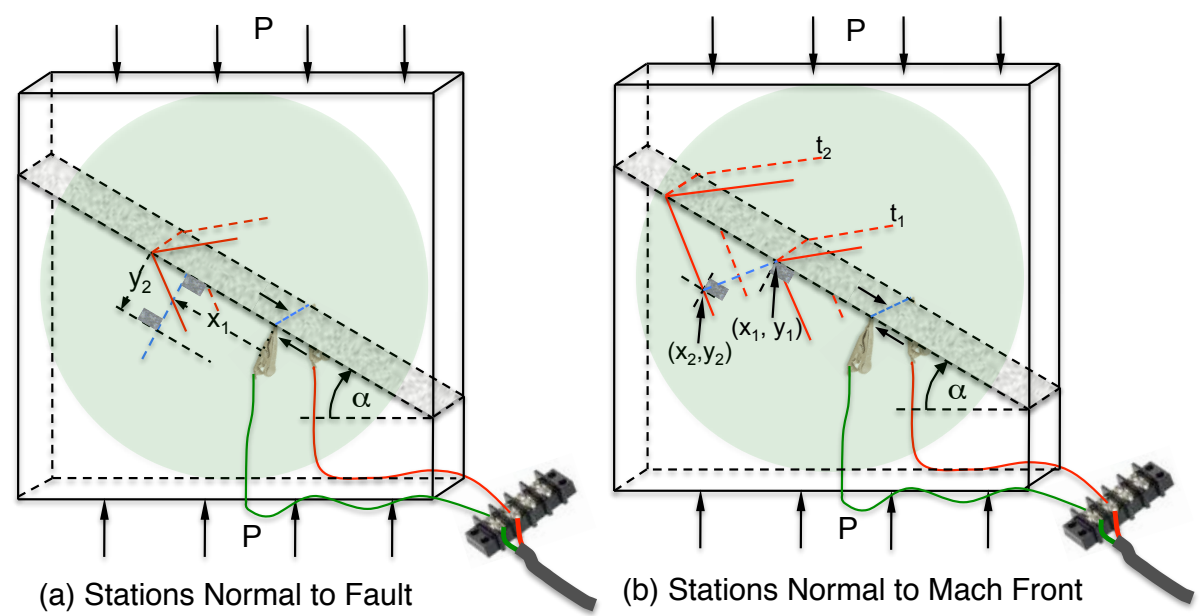

Figure 3.46: Particle velocity measurement configurations adopted in laboratory earthquake experiments

in both sub-Rayleigh and supershear experiments. Numerous other combinations of paired particle velocity measurements may also be configured depending upon the design and objectives of a given experiment.

Figure 3.46 depicts two velocity measurement station configurations, which may be adopted when working with either of the paired velocity measurement configurations depicted in Figure 3.45a and Figure 3.45b. Each configuration provides a unique approach for investigating the attenuation of supershear ground motion as a function of the FN distance $\left(y_{2}\right)$. The station configuration depicted in Figure 3.46a corresponds to the most commonly used configuration whereby the measurement station locations are aligned along a line drawn normal to the fault. The second configuration in Figure $3.46 \mathrm{~b}$ depicts a pair of measurement stations oriented along a line drawn normal to the shear Mach front. The spirit of the latter configuration centers around the notion that the velocity jump induced by the shear Mach front at the off-fault station was propagated to that point by the shear wavelets emitted from the supershear rupture tip as it swept the on-fault station.

\subsubsection{Non-dimensional analysis of particle velocity fields}

It is experimentally observed that the magnitude of particle velocities obtained in typical laboratory earthquake experiments is comparable to the magnitudes obtained from near-field natural earthquake 
velocity records. A simple non-dimensional argument is presented to explain this observation.

Based on the steady-state slip-pulse models of (Rice et al., 2005) and (Dunham and Archuleta, 2005), the proper non-dimensionalized representation of the velocity field in a medium hosting such a slip pulse is given by

$$
\frac{\mu \dot{u}}{\left(\tau_{p}-\tau_{r}\right) C_{s}} \equiv C \dot{u}=F\left(V_{r} / C_{s}, R / L\right)
$$

where $\mu$ is the shear modulus, $\tau_{p}-\tau_{r}$ is the strength drop, $\dot{u}$ is the particle velocity, $R / L$ is the ratio of the size of the process zone to slip-pulse length and $V_{r} / C_{s}$ is the rupture velocity scaled by the shear wave speed. This universal feature applies to all elastodynamic steady-state models, independent of frictional or cohesive law used (Samudrala et al., 2002a).

A typical value for the shear modulus of crustal rock is $\mu_{\text {rock }}=30 \mathrm{GPa}$, while the corresponding value for $\mathrm{H}-100$ is $\mu_{H-100}=1.96 G P a$. At mid-seismogenic depths (around $7 k m$ ) $\sigma$ is estimated to be on the order of $100 M P a$. If a Coulomb-like friction relation is assumed ( $\tau=f \sigma$, where $f$ is the friction coefficient and $\sigma$ is the normal stress), then assuming $f_{p}=0.6$ and $f_{r}=0.2$, a strength drop on the order of $40 \mathrm{MPa}$ is obtained for rock material. This then yields the value $C$ for rock to be about 0.25 . A typical observed strength drop in laboratory earthquake experiments is about $7 \mathrm{MPa}$ (assuming $\sigma=20 \mathrm{MPa}$ ), giving a value of $C$ close to 0.22 , assuming $R / L$ is similar for rupture in $\mathrm{H}-100$ and crustal rock. It stands to reason therefore, that the particle velocity records obtained in laboratory earthquake experiments should be comparable to the magnitude of ground motion velocities measured in natural earthquakes. 


\section{Chapter 4}

\section{Laboratory earthquake investigations of near-source ground motion produced by sub-Rayleigh and supershear fault ruptures}

\subsection{Introduction}

Steady-state rupture models predict that in the stable intersonic (supershear) regime $\left(\sqrt{2} C s<V_{r}<\right.$ $C_{p}$ ), the ground motion carried by shear Mach front is dominated by the fault-parallel component, whereas in the unstable intersonic rupture speed regime $\left(C s<V_{r}<\sqrt{2} C_{p}\right)$ the opposite holds true (Dunham and Archuleta, 2004; Aagaard and Heaton, 2004). In the sub-Rayleigh rupture speed regime $\left(V_{r}<C_{R}\right)$, where $C_{R}$ represents the Rayleigh wave speed, the fault-normal component of particle velocity is predicted to dominate over the fault-parallel component (Aagaard and Heaton, 2004).

The sense of particle motion along a shear Mach front was reasoned from rudimentary principles in Chapter 1 by considering the source spectrum of a simple shear dislocation. Sub-Rayleigh and supershear particle velocity signatures were then more-rigorously analyzed in Section 2.2 by plotting the particle velocity fields for the well-known 2D plane strain elastodynamic solution for a propagating shear (mode II) crack in a linear elastic half-space (Freund, 1979, 1990). Steady-state cohesive 
zone models were then examined, culminating with the general $2 \mathrm{D}$ steady-state solution obtained by (Dunham and Archuleta, 2004) for a supershear rupture pulse of finite length L, propagating at a rupture speed $V_{r}$ along a frictional fault with a prescribed shear traction $\tau(x)$ (Section 2.3.1). New theoretical findings on radiation partitioning within the supershear slip zone were gleaned from this general treatment and presented at the conclusion of Section 2.3.1. Ground motion signatures as presented by (Dunham and Archuleta, 2004) along with new theoretical insights which have stemmed from this work form the basis of the experimental investigations presented in this chapter.

The discussion on ground motion in Section 2.4 concluded by noting the limitations of the 2D steady-state models, most notably the inability of these models to predict the supershear transition and the subsequent emergence of a secondary sub-Rayleigh rupture, which trails in the wake of the leading rupture (Section 2.4). Problems of this type are better addressed by a more-general class of self-similar analytical solutions for supershear ruptures (for, e.g., Burridge, 1973; Broberg, 1994), which incorporate unsteady cohesive zone models. Experimental investigations presented in this chapter confirm the presence of the trailing Rayleigh rupture and examine the ground motion induced by this rupture following the passage of the leading supershear rupture.

Theoretical and numerical investigations of supershear ruptures in 2D (Dunham and Archuleta, 2004; Bhat et al., 2007) and in 3D (Dunham and Bhat, 2008) also predict that ground motion due to the passage of the shear Mach front is virtually unattenuated at large distances from the fault. In the 2D steady-state supershear rupture model, the shear Mach front carries ground motion unattenuated, out to infinity. In the 3D case, the Mach cone eventually succumbs to geometric dispersion whereas the field strength is predicted to decay as the inverse square-root of the fault-normal distance (BenMenahem and Singh, 1987). (Bhat et al., 2007) reasoned that the fields carried by the shear Mach front should at least be sustained out to distances comparable to the depth of the seismogenic zone. (Dunham and Bhat, 2008) followed with a numerical 3D model, which demonstrated the effects of geometric dispersion while also confirming the earlier estimation by (Bhat et al., 2007). Theoretical estimates of supershear ground motion attenuation are also supported by field observations such as anomalous ground cracking observed several kilometers from the main fault following the 2001 


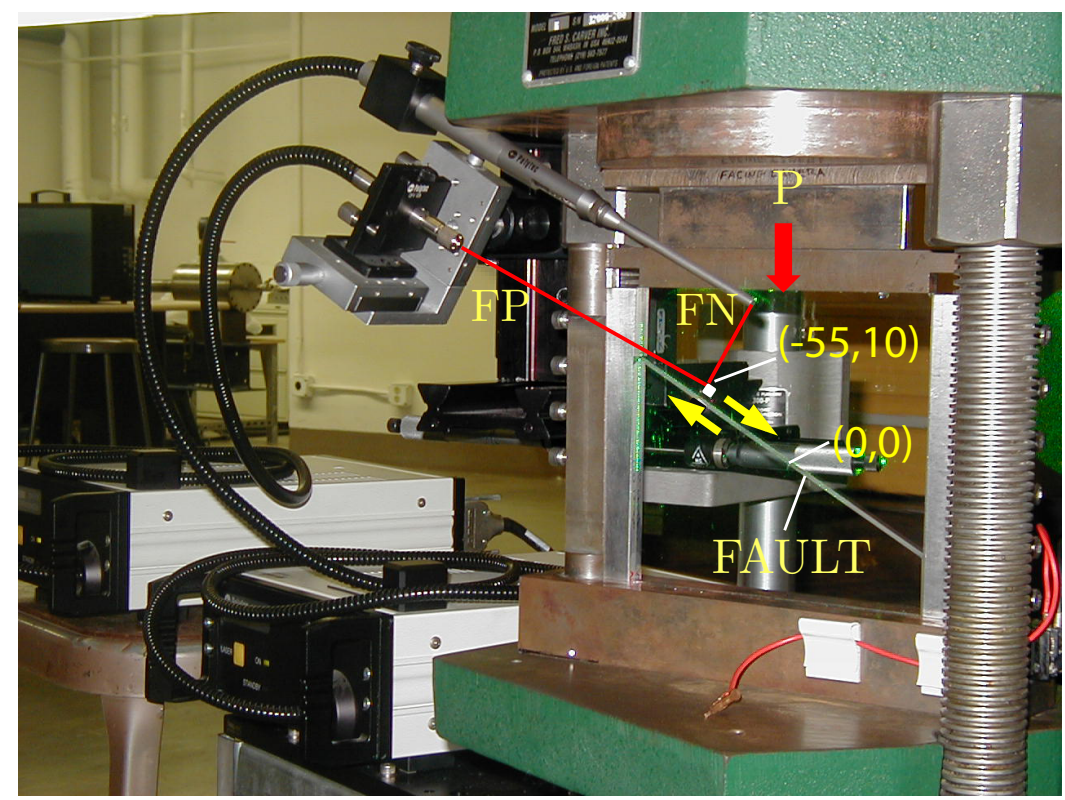

Figure 4.1: Laboratory earthquake experimental configuration used for the simultaneous measurement of fault-normal and fault-parallel particle velocity components at $(-55,10) \mathrm{mm}$

Kokoxili (Kunlun) $M_{w} 7.8$ event in Tibet (Bhat et al., 2007) and aftershocks clustered in a region away from the fault at distances comparable to the depth of the seismogenic zone following the 1999 moment magnitude $M_{w} 7.6$ Izmit earthquake (Bouchon and Karabulut, 2008). Laboratory earthquake investigations presented in this chapter address the issue of ground motion attenuation in supershear earthquakes. Supershear and sub-Rayleigh ground motion measurements obtained at various distances from the fault are examined, contrasted, and compared to theoretical predictions.

\subsection{Identification of sub-Rayleigh and supershear ground mo-}

\section{tion signatures through a frame-by-frame comparison of}

\section{two laboratory earthquake experiments}

\subsubsection{Design of experiment}

A series of laboratory earthquake experiments were conducted in order to identify and contrast the defining ground motion signatures of sub-Rayleigh and supershear fault ruptures. The exper- 
iments were facilitated by fiber-optic heterodyne laser interferometers, which provide continuous, high-bandwidth particle velocity ("ground motion") records at fixed locations on the surface of the test specimen (Section 3.14). Spatially resolved, full field images of the advancing rupture tip and the surrounding maximum shear stress field were simultaneously obtained through the use of photoelasticity and high-speed photography (Section 3.11). The experiments presented in this section contrast the results obtained from two independent laboratory earthquake experiments, one of which yielded a sub-Rayleigh rupture and the other, which resulted in a supershear rupture. In the case of the sub-Rayleigh rupture experiment (exp 09-052), a static compressive load of $P=12 \mathrm{MPa}$ ensured that the propagating rupture remained within the sub-Rayleigh rupture regime as it swept the particle velocity measurement station located at $(-55,10) \mathrm{mm}$. In the case of the supershear experiment (exp 09-047), the static-compressive load was doubled to $P=24 M P a$ in order to produce a stable supershear rupture that swept a particle velocity measurement station, also located at $(-55,10) \mathrm{mm}$.

The laboratory earthquake setup used in this series of ground motion investigations is depicted in Figure 4.1. Experiments were conducted on $6 " \times 6 " \times 3 / 8 " \mathrm{H}-100$ specimen assemblies, which featured a uniform frictional interface and a specimen fault angle of $\alpha=-29^{\circ}$ that was naturally conducive to right-lateral, crack-like ruptures (Lu, 2009). The fiber-optic interferometer probes are arranged in the (FN,FP) configuration for the simultaneous measurement of $\dot{u}_{x}(t)$ and $\dot{u}_{y}(t)$ at a fixed point on the surface of the specimen. The red lines highlight the optical path of the FP and FN probe beams, which were directed at nearly grazing incidence to the surface of the test specimen and focused onto the upper-left corner of the retro-reflective tape. The short distance between the probes and the measurement station ensured that the focused spot size was $<100 \mu \mathrm{m}$ for both beams. Scattered light directed along each respective direction was collected by the fiber-optic probes and directed to the Polytec model OFV-511 heterodyne interferometers, which are seen in the left side of the image Section 3.14. The scattered green light on the left Aluminum side support was produced by the expanded laser beam used for photoelastic high-speed imaging (Section 3.11). The incident laser beam was intentionally offset to transmit through the left side of the test specimen (viewed in 

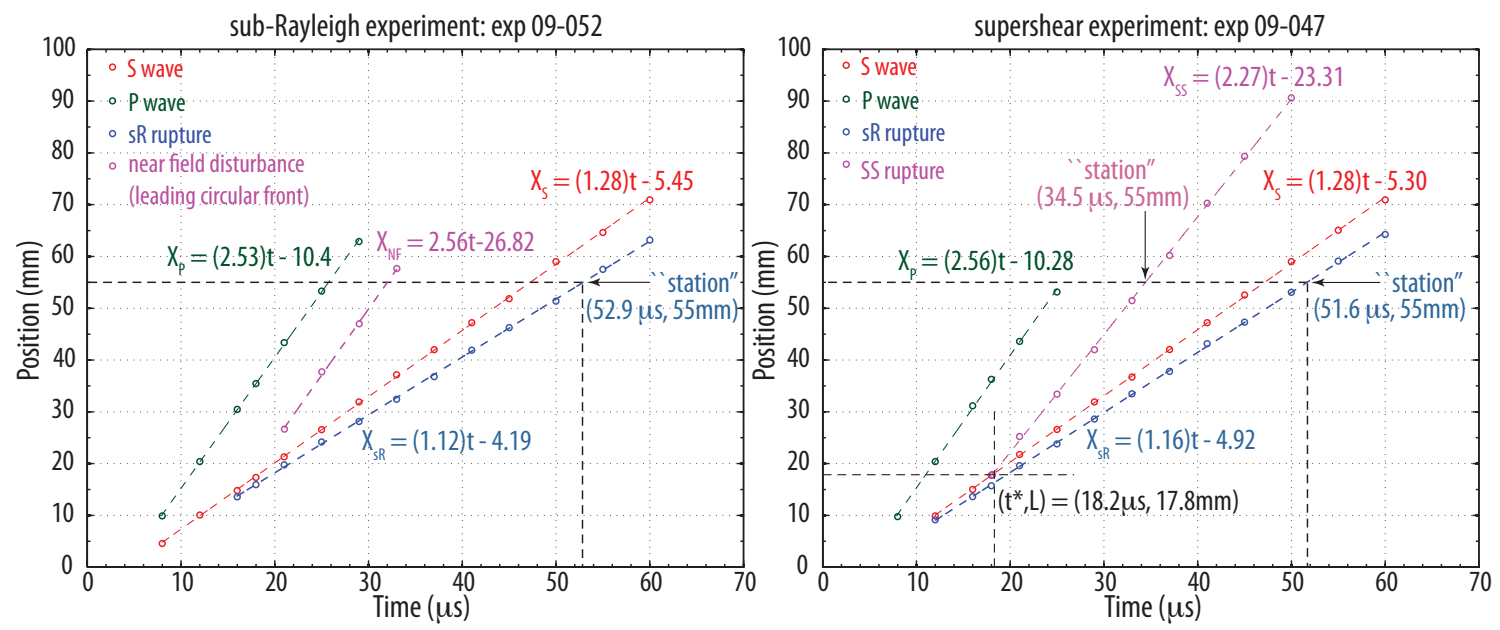

Figure 4.2: Displacement-time plots corresponding to the sub-Rayleigh and supershear experiments. Dots represent data points obtained from photoelastic image analysis and dashed lines represent a best-fit line passed through the points.

the right-lateral configuration) in order to ensure that the advancing rupture was captured in the final image frames as it swept beyond the measurement station.

A static compressive stress $P$ was applied to the specimen assembly with the aid of the manually operated hydraulic press (Carver@ model 3925) (Figure 3.7). The test specimen was placed within the mounting frame and initially constrained on all four sides, as depicted in Figure 4.1, as it was loaded to an initial static-compressive load of $P_{i}=3 M P a$. The constraint along the left side of the test specimen was then released by loosening the screws in the left pillar of the mounting frame and then disengaging the Aluminum bar from the test specimen. Close attention was paid to detect whether the plates slipped during this step. If any slip was detected, the specimen was removed from the mounting frame and the fault surfaces were either cleaned or reconditioned before attempting to reload the specimen assembly. If the plates held together then the right-side-constraint was released in a similar way. Upon removing the side constraints, the specimen assembly was then slowly loaded up to the desired static compressive stress. The experiments were then conducted using the rupture nucleation scheme as described in Section 3.3. 

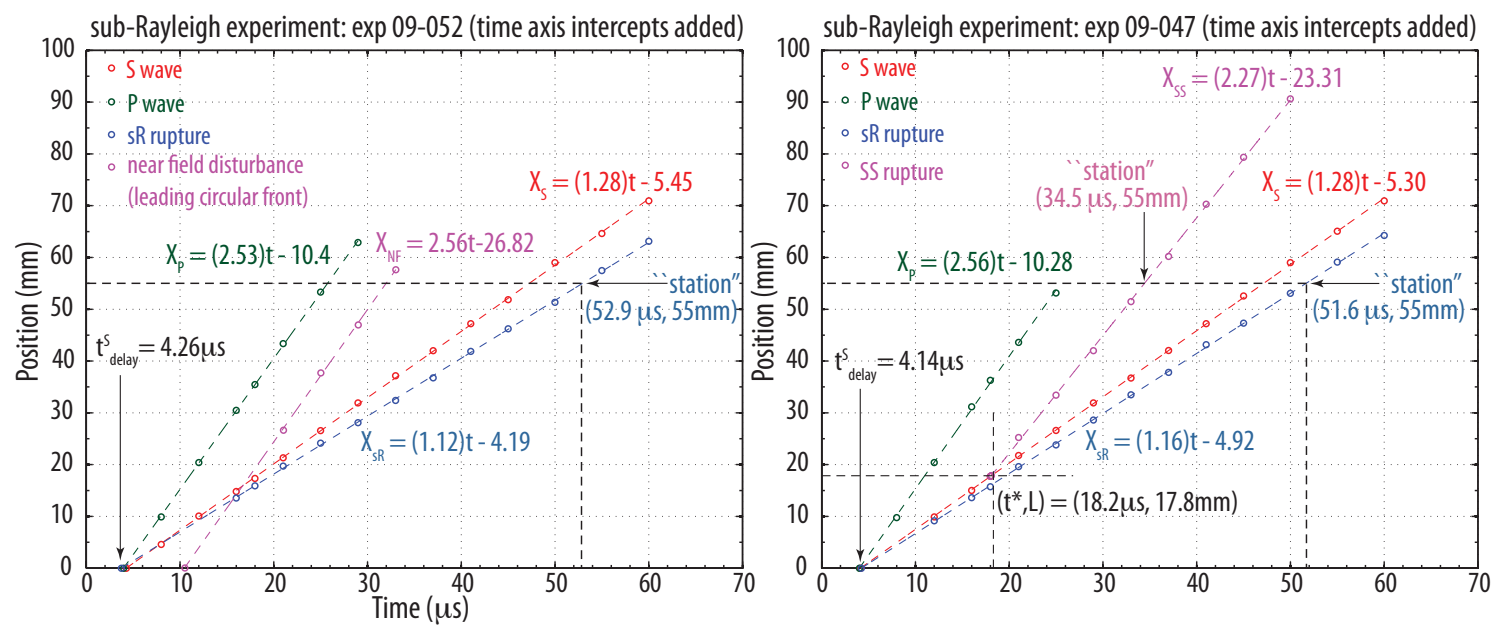

Figure 4.3: Displacement-time plots corresponding to the sub-Rayleigh and supershear experiments shown with the best-fit lines extrapolated to their respective time axis intercepts. The supershear best-fit line was extrapolated to the point where it intersects the sub-Rayleigh best-fit line.

\subsubsection{Results, analysis, and interpretation of particle velocity records and photoelastic images}

Figure 4.2 contains distance-time plots corresponding to each of the experiments. The data points upon which these curves are based were obtained through a frame-by-frame analysis of each photoelastic image sequence. The colored dots represent the actual data points obtained from the photoelastic images while the dashed lines represent a best linear fit to the data. The linear equation describing the best-fit line is displayed beside each respective curve. The excellent linear fit in each case reveals how steady the rupture speeds remained throughout the duration of the experiment. The slope of each curve naturally provides an estimate of the average speed in each case. Note that the same curves are also presented in Section 3.11.2 where they were included as an example to describe the data analysis methods used for the determination of elastic wave speeds and rupture speeds. The curves are re-presented here with one additional curve added in the sub-Rayleigh distance-time plots corresponding to a near-field disturbance, which was observed and characterized in the experiments.

Figure 4.3 displays the same identical plots with some of the best-fit lines extrapolated to their respective time axis intercepts. Note how the S-wave, P-wave, and sub-Rayleigh rupture curves 
extrapolate back to a set of closely spaced points on the time axis. The S-wave time axis intercept $\left(t_{\text {delay }}^{s}\right)$, which is displayed in each of the plots represents an estimate for the delay time between the instrumentation trigger and the emergence of the very first stress waves resulting from the ablation of the $\mathrm{NiCr}$ wire. The $\mathrm{S}$-wave curve intercept is considered to provide a more-reliable estimate of the nucleation delay time since it is based upon a larger number of data points and also because the S-wave front generally appears sharper than the $\mathrm{P}$-wave front in the images and is thus easier to identify.

Estimates for the arrival time of each rupture tip at the measurement station are also determined from the best-fit lines as indicated in the figure. Strictly speaking, the arrival time estimates correspond to the time taken by the rupture tips to reach the point $(-55,0) \mathrm{mm}$. Note that the distance is also taken to be positive in the plots for the sake of convenience even though the station was actually located at $(-55,10) \mathrm{mm}$. The supershear transition distance and time, labeled $\left(t^{*}, L\right)$ in the right-hand plot (exp 09-047), was estimated from the intersection of the best-fit lines for the supershear and S-wave plots. Additional details regarding the image analysis and the measurement procedures used to produce each of these curves is contained in Section 3.11.2.

A frame-by-frame discussion and interpretation of the sub-Rayleigh and supershear experiments is now presented. Photoelastic images obtained from the two experiments are contrasted side-byside, as depicted in Figure 4.4. The red and green arrows, superimposed on each image frame depict the orientation of the FP and FN probe beams used in these experiments. Note that the probe beam orientation in Figure 4.4 differs from the configuration depicted in Figure 4.1, whereby the FP and FN beams are now focused onto the lower-left corner of the retroreflective strip with the FN beam approaching the measurement station from below. Each photoelastic image frame is accompanied by synchronized particle velocity records, which are plotted out to $4 \mu s$ beyond the posted image frame time. The color coding of the arrows representing the probe beams are matched to the color of the corresponding particle velocity traces depicted below the image frames. A black triangle on the time axis of each particle velocity plot marks the time at which the corresponding image frame was acquired relative to the instrumentation trigger. 


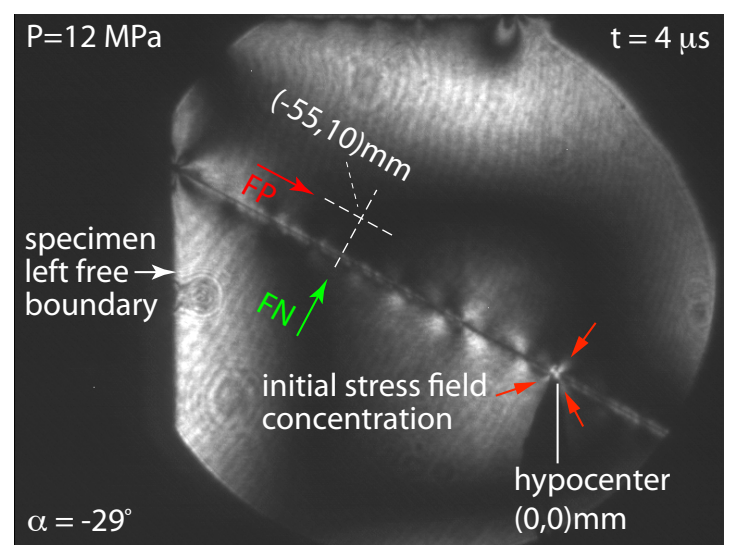

sub-Rayleigh experiment
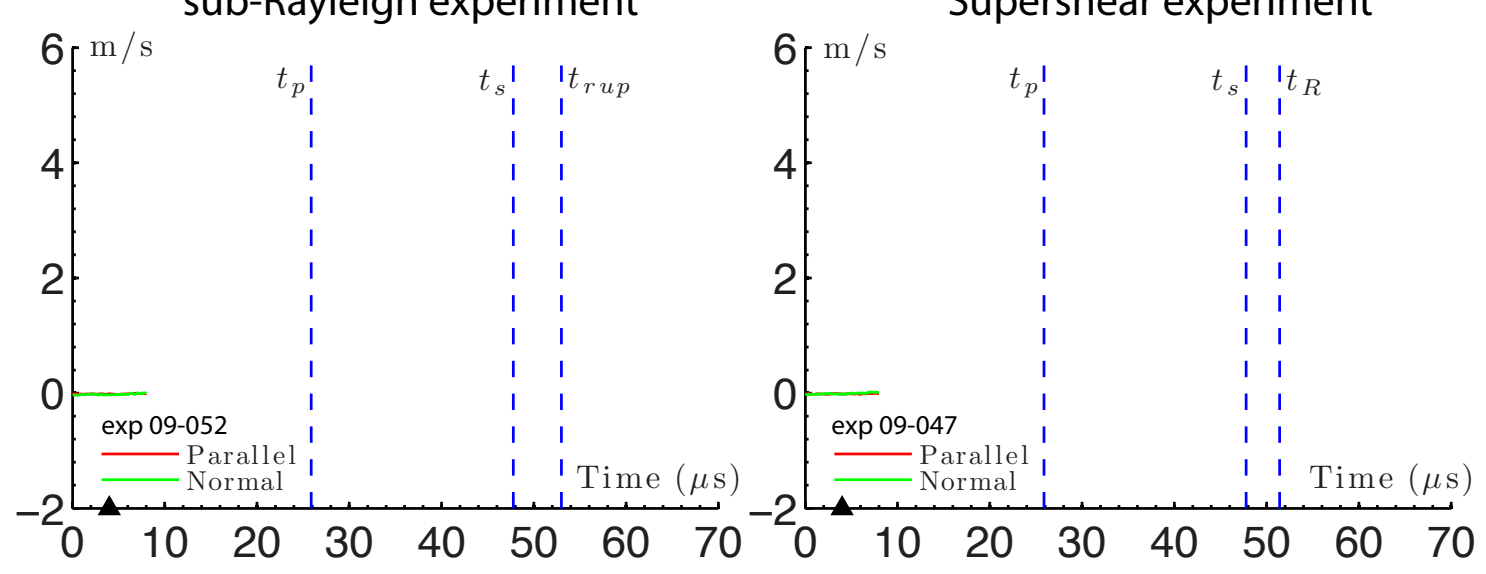

Figure 4.4: Contrast between sub-Rayleigh and supershear experiments: $(-55,10) \mathrm{mm}$ at $t=4 \mu \mathrm{s}$

The high-speed photoelastic image frames depicted in Figure 4.4 were acquired at $t=4 \mu s$ relative to the instrumentation trigger provided by the discharge circuit. A faint flash of light arising from the ablation of the $\mathrm{NiCr}$ wire filament is visible within both image frames at this time. The absence of any dynamic radiation pattern is consistent with the observed nucleation delay times $\left(t_{\text {delay }}^{s}\right)$. The actual time in the case of the sub-Rayleigh experiment is $t=-0.26 \mu s$, when corrected for the measured S-wave time delay of $t_{\text {delay }}^{s}=4.26 \mu s$. The corrected time in the case of the supershear experiment image frame corresponds to $t=-0.14 \mu \mathrm{s}$ based upon the measured S-wave time delay of $t=4.14 \mu \mathrm{s}$ in that experiment. The initial faint flash of light obviously precedes the mechanical rupture nucleation process.

The photoelastic fringe patterns provide a full-field view of the initial static (maximum shear) stress field in each experiment. The circular field of view appears truncated on the left side within 


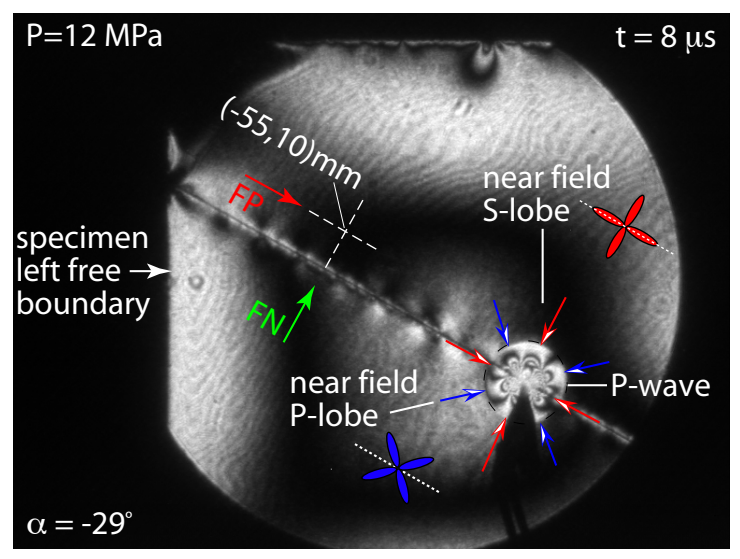

sub-Rayleigh experiment

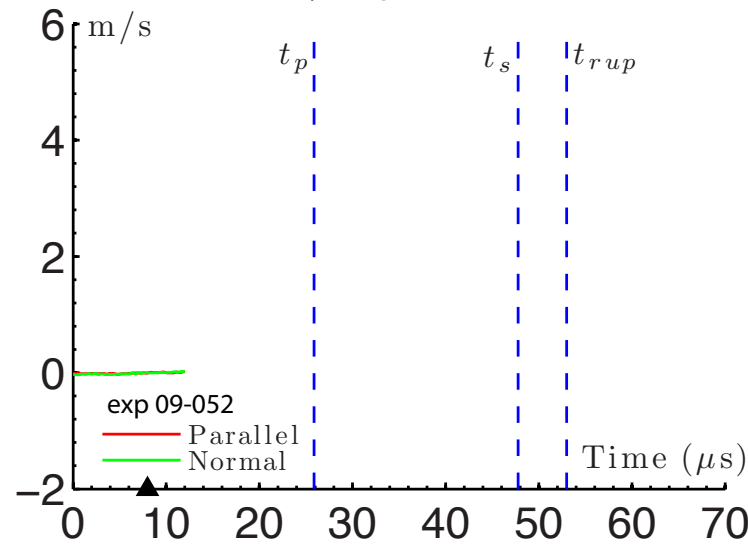

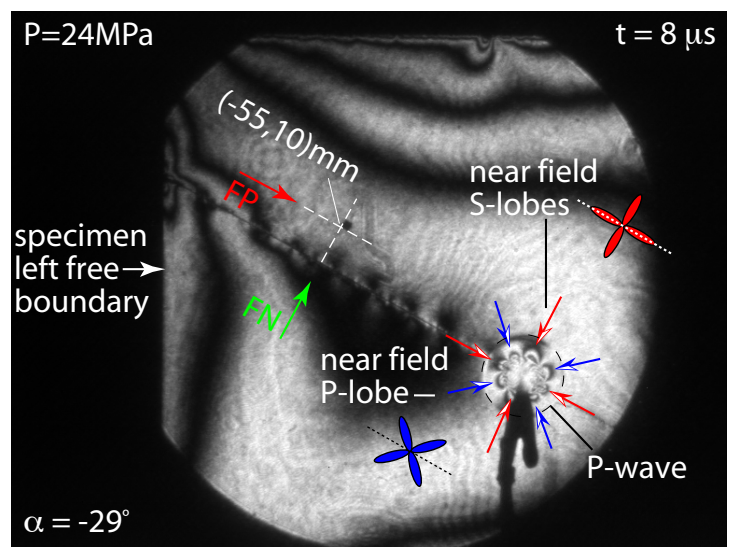

Supershear experiment

Figure 4.5: Contrast between sub-Rayleigh and supershear experiments: $(-55,10) \mathrm{mm}$ at $t=8 \mu \mathrm{s}$

each image frame due to the fact that the expanded laser beam was intentionally offset to favor that side of the specimen. The primary region of interest, located between the rupture nucleation site at $(0,0) \mathrm{mm}$ and the measurement station at $(-55,10) \mathrm{mm}$, is well-centered within each FOV. Both images display a fairly symmetric and well-centered fringe pattern bearing a somewhat "squarelike" profile, which results from the frictional constraint imposed by the top and bottom edges of the specimen mounting frame. The symmetry exhibited by the fringe patterns in each case is indicative of a properly seated specimen and a uniformly distributed static-compressive load across the region of interest. Note as well how the fringe pattern in the supershear experiment image frame exhibits very nearly $2 \times$ the number of photoelastic fringes (counted along the vertical axis from the center to top edge in each image), as it should in response to twice the applied load. Both image frames also reveal 3 faint fringe lobes, highlighted by the red arrows in Figure 4.4, which represent the 
local (maximum shear) stress field concentration surrounding the statically loaded cylindrical wire channel. These fringes are unrelated to the rupture nucleation process and are routinely observed during set up whenever the specimen is under an applied static-compressive load.

The dashed blue vertical lines labeled $t_{p}$ and $t_{s}$ in the particle velocity plots mark the anticipated arrival times of the $\mathrm{P}$-wave and S-wave fronts at the measurement station. The elastic wave arrival times at the measurement station are estimated according to $t j=\sqrt{(-55)^{2}+(10)^{2}} / C_{j}+t_{\text {delay }}^{s}$, where $\mathrm{j}=\mathrm{s}, \mathrm{p}$ designate the P-and S- elastic wave fronts. Note that one can either choose to use the wave speed estimates from the slope of the best-fit lines or from the nominal statistically averaged values (Section 3.11.2). The same applies for the nucleation delay time $t_{\text {delay }}^{s}$. The difference is expected to be very small but may be significant in some rare occasions depending upon the details of the experiment and the degree of precision required. The statistically averaged wave speed estimates $C_{p}=2.59-\mathrm{mm} / \mu \mathrm{s}$ and $C_{s}=1.284 \mathrm{~mm} / \mu \mathrm{s}$ were used to set the estimated arrival times displayed in the figures. In the case of the sub-Rayleigh experiment, estimated arrival times of $t_{p}=25.8 \mu \mathrm{s}$ and $t_{s}=47.9 \mu \mathrm{s}$ are obtained for the P- and S-wave fronts, respectively. Likewise, the values $t_{p}=25.7 \mu s$ and $t_{s}=47.8 \mu s$, are determined for the estimated arrival times of the Pand S-wave fronts in the supershear experiment. The dashed blue vertical lines labeled $t_{r u p}$ and $t_{R}$ in both image frames mark the anticipated arrival times of the sub-Rayleigh (sR) and trailing subRayleigh (TR) ruptures, respectively. Arrival time estimates for each rupture front, as determined from the equation of the best-fit lines, are highlighted in Figure 4.3.

The high-speed photoelastic image frames depicted in Figure 4.5 were acquired at $t=8 \mu s$ relative to the instrumentation trigger time. The actual time in the case of the sub-Rayleigh experiment is $t=3.74 \mu \mathrm{s}$ when corrected for the measured nucleation delay time $\left(t_{\text {delay }}^{s}\right)$, while the actual time in the case of the supershear experiment image frame corresponds to $t=3.86 \mu \mathrm{s}$.

The physical process through which the ablation of the $\mathrm{NiCr}$ wire leads to rupture nucleation is not completely understood. However, it is reasonable to assume that the plasma discharge and the resulting action of the expanding gases lead to a rapid, localized pressurization of the cylindrical wire channel and the emission of a dilatational wave front into the surrounding medium. This leading 
dilatational disturbance likely corresponds to the familiar circular P-wave front, which is typically observed in the early high-speed photoelastic image frames. Countless fault-parallel particle velocity measurements, conducted both on and off of the fault, have shown that there is very little particle displacement associated with the arrival of the leading P-wave front. The most dominant stress and particle velocity field contributions observed in the experiments appear to result from a mode II (shear) loading mechanism, which simultaneously occurs as a consequence of the resolved compressive and shear stresses applied to the pressurized wire channel as the wire is ablated.

An expanded view of each image frame in Figure 4.5 reveals eight radially symmetric "fringe lobes", which are strikingly similar to the familiar radiation patterns of the transverse (S) and radial (P) fields produced by a double couple. Note however that this is a maximum shear stress field representation of the radiation patterns as manifested by the photoelastic fringes. The radiation patterns are very likely the result of an impulsive shear loading of the cylindrical wire channel, which occurs as the $\mathrm{NiCr}$ wire is rapidly ablated.

The four fringe lobes, oriented at approximately $0^{\circ}, 90^{\circ}, 180^{\circ}$, and $270^{\circ}$ with respect to the fault are the maximum shear stress manifestation of the near-field S-lobes. The other four fringe lobes, oriented at approximately $45^{\circ}, 135^{\circ}, 225^{\circ}$, and $315^{\circ}$ correspond to the near-field P-lobes. Radiation patterns such as those displayed in Figure 4.5, and in the image frames which follow, are routinely observed during the earliest moments of virtually every experiment and bear the defining signature of a mode II dominated rupture nucleation process.

Although not as well-resolved as the radiation pattern fringe lobes, the photoelastic images in Figure 4.5 also reveal a concentrated, anti-symmetric fringe pattern, corresponding to the stress field of a mode II "crack". The characteristic dimensions of the "crack" in this instance appear to correlate with the dimensions of the discharge residue patch, which is routinely observed on the fault surfaces of the test specimens following each experiment. Note as well the absence of any dark, concentrated caustic zones a this time, which typically identify the position of the sub-Rayleigh rupture tip. The observation suggests that the critical crack length condition required to nucleate a dynamic (sub-Rayleigh) rupture has not yet been achieved. Note as well the absence of a well- 


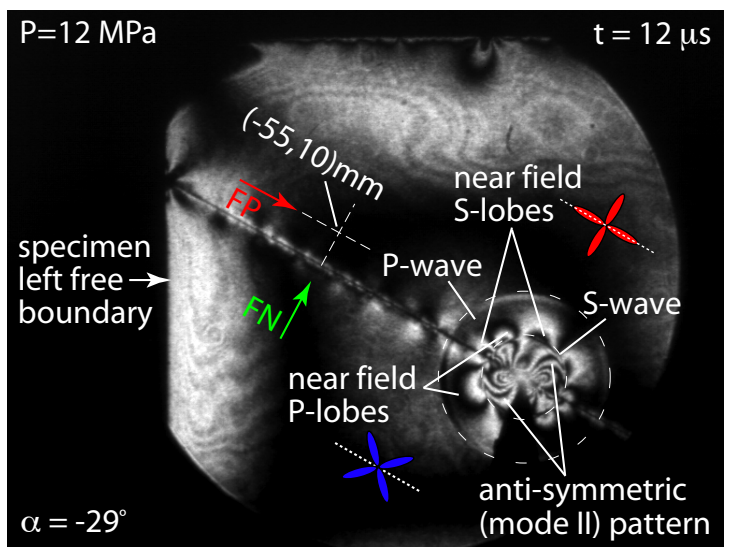

sub-Rayleigh experiment
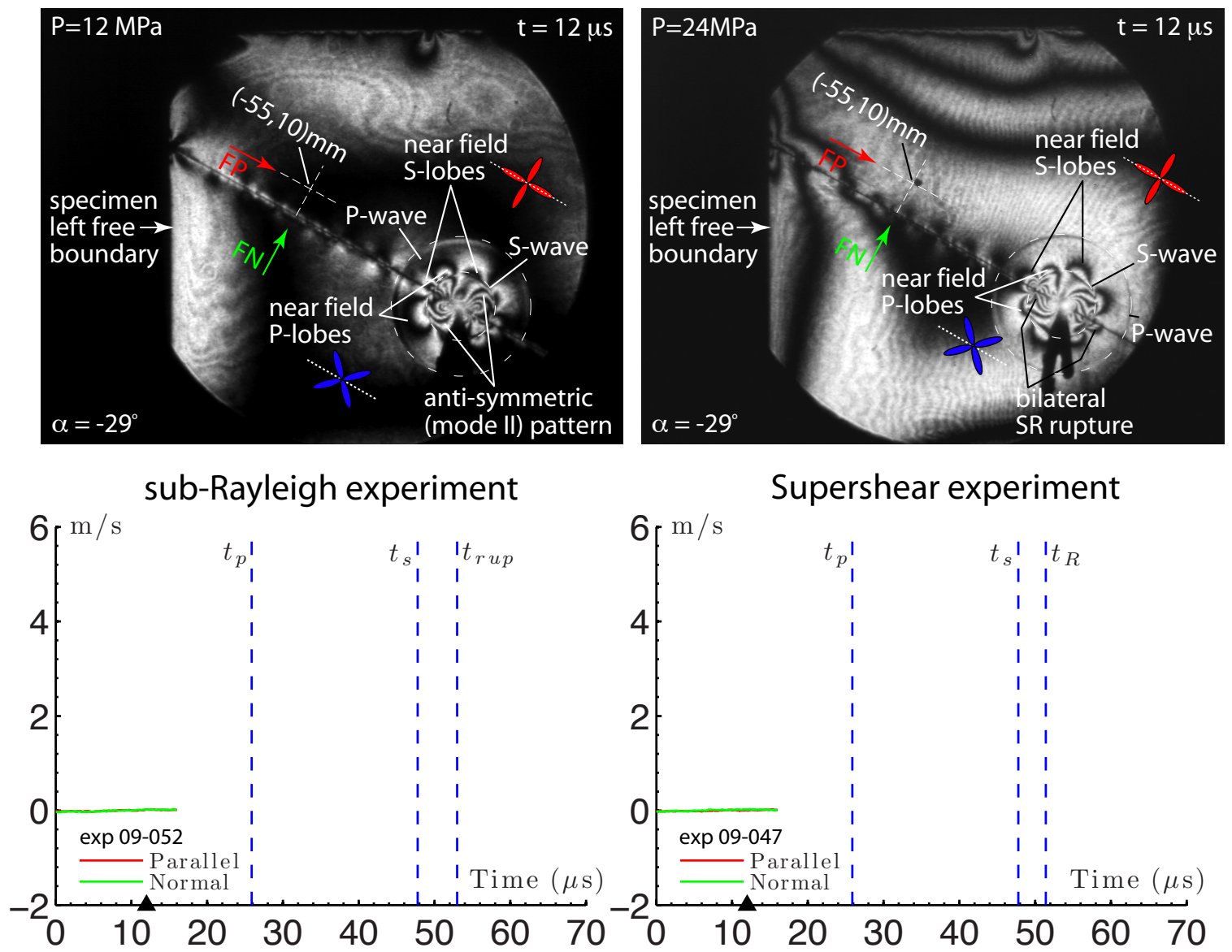

Figure 4.6: Contrast between sub-Rayleigh and supershear experiments: $(-55,10) \mathrm{mm}$ at $t=12 \mu \mathrm{s}$

defined circular S-wave frontsat this time in stark comparison to the sharp expanding P-wave front, which was observed from the outset.

The high-speed photoelastic image frames depicted in Figure 4.6 were acquired at $t=12 \mu \mathrm{s}$ relative to the instrumentation trigger time. The actual time in the case of the sub-Rayleigh experiment is $t=7.74 \mu \mathrm{s}$ when corrected for the measured nucleation delay time $\left(t_{\text {delay }}^{s}\right)$, while the actual time in the case of the supershear experiment image frame corresponds to $t=7.86 \mu \mathrm{s}$.

Sharp, circular expanding S-wave fronts are now clearly visible in both image frames. Bilateral sub-Rayleigh ruptures, as depicted in the right-hand image frame, are routinely observed in traditional laboratory earthquake experiments. Small dark caustics coinciding with the sub-Rayleigh tips are easily identified in the image. Observations acquired over many experiments appear to suggest that there is a direct correlation between the time when the sub-Rayleigh rupture tips emerge and 


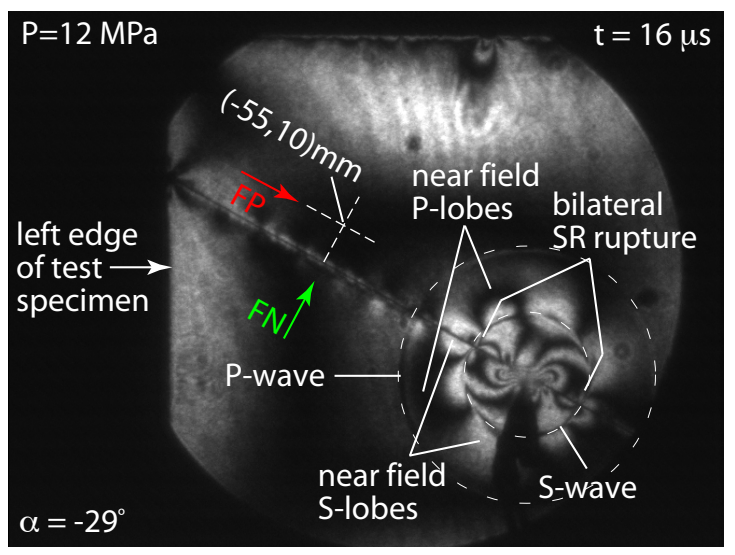

sub-Rayleigh experiment

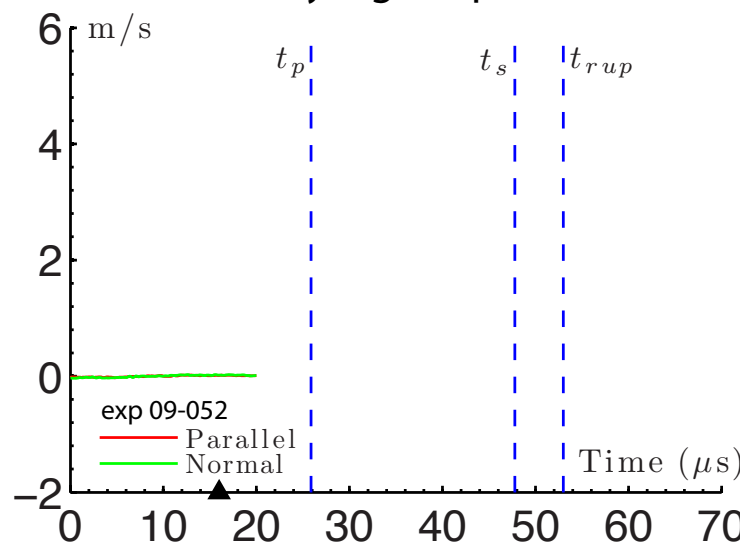

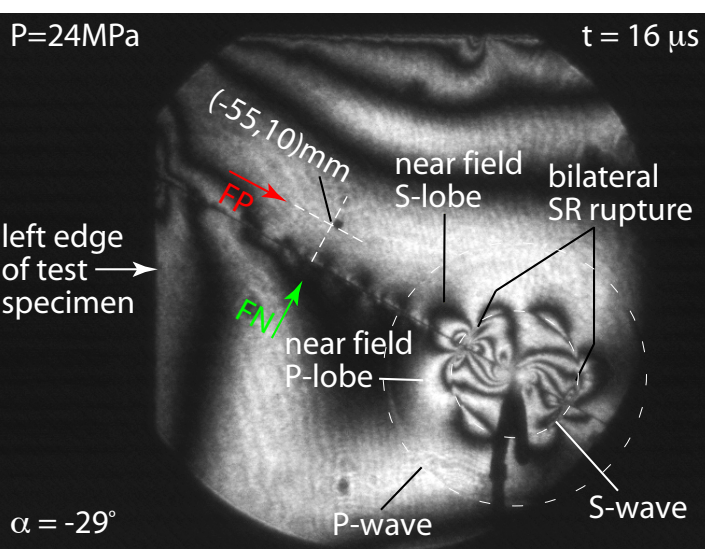

Supershear experiment

Figure 4.7: Contrast between sub-Rayleigh and supershear experiments: $(-55,10) \mathrm{mm}$ at $t=16 \mu \mathrm{s}$

the time at which the sharp expanding circular S-wave fronts are first observed. The inference here is that the familiar, sharp circular S-wave front, arises at the instant that the quasi-static mode II crack becomes unstable and the (dynamic) bilateral rupture is suddenly nucleated.

Note that a bilateral sub-Rayleigh rupture has also been nucleated at this time in the supershear experiment although the dark caustics are not nearly as visible as they are in the right-hand image. The emergence of the sub-Rayleigh ruptures by this time in both experiments conservatively bounds the rupture nucleation time to $8 \mu s<t<12 \mu s$ relative to the instrumentation trigger time or, $4 \mu s<t<8 \mu s$ when corrected for the measured nucleation delay time $\left(t_{\text {delay }}^{s}\right)$.

The radiation patterns in Figure 4.6 are now prominently displayed and clearly visible within the annulus defined by the leading circular P-wave front and the sharp trailing circular S-wave front. The anti-symmetric fringe patterns are also now clearly visible in both image frames and dominate 


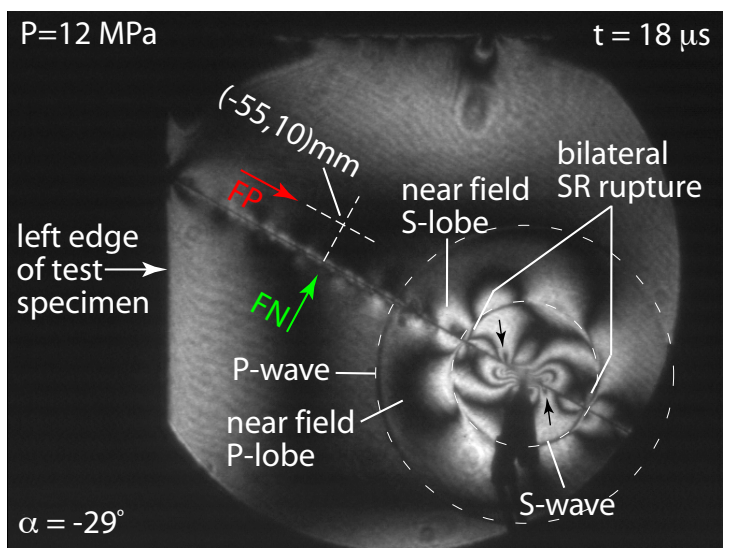

sub-Rayleigh experiment

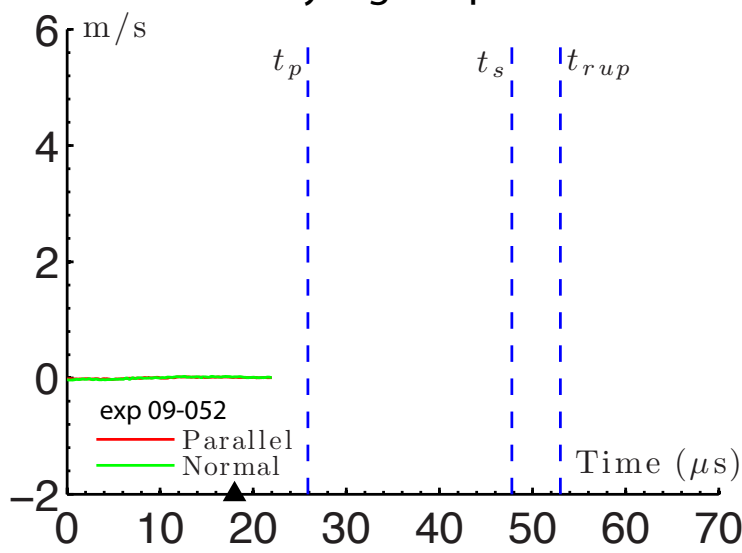

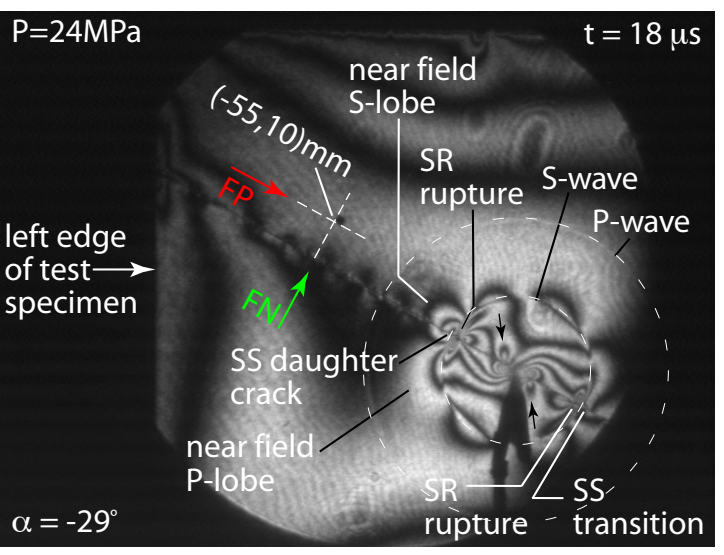

Supershear experiment

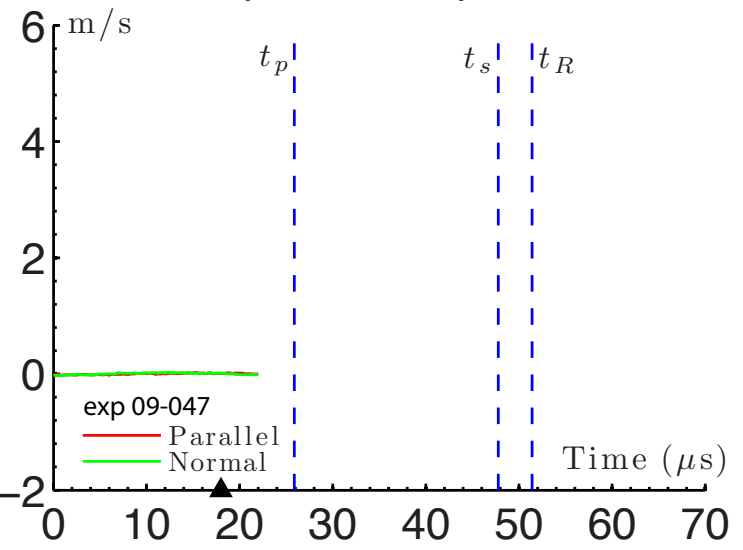

Figure 4.8: Contrast between sub-Rayleigh and supershear experiments: $(-55,10) \mathrm{mm}$ at $t=18 \mu \mathrm{s}$

the interior region bounded by the expanding circular S-wave fronts.

The high-speed photoelastic image frames depicted in Figure 4.7 were acquired at $t=16 \mu \mathrm{s}$ relative to the instrumentation trigger time. The actual time in the case of the sub-Rayleigh experiment is $t=11.74 \mu s$ when corrected for the measured nucleation delay time $\left(t_{\text {delay }}^{s}\right)$, while the actual time in the case of the supershear experiment image frame corresponds to $t=11.86 \mu s$.

The radiation patterns have continued to expand outward and remain bounded between the leading circular P-wave front and the sharp trailing circular S-wave front. Note once again the prominent leading S-field lobe in the right-hand image of Figure 4.7. The stress field within the local region just ahead of the rupture tip results from the superposition of the leading S-field radiation lobe and the local stress field produced by the sub-Rayleigh rupture tip. According to the BurridgeAndrews mechanism, a peak in the shear stress field within this local region can lead to the formation 


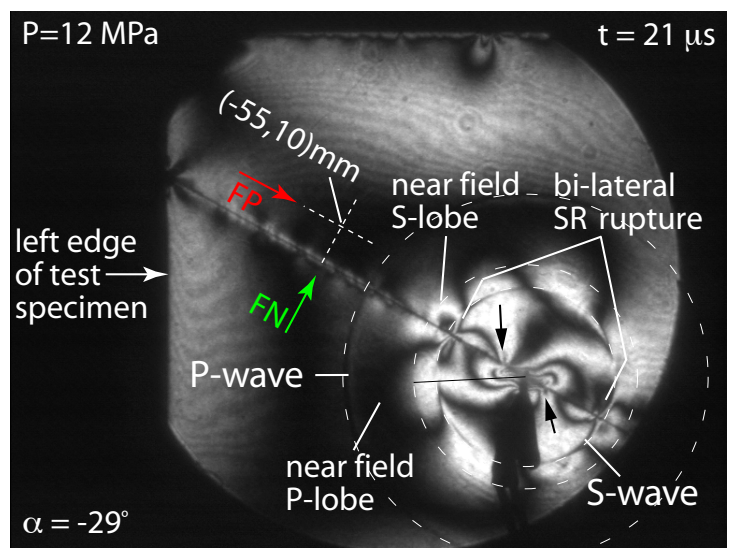

sub-Rayleigh experiment
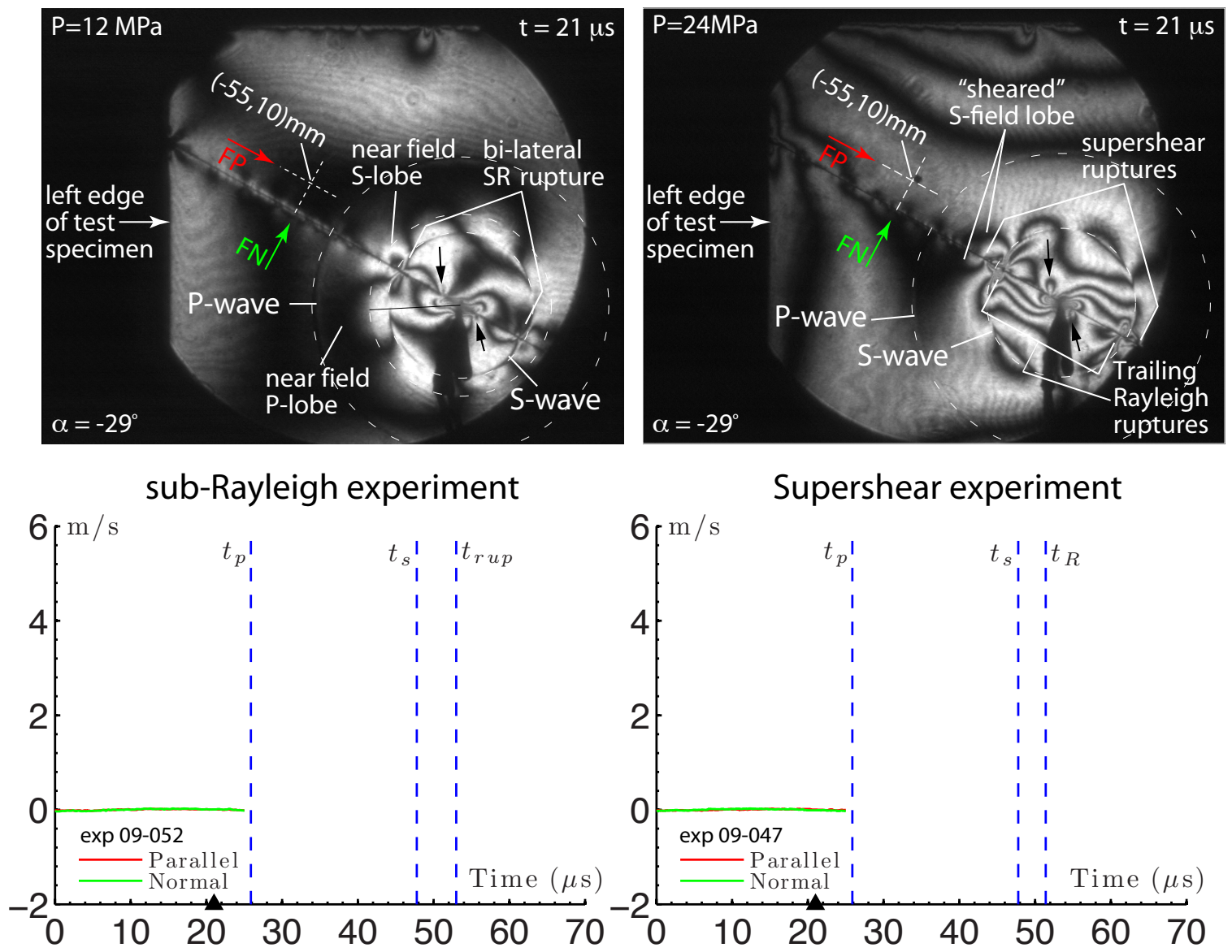

Figure 4.9: Contrast between sub-Rayleigh and supershear experiments: $(-55,10) \mathrm{mm}$ at $t=21 \mu \mathrm{s}$

of a daughter crack on the S-wave front. There is as of yet no hint of a supershear transition in the image.

The high-speed photoelastic image frames depicted in Figure 4.8 were acquired at $t=18 \mu \mathrm{s}$ relative to the instrumentation trigger time. The actual time in the case of the sub-Rayleigh experiment is $t=13.74 \mu s$ when corrected for the measured nucleation delay time $\left(t_{\text {delay }}^{s}\right)$, while the actual time in the case of the supershear experiment image frame corresponds to $t=13.86 \mu \mathrm{s}$. An expanded view and careful examination of the image frame on the right reveals a fringe discontinuity just ahead of the left traveling sub-Rayleigh rupture tip within the previously noted region where the S-field lobe and sub-Rayleigh rupture fields are superimposed. The observed "break" in the fringe signals the emergence of a daughter crack and the onset of the supershear transition. Note how the estimate of $t^{*}=18.2 \mu \mathrm{s}$ for the supershear transition time, based upon the intersection of the 


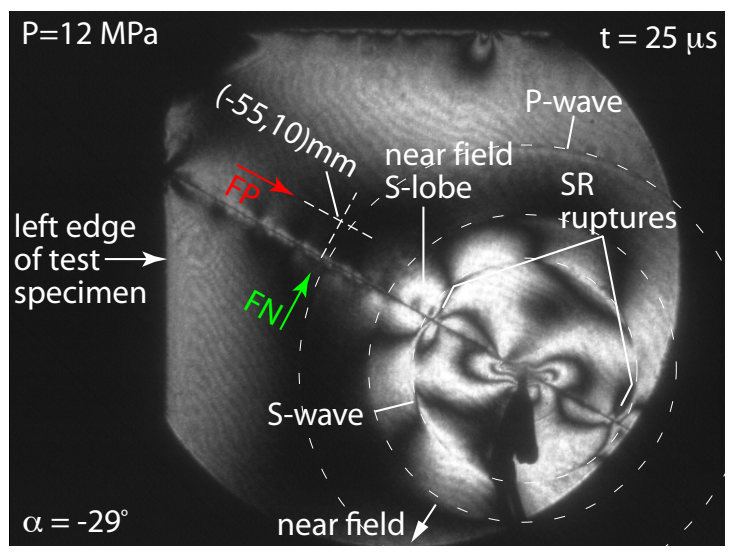

sub-Rayleigh experiment
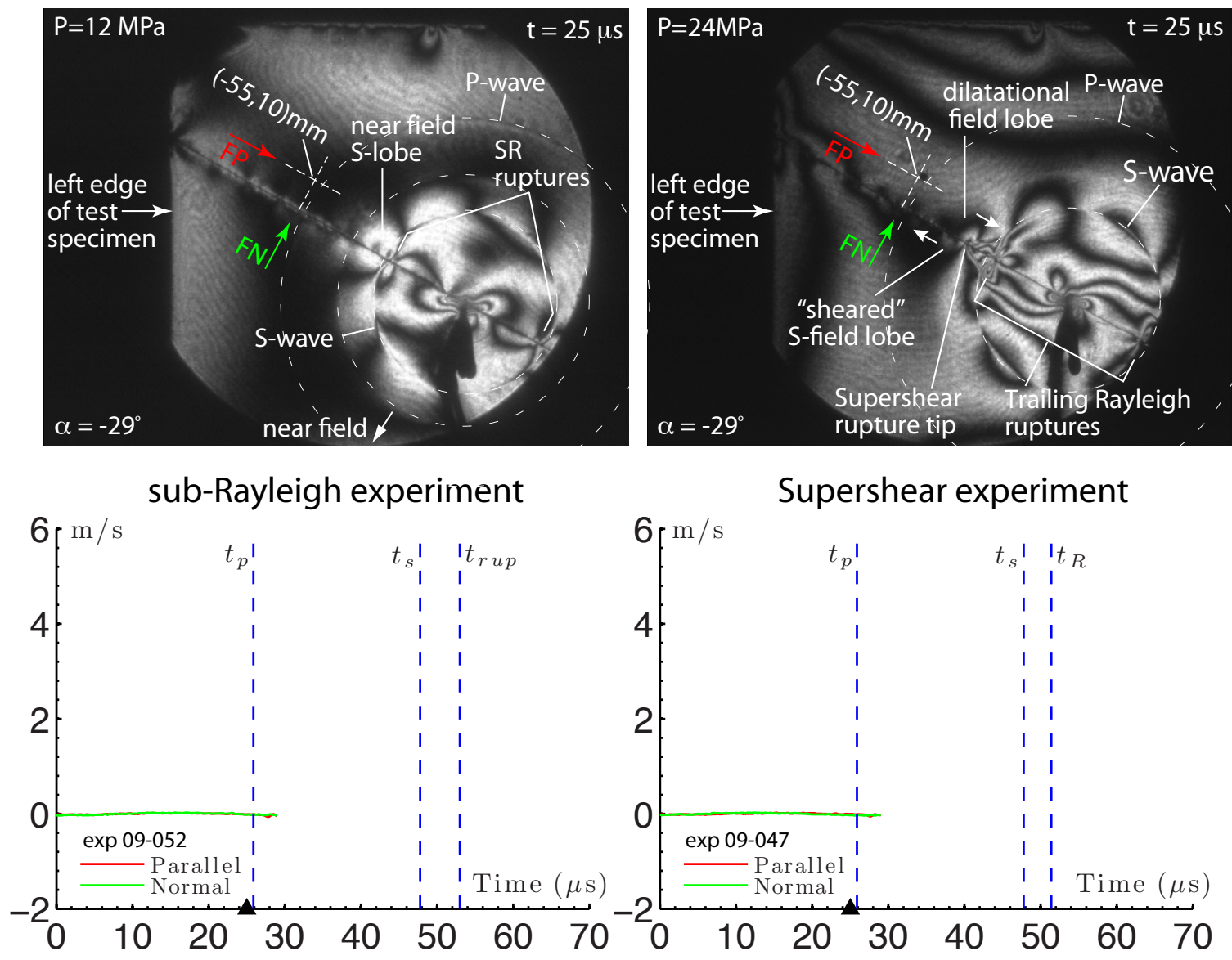

Figure 4.10: Contrast between sub-Rayleigh and supershear experiments: $(-55,10) \mathrm{mm}$ at $t=25 \mu \mathrm{s}$

best-fit lines in Figure 4.3, has yielded a value within $0.2 \mu \mathrm{sec}$ of the image frame time at which the daughter crack is first observed. The accuracy of this estimate largely stems from the steady speed maintained by the rupture throughout the duration of the experiment. Note as well the supershear transition occurring just ahead of the right traveling sub-Rayleigh rupture in the same image frame.

The pair of black arrows in each image frame in Figure 4.8 highlight the anti-symmetric fringe fringe pattern that remains centered about the hypocenter and persists even as the ruptures and elastic wave fronts propagate away. Note in particular the sharply inclined fringe loops positioned to the right and left of the hypocenter, which extend above and below the fault. The distance between the points where the fringe loops intersect the fault was found to correlate with the dimensions of the discharge residue patch, which was observed and measured on the fault surfaces after the experiment was completed. 
The high-speed photoelastic image frames depicted in Figure 4.9 were acquired at $t=21 \mu \mathrm{s}$ relative to the instrumentation trigger time. The actual time in the case of the sub-Rayleigh experiment is $t=16.74 \mu \mathrm{s}$ when corrected for the measured nucleation delay time $\left(t_{\text {delay }}^{\mathrm{s}}\right)$, while the actual time in the case of the supershear experiment image frame corresponds to $t=16.86 \mu \mathrm{s}$.

Two supershear ruptures are seen propagating in opposite directions as clearly revealed by the shear Mach cones in the right-hand figure. The trailing sub-Rayleigh rupture tips on either side of the hypocenter are also clearly identified by a dark caustic zone at the center of the fringe concentrations just behind the expanding S-wave front. Note as well how the lower portion of the leading S-field fringe lobe has been shifted forward with respect to its counterpart on the opposite side of the fault as a consequence of the right-lateral stress field perturbation introduced by the sudden emergence of the supershear rupture. All of the other prominent stress field features, including the expanding radiation patterns, remain visible at this time.

Returning now to the sub-Rayleigh experiment, note that a third dashed circle has been added in the sub-Rayleigh experiment image on the left in Figure 4.9 in order to highlight a near-field disturbance, which begins to appear at this time between the P- and S-wave fronts. A very similar feature, although not as bright, is also visible in the supershear image on the right. This phenomena is frequently captured in photoelastic images during this early portion of the experiment. The feature is characterized by a brightening of the photoelastic field between the S-wave front and the leading circular front, which bounds the disturbance. The nature of this disturbance is not fully understood at this time and it remains unclear as to whether this can be interpreted as a natural near-field effect, or whether it is a residual effect of the rupture nucleation scheme. The radius of the intermediate expanding front was carefully measured beginning with this image frame and measured in subsequent frames until it was no longer visible.

The high-speed photoelastic image frames depicted in Figure 4.10 were acquired at $t=25 \mu \mathrm{s}$ relative to the instrumentation trigger time. The actual time in the case of the sub-Rayleigh experiment is $t=20.74 \mu \mathrm{s}$ when corrected for the measured nucleation delay time $\left(t_{\text {delay }}^{s}\right)$, while the actual time in the case of the supershear experiment image frame corresponds to $t=20.86 \mu \mathrm{s}$. 


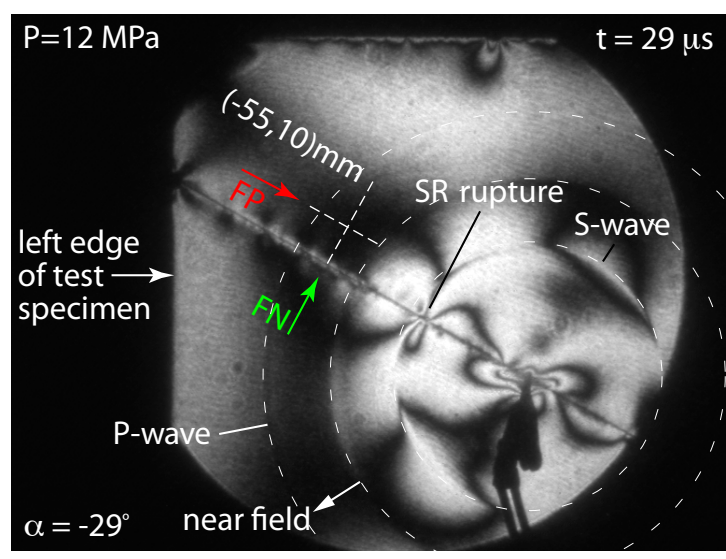

sub-Rayleigh experiment

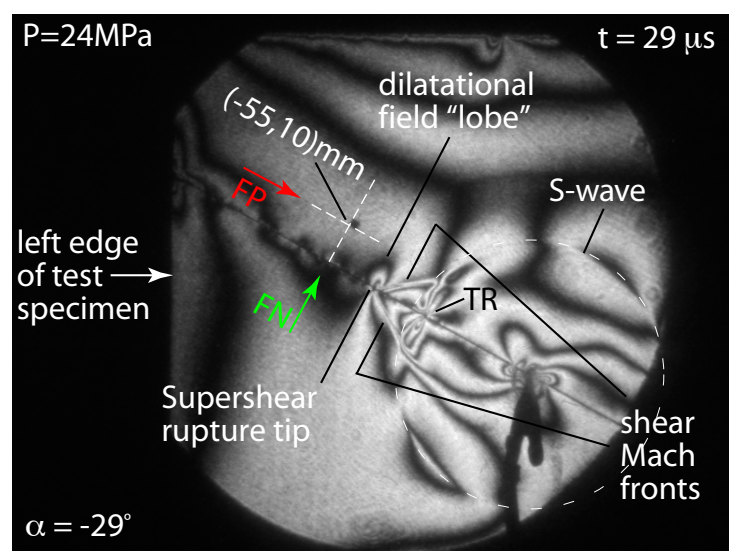

Supershear experiment

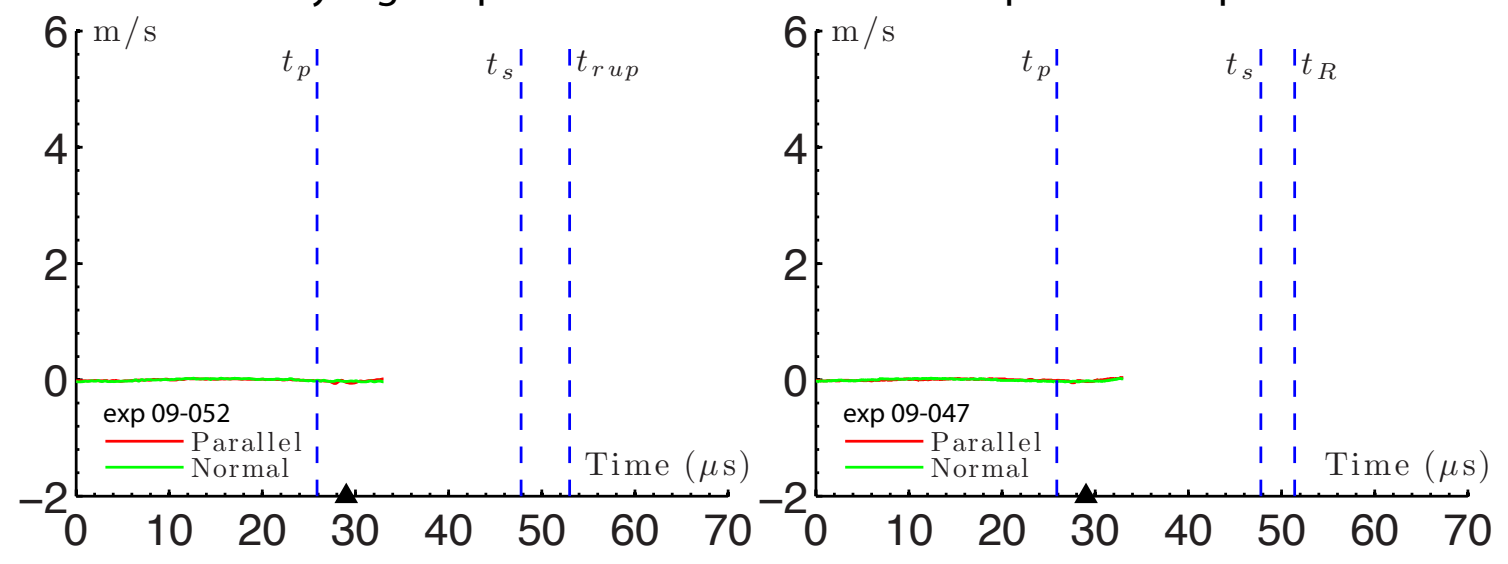

Figure 4.11: Contrast between sub-Rayleigh and supershear experiments: $(-55,10) \mathrm{mm}$ at $t=29 \mu \mathrm{s}$

The expanding circular $\mathrm{P}$-wave fronts in each image frame are now each only a few millimeters away from crossing the measurement station at $(-55,10) \mathrm{mm}$. Inspection of the supershear displacement-time curve in Figure 4.3 reveals that the rupture is propagating in the stable rupture speed regime with an average speed of $V_{s s}=2.27 \mathrm{~mm} / \mu \mathrm{s}$, which translates to $V_{s s} / C_{s}=1.77$. At the same time, the trailing sub-Rayleigh rupture is observed to propagate with an average rupture speed of $V_{T R}=1.16 \mathrm{~mm} / \mu \mathrm{s}$, which translates to $V_{T R} / C_{s}=0.91$.

A concentrated dilatational field lobe is revealed by the sharp looping profile of the dark fringe, positioned just above the supershear rupture tip in Figure 4.10. In this instance the dark fringe acts as a "carrier fringe" which exposes the sharp stress field gradient between the leading dilatational field lobe and the background stress field. The photoelastic (maximum shear stress field) manifestation of the dilatational field lobes gives the false impression that the lobe only extends to one side of 
the fault. Contour plots derived from theoretical and numerical models reveal a similar profile for the maximum shear stress field while plots of the other stress field components reveal symmetrically oriented lobes, extending above and below fault. The observed position in this instance of the maximum shear stress representation of the dilatational field lobe on the extensional of the fault correlates very well with theoretical predictions.

Meanwhile, in the sub-Rayleigh rupture experiment, the rupture continues to propagate with an average speed of $V_{s R}=1.12 \mathrm{~mm} / \mu \mathrm{s}$, which translates to $V_{s R} / C_{s}=0.88$. Note how the expanding near-field disturbance is still clearly visible in both images although far more-dominant in the subRayleigh image on the left. The particle velocity traces accompanying each image frame each remain flat up to this point in time, consistent with the fact that not even the leading P-wave fronts have reached the measurement stations at this time.

The high-speed photoelastic image frames depicted in Figure 4.11 were acquired at $t=29 \mu \mathrm{s}$ relative to the instrumentation trigger time. The actual time in the case of the sub-Rayleigh experiment is $t=24.74 \mu \mathrm{s}$ when corrected for the measured nucleation delay time $\left(t_{\text {delay }}^{s}\right)$, while the actual time in the case of the supershear experiment image frame corresponds to $t=24.86 \mu s$.

Note how the particle velocity traces in both experiments continue to remain flat up through this time even though the leading P-wave fronts have now both long since crossed the measurement station. In the case of the sub-Rayleigh rupture experiment, the sharp circular boundary of the advancing near-field disturbance continues to expand with the prominent lobes of the radiation patterns vividly displayed within the intermediate near-field region.

Over in the supershear experiment, the radiation pattern has now faded to the point where it is no longer revealed by the photoelastic fringe pattern. Sharp, straight Mach fronts are seen stemming from the supershear rupture tip along with the prominent dilatational field lobe as revealed by dark looping fringe, which wraps around the rupture tip. The pronounced contraction of the dilatational field lobe along the FP direction naturally arises as a consequence of the supershear rupture speed $\left(V_{s s}=2.27 \mu s\right)$ which is approaching $90 \%$ of the dilatational wave speed $\left(V_{s s} / C_{p}=0.89\right)$. The trailing sub-Rayleigh rupture and its surrounding stress field is also clearly visible in the wake of 


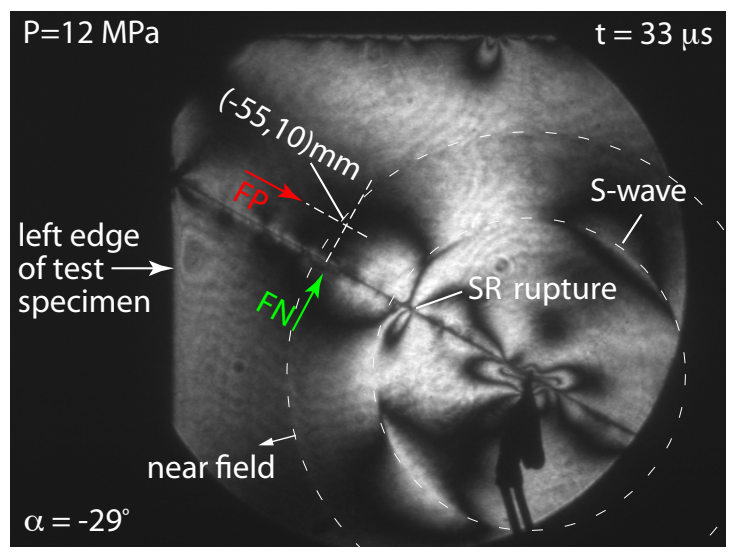

sub-Rayleigh experiment
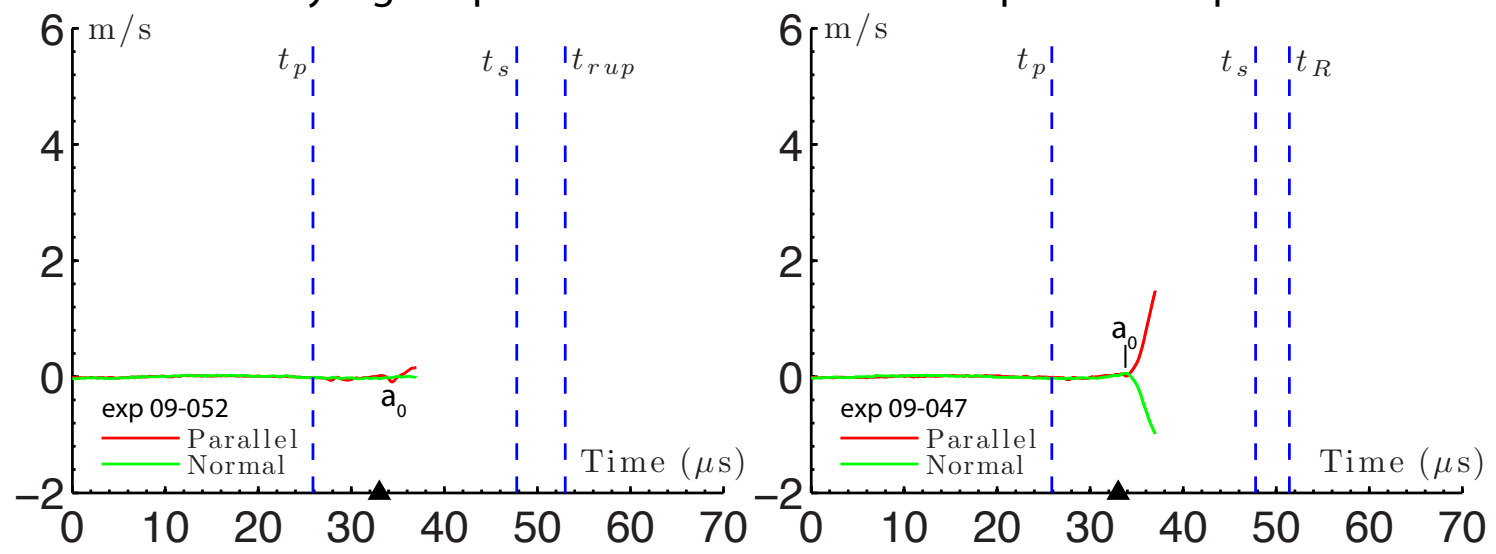

Figure 4.12: Contrast between sub-Rayleigh and supershear experiments: $(-55,10) \mathrm{mm}$ at $t=33 \mu \mathrm{s}$

the supershear rupture.

The high-speed photoelastic image frames depicted in Figure 4.12 were acquired at $t=33 \mu \mathrm{s}$ relative to the instrumentation trigger time. The actual time in the case of the sub-Rayleigh experiment is $t=28.74 \mu \mathrm{s}$ when corrected for the measured nucleation delay time $\left(t_{\text {delay }}^{s}\right)$, while the actual time in the case of the supershear experiment image frame corresponds to $t=28.86 \mu s$.

Note the distinct physical separation between the contracted dilatational field lobe and the inclined shear Mach front in the superhsear experiment image frame, which naturally widens with increased FN distance. The distinct separation between the dilatational and shear portions of the field as observed in this experiment is a unique attribute of supershear rupture fields, which has no counterpart in sub-Rayleigh rupture fields.

Pronounced jumps are now suddenly registered in the FP and FN particle velocity records in the 
supershear experiment. The noted velocity swings are clearly attributed to the leading dilatational field lobe, which sweeps the off-fault measurement station in advance of the shear Mach fronts (recall, that the particle velocity signals are plotted to a time $4 \mu \mathrm{s}$ beyond the posted image frame time). The initial velocity jumps produced by the dilatational field lobe are precursors to the arrival of the shear Mach front, which follows.

Next, recall the supershear rupture arrival time estimate of $t=34.5 \mu \mathrm{s}$, which was inferred from the distance-time curves in Figure 4.3. In actuality, this arrival time estimate predicts the time at which the supershear rupture tip will reach $(-55,0) \mathrm{mm}$. Nonetheless, note how this estimate is in very close agreement with emergence of the precursor signal at the point labeled $a_{0}$ in Figure 4.12. The excellent agreement between the on-fault arrival time of the rupture tip at $(-55,0) \mathrm{mm}$ and the off-fault arrival time of the dilatational field lobe at $(-55,10) \mathrm{mm}$ is attributed to the strong contraction of the field lobe in the FP direction and it normal orientation with respect to the fault.

Returning to the sub-Rayleigh experiment in Figure 4.12, observe how the radiation patterns in the sub-Rayleigh image have now dramatically faded compared to their appearance in the previous image frame. The expanding near-field disturbance has also just crossed the measurement station at this time. Minor fluctuations are observed in the FP signal, beginning at the point labeled $a_{0}$, which correlates with the arrival of the intermediate circular front at the measurement station. The final measurement of the expanding near-field front position was obtained from the $t=33 \mu \mathrm{s}$ image frame, leading to a total of four data points tracing back to $t=21 \mu \mathrm{s}$. The front was no longer visible in the image frames obtained beyond this time. The resulting displacement-time plot corresponding to the expanding near-field disturbance is displayed in Figure 4.3. A best-fit line passed through these points is characterized by a slope of $C_{N F}=2.56 \mathrm{~mm} / \mu \mathrm{s}$ with a time axis intercept $t_{N F}=10.48 \mu \mathrm{s}$ relative to the instrumentation trigger time. The first interesting revelation is that the intermediate-field disturbance propagates at the P-wave speed. An extra data point was added to the plot, based upon the equation of the best-fit line, in order to extrapolate the curve down to its time axis intercept. Note the offset of $\Delta t=6.22 \mu s$ between the time axis intercept of the near-field disturbance curve and the S-wave time axis intercept given by $\left(t_{\text {delay }}^{s}\right)$. 


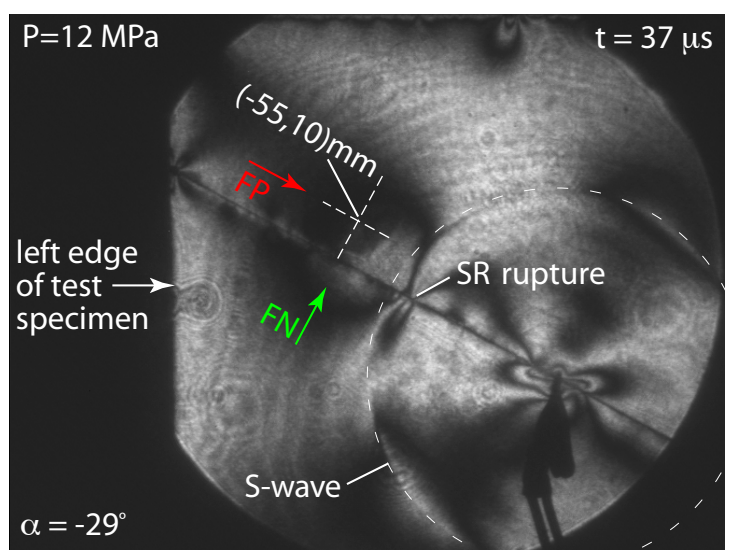

sub-Rayleigh experiment

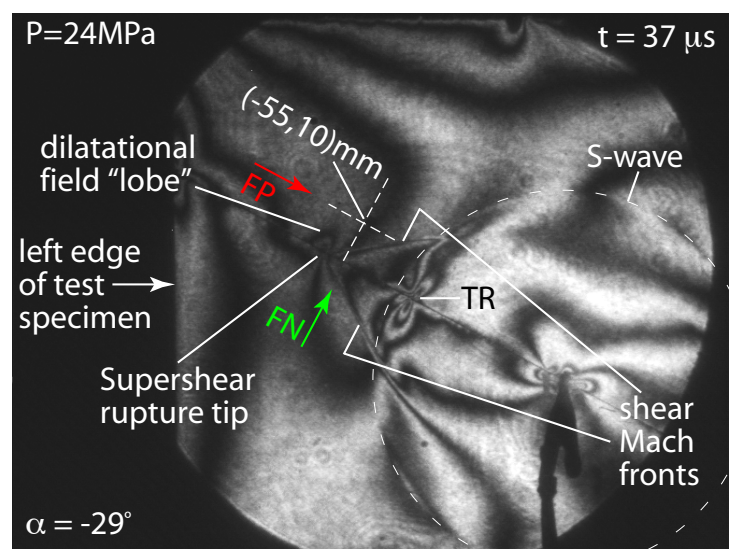

Supershear experiment

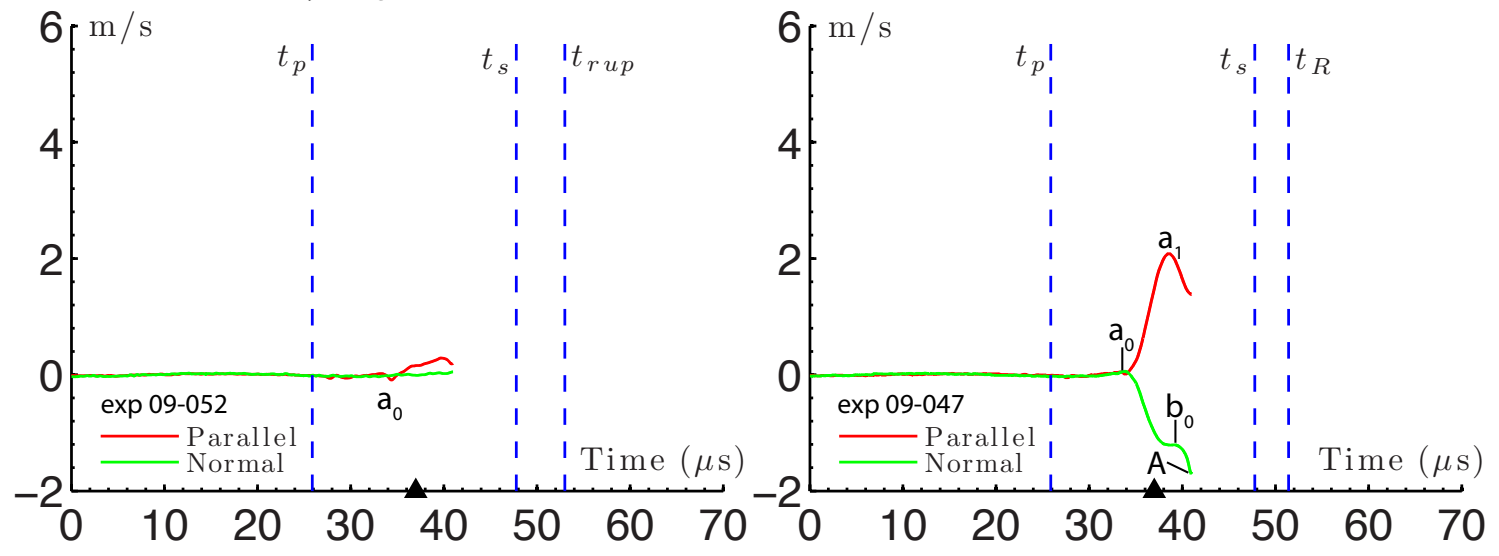

Figure 4.13: Contrast between sub-Rayleigh and supershear experiments: $(-55,10) \mathrm{mm}$ at $t=37 \mu \mathrm{s}$

The near-field disturbance was clearly initiated at a later time since it does not extrapolate back to the same common point in time. This observation, coupled with the fact that the leading front propagates at the dilatational wave speed suggests that the near-field disturbance may be a final release wave arising from the wire ablation process, perhaps at the tail end of the process as the wire channel cavity is unloaded. Evidence in support of this hypothesis is provided by the FP velocity measurement, which reveals a small positive velocity increase at the time when the station was swept by the near-field disturbance. Recall that the P-wave displacement field arising from a double couple is predicted to be nodal on the fault and should gradually increase in magnitude at off-fault positions on the extensional side of the fault until reaching a maxima at $135^{\circ}$. Furthermore, in the case of a double couple, the P-field should be directed radially outward from hypocenter and a faultparallel measurement should therefore initially register a negative velocity swing within the imposed 
coordinate system. However, note that the FP signal exhibits a gradual positive velocity increase as the near-field disturbance crossed the station. The result implies a radial loading component, which is directed back towards the hypocenter. One additional and very important observation comes from a different set of experiments where the FP motion has been monitored at points lying very close to the fault, and positioned on either side of the fault. When sliding occurs a distinct antisymmetric pair of particle velocity signals is obtained. When sliding does not occur the resulting records are matched and virtually identical within the bounds of experimental uncertainties. Paired FP measurements conducted just above and below the fault have shown that both sides of the fault move together and that no sliding is observed as the near-field disturbance crosses the measurement stations. All of these collective observations are consistent with the hypothesis that the near-field disturbance is likely a release wave phenomena in the form of a "pull back" signal which is perhaps linked to the sudden contraction of the wire channel at the tail end of the ablation process.

The high-speed photoelastic image frames depicted in Figure 4.13 were acquired at $t=37 \mu \mathrm{s}$ relative to the instrumentation trigger time. The actual time in the case of the sub-Rayleigh experiment is $t=32.74 \mu \mathrm{s}$ when corrected for the measured nucleation delay time $\left(t_{\text {delay }}^{s}\right)$, while the actual time in the case of the supershear experiment image frame corresponds to $t=32.86 \mu \mathrm{s}$.

Returning once again to the sub-Rayleigh experiment, note the continued positive climb of the FP signal starting from the point labeled as $a_{0}$ as the near-field disturbance continues to sweep across the measurement station at $(-55,10) \mathrm{mm}$. The photoelastic image appears otherwise normal in every respect with the S-wave front and sub-Rayleigh rupture continuing to propagate towards the measurement station.

A glance at the supershear image frame reveals that there is still a considerable gap between the measurement station and the shear Mach front. The supershear rupture tip and the leading dilatational field-lobe have already advanced well beyond the measurement station at this time.

Careful evaluation of the particle velocity signals reveals that the precursor signals reach their respective maxima (point $a_{1}$ ) and minima (point $A$ ) at times $t=38.5 \mu \mathrm{s}$ and $t=42.87 \mu \mathrm{s}$, respectively, (the point $A$ in the FN record is also identified with the arrival of the shear Mach front). Note 


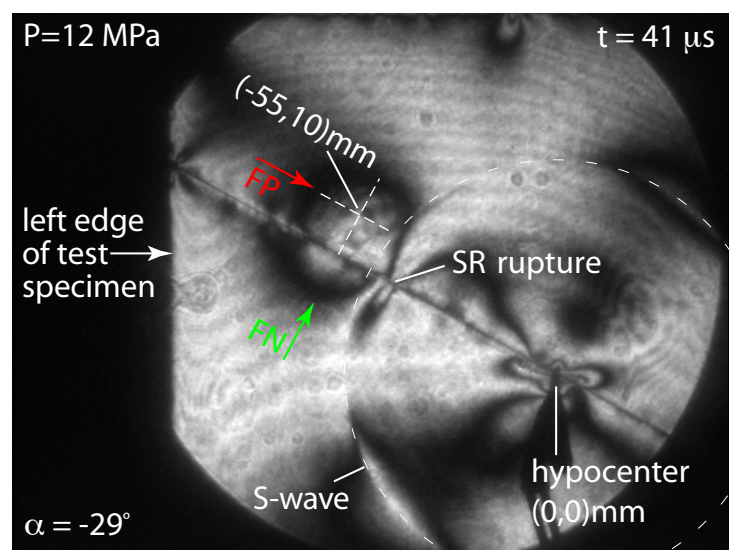

sub-Rayleigh experiment
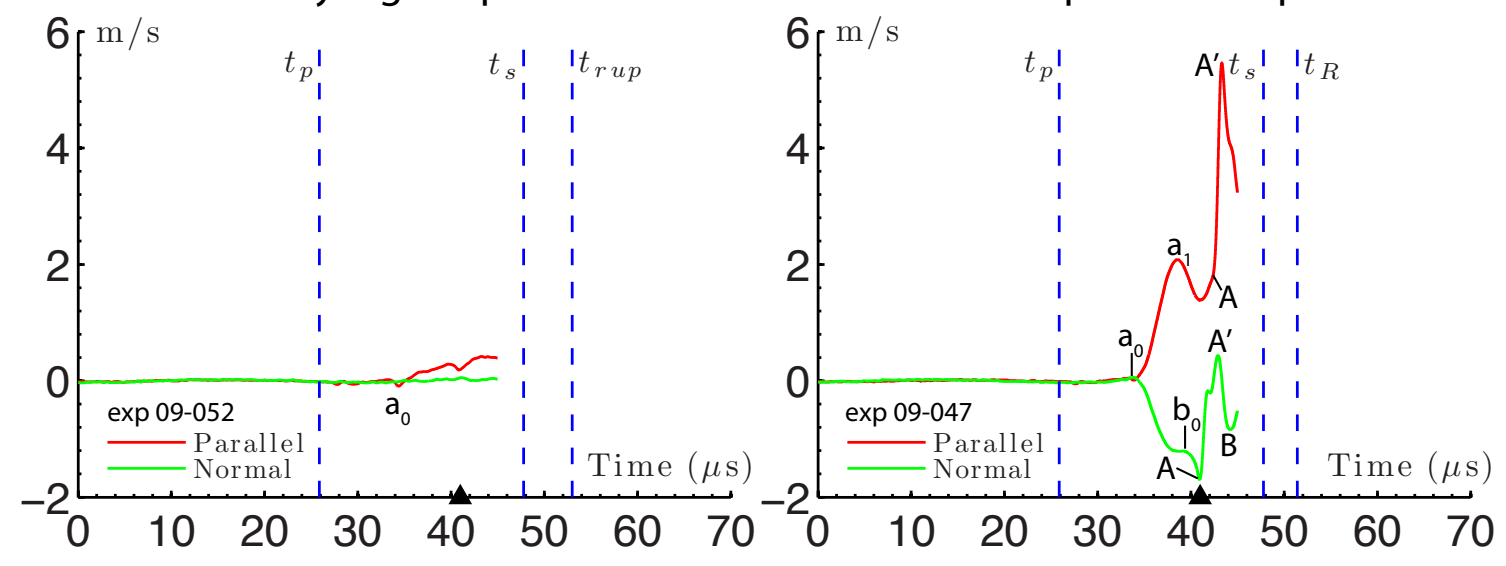

Figure 4.14: Contrast between sub-Rayleigh and supershear experiments: $(-55,10) \mathrm{mm}$ at $t=41 \mu \mathrm{s}$

that neither of these times coincide with the time when the supershear rupture tip was positioned directly below the station. The result indicates that location where dilatational field lobe components reach their respective peaks is inclined away from the fault-normal and positioned closer to the shear Mach fronts.

Note as well how the magnitude of the velocity swing between points $a_{0}$ and $a_{1}$ in the FP record is greater than the corresponding swing between points $a_{0}$ and $A$ in the FN record. This feature is also predicted by theory and reflects the circulation of the dilatational field term about the rupture tip (recall $\left.\nabla \times u^{p}=0\right)$. This is once again a unique consequence of supershear rupture field solutions, whereby the dilatational field in the region lying ahead of the shear Mach front is distinctly isolated from shear field bounded behind the Mach fronts.

The high-speed photoelastic image frames depicted in Figure 4.14 were acquired at $t=41 \mu \mathrm{s}$ 
relative to the instrumentation trigger time. The actual time in the case of the sub-Rayleigh experiment is $t=36.74 \mu \mathrm{s}$ when corrected for the measured nucleation delay time $\left(t_{\text {delay }}^{s}\right)$, while the actual time in the case of the supershear experiment image frame corresponds to $t=36.86 \mu \mathrm{s}$.

The supershear image frame captures the shear Mach front as it was just about to cross the measurement station. The particle velocity signals (plotted up to $t=45 \mu \mathrm{s}$ ) reveal how the motion along the shear Mach front is dominated by the FP component as predicted for a supershear rupture in the stable rupture speed regime $\left(\sqrt{2} C_{s}<V_{r}<C_{p}\right)$. A careful examination of the particle velocity records identifies the points labeled $A$ (at $t=42.87 \mu \mathrm{s}$ ) in the FN record and $a_{2}$ (at $t=42.45 \mu \mathrm{s}$ ) in the FP record with the arrival of the shear Mach front. The ratio between the FP and FN velocity jumps along the shear Mach front was determined by dividing the magnitude of the velocity jump between points $a_{2}$ and $A$ in the FP record by the magnitude of the velocity jump between points $A$ and $B$ in the FN record. The measurements yield the ratio $\beta_{s}^{\text {meas }}=\left|\delta \dot{u}_{x}^{s}\right| /\left|\delta \dot{u}_{y}^{s}\right|=1.686$, where $\delta \dot{u}_{x}^{s}$ and $\delta \dot{u}_{y}^{s}$ represent the measured fault-parallel and fault-normal particle velocity jumps, respectively. The average supershear rupture speed $V_{s s}=2.27 \mathrm{~mm} / \mu \mathrm{s}$ translates to a theoretical value of $\beta_{s}=\sqrt{V_{s s}^{2} / C_{s}^{2}-1}=1.458$. The particle velocity measurements thus yield an estimate for $\beta_{s}^{\text {meas }}$, which is $15.7 \%$ higher than the theoretical value of $\beta_{s}$. This particular measurement along with several other similar measurements of $\beta_{s}$ is examined in more-detail in Section 4.5.

Returning once again to the sub-Rayleigh experiment, the image frame reveals the S-wave front and sub-Rayleigh rupture continuing to advance towards the measurement station at $(-55,10) \mathrm{mm}$. The photoelastic fringe pattern looks classic in every respect. The radiation patterns have long since faded as has the brightened band induced by the near-field disturbance. Note the gentle but sustained, positive velocity increase registered by the FP component as the trailing portion of the near-field disturbance continues to cross the measurement station.

The high-speed photoelastic image frames depicted in Figure 4.15 were acquired at $t=45 \mu \mathrm{s}$ relative to the instrumentation trigger time. The actual time in the case of the sub-Rayleigh experiment is $t=40.74 \mu \mathrm{s}$ when corrected for the measured nucleation delay time $\left(t_{\text {delay }}^{s}\right)$, while the actual time in the case of the supershear experiment image frame corresponds to $t=40.86 \mu \mathrm{s}$. The 


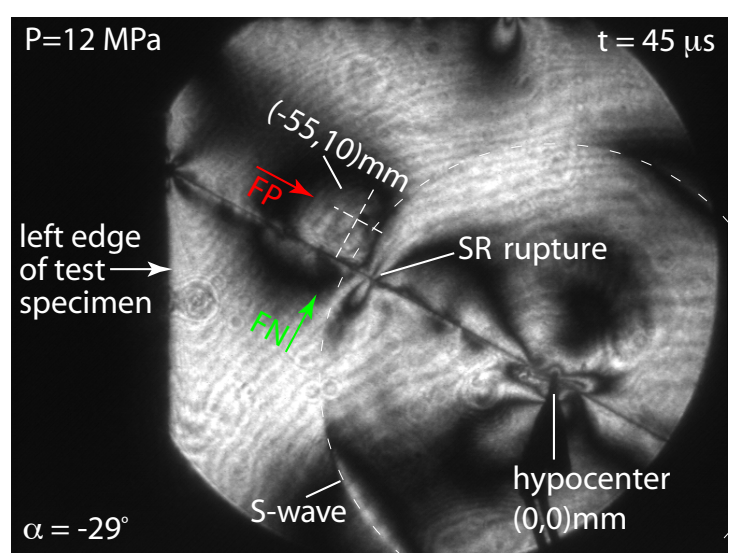

sub-Rayleigh experiment
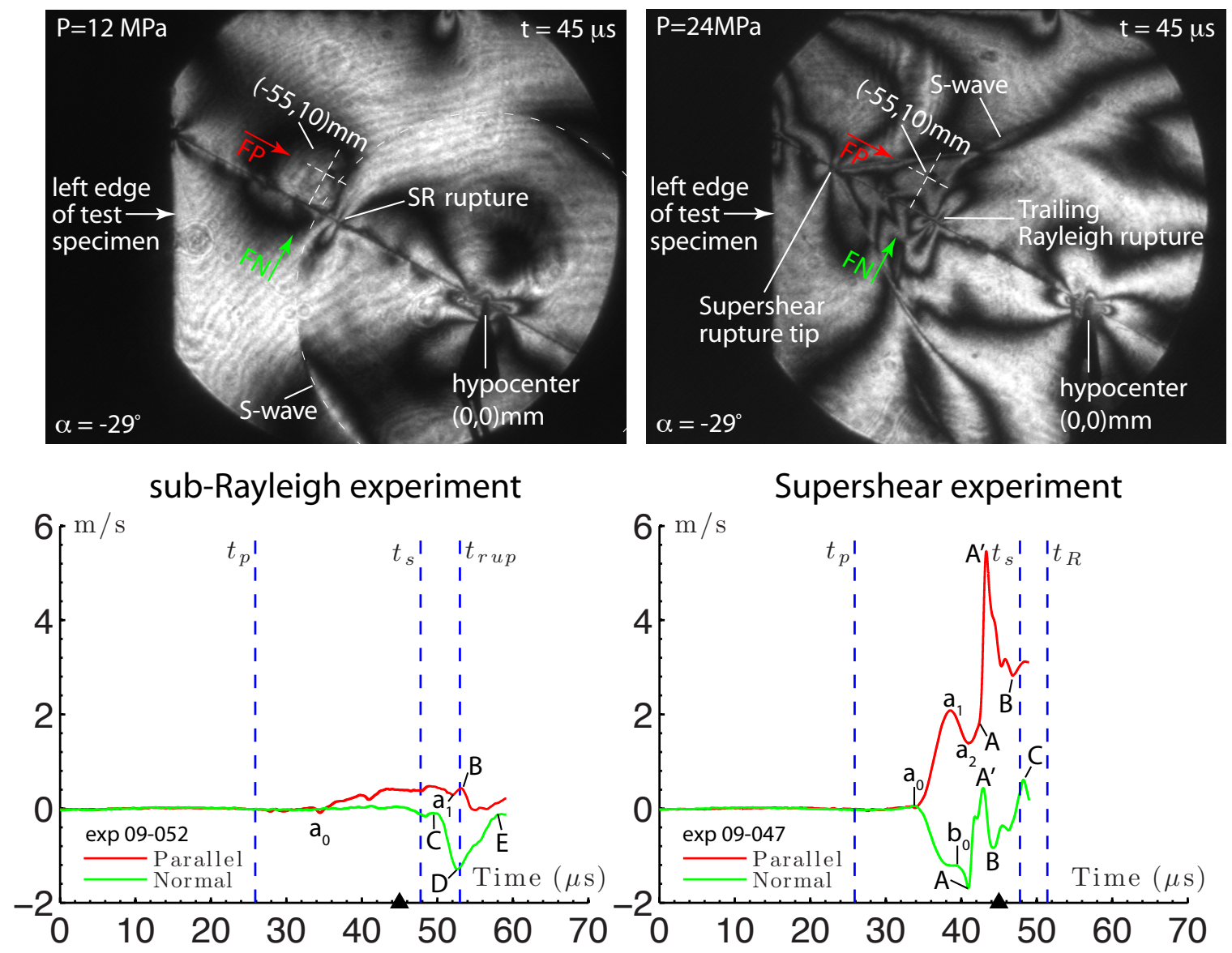

\section{Supershear experiment}

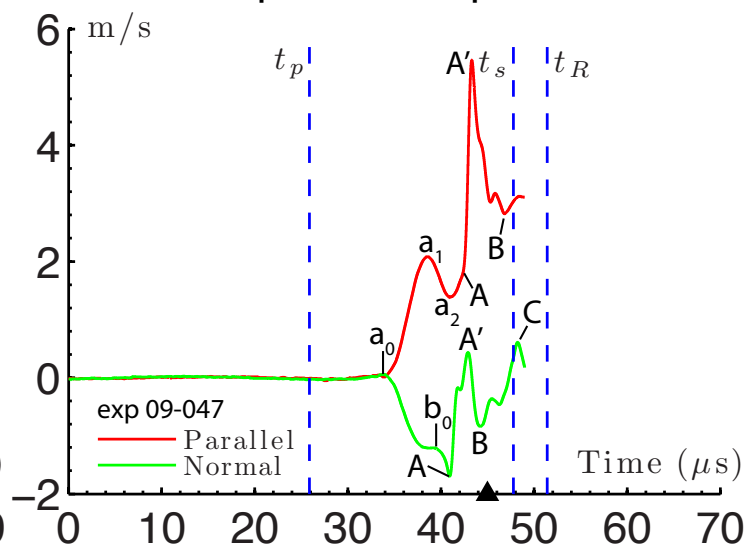

Figure 4.15: Contrast between sub-Rayleigh and supershear experiments: $(-55,10) \mathrm{mm}$ at $t=45 \mu \mathrm{s}$

high-speed image at $t=45 \mu \mathrm{s}$ captures a relatively quiescent moment in time between the passage of the shear Mach front and the pending arrival of the (secondary) sub-Rayleigh rupture. The measurement station will soon experience a second dynamic event when the extended stress field lobe of the sub-Rayleigh arrives and crosses the station.

It is interesting to note the glaring lack of a secondary signal in the supershear experiment. Close examination of the photoelastic image reveals the secondary (interior) Mach fronts, which are normally correlated to the observance of the secondary supershear signals (see Section 3.10). In this instance however, the internal secondary Mach fronts have simply not yet extended far enough in the FN direction to sweep the off-fault measurement station at $(-55,10) \mathrm{mm}$ and so the recorded signal is void of the secondary peak that is so often observed!

In the sub-Rayleigh experiment, the S-wave front is shown approaching the measurement station. 


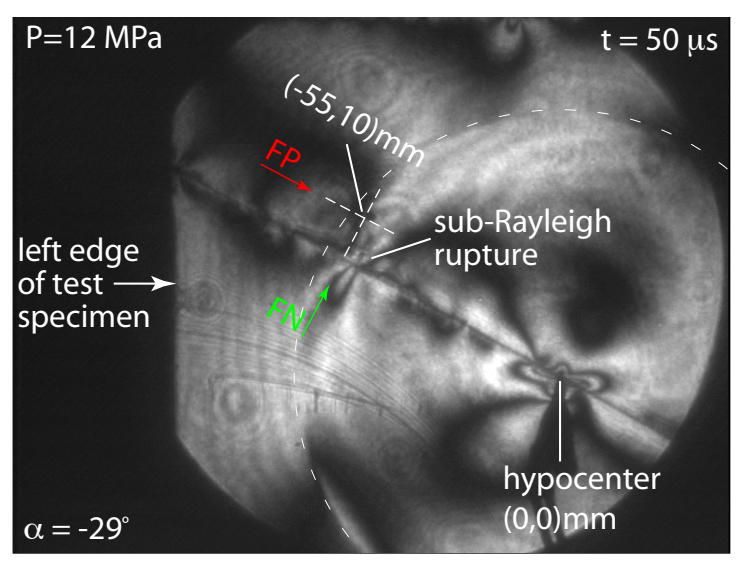

sub-Rayleigh experiment

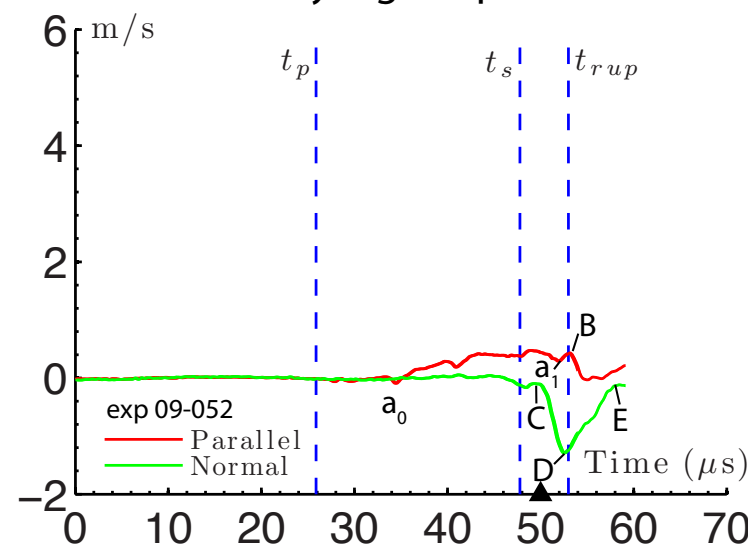

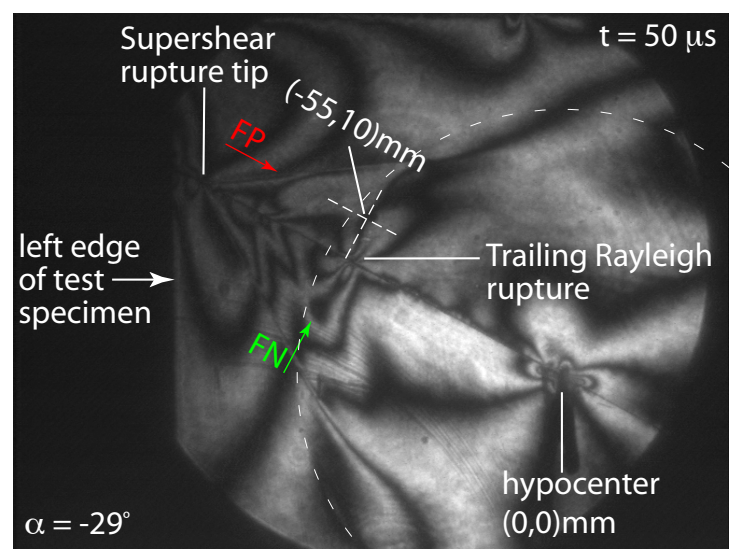

Supershear experiment

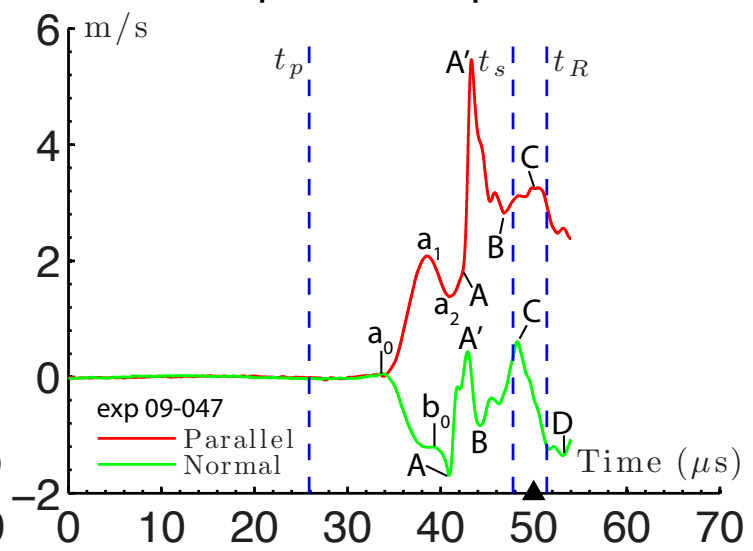

Figure 4.16: Contrast between sub-Rayleigh and supershear experiments: $(-55,10) \mathrm{mm}$ at $t=50 \mu \mathrm{s}$

The particle velocity curves, which are plotted to $t=49 \mu \mathrm{s}$ beyond the image frame time reveal how the near-field disturbance has leveled off and has had the net effect of slightly biasing the FP curve by $0.5 \mathrm{~m} / \mathrm{s}$ in advance of the S-wave front and sub-Rayleigh rupture.

The high-speed photoelastic image frames depicted in Figure 4.16 were acquired at $t=50 \mu \mathrm{s}$ relative to the instrumentation trigger time. The actual time in the case of the sub-Rayleigh experiment is $t=45.74 \mu \mathrm{s}$ when corrected for the measured rupture time delay $\left(t_{\text {delay }}^{s}\right)$, while the actual time in the case of the supershear experiment image frame corresponds to $t=45.86 \mu \mathrm{s}$.

The image frames corresponding to $t=50 \mu \mathrm{s}$ in both experiments show the extended lobe of each sub-Rayleigh rupture just beginning to cross the particle velocity measurement station at $(-55,10) \mathrm{mm}$.

The FN particle velocity signal in the sub-Rayleigh experiment exhibits a sudden sharp negative 
velocity swing of $-1.19 \mathrm{~m} / \mathrm{s}$ between the points $C$ and $D$ as the sub-Rayleigh rupture field lobe sweeps the station. The sense of motion is consistent with a right-lateral rupture at this location. An expanded view of the FP particle velocity trace for the sub-Rayleigh experiment shows a very subtle but detectable velocity increase of $0.13 \mathrm{~mm} / \mu \mathrm{s}$ between $a_{1}$ and $B$. It is difficult to ascertain whether this FP swing is due to the rupture or part of the background near-field signal. Either way, there is clearly very little FP motion transported to this off-fault location by the sub-Rayleigh field. The sub-Rayleigh velocity field is dominated by the FN component as it should be according to theory.

Meanwhile in the supershear experiment, similar features are observed within the portion of each particle velocity trace corresponding to the arrival of the trailing sub-Rayleigh rupture. A negative velocity swing of $-1.96 \mathrm{~mm} / \mu \mathrm{s}$ is exhibited by the $\mathrm{FN}$ component between the points labeled $C$ and $D$ while a small positive velocity increase of $0.434 \mu s$ is registered in the FP signal between the points $B$ and $C$. The observed dominance of the FN component and the sign each respective velocity swing are all consistent with the expected sense of particle motion induced by a right-lateral sub-Rayleigh rupture at this location.

The high-speed photoelastic image frames depicted in Figure 4.17 were acquired at $t=60 \mu s$ relative to the instrumentation trigger time. The actual time in the case of the sub-Rayleigh experiment is $t=55.74 \mu \mathrm{s}$ when corrected for the measured rupture time delay $\left(t_{\text {delay }}^{s}\right)$, while the actual time in the case of the supershear experiment image frame corresponds to $t=55.86 \mu s$.

The supershear rupture and the trailing sub-Rayleigh rupture, which follows in its wake have both long since swept through by the off-fault measurement station at $(-55,10) \mathrm{mm}$. The velocity swings within the FP and FN records, which occur between the S-wave arrival time $\left(t_{s}\right)$ and the rupture (on-fault) arrival time $\left(t_{\text {rup }}\right)$, are clearly characterized by a dominant FN velocity swing. Note how the negative velocity swing in the "trailing Rayleigh" signal mimics the observed velocity swing between points $C$ and $D$ in the $\mathrm{FN}$ record of the sub-Rayleigh rupture experiment. The positive velocity swing registered between the points $D$ and $E$ in the in the $\mathrm{FN}$ record on the right was induced by the trailing edge of the rupture field lobe. Note as well how this portion of the 


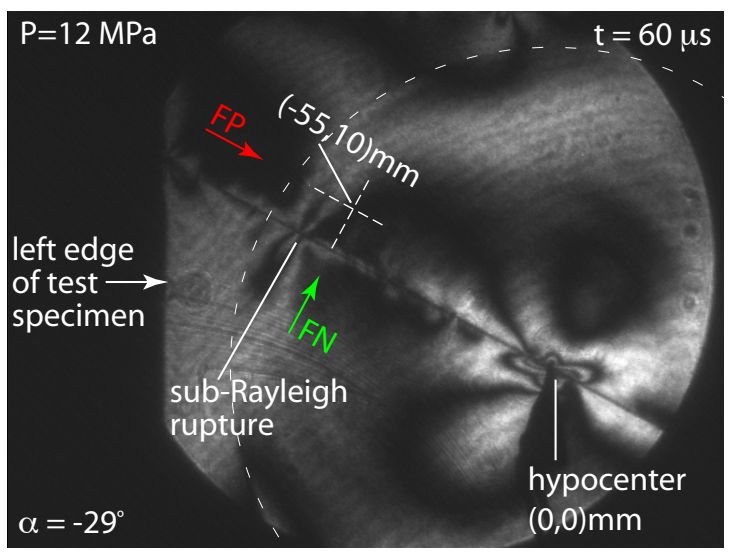

sub-Rayleigh experiment
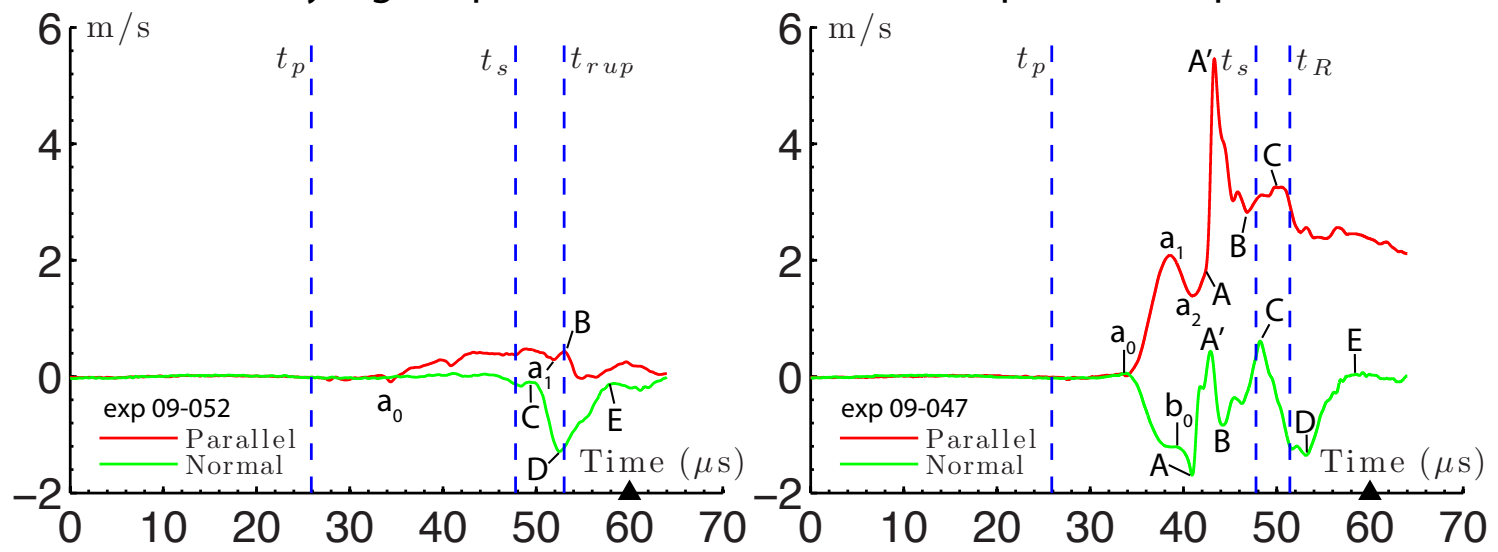

Figure 4.17: Contrast between sub-Rayleigh and supershear experiments: $(-55,10) \mathrm{mm}$ at $t=60 \mu \mathrm{s}$

record very much mimics the positive velocity swing between points $D$ and $E$ in FN record of the sub-Rayleigh rupture experiment on the left. Beyond this time, both signals proceeded to settle down to nearly constant levels.

The particle velocity traces for each experiment, plotted out to $t=64 \mu s$, provide a final graphical contrast between the sub-Rayleigh and supershear ground motion. In summary, the supershear particle velocity records are comprised of three distinct segments. The first distinct segment spans from $a_{0} \rightarrow A$ in the $\mathrm{FP}$ and $\mathrm{FN}$ records and corresponds to the passage of the leading dilatational field lobe, which precedes the arrival of the shear Mach front. The observed dominance of the FP motion in the dilatational portion of the record at this measurement location is consistent with theoretical and numerical predictions. The second distinct segment of the FP and FN records, spans between the points $A \rightarrow A^{\prime}$ and corresponds to the arrival of the shear Mach front. The observed 

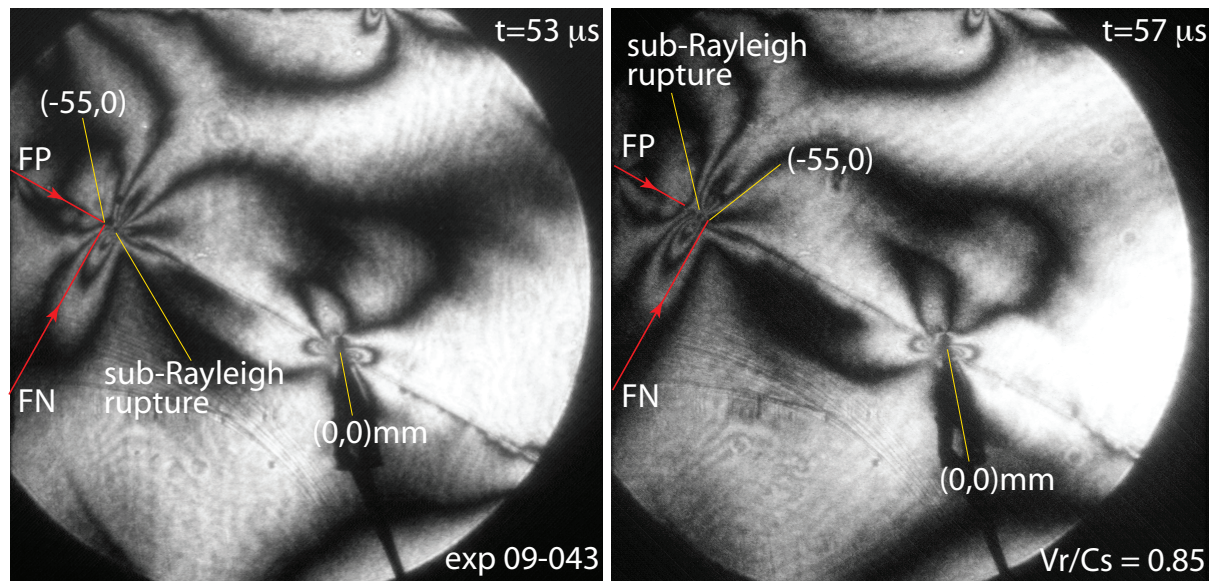

Figure 4.18: Consecutive photoelastic image frames capture the sub-Rayleigh rupture crossing the measurement station located at $(-55,0) \mathrm{mm}$. The red lines in the image highlight the optical paths of the FP and FN interferometer beams, which simultaneously monitored the particle velocity at this location.

dominance of the FP component and the noted positive velocity jumps exhibited by each component at $(-55,10) \mathrm{mm}$, are consistent with the predicted sense of motion for a stable supershear rupture $\left(\sqrt{2} C_{s}<V_{r}<C_{p}\right)$ crossing a measurement station on the extensional side of the fault. The third distinct segment of the supershear particle velocity records, corresponds to the "trailing Rayleigh" portion of the record. which spans between $C \rightarrow E$ in the FN record and the corresponding portion of the FP record starting from point $B$ and extending through point $C$. The "trailing Rayleigh" portion of the record is now dominated by the $\mathrm{FN}$ component. The observed negative velocity swing of the FN component and positive swing in the FP component at $(-55,10) \mathrm{mm}$ is consistent in every respect with the predicted sense of motion for a sub-Rayleigh rupture field on the extensional side of the fault.

The particle velocity records, which resulted in the sub-Rayleigh experiment also feature a dominant FN component as expected. Note as well that the general profile of the FN record in the sub-Rayleigh experiments mimics the "trailing Rayleigh" portion of the supershear experiment record (and vice versa). The resulting fault-parallel component in the sub-Rayleigh experiment was weak and did not extend much above the biased FP motion introduced by the near-field disturbance, which peaked at $0.48 \mathrm{~m} / \mathrm{s}$. Regardless of this fact, the experiments show that there is clearly very little FP motion transported to the off-fault locations by the sub-Rayleigh field. 


\subsection{Examination of $\left|\delta \dot{u}_{y}(x, y)\right| /\left|\delta \dot{u}_{x}(x, y)\right|$ in sub-Rayleigh par- ticle velocity records using the $(\mathrm{FN}, \mathrm{FP})$ laser interfer- ometer probe configuration}

\subsubsection{Design of experiment}

A series of sub-Rayleigh rupture experiments were conducted using the (FP,FN) fiber-optic probe configuration depicted in Figure 4.1. All tests in this particular series were conducted using $6^{\prime \prime} \times$ $6^{\prime \prime} \times 0.375^{\prime \prime} \mathrm{H}-100$ specimens, which featured a fault angle of $\alpha=-29^{\circ}$. Simultaneous particle velocity records were obtained at various distances from the fault as a sub-Rayleigh rupture and its surrounding field swept past the measurement station. A synchronized, photoelastic image sequence of the event was also obtained using high-speed photography. The precise location of the subRayleigh rupture was identified in high-speed image frames and directly correlated to the observed velocity swings exhibited by each particle velocity component. Additional details of the experimental configuration and all measurement procedures are summarized in Section 4.2.1.

\subsubsection{Results, analysis, and interpretation of particle velocity records and photoelastic images}

Figure 4.18 depicts a pair of photoelastic images obtained from a sub-Rayleigh experiment (exp09043), which was conducted with a static compressive load of $P=19 M P a$ applied to the specimen. A bilateral sub-Rayleigh rupture was produced and a $2 M P a$ reduction of the static compressive load was noted at the conclusion of the experiment as registered by the load dial indicator of the hydraulic press.

The photoelastic image frames in Figure 4.18 reveal a sub-Rayleigh rupture as it crossed the particle velocity measurement station located at $(-55,0) \mathrm{mm}$. An expanded view of the images reveals a dark, concentrated caustic zone on the fault corresponding to the position of the advancing rupture tip. The image on the left, acquired at $t=53 \mu s$, depicts the sub-Rayleigh rupture tip 


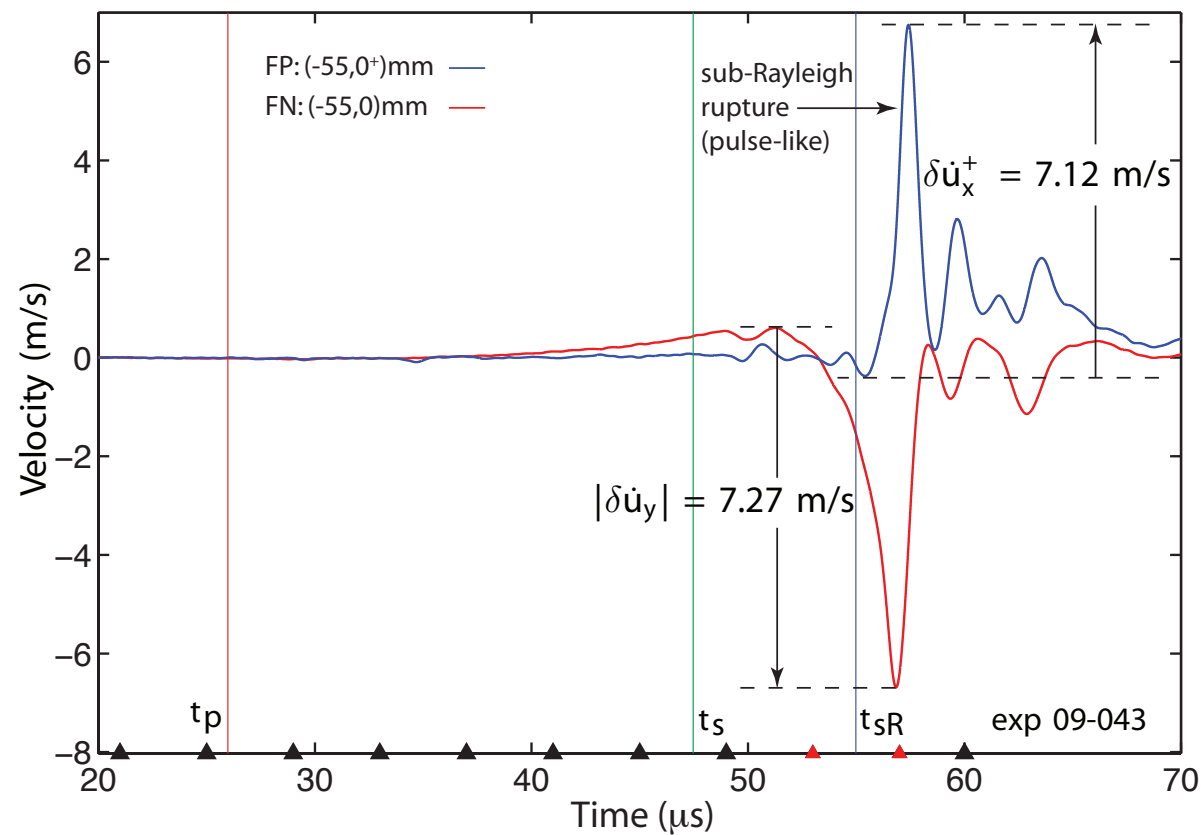

Figure 4.19: Pulse-like particle velocity profile of the sub-Rayleigh rupture portrayed in Figure 4.18. The curves are characterized by a FN component, whose magnitude is only slightly greater than the magnitude of the FP velocity signal $\left(\left|\delta \dot{u}_{y}\right| /\left|\delta \dot{u}_{x}\right|^{+}=1.02\right)$.

approaching the on-fault measurement station, while the image on the right, acquired at $t=57 \mu s$, reveals the rupture just after it crossed the station. The local maximum shear stress field surrounding the rupture tip is revealed by the prominent fringe lobes extending to either side of the fault. A faint relic of the expanding circular S-wave front is also visible in the lower-left corner of the $t=53 \mu s$ image frame. The red lines in the images highlight the optical paths of the FP and FN fiberoptic probe beams, which were focused at nearly grazing incidence onto the lower-left corner of a retro-reflective strip of tape (also visible in an expanded view). Scattered laser light was collected and interfered to generate continuous particle velocity records corresponding to $\dot{u}_{x}\left(-55,0^{+}\right)$and $\dot{u}_{y}(-55,0)$. Note that the red line corresponding to the FP beam in the right-hand image has not been extended to the station in order to avoid covering the small dark caustic zone corresponding to the sub-Rayleigh rupture tip.

Figure 4.19 depicts the continuous FP and FN particle velocity records obtained from the experiment (exp09-043). The two red triangles on the time axis correspond to the times at which the consecutive image frames in Figure 4.18 were acquired. The red and green vertical lines labeled $t_{p}$ 

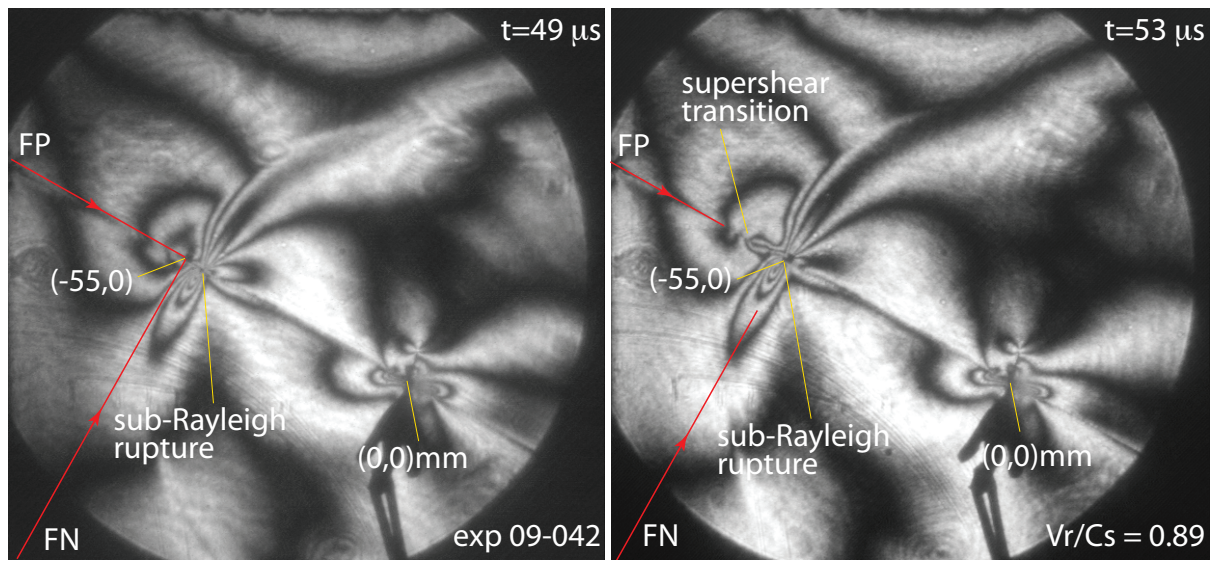

Figure 4.20: Consecutive photoelastic image frames capture the sub-Rayleigh rupture crossing the particle velocity measurement station located at $(-55,0) \mathrm{mm}$. The $t=53 \mu \mathrm{s}$ image frame reveals the sudden emergence of a daughter crack and the onset of a supershear transition.

and $t_{s}$ mark the anticipated arrival times of the leading $\mathrm{P}$ - and $\mathrm{S}$-wave fronts at the measurement station. The blue vertical line positioned at $t=55 \mu \mathrm{s}$ marks the rupture arrival time at the measurement station as determined by tracking the position of the rupture tip. The noted arrival time corresponds to an average rupture speed of $V_{r}=0.85 C_{s}$ and also correlates well with the sudden sharp velocity increase exhibited by the FP record. Note that the notation $\delta u_{1}^{+}$signifies that the FP particle velocity measurement was conducted on the extensional side of the fault at a point estimated to lie within $200 \mu m$ of the interface. The FP record in this instance appears strongly pulse-like and represents one-half of the slip function. The $(+)$ notation is dropped in the case of the FN component since the sense of motion for this component is continuous across the fault. The magnitudes of the FN and FP velocity swings, determined by

$$
\begin{gathered}
\left|\delta \dot{u}_{x}^{+}\right|=\left|\left(\dot{u}_{1}^{+}\right)_{\text {max }}-\left(\dot{u}_{x}^{+}\right)_{\text {min }}\right| \\
\left|\delta \dot{u}_{y}\right|=\left|\left(\dot{u}_{2}\right)_{\text {max }}-\left(\dot{u}_{y}\right)_{\text {min }}\right|
\end{gathered}
$$

are each noted in Figure 4.19. The "dominance" of the FN signal is observed although in this instance the FP velocity swing given by $\left|\delta u_{1}^{+}\right|=7.12 \mathrm{~m} / \mathrm{s}$ approaches to within $98 \%$ of the FN velocity swing given by $\left|\delta u_{2}\right|=7.27 \mathrm{~m} / \mathrm{s}$. 


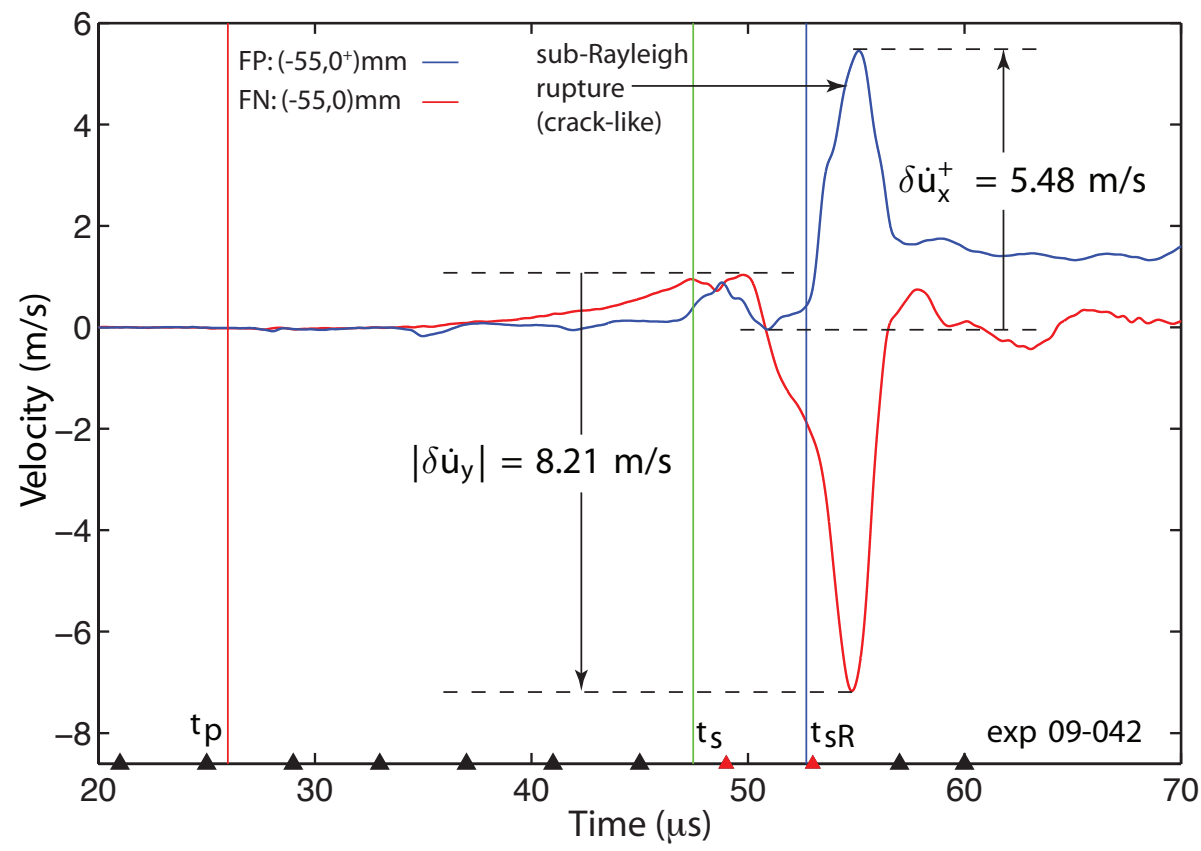

Figure 4.21: Crack-like particle velocity profile of the sub-Rayleigh rupture portrayed in Figure 4.20. The curves are characterized by a dominant FN component, whose magnitude is $1.5 \times$ greater than the magnitude of the FP velocity signal $\left(\left|\delta \dot{u}_{y}\right| /\left|\delta \dot{u}_{x}^{+}\right|=1.50\right)$.

Figure 4.20 depicts a pair of photoelastic images obtained from a second laboratory earthquake experiment (exp09-042), which was also conducted with an initial static compressive load of $P=$ $19 M P a$. A bilateral sub-Rayleigh rupture once again resulted and a $3.4 M P a$ stress drop was noted at the conclusion of the experiment.

The image frame on the left in Figure 4.20, corresponding to $t=49 \mu \mathrm{s}$, reveals a sub-Rayleigh rupture approaching the measurement station located at $(-55,0) \mathrm{mm}$. As was the case in the previous experiment, an expanded view of the image reveals a dark, concentrated caustic zone on the fault corresponding to the advancing rupture tip. The prominent local maximum shear stress field surrounding the rupture tip is revealed by the fringe pattern as is the expanding circular S-wave front, which is more-clearly visible in this experiment. An expanded view of the image on the right, acquired at $t=53 \mu \mathrm{s}$, reveals that the rupture tip has now just cleared the measurement station. A rupture arrival time estimate of $t=52.9 \mu \mathrm{s}$ was determined from the photoelastic image sequence. The $t=53 \mu \mathrm{s}$ image frame also reveals the sudden emergence of a daughter crack and the onset of a supershear transition. The image is in stark contrast to the image of the sub-Rayleigh rupture 


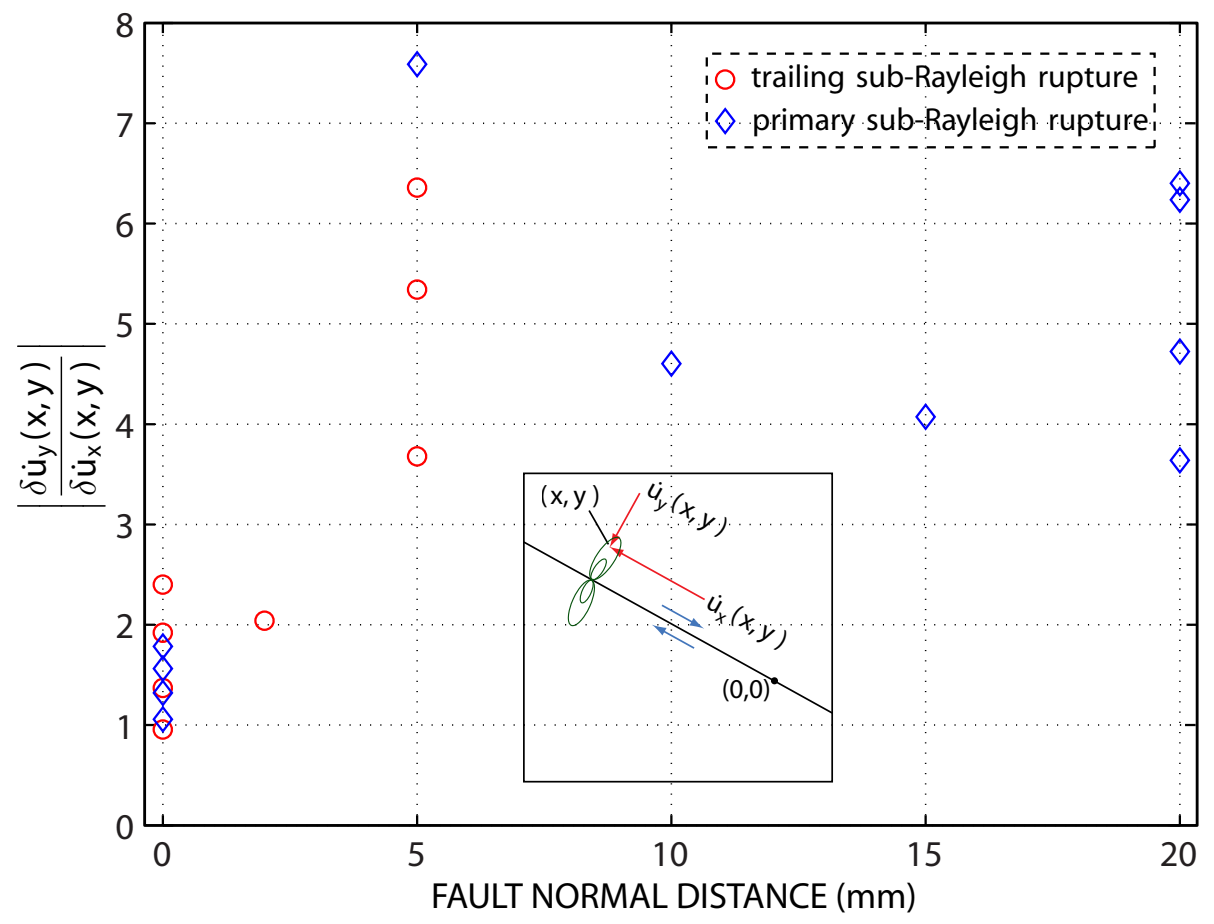

Figure 4.22: Scatter plot of the $\left|\delta \dot{u}_{y}\right| /\left|\delta \dot{u}_{x}^{+}\right|$for sub-Rayleigh and trailing-sub-Rayleigh ruptures resulting from broad range of laboratory earthquake experiments

acquired only $4 \mu s$ earlier. A closer examination of the left-hand image frame (in hindsight) reveals a subtle hint of the looming transition by the slightly distorted appearance of the interior fringe of the leading S-field radiation lobe.

Figure 4.21 depicts the continuous FP and FN particle velocity records obtained from experiment exp 09-042. As in the previous example, the two red triangles mark the times at which the consecutive image frames in Figure 4.20 were acquired. The blue vertical line positioned at $t=52.9 \mu \mathrm{s}$ marks the estimated rupture arrival time at the measurement station as determined from the rupture tip positions in the high-speed images. The noted rupture arrival time corresponds to an average rupture speed of $V_{r}=0.88 C_{s}$ and also correlates very well with the sudden sharp velocity increase exhibited by the FP record. Note as well how the rupture was crack-like in this experiment as revealed by the nearly constant sliding speed of $\dot{u}_{x} 1.3 \mathrm{~m} / \mathrm{s}$, which was sustained well after the rupture had crossed the measurement station. The particle velocity records are dominated by the FN component, which exhibits a velocity swing, which is $1.5 \times$ greater in magnitude than the velocity swing displayed by the 
FP component. The higher recorded ratio between the FN and FP velocity swings of $\left|\delta \dot{u}_{y}\right| /\left|\delta \dot{u}_{x}^{+}\right|=$ 1.5 in the case of the crack-like rupture (exp09-042) compared to $\left|\delta \dot{u}_{y}\right| /\left|\delta \dot{u}_{x}^{+}\right|=1.02$ in the case of the pulse-like rupture (exp09-043) is typical of what is experimentally observed between crack-like and pulse-like ruptures. Finally, note that the leading (shorter) peak in the FP record at $t=t_{s}$, which is attributed to the emergence of the supershear daughter crack on the leading S-wave front. The experiment demonstrates a sub-Rayleigh rupture velocity field, which is dominated by the FN component leading all the way up to the onset of a supershear transition.

The experiments (exp09-042) and (exp09-043) are representative of a broad range of laboratory earthquake experiments, which were conducted using the (FN,FP) fiber-optic laser interferometer probe configuration. The objectives of these experiments varied but they were all re-examined after the fact in order to assess the ratio between the magnitudes of the FN and FP components. Figure 4.22 is a scatter plot of the ratio $\left|\delta \dot{u}_{y}\right| /\left|\delta \dot{u}_{x}^{+}\right|$for sub-Rayleigh and trailing sub-Rayleigh ruptures, obtained from 19 separate laboratory earthquake experiments. The blue diamonds in the plot correspond to sub-Rayleigh rupture experiments and the red circles correspond to the ratio $\left|\delta \dot{u}_{y}\right| /\left|\delta \dot{u}_{x}^{+}\right|$applied to the trailing Rayleigh portion of various supershear records. The experiments were conducted at various static compressive loads, which also resulted in a wide range of stress drops. The scatterplot mainly serves to demonstrate the dominance if the FN component in subRayleigh ruptures as experimentally observed over a wide range of test conditions. As previously noted, there does appear to be an observed trend whereby the ratio $\left|\delta \dot{u}_{y}\right| /\left|\delta \dot{u}_{x}^{+}\right| \rightarrow 1^{+}$in the case of pulse-like sub-Rayleigh ruptures. 


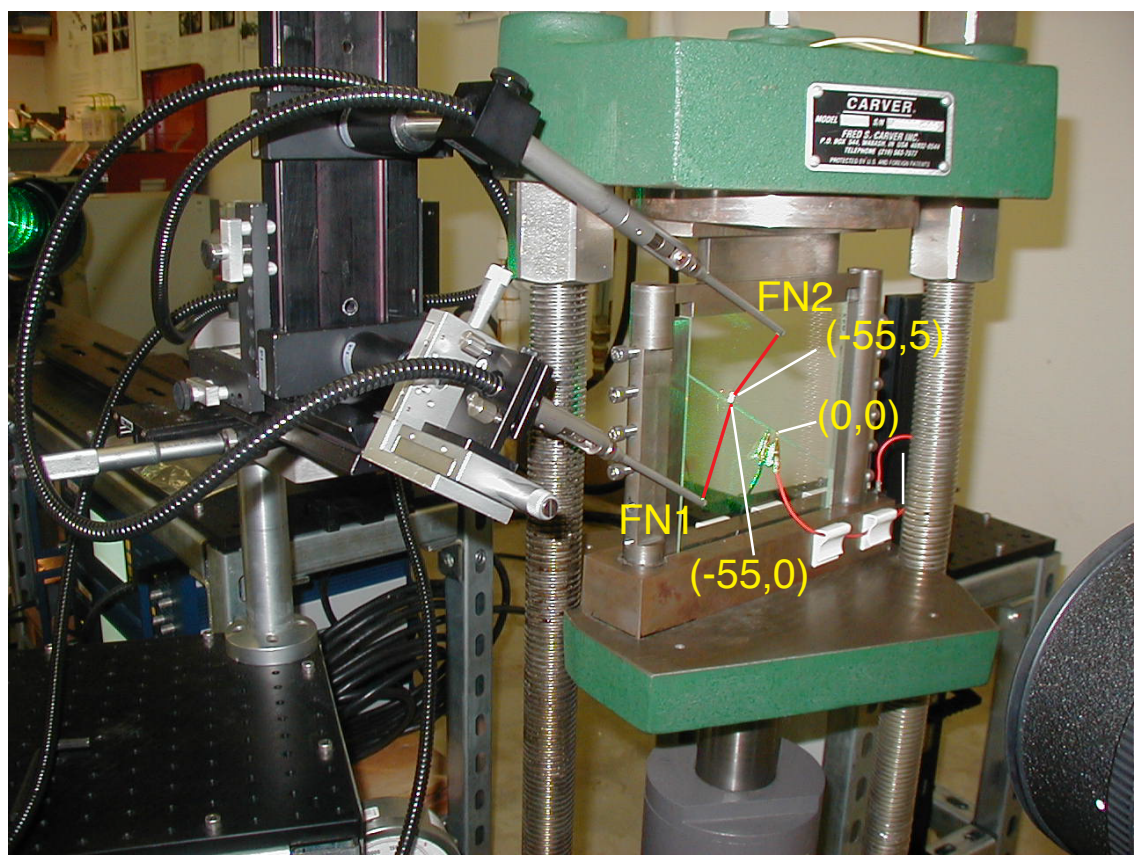

Figure 4.23: Laboratory earthquake experiment featuring the paired $\mathrm{FN}$ laser interferometer probe beam configuration for the characterization of sub-Rayleigh particle velocity field decay

\subsection{Investigation of sub-Rayleigh particle velocity field decay using an (FN1,FN2) laser interferometer probe arrange-}

\section{ment}

\subsubsection{Design of experiment}

A series of sub-Rayleigh rupture experiments were conducted using the laser interferometer probe configuration depicted in Figure 4.23. All tests in this series were conducted using a $6^{\prime \prime} \times 6^{\prime \prime} \times 0.375^{\prime \prime}$ H-100 specimens, which featured a fault angle of $\alpha=-29^{\circ}$. Simultaneous FN particle velocity records were obtained at an on-fault and an off-fault measurement station as depicted in the image. Experiments were conducted at various FN distances and the ratio of the resulting velocity swings was examined. A synchronized, photoelastic image sequence of the event was also obtained using high-speed photography. The precise location of the sub-Rayleigh rupture was identified in highspeed image frames and directly correlated to the observed velocity swings exhibited by each particle 


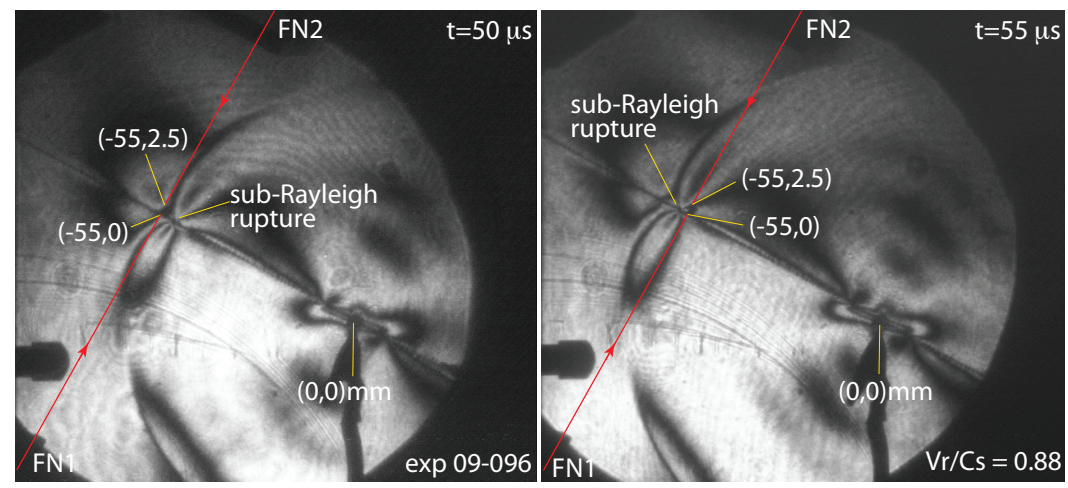

Figure 4.24: Consecutive photoelastic image frames capture the sub-Rayleigh rupture and its surrounding stress field crossing the particle velocity measurement stations located at $(-55,0) \mathrm{mm}$ and $(-55,2.5) \mathrm{mm}$. The red lines in the image highlight the optical paths of the two FN interferometer probe beams, which simultaneously monitored the particle velocity at these locations.

velocity component.

\subsubsection{Results, analysis, and interpretation of particle velocity records and photoelastic images}

Figure 4.24 depicts a pair of photoelastic images obtained from a sub-Rayleigh experiment (exp09096), which was conducted with a static compressive load of $P=11 M P a$ applied to the specimen.

A bilateral sub-Rayleigh rupture was produced and a $1 \mathrm{MPa}$ reduction of the static compressive load was noted at the conclusion of the experiment as registered by the load dial indicator of the hydraulic press.

The photoelastic image frames in Figure 4.24 reveal a sub-Rayleigh rupture and its surrounding maximum shear stress field as it crossed two closely spaced particle velocity measurement stations located at $(-55,0) \mathrm{mm}$ and $(-55,2.5) \mathrm{mm}$. An expanded view of the images reveals the familiar dark, concentrated caustic zone on the fault corresponding to the position of the advancing rupture tip in each image frame. The image on the left, acquired at $t=50 \mu \mathrm{s}$, depicts the sub-Rayleigh rupture tip approaching the on-fault measurement station, while the image on the right, acquired at $t=55 \mu \mathrm{s}$, reveals the rupture just after it crossed the on-fault station. A rupture arrival time estimate of $t=53.4 \mu \mathrm{s}$ was determined from the photoelastic image sequence. The images also show the leading edge of the prominent field lobe approaching the off-fault station at $t=50 \mu s$ and the 


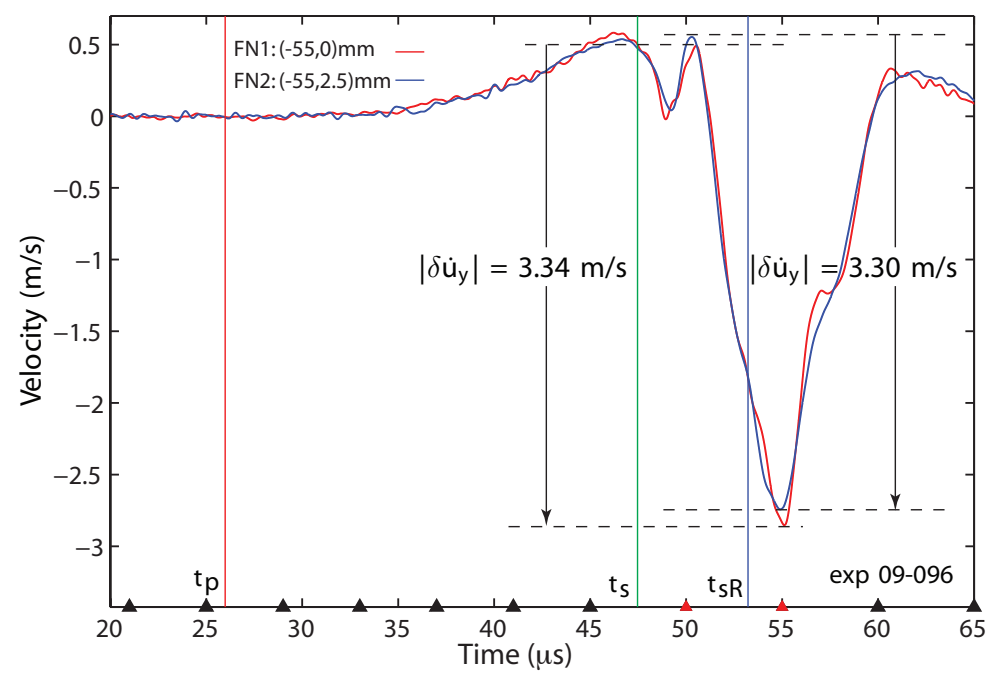

Figure 4.25: Paired FN particle velocity traces corresponding to the sub-Rayleigh rupture depicted in Figure 4.24. Virtually identical FN particle velocity profiles are observed at the on-fault and off-fault measurement stations.

trailing edge of the field lobe crossing the measurement station at $t=55 \mu \mathrm{s}$.

The red lines in the images highlight the optical paths of the FN fiber-optic probe beams, which were focused at nearly grazing incidence onto the upper and lower-left corner of the retro-reflective strip of tape (also visible in an expanded view). Scattered laser light was collected and interfered to generate continuous particle velocity records corresponding to $\dot{u}_{y}\left(-55,0^{+}\right)$and $\dot{u}_{y}(-55,2.5)$.

Figure 4.25 depicts the continuous paired FN particle velocity records obtained from the same experiment (exp09-096). The two red triangles on the time axis correspond to the times at which the consecutive image frames in Figure 4.24 were acquired. The blue vertical line positioned at $t=53.4 \mu \mathrm{s}$ marks the rupture arrival time estimate at the measurement station as determined by tracking the position of the tip. The noted arrival time corresponds to an average rupture speed of $V_{r}=0.88 C_{s}$. The two particle velocity records exhibit virtually identical profiles at the two closely spaced measurement locations and show no appreciable decay at such a short distance from the fault.

Figure 4.26 depicts a pair of photoelastic images obtained from a second laboratory earthquake experiment (exp09-073), which was conducted with an initial static compressive load of $P=7.6 M P a$. A bilateral sub-Rayleigh rupture resulted and a $0.6 M P a$ stress drop was noted at 

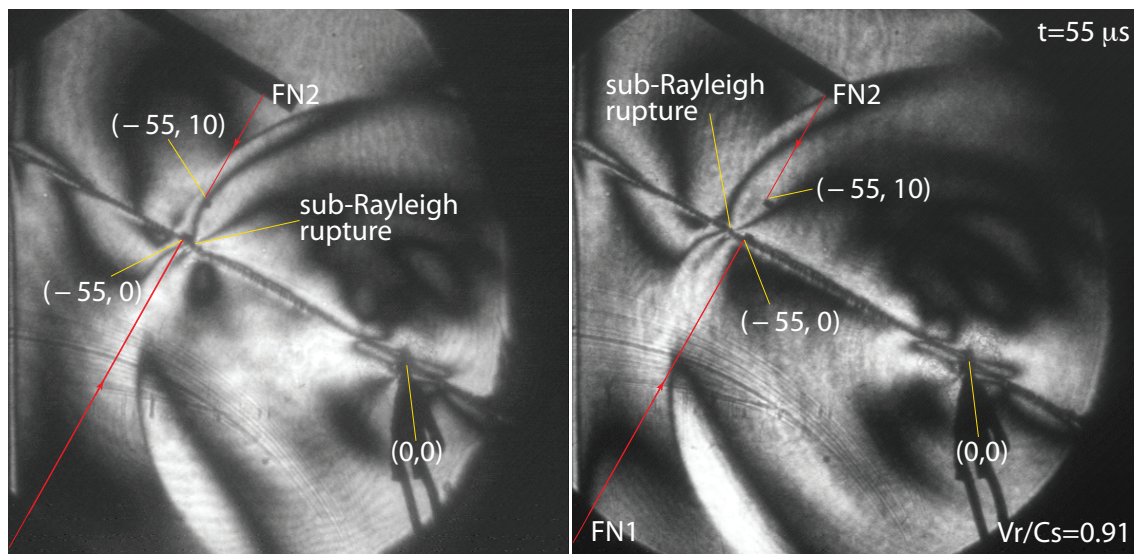

Figure 4.26: Consecutive photoelastic image frames capture the sub-Rayleigh rupture and its surrounding stress field crossing the particle velocity measurement stations located at $(-55,0) \mathrm{mm}$ and $(-55,10) \mathrm{mm}$. The red lines in the image highlight the optical paths of the two FN interferometer probe beams, which simultaneously monitored the particle velocity at these locations.

the conclusion of the experiment.

The photoelastic image frames in Figure 4.26 reveal a sub-Rayleigh rupture and its surrounding maximum shear stress field as it crossed the two particle velocity measurement stations located at $(-55,0) \mathrm{mm}$ and $(-55,10) \mathrm{mm}$. The prominent lobes of the local maximum shear stress field surrounding the rupture tip, and the expanding circular S-wave fronts are clearly visible in each image frame. The image on the left, acquired at $t=50 \mu \mathrm{s}$, depicts the sub-Rayleigh rupture tip approaching the on-fault measurement station, while the image on the right, acquired at $t=55 \mu \mathrm{s}$, reveals the rupture just after it crossed the on-fault station. A rupture arrival time estimate of $t=51.88 \mu \mathrm{s}$ was determined from the photoelastic image sequence. The images also show the leading edge of the prominent field lobe crossing the off-fault station at $t=50 \mu s$ and the trailing edge of the field lobe crossing the approaching station at $t=55 \mu \mathrm{s}$.

Figure 4.27 depicts the continuous paired FN particle velocity records obtained from the same experiment (exp09-073). The two red triangles on the time axis correspond to the times at which the consecutive image frames in Figure 4.26 were acquired. The solid red and green vertical lines labeled $t_{p}$ and $t_{s}$ mark the anticipated arrival times of the leading $\mathrm{P}$ - and $\mathrm{S}$-wave fronts at the onfault measurement station. The dashed red and green lines correspond to the S- and P-wave arrival times at the off-fault station. The blue vertical line positioned at $t=51.88 \mu \mathrm{s}$ marks the rupture 


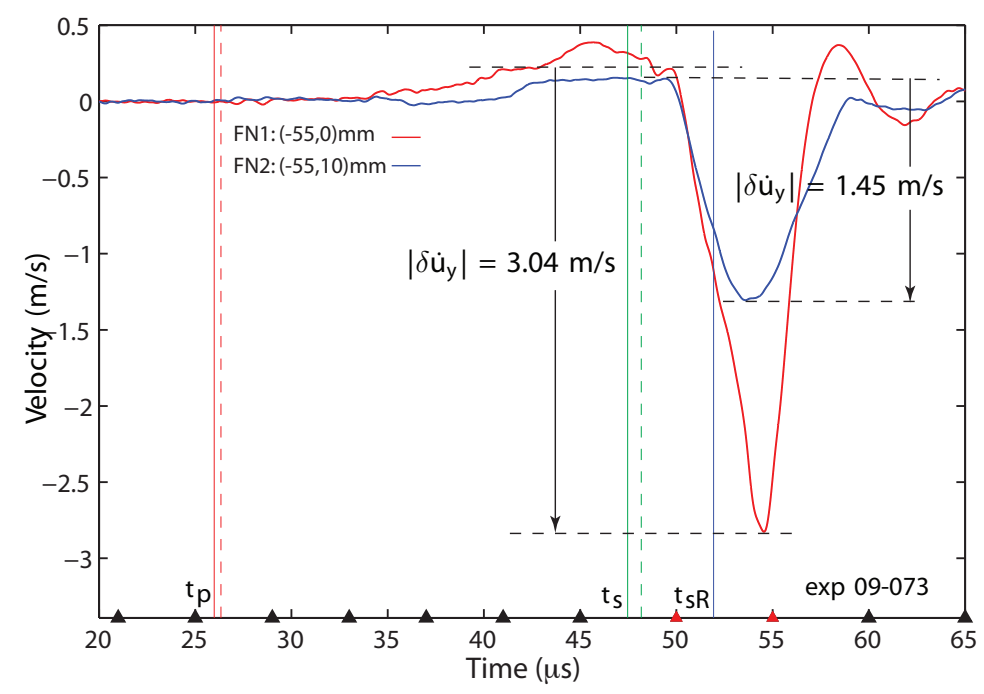

Figure 4.27: Paired FN particle velocity traces corresponding to the sub-Rayleigh rupture depicted in Figure 4.26. The FN record obtained at $(-55,10) \mathrm{mm}$ exhibits a signal amplitude which is $48 \%$ of its corresponding on-fault value.

arrival time estimate at the on-fault measurement station as determined from the photoelastic image sequence. In this case the noted arrival time corresponds to an average rupture speed of $V_{r}=0.91 C_{s}$. The two symmetric particle velocity profiles in Figure 4.27 correlate to the passage of the rupture tip and its surrounding field at the two measurement stations. The amplitude of the FN record obtained at $(-55,10) \mathrm{mm}$ is $48 \%$ of the corresponding on-fault signal amplitude.

Figure 4.28 depicts a pair of photoelastic images obtained from a third laboratory earthquake experiment (exp09-073), which was conducted with an initial static compressive load of $P=12 M P a$. A bilateral sub-Rayleigh rupture resulted and a $0.2 M P a$ stress drop in the static-compressive stress was noted at the conclusion of the experiment.

The photoelastic image frames in Figure 4.28 reveal a sub-Rayleigh rupture and its surrounding maximum shear stress field as it crossed the two particle velocity measurement stations located at $(-55,0) \mathrm{mm}$ and $(-55,24) \mathrm{mm}$. The prominent lobes of the local maximum shear stress field surrounding the rupture tip are clearly visible within each image frame along faint but detectable traces of the circular S-wave front. The image on the left, acquired at $t=50 \mu \mathrm{s}$, depicts the subRayleigh rupture tip approaching the on-fault measurement station, while the image on the right, acquired at $t=55 \mu \mathrm{s}$, reveals the rupture just after it crossed the on-fault station. A rupture arrival 


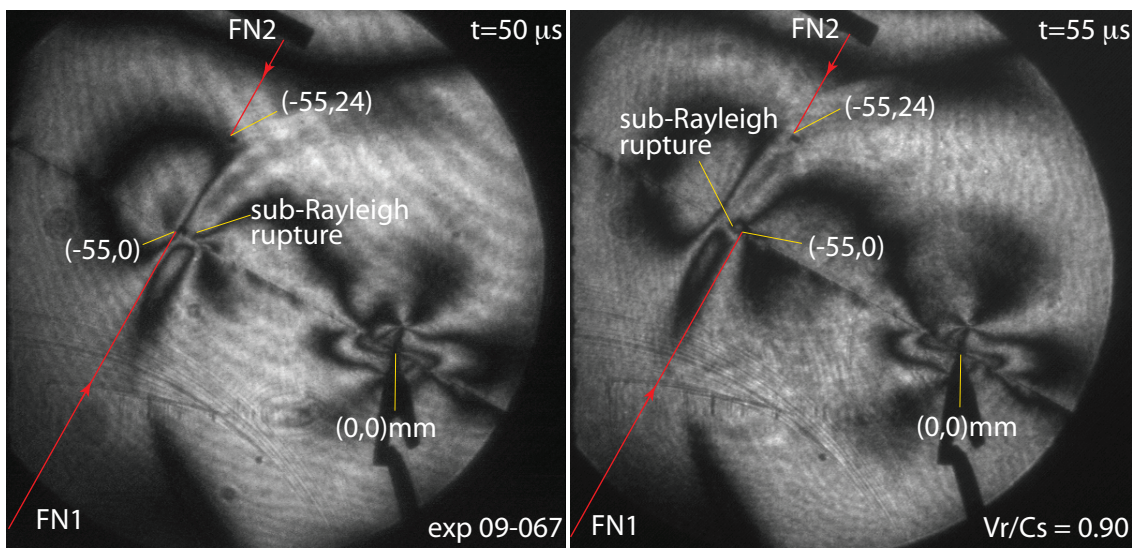

Figure 4.28: Consecutive photoelastic image frames capture the sub-Rayleigh rupture and its surrounding stress field crossing the particle velocity measurement stations located at $(-55,0) \mathrm{mm}$ and $(-55,24) \mathrm{mm}$. The red lines in the image highlight the optical paths of the two FN interferometer probe beams, which simultaneously monitored the particle velocity at these locations.

time estimate of $t=52 \mu \mathrm{s}$ was determined from the photoelastic image sequence. The images on the left reveals the S-wave front and the leading edge of the prominent field lobe approaching the off-fault station at $t=50 \mu \mathrm{s}$. The central portion of the extended field lobe is shown crossing the measurement station in the $t=55 \mu \mathrm{s}$ image frame.

Figure 4.29 depicts the continuous paired FN particle velocity records obtained from the same experiment (exp09-067). The two red triangles on the time axis correspond to the times at which the consecutive image frames in Figure 4.28 were acquired. The solid red and green vertical lines labeled $t_{p}$ and $t_{s}$ mark the anticipated arrival times of the leading $\mathrm{P}$ - and S-wave fronts at the on-fault measurement station. The dashed red and green lines correspond to the S- and P-wave arrival times at the off-fault station. The blue vertical line positioned at $t=52 \mu \mathrm{s}$ marks the rupture arrival time estimate at the on-fault measurement station as determined from the photoelastic image sequence. In this case the noted arrival time corresponds to an average rupture speed of $V_{r}=0.90 C_{s}$. The corresponding FN particle velocity profiles in Figure 4.29 correlate well with the observed passage of the rupture tip and its surrounding field at the two measurement stations. The amplitude of the FN record obtained at $(-55,24) \mathrm{mm}$ is $24 \%$ of the corresponding on-fault signal amplitude.

The three experiments exp09-096, exp09-073, and exp09-043 reflect the a span of laboratory earthquake experiments, which were conducted using the (FN1,FN2) interferometer probe config- 


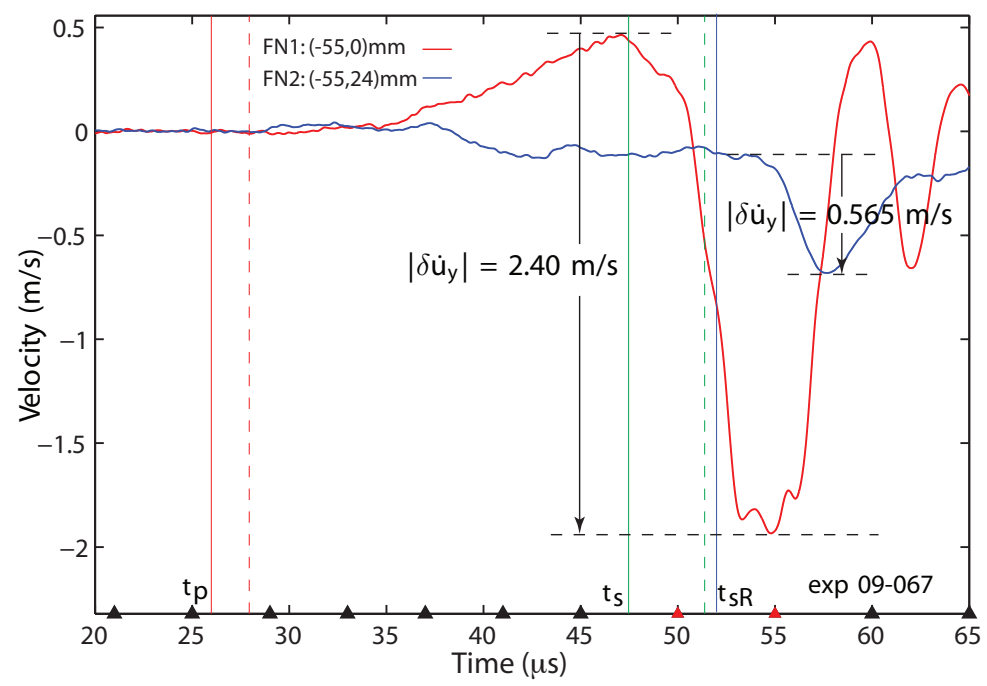

Figure 4.29: Paired FN particle velocity traces corresponding to the sub-Rayleigh rupture depicted in Figure 4.28. The FN record obtained at $(-55,24) \mathrm{mm}$ exhibits a signal amplitude which is $24 \%$ of its corresponding on-fault value

uration in order to investigate the decay of sub-Rayleigh ground motion. Figure 4.30 summarizes the results obtained from all of the sub-Rayleigh ground motion decay experiments whereby the ratio $\left|\dot{u}_{y}^{\text {off-fault }} / \dot{u}_{y}^{\text {on-fault }}\right|$ is plotted versus the FN distance. Each measurement was naturally normalized with respect to the static load and resulting load drop of the experiment from which it was obtained. The experiments were however, conducted over a range of static compressive loads and also encompass a range of global stress drops.

The inset in the figure is a simple reminder of the experimental configuration and the laser interferometer probe configuration that was used to determine the FN velocity swing at each respective measurement station. The red triangles in the figure correspond to data points while the blue curve is a simple empirical curve fit obtained using a Gaussian relationship and has no physical meaning. Note the cluster of data points corresponding to $(1 \mathrm{~mm} \leq x \leq 2.5 \mathrm{~mm})$, which consistently yielded a ratio slightly greater than unity. This is likely a manifestation of the local field associated with the process zone surrounding the rupture tip. The FN signal amplitude otherwise exhibits a rapid decay with increased FN distance as expected for a sub-Rayleigh particle velocity field. 


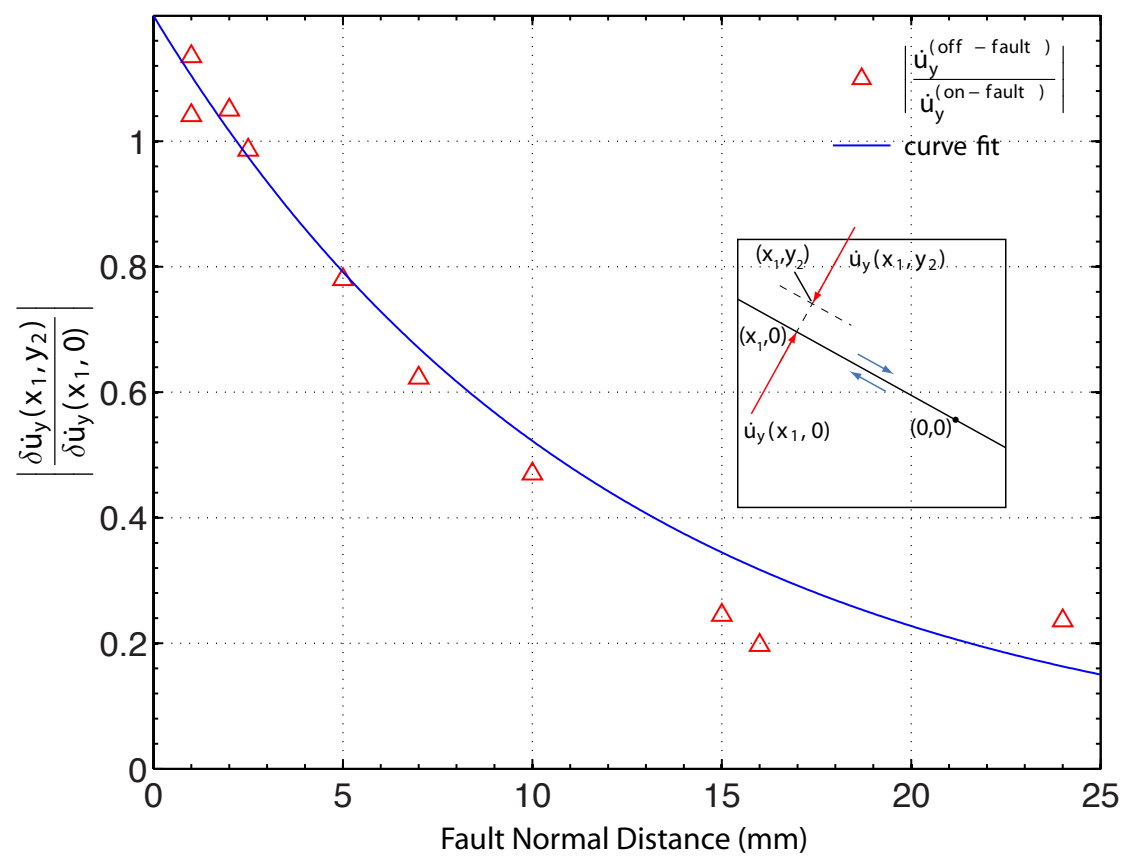

Figure 4.30: Decay of the FN signal amplitude represented by the $\left|\dot{u}_{y}^{\text {off-fault }} / \dot{u}_{y}^{\text {on-fault }}\right|$ plotted versus the FN distance. Results were obtained from 10 independent laboratory earthquake experiments using the paired FN laser interferometer probe configuration, as depicted in the inset.

\subsection{Examination of the ratio $\beta_{s}=\left|\delta \dot{u}_{x}^{s}\left(z_{s}\right)\right| /\left|\delta \dot{u}_{y}^{s}\left(z_{s}\right)\right|$ along a shear Mach front using the (FN,FP) laser interferometer probe configuration}

\subsubsection{Design of experiment}

A series of supershear experiments were conducted using the (FP,FN) laser interferometer probe configuration depicted in Figure 4.1. All tests in this particular series were conducted using $6^{\prime \prime} \times$ $6^{\prime \prime} \times 0.375^{\prime \prime} \mathrm{H}-100$ specimens, which featured a fault angle of $\alpha=-29^{\circ}$. Simultaneous particle velocity records were obtained at various distances from the fault as a supershear rupture and its surrounding field swept past the measurement station. A synchronized, photoelastic image sequence of the event was also obtained using high-speed photography. The precise location of the supershear rupture tip, leading dilatational field lobe, and shear Mach fronts were all identified in high-speed image frames and directly correlated to the observed velocity swings exhibited by each particle 


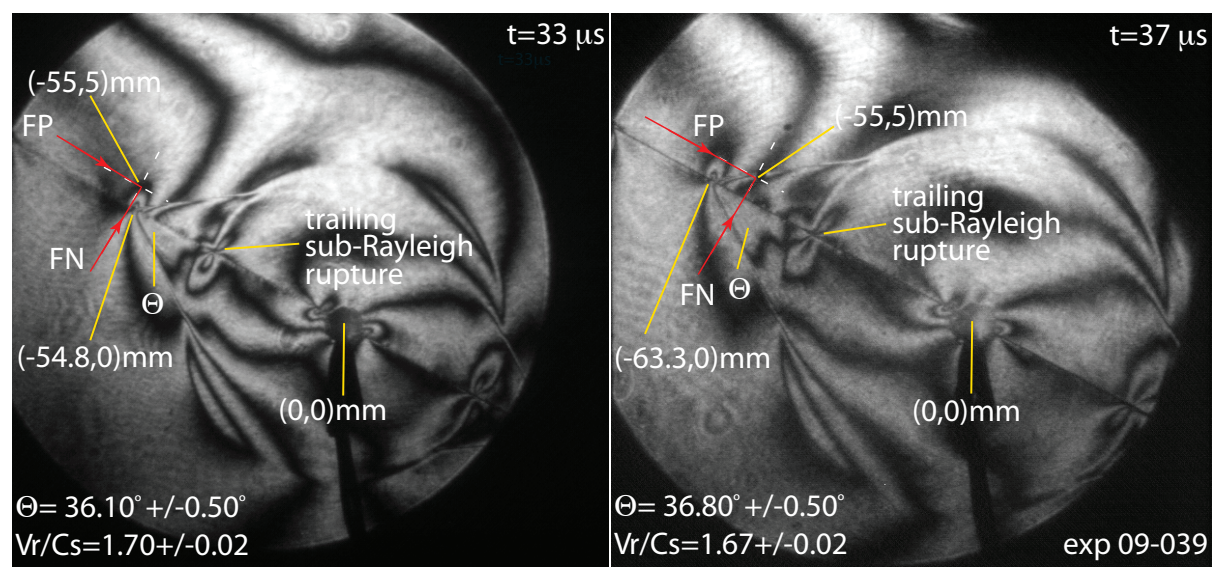

Figure 4.31: Consecutive photoelastic image frames capture a supershear rupture crossing a particle velocity measurement station positioned at $(-55,5) \mathrm{mm}$. The red lines in the image highlight the optical paths of the FN and FP interferometer probe beams, which simultaneously monitored the FN and FP particle velocity components at this location.

velocity component. The separation between the leading dilatational field lobe and the shear Mach front permits a direct measurement of the particle velocity jumps $\delta \dot{u}_{x}^{s}$ and $\delta \dot{u}_{y}^{s}$ along the shear Mach front. The ratio $\left|\delta \dot{u}_{x}^{s}\right| /\left|\delta \dot{u}_{y}^{s}\right|$ was then determined and compared to the theoretical value based upon a knowledge of the rupture speed as revealed by the high-speed image sequence. An example of this type of measurement was presented in Section 3.11.2 during the frame-by-frame evaluation of the supershear experiment (exp09-047). Nine additional supershear experiments were conducted an analyzed in a similar manner. The results from this series of experiments are presented in this section. Figure 4.31 depicts a pair of photoelastic images obtained from a supershear rupture experiment (exp09-039), which was conducted with an initial static compressive load of $P=24.8 M P a$. A bilateral sub-Rayleigh rupture resulted, which quickly transitioned to a pair of supershear ruptures, which are clearly visible in both image frames. Note as well the sharp circular S-wave front and the trailing sub-Rayleigh rupture positioned within the wake of the supershear rupture. A 3.4 MPa stress drop in the static-compressive stress was noted at the conclusion of the experiment.

The photoelastic image frames in Figure 4.28 capture the supershear rupture and its surrounding field as it crossed the particle velocity measurement station at $(-55,5) \mathrm{mm}$. The leading dilatational field lobe is clearly visible and is seen crossing the measurement station in the $t=33 \mu \mathrm{s}$ image frame. The supershear rupture tip was located at $(-54.8,0) \mathrm{mm}$ at this moment in time. The acute angle 
$(\theta)$ between the shear Mach fronts and the specimen fault was measured in order to determine the instantaneous supershear rupture speed (Section 3.11.3). An estimated measurement uncertainty of $\pm 0.50^{\circ}$ in the measurement of the Mach angle translates to a normalized rupture speed uncertainty of $\pm 0.02 \mathrm{~m} / \mathrm{s}$ through the Mach angle relationship given by

$$
V_{r} / C_{s}=\frac{1}{\sin \theta}
$$

The shear Mach front angles in the $t=33 \mu \mathrm{s}$ image frame were measured and averaged to yield a Mach angle estimate of $\theta=36.10^{\circ} \pm 0.50^{\circ}$, which translates to a normalized rupture speed of $V_{r} / C_{s}=1.70 \pm 0.02$.

The image on the right, acquired at $t=37 \mu \mathrm{s}$, captures the shear Mach front just as it crossed the measurement station. A line drawn through the sharp cusps in the upper Mach front was used to measure the Mach angle and estimate the instantaneous rupture speed (Section 3.11.3). The straight Mach front on the compressional side of the fault also facilitated an accurate measurement of the supershear rupture speed. The two Mach angles were averaged to obtain the value $\theta=36.80^{\circ} \pm 0.50^{\circ}$, which translates to a an estimated normalized rupture speed of $V_{r} / C_{s}=1.67 \pm 0.05$. The normalized rupture speed values obtained from the left and right image frames were then averaged together to obtain a final estimate for the normalized supershear rupture speed given by $V_{r} / C_{s}=1.69 \pm 0.02$. The instantaneous rupture speed estimate was also found to agree very well with the average normalized rupture speed $\bar{V}_{r} / C_{s}=1.66$ obtained through a frame-by-frame analysis of the rupture tip position spanning 3 image frames between $t=29 \mu \mathrm{s}$ and $t=37 \mu \mathrm{s}$.

Figure 4.32 depicts the FN and FP particle velocity records obtained at $(-55,5) \mathrm{mm}$ during the supershear rupture experiment (exp09-039). The red and green vertical lines at $t=26.1 \mu \mathrm{s}$ and $t=47.7 \mu \mathrm{s}$, respectively, denote the anticipated arrival times of the leading $\mathrm{P}$ - and S-wave fronts at $(-55,5 \mathrm{~mm}$. The two red triangles mark the times $t=33 \mu \mathrm{s}$ and $t=37 \mu \mathrm{s}$ when the consecutive image frames in Figure 4.31 were acquired. The blue line at $t=53.9 \mu s$ marks the anticipated arrival time of the sub-Rayleigh rupture tip at $(-55,0) \mathrm{mm}$. The dashed, black, vertical line at 


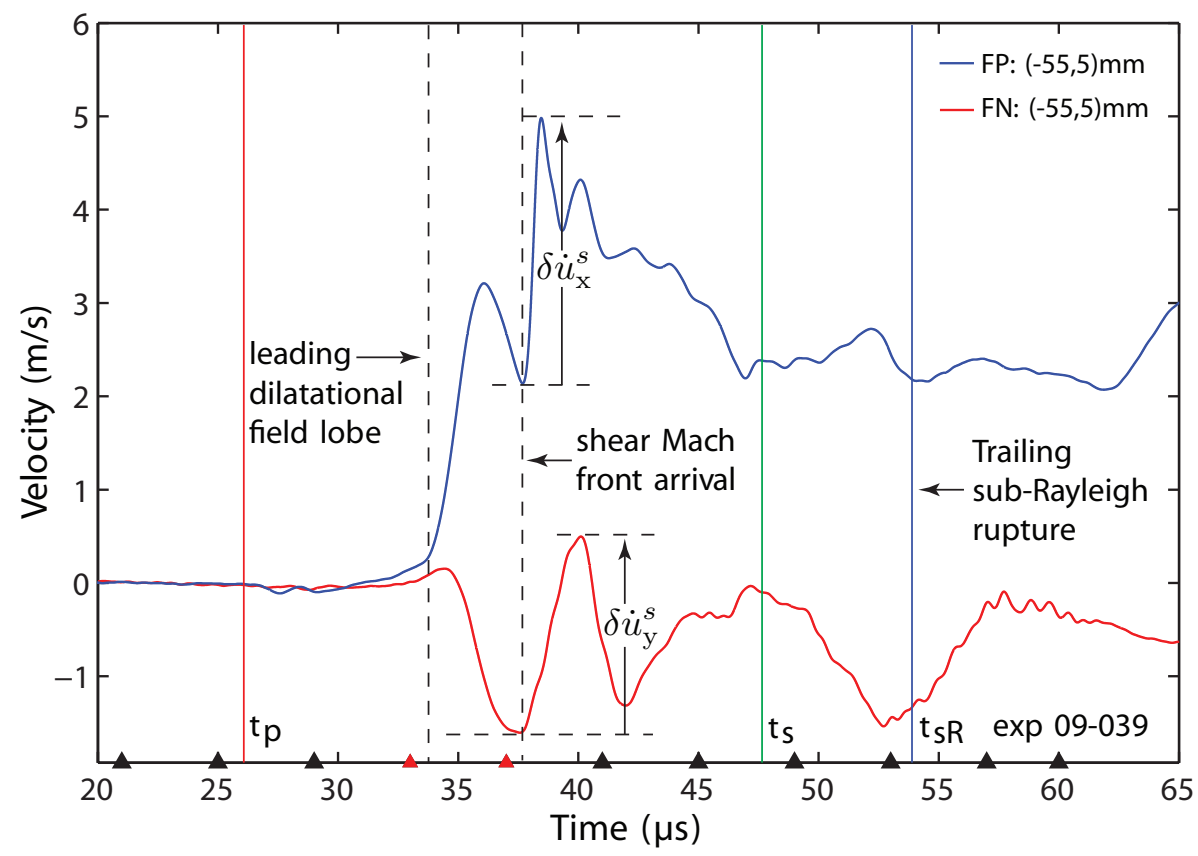

Figure 4.32: Simultaneous FN and FP particle velocity records recorded at the at the off-fault measurement positioned at $(-55,5) \mathrm{mm}$ reveal the signature of the leading dilatational field lobe followed shortly thereafter by the sharp velocity jumps associated with the arrival of the shear Mach front.

$t=33.6 \mu s$ marks the arrival of the familiar precursor signal, which is attributed to the leading dilatational field lobe. The time of the of the noted velocity jump correlates extremely well with the image of the leading dilatational field lobe shown crossing the measurement station in the left-hand $(t=33 \mu \mathrm{s})$ image frame of Figure 4.31. The second, dashed, black, vertical line at $t=37.7 \mu \mathrm{sec}$ in Figure 4.32 corresponds to the arrival of the shear Mach front at $(-55,5) \mathrm{mm}$. The noted velocity jump also correlates very well with image of the shear Mach front shown crossing the measurement station in the right-hand $(t=37 \mu s)$ image frame of Figure 4.31.

The sharp velocity jumps attributed to the passage of the shear Mach front are highlighted in Figure 4.32 and denoted as $\delta \dot{u}_{x}^{s}$ and $\delta \dot{u}_{y}^{s}$, where the superscript " $s$ " is added to emphasize the fact that the observed velocity jump corresponds to the motion carried by the shear Mach front. A detailed analysis of the particle velocity records reveals that the FP signal jumped from $\left(\dot{u}_{x}^{s}\right)_{0}=$ $2.138 \mathrm{~m} / \mathrm{s}$ to $\left(\dot{u}_{x}^{s}\right)_{f}=4.982 \mathrm{~m} / \mathrm{s}$ in the course of $0.77 \mu \mathrm{s}$, corresponding to $\delta \dot{u}_{x}^{s}=2.844 \mathrm{~m} / \mathrm{s}$ and an average acceleration of $\ddot{u}_{1}^{s}=3.7 \times 10^{6} \mathrm{~m} / \mathrm{s}^{2}$ ! In the case of the FN signal, the particle velocity 
jumped from $\left(\dot{u}_{y}^{s}\right)_{0}=-1.603 \mathrm{~m} / \mathrm{s}$ to $\left(\dot{u}_{x}^{s}\right)_{f}=0.498 \mathrm{~m} / \mathrm{s}$ in the course of $2.52 \mu \mathrm{s}$, corresponding to $\delta \dot{u}_{x}^{s}=2.101 \mathrm{~m} / \mathrm{s}$ and an average acceleration of $\ddot{u}_{1}^{s}=0.8 \times 10^{6} \mathrm{~m} / \mathrm{s}^{2}$. An experimental estimate of $\beta_{s}^{\text {meas }}=\left|\delta \dot{u}_{x}^{s}\right| / \delta \dot{u}_{y}^{s} \mid=1.354$ is thus obtained for the ratio between the FP and FN jumps along the shear Mach front. The measured value for $\beta_{s}$ may be compared to the theoretically predicted value given by

$$
\beta_{s}=\frac{\left|\delta \dot{u}_{x}^{s}\right|}{\left|\delta \dot{u}_{y}^{s}\right|}=\sqrt{V_{r}^{2} / C_{s}^{2}-1}
$$

assuming that the normalized supershear rupture speed is known. In the current experiment, the normalized rupture speed was estimated at $V_{r} / C_{s}=1.69 \mathrm{~m} / \mathrm{s} \pm 0.02 \mathrm{~m} / \mathrm{s}$ through a detailed analysis of the high-speed photoelastic image frames. The normalized rupture speed in this instance translates to a predicted value $\beta_{s}=1.362 \pm 0.025$. The value $\beta_{s}^{\text {meas }}=1.354$ obtained from the particle velocity records is found to be in excellent agreement with the theoretical and lies well within the range of estimated measurement uncertainty. The "error" in this case is $0.64 \%$ assuming that the normalized rupture speed value is precisely known.

The supershear experiment (exp09-039) presented here is representative of a series of supershear experiments exhibiting a broad range of rupture speeds that were analyzed in a similar manner. Estimates for $\beta_{s}$ obtained from each of these experiments are plotted against the corresponding normalized rupture speed in Figure 4.33. The individual data points are color coded according to the FN distance of the measurement station in the experiment. The red curve represents $\beta_{s}=$ $\sqrt{V_{r}^{2} / C_{s}^{2}-1}$. Eight out of the ten supershear experiments yielded a value for $\beta_{s}^{\text {meas }}$ that was found to be within $3.44 \%$ of the theoretically predicted value. This includes (exp09-039), which was within $0.64 \%$ of the theoretical curve. The error estimate naturally assumes that the rupture speed is precisely known. There are two noted "outliers", in the plot which were within $9 \%$ and $15.6 \%$ of the theoretical curve. Recall that the latter value corresponds to the measurement obtained from the supershear experiment analyzed in Section 4.2.2. In both cases the rupture speed was confidently estimated as it was for the other eight experiments. The noted difference between these experiments and the remaining eight was the noted presence of the near-field disturbance, as discussed in Section 4.2.2, which was strongly superimposed upon the measurement. Recall that the 


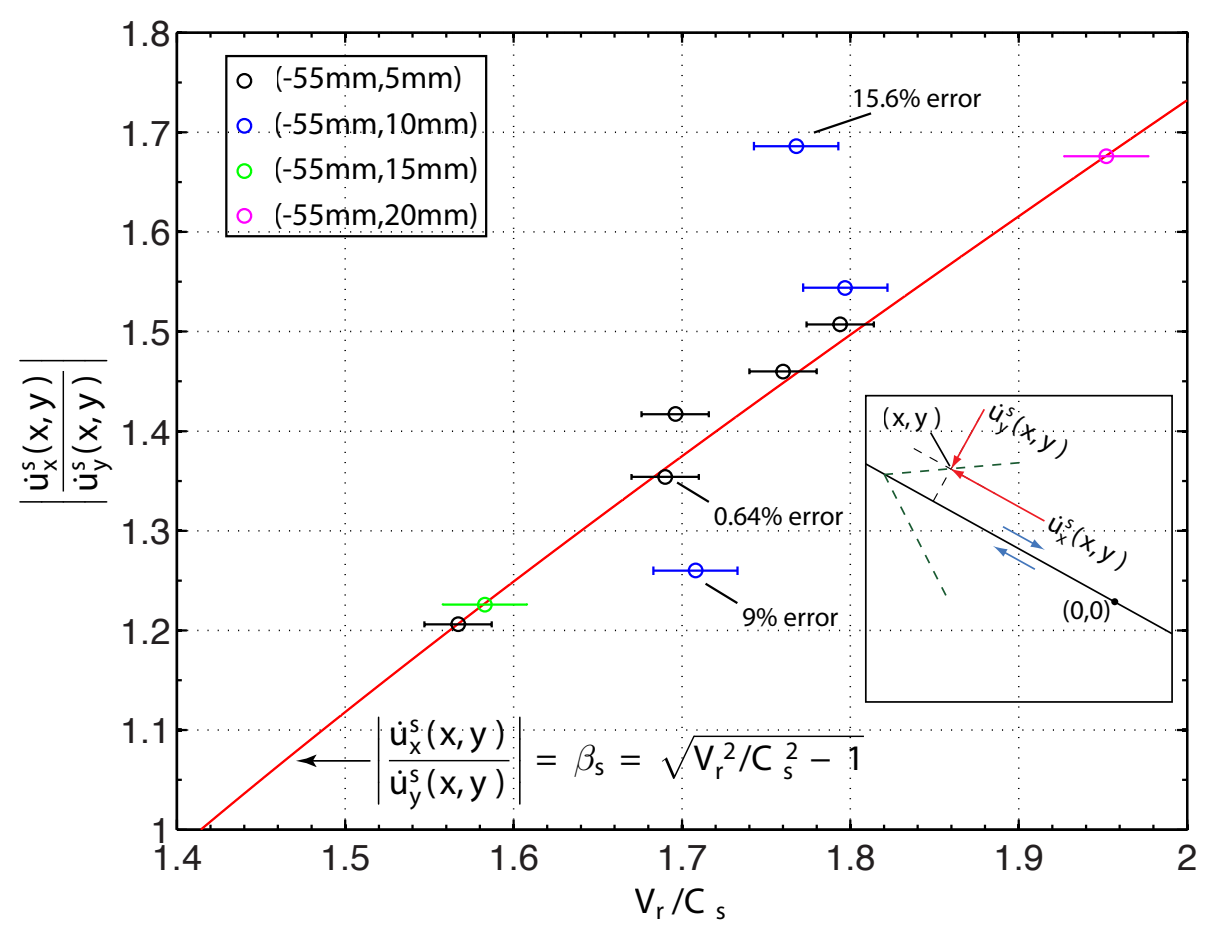

Figure 4.33: Measurements of $\beta_{s}$ determined from supershear experiments plotted versus the normalized rupture speed. Excellent agreement between theory and experiment is demonstrated across a broad range of supershear rupture speeds, which nearly span the entire stable rupture speed regime.

speed of the advancing near-field front was measured and shown to propagate at the dilatational wave speed. The common trend between the other eight "known good" experiments is that they all transitioned relatively early and thus arrived at the measurement well before the peak in the nearfield disturbance reached the measurement station. This appears to be the only scenario whereby reliable measurements of this type can be conducted given the current state of the rupture nucleation scheme. Bearing all of these points and observations in mind, the experimental results summarized by the plot in Figure 4.33 represent excellent agreement between theory and measurement. The results demonstrate that the ratio between the magnitudes of the FP and FN components along the shear Mach front is preserved out to a considerable distance from the fault (note the data point obtained at $y=20 \mathrm{~mm}$ ). Results also validate the theoretical prediction for the ratio given by $\beta_{s}=\left|\delta \dot{u}_{x}^{s}\right| / \delta \dot{u}_{y}^{s} \mid=\sqrt{V_{r}^{2} / C_{s}^{2}-1}$ across a broad range of normalized rupture speeds, which nearly encompass the entire stable supershear rupture speed regime. 


\subsection{Examination of the partitioning relationship between the motion recorded at the fault and the motion propagated by a shear Mach front into the surrounding medium.}

A series of supershear experiments were conducted using the (FP1,FP2) laser interferometer probe configuration depicted in Figure 4.34. Off-fault particle velocity records were obtained at various distances from the fault as a supershear rupture and its surrounding field swept the on- and off-fault measurement stations. The magnitude of the off-fault velocity jump carried by the shear Mach front was successfully measured in the experiments. Corresponding on-fault velocity jumps were also obtained in the same experiments. The ratio of the measured velocity jumps was then compared to the theoretical value predicted by

$$
\frac{\dot{u}_{x}^{s}\left(z_{s}\right)}{\dot{u}_{x}\left(x, 0^{+}\right)}=\frac{\beta_{s}^{2}-1}{\beta_{s}^{2}+1}
$$

based upon an independent measurement of the rupture speed, which was obtained from high-speed photoelastic images.

All tests in this series were conducted using traditional $6^{\prime \prime} \times 6^{\prime \prime} \times 0.375^{\prime \prime} \mathrm{H}-100$ specimens, which featured a fault angle of $\alpha=-29^{\circ}$. A synchronized, photoelastic image sequence of the event was also obtained using high-speed photography. The precise location of the supershear rupture tip, leading dilatational field lobe, and shear Mach fronts were all precisely identified in the high-speed image frames and directly correlated to the observed velocity swings exhibited by each particle velocity record.

The key to this particular series of experiments is the natural separation, which occurs between the leading dilatational field lobe and the shear Mach front at observation points located away from the specimen fault. Recall that the same principle was leveraged in order to investigate the ratio between the FP and FN velocity swings $\left(\dot{u}_{x}^{s}\right.$ and $\dot{u}_{y}^{s}$ ) along the shear Mach front (Section 4.5). The natural separation of the two fields with increased FN distance is demonstrated in Figure 4.35. The figure depicts a set of five FP records, obtained at various distances from the fault during separate 


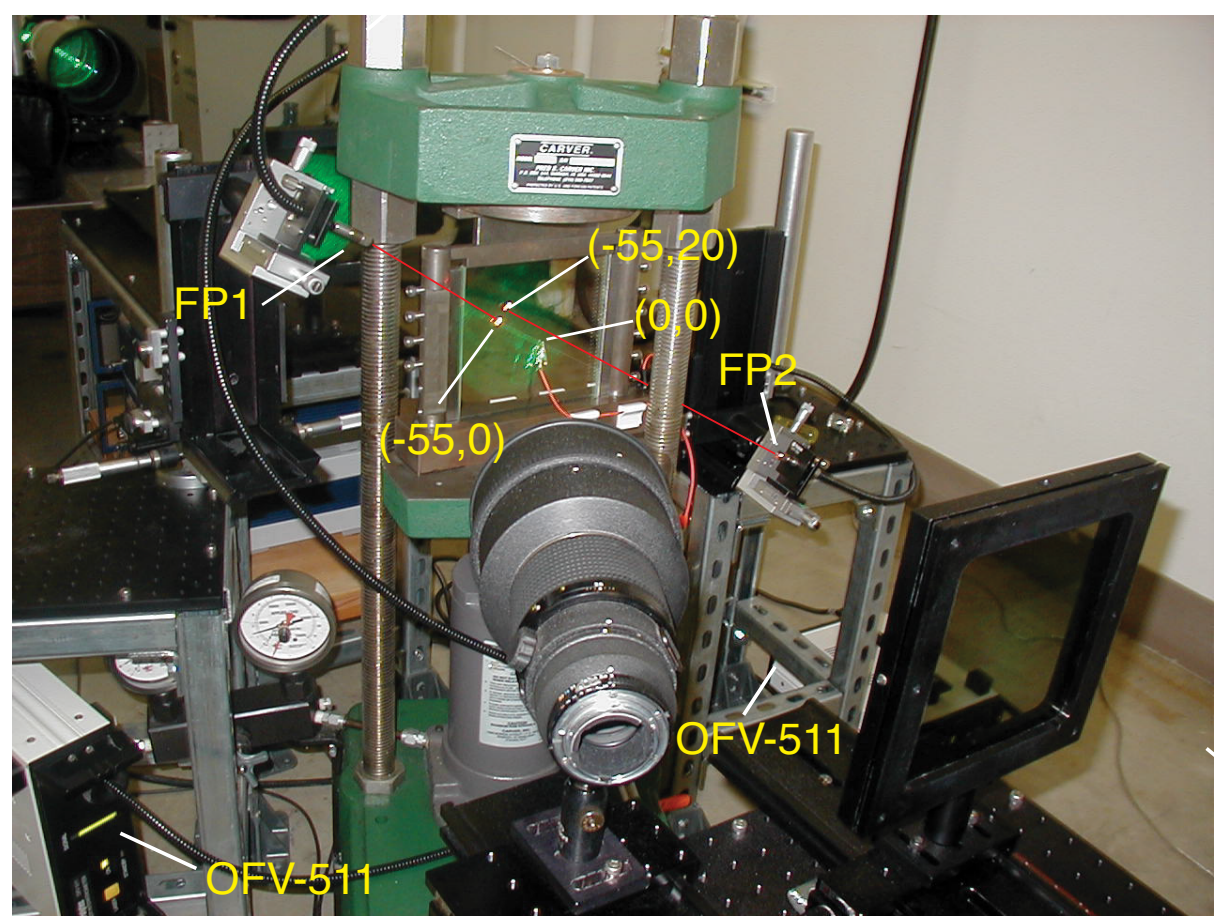

Figure 4.34: Laboratory earthquake experiment featuring the (FP1,FP2) laser interferometer probe beam configuration for the simultaneous measurement of fault-parallel particle velocity components on the fault and away from the fault

super shear experiments. The experiments exhibited different stress drops, supershear transition distances, and rupture speeds and so the stacked plots are not equivalent to having five simultaneous FP measurements from the same experiment. Nevertheless, the results clearly demonstrate the increased separation between the leading dilatational field lobe and the shear Mach front with increased FN distance. The lowest panel in Figure 4.35 corresponds to supershear experiment (exp 09-013), which featured the simultaneous measurement of FP and FN particle velocity records at at an on-fault station located at $\left(-40,0^{+}\right) \mathrm{mm}$. The coordinate description implies the laser beams were focused, slightly above the fault (within $100-200 \mu m$ of the fault). A sharp velocity jump near $t=30 \mu \mathrm{s}$ resulted from the superimposed combination of the leading dilatational field and the supershear rupture front. An expanded view of the curve along with a comparison to the high-speed image sequence reveals that the leading portion of the dilatational field lobe arrived $t=28.0 \mu \mathrm{s}$ and was quickly followed by the arrival of the supershear rupture at $t=29.5 \mu \mathrm{s}$. Although not resolvable at this scale, there is a notable difference in the slopes of the records corresponding to the time at 


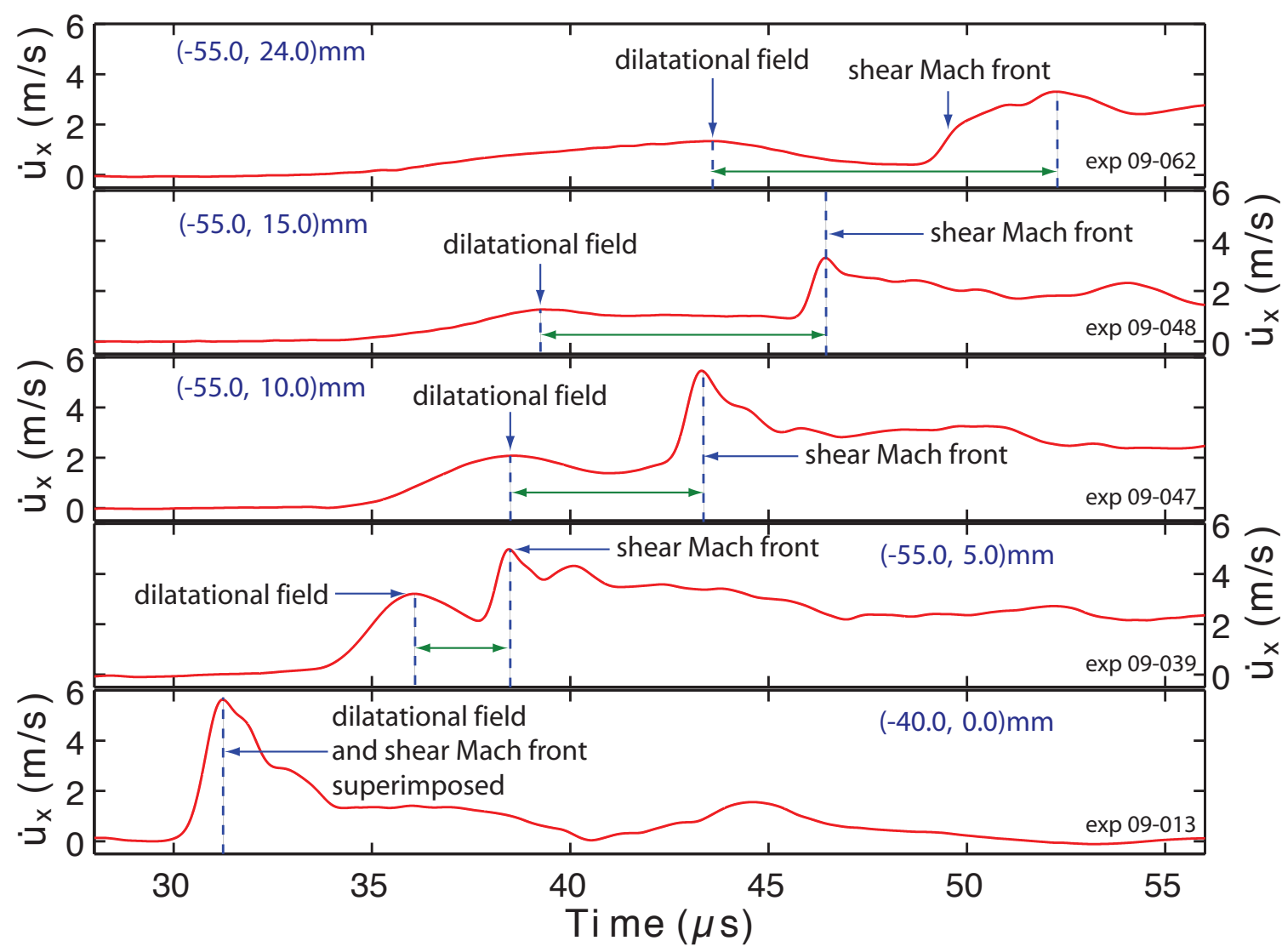

Figure 4.35: Series of FP particle velocity records obtained in five separate supershear experiments showing the progressive separation between the leading dilatational field lobe and the shear Mach front with increased FN distance

which the leading portion of the dilatational field swept the station and the portion of the curve dominated by the arrival of the shear Mach front. These features are examined in great detail in Section ?? when the same record is presented again in order to demonstrate a new type of dynamic stress measurement. Finally, note the second peak in the record at $t=44.6 \mu \mathrm{s}$, which corresponds to the peak sliding speed of the trailing Rayleigh rupture, which arrived at $t=40.4 \mu \mathrm{s}$.

The FP record presented in the second panel from the bottom corresponds to the supershear experiment (exp 09-039), which was analyzed in the previous section and used to examine the predicted relationship between the FP and FN velocity jumps $\left(\beta_{s}=\delta \dot{u}_{x}^{s} / \delta \dot{u}_{y}^{s}\right)$ along the shear Mach front. The particle velocity trace demonstrates that there is already a considerable separation between the two fields at $y=5 \mathrm{~mm}$, which enables a reliable estimate of $\dot{u}_{x}^{s}$ and $\dot{u}_{y}^{s}$ along the shear Mach front. 


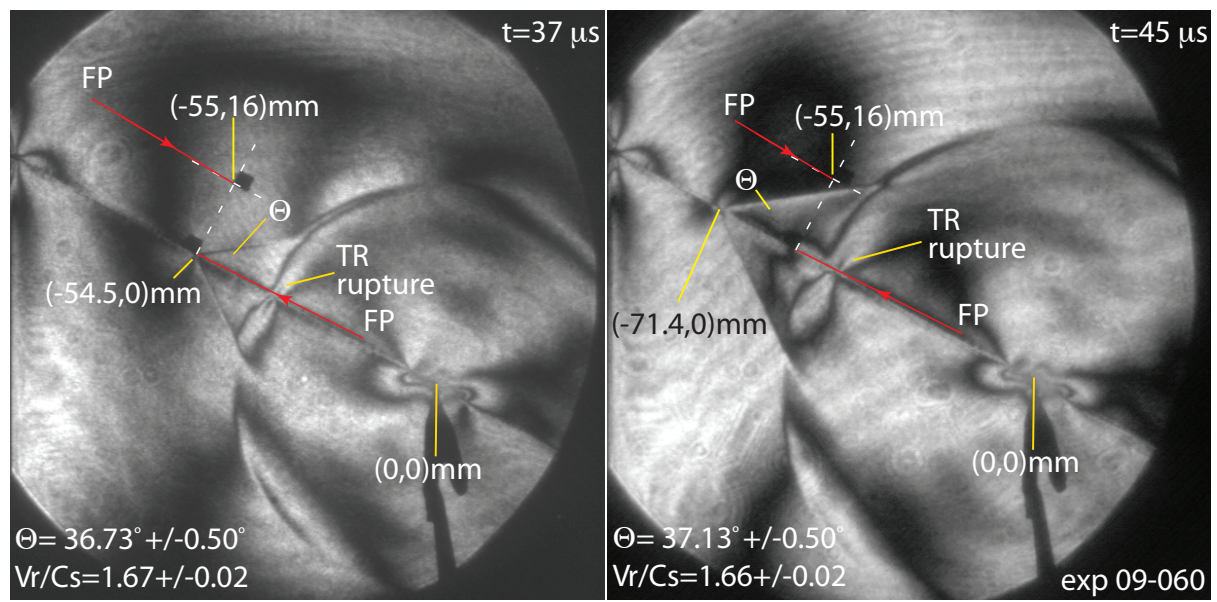

Figure 4.36: Photoelastic image frames capture the supershear rupture at two different times in the experiment as the rupture tip and shear Mach front were about to cross the on- and off-fault measurement stations at $\left(-55,0^{+}\right) \mathrm{mm}$ and $(-55,16) \mathrm{mm}$. Mach angle measurements yield an estimate of $V_{r} / C_{s}=1.67$ for the normalized rupture speed.

The third FP record corresponding to supershear experiment (exp 09-047), which was conducted at $y=10 \mathrm{~mm}$ was also previously examined in Section 4.2.1 through a frame-by-frame comparison with a sub-Rayleigh rupture experiment.

The fourth FP record corresponding to (exp 09-048) was also used in the investigation presented in the previous section and corresponds to the data point marked by the green symbol positioned at $(1.583,1.226)$ in Figure 4.33 .

The fifth and final record corresponding to supershear experiment (exp 09-062) was conducted using the $(\mathrm{FP} 1, \mathrm{FP} 2)$ arrangement with a remote off-fault station positioned at $(-55,24) \mathrm{mm}$. This results of this experiment are presented in the next section. The obvious and most striking feature of the curves in Figure 4.35 is the sustained amplitude of the FP velocity jump carried by the shear Mach front with increased FN distance even as far away as $y=24 \mathrm{~mm}$, which represents a considerable distance in a laboratory earthquake experiment. Note as well the pronounced broadening and eventual decay of the leading dilatational field lobe in comparison to the sharp rise time sustained by the shear Mach front.

The curves presented in Figure 4.35 are representative of a set of experiments that were conducted using the (FP1,FP2) laser interferometer configuration. The plots were chosen simply to demonstrate 


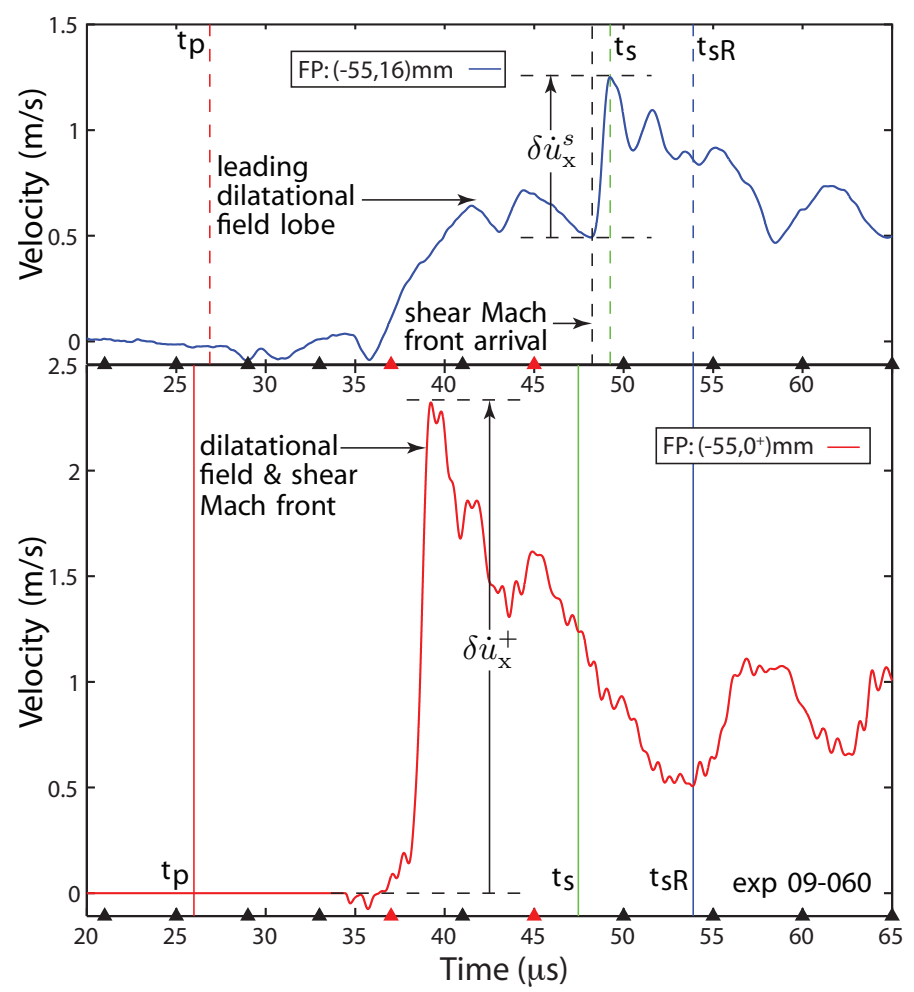

Figure 4.37: FP particle velocity records obtained the on- and off-fault measurement stations located at $\left(-55,0^{+}\right) \mathrm{mm}$ and $(-55,16) \mathrm{mm}$ during supershear experiment (exp 09-060)

the separation of the fields with increased FN distance regardless of the fact that some of them were obtained from a different series of experiments, which involved the (FN,FP) arrangement as described in Section 4.2.1.

\subsubsection{Results, analysis, and interpretation of particle velocity records and photoelastic images}

The results from three supershear experiments are presented, which are representative of a broader group of similar experiments that were conducted using the paired FP measurement configuration depicted in Figure 4.34 .

The photoelastic image frames in Figure 4.36 correspond to a supershear experiment (exp 09060). The images capture the supershear rupture at two different times in the experiment as the rupture tip and shear Mach front were about to cross the on- and off-fault measurement stations. The red lines highlight the optical paths of the two laser interferometer probe beams that were 

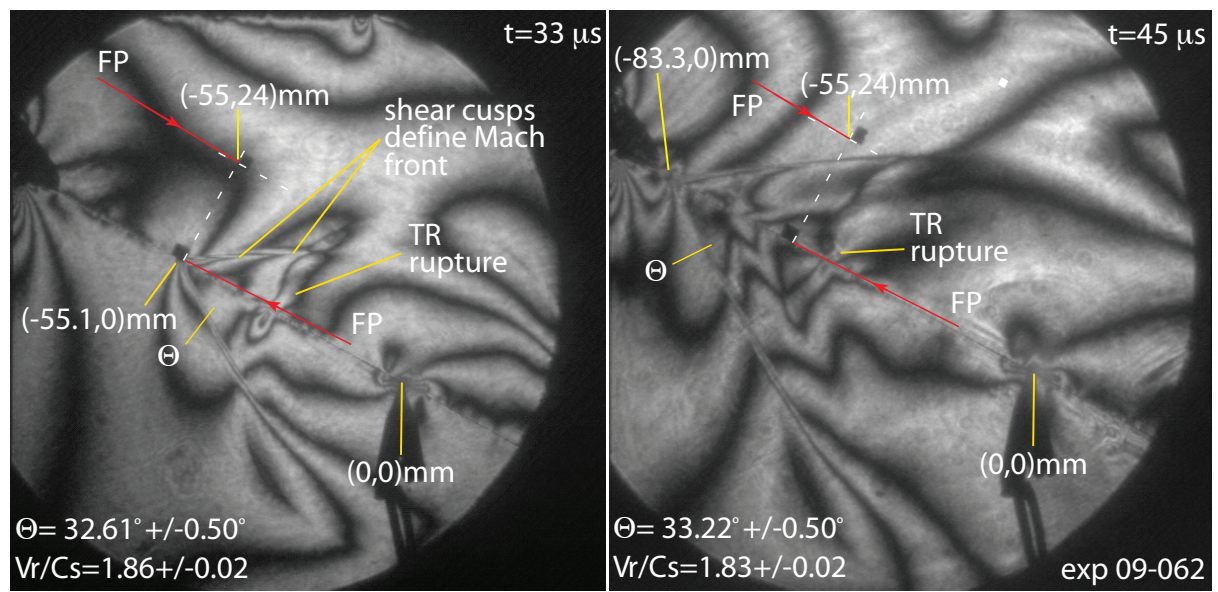

Figure 4.38: Photoelastic image frames capture the supershear rupture at two different times in the experiment as the rupture tip and shear Mach front were about to cross the on- and off-fault measurement stations at $\left(-55,0^{+}\right) \mathrm{mm}$ and $(-55,24) \mathrm{mm}$. Mach angle measurements yield an estimate of $V_{r} / C_{s}=1.85$ for the normalized rupture speed.

used to monitor the FP particle velocity component at each measurement station. The supershear rupture tip in Figure 4.36 is shown approaching the on-fault measurement station at $(-55,0) \mathrm{mm}$ in the $t=37 \mu \mathrm{s}$ image frame, while the shear Mach front on the extensional side of the fault in the $t=45 \mu \mathrm{s}$ image frame as it about to cross the measurement station at $(-55,16) \mathrm{mm}$. The images reveal extraordinarily sharp and straight shear Mach fronts, which are inclined at virtually identical angles as measured with respect to the fault. Note as well the sharp outline of the expanding S-wave front and the field lobes of the trailing Rayleigh rupture positioned in the wake of the supershear rupture. The measured Mach angles between the two frames indicate the rupture exhibited a very steady speed during this time. The normalized rupture speed of $V_{r} / C_{s}=1.67$ also establishes that the rupture was propagating within the stable rupture speed regime $\left(\sqrt{2} C_{s}<V_{r}<C_{p}\right)$.

Figure 4.37 displays the on-fault particle velocity record obtained at $\left(-55,0^{+}\right)$in the lower panel and the off-fault particle velocity record obtained at $(-55,16) \mathrm{mm}$ in the upper panel. The red and green vertical lines labeled $t_{p}$ and $t_{s}$ represent the anticipated arrival times of the $\mathrm{S}$ - and $\mathrm{P}$-waves at the measurement stations. The vertical blue line labeled $t_{R}$ corresponds to the arrival time of the trailing Rayleigh rupture. The noted velocity jumps labeled $\delta \dot{u}_{x}^{+}$and $\delta \dot{u}_{x}^{s}$ in Figure 4.36 correspond to

$$
\delta \dot{u}_{x}^{+}=\dot{u}_{x}^{\max }\left(-55,0^{+}\right)-\dot{u}_{x}^{\min }\left(-55,0^{+}\right)=2.395 \mathrm{~m} / \mathrm{s}
$$




$$
\delta \dot{u}_{x}^{s}=\left[\dot{u}_{x}^{s}(-55,16)\right]_{\max }-\left[\dot{u}_{x}^{s}(-55,16)\right]_{\min }=0.759 \mathrm{~m} / \mathrm{s}
$$

The measured values for the on-fault and off-fault velocity jumps yield a value for the measured ratio of $\delta \dot{u}_{x}^{s} / \delta \dot{u}_{x}^{+}=0.317$. Application of Eq. 4.5 using the normalized rupture speed estimate of $V_{r} / C_{s}=1.67$ predicts a ratio of $\delta \dot{u}_{x}^{s} / \delta \dot{u}_{x}^{+}=0.283$. The measured value in this example is found to be $12.0 \%$ higher than the predicted value.

The photoelastic image frames in Figure 4.38 correspond to a 2nd supershear experiment (exp 09062). The high-speed images capture the supershear rupture at two different times in the experiment as the rupture tip and shear Mach front approached the on- and off-fault measurement stations at $\left(-55,0^{+}\right) \mathrm{mm}$ and $(-55,24) \mathrm{mm}$. The red lines once again highlight the optical paths of the two laser interferometer probe beams that were used to monitor the FP particle velocity component at each measurement station. The supershear rupture tip is shown arriving at the on-fault measurement station at $(-55,0) \mathrm{mm}$ in the $t=33 \mu \mathrm{s}$ image frame, while the shear Mach front on the extensional side of the fault is still clearly several millimeters shy of the measurement station at $(-55,24) \mathrm{mm}$. The images also reveal shear Mach fronts defined by sharp cusps in the fringe pattern, which were used to measure the Mach angle (Section 3.11.3). The measured Mach angles yield an average normalized rupture speed of $V_{r} / C_{s}=1.85$. Note as well the secondary internal Mach fronts, which are clearly visible in the second image frame. These are believed to arise as a consequence of the finite specimen thickness as discussed in Section 3.10 and their arrival at the station correlates with the observed sharp secondary peaks in the on-fault particle velocity record.

Figure 4.39 displays the on-fault particle velocity record obtained at $\left(-55,0^{+}\right)$in the lower panel and the off-fault particle velocity record obtained at $(-55,24) \mathrm{mm}$ in the upper panel. The red and green vertical lines labeled $t_{p}$ and $t_{s}$ represent the anticipated arrival times of the S- and P-waves at the measurement stations. The vertical blue line labeled $t_{R}$ corresponds to the arrival time of the trailing Rayleigh rupture. The velocity jumps labeled $\delta \dot{u}_{x}^{+}$and $\delta \dot{u}_{x}^{s}$ in Figure 4.41 correspond to

$$
\delta \dot{u}_{x}^{+}=\dot{u}_{1}^{\max }\left(-55,0^{+}\right)-\dot{u}_{x}^{\min }\left(-55,0^{+}\right)=6.998 \mathrm{~m} / \mathrm{s}
$$




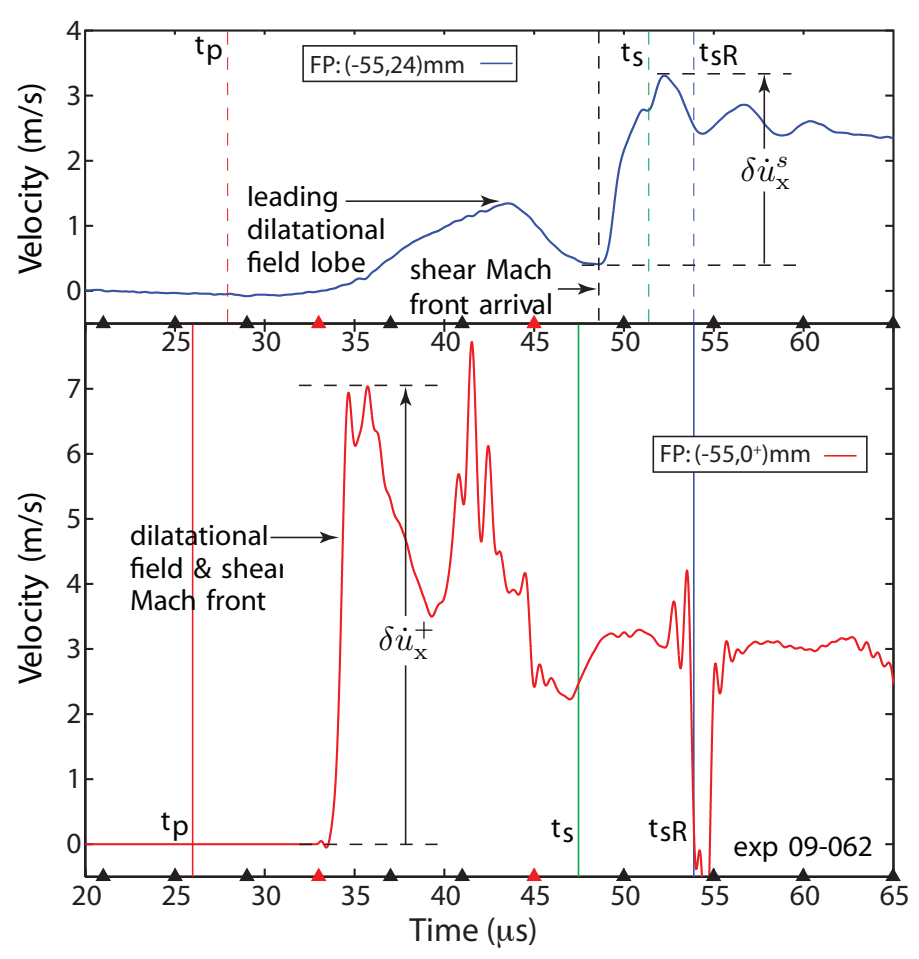

Figure 4.39: FP particle velocity records obtained the on- and off-fault measurement stations located at $\left(-55,0^{+}\right) \mathrm{mm}$ and $(-55,24) \mathrm{mm}$ during supershear experiment (exp 09-062).

$$
\delta \dot{u}_{x}^{s}=\left[\dot{u}_{x}^{s}(-55,24)\right]_{\max }-\left[\dot{u}_{x}^{s}(-55,24)\right]_{\min }=2.898 \mathrm{~m} / \mathrm{s}
$$

The measured values for the on-fault and off-fault velocity yield $\delta \dot{u}_{x}^{s} / \delta \dot{u}_{x}^{+}=0.414$. Application of Eq. 4.5 using a normalized rupture speed estimate of $V_{r} / C_{s}=1.85$ predicts a ratio of $\delta \dot{u}_{x}^{s} / \delta \dot{u}_{x}^{+}=0.416$. The measured value in this example is found to be $0.36 \%$ higher than the predicted value.

The photoelastic image frames in Figure 4.41 correspond to a slightly different configuration whereby the measurement stations were oriented along a line lying perpendicular to the anticipated normal to the shear Mach fronts (Section 3.14.2). The image frames capture the supershear rupture at two different times in the experiment as the rupture tip and shear Mach front approached the on- and off-fault measurement stations at $\left(-55,0^{+}\right) \mathrm{mm}$ and $(-62,10) \mathrm{mm}$. The station positions appear to be well aligned with the normal to the shear Mach front. The red lines highlight the optical paths of the two laser interferometer probe beams that were used to monitor the FP particle velocity component at each measurement station. The dilatational field lobe has already crossed the on-fault station at $(-55,0) \mathrm{mm}$ in the $t=37 \mu \mathrm{s}$ image frame, while the shear Mach front on the 


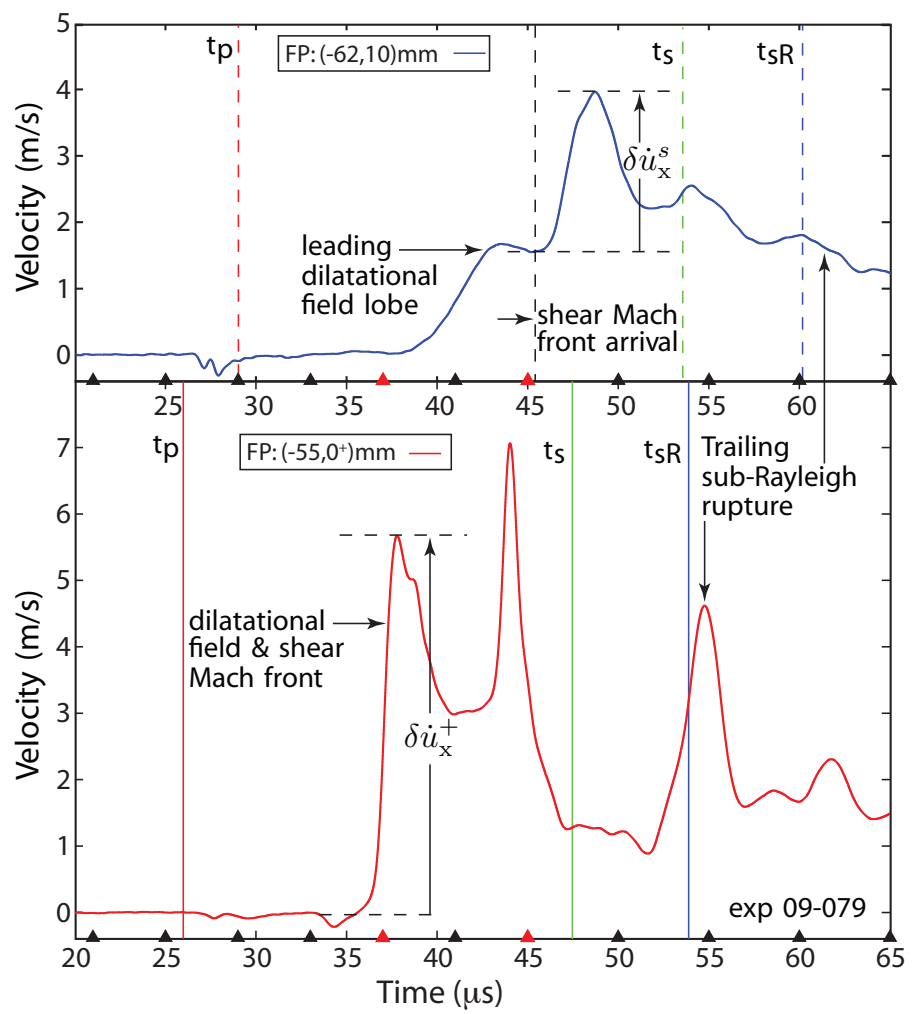

Figure 4.40: FP particle velocity records obtained the on- and off-fault measurement stations located at $\left(-55,0^{+}\right) \mathrm{mm}$ and $(-62,10) \mathrm{mm}$ during supershear experiment (exp 09-079). Measurement stations were positioned along a line, which was very nearly aligned with the normal to the shear Mach front.

extensional side of the fault is captured just as it arrives at the measurement station positioned at $(-62,10) \mathrm{mm}$. The measured Mach angles in both frames were nearly identical and correspond to a normalized rupture speed of $V_{r} / C_{s}=1.81$. Note the internal secondary Mach front, which is visible in the $t=45 \mu \mathrm{s}$ image frame. The arrival of the secondary Mach front at $(-55,0) \mathrm{mm}$ correlates with the sharp secondary peak seen in the on-fault particle velocity record.

Figure 4.39 displays the on-fault particle velocity records obtained at $\left(-55,0^{+}\right)$in the lower panel and the off-fault particle velocity record obtained at $(-62,10) \mathrm{mm}$ in the upper panel. The red and green vertical lines labeled $t_{p}$ and $t_{s}$ represent the anticipated arrival times of the S- and P-waves at the measurement stations. The vertical blue line labeled $t_{R}$ corresponds to the arrival time of the trailing Rayleigh rupture. The velocity jumps labeled $\delta \dot{u}_{x}^{+}$and $\delta \dot{u}_{x}^{s}$ in Figure 4.41 correspond to

$$
\delta \dot{u}_{x}^{+}=\dot{u}_{x}^{\max }\left(-55,0^{+}\right)-\dot{u}_{x}^{\min }\left(-55,0^{+}\right)=5.670 \mathrm{~m} / \mathrm{s}
$$




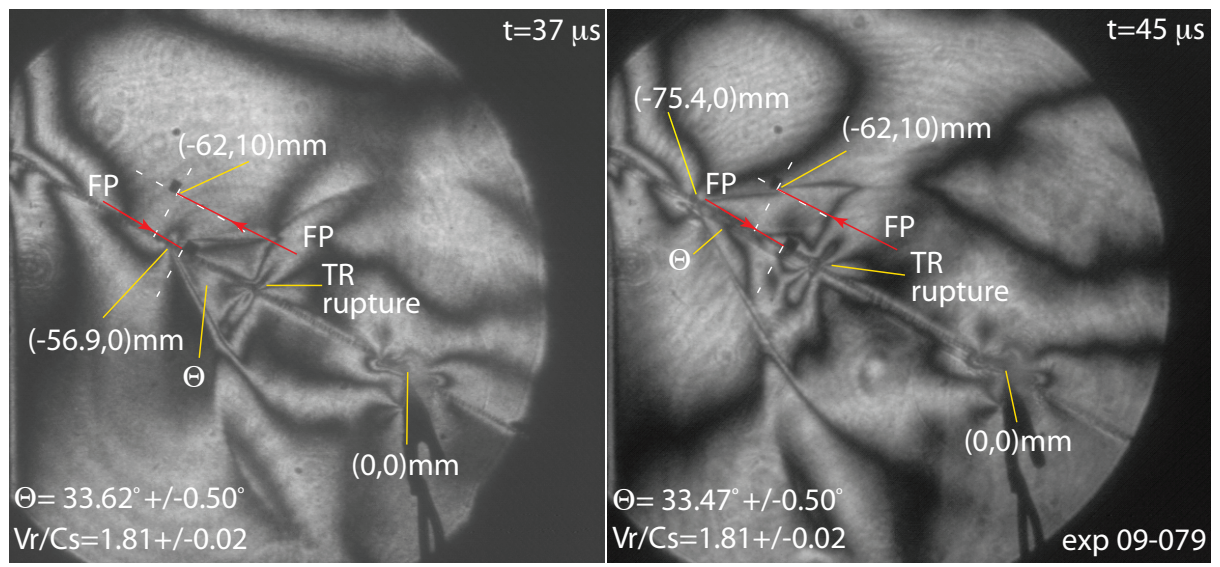

Figure 4.41: Photoelastic image frames capture the supershear rupture at two different times in the experiment as the rupture tip and shear Mach front were about to cross the on- and off-fault measurement stations at $\left(-55,0^{+}\right) \mathrm{mm}$ and $(-62,10) \mathrm{mm}$. Mach angle measurements yield an estimate of $V_{r} / C_{s}=1.81$ for the normalized rupture speed.

$$
\delta \dot{u}_{x}^{s}=\left[\dot{u}_{x}^{s}(-62,10)\right]_{\text {max }}-\left[\dot{u}_{x}^{s}(-62,10)\right]_{\min }=2.396 \mathrm{~m} / \mathrm{s}
$$

The measured values for the on-fault and off-fault particle velocities yield $\delta \dot{u}_{x}^{s} / \delta \dot{u}_{x}^{+}=0.423$. Application of Eq. 4.5 using a normalized rupture speed estimate of $V_{r} / C_{s}=1.85$ predicts a ratio of $\delta \dot{u}_{x}^{s} / \delta \dot{u}_{x}^{+}=0.390$. The measured value in this example is found to be $8.5 \%$ higher than the predicted value.

Figure 4.42 summarizes the results obtained from 11 supershear experimental records including the three records, which have been presented in detail. The blue curve corresponds to theoretical partitioning ratio between the FP record measured at the fault and the FP component along the shear Mach front. The curve spans from a value of zero at $V_{r} / C_{s}=\sqrt{2}$, to a maximum value of 0.5 at a normalized wave speed of $V_{r} / C_{s}=2$. The inset in the figure is a reminder of the measurements that were conducted in order to obtain the colored data points, which populate the plot. Nine of the data points were collected using two measurement stations that were aligned normal to the fault while the remaining three tests were conducted using a pair of stations that were aligned along a line lying normal to the advancing shear Mach front. The data points are seen to span across a broad range of rupture speeds, which nearly span the entire stable rupture speed regime. A very reasonable match is seen between the measured ratios and the theoretical partitioning curve. Note as well the 


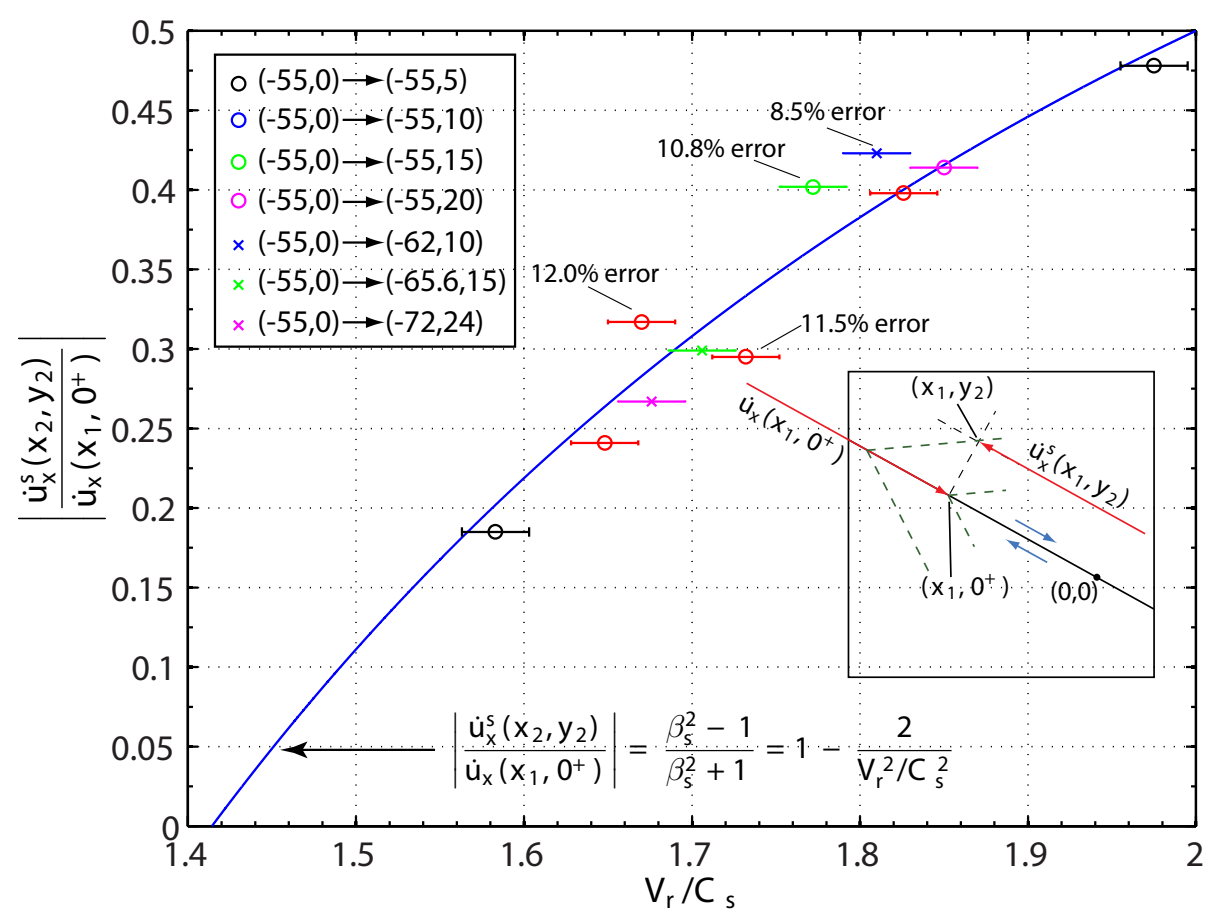

Figure 4.42: Plot of the ratio $\dot{u}_{x}^{s} / \dot{u}_{x}\left(x, 0^{+}\right)$obtained from 11 supershear experimental records poltted against the normalized wave speed $V_{r} / C_{s}$. Results compare quite favorably with theoretical partitioning relationship corresponding to the blue curve.

span of FN distances over which the tests were conducted and how the results are independent of the FN distance. The results demonstrate the partitioning of the on-fault FP particle velocity component onto the shear Mach front and the manner through which the slip function is carried deep into the surrounding medium by the shear Mach front. 


\section{Chapter 5}

\section{Scaling Relationships between Laboratory Earthquake Measurements and Real-Earth Ground Motion Records}

\subsection{The November 3, $2002\left(M_{w} 7.9\right)$ Denali, AK earthquake}

The $2002\left(M_{w} 7.9\right)$ Denali fault earthquake was the largest strike-slip rupture to take place on the North American continent in over 150 years and was rivaled in magnitude only by the $1906\left(M_{w} 7.8\right)$ San Francisco earthquake and the $1857\left(M_{w} 7.9\right)$ Fort Tejon earthquake. Its total rupture length of $334 \mathrm{~km}$, average slip of $4.9 \mathrm{~m}$, and maximum slip of $8.8 \mathrm{~m}$, rank it amongst the largest shallow-crust earthquakes recorded anywhere in the world throughout the past two centuries (Haeussler et al., 2004). Due in large measure to its remote location within south- central Alaska, there was very little damage to modern infrastructure and fortunately no loss of human life. Field evidence and ground motion data from this event have, however, provided seismologists with a rare and extraordinary opportunity to study a large, shallow crust, strike-slip earthquake, which is in many ways an analogue to the earthquakes which are known to occur along the San Andreas fault (Haeussler et al., 2004).

The Denali and Totschunda faults, depicted in Figure 5.1, are the principal elements in an intracontinental right-lateral strike-slip fault system that curves across Alaska and western Canada and passes through the Alaska Range (Haeussler et al., 2004). Collision of the Yakutat block with 


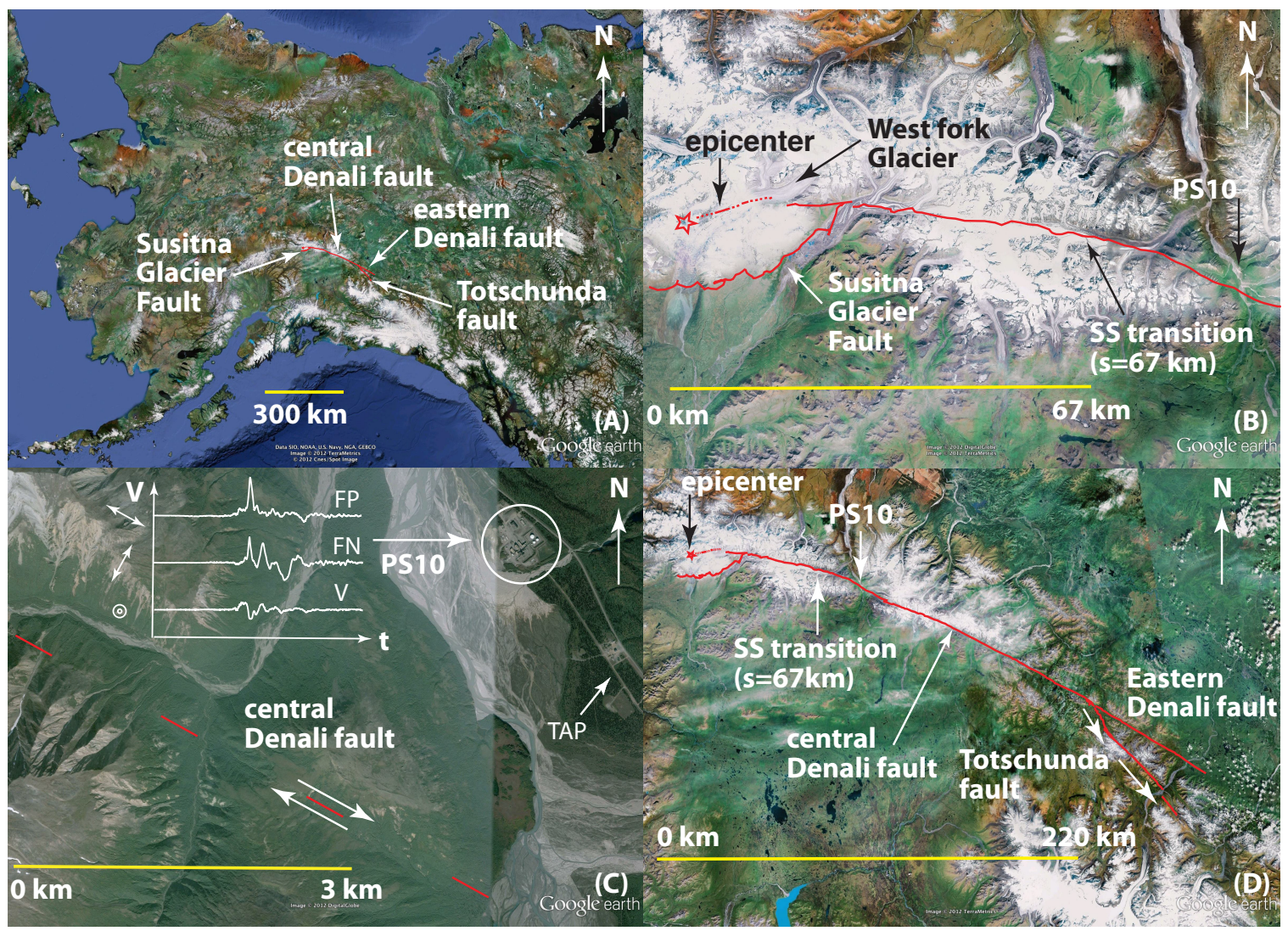

Figure 5.1: Denali fault image montage: (a) Susitna Galcier, Denali, and Totschunda fault systems, (b) strike-slip fault segment extending from epicenter to Pump Station 10 located along the Alaska pipeline, (c) closeup view of central Denali fault and Pump Station 10, (d) Denali strike-slip segment and branch onto Totschunda fault (images obtained using Google earth Pro)

the North American plate, as depicted in Figure 5.2, is believed to be the driving force of the movements along the Denali fault system (Eberhart-Phillips et al., 2003). The collision is driven by northwest-dipping subduction at nearly $5 \mathrm{~m}$ per century along the Aleutian megathrust (Hansen and Ratchkovski, 2004).

The Denali earthquake occurred at 22:12:41 (UTC) on 3 November 2002, with the hypocenter located at a depth of $4.2 \mathrm{~km}$ at $63.514 \mathrm{~N}, 147.453 \mathrm{~W}$ (Martirosyan et al., 2004). The rupture began along a $40 \mathrm{~km}$-long segment of the previously unknown Susitna Glacier thrust fault (Figure 5.1b). Thrusting on the Susitna Glacier fault raised the north side relative to the south (Haeussler et al., 2004). The resulting scarp heights were typically $1-3 m$, with a maximum of $4.3 m$ (Craw et al., 2003). The rupture then transferred to the Denali strike-slip fault system and propagated $218 \mathrm{~km}$ 


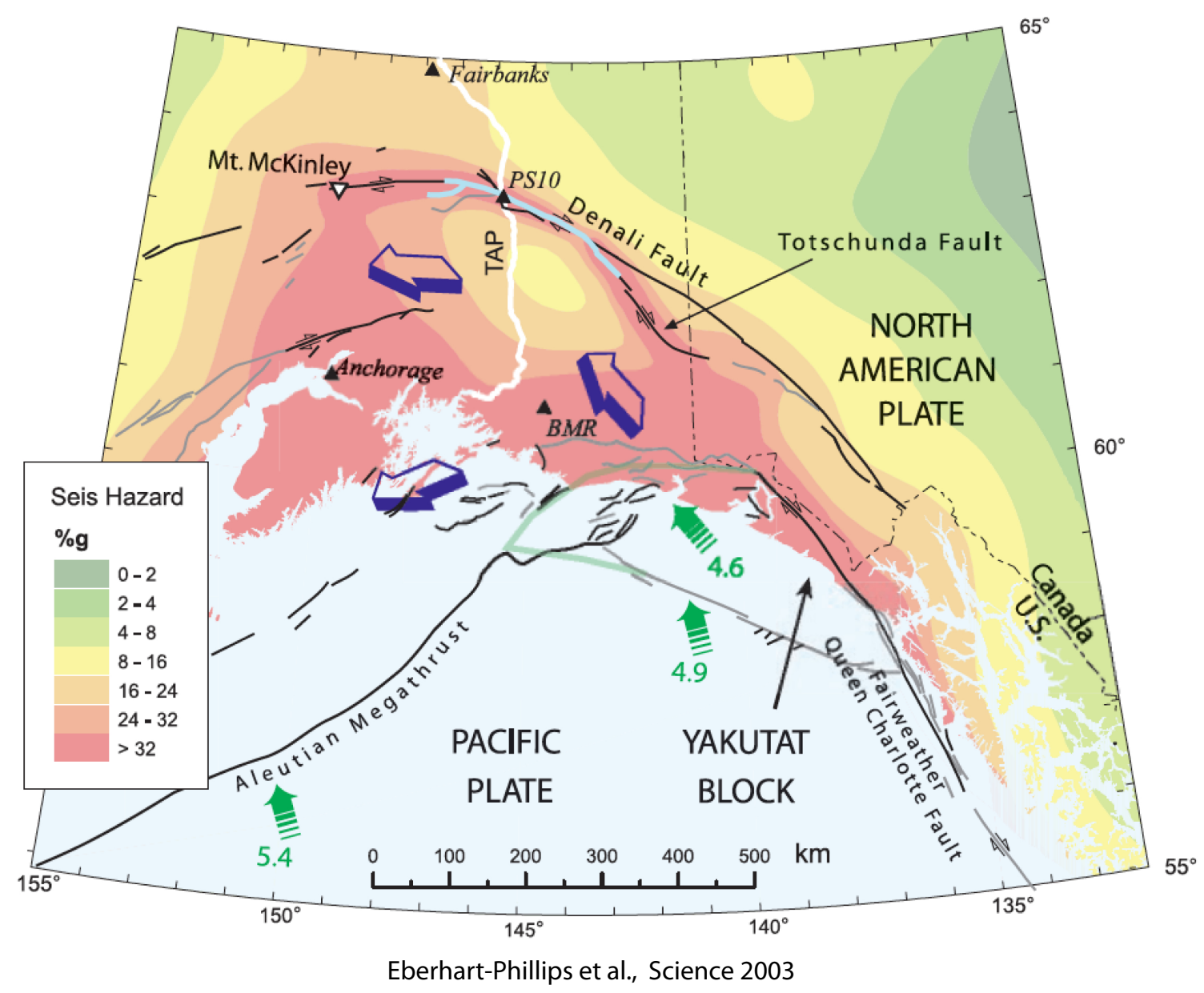

Figure 5.2: From Eberhart-Phillips et al., 2003: Plate tectonic setting of southern Alaska and major tectonic elements. The Pacific and North American plates converge at $5.4 \mathrm{~cm} /$ year beneath Anchorage, and the Yakutat block collides with North America independently. Plate motion is indicated by green arrows. Blue open arrows schematically show lateral movement of broad region south of the Denali fault.

from west to east along the central Denali fault. Post-earthquake surveys revealed an average rightlateral offset of approximately $5 \mathrm{~m}$ and a maximum offset of $8.8 \mathrm{~m}$ near the eastern end of the fault, approximately $190 \mathrm{~km}$ from the epicenter. The rupture eventually branched off of the Denali fault and stepped over onto the Totschunda fault (Figure 5.1c) where it propagated for an additional 76 $\mathrm{km}$ with an average slip of $3 m$ before finally arresting (Haeussler et al., 2004).

The central Denali fault ruptured beneath the Trans-Alaska pipeline (TAP), which is located approximately $85 \mathrm{~km}$ east of the earthquake epicenter. Post-earthquake surveys indicated that up to $5.8 \mathrm{~m}$ of right-lateral slip was experienced across the TAP fault zone. The resulting fault 
displacement tested the limits of a compliant pipeline support structure, which was designed to accommodate up to $6.1 \mathrm{~m}$ of lateral displacement and $1.5 \mathrm{~m}$ of vertical slip (Honegger et al., 2004). The pipeline support structure withstood the large fault zone displacement and prevented oil from being spilled into the surrounding environment. The success of the TAP support structure at the Denali fault zone crossing represents a major engineering triumph and is a credit to WoodwardLundgren Associates (1974) who successfully estimated the magnitude of slip in a future Denali fault rupture (Haeussler et al., 2004).

The second major significance of the TAP Denali fault crossing involves a set of "celebrated" near-source ground motion records obtained at Pump Station 10 (PS10), positioned at $63.424 N$, 145.763 $W$ along the TAP and located just $3 \mathrm{~km}$ north of the fault. The accelerometer recording station at PS10 is part of the accelerograph network operated by the Alyeska Pipeline Service Company. (Ellsworth et al., 2004) conducted a thorough analysis and calibration of the PS10 instrumentation and re-processed the signals in order to recover the long-period $(>10 s)$ ground motions. A set of instrument-corrected acceleration, velocity, and displacement time records were obtained and properly rotated into the fault-normal (FN) and parallel (FP) directions. The FP, FN, and vertical (UP) velocity records are depicted in Figure 5.3a and represented by the thicker (gray) curves within each respective panel.

Forward modeling of the instrument-corrected ground motion records lead (Ellsworth et al., 2004) to conclude that a supershear burst occurred along a $38 \mathrm{~km}$ segment of the fault, which was nearly centered about PS10. The ground motion records were best matched by the model if a normalized rupture speed of $V_{r} / C_{s}=1.5$ (i.e., about $5.3 \mathrm{~km} / \mathrm{s}$ at $5 \mathrm{~km}$ depth) was assumed across the supershear segment. The model predicted a normalized sub-Rayleigh rupture speed of $V_{r} / C_{s}=0.66$ over a $67 \mathrm{~km}$ stretch between the epicenter and the point of supershear transition. The model estimated a normalized rupture speed jumped to $V_{r} / C_{s}=1.5$ within the supershear interval followed by a decrease to a normalized sub-Rayleigh rupture speed of $V_{r} / C_{s}=0.85$ for points beyond the terminus of the supershear interval (i.e., distances $>20 \mathrm{~km}$ east of PS10). Synthetic ground motion predictions are depicted by the thinner (black) curves in Figure 5.3a. The curves do 


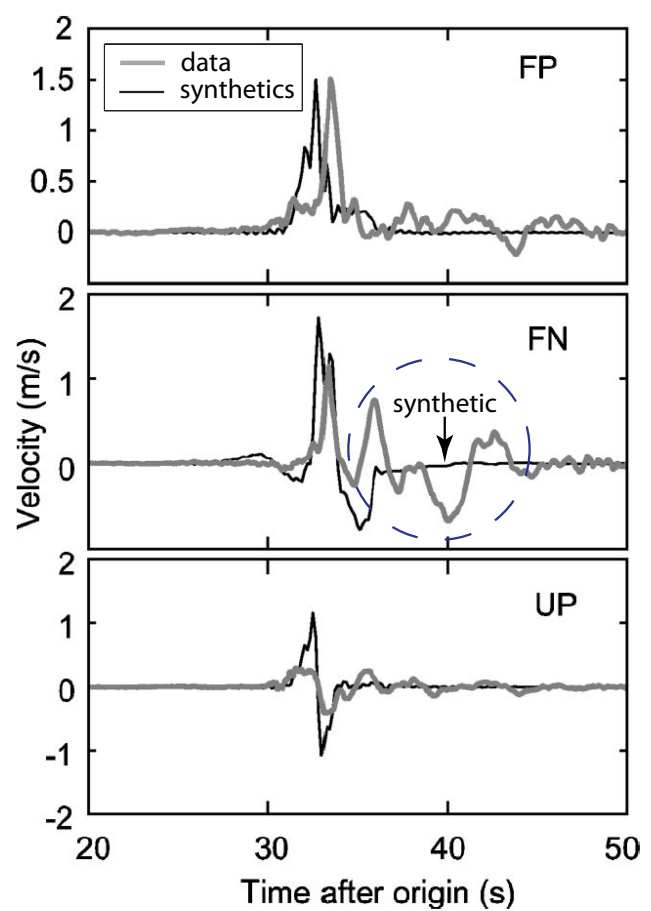

(a) Ellsworth et al., 2004

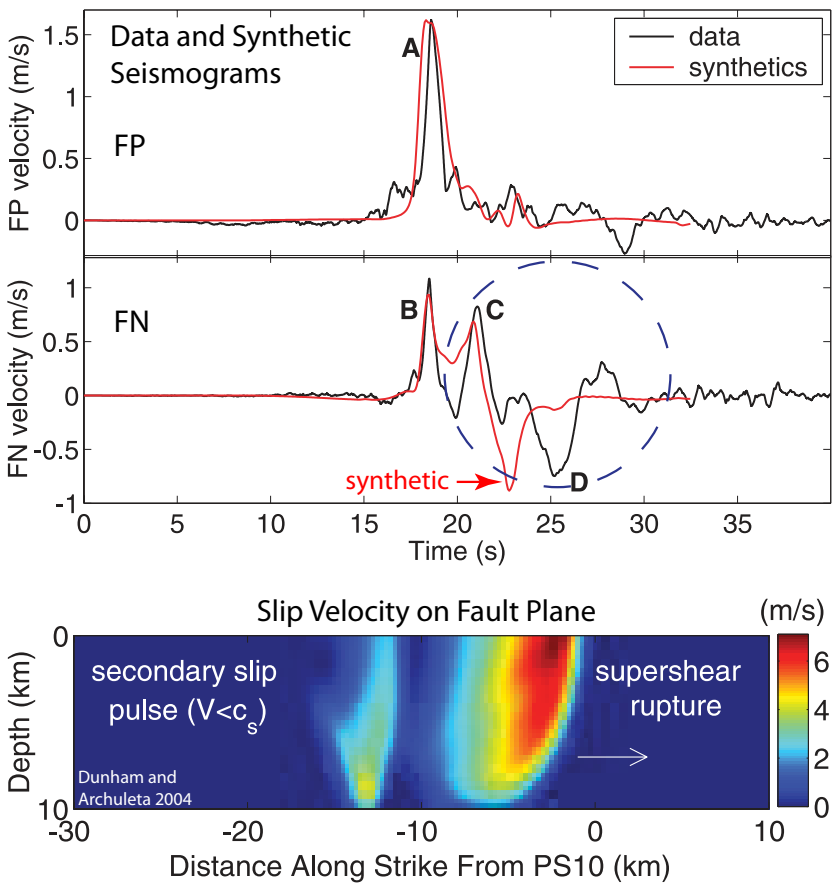

(b) Dunham and Archuleta 2004

Figure 5.3: (a) From (Ellsworth et al., 2004): comparison of fault-parallel (top), fault-normal (middle) and vertical (bottom), PS10 data (gray) and synthetic (black) ground velocity predictions (b) From (Dunham and Archuleta, 2004)l: Comparison between recorded and synthetic ground motions at PS10 for dynamic model featuring slip weakening with healing feature, and slip to $10 \mathrm{~km}$ depth

a reasonable job in capturing the general profile of the leading portions of the FP, FN, and vertical (UP) records although the synthetic vertical curves tend to over-predict the peak vertical ground velocity. Time in these plots is measured relative to the origin time of the earthquake. The biggest shortcoming of the model was its inability to capture the prominent secondary pulses in the FN ground motion record, which are circled in Figure 5.3a. Nevertheless, the careful processing of the instrument-corrected PS10 data and the interpretation of these remarkable ground motion records represents a major seismological finding and the most direct evidence ever gathered for the existence of a supershear earthquake rupture.

(Dunham and Archuleta, 2004) noted specific features in the PS10 ground motion records, which he recognized as characteristic ground motion signatures of a supershear rupture. The first feature of note involves the $\mathrm{FP}$ velocity pulse (labeled $A$ in the figure) which is approximately $1.5 \times$ greater 
in magnitude than the corresponding FN velocity pulse (labeled $B$ ). The second unique feature corresponds to the set of pronounced velocity swings between points labeled $C$ and $D$ in the FN record, which (Ellsworth et al., 2004) was unable to replicate using his simple model. (Dunham and Archuleta, 2004) reasoned that the secondary pulses in the FN record resulted from rupture acceleration during the supershear transition and the release of Rayleigh waves during this phase that combine to produce a secondary slip-pulse, which trails the primary supershear rupture. A spontaneous dynamic rupture propagation model was developed which incorporated a slip weakening friction law with a built-in healing mechanism (Nielsen et al., 2000). The black curves in Figure 5.3b, reproduced here from (Dunham and Archuleta, 2004), represent the filtered Pump Station 10 ground motion records, while the superimposed red curves correspond to synthetic ground motion predictions from the dynamic rupture model. Note that time in Figure 5.3b is now measured relative to arrival of the first P-waves at PS10. The synthetic ground motion predictions match up well with the leading portions of the FP and FN PS10 ground motion records. The dynamic model also successfully predicted the emergence of the secondary slip-pulse as manifested by the velocity swings in synthetic FN curve, which mimic the velocity swings between the points labeled $C$ and $D$ in the PS10 FN record. Note as well how this same feature is also revealed by the leading and trailing slip velocity color contour plots in Figure 5.3b. Emphasis was placed on matching the width of the supershear rupture pulses (labeled $A$ and $B$ ) at the expense of truncating the width of the trailing secondary slip-pulse. The fact that the synthetic curves fail to match up as well with trailing portion in the FN record was attributed to complex frictional faulting mechanisms not captured by the assumed frictional law (Dunham and Archuleta, 2004).

Motivated by the success of the dynamic simulations and the physically based interpretation of the secondary slip-pulse, an attempt was made to replicate the PS10 scenario using the laboratory earthquake arrangement. Experiments were conducted using the new 3D specimen fault geometry (Section 3.9), which offers control of the supershear transition distance $x=L_{T}$. The new specimen design also promotes healing of the rupture signal through a stopping phase produced by the locked boundary where the width of the frictional fault is suddenly reduced. 
PS10 ground motion was mimicked by particle velocity records obtained at a pre-defined (scaled) location on the compressional side of the fault, as a supershear rupture swept past the measurement station from left to right (west-to-east). Both sub-Rayleigh and supershear laboratory earthquake experiments were conducted using the "Denali PS10" experimental configuration in order to compare and contrast the resulting particle velocity signatures. The measurement station coordinates $(57,3) \mathrm{mm}$ were selected a priori through scaling relationships, which are derived and rationalized in the following section. The resulting supershear particle velocity records from the experiment were then scaled through six orders of magnitude from microseconds to seconds in order to appropriately contrast the experimental results with the Denali PS10 ground motion records. Additional details of the experiments and experimental parameters used in the scaling analysis are provided in Section 3.9 following the discussion on scaling.

\subsection{Development of a scaling relationship}

Consider the geometry shown in Figure 5.4, which depicts a fault where sub-Rayleigh rupture nucleates at the origin and transitions to a supershear rupture at $x=L_{T}$. The measurement station is located at $(x, y)$. Let $V_{r}(x)$ represent the rupture velocity history along the fault. The time taken for the rupture to arrive at $(x, 0)$ is then given by

$$
t_{S S}=\int_{0}^{L_{T}} \frac{d x}{V_{r}(x)}+\int_{L_{T}}^{x} \frac{d x}{V_{r}(x)}
$$

Note that integral is expressed in the above form in order to separate the sub-Rayleigh and the supershear rupture portions of the rupture velocity history.

By the time the rupture arrives at $(x, 0)$ it is has already transitioned to supershear rupture and is assumed to have a well-developed shear Mach front, as indicated in Figure 5.4, which advances at the S-wave speed along a direction normal to itself (Figure 5.4). The time taken for the shear Mach front to reach the measurement station at $(x, y)$ is given by $y \cos \theta / C_{s}$ where $\sin \theta=C_{s} / V_{r}$. Hence, 


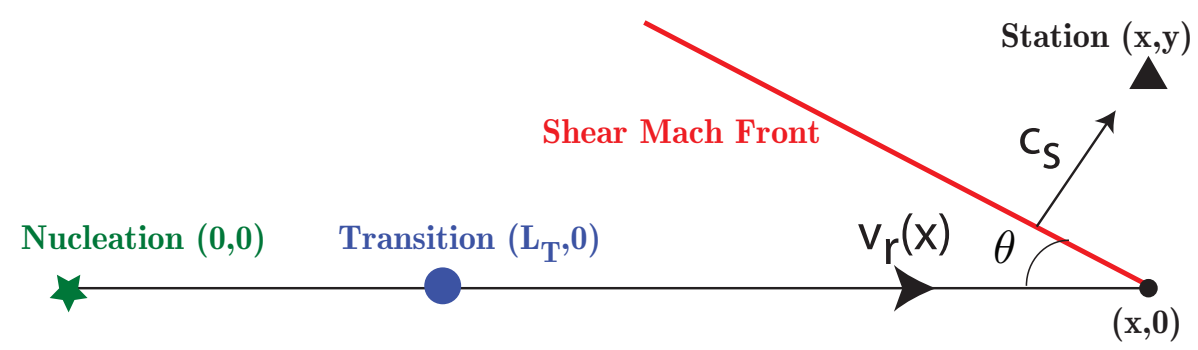

Figure 5.4: Laboratory earthquake scaling geometry

the time taken for the main rupture information to reach the measurement station is given by

$$
t_{S S}=\int_{0}^{L_{T}} \frac{d x}{V_{r}(x)}+\int_{L_{T}}^{x} \frac{d x}{V_{r}(x)}+\frac{y \cos \theta}{C_{s}}
$$

Next, consider the time taken for the trailing sub-Rayleigh rupture (pulse) to arrive at the measurement station. The analysis assumes that the rupture propagates with a rupture speed $V_{r}(x)$ from the nucleation site $(0,0)$ up until the supershear transition location at $x=L$, after which it propagates exactly at the Rayleigh wave speed $V_{r}=C_{R}$. It is also assumed that the measurement station is positioned relatively close to the fault such that when the trailing sub-Rayleigh rupture arrives at $(x, 0)$, its vertically extended field lobes simultaneously sweep the off-fault station at $(x, y)$. Thus,

$$
t_{R}=\int_{0}^{L_{T}} \frac{d x}{V_{r}(x)}+\int_{L_{T}}^{x} \frac{d x}{C_{R}}
$$

The difference in the arrival times at the measurement $(\mathrm{x}, \mathrm{y})$ between the shear Mach front and the extended field lobe of the trailing sub-Rayleigh pulse, $\left(\Delta t^{S S-T R}\right)$, is therefore given by

$$
\Delta t^{S S-T R}=\frac{x-L_{T}}{C_{R}}-\int_{L_{T}}^{x} \frac{d x}{V_{r}(x)}-\frac{y}{C_{s}} \sqrt{1-\frac{C_{s}^{2}}{V_{r}^{2}}} .
$$

Assuming that $V_{r}(x)$ is constant between the supershear transition location at $\left(L_{T}, 0\right)$ and its arrival at the on-fault position $(x, 0)$ then leads to,

$$
x=L_{T}+C_{s} \Delta t^{S S-T R}+y \sqrt{1-\frac{C_{s}^{2}}{V_{r}^{2}}}\left(\frac{C_{s}}{C_{R}}-\frac{C_{s}}{V_{r}}\right)^{-1} .
$$




\section{1}

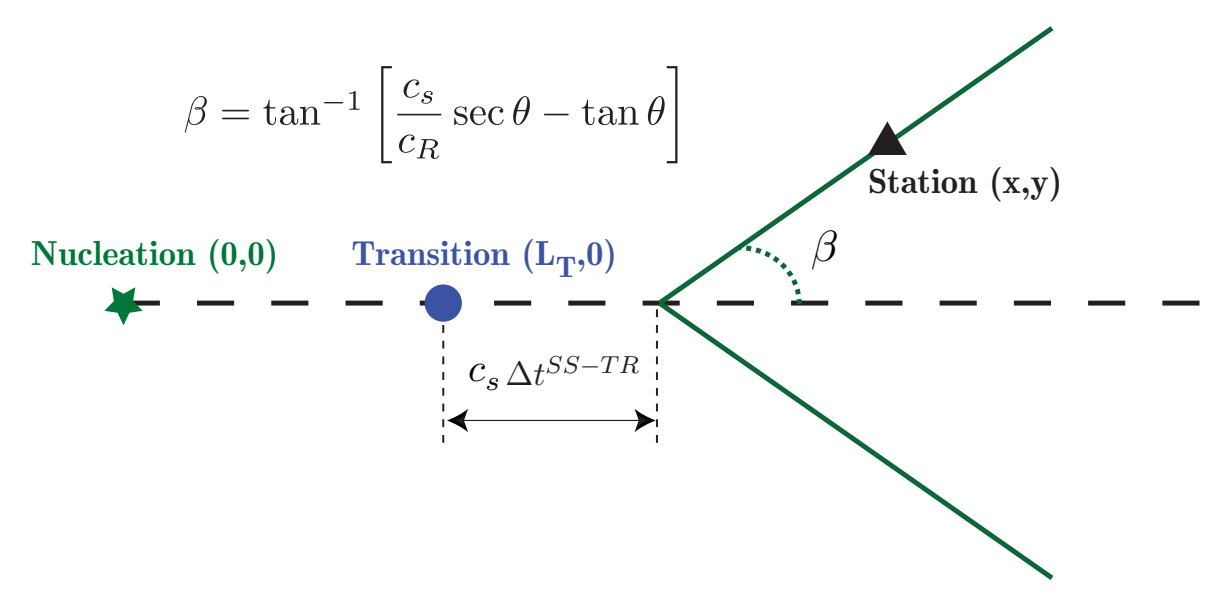

Figure 5.5: Locus of stations with the same $\Delta t^{S S-T R}$, assuming a fixed transition length and rupture velocity

It becomes convenient at this point in the derivation to define the following quantities,

$$
\tan \beta=\left(\frac{C_{s}}{C_{R}}-\frac{C_{s}}{V_{r}}\right) \quad ; \quad \sin \theta=\frac{C_{s}}{V_{r}} \quad ; \quad L^{*}=\left(L_{T}+C_{s} \Delta t^{S S-T R}\right) \tan \beta
$$

Solving for $y$ from the Eq. 5.5 then yields,

$$
y=x \tan \beta-L^{*}
$$

The relationship represents a unique locus of points given by a straight line inclined at an angle $\beta$, which intersects the fault at $L_{T}+C_{s} \Delta t^{S S-T R}$, over which the time difference given by Eq. 5.4 is preserved. (See Figure 5.5).

Geometric scaling is imposed in order to constrain the solution given by Eq. 5.7. Although numerous options may be considered, the geometric aspect ratio beyond the supershear transition location is retained and used to constrain the scaling relationship, i.e.,

$$
\left.\frac{y^{P S 10}}{\left(x^{P S 10}-L_{T}^{P S 10}\right)}=\frac{y^{e x p}}{\left(x^{e x p}-L_{T}^{e x p}\right)}=\frac{1}{6}=S_{L} \quad \text { (one option }\right)
$$

Laboratory earthquake records are scaled to the supershear ground motion records acquired at Pump 
Station 10 during the $2002 M_{w} 7.9$ Denali earthquake. In the case of Pump Station 10 (PS10), $y=3 \mathrm{~km}, x-L_{T}=18 \mathrm{~km}$ (Ellsworth et al., 2004). Substituting the geometric constraint given by Eq. 5.8 into Eq. 5.7 yields the equivalent coordinates on the surface of the laboratory earthquake test specimen.

$$
x^{e x p}=\frac{L^{*, e x p}-L_{T}^{e x p} S_{L}}{\tan \beta^{\exp }-S_{L}} \quad ; \quad y^{e x p}=S_{L}\left(x^{e x p}-L_{T}^{e x p}\right)
$$

$$
\text { where } L^{*, e x p}=\left(L_{T}^{\text {exp }}+C_{s}^{e x p} \Delta t^{S S-T R, \exp }\right) \tan \beta^{\text {exp }} \quad ; \quad \tan \beta^{\text {exp }}=\left(\frac{C_{s}^{e x p}}{C_{R}^{\text {exp }}}-\frac{C_{s}^{e x p}}{V_{r}^{\text {exp }}}\right)
$$

Strictly speaking, the above expressions determine a geometrically equivalent station, in terms of the arrival times of the supershear rupture and the trailing Rayleigh rupture, for a medium with different elastic properties than crustal rock. Note that in order to effectively apply the scaling relationships to laboratory earthquake data, the transition length, $L_{T}^{e x p}$, and the difference in the arrival times between the two ruptures, $\Delta t^{S S-T R, e x p}$, must be known in advance.

Recent experimental advancements using a 3D specimen fault geometry (Section 3.9) ensure that the supershear transition length is very well controlled and can be predicted with a high-degree of confidence. In order to determine $\Delta t^{S S-T R, e x p}$, the following temporal scaling argument is invoked. The temporal scale is established by the ratio of the temporal width of the trailing Rayleigh portion of the laboratory (FN) record and temporal width of the trailing Rayleigh portion of the actual PS10 (FN) ground motion record. The temporal scaling factor can then be used to stretch one trailing Rayleigh signature onto the other and vice versa. This approach requires confidence in the repeatability of the temporal width of the trailing Rayleigh rupture, $t^{T R, e x p}$, obtained in the experiments. The temporal width of the trailing Rayleigh rupture is found to be quite repeatable assuming specimen preparation procedures and all critical test conditions remain unchanged. Recent experiments using the 3D specimen fault geometry have yielded a temporal width for the trailing Rayleigh portion of the FN record given by $17.8 \mu \mathrm{s}$. The corresponding temporal width of the trailing Rayleigh portion of the (FN) Denali PS10 record, $t^{T R, P S 10}$ is $6.88 \mathrm{~s}$. The temporal scaling 
factor, $S_{T}$, is therefore determined to be

$$
S_{T}=\frac{t^{T R, P S 10}}{t^{T R, \exp }}=\frac{6.88 \mathrm{~s}}{17.8 \mu \mathrm{s}}=3.865 \times 10^{5}
$$

The temporal scaling factor may then be used to determine the delay time $\Delta t^{S S-T R, e x p}$ based on the corresponding value for PS10, which is estimated to be $4 s$ from the actual ground records. Thus,

$$
\Delta t^{S S-T R, \exp }=\frac{\Delta t^{S S-T R, P S 10}}{S_{T}}=\frac{4 \mathrm{~s}}{3.865 \times 10^{5}}=10.3 \mu \mathrm{s}
$$

Substituting these values into Eq. 5.9 along with $L_{T}^{\text {exp }}=45 \mathrm{~mm}, C_{s}^{\text {exp }}=1.3 \mathrm{~km} / \mathrm{s}, C_{R}^{\text {exp }}=$ $0.9 C_{s}^{e x p}$ and $V_{r}^{e x p}=1.7 C_{s}$, one obtains $x^{e x p} \approx 57 \mathrm{~mm}$ and $y^{\text {exp }} \approx 3 \mathrm{~mm}$. Note that the parameters for $L_{T}, C_{s}, C_{R}$, and $V_{r}$ are all determined from experimental trials prior to the converging on the final experiment, which is then scaled.

Once the experimental records are obtained they are then subjected to temporal scaling using the relation, $t^{\text {scaled }}=t^{e x p} \times S_{T}$. The spatial scaling and geometric constraint used to design the experiment automatically ensures that the arrival times of the supershear and trailing sub-Rayleigh ruptures in the scaled laboratory record will register with the arrival times of the equivalent features in the actual PS10 ground motion record.

As a final step, the magnitudes are scaled by the peak to peak swing in the trailing Rayleigh record, which is also justified by the dimensional scaling argument presented in Section 3.14.3.

\subsection{Scaled laboratory earthquake simulation of the $2002 M_{w} 7.9$ Denali fault, PS10 ground motion recordings}

Scaled PS10 laboratory earthquake experiments were conducted using $8^{\prime \prime} \times 8^{\prime \prime} \times 0.5^{\prime \prime}$ (nominal thickness) H-100 specimen assemblies featuring a 3D fault geometry and a specimen fault angle $\alpha=-26^{\circ}$. Figure 5.6 depicts a close-up view of an actual test specimen shown mounted in the 


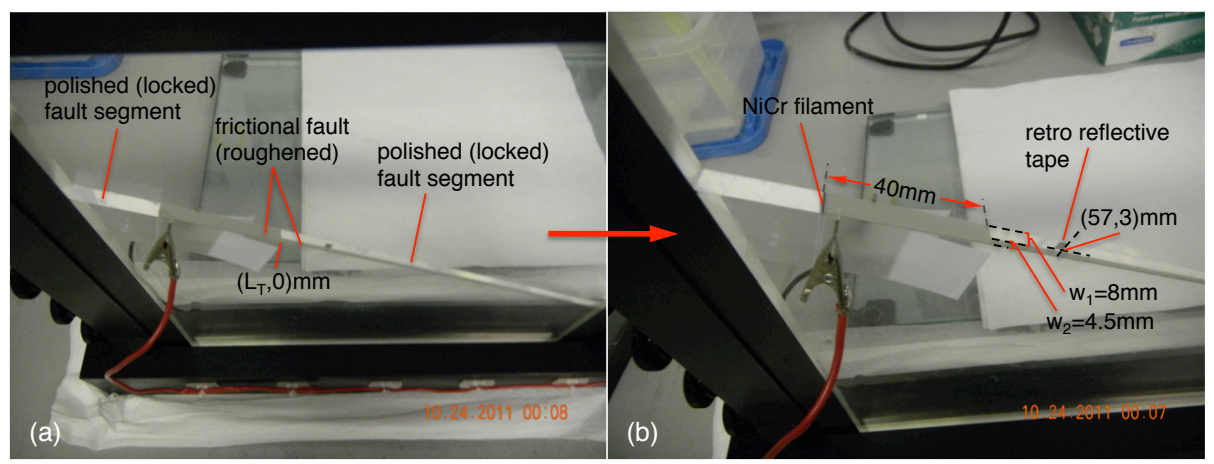

Figure 5.6: Close-up view of H-100 specimen prepared with $3 \mathrm{D}$ fault geometry used in scaled PS10 laboratory earthquake experiments

specimen frame and ready for testing. The same image is also shown in (Section 3.9), where specific details of the new 3D specimen fault geometry are addressed. The fault segment to the left of the nucleation site corresponds to a $25.4 \mathrm{~mm} \times 12.5 \mathrm{~mm}$ interface formed by two polished surfaces. The intent is to inhibit rupture propagation to the left through contact bonding of the flat polished surfaces of the short fault segment under the applied static compressive load. The $40 \mathrm{~mm}$ roughenedfault-segment to the right of the polished section is referred to as the nucleation patch. The $\mathrm{NiCr}$ filament channel was milled within $0.5 \mathrm{~mm}$ of the boundary between the short polished segment and the nucleation patch on the roughened side of the boundary. The $\mathrm{NiCr}$ wire is shown temporarily held in place by a strip of cellophane tape on either side of the specimen as is customary prior to loading the specimen in the hydraulic press. The coordinates $\left(L_{T}, 0\right)=(40,0) \mathrm{mm}$ correspond to a point on the surface of the test specimen, at the end of the nucleation patch, where the roughened portion of the fault is abruptly reduced in width from $t=12.5 \mathrm{~mm}$ to $w_{2}=4.5 \mathrm{~mm}$ as depicted in Figure 5.6. The adjacent polished fault section of width $w_{1}=8 \mathrm{~mm}$ is also expected to adhere under an applied static compressive load and act as a high-strength barrier, which resists rupture propagation and triggers a sub-Rayleigh-to-supershear transition. The retro-reflective tape strip used for the particle velocity measurements was positioned with its lower-right corner at the scaled PS10 location $(57,3) \mathrm{mm}$, based upon the expectation that a supershear transition will occur close to $x=L_{T}$. The results from several experiments conducted in advance were also used to estimate that a critical load $P_{c}>30 \mathrm{MPa}$ is required to trigger a supershear rupture at the high-strength 


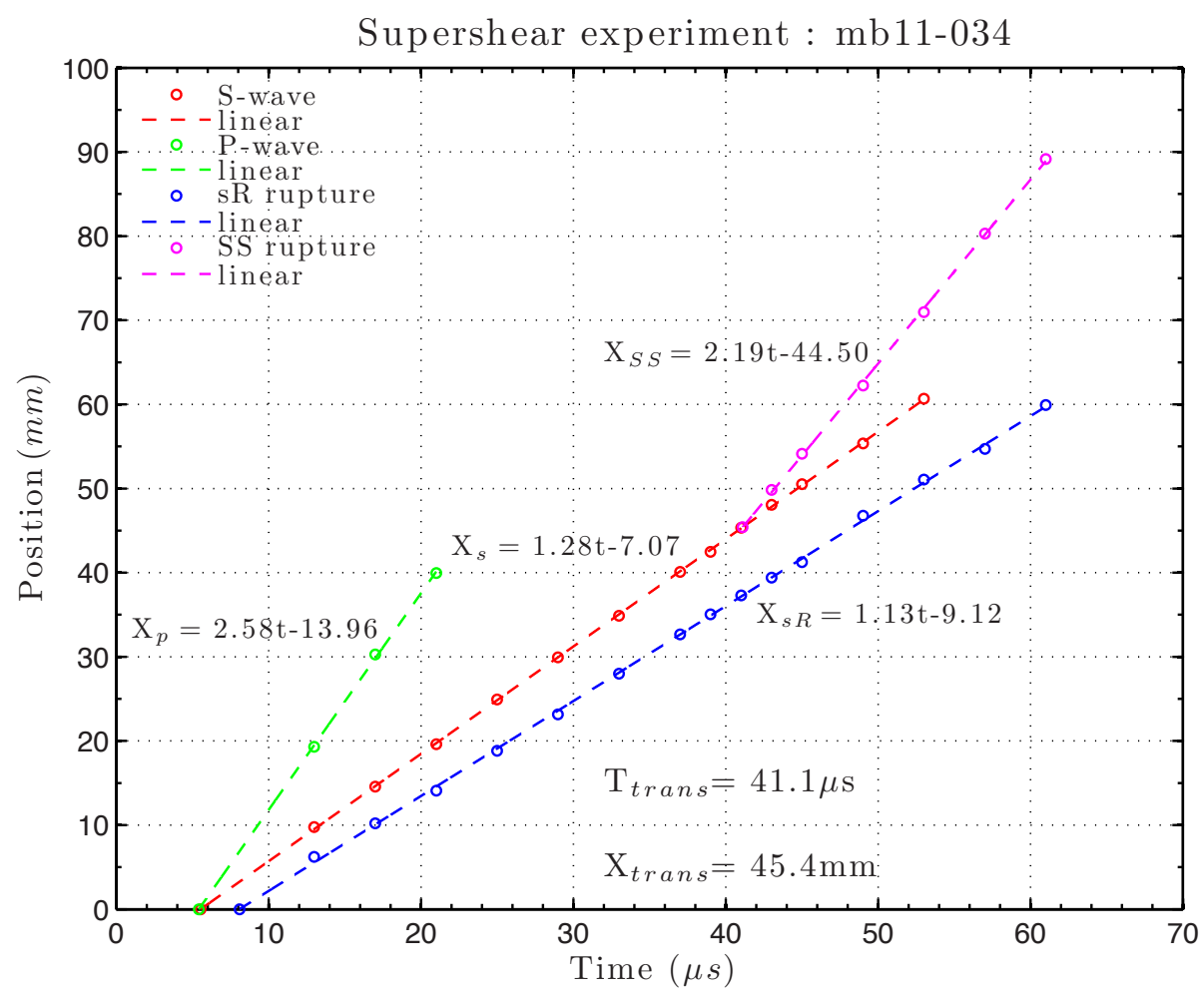

Figure 5.7: Distance-time plots obtained from the scaled $2002 M_{w} 7.9$ Denali, PS10 supershear experiment (exp11-034)

barrier.

The scaled PS10 supershear experiment (exp11-034) was conducted under a static compressive load of $P=31 M P a$. Three laser interferometer probe beams were focused onto the measurement station at $(57,3) \mathrm{mm}$ in order to simultaneously monitor the FP, FN, and vertical (V) particle velocity components at this location (Section 3.14). Synchronized, high-speed photoelastic images were simultaneously acquired in order to obtained a spatially resolved, full field view of the dynamic event.

\subsubsection{Distance-time plots resulting from the scaled PS10 experiment}

The distance-time plots obtained from the scaled PS10 supershear experiment (exp11-034) are depicted in Figure 5.7. The elastic wave speed plots yielded a $\mathrm{P}$-wave speed estimate of $C_{p}=$ $2.58 \mathrm{~mm} / \mu \mathrm{s}$ and an S-wave speed estimate of $C_{s}=1.28 \mathrm{~mm} / \mu \mathrm{s}$. S-wave and P-wave delays of $5.4 \mu s$ and $5.5 \mu s$, respectively, were obtained from time axis intercepts. Note that the elastic wave 
speed values are exactly as expected but the delay appears longer than the statistically averaged value given by $t_{s}^{\text {delay }}=4.45 \mu \mathrm{s}$. The blue curve in Figure 5.7 correponds to the sub-Rayleigh rupture distance-time curve. The slope of the best-fit line yields an average rupture speed of $V_{r}=1.13 \mathrm{~m} / \mathrm{s}$ corresponding to a normalized rupture speed of $V_{r} / C_{s}=0.88$. The equation of the best-fit line yields an estimated station crossing time of $t=58.5 \mu \mathrm{s}$ and a rupture delay time of $t_{\text {delay }}^{\text {rup }}=8.07 \mu \mathrm{s}$ corresponding to its time axis intercept. The supershear rupture displacement-time curve is also displayed in Figure 5.7. The data points correspond to estimated positions of the tip of the leading shear Mach cone in each image frame. The slope of the best-fit line reveals an average rupture speed $V_{r}=2.19 \mathrm{~mm} / \mu \mathrm{s}$ corresponding to a normalized rupture speed given by $V_{r} / C_{s}=1.71$. The location and time of the supershear transition were estimated by considering the intersection between the supershear and S-wave displacement-time curves. The intercept yields a transition time of $t^{*}=41.1 \mu \mathrm{s}$ and a transition distance $L_{T}=45.6 \mathrm{~mm}$. The equation of the line also reveals that the supershear rupture tip arrived at $x=57 \mathrm{~mm}$ at $t_{s s}=46.3 \mu \mathrm{s}$. An estimate of $t_{M F}=48.3 \mu s$ is obtained for the shear Mach front station-crossing-time at $(57,3) \mathrm{mm}$ by considering the Mach angle of $\theta=35.77^{\circ}$ resulting from the normalized rupture speed $V_{r} / C_{s}=1.71$. The measured value $\Delta t_{\text {meas }}^{S S-T R, \exp }=58.5 \mu s-48.3 \mu s=10.2 \mu s$ is found to be within $0.1 \mu s$ of the scaled value for $\Delta t^{S S-T R, \exp }$ as given by Eq. 5.11.

\subsubsection{High-speed photoelastic image sequence obtained during scaled su- pershear PS10 experiment}

The photoelastic image sequence in Figure 5.8 provides a spatially-resolved, frame-by-frame view of the scaled PS10 supershear experiment (exp11-034). An expanded view of Figure 5.8a, obtained at $t=39 \mu \mathrm{s}$, reveals a small caustic zone corresponding to a unilateral sub-Rayleigh rupture, which propagated to the right. The dark, circular caustic zone at $(40,0) \mathrm{mm}$ corresponds to the stress concentration at the boundary between the roughened and polished (locked) fault segments. The long cylindrical shadow seen in each of the image frames was produced by the side exit probe, which was used to direct the vertical (V) laser interferometer probe beam to the measurement station at 

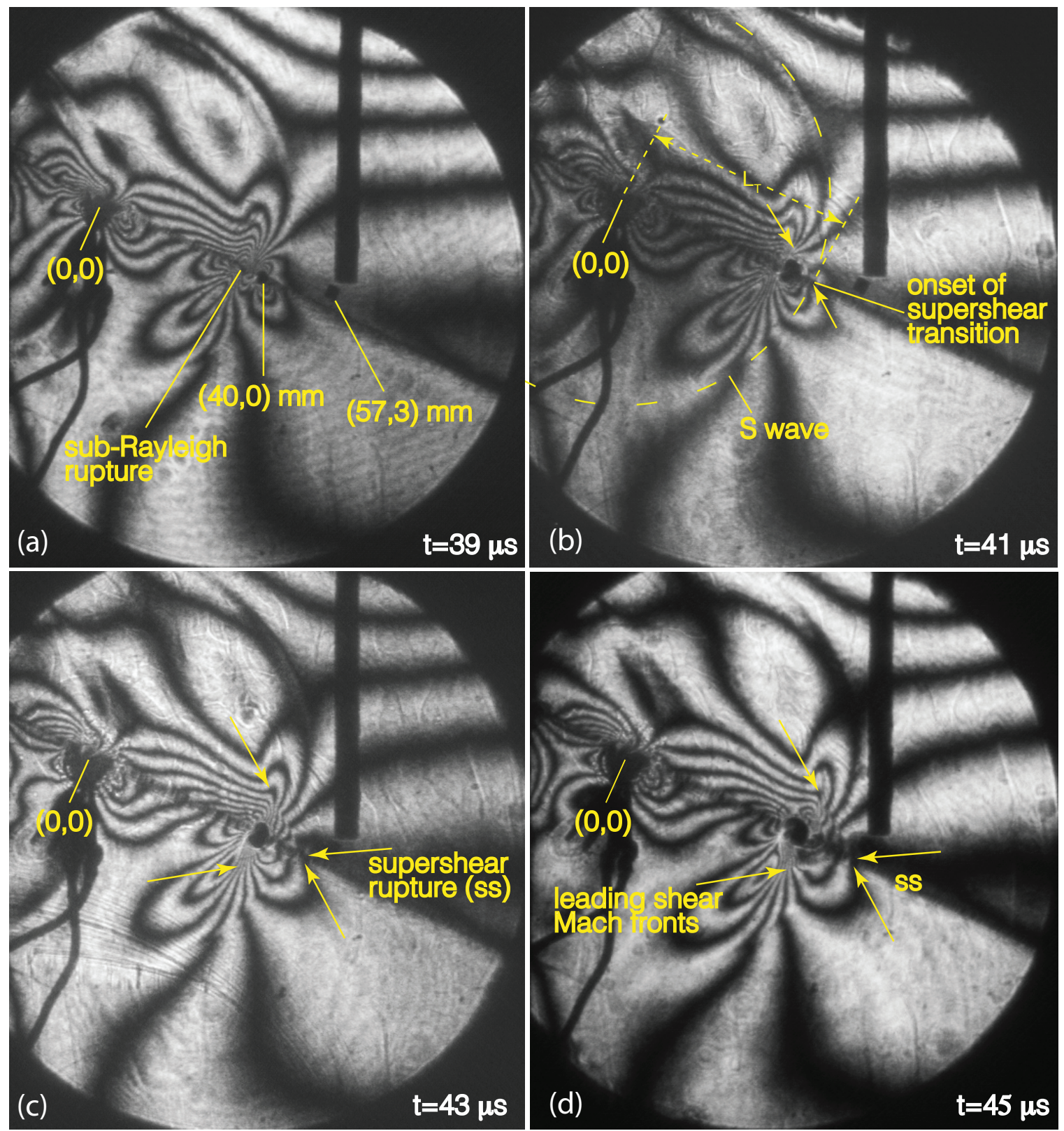

Figure 5.8: High-speed photoelastic image sequence of the sub-Rayleigh-to-supershear transition acquired during the scaled $2002 M_{w} 7.9$ Denali, PS10 laboratory earthquake experiment

$(57,3) \mathrm{mm}$.

Figure 5.8b depicts the sub-Rayleigh rupture as it encountered the boundary of the locked fault segment. The increased diameter of the caustic zone at $(40,0) \mathrm{mm}$ reveals a stress build-up which occurred as the advancing sub-Rayleigh rupture field was superimposed upon the pre-existing static stress field at the boundary of the locked fault segment. A supershear transition was triggered by 
the locked fault segment, which acted as a high-strength barrier, or, alternatively, as a patch of higher pre-stress (Dunham et al., 2003; Liu and Lapusta, 2008). Close examination of Figure 5.8b, also reveals the faint trace of a shear Mach front (between the tips of the two yellow arrows) and the onset of a supershear transition. The faint tip of the emerging supershear rupture coincides with the extended circular arc of the expanding S-wave front. The image frame time $\left(t^{*}=41 \mu s\right)$ thus provides a direct estimate of the supershear transition, which is in excellent agreement with the earlier estimate of $t^{*}=41.1 \mu s$ obtained from the intersection of the supershear rupture and S-wave front distance-time plots (Figure 5.7).

Well-formed shear Mach fronts are clearly visible in Figures 5.8c $(t=43 \mu s)$ and 5.8d $(t=45 \mu s)$. Note how the shear Mach fronts are mainly confined within the interior region bounded by the expanding S-wave front. This suggests that the supershear rupture emerged following a build-up of local stress over a finite period of time during which the S-wave front continued to expand radially outward. The observed supershear transition in the scaled PS10 experiment is notably different than the Burridge-Andrews type transition mechanism discussed in Section 3.12 and depicted in Figure 3.42. The results are best modeled by theoretical and numerical predictions of (Dunham et al., 2003; Liu and Lapusta, 2008) who considered alternative supershear transition mechanisms triggered by a high-strength barrier or a patch of higher pre-stress.

An expanded view of Figure 5.9a $(t=49 \mu s)$ reveals that the shear Mach front on the compressional side of the fault has just cleared the measurement station. The position of the shear Mach front with respect to the measurement station at this time correlates with the previously noted estimate of $t_{M F}=48.3 \mu \mathrm{s}$ for the shear Mach front station crossing. A pronounced circular caustic zone, corresponding to the trailing sub-Rayleigh rupture tip, is also clearly visible in the wake of the supershear rupture.

Figure $5.9 \mathrm{~b}(t=53 \mu \mathrm{s})$ reveals a striking image of a supershear rupture with leading and trailing shear Mach fronts, which bound the supershear slip zone. The distance between the tips of the leading and trailing Mach cones is estimated to be $5.7 \mathrm{~mm}$, which translates to a steady-state time interval of $2.6 \mu \mathrm{s}$ based upon the measured rupture speed of $V_{r}=2.19 \mathrm{~mm} / \mu \mathrm{s}$. This observation 


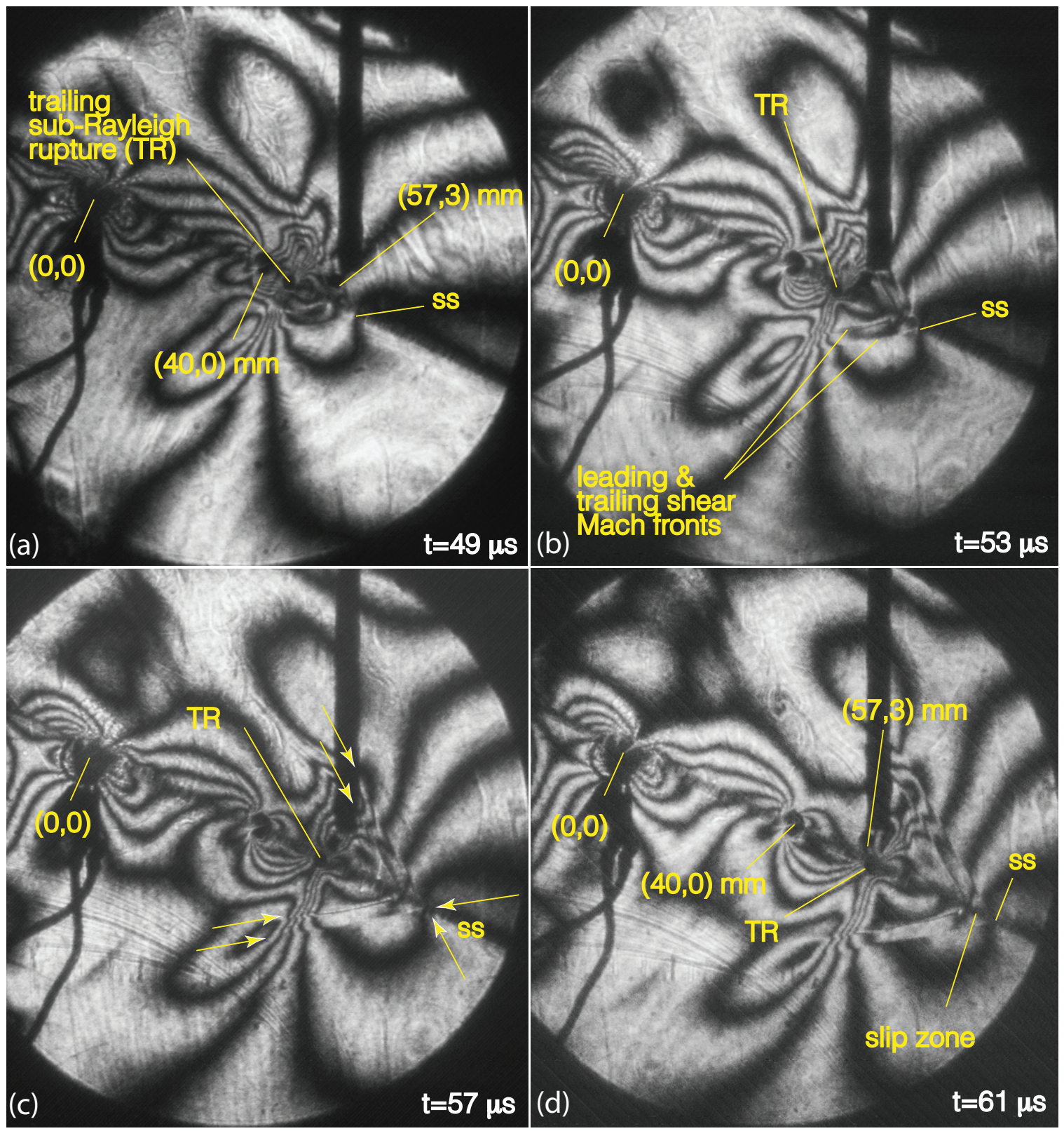

Figure 5.9: Image frame sequence following the superhsear transition captures the supershear rupture as it crosses the station followed by the emergence of the trailing Rayleigh rupture in the wake of the leading rupture. The two closely spaced shear Mach fronts define the supershear slip zone.

will be re-addressed shortly when the particle velocity traces for this experiment are examined.

Note the concentrated and elongated fringe pattern that surrounds the trailing Rayleigh (TR) rupture tip in Figure 5.9b $(t=53 \mu \mathrm{s})$ and how different it appears compared to the fringe pattern surrounding the TR in the previous $(t=49 \mu s)$ image frame. This feature is not typically observed when using the traditional laboratory earthquake specimen fault geometry. The observed differences 


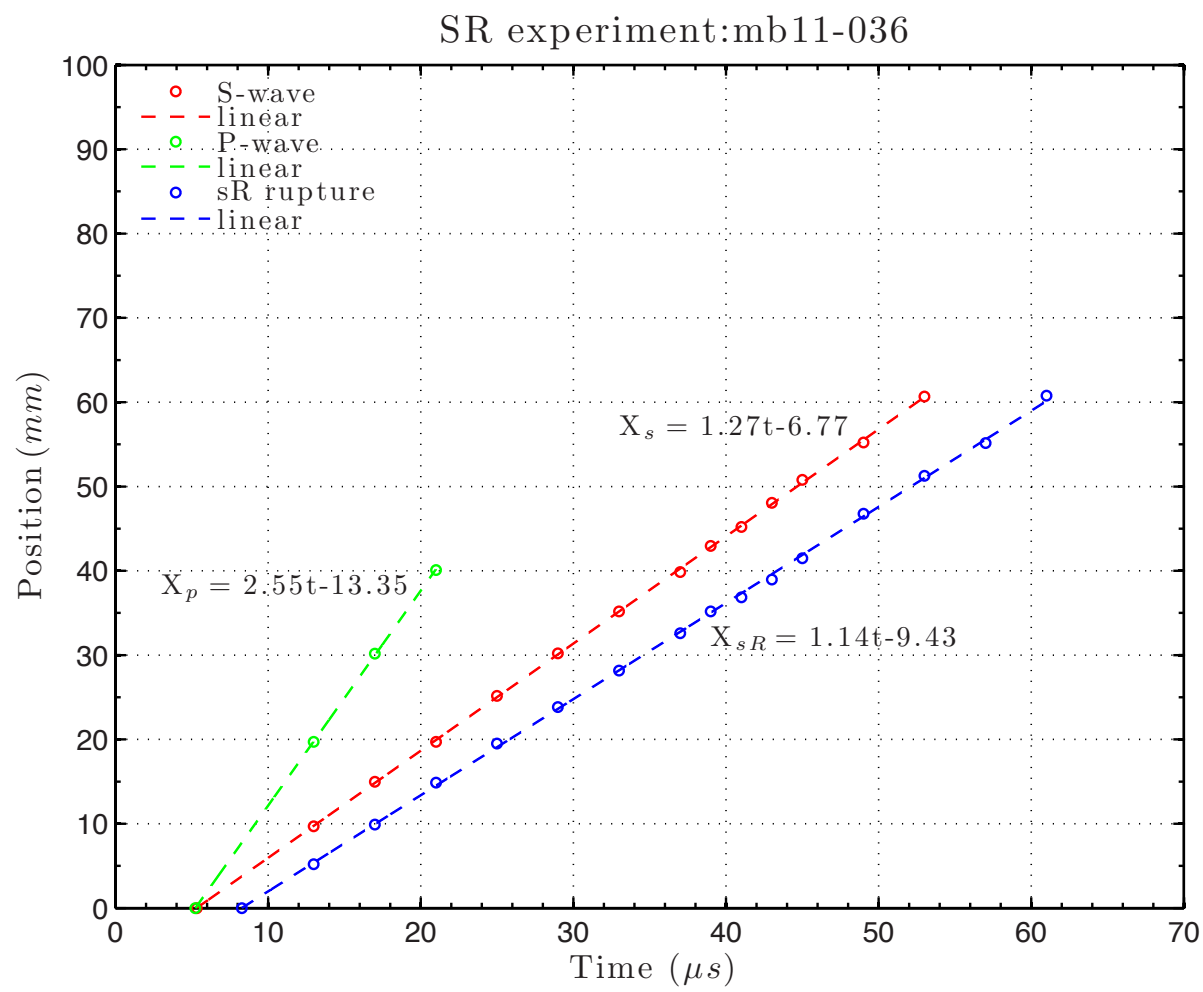

Figure 5.10: Distance-time plots obtained from the scaled $2002 M_{w} 7.9$ Denali, PS10 sub-Rayleigh experiment (exp11-036)

between the local stress fields surrounding the sub-Rayleigh and trailing Rayleigh ruptures on either side of $(40,0) \mathrm{mm}$ is likely a consequence of the asymmetrical nature of the fault, which is partially locked at depth. The final two image frames in Figures 5.9c $(t=57 \mu s)$ and $5.9 \mathrm{~d}(t=61 \mu s)$ reveal sharp images of the supershear slip zone between the leading and trailing supershear Mach fronts. The image frames also capture the TR rupture as its swept across the off-fault station at $(57,3) \mathrm{mm}$.

\subsubsection{Distance-time plots resulting from the scaled sub-Rayleigh PS10 experiment}

A second experiment (exp11-036) was conducted at a static compressive load of $P=28 M P a$ (below the experimentally determined threshold) in order to avoid a supershear transition and generate a set of sub-Rayleigh particle velocity records that could be contrasted against the results obtained in the supershear experiment (exp11-034). The test specimen used in the sub-Rayleigh experiment 
featured an identical fault geometry and had its fault surfaces prepared in an identical manner.

The displacement time plots for the scaled sub-Rayleigh PS10 experiment (exp11-036) are depicted in Figure 5.10. The elastic wave front displacement-time plots yield a P-wave speed estimate of $C_{p}=2.55 \mathrm{~mm} / \mu \mathrm{s}$ and an $\mathrm{S}$-wave speed estimate of $C_{s}=1.27 \mathrm{~mm} / \mu \mathrm{s}$. S-wave and $\mathrm{P}$-wave delays of $5.3 \mu \mathrm{s}$ and $5.2 \mu \mathrm{s}$, were, respectively, obtained from time axis intercepts. The wave speed values are well within the range of expected values but once again measured delay time appears

slightly longer than the statistically averaged value given by $t_{s}^{\text {delay }}=4.45 \mu \mathrm{s}$. The precise nature of the $\mathrm{S}$ and $\mathrm{P}$-wave delay is not well understood but it stands to reason that the increased specimen thickness and hence the increased length of the NiCr filament length (and hence resistance) may have contributed to slightly increased delay time. The blue curve in Figure 5.10 corresponds to the sub-Rayleigh rupture distance-time curve. The slope of the best-fit line yields an average rupture speed estimate of $V_{r}=1.14 \mathrm{~m} / \mathrm{s}$ corresponding to a normalized rupture speed of $V_{r} / C_{s}=0.89$. The equation of the best-fit line yields an estimated station crossing time of $t=58.3 \mu \mathrm{s}$ and a rupture delay time of $t_{\text {delay }}^{\text {rup }}=8.27 \mu \mathrm{s}$ corresponding to its time axis intercept.

\subsubsection{Analysis and discussion of particle velocity records obtained from the scaled PS10 laboratory earthquake experiments}

Figure 5.11 displays the particle velocity records obtained from both experiments with the subRayleigh particle velocity records depicted on the left and the supershear records depicted on the right. Each set of particle velocity records is accompanied by a photoelastic image acquired at $t=61 \mu \mathrm{s}$. The red lines superimposed upon the high-speed photoelastic images represent the optical paths of the FN and FP laser interferometer probe beams used in the experiment. The dark cylindrical feature stemming from the top of each image corresponds to the shadow of the side exit probe, which directed the vertical beam to the measurement station. Note that the subRayleigh and trailing Rayleigh ruptures have each already crossed their respective measurement stations at $t=61 \mu \mathrm{s}$. Note as well the general similarities between the local stress field surrounding the sub-Rayleigh image in the left-hand image and the trailing Rayleigh rupture in the right-hand 

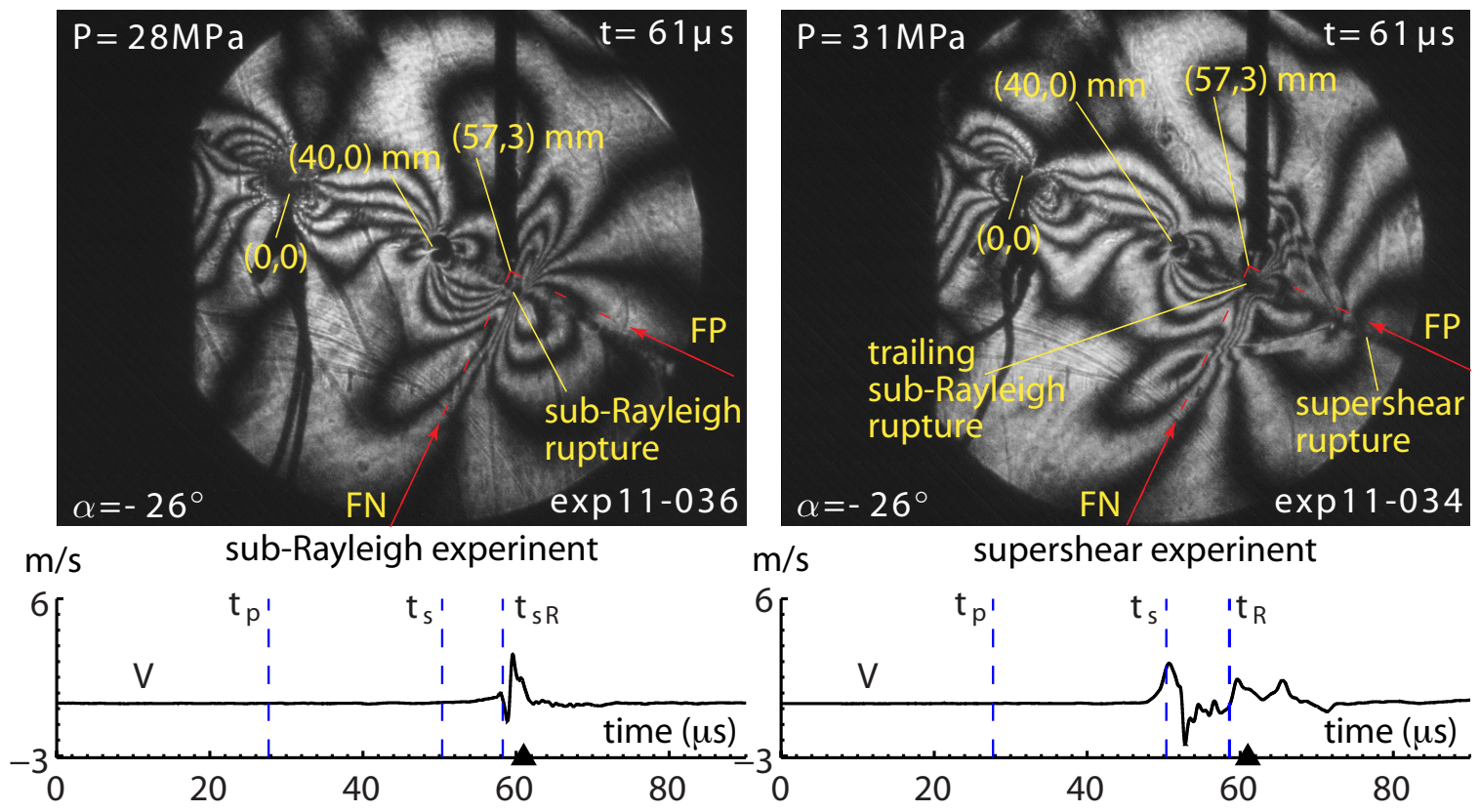

$\mathrm{m} / \mathrm{s}$

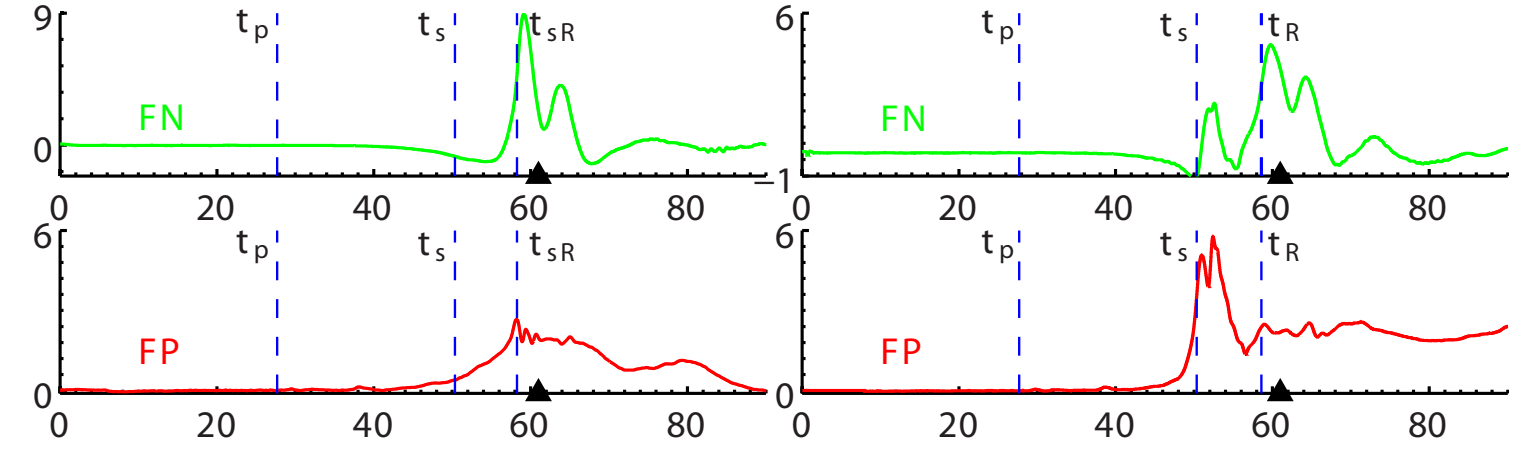

Figure 5.11: Comparison between the sub-Rayleigh and supershear laboratory earthquake experiments conducted using the scaled $2002 M_{w} 7.9$ Denali PS10 configuration

image.

The dashed blue vertical lines in the particle velocity plots designate the anticipated arrival times of the P-wave, S-wave, and respective sub-Rayleigh ruptures in each experiment. The black triangle on the time axis marks the time at which the photoelastic image above was acquired with respect to the instrumentation trigger. Note as well that the range of the particle velocity axes varies from component to component, which required in order to properly reveal the defining features within each individual record. 


\subsubsection{Analysis and discussion of sub-Rayleigh particle velocity records obtained in (exp11-036)}

The sub-Rayleigh particle velocity records obtained from the scaled PS10 configuration are dominated by the FN record, as expected. The time at which the sharp velocity increase is registered correlates very well with the estimated rupture arrival time $t_{s R}=58.3 \mu \mathrm{s}$.

The velocity jump in the vertical record also correlates with the estimated rupture arrival time of $t_{s R}=58.3 \mu \mathrm{s}$. The steep, positive jump exhibited by the vertical component in the sub-Rayleigh experiment is consistent with the expected sense of motion produced by a right lateral rupture on the compressional side of the fault, where the surface is expected to bulge outward in the positive direction as the rupture sweeps across the station. An anti-symmetric profile is anticipated on the extensional side.

Interestingly, the FP velocity component begins to increase well in advance of theoretical S-wave front arrival time (in stark contrast to the other two records, and also exhibits a long pulse-like profile, which extends for nearly $50 \mu \mathrm{s}$. Note however, how the peak in the FP record correlates with the estimated arrival time of the rupture tip. The initial (early) FP motion likely arises from radiation emitted from the high-strength barrier, which arrives in advance of the main rupture.

The long pulse-width exhibited by the FP record may be attributed to the fact that the original sub-Rayleigh rupture was crack-like as it approached the high-strength barrier. The healing mechanism eventually converted the crack-like velocity profile to a slip-pulse with a broad temporal width. It was reasoned that a lower static-compressive load may have produced a pulse-like rupture from the very start, which would necessarily emerge beyond the high-strength barrier as a pulse-like rupture of with a much shorter temporal width. This hypothesis was later confirmed through a separate experiment, conducted at lower static compressive stress, which resulted in a pulse width on the order to $20 \mu s$. The observations and hypotheses regarding the nature of the long slip-pulse in the sub-Rayleigh experiment (exp11-036) are relevant to the results obtained in the supershear experiment since they apply equally well to the trailing Rayleigh rupture. This point will be further clarified in the next section when the particle velocity traces for the supershear experiment 
(exp11-034) are examined.

\subsubsection{Analysis and discussion of supershear particle velocity records ob- tained in (exp11-034)}

The particle velocity records obtained from the supershear experiment (exp11-034) are depicted on the right-hand side of Figure 5.11. The dashed blue vertical lines labeled $t_{p}$ and $t_{s}$ mark the anticipated arrival times of the $\mathrm{S}$ and $\mathrm{P}$-wave fronts while the lines labeled $t_{s R}$ and $t_{R}$ mark the anticipated arrival times of the sub-Rayleigh and trailing Rayleigh rupture tips at $x=57 \mathrm{~mm}$.

When interpreting the supershear particle velocity records It is important to note that the dilatational and shear field contributions never fully separate at this short distance from the fault. The resulting particle velocity records generally represent a superposition of the dilatational field, which circulates around the rupture tip, and the shear radiation field confined within the region bounded by the shear Mach fronts.

The leading portions of the supershear particle velocity records are dominated by the FP record, as expected, which is characterized by a pronounced double-peak. The first local maxima, which peaks near $t=t_{s}$, is attributed to the leading dilatational field lobe, which encircles the supershear rupture tip. The second sharp velocity jump which follows is correlated to the arrival of the shear Mach front. Hence, although the two fields do not fully separate, the arrival times of their respective maxima are still resolved within the record.

The leading portion of the supershear FN record exhibits a more subtle double-peak is also linked to the arrival of the leading dilatational field and the shear Mach front. The anticipated negative velocity swing of the FN component along the shear Mach front is overwhelmed by the dilatational field, which dominates the sense of motion at such a short distance from the fault.

The trailing portion of the FN record, which follows the arrival of the supershear rupture bears striking similarities to the FN signal obtained in the sub-Rayleigh experiment. Although the signals exhibit different amplitudes, the two records are otherwise extremely similar in form.

The initial pulse-like portion of the supershear vertical record, which peaks near $t=t_{s}$, is 
correlated to the arrival of the dilatational field. This is followed by an abrupt change of slope and a negative velocity dip, which is attributed to the arrival of the shear Mach front. the time corresponding to the abrupt slope change provides an excellent estimate for the arrival time of the shear Mach front, which also correlates quite well with the noted arrival times of the Mach front in the FP and FN records.

A detailed examination of the FP record obtained in the supershear experiment reveals that the dilatational signal peaked at $t=51 \mu s$ while the peak linked to the shear Mach front, occurred at $t=52.41 \mu \mathrm{s}$. The two peaks are therefore separated by $1.41 \mu \mathrm{s}$, which is notably less than the previously estimated time interval of $t=2.6 \mu \mathrm{s}$ between the tips of the leading and trailing Mach cones. The analysis demonstrates that the two initial peaks in the FP record are therefore not correlated to the passage of the leading and trailing shear Mach fronts.

The FP signal dropped by $76 \%$ from its peak value of $5.77 \mathrm{~m} / \mathrm{s}$ (at $t=52.41 \mu \mathrm{s}$ ) down to 1.37 $\mathrm{m} / \mathrm{s}$ at $t=56.58 \mu \mathrm{s}$, after which it began to climb again. If the $2.6 \mu s$ steady-state time estimate between the tips of the two Mach cones is attributed to a supershear slip-pulse (slip zone), then healing of the supershear signal should have been observed near $t=52.41 \mu s+2.60 \mu s=55 \mu s$. This clearly did not occur and a crack-like rupture resulted instead. The central question is why did the supershear pulse fail to completely heal?

Next, note the complete absence of any secondary supershear signals in the particle velocity records (section 3.10). Such signals typically accompany secondary shear Mach fronts as previously addressed in (Section 3.10). The main point here is that the sharp, trailing shear Mach fronts captured in the scaled PS10 superhsear image sequence are not accompanied by the usual (anomalous) secondary supershear particle velocity signals. This observation suggests that the trailing Mach fronts captured in the PS10 supershear experiment are not an artifact of the plate thickness (section 3.10). The results appear to validate the new $3 \mathrm{D}$ fault geometry and the mechanism through which it suppresses any sharp signals that might otherwise arrive from the free surface on the opposite side of the specimen. The trailing Mach front, which is so clearly visible in the image sequence may very well denote the tail end of slip zone and the healing phase of the supershear rupture. The 
central question is why did the supershear pulse fail to completely heal? As it turns out, the most significant clue comes from the FP record in the sub-Rayleigh rupture experiment.

A plausible explanation is obtained by appealing to the FP record obtained in the scaled PS10 sub-Rayleigh rupture experiment (exp11-036), which was previously examined in Section 5.3.5. Figure $5.9 \mathrm{a}$ shows how closely spaced the two ruptures were to each other as the leading and trailing shear Mach fronts were crossing the measurement station at $(57,3) \mathrm{mm}$. Recall from Section 5.3.5) how the FP signal in the sub-Rayleigh experiment exhibited an early velocity increase, which began in advance of the nucleation S-wave arrival $\left(t=t_{s}\right)$. Note as well that the sub-Rayleigh experiment was conducted under a static compressive load of $P=28 M P a$, which was only $2 M P a$ lower than the static-compressive load used in the supershear experiment. In light of these points, it is quite reasonable to assume that a similar FP signal contribution arose from the trailing Rayleigh rupture, which was superimposed upon the primary supershear signal during this same period of time during the supershear experiment. Hence, as the supershear slip-pulse was healing there was a superimposed (increasing) FP velocity signal from the leading edge of the TR rupture which proceeded to offset the velocity drop imposed by the trailing edge of the supershear slip-pulse.

The visual evidence of the double shear Mach fronts captured in the photoelastic image sequence is compelling. The collective particle velocity data sets indicate that the trailing shear Mach front was not an artifact of the finite specimen thickness but was instead the healing phase of a supershear slip pulse. It also likely that the FP particle velocity signal may have resulted in a perfect slip-pulse had the measurement station been positioned further away from the high-strength barrier. This would provide enough time for the supershear rupture to sweep the measurement station and have the FP signal fully heal before any concentrated radiation from the high-strength barrier arrived to offset the healing process. The experiments demonstrate the potential of the new 3D fault geometry. Continued development and refinement of the new technique may very well lead to the repeatable generation of supershear slip-pulses in laboratory earthquake experiments. This could enable new and unprecedented laboratory earthquake investigations. 

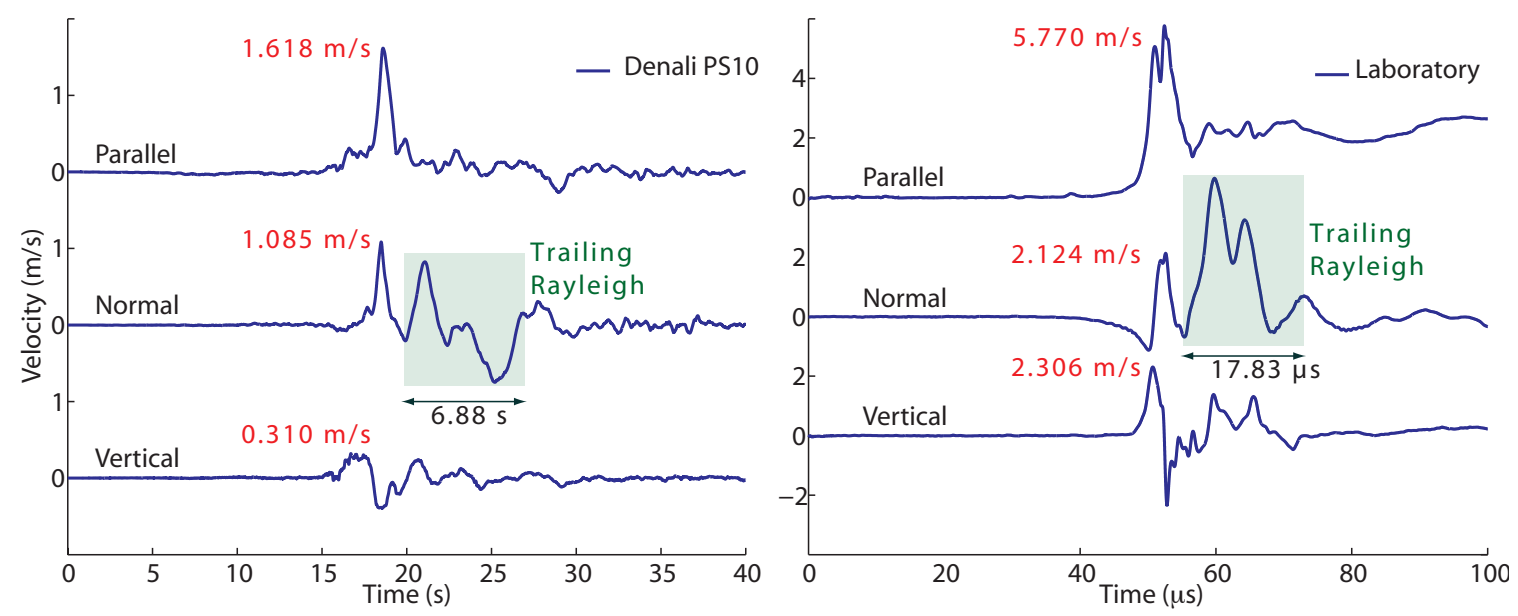

Figure 5.12: Denali supershear event Recorded at PS10 (left) and Laboratory record obtained at the scaled measurement station located at $(57,3) \mathrm{mm}$ (right)

\subsection{Scaling of laboratory earthquake supershear experiment and direct comparison with PS10 ground motion records}

Figure 5.12 depicts the PS10 ground motion records on the left and the particle velocity records from the scaled supershear laboratory experiment (exp11-034) on the right. Note that the time axis in the PS10 plots is measured with respect to the $\mathrm{P}$-wave arrival while the time axis in the experimental plots is measured with respect to the instrumentation trigger time. The temporal widths of the trailing Rayleigh portion of each record are identified and labeled as shown. The ratio of the temporal widths between the two records was used to set the temporal scaling factor in accordance with Eq. 5.10. Spatial scaling was accomplished a priori through the design of the experiment in accordance with Eq. 5.9 and ensures that relative arrival times between the supershear and trailing Rayleigh ruptures is preserved when the temporal scaling factor is applied. Peak velocity values for each particle velocity component and the ground motion records are also noted in anticipation of amplitude scaling. As a final step, the magnitudes are scaled by the peak to peak swing in the trailing Rayleigh record, which is also justified by the dimensional scaling argument presented in (Section 3.14.3).

In general, the pulse-like signature obtained in the sub-Rayleigh experiment and the crack-like 


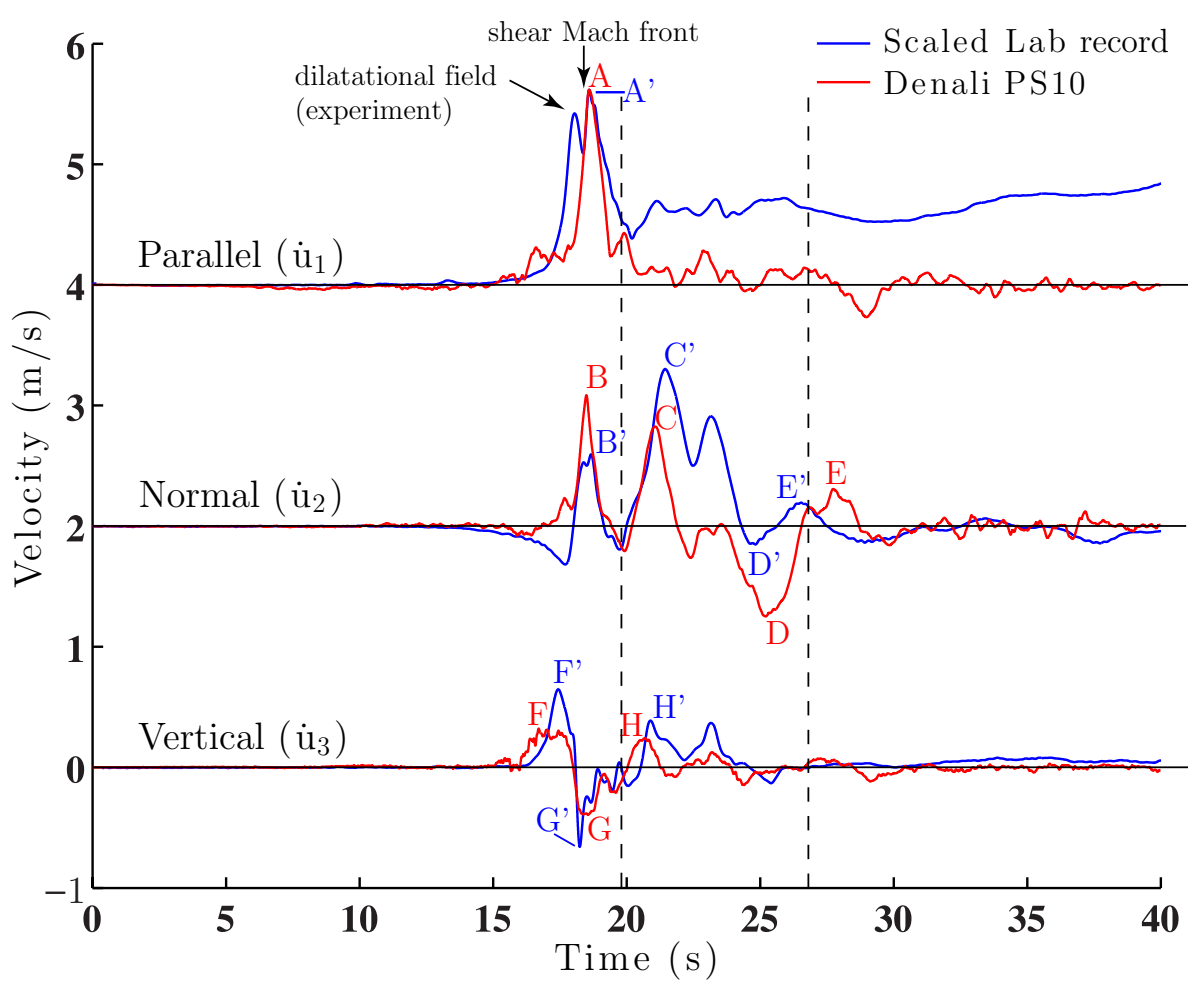

Figure 5.13: Scaled experimental supershear record compared with Denali PS10 record

result of the supershear experiment is consistent with previous experiments, which demonstrate how dynamic shear ruptures on interfaces that are pre-stressed in compression and in shear systematically vary from crack-like to pulse-like in accordance with the non-dimensional shear pre-stress or absolute stress levels (Lu et al., 2007, 2010). Experimental results are also consistent with theory of pulse-like and crack-like shear ruptures on velocity weakening interfaces (Zheng and Rice, 1998; Samudrala et al., 2002a).

The scaling relationships presented in Section 5.2 were applied to the laboratory records to produce the blue curves in Figure 5.13, which are shown superimposed upon the actual Denali PS10 ground motion records. Note that the laboratory records, which originally spanned a time interval of $100 \mu s$ have now been stretched through six orders of magnitude in time to match up with the Pump Station 10 ground motion records. Time is plotted relative to the $\mathrm{P}$-wave arrival time at PS10. The dashed vertical lines extending through the plots correspond to the $6.88 \mathrm{~s}$ time interval of the trailing Rayleigh portion of the record that was used to set the temporal scaling factor. 
Each point labeled (A' - H') in the laboratory particle velocity records has a corresponding point $(\mathrm{A}-\mathrm{H})$ in the PS10 ground motion records and vice versa. Apart from the fact that the experimental record is crack-like, the scaled records much up quite well with the PS10 ground motion records capture all of the prominent signatures. The correct sense of motion is properly observed between the corresponding portions of the records and is consistent with the choice of a properly scaled measurement station positioned on the compressional side of the fault located to the right of the nucleation site $(x>0)$.

The laboratory FP record (blue curve) exhibits an initial peak, which is attributed to the leading dilatational field lobe. The resulting ground motion at such a short distance from the fault is heavily influenced by the dilatational field, which curls around the rupture front, and there is never a sustained separation between the two field contributions as the rupture and its surrounding field sweeps across the station. The dominance of the FP component over the FN component ( $A$ vs. B) and $\left(A^{\prime}\right.$ vs. $\left.B^{\prime}\right)$ is observed in the early portion of the experimental records although the $1.5 \times$ ratio exhibited by the PS10 records was not captured by the experimental records. Both records become quiescent following the passage of the primary supershear rupture.

Note that the PS10 fault-parallel record does not exhibit a dilatational field peak prior to the arrival of the shear Mach front. This could be attributed to the fact that the lab experiment is still largely 2D-like by nature in that there is no significant spatial variation of the rupture through depth. This point is bolstered by the results of the dynamic 3D calculations by (Dunham and Archuleta, 2004) which do not distinguish between the leading dilatational field and the shear Mach front.

The experimental FN record exhibits some striking similarities with the corresponding PS10 record, particularly between the points labeled $C^{\prime} \rightarrow D^{\prime} \rightarrow E^{\prime}$ in the laboratory FN record and the corresponding velocity swings spanning from $C \rightarrow D \rightarrow E$ in the PS10 FN ground motion record. The magnitude of the relative velocity swings between $C^{\prime} \rightarrow D^{\prime}$ and $C \rightarrow D$ were forced to match as part of the scaling process. This then established the amplitude scaling which was applied to the FP and V records.

As noted by (Dunham and Archuleta, 2004) the almost antisymmetric nature of this part of the 
record at PS10 reveals that the trailing Rayleigh disturbance was pulse-like. However, since a cracklike rupture resulted in the experiment, the trailing Rayleigh disturbance failed to completely heal and hence the record is not as antisymmetric. Nevertheless, the result captures the same general features and sense of motion observed in the corresponding portion of the PS10 FN ground motion record, and provides strong experimental confirmation that this portion of the PS10 record was indeed attributed to the passage of a trailing Rayleigh rupture.

There is also a very nice match between the vertical record obtained in the laboratory earthquake spanning between the points $F^{\prime} \rightarrow H^{\prime}$. and the PS!0 ground motion record spanning the interval defined by between the points labeled $F \rightarrow H$. The result suggests that the near tip fields were largely responsible for the recorded ground motion and that depth effects played a far lesser role in the process.

Finally, it is acknowledged that the PS10 recordings feature a pulse-like rupture and that the supershear experiment presented here resulted in a crack-like rupture. This point was addressed in considerable detail in the previous section where arguments were presented to support the claim that such experiments can, under appropriate conditions, produce pulse-like supershear ruptures. The fact that the experimental record failed to produce a perfect healing phase is inconsequential when viewed within the broader objectives of the investigation. The results presented here should be viewed as a physical analog to numerical modeling simulations of the PS10 ground motion records (Ellsworth et al., 2004; Dunham and Archuleta, 2004). The overall goal was to capture the prominent supershear ground motion signatures and offer a physical basis for the design of the experiment and the interpretation of the results. Viewed from this perspective, the experiments as presented were quite successful and comparable in many respects to the best numerical modeling attempts offered up to this point in time. In summary, the unique near field signatures in the ground motion records for both sub-Rayleigh and supershear ruptures have been identified. Building upon previous work by (Ellsworth et al., 2004; Dunham and Archuleta, 2004), a rigorous theoretical and numerical argument regarding the supershear ground motion signatures has been presented. Experiments clearly show that the dominance of the fault-parallel velocity component in the rupture speed regime 
$\left(\sqrt{2} C_{s}<V_{r} \leq C_{p}\right)$ is observed.

Experiments have validated that a rupture traveling at supershear speeds exhibits the following signatures, many of which are predicted by theory:

- A dilatational precursor, dominant in the fault-parallel component, that arrives in the ground motion records prior to the arrival of the Mach front.

- Dominance of the fault-parallel component over the fault-normal component in the supershear speed regime $\left(\sqrt{2} C_{s}<V_{r} \leq C_{p}\right)$.

- Trailing Rayleigh rupture behind the main rupture tip with a dominant fault-normal component. This produces a ground-shaking signature qualitatively similar to a sub-Rayleigh rupture.

Experiments were also conducted to replicate the 2002 Denali $M_{w} 7.9$ ground motions as recorded at Pump Station 10 located $85 \mathrm{~km}$ east of the hypocenter and just $3 \mathrm{~km}$ north of the fault. The results of the experiments validate the hypothesis put forth by (Ellsworth et al., 2004; Dunham and Archuleta, 2004) that a segment of the Denali strike slip fault experienced a supershear burst.

The direct practical consequence of the above observations are that a near field station will first experience the primary fault-parallel (FP) shaking due to the arrival of the supershear rupture fields, followed by a primary fault-normal (FN) shaking linked to the trailing Rayleigh rupture. Structures located near a fault hosting such a transition will effectively experience two separate, closely timed earthquake events characterized by different forms of ground-shaking. The timing between these two occurrences will depend on the location of the near field station relative to the point of sub-Rayleigh-to-supershear transition. 


\section{Chapter 6}

\section{Dynamic Stress Measurements within the Supershear Slip Zone}

Consider the scenario where simultaneous measurements of the fault-normal (FN) and fault-parallel (FP) particle velocity components are conducted at a point $\left(x, 0^{-}\right)$or $\left(x, 0^{+}\right)$located arbitrarily close to a frictional fault. In a laboratory earthquake experiment this constitutes focusing two laser interferometer probe beams onto a location positioned within $100-200 \mu m$ of the specimen fault as depicted in Figure 6.1. Measurements of this nature are herein referred to as "near-fault" particle velocity measurements and can also involve paired FP measurements whereby the probe beams are focused on either side of the fault and separated by an extremely short distance on the order of the focused laser beam spot diameter.

The scope of this chapter addresses a discovered relationship, pertaining mainly to supershear ruptures, which relates the near-fault particle velocity records to the evolution of the dynamic stress components at the same location. The observed relationship is derived from the general 2D steadystate supershear solution by (Dunham and Archuleta, 2005), which is summarized in Section 2.3.1. The new technique enables the direct measurement of $\sigma_{x x}$ and $\sigma_{x y}$ at the specimen frictional interface as a supershear rupture sweeps across the measurement station. A knowledge of the elastic wave speeds $\left(C_{s}, C_{p}\right)$, density $(\rho)$, and shear modulus $(\mu)$ of the test specimen are required along with an accurate estimate of the supershear rupture speed $\left(V_{r}\right)$. Each of these physical variables, apart from the density of $\mathrm{H}-100$, is accurately estimated within each experiment through the analysis of synchronized, high-speed photoelastic images as detailed in Section 3.11.2. 


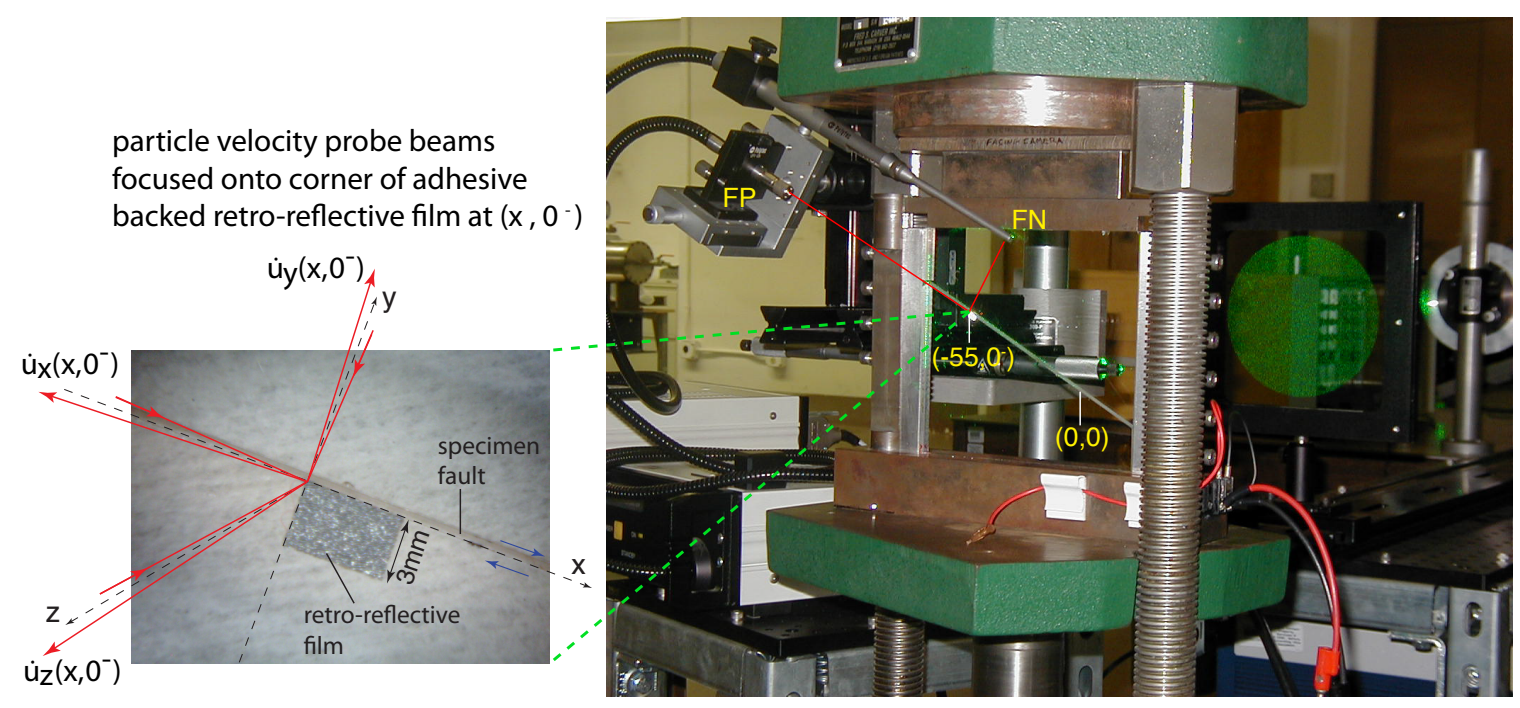

Figure 6.1: Laboratory earthquake experimental configuration used for simultaneous measurement of fault-normal and fault-parallel particle velocity measurements at a near-fault measurement station positioned at $\left(-55,0^{-}\right) \mathrm{mm}$

Stress measurements derived from particle velocity components at the frictional fault enable a unique class of dynamic friction investigations. The theoretical basis of the new measurement technique is presented in detail. The dynamic stress relationships were applied to a subset of supershear experiments, which featured near-fault particle velocity measurements using the (FN,FP) laser interferometer configuration. Stress measurements and dynamic friction investigations obtained from representative supershear experiments are presented in detail.

\subsubsection{Experimental verification of anti-symmetric fault-parallel particle velocity records in laboratory earthquake experiments}

Antisymmetry of the FP particle velocity component was invoked in Section 2.3.2 and will be further invoked in the derivation of the stress-particle velocity relationships developed later in the chapter. Experimental verification of antisymmetry in laboratory earthquake particle velocity records is demonstrated by examining the results obtained from two independent supershear rupture experiments. The results also demonstrate how in many instances a single fault-parallel particle velocity record can be used to accurately estimate the slip velocity. 
As previously discussed in Section 2.3.2, the slip velocity is defined by

$$
\Delta \dot{u}_{x}(x, 0)=\dot{u}_{x}\left(x, 0^{+}\right)-\dot{u}_{x}\left(x, 0^{-}\right)
$$

where the notation $\dot{u}_{x}\left(x, 0^{ \pm}\right)=\lim _{y \rightarrow 0^{ \pm}} \dot{u}_{x}\left(z_{s}\right)$ denotes the particle velocities at a pair of closely spaced points positioned just above and below the fault. The assumption that the fault-parallel particle velocity component is anti-symmetric, i.e., $\dot{u}_{x}\left(x, 0^{+}\right)=-\dot{u}_{x}\left(x, 0^{-}\right)$, leads to an alternative description of the slip velocity given by

$$
\begin{aligned}
& \Delta \dot{u}_{x}(x, 0)=2 \dot{u}_{x}\left(x, 0^{+}\right) \\
& \Delta \dot{u}_{x}(x, 0)=-2 \dot{u}_{x}\left(x, 0^{-}\right) .
\end{aligned}
$$

Eq. 6.2 implies that the slip velocity can be determined from a single near-fault particle velocity record $\left(\mathrm{FP}^{+}\right.$or $\left.\mathrm{FP}^{-}\right)$. Care must be exercised, however, when "blindly" applying Eq. 6.2 to a FP particle velocity record since any non-zero portion of the record will be effectively doubled. Strictly speaking, one should first identify which portions of the particle velocity record which correspond to interfacial sliding and apply Eq. 6.2 only to those portions. The use of paired fault-parallel records is recommended whenever possible. Experience gained from the analysis of anti-symmetric FP records can then be leveraged to estimate the arrival time of the slip-pulse in cases when only a single FP measurement is obtained.

Antisymmetry of the fault-parallel particle velocity component has been been observed and verified in laboratory earthquake experiments through paired fault-parallel measurements conducted at two closely spaced points positioned above and below the specimen fault. Figure 6.2 depicts the results obtained from one such supershear experiment (exp10-036). The experiment was conducted using a traditional $6^{\prime \prime} \times 6^{\prime \prime} \times 0.375^{\prime \prime} \mathrm{H}-100$ specimen with uniformly roughened fault surfaces with a fault angle of $\alpha=-26^{\circ}$ and a static-compressive load $P=31 \mathrm{MPa}$.

The two high-speed photoelastic images reveal the positions of the supershear and trailing Rayleigh rupture fronts at $t=45 \mu \mathrm{s}$ and $t=55 \mu \mathrm{s}$ and establish temporal correlation between 

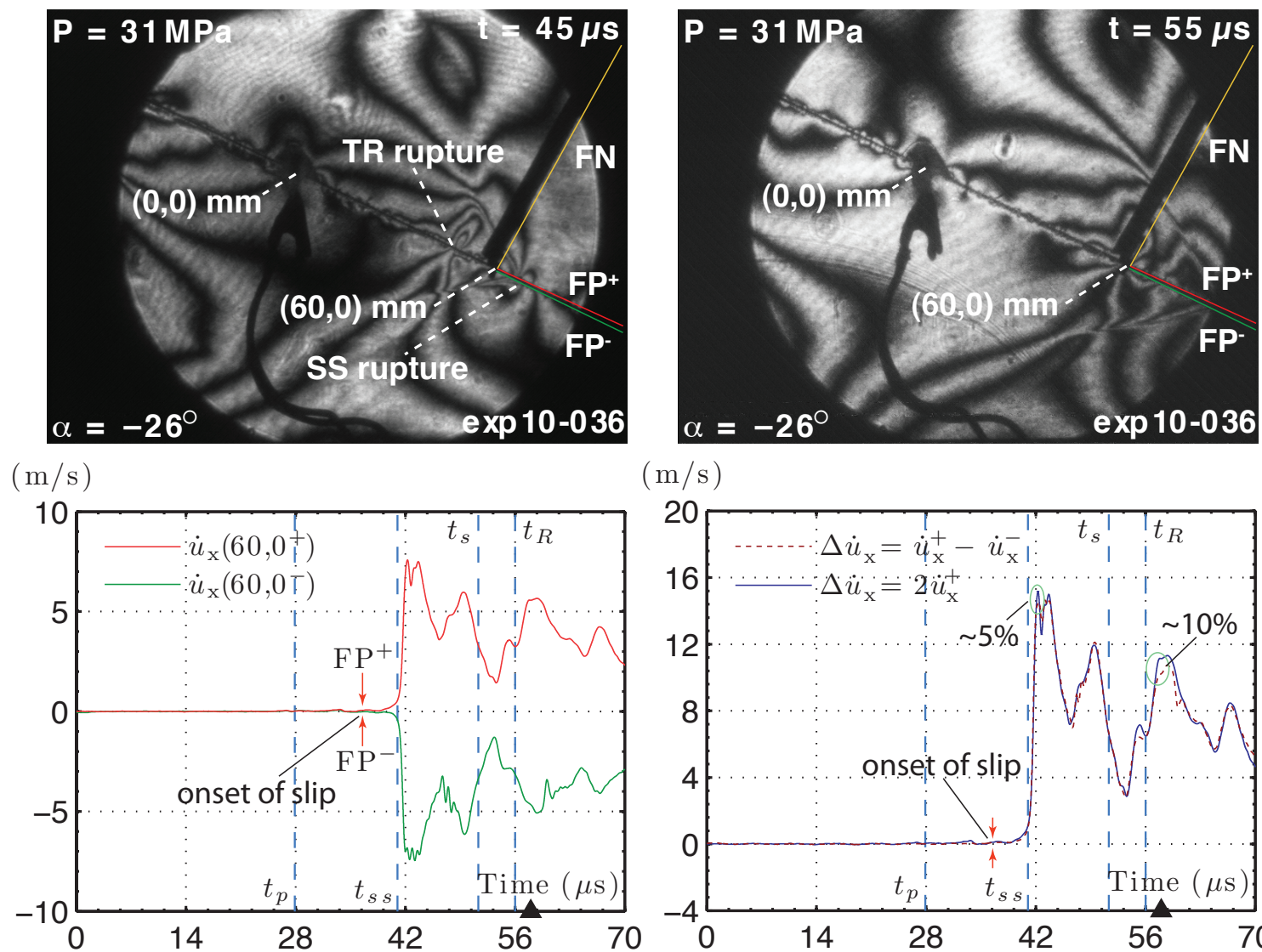

$(\mathrm{m} / \mathrm{s})$

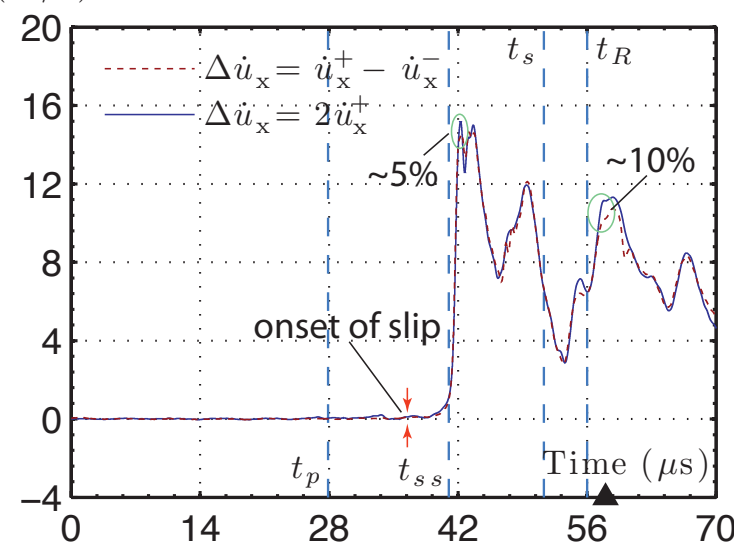

Figure 6.2: Supershear rupture experiment (exp10-036) featuring anti-symmetric fault-parallel particle velocity records $\mathrm{FP}^{+}\left(\dot{u}_{x}\left(60,0^{+}\right)\right.$and $\mathrm{FP}^{-}\left(\dot{u}_{x}\left(60,0^{-}\right)\right.$.

the location of the two rupture fronts and their noted arrival times and velocity jumps in the particle velocity records. The thin green and red lines aligned parallel to the fault in the photoelastic images represent the optical paths of the FP probe beams that were focused at $\left(60,0^{-}\right) \mathrm{mm}$ and $\left(60,0^{+}\right) \mathrm{mm}$, respectively. The yellow line aligned perpendicular to the fault represents the optical path of the FN beam, which was focused on the upper edge of the lower retro-reflective tape section corresponding to $\left(60,0^{-}\right) \mathrm{mm}$. The dark cylindrical shadow in the image was formed by the side exit probe which directed the vertical particle velocity probe beam to focus at $\left(60,0^{+}\right) \mathrm{mm}$.

The lower-left panel in Figure 6.2 depicts the fault-parallel particle velocity records $F P^{+}$and $F P^{-}$, obtained at $\left(60,0^{+}\right) \mathrm{mm}$ and $\left(60,0^{-}\right) \mathrm{mm}$, respectively. Dashed blue vertical lines labeled $t_{p}, t_{s}, t_{s s}$, and $t_{R}$ mark the arrival of the $\mathrm{P}$-wave, $\mathrm{S}$-wave, supershear rupture, and trailing Rayleigh rupture (TR), respectively, at the near-fault measurement stations. The onset of slip (estimated at 
$t=37.3 \mu \mathrm{s})$ is determined by noting the point where the two antisymmetric FP records just begin to separate. The two red arrows in the lower-left panel highlight this point. Antisymmetry between the two fault-parallel particle velocity records is clearly evident. Minor differences in magnitude between the two records are attributed to the fact that the laser beams were not precisely focused at the same distance from the fault. Additional subtle differences in high frequency content between the two records is attributed to differences in texture and light scattering efficiency at the opposing corners of the two retro-reflective tape strips.

The plots depicted in the lower-right panel in Figure 6.2 were obtained by applying Eqs. 6.1, 6.2 to the entire experimental record. The dashed red line corresponds to the slip velocity estimate obtained by subtracting the two signals in accordance with Eq. 6.1 while the solid blue line corresponds to the estimate obtained using Eq. 6.2, which assumes antisymmetry. The pair of red arrows in the lower-right panel highlight the onset of slip and correspond to the same time highlighted by the two red arrows in the lower-left panel. Note that slip velocity as defined here encompasses the entire period spanned by the leading supershear portion, the secondary (anomalous) supershear signal, and the trailing sub-Rayleigh rupture segment of the experimental curve. The plots reveal that the interfacial sliding occurred throughout this entire period.

The obvious overlap and the strong correlation between the two slip velocity curves in the lowerright panel of Figure 6.2 demonstrates the antisymmetry of the FP component. High frequency variations between the records contribute to small differences between the two curves. The largest difference between the two curves in the supershear portion of the records occurs at the first peak near $t=42 \mu \mathrm{s}$ where the solid blue curve overshoots the dashed red curve by approximately $5 \%$. The biggest difference between the two records over the course of the entire experiment occurs around $t=55 \mu s$ while the trailing sub-Rayleigh rupture was sweeping across the measurement stations. An estimated $10 \%$ overshoot of the solid blue curve with respect to the dashed red curve is partially attributed to the dominant FN component which displaced the fault upward and thus shortened the distance between the measurement station and the $F P^{+}$beam while simultaneously increasing the distance between the $F N^{-}$beam and its respective measurement station positioned below the 


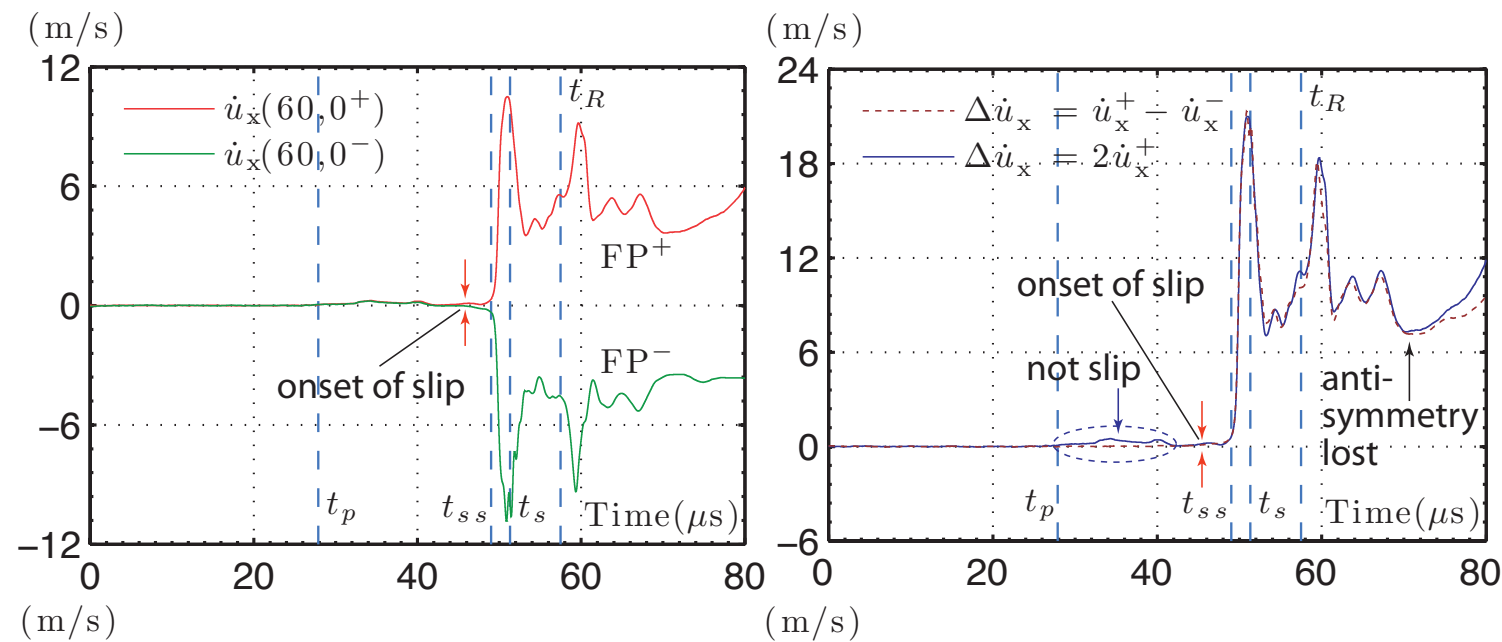

$(\mathrm{m} / \mathrm{s})$

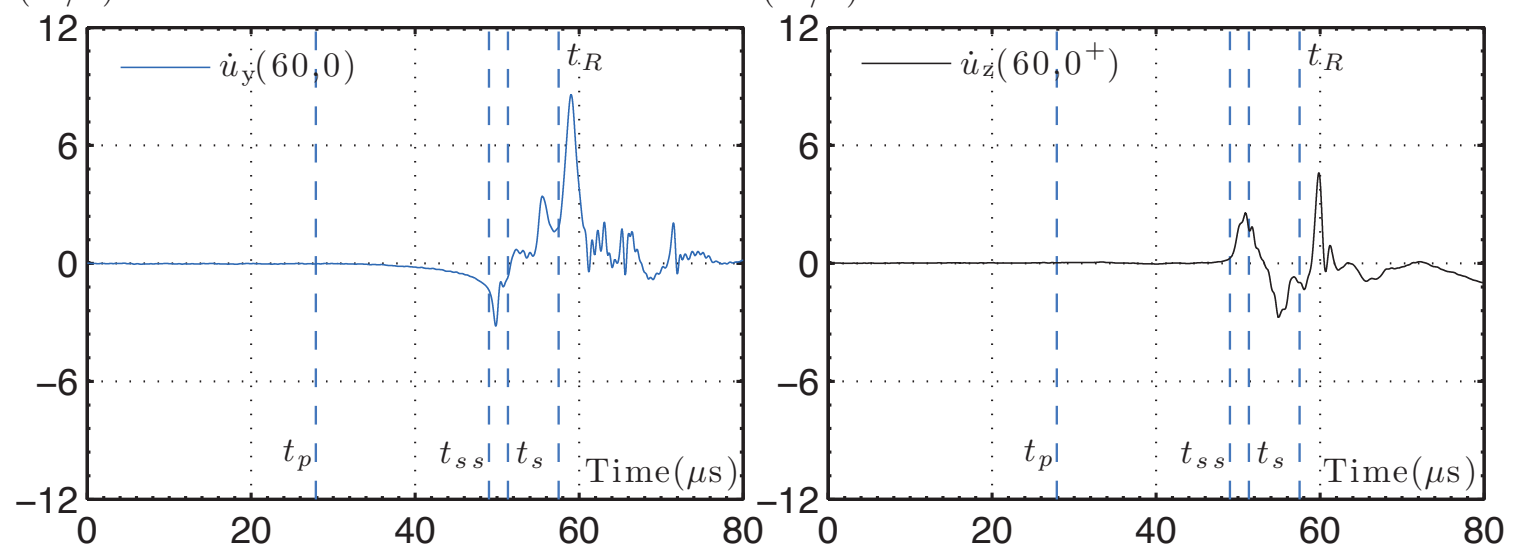

Figure 6.3: Supershear rupture experiment (exp10-041) featuring anti-symmetric fault-parallel particle velocity records $\mathrm{FP}^{+}\left(\dot{u}_{x}\left(60,0^{+}\right)\right.$and $\mathrm{FP}^{-}\left(\dot{u}_{x}\left(60,0^{-}\right)\right.$along with $\mathrm{FN}\left(\dot{u}_{y}(60,0)\right.$ and vertical $\left(\dot{u}_{z}\left(60,0^{+}\right)\right.$records acquired at the same measurement station locations.

fault. Nonetheless, the results reveal an excellent overall correlation between the two slip velocity estimates and effectively demonstrate how a single near-fault particle velocity measurement can be relied upon to make a very reasonable estimate of the slip velocity.

The particle velocity records from a second supershear experiment (exp10-041) are depicted in Figure 6.3. The experiment was conducted under a static-compressive load of $P=30 \mathrm{MPa}$ using a traditional $6^{\prime \prime} \times 6^{\prime \prime} \times 0.375^{\prime \prime} \mathrm{H}-100$ specimen with uniformly roughened fault surfaces and a fault angle $\alpha=-26^{\circ}$. Four particle velocity probe beams were used in the experiment in order to produce simultaneous recordings of the fault-parallel components $\left(F P^{+}, F P^{-}\right)$, vertical component $V$, and fault-normal component $F N$.

Paired fault-parallel particle velocity measurements were conducted at a pair of closely spaced 
measurement stations located at $\left(60,0^{+}\right) \mathrm{mm}$ and $\left(60,0^{-}\right) \mathrm{mm}$. The upper-left panel in Figure 6.3 depicts the resulting anti-symmetric fault-parallel particle velocity records, $F P^{+}$and $F P^{-}$, which resulted from the experiment. An extraordinary degree of antisymmetry between the two records is revealed by the excellent overlap and strong overall correlation between the two slip velocity estimates (dashed red and solid violet curves) depicted in the upper right-hand panel of Figure 6.3. The two red arrows in the upper-left panel of Figure 6.3 indicate the onset of slip, which is identified by the point at which the two curves just begin to separate. The same point in time is highlighted in the upper-right hand panel where the slip velocity curves begins to pull away from the horizontal axis $\left(\Delta \dot{u}_{x}(x, 0)=0\right)$. The black arrow on the right-hand side in the upper-right panel reveals the point where antisymmetry between the curves is lost, presumably due to the arrival of boundary waves reflected from the right-hand side of the specimen.

The circled portion of the slip velocity $\left(\Delta \dot{u}_{x}=2 \dot{u}_{x}^{+}\right)$in the upper-right panel of Figure 6.3 highlights an example where the use of Eq. 6.2 doubled a non-zero portion of the $F P^{+}$particle velocity record that did not result from interfacial sliding. Although the magnitude of this contribution appears to be small compared to the actual sliding portion of the record, the example nonetheless highlights how blindly applying Eq. 6.2 to a single FP particle velocity record can lead to an erroneous description of the slip velocity. Strictly speaking, Eq. 6.2 should only be applied to a specific portions of the curve which correspond to interfacial slip.

The two examples highlighted in this section reveal how the onset of slip can be detected through close inspection of the curves. The ideal scenario involves paired FP measurements, which leave little doubt as to when interfacial slip ensued. The results presented here demonstrate how it then becomes possible, with sufficient experience, to conduct similar measurements using a single FP probe beam and properly identify the sliding portion of the particle velocity record. 


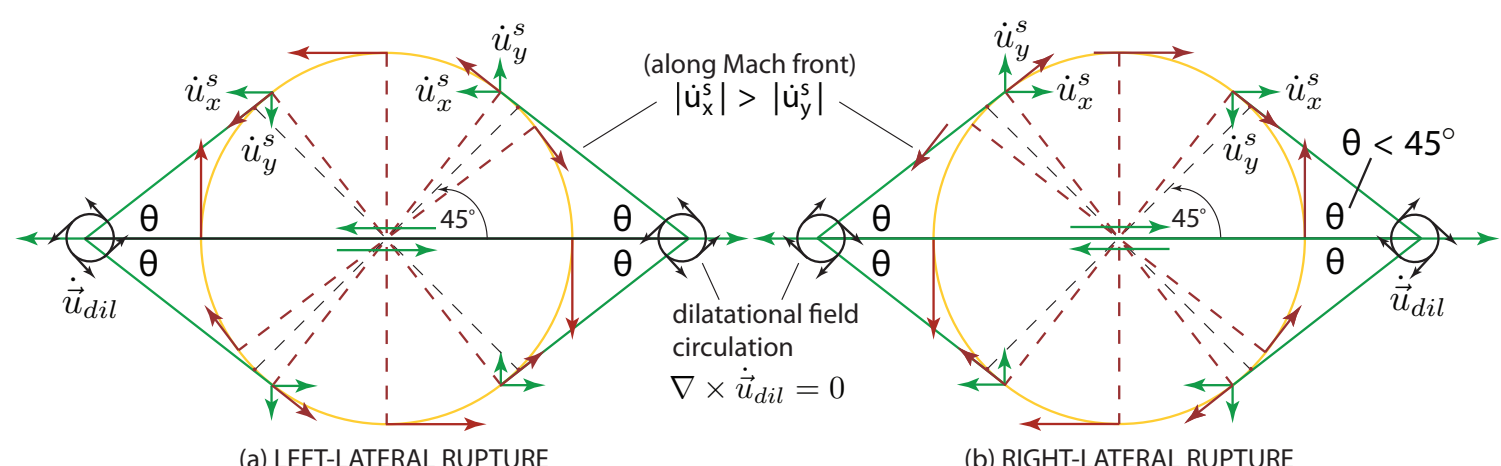

(a) LEFT-LATERAL RUPTURE

(b) RIGHT-LATERAL RUPTURE

stable supershear rupture speed regime: $\sqrt{2} C_{s}<V_{r}<C_{p}$

Figure 6.4: (a) Sense of particle displacement for (stable) left-lateral supershear rupture rupture (b)

Sense of particle displacement for (stable) left-lateral supershear rupture rupture

\subsubsection{Summary of particle displacement for right- and left-lateral super- shear ruptures}

The diagrams depicted in Figure 6.4 summarize the expected sense of particle motion for the dilatational and shear fields surrounding stable, right- and left-lateral supershear ruptures. Figure 6.4(a) depicts the sense of particle motion resulting from a left-lateral rupture propagating to the right or left while Figure 6.4(b) indicates the corresponding sense of motion resulting from a right-lateral rupture.

The large circle in each diagram represents an S-wavelet emitted by a shear dislocation positioned at the origin. The sense of motion along the circular wavefront, as depicted by the red arrows, corresponds with the solution for the far-field radiation pattern resulting from a shear dislocation, also commonly referred to as the double-couple solution in an infinite homogeneous medium (Chapter 1). The dashed black lines oriented at $\pm 45^{\circ}$ with respect to the horizontal axis intersect the Swavefronts at the nodal points where $\vec{u}^{s}=0$. The direction indicated by the red arrows also highlight how the sense of particle motion reverses at the nodal positions. Shear Mach fronts from a stable supershear rupture will always be tangent to its constituent S-wavelets at points located within the upper and lower octants (Chapter 1) of the expanding circular fronts. The sense of particle motion along a shear Mach front is set by $\dot{\vec{u}}^{s}$ along the S-wavelet at the point of tangency. 
The particle motion along a shear Mach front is decomposed into fault-parallel and fault-normal particle velocity components $\left(\dot{u}_{x}^{s}\right.$ and $\left.\dot{u}_{y}^{s}\right)$ represented by the green arrows. The smaller black circles centered around the tip of the Mach cones in each of the diagrams indicates the sense of particle motion of the dilatational field $\left(u^{p}\right)$, which circulates around the rupture tip (recall $\nabla u^{p}=0$ ).

The previously examined particle velocity records in Figure 6.3 corresponding to the supershear experiment (exp10-041) also serve to demonstrate the sense of particle motion associated with a right-lateral supershear rupture as predicted in Figure 6.4(b). First, note the positive velocity jump exhibited by $\dot{u}_{x}\left(x, 0^{+}\right)$upon arrival of the right-traveling supershear rupture and the opposite (antisymmetric) velocity swing exhibited by $\dot{u}_{x}\left(x, 0^{-}\right)$. The observed particle motion is just as expected for a shear Mach front associated with a stable right-lateral supershear rupture and in agreement with the direction of the green FP vectors labeled $\dot{u}_{x}^{s}$ in Figure 6.4(b). Next, note the negative velocity jump exhibited by $\dot{u}_{y}\left(x, 0^{-}\right)$upon arrival of the supershear rupture. This observation is also in agreement with the predicted sense of motion along a shear Mach for a stable right-lateral supershear rupture as indicted by the direction of the green FN vectors labeled $\dot{u}_{x}^{s}$ in Figure 6.4(b).

Note as well how the vertically oriented red arrow drawn tangent to the S-wavelet in Figure 6.4(b) indicates the correct sense of particle motion and dominance of the FN component in the case of a right-lateral sub-Rayleigh rupture (Chapter 1). This feature is easily verified by examining the trailing Rayleigh portions of the particle velocity records in Figure 6.3. The FN signal dominates over the FP signal in magnitude within the trailing sub-Rayleigh portion of the record and also exhibits a positive velocity jump, as expected, for a right-lateral rupture. The observed sense of motion is in complete agreement with the direction of the vertically oriented arrow in Figure 6.4(b).

The clockwise sense of particle motion of the dilatational field which circulates around the supershear rupture tip cannot be discerned from the particle velocity records at such a close distance from the fault.

Finally, although Figure 6.4(b) cannot predict the sense of vertical particle motion, it is still possible to infer the correct sense of particle motion by considering the sign of the horizontal stress 
component. In the case of a right-lateral rupture points $\left(x, 0^{+}\right)$experience compression while points $\left(x, 0^{-}\right)$experience tension. Hence, as a general rule of thumb we should expect positive velocity swings associated with the arrival of the supershear and trailing sub-Rayleigh ruptures when the vertical particle velocity probe beam is focused at a points $\left(x, 0^{+}\right)$and negative velocity swings when it is focused on the opposite side of the fault. Examination of the vertical particle velocity record plotted in the lower-right panel of Figure 6.3 reveals that positive velocity jumps were registered upon arrival of the supershear and trailing sub-Rayleigh ruptures, which is consistent with the fact that the vertical particle velocity probe beam was focused at $\left(60,0^{+}\right) \mathrm{mm}$.

\subsubsection{Extension of 2D steady-state supershear slip-pulse solution to left- traveling ruptures}

The 2D steady-state supershear slip-pulse solution by (Dunham and Archuleta, 2005) was originally cast for a right-lateral rupture that was assumed to propagate to the right $(x>0)$. The dilatational and shear field contributions appear as separate terms in the final stress and particle velocity field expressions as a consequence of the Poisson displacement field representation which was adopted in the derivation. The leading term (function of $z_{p}$ ) in the field expressions corresponds to the dilatational field which is centered around the supershear rupture tip while the second term (function of $\left(z_{s}\right)$ corresponds to the shear field contribution within the region bounded by the leading and trailing shear Mach fronts.

Laboratory earthquake experiments can involve either right- or left-traveling ruptures depending upon the objectives and physical circumstances of a given experiment. The stress and particle velocity field expressions must therefore be adjusted in order to process particle velocity records corresponding to a left-traveling rupture. Adjustments to the stress and particle velocity field expressions for a right-lateral supershear rupture are implemented in accordance with Figure 6.4(b). The relationships given by Eq. 6.3

$$
\dot{u}_{x}(x, y)=\dot{u}_{x}(-x, y) \quad ; \quad \dot{u}_{y}(x, y)=-\dot{u}_{y}(-x, y)
$$


between the fault-parallel and fault-normal velocity components to the right- and left-of-center are confirmed by inspection of Figure 6.4(b).

The first adjustment to the $2 \mathrm{D}$ steady-state supershear slip-pulse solutions involves changing $x \rightarrow|x|$ in the definitions of $z_{s}$ and $z_{p}$, i.e., $z_{p}=|x|+i \alpha_{p} y$ and $z_{s}=|x|+\beta_{s} y$. This nearly takes care of everything except for the minus sign between the left- and right-traveling fault-normal expressions, which is easily handled by introducing the $\operatorname{sgn}(x)$ function in the fault normal particle velocity expression. The modified particle velocity field expressions are then given by

$$
\begin{gathered}
\dot{u}_{x}=-\frac{V_{r}}{\mu}\left[\frac{1}{2 \alpha_{p}} \Im N\left(z_{p}\right)+\frac{\beta_{s}^{2}-1}{4 \alpha_{p}} \Im N\left(z_{s}\right)\right] \\
\dot{u}_{y}=-\frac{V_{r}}{\mu}\left[\frac{1}{2} \Re N\left(z_{p}\right)-\operatorname{sgn}(y) \frac{\beta_{s}^{2}-1}{4 \alpha_{p} \beta_{s}} \Im N\left(z_{s}\right)\right] \operatorname{sgn}(x)
\end{gathered}
$$

where the variables $z_{p}, z_{s}$ are now given by

$$
z_{p}=|x|+i \alpha_{p} y \quad ; \quad z_{x}=|x|+\beta_{s} y
$$

and

$$
N(z)=-\frac{\sin (\pi q)}{\pi} z^{1-q}(z+L)^{q} \int_{-L}^{0} \frac{\tau(w)-\sigma_{x y}^{0}}{(-w)^{1-q}(w+L)^{q}(w-z)} d w
$$

where $N(z)$ represents an analytic function of the form described in Section 2.3. The adjusted stress field expressions are adjusted in a similar manner and given by

$$
\begin{gathered}
\sigma_{x x}=\left[\frac{1+\beta_{s}^{2}+2 \alpha_{p}^{2}}{2 \alpha_{p}} \Im N\left(z_{p}\right)+\frac{\beta_{s}^{2}-1}{2 \alpha_{p}} \Im N\left(z_{s}\right)\right] \operatorname{sgn}(x) \\
\sigma_{x y}=\Re N\left(z_{p}\right)+\left[\frac{\left(\beta_{s}^{2}-1\right)^{2}}{4 \alpha_{p} \beta_{s}} \Im N\left(z_{s}\right)\right] \operatorname{sgn}(y) \\
\sigma_{y y}=\left[\frac{\beta_{s}^{2}-1}{2 \alpha_{p}} \Im\left[N\left(z_{p}\right)-N\left(z_{s}\right)\right]\right] \operatorname{sgn}(x)
\end{gathered}
$$

where the use of $\operatorname{sgn}(x)$ in Eqs. 6.8, 6.10 properly adjusts the solutions for compressional and tension within the four rupture quadrants, i.e., $\sigma_{x x}(x>0, y>0)<0, \sigma_{x x}(x>0, y<0)>0$; 
$\sigma_{x x}(x<0, y<0)<0, \sigma_{x x}(x<0, y>0)>0$, and $\sigma_{y y}(x>0, y>0)>0, \sigma_{y y}(x>0, y<0)<0$ $\sigma_{y y}(x<0, y<0)>0, \sigma_{y y}(x<0, y>0)<0$.

Note that the adjusted equations only apply to a right-lateral rupture as originally formulated by (Dunham and Archuleta, 2005). The adjustment for left-lateral ruptures, although not considered here, simply involves the insertion of a minus sign. Unless otherwise noted, the adjusted equations and any derived relationships which follow from these expressions will strictly apply to right-lateral ruptures.

\subsubsection{Relationship between the slip velocity $\Delta \dot{u}\left(z_{s}\right)$ and $\Im N\left(z_{s}\right)$}

For an observation point positioned arbitrarily close to the fault, $\lim _{y \rightarrow 0^{ \pm}}(z)=x$, in which case $z_{p}=$ $z_{s}=x$. Similarly, we can write $\lim _{y \rightarrow 0^{ \pm}} N\left(z_{p}\right)=\lim _{y \rightarrow 0^{ \pm}} N\left(z_{s}\right)=N_{ \pm}(x)$. The fault-parallel particle velocity (Eq. 6.4), evaluated at two closely spaced points $\left(x, 0^{ \pm}\right)$, positioned just above and below the fault, is thus given by

$$
\begin{aligned}
& \dot{u}_{x}\left(x, 0^{+}\right)=-\frac{V_{r}}{\mu}\left[\frac{1}{2 \alpha_{p}} \Im N_{+}(x)+\frac{\beta_{s}^{2}-1}{4 \alpha_{p}} \Im N_{+}(x)\right] \\
& \dot{u}_{x}\left(x, 0^{-}\right)=-\frac{V_{r}}{\mu}\left[\frac{1}{2 \alpha_{p}} \Im N_{-}(x)+\frac{\beta_{s}^{2}-1}{4 \alpha_{p}} \Im N_{-}(x)\right] .
\end{aligned}
$$

The temptation to combine common terms is resisted for the time being. Note as well the use of the variable $x$ instead of $|x|$ as discussed in the previous section. It should be understood that $x \rightarrow|x|$ for a left-traveling rupture.

Next, invoking the mode II antisymmetry condition $\dot{u}_{x}\left(x, 0^{+}\right)=-\dot{u}_{x}\left(x, 0^{-}\right)$, or, equivalently $\Im N\left(x, 0^{+}\right)=-\Im N\left(x, 0^{-}\right)$, allows us to recast Eq. 6.11 as

$$
\begin{aligned}
& \dot{u}_{x}\left(x, 0^{+}\right)=-\frac{V_{r}}{\mu}\left[\frac{1}{2 \alpha_{p}} \Im N_{+}(x)+\frac{\beta_{s}^{2}-1}{4 \alpha_{p}} \Im N_{+}(x)\right] \\
& \dot{u}_{x}\left(x, 0^{-}\right)=\frac{V_{r}}{\mu}\left[\frac{1}{2 \alpha_{p}} \Im N_{+}(x)+\frac{\beta_{s}^{2}-1}{4 \alpha_{p}} \Im N_{+}(x)\right] .
\end{aligned}
$$

Substituting Eqs. 6.12 into the general slip velocity expression given by Eq. 6.1 and re-invoking the 
mode II antisymmetry relation $\left[\Im N\left(x, 0^{+}\right)=-\Im N\left(x, 0^{-}\right)\right]$then yields

$$
\Delta \dot{u}_{x}(x, 0)=\mp \frac{2 V_{r}}{\mu} \frac{\beta_{s}^{2}+1}{4 \alpha_{p}} \Im N_{ \pm}(x)
$$

Substitution of the antisymmetric forms of the slip velocity (Eq. 6.2) into Eq. 6.13 leads to

$$
\dot{u}_{x}\left(x, 0^{ \pm}\right)=-\frac{V_{r}}{\mu} \frac{\beta_{s}^{2}+1}{4 \alpha_{p}} \Im N_{ \pm}(x)
$$

which is then solved for $\Im N_{ \pm}(x)$ and recast as

$$
\Im N_{ \pm}(x)=-\frac{4 \mu}{V_{r}} \frac{\alpha_{p}}{\beta_{s}^{2}+1} \dot{u}_{x}\left(x, 0^{ \pm}\right)
$$

Equation 6.15 is an important result which reveals how a single fault-parallel particle velocity measurement $\left[\dot{u}_{x}\left(x, 0^{ \pm}\right)\right]$conducted very close to the fault can provide an experimental estimate of $\Im N_{ \pm}(x)$.

Finally, note the general form for the slip velocity, given by Eq. 6.16, which is obtained by invoking $\Delta \dot{u}_{x}(x, 0)=\Delta \dot{u}\left(z_{s}\right)$ and $\Im N_{ \pm}(x)=\Im N\left(z_{s}\right)$, since $z_{s}=x+\beta_{s} y$ defines a characteristic (shear Mach front) extending from the fault out to infinity.

$$
\Delta \dot{u}_{x}\left(z_{s}\right)=\left[-\frac{2 V_{r}}{\mu} \frac{\beta_{s}^{2}+1}{4 \alpha_{p}} \Im N\left(z_{s}\right)\right] \operatorname{sgn}(y)
$$

Equation 6.16 can be directly substituted into Eqns. 6.4, 6.5 to show that the shear contribution to both of the particle velocity field components scales directly with the slip velocity as expressed by Eq. 6.17 and Eq. 6.18 (Dunham and Archuleta, 2005).

$$
\begin{gathered}
\dot{u}_{x}=-\frac{V_{r}}{\mu}\left[\frac{1}{2 \alpha_{p}} \Im N\left(z_{p}\right)\right]+\operatorname{sgn}(y) \frac{\beta_{s}^{2}-1}{2\left(\beta_{s}^{2}+1\right)} \Delta \dot{u}_{x}\left(z_{s}\right) \\
\dot{u}_{y}=\left[-\frac{V_{r}}{\mu} \frac{1}{2} \Re N\left(z_{p}\right)-\frac{1}{2 \beta_{s}} \frac{\beta_{s}^{2}-1}{\beta_{s}^{2}+1} \Delta \dot{u}_{x}\left(z_{s}\right)\right] \operatorname{sgn}(x)
\end{gathered}
$$




\subsubsection{Relationship between the slip velocity $\Delta \dot{u}\left(z_{s}\right)$ and $\Re N\left(z_{p}\right)$}

Equation 6.18 is solved for $\Re N\left(z_{p}\right)$ and recast as

$$
\Re N\left(z_{p}\right)=-\frac{2 \mu}{V_{r}}\left[\frac{\dot{u}_{y}}{\operatorname{sgn}(x)}+\frac{1}{2 \beta_{s}} \frac{\beta_{s}^{2}-1}{\beta_{s}^{2}+1} \Delta \dot{u}_{x}\left(z_{s}\right)\right] .
$$

In the limit that the observation point approaches the fault, Eq. 6.19 assumes the form given by

$$
\Re N_{ \pm}(x)=-\frac{2 \mu}{V_{r}}\left[\frac{\dot{u}_{y}\left(x, 0^{ \pm}\right)}{\operatorname{sgn}(x)}+\frac{1}{2 \beta_{s}} \frac{\beta_{s}^{2}-1}{\beta_{s}^{2}+1} \Delta \dot{u}_{x}(x, 0)\right] .
$$

Substituting the antisymmetric form of the slip velocity (Eq. 6.2) yields a second critical relationship given by

$$
\Re N_{ \pm}(x)=-\frac{2 \mu}{V_{r}}\left[\frac{\dot{u}_{y}\left(x, 0^{ \pm}\right)}{\operatorname{sgn}(x)}+\frac{\operatorname{sgn}(y)}{\beta_{s}} \frac{\beta_{s}^{2}-1}{\beta_{s}^{2}+1} \dot{u}_{x}\left(x, 0^{ \pm}\right)\right] .
$$

Equation 6.21 reveals how the simultaneous measurement of $\dot{u}_{x}\left(x, 0^{ \pm}\right)$and $\dot{u}_{y}\left(x, 0^{ \pm}\right)$can lead to a direct estimate of $\Re N_{ \pm}(x)$ and thus the complete characterization of the complex function $N_{ \pm}(x)$ when combined with $\Im N_{ \pm}(x)$ through Eq. 6.15. This in turn enables the complete characterization of the stress tensor within the slip zone in accordance with the $2 \mathrm{D}$ steady-state assumptions of the supershear slip-pulse solution.

Measurements of $\Im N\left(z_{s}\right)$ and $\Re N\left(z_{p}\right)$ using Eqs. 6.15, 6.21 necessarily require a knowledge of the rupture speed $V_{r}$, the elastic wavespeeds $C_{p}, C_{s}$, and the shear modulus $\mu$. The elastic wave speeds $C_{s}$ and $C_{p}$ are conveniently obtained through the analysis of high-speed photoelastic image frames. The shear modulus is thus also directly estimated through the relation $\mu=\rho C_{s}^{2}$, assuming that the density $(\rho)$ of H-100 is known Section 3.11.2.

\subsubsection{Experimental estimation of $\sigma_{x x}$ from fault-parallel particle velocity records}

The 2D steady-state supershear slip-pulse solution results from a mixed boundary value problem where it is assumed that two identical isotropic linear elastic half-spaces, having a shear modulus $\mu$, 
and $\mathrm{P}$ - and S-wave speeds $C_{p}$ and $C_{s}$, are joined along the interface (fault) $y=0$. The stress field boundary conditions are given by

$$
\begin{aligned}
& \sigma_{y y}(-\infty<x<\infty, 0)=0 \\
& \sigma_{x y}(-L<x<0,0)=\tau(x)-\tau_{0} .
\end{aligned}
$$

and the only two nonzero stress components along the fault are $\sigma_{x x}$ and $\sigma_{x y}$. The nature of the stress field solutions within the supershear slip zone is examined by once again considering the limit as the observation point approaches the fault, in which case $\lim _{y \rightarrow 0^{ \pm}}(z)=x$ implies $z_{p}=z_{s}=\left(x, 0^{ \pm}\right)$ and $\lim _{y \rightarrow 0^{ \pm}} N\left(z_{p}\right)=\lim _{y \rightarrow 0^{ \pm}} N\left(z_{s}\right)=N_{ \pm}(x)$.

At points $\left(x, 0^{ \pm}\right)$positioned close to the fault, Eq. 6.8 assumes the limiting form given by

$$
\sigma_{x x}\left(x, 0^{ \pm}\right)=\left[\frac{1+\beta_{s}^{2}+2 \alpha_{p}^{2}}{2 \alpha_{p}} \Im N_{ \pm}(x)+\frac{\beta_{s}^{2}-1}{2 \alpha_{p}} \Im N_{ \pm}(x)\right] \operatorname{sgn}(x)
$$

Substituting Eq. 6.15 into Eq. 6.23 along with the explicit expressions for $\alpha_{p}$ and $\beta_{s}$ (Eq. 2.10) yields

$$
\sigma_{x x}\left(x, 0^{ \pm}\right)=\left[-\frac{4 \mu}{V_{r}}\left(1-C_{s}^{2} / C_{p}^{2}\right) \dot{u}_{x}\left(x, 0^{ \pm}\right)\right] \operatorname{sgn}(x)
$$

Equation 6.24 is of the form $\sigma_{x x}\left(x, 0^{ \pm}\right)=\epsilon\left(x, 0^{ \pm}\right) E$, where $\epsilon_{x x}\left(x, 0^{ \pm}\right)$is the horizontal strain component which emerges through the steady-state differential relationship given by

$$
\frac{\partial u_{x}\left(x, 0^{ \pm}\right)}{\partial t}=\frac{\partial u_{x}\left(x, 0^{ \pm}\right)}{\partial x} \frac{\partial x}{\partial t}=-V_{r} \epsilon_{x x}\left(x, 0^{ \pm}\right)
$$

and

$$
E=4 \mu\left(1-C_{s}^{2} / C_{p}^{2}\right)
$$

represents the elastic modulus of the surrounding medium. Next, recall that the shear wavespeed 
$\left(C_{s}\right)$ is given by the universal expression

$$
C_{s}=\sqrt{\mu / \rho}
$$

where $\mu$ is the elastic modulus and $\rho$ represents the material density. The value of the dilatational wavespeed $\left(C_{p}\right)$ is bounded between plane stress and plane strain extremes. Under plane stress conditions the dilatational wavespeed is given by

$$
C_{p}=\sqrt{\frac{E}{\rho(1-\nu)(1+\nu)}}
$$

where $\nu$ represents Poisson's ratio. The more familiar plane strain form of the dilatational wavespeed given by 6.29 is recovered by replacing $E \rightarrow E /\left(1-\nu^{2}\right)$ and $\nu \rightarrow \nu /(1-\nu)$ in Eq. 6.28.

$$
C_{p}=\sqrt{\frac{E(1-\nu)}{\rho(1+\nu)(1-2 \nu)}}
$$

As previously noted, the shear and dilatational wavespeeds, $C_{s}$ and $C_{p}$, can be directly measured in laboratory earthquake experiments (Section 3.11.2). The shear modulus is also readily estimated through Eq. 6.27, assuming that the density ( $\rho$ ) of H-100 is known (Section 3.11.2). Measured values of $C_{p}, C_{s}$, and $\mu$ may then be substituted directly into Eq. 6.26 thus eliminating the reliance upon tabulated material properties and relaxing the need to invoke strict assumptions of plane stress or plane strain.

\subsubsection{Experimental estimation of $\tau(x)-\tau_{0}$ within the supershear slip zone}

$$
(-L<x<0)
$$

At points $\left(x, 0^{ \pm}\right)$positioned close to the fault, Eq. 6.9 assumes the limiting form given by

$$
\sigma_{x y}\left(x, 0^{ \pm}\right)=\Re N_{ \pm}(x)+\left[\frac{\left(\beta_{s}^{2}-1\right)^{2}}{4 \alpha_{p} \beta_{s}} \Im N_{ \pm}(x)\right] \operatorname{sgn}(y)
$$


Invoking the boundary condition (Eq. 6.22) allows us to recast Eq. 6.30 as

$$
\tau(x)-\tau_{0}=\Re N_{ \pm}(x)+\left[\frac{\left(\beta_{s}^{2}-1\right)^{2}}{4 \alpha_{p} \beta_{s}} \Im N_{ \pm}(x)\right] \operatorname{sgn}(y)
$$

where $\tau(x)=\tau\left(x, 0^{ \pm}\right)$represents an estimate of the shear traction within the supershear slip zone and the variable $\tau_{0}$ is the initial shear stress on the fault. Recall that in the case of a laboratory earthquake experiment $\tau_{0}=P \sin \alpha \cos \alpha$, where $P$ is the imposed by the static compressive load and $\alpha$ represents the specimen fault angle. A closer examination of Eq. 6.31 reveals that the factor in braces is nothing more than the familiar speed dependent factor $q$ (Section 2.2.2, Eq. 2.46) in disguise, i.e.,

$$
\cot \pi q=\frac{\left(\beta_{s}^{2}-1\right)^{2}}{4 \alpha_{p} \beta_{s}}
$$

which can be substituted into Eq. 6.31 resulting in a more compact form given by

$$
\tau(x)-\tau_{0}=\Re N_{ \pm}(x)+\operatorname{sgn}(y) \cot (\pi q) \Im N_{ \pm}(x)
$$

Experimental estimates of $\tau(x)$ are thus obtained through Eq. 6.33 using measured values of $\Re N_{ \pm}(x)$ and $\Im N_{ \pm}(x)$ obtained from particle velocity records through Eqs. 6.15 and 6.21, respectively. The variable $q$ can also be well estimated through the analysis of high-speed photoelastic images, which provides direct measurements of the elastic constants, and rupture speed. The only required material property that cannot be directly determined from the experiment is the density of the specimen material (H-100). This is a minor concern since the density of H-100 has been well characterized can be easily determined if deemed necessary.

An explicit solution for $\tau(x)$, given by Eq. 6.34, is obtained through direct substitution of Eqs. 6.15, 6.21 into Eq. 6.33

$$
\tau(x)-\tau_{0}=-\frac{2 \mu}{V_{r}}\left[\frac{\dot{u}_{y}\left(x, 0^{ \pm}\right)}{\operatorname{sgn}(x)}+\operatorname{sgn}(y) \frac{\beta_{s}^{2}-1}{2 \beta_{s}} \dot{u}_{x}\left(x, 0^{ \pm}\right)\right]
$$

This particular is most useful since it provides greater insight into the role that each particle velocity 


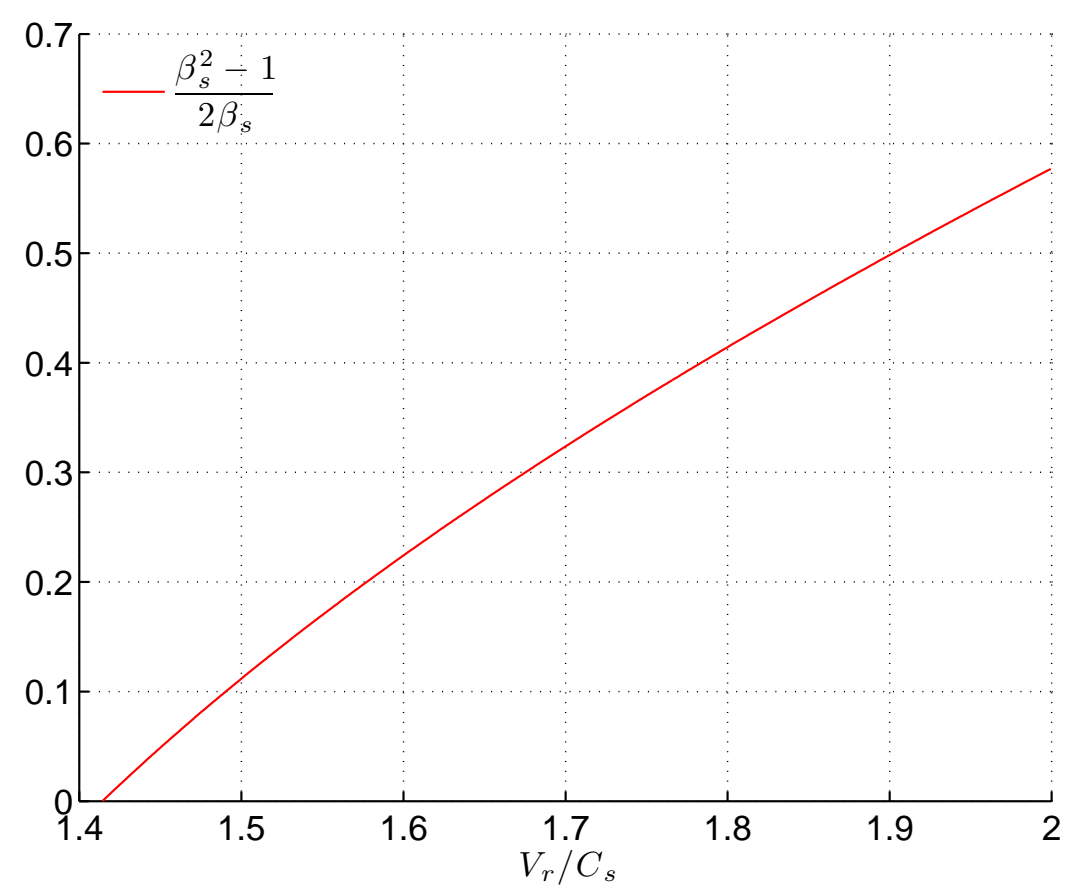

Figure 6.5: Plot of speed dependent weighting factor $(B)$ versus the normalized rupture speed

plays in the estimation of $\tau(x)$. Eq. 6.34 immediately reveals that contribution of the fault-parallel component is weighted by a non-dimensional rupture-speed-dependent factor given by

$$
B=\frac{\beta_{s}^{2}-1}{2 \beta_{s}}
$$

A plot of the fault-parallel weighting factor $B=B\left(V_{r} / C_{s}\right)$ is displayed in Fig 6.5.

\subsubsection{Alternative derivation for $\tau(x)-\tau_{0}$ within the supershear slip zone $(-L<x<0)$ using the integral equation for $N(z)$}

The mode II symmetry properties and the shear traction boundary condition given by Eq. 6.22 are combined to define a Hilbert problem given by

$$
N_{+}(x)=e^{-2 \pi i q} N_{-}(x)+2 i \sin (\pi q) e^{-i \pi q}\left[\tau(x)-\tau_{0}\right] .
$$


(Dunham and Archuleta, 2005). The solution to the Hilbert problem is the analytic function $N(z)$ given by Eq. 6.7. Within the slip zone $(-L<x<0)$, the complex integral assumes the form given by (Eq. 6.37)

$$
N_{+}(x)=\frac{\sin (\pi q)}{\pi} e^{-i \pi q}\left[(-x)^{1-q}(x+L)^{q} \int_{-L}^{0} \frac{\tau(w)-\tau_{0}}{(-w)^{1-q}(w+L)^{q}(w-x)} d w+i \pi\left[\tau(x)-\tau_{0}\right]\right]
$$

where $N_{+}(x)$ is a complex expression containing a real integral. Euler's formula may then be applied to Eq. 6.37 in order to extract the real and imaginary parts of the complex expression, given by

$$
\begin{aligned}
& \Re\left[N_{+}(x)\right]=\frac{1}{\pi} \sin \pi q \cos \pi q(-x)^{1-q}(x+L)^{q} \int_{-L}^{0} \frac{\left[\tau(w)-\tau_{0}\right]}{-w^{1-q}(w+L)^{q}(w-x)} d w+\sin ^{2} \pi q\left[\tau(x)-\tau_{0}\right] \\
& \Im\left[N_{+}(x)\right]=\frac{-1}{\pi} \sin ^{2} \pi q(-x)^{1-q}(x+L)^{q} \int_{-L}^{0} \frac{\left[\tau(w)-\tau_{0}\right]}{-w^{1-q}(w+L)^{q}(w-x)} d w+\sin \pi q \cos \pi q\left[\tau(x)-\tau_{0}\right]
\end{aligned}
$$

Recall that value of $q$ (Eq. 6.32) can be readily calculated for any supershear experiment assuming that the elastic wave speeds are known and the rupture speed $V_{r}$ is accurately determined during the time period of interest. It has also been shown that $\Re N_{+}(x)$ and $\Im N_{+}(x)$ can be experimentally determined through Eqs. 6.21 and 6.15 using simultaneous FN and FP particle velocity measurements conducted at a point positioned close to the fault. Assuming that each of these quantities are accurately determined, Eq. 6.38 and Eq. 6.39 effectively constitute a system of two equations in two unknowns, where the unknown quantities correspond to $\tau(x)$ and the real integral expression, which is evaluated across the slip zone $(-L<x<0)$.

Solving the system of equations recovers the same solution for $\tau(x)$ given by Eq. 6.33

$$
\tau(x)-\tau_{0}=\Re N_{+}(x)+\cot (\pi q) \Im N_{+}(x)
$$

It is interesting to contrast the two solution paths which both lead to Eq. 6.33 (and hence Eq. 6.34). The first solution path for $\tau(x)$ was based upon Eq. 6.30 which emerged as a solution to the mixed boundary value through enforcement of the boundary condition $\sigma_{y y}(-\infty<x<\infty, 0)=0$. The 


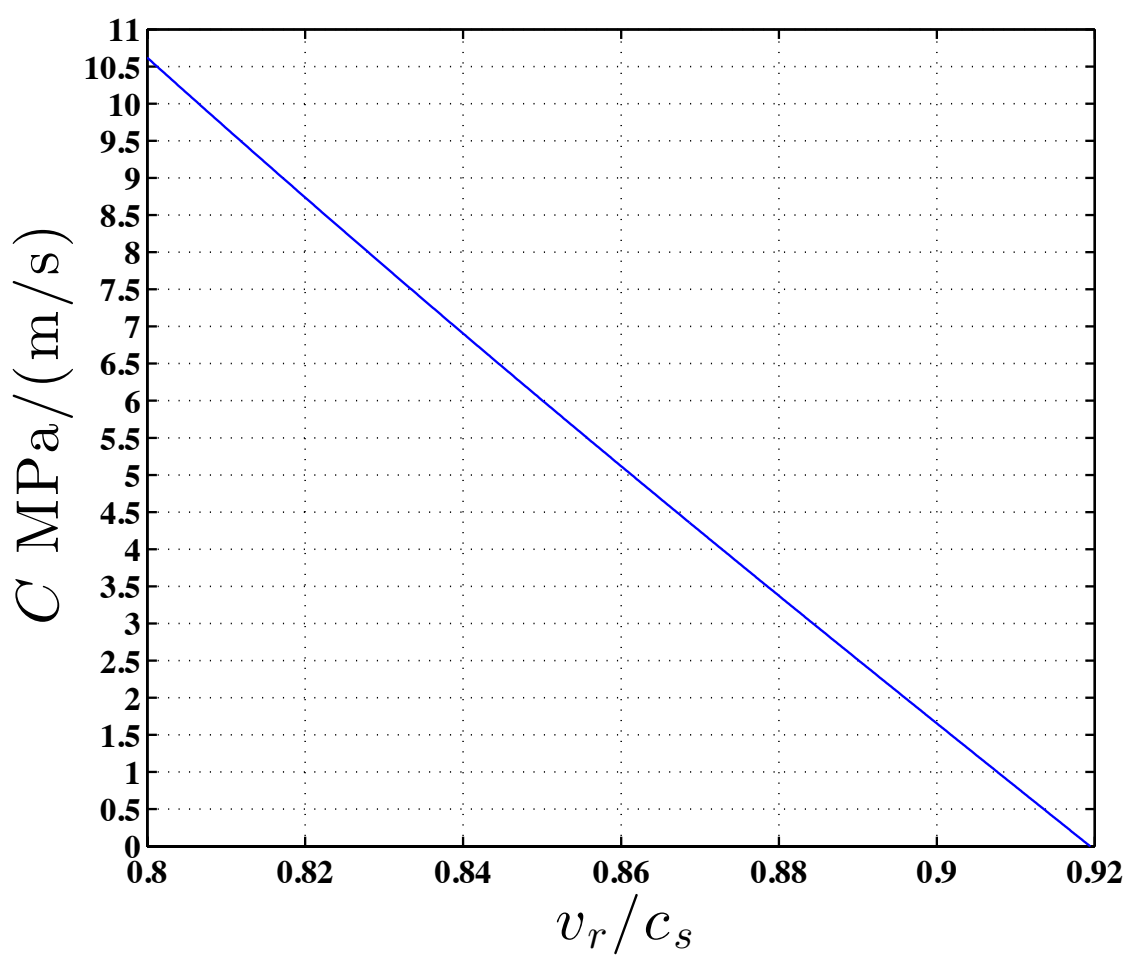

Figure 6.6: Coefficient $C$ in Eq. 6.46 plotted as a function of the normalized rupture speed $V_{r} / C_{s}$

second solution path for $\tau(x)$ relies solely upon $N(z)$ which in turn traces back to the shear traction boundary condition $\sigma_{x y}(-L<x<0,0)=\tau(x)-\tau_{0}$ from which the Hilbert problem was originally formulated. The two seemingly independent derivation paths lead to the same result.

Finally, note as well that the simultaneous solution of Eqs. 6.38, 6.39 also provides a means of evaluating the real integral

$$
I_{+}(x)=\frac{1}{\pi}(-x)^{1-q}(x+L)^{q} \int_{-L}^{0} \frac{\tau(w)-\tau_{0}}{(-w)^{1-q}(w+L)^{q}(w-x)} d w
$$

which is common to both expressions. Solving the simultaneous pair of equations yields s

$$
I_{+}(x)=\cot (\pi q) \Re N_{+}(x)-\Im N_{+}(x)
$$

The result is simply noted in the event that there is a useful physical interpretation associated with this integral expression. 


\subsubsection{Determination of $\tau(x)-\tau_{0}$ within a sub-Rayleigh slip zone $(-L<$}

$$
x<0)
$$

(Bhat, 2012) has recently derived an analogous near-fault stress field solution which is applicable to sub-Rayleigh rupture velocity fields. From (Rice et al., 2005) we have $M(z)$ (which is the same as $N(z)$ from (Dunham and Archuleta, 2005) when you set $q=0.5$ )

$$
\begin{aligned}
M(z) & =-\frac{1}{\pi} z^{1 / 2}(z+L)^{1 / 2} \int_{-L}^{0} \frac{\tau(w)-\tau_{0}}{(-w)^{1 / 2}(w+L)^{1 / 2}(w-z)} d w \\
\tau_{x y} & =\tau_{0}^{0}+\frac{1}{D}\left\{4 \alpha_{s} \alpha_{p} \Re M\left(z_{p}\right)-\left(1+\alpha_{s}^{2}\right)^{2} \Re M\left(z_{s}\right)\right\}
\end{aligned}
$$

where $D=4 \alpha_{s} \alpha_{p}-\left(1+\alpha_{s}^{2}\right)^{2}$ is the Rayleigh function. (Bhat, 2012) has extended these result for velocity fields and shown that

$$
\begin{aligned}
& \dot{u}_{x}=-\frac{v_{r}}{\mu D}\left\{2 \alpha_{s} \Im M\left(z_{p}\right)-\alpha_{s}\left(1+\alpha_{s}^{2}\right) \Im M\left(z_{s}\right)\right\} \\
& \dot{u}_{y}=-\frac{v_{r}}{\mu D}\left\{2 \alpha_{s} \alpha_{p} \Re M\left(z_{p}\right)-\left(1+\alpha_{s}^{2}\right) \Re M\left(z_{s}\right)\right\}
\end{aligned}
$$

It becomes immediately apparent by inspection that $\tau(x)$ and $\dot{u}_{y}$ are virtually identical in form apart from differences in their respective scaling factors. The nature of the stress field solution within the sub-Rayleigh rupture pulse slip zone is examined by considering the limiting form of these expressions as the observation point approaches the fault, in which case $\lim _{y \rightarrow 0^{ \pm}}(z)=x$ implies $z_{p}=z_{s}=\left(x, 0^{ \pm}\right)$and $\lim _{y \rightarrow 0^{ \pm}} M\left(z_{p}\right)=\lim _{y \rightarrow 0^{ \pm}} M\left(z_{s}\right)=M_{ \pm}(x)$. This leads to the remarkable result given by Eq. 6.46 that the limiting form of $\tau(x)$ at the fault is a scaled replicate of $\dot{u}_{y}\left(x, 0^{ \pm}\right)$.

$$
\tau_{x y}-\tau_{0}=C\left(\mu, v_{r}, C_{s}, C_{p}\right) \dot{u}_{y}
$$

where

$$
C=\left\{\frac{-\mu D}{v_{r}\left[2 \alpha_{s} \alpha_{p}-\left(1+\alpha_{s}^{2}\right)\right]}\right\} .
$$


As an example we may consider $\mu=30 \mathrm{GPa}, \rho=2700 \mathrm{~kg} / \mathrm{m}^{3}$ we get $C_{s}=3.3 \mathrm{~km} / \mathrm{s}$ and $C_{p}=5.8$ $\mathrm{km} / \mathrm{s}$, which then leads to the plot of $C$ vs. $V_{r} / C_{s}$ plotted in Figure 6.6.

\subsubsection{Dynamic stress measurements in laboratory earthquake experi- ments}

Dynamic stress estimates are obtained from laboratory earthquake particle velocity records by invoking a local steady-state argument which assumes that the rupture speed swept across the measurement station at constant speed. The analysis also takes advantage of the $6 \mu s$ (nominal) time interval following the arrival of the supershear rupture during which no S-waves arrive from the specimen boundaries and nearly semi-infinite conditions prevail (Section 3.10). The observation window is also generally greater than the temporal width of a supershear slip-pulse or at the very least envelopes the primary portion of the resulting supershear particle velocity profile. Application of Equations 6.24 and 6.34 within the observation window yield estimates for the normal stress $\sigma_{x x}\left(x, 0^{ \pm}\right)$and the shear stress distribution $\tau(x, 0)$ within the supershear slip zone $(-L<x<0)$, where $L$ represents the length of the supershear pulse. The method may also be applied to crack-like ruptures under the additional assumption that the locally steady-state portion of the record which is analyzed represents only a small fraction of a much longer supershear pulse width which extends well beyond the observation window. Note that the final result in either case can either be expressed in terms of time (i.e., temporal evolution form) or distance (i.e., spatial distribution form) since time

and space are interchangeable under the steady-state assumption and the Galilean transformation $x=X-V_{r} t$.

Simultaneous near-fault particle velocity records are obtained using a FN/FP fiber-optic probe arrangement such as the one depicted in Figure 6.1. Particle velocity records are typically acquired at $2.5 \mathrm{Gs} / \mathrm{sec}$, which translates to $0.4 \mathrm{~ns}$ per data point. A $6 \mu \mathrm{s}$ time interval selected from the record is composed of $15 \mathrm{~K}$ data points and is thus well sampled. Particle velocity measurements are complemented by synchronized high-speed photoelastic images of the supershear rupture and it's surrounding maximum shear stress field. The Cordin 220 gated-intensified camera used in the 
experiments features inter-frame times ranging from $10 \mathrm{~ns}-10 \mathrm{~ns}$ and exposure (integration) times ranging from $10 \mathrm{~ns}-1 \mathrm{~ms}$. A total of 16 image frames are acquired during an experiment with typical inter-frame times in the range of $1-4 \mu s$ and exposure times of $50 \mathrm{~ns}$. An accurate estimate of the rupture speed $V_{r}$ is obtained either by analyzing the position versus time of the supershear rupture tip or through measurements of the shear Mach front angle. Images of the leading P- and S-wavefronts also provide a direct estimate of the elastic wave speeds $C_{s}$ and $C_{p}$, and thus all of the elastic constants which are required by the stress-velocity relationships (Eqs. 6.24, 6.34).

The new technique also enables the investigation of dynamic friction in laboratory earthquake experiments. The measurement of $\tau(x)-\tau_{0}$ within the supershear slip zone, coupled with an independent measurement of the resolved normal and shear tractions $\left(\sigma_{y y}^{0}\right.$ and $\left.\tau_{0}\right)$ imposed on the fault, enable the direct characterization of the dynamic coefficient $f_{d}=\tau(x) / \sigma_{y y}^{0}$. Other frictional faulting relationships such as the variation of $\tau(x)$ (or $f_{d}$ ) with the accumulated slip $\Delta u(t)$ or it's dependency upon the rate of slip $\left(\Delta \dot{u}_{x}\right)$ are also directly obtained from the particle velocity measurements.

The dynamic stress measurement technique as presented is not necessarily restricted to the use of transparent, photoelastic materials such as H-100. Alternative high-speed imaging techniques may also be considered such as Digital Image Correlation (DIC) (Sutton, 2008) in the event that opaque materials such as crustal rock are investigated. Fiber optic laser interferometers may also be complemented by an array of closely spaced strain gages or piezoelectric sensors positioned close to fault which can be used to track the rupture at these locations and thus provide a reliable estimate of the rupture speed history (Wu et al., 1972; Schubnel et al., 2011). P- and S-wave speeds may also be determined in a similar manner assuming that the bonded transducers are sensitive to the arrival of the elastic wavefronts.

Finally, the 2D stress-velocity relationships need not be limited to laboratory earthquake investigations but are perhaps even applicable to seismological ground motion records obtained close to a fault during a supershear earthquake. This would also assume that depth effects play a minor role and that the ground motion records are largely a consequence of the local rupture field. Such 

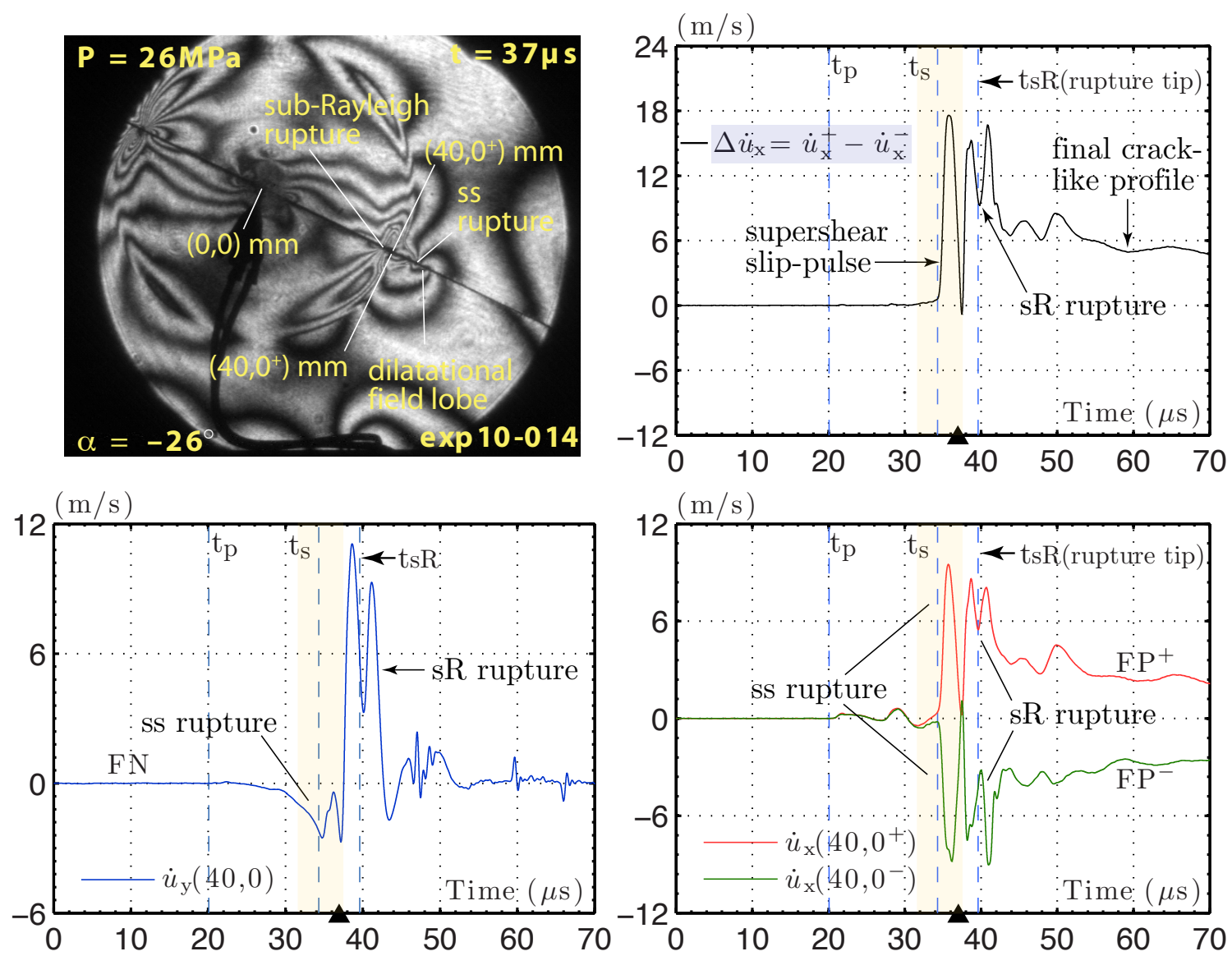

Figure 6.7: Supershear rupture experiment (exp10-014) featuring an anti-symmetric slip-pulse followed by crack-like trailing sub-Rayleigh rupture

a scenario could provide an unprecedented opportunity to investigate frictional faulting processes within the Earth'a shallow crust.

\subsubsection{Dynamic stress and friction investigations of a supershear slip- pulse (exp010-014)}

Figure 6.7 summarizes the particle velocity records obtained from a laboratory earthquake experiment (exp10-014) which resulted in a supershear slip-pulse. The experiment was conducted under a static-compressive load of $P=29 M P a$ using a traditional $6^{\prime \prime} \times 6^{\prime \prime} \times 0.375^{\prime \prime} \mathrm{H}-100$ specimen with a fault angle $\alpha=-26^{\circ}$. Three particle velocity probe beams were used to produce simultaneous near-fault recordings of the fault-parallel components $\dot{u}_{x}\left(40,0^{+}\right), \dot{u}_{x}\left(40,0^{-}\right)$and the fault-normal 
component $\dot{u}_{y}\left(40,0^{+}\right)$. Note that the \pm designation is not critical in the case of the FN component since the sign of $\dot{u}_{y}$ does not change across the fault. In cases where the \pm sign convention is used for $\dot{u}_{y}$, it is merely to designate the side of the fault where the measurement was conducted. The near-fault particle velocity records are interpreted and analyzed using Eqs. 6.24 and 6.34 in order to estimate $\sigma_{x x}\left(40,0^{+}\right)$and $\tau(40,0)$ from the slip-pulse portion of the particle velocity records.

The photoelastic image displayed in the upper-left panel of Figure 6.7 was acquired at $t=$ $37 \mu s$ and corresponds to the first of eight image frames that were acquired by the second high speed camera. The first eight image frames were unfortunately not obtained due to an equipment malfunction of the first high-speed camera and so the sub-Rayleigh-to-supershear transition was not recorded. The labeled near-fault measurement stations at $\left(40,0^{+}\right) \mathrm{mm}$ and $\left(40,0^{-}\right) \mathrm{mm}$ and the hypocenter at $(0,0)$ provide a length scale calibration for the image displayed in Figure 6.7. An expanded view of the image reveals a small, circular caustic zone which identifies the position of the supershear (ss) rupture front (tip). Looped fringes reveal the concentrated dilatational field which circulates and extends out beyond the supershear rupture tip. The trailing sub-Rayleigh rupture tip is also identified by a caustic zone between the prominent field lobes which extend to either side of the fault.

The first image acquired at $t=37 \mu s$ reveals the supershear rupture positioned well beyond the measurement stations at $\left(40,0^{ \pm}\right) \mathrm{mm}$. A normalized rupture speed estimate of $V_{r} / C_{s}=1.73 \pm 0.03$ was obtained from the angle of the shear Mach front on the lower (extensional) side of the fault. An average supershear rupture speed estimate was also obtained by tracking the position of the caustic zones within each of the high-speed image frames. The resulting position-time plot of the supershear rupture is displayed in Figure 6.8. The five data points which define the curve correspond to the position of the rupture tip at $t=37,41,45,50$, and $55 \mu \mathrm{s}$. The dashed blue line represents a best-fit line to the data and is represented by the linear relationship $x=2.18 t-31.95$. A strong linear fit to the raw data $\left(R^{2}=0.99987\right)$ also implies that the supershear rupture speed remained reasonably constant throughout the extended time interval. The resulting slope of the best-fit line $\left(V_{r}=2.18 \mathrm{~m} / \mathrm{s}\right)$ corresponds to an average normalized rupture speed of $\bar{V}_{r} / C_{s}=1.70$, which just falls 


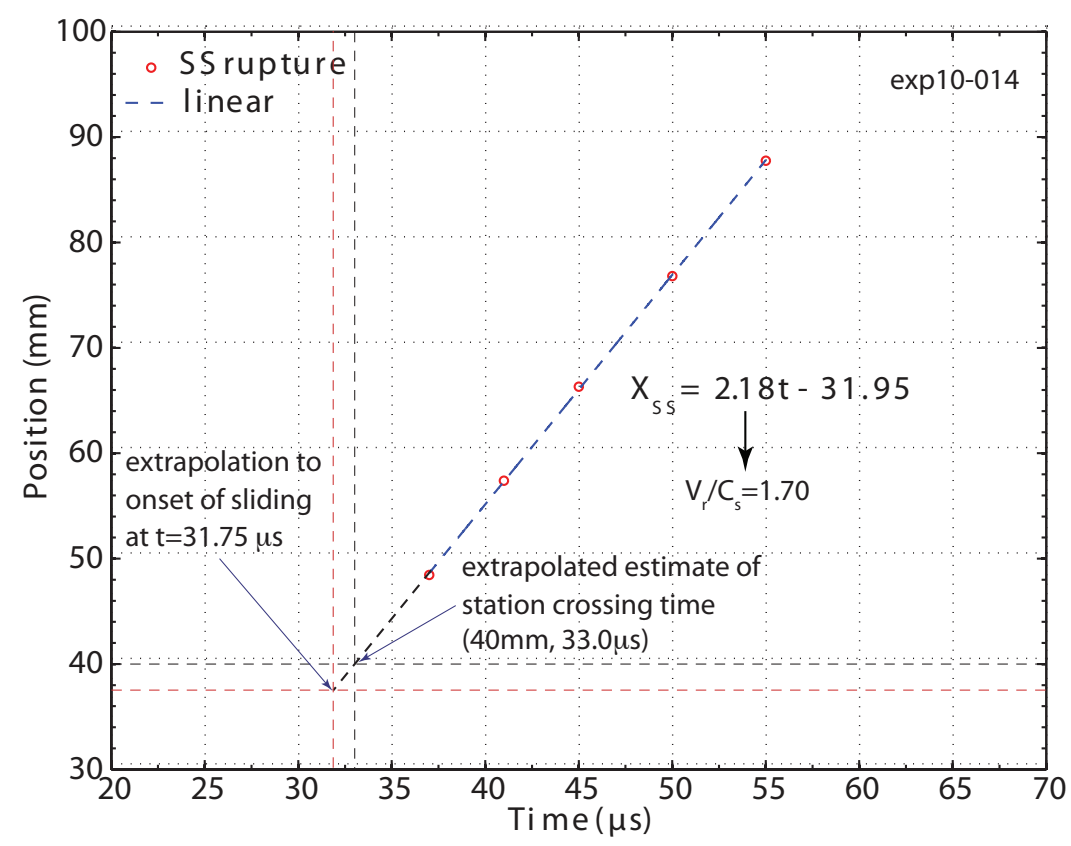

Figure 6.8: Position versus time of the supershear rupture tip determined from the photoelastic image sequence. Extrapolation of the best-fit line yields an estimated station crossing time of $t=33.0 \mu \mathrm{s}$. Extrapolation to $t=31.75 \mu \mathrm{s}$ (onset of sliding) reveals how the rupture tip (caustic) had not yet reached $(40,0) \mathrm{mm}$ at this time.

within the error bound for the rupture speed estimate obtained from the Mach angle measurement in the $t=37 \mu \mathrm{s}$ image frame.

The extrapolation of the best-fit line to $x=40 \mathrm{~mm}$ indicates that the supershear rupture tip crossed the measurement stations at $t^{*}=33.0 \mu \mathrm{s}$. The estimate is bounded by considering the earliest and latest possible times that the supershear rupture tip could have crossed the measurement station based upon its position at $x=48.439 \mathrm{~mm}$, as determined from the $t=37 \mu \mathrm{s}$ image frame. The upper bound is obtained by assuming an average rupture speed of $\bar{V}_{\max }=2 C_{s}$ (however unrealistic) as the rupture traversed from $x=40 \mathrm{~mm} \rightarrow x=48.439 \mathrm{~mm}$. A simple calculation then yields a station crossing time estimate of $t^{\text {upper }}=33.7 \mu \mathrm{s}$. A lower bound on the estimate is similarly obtained by assuming $\bar{V}_{\max }=\sqrt{2} C_{s}$ as the rupture traversed the interval, which leads to a station crossing time estimate of $t^{\text {lower }}=32.35 \mu \mathrm{s}$. Bearing these two extremes in mind leads to a conservative station crossing time estimate of $t^{*}=33 \pm 0.7 \mu \mathrm{s}$.

Near-fault particle velocity traces acquired during the experiment are displayed in the remaining three panels of Figure 6.7. The dashed vertical lines in each of the plots represent the estimated 
arrival times of the leading $\mathrm{P}$-wave, S-wave, and the trailing sub-Rayleigh rupture at $(40,0) \mathrm{mm}$. A black triangle positioned at $t=37 \mu s$ marks the time at which the photoelastic image in the upper-left panel was obtained. Fault-parallel velocity records $\left(F P^{+}\right.$and $\left.F P^{-}\right)$are displayed in the lower-right panel. The most obvious and striking feature exhibited by the records is the supershear slip-pulse highlighted within the rectangular shaded region. The same corresponding time interval is highlighted in the FN plot depicted in the lower-left panel. The dominance of the FP component, and the noted sign of the velocity jumps within the shaded regions of each respective record, are consistent with that of a stable $\left(\sqrt{2} C_{s}<V_{r}<C_{p}\right)$, right-lateral, supershear slip-pulse propagating to the right (Figure $6.4 \mathrm{~b})$. The stable rupture speed scenario revealed by the particle velocity records is also consistent with the normalized rupture speed estimate $\left(V_{r} / C_{s}=1.72 \pm 0.02\right)$ inferred through the analysis of the high-speed image sequence. The absence of any strong secondary supershear signals may be related to the fact that the supershear transition occurred so late in the experiment. It is also possible that secondary signals are superimposed upon the sub-Rayleigh portion of the record. This is largely inconsequential to the experiment since the supershear slip-pulse is the primary focus of the investigation.

The slip velocity $\Delta \dot{u}_{x}(40,0)$ is plotted in the upper-right panel of Figure 6.7 . The curve was obtained by subtracting the two antisymmetric records in accordance with Eq. 6.1. The resulting curve displays the supershear slip-pulse (highlighted within the shaded region), followed by a cracklike interfacial sliding signature of the trailing sub-Rayleigh rupture. A close examination of the plot reveals that the onset of slip occurred near $t_{0}=31.75 \mu \mathrm{s}$. The supershear slip-pulse curve exhibits a very gradual and steady increase until $t=34.3 \mu \mathrm{s}$ at which point a steep velocity jump is suddenly registered and the relative sliding rises to a peak value of $17.5 \mathrm{~m} / \mathrm{s}$ in the course of $1.4 \mu s$. The sharp velocity increase is then immediately followed by a rapid deceleration along the tail end of the slip pulse leading to momentary healing of the fault surfaces at $t=37.4 \mu \mathrm{s}$. The slight overshoot below $0 \mathrm{~m} / \mathrm{s}$ s an artifact due to the slight differences between the two records and is clearly unphysical as it would otherwise imply a reversal of the sliding direction.

The photoelastic images in Figure 6.9 show the local supershear rupture fields and the rupture 

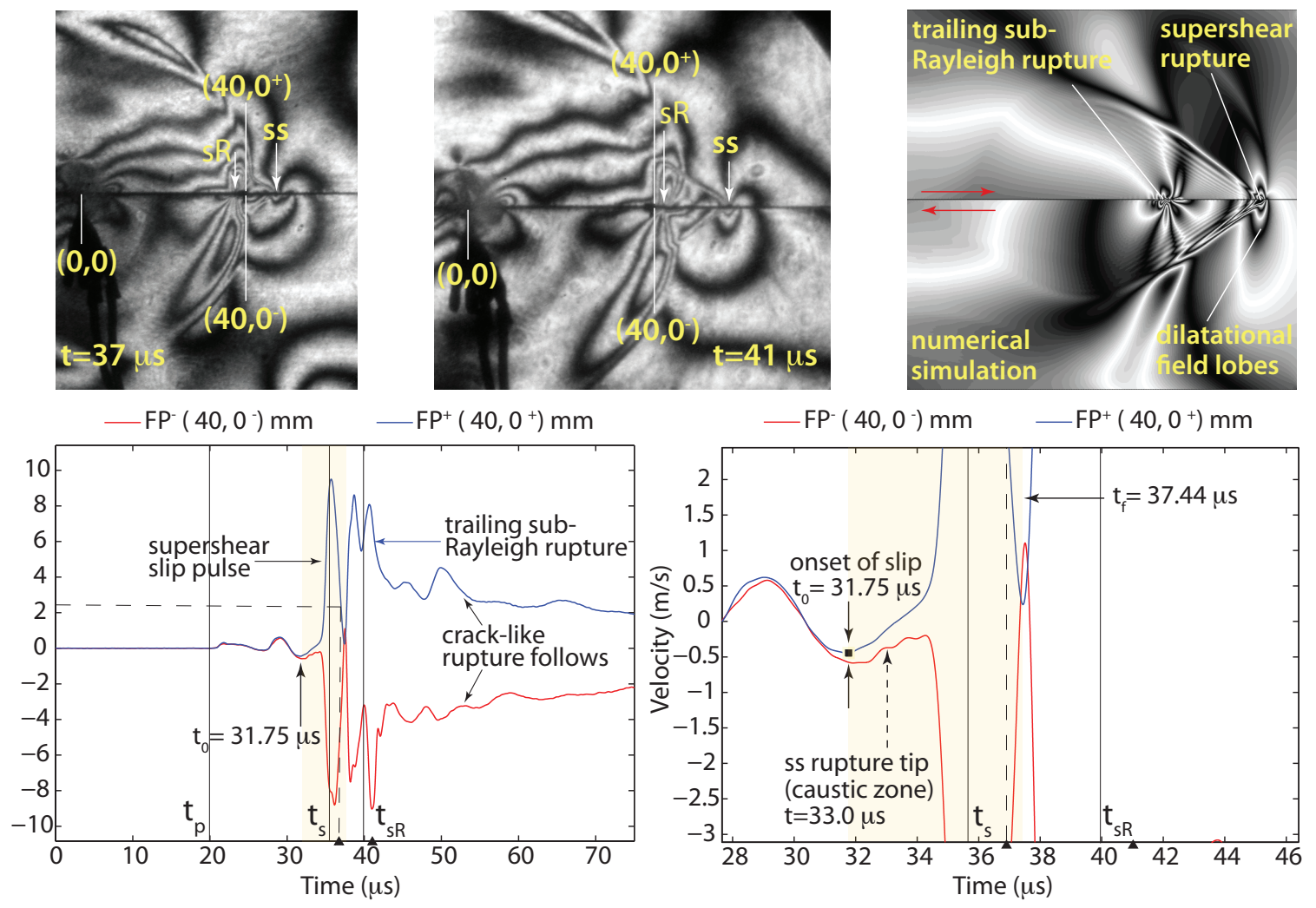

Figure 6.9: (a) Expanded views of photoelastic images from supershear experiment (exp10-014) reveal the prominent dilatational field lobes extending out beyond the primary rupture tip which perturb the fault in advance of the primary rupture (b) Numerical simulation of photoelastic field obtained using ABAQUS validates and highlights the primary features of the supershear rupture field observed in experiments (c) Magnified view of the fault-parallel traces highlights the onset of slip and gradual ramping of the interfacial sliding speed prior to the arrival of the primary supershear rupture

tip positions at $t=37 \mu \mathrm{s}$ and $t=41 \mu \mathrm{s}$. The high-speed image frames are well synchronized with the particle velocity records as can be seen by noting how the first maxima at $t=38.7 \mu \mathrm{s}$ in the sub-Rayleigh portion of the particle velocity records correlates with the estimated station crossing time of the sub-Rayleigh rupture in the vicinity of $t=39 \mu \mathrm{s}$. The leading dilatational field lobes are identified in the images by the looping fringes which appear to circulate around the supershear rupture tip.

The contour plot on the right in Figure 6.9 was obtained from a 2D plane stress finite element simulation using a commercial code, ABAQUS. The simulation was conducted for the model material (Homalite-100) and assumed a slip-weakening frictional constitutive description with $D_{c}=10 \mathrm{~mm}$, $f_{s}=0.8$, and $f_{d}=0.2$ (Bhat, 2012). The numerical simulation is included alongside the photoelastic 
images in order to validate and highlight the primary features of the supershear rupture field, namely the sub-Rayleigh and supershear rupture fields and the dilatational field lobes which exhibit a similar form in the immediate vicinity of the supershear rupture tip.

The curves displayed in the lower-right panel of Figure 6.9 correspond to an expanded view of the antisymmetric $\mathrm{FP}^{+}$and $\mathrm{FP}^{-}$velocity traces. The expanded records reveal how the $\mathrm{FP}^{+}$and $F P^{-}$curves undulated and shifted together from $27.6 \mu s \rightarrow 31.75 \mu s$ prior to the onset of slip. Note how this portion of the particle velocity records is naturally cancelled out in the slip velocity plot depicted in Figure 6.7. The onset of slip at $t=31.75 \mu \mathrm{s}$, which was initially identified in the slip velocity curve in Figure 6.7 corresponds to the labeled point in the plot where the curves clearly begin to separate.

As previously discussed, the equation of the best-fit line in Figure 6.8 predicts a measurement station crossing time of $t^{*}=33.0 \pm 0.7 \mu \mathrm{s}$. Recall as well that the estimate bound of $\pm 0.7 \mu \mathrm{s}$ is extremely conservative in this instance and that it is highly unlikely that the rupture swept the station at either of the two rupture speed extremes form which the bounds were determined. It is far more likely that the rupture swept across $(40,0)$ at a time much closer to $t^{*}=33.0 \mu s$, which sits between the onset of sliding at $t=31.75 \mu \mathrm{s}$ and the instant when the sharp velocity jump was registered at $t=34.3 \mu \mathrm{s}$. Note as well how the extrapolation of the best-fit line to $t=31.75 \mu \mathrm{s}$ (onset of sliding time) in Figure 6.8 reveals that the supershear rupture tip had not yet reached the measurement station at this time.

The results collectively imply that the onset of slip and the gradual, steady increase of the sliding speed observed prior to the sharp jump at $t=34.3 \mu \mathrm{s}$ must therefore be linked to the leading dilatational field which perturbs the fault in advance of the primary rupture. The primary supershear rupture immediately follows to induce additional preliminary sliding before the fault surfaces ultimately give way and the sharp slip velocity pulse is registered. 

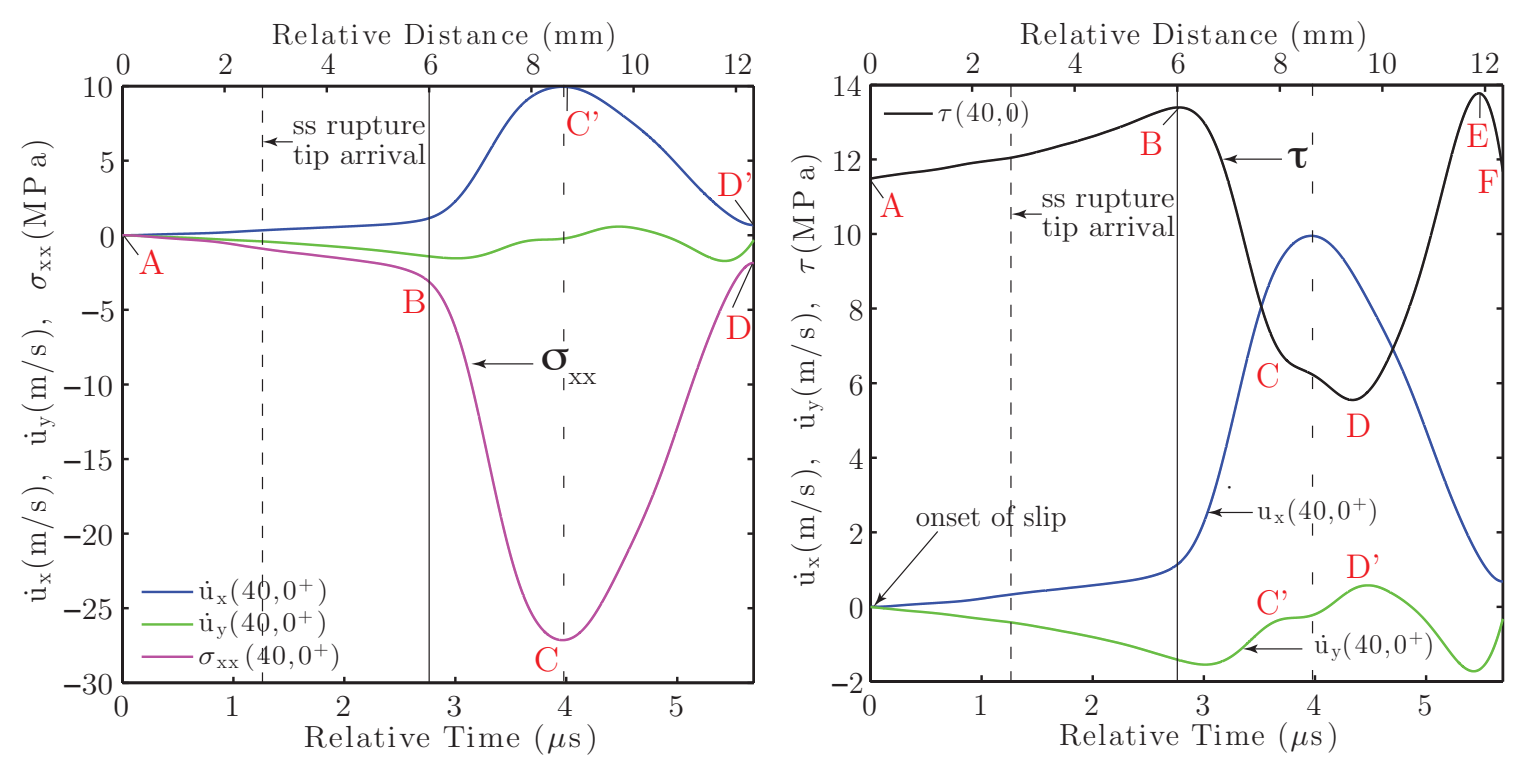

Figure 6.10: Temporal evolution and spatial distribution of dynamic stress components $\sigma_{x x}$ and $\tau$ accompanied by the FP and FN particle velocity records corresponding to the supershear slip-pulse recorded in exp10-014.

\subsubsection{Estimates of $\sigma_{x x}$ and $\tau$ obtained from the supershear slip-pulse particle velocity records}

The stress-velocity relationships given by Eqs. 6.24 and Eq. 6.34 were applied to the supershear slippulse portion of the particle velocity records in order to investigate the frictional faulting process. The time interval $\Delta t=5.69 \mu \mathrm{s}$ spanning between $t_{0}=31.75 \mu \mathrm{s}$ and $t=37.44 \mu \mathrm{s}$ was selected as the observation window for the dynamic stress analysis. The plots in Figure 6.10 display the resulting profiles of the normal stress component $\sigma_{x x}$ and the shear stress $\tau$ at the near-fault location. The resulting curves are accompanied by the fault-parallel and fault-normal particle velocity records $\dot{u}_{x}\left(40,0^{+}\right)$and $\dot{u}_{y}\left(40,0^{+}\right)$from which they were derived.

The time axis in Figure 6.10 corresponds to $t^{\prime}=t-t_{0}$ where $t$ represents the time in the original records and $t_{0}=31.75 \mu \mathrm{s}$ corresponds to the onset of slip as previously discussed. The final point in the interval $t_{f}=5.69 \mu \mathrm{s}$ corresponds to the end of the slip pulse at $t=37.44 \mu \mathrm{s}$. An equivalent steady-state length scale corresponding to $x=V_{r} t$, where $V_{r}=1.7 C_{s}$, is also displayed along the top of the plots. Note as well that the scale of the vertical axis applies equally well to velocity in $(\mathrm{m} / \mathrm{s})$ and stress in $(M P a)$. 
Before conducting the stress analysis it was noted that the original particle velocity records were each slightly biased away from $0 \mathrm{~m} / \mathrm{s}$ at $t=31.75 \mu \mathrm{s}$. Two distinct perturbations of the velocity records occurred prior to the onset of slip. The first perturbation of the records commencing at $t=21 \mu s$ and is likely attributed to the near-field disturbance stemming from rupture nucleation, which was previously addressed in Section 4.2.2. A second, slightly stronger perturbation of the records commencing at $t=27.5 \mu \mathrm{s}$ is characterized by a pulse-like undulation of the $F P^{+}$and $F P^{-}$ curves leading up to the onset of slip at $t=31.75 \mu \mathrm{s}$. The FN particle velocity begins to steadily decrease at $t=29 \mu \mathrm{s}$ coinciding with the time at which the pulse-like undulation in the FP records reaches its peak. The latter perturbations are not tyically observed in typical laboratory earthquake experiments and may be a residual effect of the supershear transition which occurred just prior to the time during the which the measurements were obtained. The noted perturbations resulted in a bias of $-0.9813 \mathrm{~m} / \mathrm{s}$ in the $\mathrm{FN}$ record and $0.4446 \mathrm{~m} / \mathrm{s}$ in the $\mathrm{FP}$ record at $t=31.75 \mu \mathrm{s}$. A decision was made to eliminate the initial bias and shift each of the particle velocity records such that $\dot{u}_{x}\left(40,0^{+}\right)=\dot{u}_{y}(40,0)=0 \mathrm{~m} / \mathrm{s}$ at $t_{0}=31.75 \mu \mathrm{s}$. The bias-adjusted particle velocity records corresponding to the slip-pulse portion of the experimental record are displayed in Figure 6.10.

Figure 6.10 displays a plot of $\sigma_{x x}$ and $\tau$ corresponding to the slip-pulse portion of the supershear record as determined by Eqs. 6.24 and 6.34. The calculations assumed a rupture speed of $V_{r}=$ $2176 \mathrm{~m} / \mathrm{s}$ (recall $V_{r} / C_{s}=1.7$ ) and a shear modulus value of $\mu=1.96 \mathrm{GPa}$ for $\mathrm{H}-100$ as determined from the shear wave speed measurements. The dashed vertical lines positioned at $t=1.25 \mu s$ in each of the plots mark the estimated arrival time of the supershear rupture tip at the measurement stations $\left(40,0^{ \pm}\right) \mathrm{mm}$. The two resulting stress pulses appear naturally synchronized, as they should be, with the slip velocity pulse depicted in Figure 6.7 .

The points labeled $A$ and $B$ in each of the plots highlight the initial period during which an initial, gradual ramping of the shear and compressive stresses is observed. The observed increase naturally correlates with the initial steady ramping of the sliding speed exhibited by the slip velocity plot in Figure 6.7 and the expanded plot of the antisymmetric FP traces in Figure 6.9. A $16.4 \%$ increase of the shear stress is registered as the stress level climbs from an initial level of $\sigma_{x y}^{0}=$ 
11.48 MPa (resolved shear traction imposed by the static-compressive load) to a peak value of $\tau_{\max }=13.36 \mathrm{MPa}$ at $t=2.75 \mu \mathrm{s}$. The compressive undergoes a slightly more modest increase of $3 M P a$ during the same time interval.

Note, as was previously observed in the FP records, that the initial peak in the shear stress curve, and the sharp slope change exhibited by compressive stress at the points labeled B, occur well after the arrival of the supershear rupture tip at $(40,0) \mathrm{mm}$. Both curves then undergo a precipitous drop in stress commencing at point B which also naturally coincides with sudden the sharp jump in the slip velocity. The initial behavior exhibited by the shear stress curve suggests that sliding is initially resisted by the fault surfaces, until a critical fictional strength limit is reached, at which point the surface traction rapidly drops and the sliding speed abruptly increases. A simultaneous jump in the compressive stress immediately follows in response to the sharp velocity jump registered by the FP component.

The resulting compressive stress-pulse reaches a peak value of $\sigma_{x x}^{\max }=-27 \mathrm{MPa}$ (point $\mathrm{C}$ ) which is comparable in the magnitude to the initial static-compressive load $(P=29 M P a)$. An extensional stress $\left(\sigma_{x x}>\right)$ of the same magnitude and temporal width must therefore be registered at $\left(40,0^{-}\right) \mathrm{mm}$ on the opposite side of the fault. It is perhaps not surprising then that a series of wing cracks running nearly perpendicular to the fault were observed and noted in the extensional quadrant on the other side of the specimen (opposite side with respect to the hypocenter). It is entirely conceivable that the left-traveling supershear rupture exhibited a stress pulse of comparable or slightly greater magnitude and that the peak extensional stress either exceeded the spall strength of the H-100 specimen or triggered pre-existing flaws to grow at the location where the cracks were observed. Unfortunately, this region was well outside of the camera's field of view and so it was not possible to record the left traveling (bi-lateral) supershear rupture and the mode I cracks as they were nucleated.

The steepest drop in the shear stress curve between the points labeled $B$ and $C$ correlates with the sharp velocity increase registered by the steepest portion of the $F P^{+}$record. A noticeable change in the slope of the shear stress curve is also observed between the points labeled $C$ and 


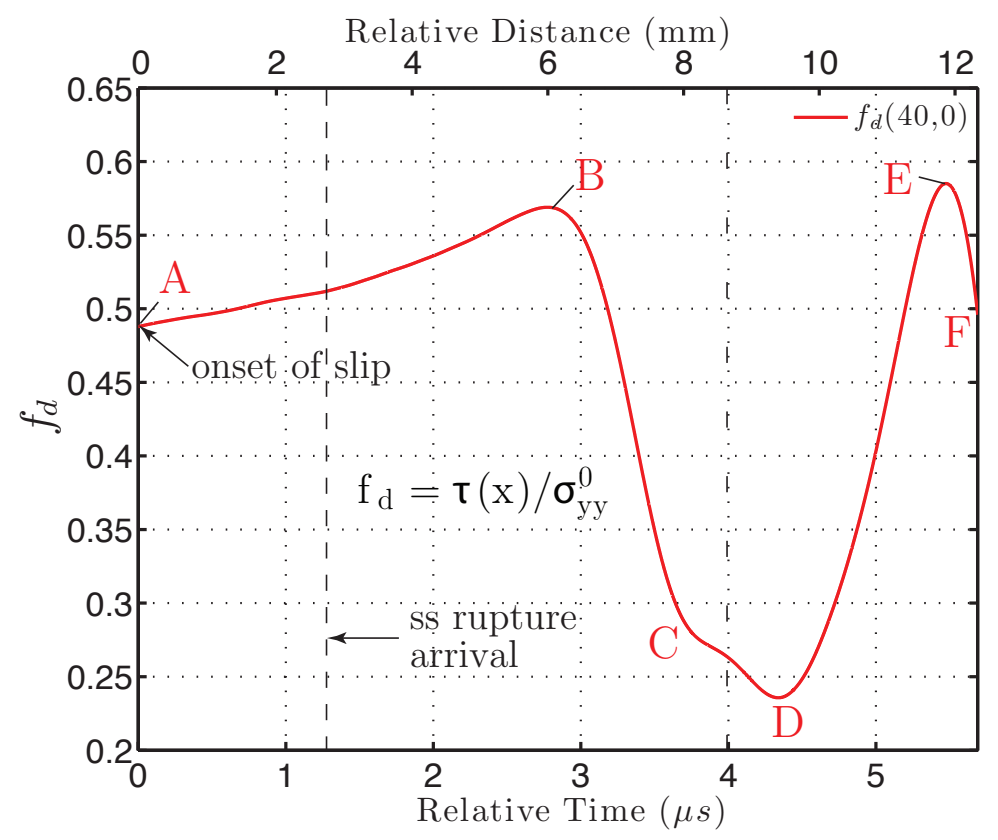

Figure 6.11: Temporal evolution and spatial distribution of the dynamic coefficient of friction $f_{d}$ obtained from supershear slip-pulse in exp10-014

$D$ as the the shear stress approaches its minimum value of $\tau_{\min }=5.5 \mathrm{MPa}$ at $t=4.338 \mu \mathrm{s}$ (relative distance $=9.42 \mathrm{~mm}$ ). The noted drop in stress corresponds to a $52 \%$ drop compared to the resolved shear traction of $\sigma_{x y}^{0}=11.5 \mathrm{MPa}$, and a $59 \%$ drop compared to the peak shearing resistance level at point $B$. The shear stress begins to rapidly recover after this point. Note as well how the recovery which commences at point $D$ does not coincide with the time at which the $\mathrm{FP}^{+}$reaches its peak but matches up instead with point $D^{\prime}$ in the FN record well after the FP has already begun to slow down.

The steep monotonic increase of the shear traction between the points labeled $D$ and $E$ naturally correlates well with the steep negatively sloped portions of then FP record along the tail end of the slip-pulse. Note how the shear traction momentarily reaches a peak value at point $E$ which is comparable to the prior peak value of the shear traction observed at point $B$. The shear stress curve then takes a final small downturn from point $E$ on its way to a nearly complete recovery as $\mathrm{FN}$ particle velocity exhibits a final positive increase toward $0 \mu s$.

Figure 6.11 depicts a plot of the dynamic friction coefficient $f_{d}$. The result is obtained by 
simply scaling $\tau$ by the resolved normal traction $\sigma_{y y}^{0}=P \sin \alpha \cos \alpha$ where $P$ represents the static compressive load and $\alpha$ represents the specimen fault angle. In the case of supershear experiment (exp10-014), a static-compressive load of $P=29 M P a$ and a specimen fault angle $\alpha=-26^{\circ}$ translate to a resolved normal traction $\sigma_{y y}^{0}=23.54 \mathrm{MPa}$.

The evolution of the dynamic friction coefficient naturally exhibits all of the same general features which were previously described in relation to the plot of $\tau$ in Figure 6.10. The value of the friction coefficient starts out at $f_{d}^{0}=0.487$, which naturally corresponds to $\tan 26^{\circ}$. Note that the plot starts out at this value as a result of the initial bias correction and that the initial value would otherwise be different had the initial bias not been eliminated from the particle velocity records. An increase of the friction coefficient was registered between points $A$ and $B$ during the initial ramping period which resulted in a $16.6 \%$ increase to a peak value of $f_{d}=0.568$ at $t=2.75 \mu \mathrm{s}$. This was immediately followed by an abrupt reduction of the friction coefficient as the initial shearing resistance of the fault surfaces was overcome. The friction coefficient dropped by $59 \%$ to a minimum value of $f_{d}=0.231$ at $t=4.338 \mu \mathrm{s}$, which is labeled as point $D$ in Figure 6.11 . The curve then rapidly climbed to a peak value of $f_{d}=0.584$ at point $E$ as the frictional resistance was recovered. Note that the value of the friction coefficient at point $E$ is only slightly higher than the previous peak value of $f_{d}=0.568$ registered at point $B$. The curve then took a final downturn and terminated at the point labeled $F$ corresponding to the end of the slip-pulse. The final value of $f_{d}=0.482$ represents a nearly complete recovery of the frictional strength.

Figures $6.12 \mathrm{a}$ and $6.12 \mathrm{~b}$ examine the relationship between the slip velocity and shear stress distribution within the supershear slip zone. The dashed black vertical line positioned at $t=1.25 \mu \mathrm{s}$ in the plots denotes the estimated supershear rupture arrival time as previously noted. Note that the slip velocity function displayed in Figure 6.12a was obtained using Eq. 6.2 and thus slightly overestimates the peak slip velocity. The resulting curve is otherwise identical in all respects to the original slip velocity function displayed in Figure 6.7. The dashed green vertical line positioned at $t=3.95 \mu \mathrm{s}$ in Figure 6.12a denotes the time when the peak sliding speed was registered and the sliding speed began to rapidly decrease along the healing phase of the supershear slip-pulse. 
Figure $6.12 \mathrm{~b}$ depicts a plot of the shear stress vs. the slip velocity. The blue portion of the curve represents the stress vs. slip velocity relationship along the rising portion of the supershear slippulse. The starting point naturally coincides with the onset of slip as labeled in the figure. Velocity strengthening behavior is observed during the initial, gradual, ramping profile labeled between the points $A$ and $B$ in Figures 6.10 and 6.11. The curve then changes slope and begins a long decrease until it intersects the horizontal dashed green line which marks the point at which the slip velocity reached its peak value of nearly $20 \mathrm{~m} / \mathrm{s}$. The relationship between the shear stress and the slip velocity along this portion of the curve represents velocity weakening behavior exhibited by the H-100 fault surfaces.

The evolution of the shear stress corresponding to the tail (falling edge) of the supershear slip pulse is represented by the red portion of the curve in Figure $6.12 \mathrm{~b}$. The sharp change in slope between the green and red curves simply corresponds to the peak of the slip velocity curve and the point at which the velocity begins to rapidly decrease. The initial short segment of the red curve reveals how the shear stress continued to drop by approximately $0.5 \mathrm{MPa}$ even as the slip velocity was already rapidly decreasing, i.e., velocity weakening behavior continued. The curve then briefly levels out and transitions to a velocity strengthening phase. A rapid recovery of the frictional strength is observed which corresponds with the portion of the curve between the points $D$ and $E$ in the shear stress curve (Figure 6.10). The shear stress ultimately peaks at point $E$ in Figure 6.10 and then reverses slope one last time into a final velocity weakening phase where it then gradually decreases toward a nearly complete recovery of the initial resolved shear traction $\tau_{0}=\sigma_{x y}^{0}$.

Figures $6.12 \mathrm{c}$ and $6.12 \mathrm{~d}$ examine the relationship between the accumulated slip and shear stress distribution within the supershear slip zone. The accumulate slip curve displayed in $6.12 \mathrm{c}$ was obtained through numerical integration of the slip velocity function plotted in Figure 6.12a. A total accumulated slip of $36.2 \mu \mathrm{m}$ resulted from the passage of the supershear slip pulse at $(40,0)$. As with the previous plots, the dashed black vertical lines positioned at $t=1.25 \mu \mathrm{s}$ denote the estimated supershear rupture arrival time at $(40,0) \mathrm{mm}$. The dashed green lines denote the point within each plot corresponding to the peak in the slip velocity. 

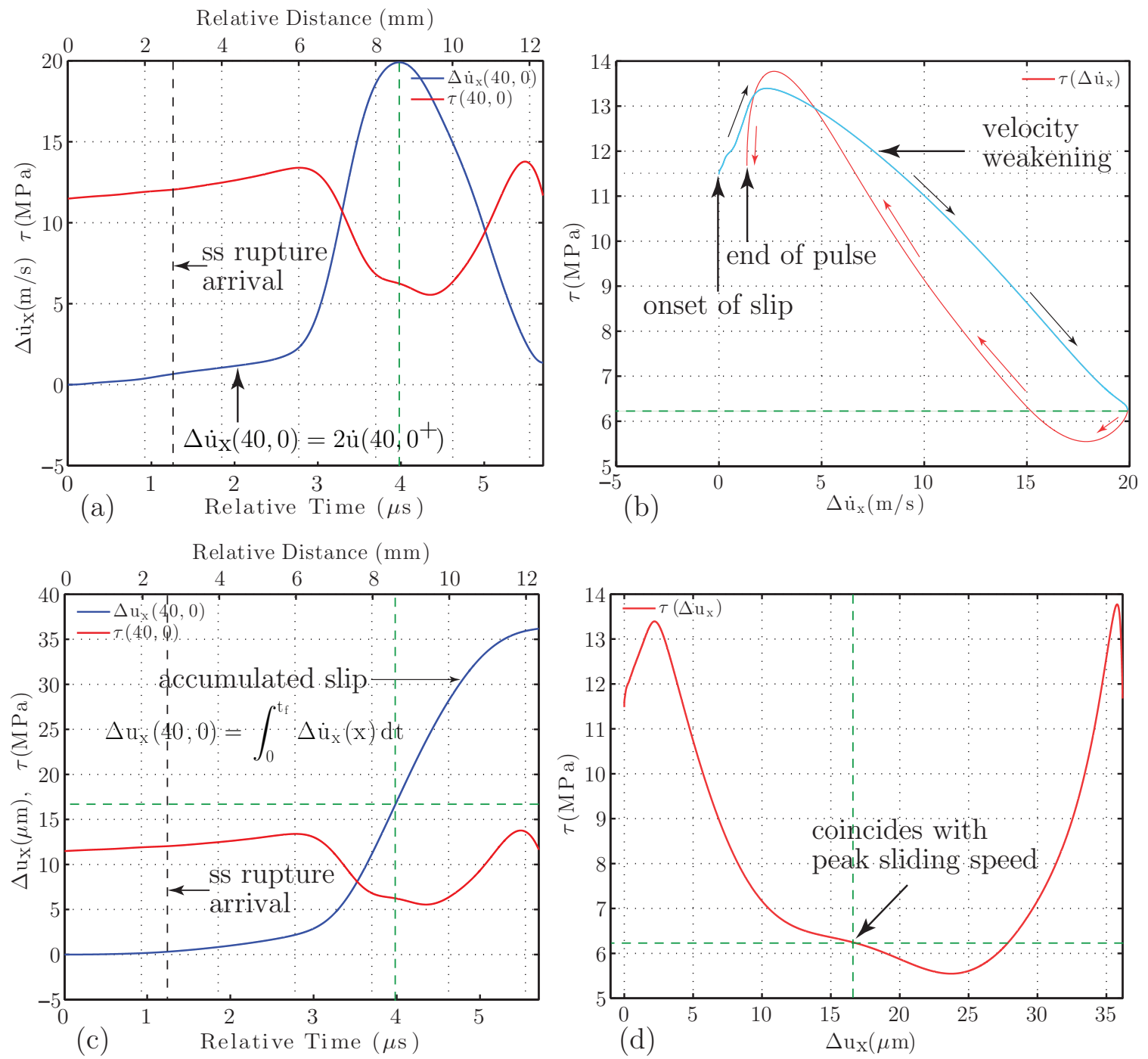

Figure 6.12: (a) Superimposed plots of the shear stress distribution and slip velocity corresponding to the supershear slip-pulse from exp10-014 (b) Plot of shear stress vs. slip rate (c) Superimposed plots of the shear stress distribution and accumulated slip corresponding to the supershear slip-pulse from exp10-014 (d) Plot of shear stress vs. accumulated slip

A plot of the shear stress vs. the accumulated slip is displayed in $6.12 \mathrm{~d}$. The initial positively sloped segment leading to the peak shear stress $\tau_{\max }=13.36 \mathrm{MPa}$ corresponds to the previously noted velocity-strengthening phase observed during the first $2.5 \mu \mathrm{s}$ of the experimental records. Note how only $2.2 \mu \mathrm{m}$ of slip was accumulated as the frictional resistance built up to its initial peak value. Each of the observed slope changes and the overall character of the curve are easily reconciled by appealing to the previous descriptions regarding the temporal evolution and spatial distribution of the shear stress. 


\section{Bibliography}

B. T. Aagaard and T. H. Heaton. Near-source ground motions from simulations of sustained intersonic and supersonic fault ruptures. Bull. Seismol. Soc. Amer., 94:2064-2078, Dec 2004.

K. Aki and P. G. Richards. Quantitative Seismology. University Science Books, 2002.

D. J. Andrews. Rupture velocity of plane strain shear cracks. J. Geophys. Res., 81(B32):5679-5689, 1976.

ANSI/ASMEB46.1-2009. Surface texture (surface roughness, waviness and lay). Measurement standard., American Society of Mechanical Engineers (ASME), 2009.

R. J. Archuleta. A faulting model for the 1979 Imperial Valley earthquake. J. Geophys. Res., 89 (B6):4559-4586, 1984.

G. I. Barenblatt. The mathematical theory of equilibrium cracks in brittle fracture. Adv. Appl. Mech., 7(2):55-129, 1962.

A. Ben-Menahem and S. J. Singh. Supershear accelerations and Mach-waves from a rupturing front : Theoretical model and implications. J. Phys. Earth, 35:347-365, 1987.

H. S. Bhat. Stress evolution from sub-Rayleigh velocity field. private communication, April 2012.

H. S. Bhat, R. Dmowska, G. C. P. King, Y. Klinger, and J. R. Rice. Off-fault damage patterns due to supershear ruptures with application to the $2001 M_{w} 8.1$ Kokoxili (Kunlun) Tibet earthquake. J. Geophys. Res., B06301, 2007. doi: 10.1029/2006JB004425.

H. S. Bhat, R. L. Biegel, A. J. Rosakis, and C. G Sammis. The effect of asymmetric damage on 
dynamic shear rupture propagation ii: With mismatch in bulk elasticity. Tectonophysics, 493: 263-271, May 2010. doi: 10.1016/j.tecto.2010.03.016.

R. L. Biegel, H. S. Bhat, C. G. Sammis, and A. J. Rosakis. The effect of asymmetric damage on dynamic shear rupture propagation i: No mismatch in bulk elasticity. Tectonophysics, 493: 254-262, May 2010. doi: 10.1016/j.tecto.2010.03.020.

M. Bouchon and H. Karabulut. The aftershock signature of supershear earthquakes. Science, 320 (5881):1323, 2008.

M. Bouchon and M. Vallee. Observation of long supershear rupture during the magnitude 8.1 Kunlunshan earthquake. Science, 301:824-826, Aug 2003.

M. Bouchon, M. P. Bouin, H. Karabulut, M. N. Toksoz, M. Dietrich, and A. J. Rosakis. How fast is rupture during an earthquake? New insights from the 1999 Turkey earthquakes. Geophys. Res. Lett., 28:2723-2726, Jul 2001.

M. Bouchon, M. N. Toksoz, H. Karabulut, M. P. Bouin, M. Dietrich, M. Aktar, and M. Edie. Space and time evolution of rupture and faulting during the 1999 Izmit (Turkey) earthquake. Bull. Seismol. Soc. Amer., 92:256-266, Feb 2002.

M.P. Bouin, M. Bouchon, H. Karabulut, and M. Aktar. Rupture process of the 1999 November 12 Duzce (Turkey) earthquake deduced from strong motion and global positioning system measurements. Geophys. J. Int, 159:207-211, 2004.

K. B. Broberg. On transient sliding motion. Geophys. J. Roy. Astr. Soc, 52:397-432, 1978.

K. B. Broberg. The near-tip field at high crack velocities. Int. J. Fract., 39(1):1-13, 1989.

K. B. Broberg. Intersonic bilateral slip. Geophys. J. Int., 119:706-714, Dec 1994.

K. B. Broberg. Cracks and Fracture. Academic Press, 1999.

R. Burridge. Admissible speeds for plane-strain self-similar shear cracks with friction but lacking cohesion. Geophys. J. Roy. Astron. Soc, 35:439-455, 1973. 
R. Burridge, G. Conn, and L. B. Freund. Stability Of a rapid mode-II shear crack with finite cohesive traction. J. Geophys. Res, 84:2210-2222, 1979.

T. Chi, R. Ballinger, R. Olds, and M. Zecchino. Surface texture analysis using Dektak stylus profilers. Application note., Veeco Instruments Inc., 2004.

D. Coker and A. J. Rosakis. Experimental observations of intersonic crack growth in asymmetrically loaded unidirectional composite plates. Philos. Mag. A-Phys. Condens. Matter. Struct. Defect. Mech., 81:571-595, Mar 2001.

D. Coker, A. J. Rosakis, and A. Needleman. Dynamic crack growth along a polymer compositehomalite interface. J. Mech. Phys. Solids, 51:425-460, Mar 2003.

P. A. Craw, A. J. Crone, P. J. Haeussler, S. F. Personius, and L. A. Staft. The Susitna Glacier thrust fault-characteristics of ruptures that initiated the Denali fault earthquake. Eos Trans. $A G U$, abstract S11B-01, 2003.

S. Das. The need to study speed. Science, 317(5840):905-906, 2007.

B.V. Deryagin. P. N. Lebedev's ideas on the nature of molecular forces. Soviet Physics USPEKHI, 10(1):108-111, 1967.

D. S. Dugdale. Yielding of steel sheets containing slits. J. Mech. Phys. Solids, 8:66-75, 1960.

E. M. Dunham and R. J. Archuleta. Evidence for a supershear transient during the 2002 Denali fault earthquake. Bull. Seismol. Soc. Amer., 94:S256-S268, Dec 2004.

E. M. Dunham and R. J. Archuleta. Near-source ground motion from steady state dynamic rupture pulses. Geophys. Res. Lett., 32, Feb 2005.

E. M. Dunham and H. S. Bhat. Attenuation of radiated ground motion and stresses from three-dimensional supershear ruptures. J. Geophys. Res., 113(B08319), 2008. doi: 10.1029/ 2007JB005182. 
E. M. Dunham, P. Favreau, and J. M. Carlson. A supershear transition mechanism for cracks. Science, 299:1557-1559, Mar 2003.

D. Eberhart-Phillips, P. J. Haeussler, J. T. Freymueller, A. D. Frankel, C. M. Rubin, P. Craw, N. A. Ratchkovski, G. Anderson, G. A. Carver, A. J. Crone, T. E. Dawson, H. Fletcher, R. Hansen, E. L. Harp, R. A. Harris, D. P. Hill, S. Hreinsdottir, R. W. Jibson, L. M. Jones, R. Kayen, D. K. Keefer, C. F. Larsen, S. C. Moran, S. F. Personius, G. Plafker, B. Sherrod, K. Sieh, N. Sitar, and W. K. Wallace. The 2002 Denali fault earthquake, Alaska: A large magnitude, slip-partitioned event. Science, 300:1113-1118, May 2003.

W. L. Ellsworth and L. Chiaraluce. Supershear during nucleation of the 2009 M 6.3 L'Aquila, Italy Earthquake. Eos Trans. AGU, 90(52), Fall Meet. Suppl.:Abstract U13B-0068, 2009.

W. L. Ellsworth, M. Celebi, J. R. Evans, E. G. Jensen, R. Kayen, D. J. Metz, M. C. Nyman, J.W. Roddick, Spudich P., and C. D. Stephens. Near-field ground motion of the 2002 Denali fault, Alaska, earthquake recorded at pump station 10. Earthquake Spectra, 20(3):597—615, 2004.

L. B. Freund. Mechanics of dynamic shear crack-propagation. J. Geophys. Res, 84(B5):2199-2209, 1979.

L. B. Freund. Dynamic Fracture Mechanics. Cambridge University Press, Cambridge, 1990.

H. Gao, Y. Huang, P. Gumbsch, and A. J. Rosakis. On radiation-free transonic motion of cracks and dislocations. J. Mech. Phys. Solids, 47:1941-1961, 1999.

K. F. Graff. Wave Motion in Elastic Solids. Dover Publications, Inc.,, 1991.

W. A. Grifith, A. J. Rosakis, D.D. Pollard, and C. W. Ko. Dynamic rupture experiments elucidate tensile crack development during propagating earthquake ruptures. Geology, 37:795-798, 2009.

P. J. Haeussler, Schwartz. D. P., T.E. Dawson, H.D. Stenner, J. J. Lienkaemper, F. Cinti, P. Montone, B. Sherrod, and P. Craw. Surface rupture of the 2002 denali fault, alaska, earthquake and comparison surface rupture of the 2002 denali fault, alaska, earthquake and comparison with other strike-slip ruptures. Earthquake Spectra, 20(3):565—578, August 2004. 
R. A. Hansen and N. A. Ratchkovski. Seismological aspects of the 2002 Denali fault, Alaska, earthquake. Earthquake Spectra, 20(3):555-563, 2004.

D. G. Honegger, Nyman D. J., E. R. Johnson, L. S. Cluff, and S. P. Sorenson. Trans-Alaska pipeline system performance in the 2002 Denali fault, Alaska, earthquake. Earthquake Spectra, 20(3): $707-738,2004$.

Y. Ida. Cohesive force across tip of a longitudinal-shear crack and Griffiths specific surface-energy. J. Geophys. Res, 77:3796-3805, 1972.

M. Kavaturu, A. Shukla, and A. J. Rosakis. Intersonic crack propagation along interfaces: Experimental observations and analysis. Exp. Mech., 38:218-225, Sep 1998.

A. S. Kobayashi, editor. Handbook of Experimantal Mechanics. Wiley, 1993.

B. V. Kostrov. Unsteady propagation of longitudinal shear cracks. J. Appl. Math. Mech, 30:1241$1248,1966$.

B. V. Kostrov and L. V. Nikitin. Some general problems of mechanics of brittle fracture. Arch. Mech., 22(6):749-776, 1970.

B. V. Kostrov, L. V. Nikitin, and L. M. Flitman. The mechanics of brittle fracture. Izv. Akad. Nauk SSSR, Mekh. Tverd. Tela, 3:112-125, 1969.

J. Lambros and A. J. Rosakis. Shear dominated transonic interfacial crack-growth in a bimaterial. 1. Experimental-observations. J. Mech. Phys. Solids, 43:169-188, Feb 1995.

C. Liu, Y. Huang, and A. J. Rosakis. Shear dominated transonic interfacial crack-growth in a bimaterial. 2. Asymptotic fields and favorable velocity regimes. J. Mech. Phys. Solids, 43:189206, Feb 1995 .

Y. Liu and N. Lapusta. Transition of mode ii cracks from sub-rayleigh to intersonic speeds in the presence of favorable heterogeneity. J. Mech. Phys. Solids, 56:25-50, 2008. 
X. Lu. Combined Experimental and Numerical Study of Spontaneous Dynamic Rupture on Frictional Interfaces. PhD thesis, California Institute of Technology, 2009.

X. Lu, N. Lapusta, and A. J. Rosakis. Pulse-like and crack-like ruptures in experiments mimicking crustal earthquakes. P. Natl. Acad. Sci. USA, 104(48):18931-18936, 2007. doi: 10.1073/pnas. 0704268104 .

X. Lu, N. Lapusta, and A. J. Rosakis. Analysis of supershear transition regimes in rupture experiments: the effect of nucleation conditions and friction parameters. Geophys. J. Int., 177(2): 717-732, 2009.

X. Lu, N. Lapusta, and A.J. Rosakis. Pulse-like and crack-like dynamic shear ruptures on frictional interfaces: experimental evidence, numerical modeling, and implications. Int. J. Fract., pages 1-13, 2010. doi: 10.1007/s10704-010-9479-4.

G. Lykotrafitis and A. J. Rosakis. Sliding along frictionally held incoherent interfaces in homogeneous systems subjected to dynamic shear loading: a photoelastic study. Int. J. Fract., 140:213-233, Jul 2006a.

G. Lykotrafitis and A. J. Rosakis. Dynamic sliding of frictionally held bimaterial interfaces subjected to impact shear loading. Proc. R. Soc. A-Math. Phys. Eng. Sci., 462:2997-3026, Oct 2006b.

G. Lykotrafitis, A. J. Rosakis, and G. Ravichandran. Particle velocimetry and photoelasticity applied to the study of dynamic sliding along frictionally-held bimaterial interfaces: Techniques and feasibility. Exp. Mech., 46:205-216, Apr 2006a.

G. Lykotrafitis, A.J. Rosakis, and G. Ravichandran. Self-healing pulse-like shear ruptures in the laboratory. Science, 313(5794):1765-1768, 2006b.

R. Madariaga. High-frequency radiation from crack (stress drop) models of earthquake faulting. Geophys. J. Roy. Astron. Soc, 51:625-651, 1977.

A. Martirosyan, R. Hansen, and N. Ratchkovskia. Strong-motion records of the 2002 denali fault, alaska, earthquake. Earthquake Spectra, 20(3):579—596, 2004. 
M. Mello, H.S. Bhat, A.J. Rosakis, and H. Kanamori. Identifying the unique ground motion signatures of supershear earthquakes: Theory and experiments. Tectonophysics, 493(3-4):297-326, 2010.

N. I. Muskhelishvili. Singular integral equations: Boundary problems of function theory and their application to mathematical physics. P. Nordoff, Groningen, 1953.

Newport. Application Note 26: Variable Attenuator for Lasers. Technical report, Newport Optics Corporation, 2006.

S. B. Nielsen, J. M. Carlson, and K. B. Olsen. Influence of friction and fault geometry on earthquake rupture. J. Geophys. Res., 105:6069-6088, 2000.

M. Ohnaka. A constitutive scaling law and a unified comprehension for frictional a constitutive scaling law and a unified comprehension for frictional slip failure, shear fracture of intact rock, and earthquake rupture. J. Geophys. Res., 108(B2), 2003.

A. C. Palmer and J. R. Rice. Growth of slip surfaces in progressive failure of over-consolidated clay. Proc. R. Soc. London. Ser. A-Math. Phys. Eng. Sci., 332:527-548, 1973.

K. Ramesh. Photoelasticity. In W.N. Sharpe Jr., editor, Springer Handbook of Experimental Solid Mechanics, chapter 25. Springer, 2008.

J. R. Rice, C. G. Sammis, and R. Parsons. Off-fault secondary failure induced by a dynamic slip pulse. Bull. Seismol. Soc. Amer., 95(1):109-134, 2005.

D. P. Robinson, C. Brough, and S. Das. The $M_{w}$ 7.8, 2001 Kunlunshan earthquake: Extreme rupture speed variability and effect of fault geometry. J. Geophys. Res., 111(B8), Aug 2006.

A. J. Rosakis. Intersonic shear cracks and fault ruptures. Adv. Phys., 51:1189-1257, Jun 2002.

A. J. Rosakis, O. Samudrala, R. P. Singh, and A. Shukla. Intersonic crack propagation in bimaterial systems. J. Mech. Phys. Solids, 46:1789-1813, Oct 1998. 
A. J. Rosakis, O. Samudrala, and D. Coker. Cracks faster than the shear wave speed. Science, 284: 1337-1340, May 1999.

A. J. Rosakis, H. Kanamori, and K. W. Xia. Laboratory earthquakes. Int. J. Fract., 138:211-218, Mar 2006.

A. J. Rosakis, K. W. Xia, G. Lykotrafitis, and H. Kanamori. Treatise in Geophysics, volume 4, chapter Dynamic shear rupture in frictional interfaces: speeds, directionality and modes, pages 153-192. Elsevier, 2007.

C. E. Rousseau and A. J. Rosakis. Dynamic path selection along dynamic path selection along branched faults: Experiments involving dynamic path selection along branched faults: Experiments involving sub-rayleigh and supershear ruptures. J. Geophys. Res., 114(B08303, doi:10.1029/2008JB006173), 2009.

O. Samudrala and A. J. Rosakis. Effect of loading and geometry on the subsonic/intersonic transition of a bimaterial interface crack. Eng. Fract. Mech., 70:309-337, Jan 2003.

O. Samudrala, Y. Huang, and A. J. Rosakis. Subsonic and intersonic shear rupture of weak planes with a velocity weakening cohesive zone. J. Geophys. Res., 107(B8), Aug 2002a. doi: 10.1029/ 2001JB000460.

O. Samudrala, Y. Huang, and A. J. Rosakis. Subsonic and intersonic mode II crack propagation with a rate-dependent cohesive zone. J. Mech. Phys. Solids, 50:1231-1268, Jun 2002b.

A. Schubnel, S. Nielsen, S. Vinciguerra, and S. Rao. Photo-acoustic study of subshear and supershear ruptures in the laboratory. Earth and Planetary Science Letters, 308(3-4):424-432, 2011.

S. K. Singh, Pacheco J., F. Courboulex, and D. A. F. Novelo. Source parameters of the Pinotepa Nacional, Mexico, earthquake of 27 march 1996, $(\mathrm{Mw}=5.4)$ estimated from near-field recordings of a single station. Journal of Sesimology, 1(1):39-45, 1997.

S. G. Song, G. C. Beroza, and P. Segall. A unified source model for the 1906 San Francisco earthquake. Seismological Society of America, 98(2):823-831, 2008. 
M. Sutton. Digital Image Correlation for Shape and Deformation Measurements. In W.N. Sharpe Jr., editor, Springer Handbook of Experimental Solid Mechanics, chapter 20. Springer, 2008.

S. P. Timoshenko and S. Woinowsky-Krieger. Theory of plates and shells. McGraw-Hill International Editions, 2nd edition, 1959.

H. Tippur. Dynamic Fracture Mechanics, chapter 4. World Scientific Publishing Co. Pte. Ltd., 2006.

F. T. Wu, K. C. Thomson, and H. Kuenzler. Stick-slip propagation velocity and seismic source mechanism. Bull. Seismol. Soc. Am., 62(6):1621-1628, December 1972.

K. W. Xia. Laboratory investigations of earthquake dynamics. PhD thesis, California Institute of Technology, 2005.

K. W. Xia, A. J. Rosakis, and H. Kanamori. Laboratory earthquakes: The sub-Rayleigh-tosupershear rupture transition. Science, 303:1859-1861, Mar 2004.

K. W. Xia, A. J. Rosakis, and H. Kanamori. Supershear and sub-Rayleigh-to-supershear transition observed in laboratory earthquake experiments. Exp. Tech., 29:63-66, May 2005a.

K. W. Xia, A. J. Rosakis, H. Kanamori, and J. R. Rice. Laboratory earthquakes along inhomogeneous faults: Directionality and supershear. Science, 308:681-684, Apr 2005b.

G. Zheng and J. R. Rice. Conditions under which velocity-weakening friction allows a self-healing versus a cracklike mode of rupture. Bull. Seismol. Soc. Am., 88(6):1466-1483, 1998. 